\title{
1928
}

\section{ПЕРААІК НАСЕНЬНЯ,}

ЯКОЕ ПРАПАНУЕШША ДА АБМЕНУ БАТАНІЧНЫМ САДАМ БЕ ААРУСКАЙ ДЗ ЯРЖАЎНАЙ АКАДЭМ І $::::::::$ СЕАЬСКАЕ ГАСПАДАРКі :::::::::

\section{DELECTUS SEMINUM,}

ANNO MCMXXVII (PARTIM AC MCMXXV ET MCMXXVI) COLLECTORUM, QUAE HORTUS BOTANICUS ACADEMIAE AGRONOMICAE REI PUBLICAE ALBORUTHENICAE PRO MUTUA COMMUTATIONE OFFERT

\section{ПЕРЕЧЕНЬ СЕМЯН,}

ПРЕДААГАЕМЫХ В ОБМЕН БОТАНИЧЕСКИМ САДОМ БЕАОРУССКОЙ ГОСУДАРСТВЕННОЙ АКАДЕМИИ :: :::::: СЕАЬСКОГО ХОЗяЙСТВА ::::::::

3 „Запісак Беларускай Дзяржаўвай Акадәміi С. Г., т. VII Gorky. R. P. Alborutheniae (U. S. S. R.)

- ГОРкі, БССе ДРУКАРНЯ АКАДаМЫ,

1928

FEE 291928 
Горрайліт6ех

Ne 79

3axas Ne 189

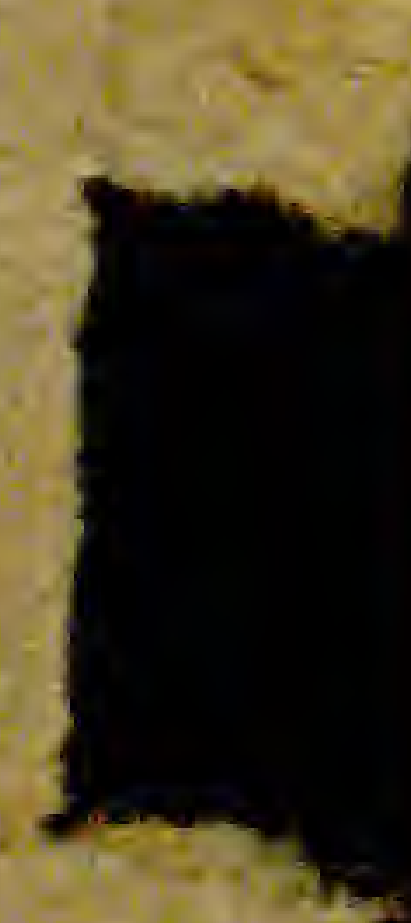




\section{A b b reviationes:}

*-paulum; C-Caucasus; W-Wladiwostok, G-Gorky; c-planta sub divo in Horto Botanico Academiae culta.

Numerus $(25,26,27)$, nomina plantarum sequens, annum, quo semina lecta sunt, significat.

Скарачэньні і ўмоўныя знакі:

Зоркай (*) абазначана насеньне, якое маеџца ў нязначнай колькасьџі. C-насеньне, якое сабрана на Каўказе; W-навакол Владзівастоку; G-навакол Горак; с-з расьлін, якія разводзяџша ў вадкрытым грунце ў Батанічным Садзе Акадәміi.

Лічбы $(25,26,27)$ - якія надрукаваны пасьля назоваў расьлін, азначаюџь гады збору насеньня.

\section{Сокращения и условные обозначения:}

Звездочкой (*) обозначены семена, имеюшиеся в малом количестве. С-семена, собранные на Кавказе; W-в окрестностях Владивостока; G-в окрестностях Горок; с-c растений, культивируемых в Ботаническом Саду Академии в открытом грунту.

Џыфры (25, 26, 27), помещенные за названиями растений, означают год сбора семян. 



\section{Pteridophyta.}

Poly $\rho$ odiacea e.

1. Aspidium Filix mas Sw. \& G. 27 2.* $\quad$ spinulosum $S w .4$ G. 27

3. Asplenium Filix femina Bernh. 4 G. 27

4. Blechnum brasiliense Desv. 4 c. 27
5. Osmunda cinnamomea $L .4$ W. 26 6.* Phegopteris Dryopteris Fèe. ${ }_{4}$ G. 27

7. Pteris aquilina L. 4 G. 27

8. ${ }^{*}$ Scolopendrium vulgare $\mathrm{Sm} .4$ c. 27

9. Struthiopteris germanica Willd. 4 c. 27

\section{Monocotyledone a e.}

Alis mataceae C. Rich. 10. Alisma Plantago L. 4 M.

$$
\text { Aracea e Juss. }
$$

11.* Calla palustris L. 4 G. 27

Commelinaceae Reich.

12. Commelina coelestis. $W$. $\odot$ c. 27

13. $\#$ communis $L$. $\odot$ c. 27

14. " tuberosa L. 4 c. 27

15. Tinantia fugax Scheidw. 4 c. 27

Cyperaceae J. St. Hil.

16.* Carex ampullacea Good. 4 c. 26

17. " hirta L. 4 c. 25

18. ” leporina $L .4$ M.

19.* ” muricata $L .4$ c. 25

20. ” pallescens L. 4 c. 27

21.* ” vesicaria $L .4 \mathrm{M}$.

22. ” vulgaris $F r .4$ c. 27

$23 . \quad$ vulpina $L .4$ c. 26

24. Cyperus esculentus L. 4 c. 27

25. Eriophorum latifolium Hoppe. 4

G. 26

26. Scirpus silvaticus $L .4$ c. 26

Gramineae Juss.

27. Agropyrum caninum P. Beauv. 2 c. 27

28. „ glaucum Roem. et. Schult. 4 c. 27

29. \# repens P. Beauv. 4

30. Agrostis alba L. 4 c. 27 c. 27

31. " canina $L .4$ c. 27

32. " vulgaris With. 4 c. 27

33. Aira capillaris Host. $\odot$ c. 27

$34{ }^{*}$ ” caryophyllea $L . \odot$ c. 27
35. Alopecurus geniculatus $L$. $\odot$ c. 27

36. ” pratensis L. 4 c. 27

37. $\quad$ ventricosus Pers. 4 c. 26

38. Andropogon Ischaemum L. 4

39. Anthoxanthum odoratum c. 27 c. 27

40. Apera spica venti P. Beauv. $\odot$ c. 27

41. Arrhenatherum elatius $M$. et. $K$. 4 c. 27

42.* Atropis convoluta Gris. 4 c. 27

43.* ” distans Gris. 4 c. 27

44. Avena barbata Brot. $\odot$ c. 27

45. ” pubescens L. 4 c. 27

46. ” strigosa Schreb. $\odot$ c. 27

47. Beckmannia eruciformıs Host. 4

G. 26

48. Brachypodium silvaticum $R$. et. $S$. 4 c. 27

49.,$\quad$ ramosum $R$. et. $S$.

50. Briza maxima $L . \odot$ c. 27

51. " media L. 4 c. 27

52. " minor $L . \odot$ c. 27

$53 *^{*} \quad$ " virens $L$. c. 27

54. Bromus arvensis L. $\odot$ c. 27

55. " commutatus Schrad. $\odot, \odot$

56. \# hordeaceus $L . \odot, \odot$ c. 27

57. " inermis Leyss. 4 c. 27

58. ” rubens $L . \odot$ c. 25

59. " secalinus $L . \odot, \odot$ c. 27

60 . ” squarrosus $L . \odot, \odot$ c. 27

61. ” sterilis $L . \odot, \odot$ c. 27

62. ” tectorum L. $\odot$ c. 27

63. Calamagrostis Epigeios Roth.

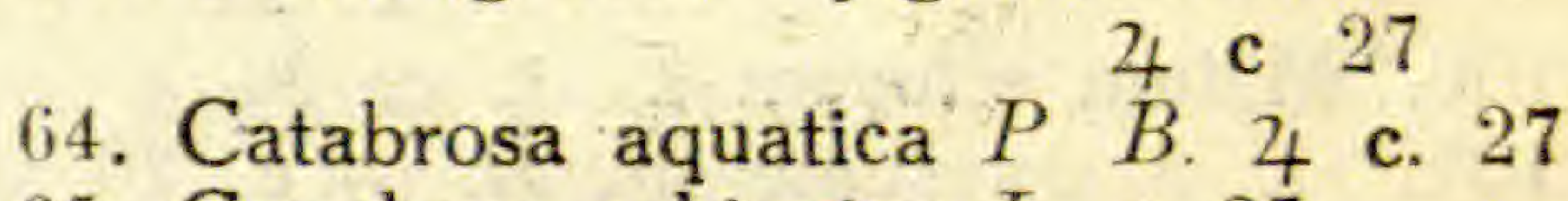

65. Cenchrus echinatus $L$ c. 27

66. $\quad$ montanus Nees. c. 27 
67. Chloris barbata $S w$. c. 27

68. Cinna glomerata L. c. 27

69. \# mexicana Beauv. 4 c. 27

70. Coix lacryma Jobi $L$. $\odot$ c. 27

71. Cynosurus cristatus L. 4 c. 27

72. „ echinatus L. 4 c. 27

73. Dactylis Aschersoniana Graebn. 4 c. 27

74. $\quad$ glomerata L. 4 c. 27

75. Deschampsia caespitosa P. B. 4 c. 27

76. flexuosa Trin. 4 c. 26

77.* Dinebra arabica Jacq. c. 27

78. Eleusine indica Grtn. $\odot$ c. 27

79. Elymus giganteus Vahl. 4 c. 27

80. \# sibiricus L. 4 c. 27

81. Eragrostis minor Host. $\odot$ c. 27

82. $” \quad$ pilosa P. B. $\odot$ c. 27

83. Eriochloa villosa Knth. • c. 27

84. Festuca arundinacea Schr. 4 c. 27

85. " Beckeri Hackel. 4 c. 27

86. " gigantea Vill. 4 c. 27

87. " heterophylla Lam. 4

88. " ovina L. 4 c. 27

89. " pratensis Huds. 4 c. 27

90. " rubra L. 4 c. 27

91.* Gastridium australe P. B. $\odot$ c. 27

92. Glyceria spectabilis $M$. et. K. 4

93. Hierochloë odorata Wahlb. c. 27

94. Holcus lanatus $L .2$ c. 27 c. 27

95. Hordeum jubatum L. 4 c. 27

96. $"$ secalinum Schreb. 4 c. 27

97. Koeleria gracilis Pers. 4 c. 27 98. Lagurus ovatus $L$. $\odot$ c. 27

99. Lasiagrostis splendens Kunth. 4 c. 27

100. Leersia oryzoides $S w .4$ c. 27

101. Lolium remotum Schrank. ๑ c. 27

102. ” temulentum L. $\odot$ c. 27

103. Melica altissima L. 4 c. 27

104. " nutans L. 4 c. 27

105.* " transsilvanica Schur. 4

106.* Milium effusum $L .2$ c. 27

c. 27

107.* " vernale $M$. B. $\odot$ c. 26

108. Miscanthus sacchariflorus Hack.
109. Nardus stricta $L .4$ c. 27

110. Oryzopsis holciformis Richt. 4

c. 27

111. Panicum calyfornicum Benth. $\odot$

112 c. 27

113. " crus galli L. $\odot$ c. 27

114. " frumentaceum Fr. et. Cav. $\odot$ c. 27

115.,$\quad$ germanicum c. 27

116.* " lineare Krock. $\odot$ c. 27

117., miliaceum L. $\odot$ c. 27

118. Paspalum digitaria Poir. 4 c. 26

119. Phalaris arundinacea L. 4 c. 27

120. " ” fol. var., v.

Iutescens 4 c. 27

121. " canariensis L. $\odot$ c. 27

122. Phleum Boehmeri Wib. 4 c. 27

123. ” pratense L. 4 c. 27

124. Poa alpina L. 4 c. 27

125. „ annua $L . \odot$ c. 27

126. " bulbosa L. 4 c. 26

127. ” compressa L. 4 c. 27

128. " glauca Vahl. 4 c. 27

129. " nemoralis L. 4 c. 27

130. ” palustris L. 4 c. 27

131. ” pratensis L. 4 c. 27

132. " trivialis L. 4 c. 27

133.* Polypogon monspeliensis Desf.

134. Scleropoa rigida Griseb. $\odot$ c. 27

135. Setaria glauca $P . B . \odot$ c. 25

136. ” viridis P. B. $\odot$ c. 27

137. Sieglingia decumbens Bernh. 4

G. 27

138. Stipa capillata L. 4 c. 27

139. Tragus racemosus Desf. $\odot$ c. 25

140. Trisetum flavescens $P$. B. 4

c. 27

141. ” rigidum Trin 4 c. 26

142.*,$\quad$ sibiricum Rupr. 4 c. 26

143. Triticum cylindricum Cesati. $\odot$

M. 26

\section{Iridaceae Juss.}

144. Gladiolus imbricatus L. 4 c. 27

145. Iris Fischeriana Hort. 4 c. 27

146. " halophila Pall. 4 c. 27

147. " Pseudacorus L. 4 c. 27

148. - setosa Pall. 4 c. 27

149. "sibirica L. 4 c. 27

150. \# spuria L. 4 c. 27

151. Sisyrinchium anceps Cav. 4 c. 27 


\section{Juncaceae Vent.}

152. Juncus alpinus Vill. 4 c. 27

153. " balticus Willd. 4 c. 27

154.* ${ }^{*}$ bufonius $L$. $\odot$ c. 26

155. " compressus Jacq. 4 c. 27

156. $\quad$ effusus L. 4 c. 27

157. " filiformis L. 4 c. 24

158. " lampocarpus Ehrh. 4 c. 24

159. Luzula nivea $D . C .7$ c. 27

160. ” pilosa Willd. 4 G. 27

Juncaginaceae $\mathrm{R}$ ich.

161. Scheuchzeria palustris L. 4 G. 27

\section{Liliaceae Scop.}

162. Allium albidum Fisch. 4 c. 27

163. " angulosum L. 4 c. 27

164. ” coeruleum Pall. 4 c. 27

165. " fistulosum L. 4 c. 27

166. \# globosum Red. 4 c. 27

167. " obliquum L. 4 c. 27
168. Allium odorum L. 4 c. 27

169. " schoenoprasum L. 4 c. 27

170. " $\quad$ senescens L. 4 c. 27

171.* " ursinum L. 4 c. 26

172. Anthericum Liliago L. 4 c. 27

173. Asparagus officinalis L. 4 c. 27

174. Asphodelus tenuifolius Cav. 4 c. 27

175. Bulbine annua Willd. $\odot$ c. 27

176.* Convallaria majalis L. 4 c. 27

177.* Galtonia candicans Dene. 4 c. 27

178. Majanthemum bifolium D.C. $4 \mathrm{M}$.

179. Muscari racemosum Mill. 4 c. 27

180. Ornithogalum narbonense L. 4

c. 27

181. Paris quadrifolia $L .4$ M.

182. Polygonatum officinale All. 4 c. 27

183.* Puschkinia scilloides Adans. 4 c. 27

184. ${ }^{*}$ Scillia bifolia L. 4 c. 27

185. Zygadenus sibiricus A. Gray 4

c. 27

\section{Dicotyled o ne a e.}

Aceraceae $\mathrm{Neck}$.

186. Acer platanoides L. $\hbar$ G. 27

187. „ tataricum L. $\hbar$ G. 27

A izoacea e.

188. Mesembrianthemum tricolor c. 27

Amaranthaceae Juss.

189. Amaranthus Blitum L. $\odot$ c. 27

190. $\quad$ caudatus $L$. $\odot$ c. 27

191. " $"$ deflexus L. 4 c. 26

192.,$\quad$ retroflexus $L$. $\odot$ c. 27

193. " gangeticus L. $\odot$ c. 26

Ampelidaceae H. B. K.

194. Vitis amurensis Rupr. 万 W. 26

Aristolochiaceae Blume.

195. Asarum europaeum L. 4 c. 27.
Asclepiadaceae Lindl. 196. Asclepias Cornuti Decsn. 4 c. 27 197. Cynanchum Vincetoxicum $R$. Br. 4 c. 26

Balsaminaceae S. F. 198. Impatiens glanduligera Royle. $\odot$ c. 27

199.* " noli tangere L. $\odot$ c. 27

Berberidaceae Torr. et. Gray. 200. Berberis vuIgaris L. $\hbar$ G. 27

Betulaceae Rich.

201. Corylus Avellana L. $\hbar$ G. 27 202. \# Colurna L. $\hbar$ c. 27

Borraginace a e S. F. 203. Anchusa italica Retz. - c) 26 204. officinalis L. 4 c 27 
205. Borrago officinalis $L$. $\odot$ c. 27

206. Cerinthe minor $L$. $\odot$ c. 27

207. Cynoglossum officinale L. 4

c. 27

208. Echium altissimum Jacq. $\odot$ c. 26

209. vulgare $L$. .) c. 26

210. Heliotropium europaeum $L$. $\odot$

211.

c. 27

peruvianum L. 4

212. Lappula Myosotis Moench. $\odot$ c. 26

213. Lindelofia spectabilis Lehm. 4 c. 27

214. Lithospermum officinale $L .4$ c. 27

215. Lycopsis arvensis $L$. $\odot$, $\odot$ c. 27 216. Myosotis arenaria Schrad. $\odot, \odot$

c. 25

217. " intermedia Link. $\odot$, $\odot$,

218. 4 c. 26

c. 27

versicolor Sw. c. 26

221. Omphalodes linifolia Moench. $\odot$ c. 27

222. Pulmonaria officinalis $L .2$ c. 26 223. Symphytum officinale $L .4$ c. 27

Campanulaceae Juss.

224. Campanula carpathica Jacq. 4 c. 27

225. " " cervicaria $L . \odot, 2 \mathrm{M}$.

226. " " Correvoniana Hort. 4

c. 27

227. " glomerata L. 2 c. 26

228. " ", L. v. acaulis 4 c. 27

229. " grandis Fisch. et. Mey.

$230 . \quad 4$ c. 27

c. 27

233. " Medium L. $\odot$ c. 27

234. $\quad \Longrightarrow \quad$ persicifolia L. 4 c. 27

235. " pulcherrima Schrank. et. Zeyh. 4 c. 27

236. , , rapunculoides L. 4

237. c. 27

cordifolia C. Koch. 4 c. v. 27
238.* Campanula Rapunculus $L$. $\odot$ c. 27

239. ” rotundifolia L. 4 c. 27

240. " " L. v. Hostii. 4 c. 27

241. " $\quad$ sibirica $L . \odot$ c. 27

242. " $\quad$ Steveni $M . B .4$ c. 27

243. " trachelium L. 4 M.

244.*,$\quad$ Van Houttei Carr.

245.* Codonopsis ovata Benth. 4 c. 27

246. " silvestris Kom. 4

247.* $\quad$ viridiflora $\stackrel{\text { c. } 27}{\text { Maxim }}$

c. 27

248. Jasione montana $L$. $\odot$ c. 24 249.*Laurentia Michelii $A$. D. C. $\odot$ c. 27

250. Lobelia erinus $L$. $\odot$ c. 27

251. , inflata $L . \odot$ c. 27

252. ", syphilitica L. 4 c. 27

253. Phyteuma spicatum L. 4 M. 25

254. Platycodon grandiflorum $D$. C.

255. Specularia hybrida $D . \stackrel{4}{\text { C. }} \odot 27$

256. " Speculum D. C. .

c. 27

257. Symphyandra Hoffmannii Pant.

258. Wahlenbergia pendula Schrad.

ค c. 27

Cannabaceae Endl.

259. Humulus Lupulus L. 4 c. 27

Caprifoliaceae Vent.

260. Sambucus nigra $L$. $\hbar$ c. 25

261. " racemosa L. 方 G. 27

262. Symphoricarpus racemosa Mchx.

263. Viburnum Lantana $L$ क c. 27

264. " Opulus L. $\hbar$ G. 27

Caryophyllaceae Juss.

265. Agrostemma Githago L. $\odot$ c. 27

266. Alsine caucasica Boiss. 4 c. 25

267. ", pinifolia Fenzl. 4 c. 25

268. Arenaria graminifolia Schrad. 4

269 c. 27

270. " longifolia M. B. 4 c. 27 
271. Cerastium arvense L. 4 c. 27

272. " caespitosum Gilib. 4 c. 26

273. Corrigiola littoralis L. $\odot$ c. 27 274. Cucubalus baccifer L. 4 c. 27 275. Dianthus arenarius L. 4 c. 27 276. " $\quad$ barbatus L. 4 c. 27 277.* " " Borbasii Vandas. 4 c. 27

278.* " campestris M. B. 4 c. 27 279. " capitatus D. C. 4 c. 27

280. - Caryophyllus L. 4 c. 27

281. " chinensis L. $\odot$ c. 27

282. " collinus Waldst. et. Kit. 2 c. 27

283. " deltoides L. 4 c. 27 284. " giganteus D.' Urv. 4 c. 27

285. " Heddewigi Hort. $\odot$ 286. lociniatus c. c. 27 287. " " plumarius L. 4 c. 27 288. Elisanthe noctiflora Rupr. $\odot$ c. 27

289. Gypsophila elegans M. B. ๑ c. 27 290. , muralis L. $\odot$ c. 26 291.* Herniaria glabra L. 4 c. 26 292. Holosteum umbellatum L. $\odot$, $\odot$ c. 27

293. Lychnis chalcedonica L. 4 c. 27 294. " Flos cuculi L. 4 c. 27 295. Melandryum album Garcke. $\odot$, $\odot$ c. 27

296. Sagina nodosa Fenzl. 4 c. 25 297. " procumbens L. 4 c. 27 298. Saponaria officinalis L. 4 c. 26 299. Scleranthus annuus $L$. $\odot$ c. 27 300 . perennis L. 4 c. 26

301. Silene Armeria L. $\odot$ c. 27

302. " Brotherana Som. Lev. c. 27

303. ", bupleuroides L. 4 c. 26

304. " " commutata Guss. c. 26

305. , , compacta Fisch. $\odot$ c. 26

306. " conica L. $\odot$ c. 27

307 " conoidea L. $\odot$ c. 27

308. " " L. f. cleistogama Troitzky. $\odot$ c. 27

309. " chlorantha Ehrh. 4 c. 26

310. " gallica L. $\odot$ c. 27

311. ", italica Pers. 4 c. 26

312.* " multiflora Pers. 4 c. 25

313. " Otites $S m . \odot, 4$ c. 26

314. " saxatilis Sims. c. 27
315.* Silene sibirica Pers. 4 c. 26 316. ", tatarica Pers. 4 c. 27

317. " venosa Aschers. 4 c. 27

318. " wolgensis Pers. 4 c. 26

319. Spergula arvensis L. ๑ c. 27

320. Spergularia campestris Aschers.

$321 . \odot$, $\odot$ c. 26

322. Stellaria graminea L. 4 c. 27

323.* , Holostea L. 4 c. 25

324. Tunica prolifera Scop. ¿ c. 27

325. " Saxifraga Scop. 4 c. 27

326. Vaccaria pyramidata Medik. $\odot$

c. 27

327. Viscaria viscosa Aschers. 4 c. 27

Celastraceae Lindl.

328. Evonymus europaeus $L$. $\hbar$ c. 27 329. ", verrucosus Scop. $\frac{\hbar}{2}$

G. 27

\section{Chenopodiaceae Less.}

330. Atriplex hastatum L. ๑ c. 27 331. " hortense L. ๑ c. 27

332. Beta patellaris Moq. ๑) c. 27 333. Chenopodium album L. ๑ c. 27 334. 335 .

336. 337. $\quad " \quad$ polyspermum $L$. " Botrys L. $\odot$ c. 27 " foliosum Schrad. ○. c. 27

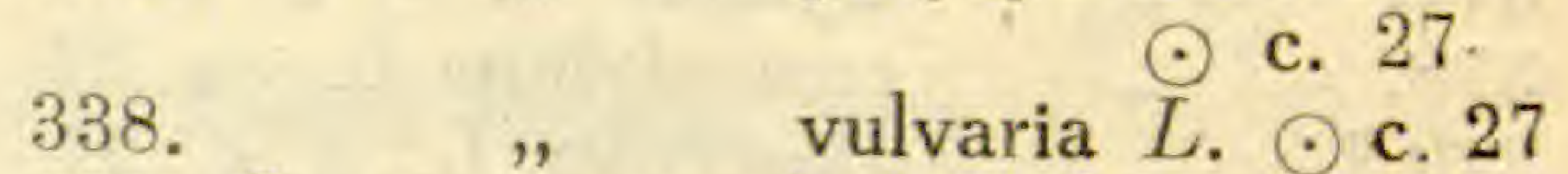
339. Corispermum hyssopifolium L. $\odot$ c. 26 340. Herniaria incana Lam. 4 c. 27 341. Kochia arenaria Roth. ๑ c. 27 342. " trichophilla $\odot$ c. 27 343. Monolepis trifida Schrad. $\odot$ c. 26 344. Spinacia oleracea L. ๑ c. 27 345. " tetrandra Stev. $\odot$ c. 27

$$
\text { Cistaceae Dun. }
$$

346. Helianthemum Chamaecistus Mill. 4 c. 27

\section{Compositae Adans.}

347. Achillea Millefolium L. 4 c. 27 348. " nobilis L. 4 c. 27 
349. Achillea Ptarmica L. 4 c. 27

350. Acroclinum roseum $\odot$ c. 27

351. Ageratum mexicanum Sims. $\odot$

c. 27

352. Anacyclus officinarum Hayne

c. 27

353. Anthemis altissima $L$. $\odot$ c. 27

354. " arvensis $L$. $\odot$ c. 27

355. " $\quad$ ruthenica $M . B . \odot$ c. 27

356. Arctotis grandis Thunb. $\odot$ c. 27

357. Arnica montana L. 4 c. 27

358. Artemisia Absinthium L. 4 c. 27

359. " campestris L. 4 c. 27

360. ", vulgaris L. 4 c. 27

361. Aster alpinus L. 4 c. 27

362. " salicifolius Scholl. 4 c. 27

363. Baeria coronaria A. Gray. ๑ c. 26

364. Bellis perennis L. 4 c. 27

365. Bidens cernuus $L$. $\odot \mathrm{M}$

366. ", leucantha Willd. ๑ c. 27

367. ", tripartitus L. $\odot$ c. 27

368. Buphtalmum speciosum Schreb.

4 c. 27

369. Calendula officinalis $L$. $\odot$ c. 27

370. Callistephus chinensis Nees. $\odot$ c. 27

371. Carduus crispus L. 4 c. 27

372. Carlina vulgaris $L$. $\odot$ c. 27

373. Carpesium cernuum L. 4 c. 27

374. Carthamus tinctorius $L$. $\odot$ c. 26

375. Centaurea axillaris Willd. 4 c. 27

376. " $"$ bella Trtv. c. 27

377. " Cyanus L. ¿ c 27

378.,$\quad$ crocodylium $L . \odot$ c. 27

379.* " glastifolia L. 4 c. 27

380. " Jacea L. 4 c 27

381.* " Koenigii Sosn. c. 27

382. " maculosa Lam. *. c. 27

383. " $\quad$ reflexa Lam. 4 c. 27

384. " Scabiosa L. 4 c. 27

385 * " $\quad$ salmantica L. $\odot$ c. 27

386. $\quad$ solstitialis $L . \odot$ c. 24

$387 *$ Chondrilla juncea $L .2$ c. 27

388. Chrysanthemum bipinnatum L. 4

389 . c. 27

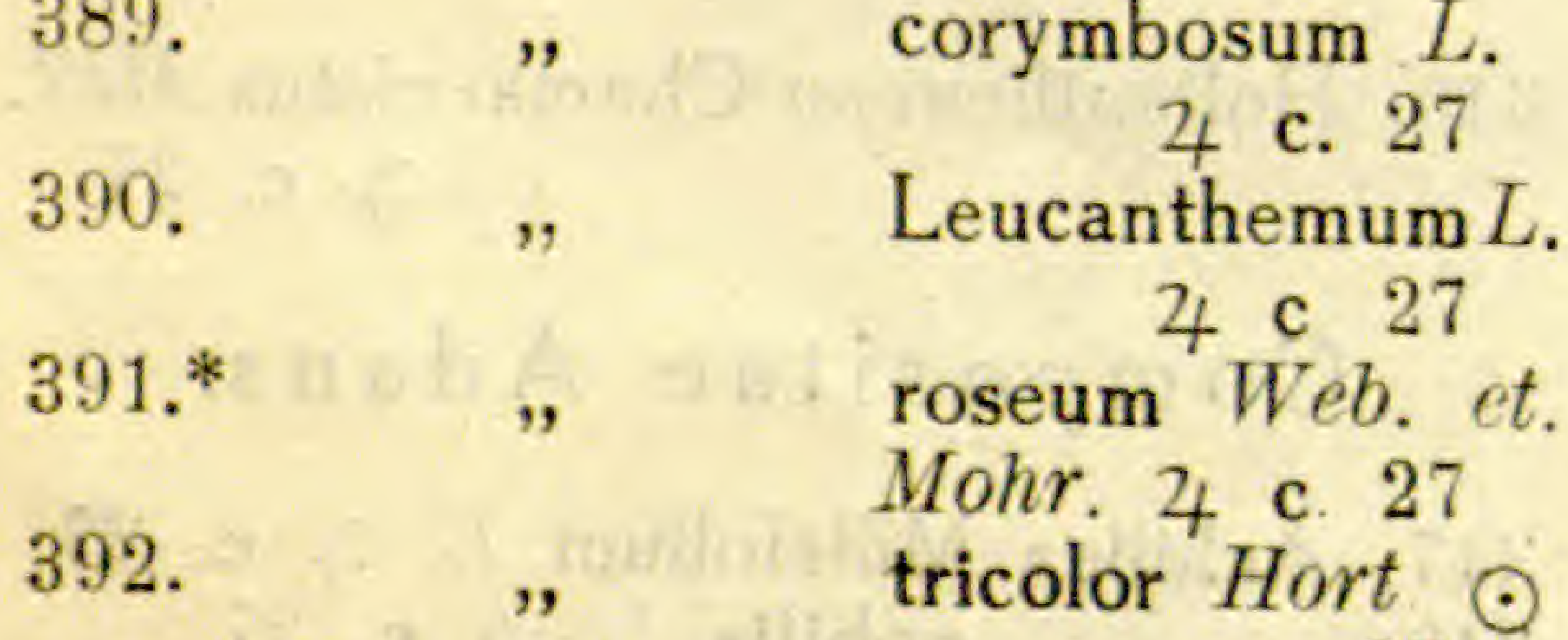

c. 27
393. Chrysanthemum viscosum Desf.

$\odot$ c. 27

394. Cichorium Intybus $L .2$ c. 27

395. Cirsium Acarna Moench. $\odot$ c. 25

396. ",$\quad$ arvense Scop. 4 M.

$397 .^{*} \quad$, palustre Scop. $\odot$ c. 25

398. ", rivulare Link. 4 c. 24

399. Cnicus Benedictus $L$. $\odot$ c 27

400. Coreopsis coronata Hooker. c. 27

401. „, peltata L. ○ c. 27

402. Cosmea bipinnata Willd. $\odot$ c. 27

403. Crepis biennis $L$. $\odot$ c. 27

404. „ , foetida L. $\odot$ c. 27

405. " paludosa Moench. 4 c. 27

406. " parviflora Desf. $\odot$ c. 27

407. „, pulchra L. $\odot$ c. 27

408. " sibirica L. 4 c. 27

409. " tectorum $L$. $\odot$ c. 27

410. Crupina vulgaris Cass ๑ c. 27

411. Dahlia coccinea Cav. 4 c. 27

412. " variabilis Desf. 4 c. 27

413. Dimorphotheca pluvialis Moench.

$\odot$ c. 27

414. Echinops globifer Janka. 4 c. 27

415. Erigeron acer L. $\odot, 4$ c. 27

416.* , alpinus L. 4 c. 27

417.* ", canadensis $L . \odot$ c. 25

418. Eupatorium cannabinum L. 4

c. 27

419. Felicia tenella Ness. $\odot$ c. 27

420. Filago spathulata Presl. $\odot$ c. 27

421. Gaillardia picta Sweet. $\odot$ c. 27

422. Galinsoga parviflora Cav. ๑) c. 27

423. Gnaphalium silvaticum L. 4 c. 24

424. ", vira-vira Molina $\odot$

c. 27

425. Guizotia oleifera D. C. $\odot$ c. 26

426. Hedypnois cretica Willd. $\odot$ c. 25

427. Helenium Hoopesii A Gray. 4

428. Helianthus cucumerifolius Torr.

et.Gray. $\odot$ c. 27

429. " globosus $\odot$ c. 27

430. Helichrysum arenarium Moench.

431. Helipterum roseum $\stackrel{4}{4}$ c. 27

c. 24

432. Hieracium Pilosella L. 4 c. 27

433. ,. pratense Tausch. 4

434. c. 27

435. " silvaticum L. 4 M.

436. " $\quad$ umbellatum L. 4 c. 27 
437. ${ }^{*}$ Hyoseris lucida $L . \odot$ c. 27

438. Hypochoeris radicata $L .4$ c. 27 439. Inula conyza $D . C . \odot, 4$ c. 27

440. „ Helenium L. 4 c. 27

441. „ magnifica Lipsky. c. 27

442. " oculus christi L. 4 c. 27

443. Jurinea cyanoides Rchb. 4 c. 27

444. Kalbfussia Salzmannii Schulz, 4 c. 26

445. Lactuca sativa $L$. $\odot$ c. 26

446. „ viminea Presl. $\odot$ c. 26 447. Lampsana communis L. $\odot$ c. 27 448. " grandiflora M. B. 4 c. 27

449. Lappa amplissima Boiss. -) c. 25 450. " tomentosa Lam. $\odot$ G. 26 451. Lasiospermum pedunculare Lag. ค c. 27

452. Layia Douglasii Hook, et. Arn.

453. Leontodon autumnalis $\stackrel{\odot}{\text { c. } 27}$ c. 27 454. Liatris spicata Willd. 4 c. 25 455. Madia sativa Mol. $\odot$ c. 27

456. Matricaria Chamomilla L. ๑) c. 27 457. " inodora $L . \odot$, $\odot$ c. 27 458. , , suaveolens Buchen. $\odot$ c. 27

459. Notobasis syriaca Coss. (-) c. 26 460. Onopordon Acanthium L. .) c. 27 461.* Petasites officinalis Moench. 4 G. 26

462. Picris hieracioides $L . *, 4$ M. 24 463. Psephellus dealbatus Boiss. c. 27 464. hypoleucus Boiss. c. 27 465. Pulicaria dysentherica Gaertn. 4 c. 24

466. Pyrethrum Parthenium Sm. 4 c. 27

467. „ roseum M. B. 4 c. 27 468. Rhagadiolus hedypnois $F$. et. $M$. (.) 27

469. " stellatus D.C. $\odot$ c. 24 470. Rudbeckia hirta $L . \odot$, $\odot$ c. 27 471. " laciniata L. 4 c. 27 472. Scorzonera eriosperma M. B. 4 c. 27

473. $\quad$ hispanica L. 4 c. 27

474. laciniata $L . \odot, 2$ c. 26

475. Senecio erraticus Bertol. -) c. 27 476. " Jacobaea $L .4$ c. 27 477. " ligularia Hook. 4 c. 27 478.* " vulgaris $L . \odot, \odot$ c. 25 479. Serratula coronata $L .2$ c. 27
480. Serratula heterophylla Desf. 4 c. 27 $4810^{*} \quad, \quad$ radiata $M . B .4$ c. 26 482. Siegesbeckia orientalis $L$. $\odot$ c. 26 483. Silybum Marianum Gaertn. $\odot, \odot$ c. 27

484. Solidago virga-aurea L. 4 c. 27 485. Sonchus arvensis L. 4 c. 27 486. , asper Hill. $\odot$ c. 27 487. \# oleraceus L. ๑ c. 27 488. Tagetes erecta $L$. $\odot$ c. 27 489. " patula $L . \odot$ c. 27 490. Tanacetum pseudachillea $C$. Winkl. 4 c. 27 491. vulgare L. 4 c. 27
492. Taraxacum bessarabicum Hand493. Mzt. 4 c. 27 c. 27 494. " , officinale Wigg. 4 G. 27

495. Tragopogon brevirostris $D . C . \odot$ 496. $"$ coloratus C. $A . M$
c. 27

497. " major Jacq. .) c. 27 498. „ porrifolius L. c. 27 499. " pratensis L. .. c. 27 500. Urospermum picroides F. W. Schmidt. ๑) c. 27

501. Xanthium spinosum L. (.) c., 27

502. Ximenesia encelioides Cav.

$$
\text { c. } 27
$$

503. Zinnia elegans Jacq. (.) c. 27

Convolvulaceae Vent. 504. Convolvulus Scammonia L. 4 c. 27

505. , , tricolor L. $\odot$ c. 27

506. " " undulatus Cav. $\odot$ c. 27

507. Cuscuta europaea L. ๑ G. 24

508. Ipomoea purpurea Lam. ( c. 27 509. Pharbitis hederacea Chois. $\odot$

$$
\text { c. } 27
$$

\section{Crassulaceae D. C.}

510. Sedum acre L. 4 c. 27

511. " glaucum W. et. K. 4 c. 27

512. , kamtschaticum $\%$. et. $M$. 4 c. 27 
513. Sedum purpureum Link. 4 c. 27

514. " spurium M. B. 4 c. 27

\section{Cruciferae Juss.}

515. Aethionema Buxbaumii $D . C$. $\odot$ c. 27

516. Alyssum desertorum Steph. $\odot$ c. 27

517. „ linifolium Steph. $\odot$ c. 26 518. \# repens Baumg. 4 c. 27 519. Arabis albida Stev. 4 c. 27

520. " hirsuta Scop. $\odot, 4$ c. 27 521. " laxa Sibth. et. Sm. c. 27 522. Barbarea stricta Andrz. $\odot$ M. 24 523. " vulgaris $R$. Br. $\odot$ c. 26 524. Berteroa incana D.C. $\odot, \odot$ c. 27 525. Biscutella cicchoriifolia Loisl. $\odot$ c. 27

526. Brassica cernua Matsum. $\odot$ c. 27 527.* " juncea Czern. $\odot$ c. 26 528. " " quadrivalvis Hoorf et. Thom. $\odot$ c. 26

529. „ nigra Koch. $\odot$ c. 26

530. Bunias orientalis L. 4 G. 27

531. Camelina sativa Crantz. $\odot$ c. 27 532. Capsella bursa pastoris Moench. $\odot$ G. 27

533. Carrichera Vella $D . C . \odot$ c. 27

534. Cheiranthus nanus Merk. $\odot$ c. 27

535. Chorispora tenella D. C. $\odot$ c. 27

536. Cochlearia officinalis $L .4$ c. 27

537. Conringia orientalis Dum. $\odot$ c. 27

538. Descurainia Sophia Webb. et. Berth. $\odot$ c. 27

539. Diplotaxis muralis $D$. C. $\odot$ c. 27 540. Erysimum cheiranthoides $L$. $\odot$

G. 27

541. " cuspidatum D. C. $\odot$

542. " pulchellum J. Gay. 4

543. $\quad \quad \quad$ suffruticosum c. 27

544.* Eruca sativa Lam. $\odot$ c. 26

545. Erucastrum elongatum L. 4 c. 26

546. Farsetia clypeata $R$. Br. 4 c. 26

547. $\quad$ eriocarpa D. C. 4 c. 27

548. Hesperis fragrans Fisch. 4 c. 27

549. \# lutea Maxim. $\odot$ c. 27

550. matronalis $L .4$ c. 27

551. Iberis pectinata Boiss. $\odot$ c. 27

552. " sempervirens $L .4$ c. 27
553. Iberis umbellata $L$. $\odot$ c. 27

554. Isatis tinctoria $L . \odot$ c. 27

555. Lepidium Draba L. 4 c. 27

556. $" \quad$ ruderale L. $\odot$ M. 24

557. " sativum L. $\odot$ c. 27

558. Lunaria biennis Moench. $\odot$ c. 27

559.* " redeviva $L .4$ c. 27

560. Malcolmia africana Ait. $\odot$ c. 26

561. Matthiola annua Sweet. $\odot$ c. 27

562. " bicornis $\odot$ c. 27

563. Melanosinapis communis Spenn.

○) c. 27

564. Neslia paniculata Desv. $\odot$ c. 27

565. Raphanus Raphanistrum L. $\odot$

G. 26

566. Rapistrum rugosum $A l l . \odot$ c. 27

567. Senebieria Coronopus Porr. $\odot$

568. Sinapis alba $L$. $\odot$ c. 27

569. " apula Tenore. $\odot$ c. 27

570. Sisymbrium Irio L. 4 c. 27

571. $\quad$ officinale Scop.

c. 27

572. ${ }^{*}$ Stenophragma Thalianum Celak.

๑ G. 25

573. Thlaspi arvense $L . \odot$ G. 27

574. Turritis glabra $L . \odot$ c. 27

\section{Cucurbitaceae Holl.}

575. Bryonia alba $L .4$ c. 27

576.* Citrullus Colocynthis Schr. $\odot$

$$
\text { c. } 26
$$

577. Cucumis Chito Naud. $\odot$ c. 27

578. \# prophetarum L. $\odot$ c. 26

579. Cucurbita maxima Duch. $\odot$ c. 27

580. " melanosperma $A$. $B r$.

581. " Melopepo L. $\odot$ c. 27

582. " " napolitana Reg. $\odot$ c. 27

583. " $\quad$ Pepo L. $\odot$ c. 27

584. " verrucosa $L . \odot$ c. 27

585. Cyclanthera pedata Schrad. c. 27 586.* Ecballium elaterium Rich. $\odot$ c. 25 587. Lagenaria vulgaris Ser. $\odot$ c. 27

588. Melothria cucumerina Naud. c. 27 589 . " punctata Cogn. c. 27

590. Sicyos angulata $L$. $\odot$ c. 27

Dat iscace a e.

591. Datisca eannabina L. 4 c. 27 


\section{Dipsacaceae D. C.}

592.* Cephalaria media Litw. c. 27 593. syriaca Schrad. 4

c. 25

594. Dipsacus laciniatus $L$. $\odot$ c. 26 595. Knautia arvensis Coult. 4 c. 26 596. Pterocephalus plumosus Coult. $\odot$ c. 26

597. Scabiosa atropurpurea $L$. $\odot$ c. 27 598. ” caucasica $M . B .4$ c. 27 599. " Columbaria L. 4 c. 27 $600 . \quad$ ochroleuca L. 4 c. 27 601. Succisa praemorsa Aschers. $4 \mathrm{M}$.

Empetraceae Lindl. 602. Empetrum nigrum L. $\hbar$ G. 27

\section{Ericaceae Lindl.}

603.* Calluna vulgaris Salisb. 5 G. 27

Euphorbiaceae J. St. Hil.

604. Euphorbia Esula L. 4 G. 27

$605 . \quad \cdots \quad$ graeca Boiss. et Sprun. 606. „ helioscopia $\odot$ L. $\odot$ c. 27 607.* ", humifusa Willd. $\odot$ c. 27 608. $\quad$, uralensis Fisch. 4 c. 27 609. Mercurialis annua $L$. $\odot$ c. 26 610. Ricinus communis $L$. $\odot$ c. 27

\section{Gentianaceae Dumort.}

611. Gentiana cruciata L. 4 c. 27 612. " Pneumonanthe L. 4 c. 27

613. Menyanthes trifoliata L. 4 G. 26

Geraniaceae J. St. Hil. 614. Erodium cicutarium L. Herit. $\odot$ c. 27

615. \# gruinum Ait. $\odot$ c. 27 616. Geranium dissectum $L$. $\odot$ c. 27 617. " divaricatum Ehrh. 4 c. 27

618. " ibericum Cav. 4 c. 24 619. " molle $L$. $\odot$ c. 27
620. Geranium palustre L. 4 c. 27 621. " pratense L. 4 c. 27 622. " $\quad$ pusillum $L . \odot$ c. 27 623. " Robertianum L. $\odot$ $624 .^{*} \quad " \quad$ sanguineum $L$ L. 4 c. 27
Globulariaceae D. C. 625. Globularia trichosantha $F$. et. $M$. 4 c. 27

\section{Guttiferae Endl.}

626. Hypericum Ascyron L. 4 c. 27 627. " montanum L. 4 c. 27 628. " $\quad$ perforatum L. 4 c. 27 629. " $\quad$ quadrangulum L. 4 G. 27

Hydrophyllaceae.

630. Nemophila maculata Benth. $\odot$ c. 27

631. Phacelia bipinnatifida Micha 4 632. divaricata A Gray. 633. \# tanacetifolia Bentham. • c. 27

\section{L a b i a t a e Juss.}

634. Ajuga reptans L. 4 c. 27 635 . " " L. var. albiflora Tin. 4 c. 27

636. Ballota nigra L. 4 c. 27 637. Betonica officinalis $L .4$ c. 27 638. Brunella vulgaris $L .4$ c. 27 639.* Coleus lanuginosus Hochst. et. Benth. c. 27 640. Dracocephalum moldavica $L$. $\odot$ 641. c. 27 Ruyschiana $L . \odot$ c. 27

642. Elsholtzia cristata Willd. $\odot$ c. 27 643. Eremostachys laciniata Bge. 4 c. 27

644. Galeopsis speciosa Mill. $\odot$ c. 27 645 . , Tetrahit L. $\odot$ c. 27 646. Glechoma hederacea L. 4 c. 25 647.* Horminium pyrenaicum L. 4 c. 27 
648. Hyssopus officinalis $L . \hbar$ c. 27 649. Lallemantia iberica Fish. et. Mey.

$$
\odot \text { c. } 27
$$

650. " peltata F. et. M. $\odot$

651. Lamium album L. 4 c. 27

652. " amplexicaule $L . \odot$, $\odot$

653.* , Galeobdolon Crantz. 4

654. " maculatum L. 4 c. 27

655. " purpureum L. $\odot, \odot$ G. 27

656. Leonurus Cardiaca L. 4 c. 27

657. " Marrubiastrum L. $\odot, \odot$ c. 26

658. Lycopus europaeus $L .4$ c. 27 659. ” exaltatus L. 4 c. 27 660. Marrubium vuIgare L. 4 c. 27 661. Melissa officinalis L. 4 c. 27 662. Mentha arvensis L. 4 c. 27 663.* " longifolia Huds. 4 c. 27 664. " piperita L. 4 c. 27 665. $\quad$ Pulegium L. $\odot$ c. 27 666. Monarda fistulosa L. 4 c. 27 667. Nepeta Cataria L. 4 c. 27 668. " grandiflora $M . B .4$ c. 27 669. „, macrantha Fisch. 4 c. 27 670. " nuda L. 4 c. 27

671. Ocimum Basilicum L. $\odot$ с 27 672. Origanum vulgare $L .4$ c. 27 673. Phlomis pungens Willd. 4 c. 27 674. " tuberosa L. 4 c. 27 675. Pycnanthemum lanceolatum Pursh.

676. Salvia Aethiopis L. 4 c. 27

677. ', azurea Lam. 4 c. 27

678. " dumetorum Andrz c. 27

679. „ glutinosa L. 4 c. 27

680.,$\quad$ nemorosa L. 4 c. 27

681. " nilotica Murr. $\odot$ c. 27

682. " nutans L. 4 c. 27

683. " officinalis $L . \hbar$ c. 26

684. " scabra Thunb. 万 c. 27

685. \#. Sibthorpii Sm. 4 c. 27

686. " " triangularis Thunb. 万

687. „ Verbenaca L. 4 c. 27 688. " verticillata L. 4 c. 27 689. Satureja Clinopodium Caruel. 4 c. 27 690. egatum "Mort. 4 c. 27
691. Satureja hortensis L. $\odot$ c. 27 692. \# serpyllifolia Briq. $\hbar$ c. 27 693. Scutellaria altissima L. 4 c. 25 694. „, galericulata L. 4 c. 27 $695 . \quad$, orientalis $L .4$ c. 27 696. Sideritis montana $L$. $\odot$ c. 26 697. . Stachys annua L. $\odot$ c. 27 698.* ", cretica Sibth. et. Sm. 4

699. , lanata Jacq. 4 c. 27

700. , palustris L. 4 c. 27

701. ", recta L. 4 c. 27

702. ",$\quad$ sideritoides C. Koch.

703. , silvatica $L .2$ c. 27

704. Teucrium Chamaedrys L. 4 c. 27

705. " hyrcanicum L. 4 c. 27 706 * \# orientale $L .4$ c. 27 707. Thymus glaber Mill. 4 c. 27

708. Zizyphora persica Boiss. ๑ c. 27

\section{Leguminosae Juss.}

709. Amorpha fruticosa $L . \hbar$ c. 27

710. Astragalus glycyphyllus $L .4$ c. 27

711.* " Onobrychis $L .4$ c. 27

712. ", sulcatus L. 4 c. 27

713. ,, vulpinus Willd. 4 c. 25

714.* Caragana frutescens $D . C . \overline{2}$ c, 27

715. Cicer arietinum L. $\odot$ c. 26

716. Cytisus scoparius Link. 万 M. 25

717. Colutea orientalis Mill. $\hbar$ c. 27

718. Coronilla emeroides Boiss $\hbar$ c. 27

719 . " varia $L .4$ c. 27

720. Dolichos Lablab L. $\odot$ c. 27

721. Dorycnium herbaceum Vill. $\hbar$ c. 27

722. Ervum Lens L. $\odot$ c. 27

723. Galega orientalis Lam. 4 c. 27

724. Genista tinctoria $L$. $\hbar$ c. 27

725. Glycyrrhiza echinata L. 4 c. 27

726. Hedysarum obscurum L. 4 c. 25

727. Lathyrus Aphaca L. (- c. 27

728. " canescens Taubert. 4 c. 27

729. " montanus Bernh. 4 c. 27

730. " ochrus D. C. $\odot$ c. 27

731. " odoratus L. $\odot$ c. 27

732. " $\quad$ palustris L. 4 c. 27

733. " pratensis L. 4 c. 27

$734 . \quad$, sativus $L . \odot$ c. 27 . 
735. Lathyrus silvestris $L .4$ c. 27

736. " sphaericus Retz. $\odot$ c. 27

737. " vernus Bernh. 4 c. 27

738. Lotus corniculatus L. 4 c. 27

739. „ tetragonolobus $L$. $\odot$ c. 27

740. Lupinus leporinus c. 27

741. ” mutabilis $S w$. c. 27

742. $\quad$ perennis L. 4 c. 27

743. ” pilosus Murr. $\odot$ c. 27

744. $\quad$ sp. fl. albo c. 27

745. Medicago lupulina $L . \odot, 4$ c. 27

746. " sativa L. 4 c. 27

747. Melilotus albus Desr. ^. c. 27

748. ” officinalis Desr. .) c. 26

749. $\quad$ wolgicus Poir. .) c. 27

750.* Mimosa pudica $L . \odot 27$

751. Onobrychis viciaefolia Scop. 4

c. 25

752. Ononis hircina Jacq. 4 c. 27

753. Ornithopus compressus $L$. $\odot$ c. 27

754. Pisum arvense L. $\odot$ c. 24

755. „ elatius $M$. B. c. 27

756. ” Jomardi Schrank. $\odot$ c. 27

757. sativum $L . \odot$ c. 24

758. Robinia pseudacacia $L$. 古 c. 27

759. Scorpiurus subvillosa $L$. $\odot$ c. 27

760. vermiculata $L . \odot$ c. 27

761. Trifolium agrarium $L$. $\odot$ c. 2 ,

762. „ angustifolium $L . \odot$ с 27

763.,$\quad$ arvense $L . \odot$ c. 27

764. ” fragiferum L. 4 c. 27

765.* " ", hybridum L. 4 c. 27

766. " " Lupinaster $L \quad 4$ c. 27

767. $\quad$ medium L. 4 c. 27

768. " " montanum L. 4 c. 27

769. " " pratense L. 4 c. 27

$770 . \quad " \quad$ procumbens $L . \odot$ c. 27

771. ” repens $L .4$ c. 27

772. ", resupinatum $L . \odot, \odot$

c. 27

773. „ striatum L. $\odot$ c. 27

774. Trigonella coerulea Ser. $\odot$ c. 27

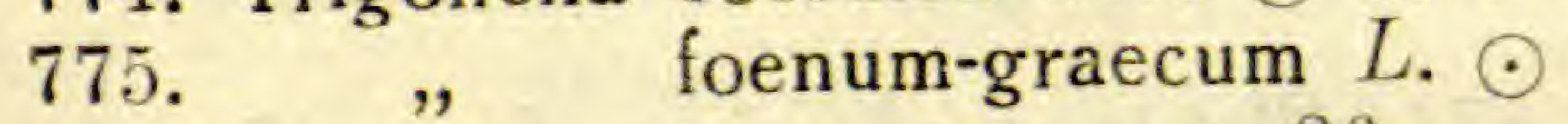

$$
\text { c. } 26
$$

776. „, orthoceras Kar. et.Kir.

$$
\odot \text { c. } 27
$$

777. Vicia angustifolia Roth. $\odot$ c. 27

778. ” cracca L. 4 c. 27

779. „ Ervilia Willd. $\odot$ c. 27

780. „ Faba L. $\odot$ c. 27

781. ” hirsuta G. Koch. $\odot$ c. 27

782. ” narbonensis $L . \odot$ c. 27

783. " picta Fisch. $\odot$ c. 27
784. Vicia sativa $L$. (sem. alb.) $\odot$

c. 27

785. ", sepium L. 4 c. 27

786. „ unijuga $A$. Br. 4 c. 27

Linaceae Dumort.

787. Linum Lewisii Pursh. 4 c. 27

788. \# nervosum W. K. 4 c. 27

789. " perenne L. 4 c. 27

790. „ usitatissimum L. $\odot$ c. 27

Lo a s a e a e.

791. Blumenbachia Hieronymi Urb. $\odot$ c. 27

792. Loasa bryoniaefolia Schrad. $\odot$

793. „, triphylla Juss. $\odot$ c. 27

794. „ urens Juss. $\odot$ c. 27

Lythraceae Lindl.

795. Cuphea lanceolata Ait. $\odot$ c. 27

796. Lythrum salicaria $L$. 4 -c. 27

797.* Nesea salicifolia $H$. B. et. $K$.

798. Peplis Portula $L . \odot$ G. $2^{5} 7^{27}$

Malvaceae Juss.

799. Abutilon Avicennae Gaertn. $\odot$ c. 27

800. Althaea ficifolia Cav. 4 c. 27

801. \# officinalis $L .4$ c. 27

802.,$\quad$ rosea Cav. 4 c. 27

803. Anoda cristata Schlecht. $\odot$ c. 27

804. Hibiscus Trionum L. $\odot$ c. 27

805. Lavatera thuringiaca L. 4 c. 27

806. " trimestris $L . \odot$ c. 27

807. Malope grandiflora $\odot$ c. 27

808. Malva Alcea L. 4 c. 27

809. " crispa $L . \odot, \odot$ c. 27

$810 . \quad$, parviflora $L . \odot$ c. 26

811. " rotundifolia $L$. $\odot$ c. 27

812. " $\quad$ silvestris $L$. $\odot$ c. 27

813.* Malvastrum peruvianum A. Gray

ค c. 27

814.* Pavonia hastata Cav. 4 c. 27

Moraceae Lindl.

815. Ficus carica L. 5 C. 24

816. Morus alba $L . \hbar$ c. 27 
Nyctaginaceae.

817. Mirabilis Jalappa $L$. $\odot$ c. 27 818. " viscosa Cav. $\odot$ c. 27 819. " Wrightii Dene $\odot$ c. 27

\section{Oleaceae Lindl.}

820. Fraxinus exceIsior $L . \hbar$ G. 27 821. Ligustrum vulgare $L$. 5 c. 27 822. Syringa vulgaris $L . \hbar$ G. 27

\section{Onagraceae Lindl.}

823. Boisduvalia densiflora Wats. $\odot$

824. ” Douglasii Spach. 27

\section{c. 27}

825. Chamaenerium angustifolium Scop.

826. Clarkia elegans Dough 425 827.* Circaea alpina $L .4$ c. 27 828. Epilobium hirsutum L. 4 c. 27 829. " Lamyi F. Schultz 830 c. 27 831. " montanum L. 4 c. 27 palustre L. 4 G. 27 pedunculare A. Cunn 833. Eucharidium grandiflorum F.et.M. ๑) 27

834. Godetia grandiflora Lindl. $\odot$ c. 27 835. Oenothera biennis $L . \odot$ c. 27 836.* " fruticosa $L .2$ c. 27 837. " " gigas De Vries c. 27 838. ” grandiflora Ait. $\odot$ c. 27 839. $\quad$ muricata $L . \odot$ c. 27 840 . " $\quad$ odorata Jacq. $\odot$ c. 27 841. " pumila $L .4$ c. 27 842. " $\quad$ rosea Ait 4 c. 26 843. $\quad$ " tetraptera Cav. $\odot$ c. 27 844. $" \quad$ triloba Nuttal. $\odot$ c. 27

\section{Oxalidaceae Lindl.}

845. Oxalis corniculata $L . \odot$, $\odot$ c. 27 846. " stricta L. 4 c. 27

\section{Papaveraceae Juss.}

847. Argemone mexicana $L$. $\odot$ c. 27 848. Chelidonium majus L. 4 G. 27 849. Corydalis glauca Purch. $\odot$ c. 27
850. Corydalis sempervirens Pers. $\odot$ c. 27 851. " vaginalis c. 27

852. $\quad$ vesicaria Pers. $\odot$ c. 27

853. Dicranostigma Franchetianum Fedde. c. 27

854. Eschscholtzia californica Cham. () c. 27

855. Fumaria officinalis $L$. $\odot$ G. 26 856.* Glaucium corniculatum Curt. $\odot$ c. 26

857. „ flavum Crantz. $\odot$ c. 27 858. Hypecoum grandiflorum Benth.

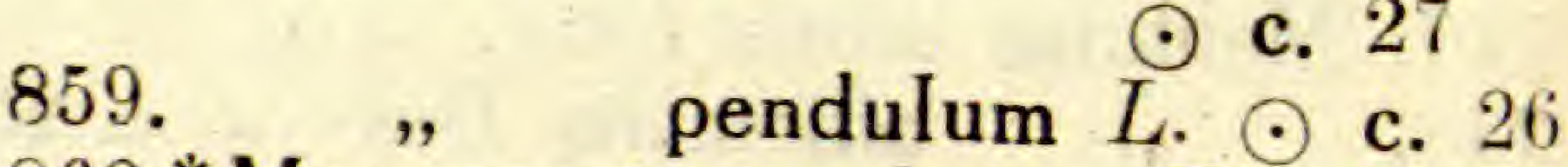
860 . Meconopsis cambrica Vig. 4

861. Papaver alpinum L. 4 c. 26 862. " argemone $L$. $\odot$ c. 27 863. " $\quad$ dubium $L . \odot$ c. 27 864.* " $\quad$ fugax Poir. $\odot$ c. 27 865. " orientale $L .2$ c. 27 866.* " radicatum Rottb. c. 27 867. " Rhoeas L. $\odot$ c. 27 868. " somniferum L. $\odot$ c. 27

\section{Passifloraceae.}

869.* Passiflora adenopoda Moc.et. Sess.

edulis Sims. ₹ 27

871.* "

Philadel $\rho$ haceae.

872. Philadelphus coronarius $L$. $\hbar$ c. 27

Phytolaccaceae.

873. Phytolacca decandra L. 4 c. 27

Plantaginaceae Lindl.

874. Plantago altissima L. c. 27 875.* " arborescens Poir. 4 c. 27

876.* " Brownii Rafin. c. 27 877. " Candollei Rafin. c. 27 878. " Cornuti Gouan. 4 c. 27 879. " Cynops L. 4 c. 27 880. $\quad$ " indica L. c. 27 881. " lanceolata L. 4 c. 27 882. " major L. 4 c. 27 
883. Plantago maritima L. 4 c. 27 884. " media L. 4 c. 27 885. " Psyllium L. ๑ c. 27 886. " $\quad$ ramosa Aschers. $\odot .27$

\section{Plumbaginaceae Lindl.}

887. Plumbagella micrantha Ledeb. 4 888. Statice tatarica L. 4 c. 27

\section{Polemoniaceae D. C.}

889.* Cobaea scandens Cav. c. 26 890. Collomia Iinearis Nutt. ๑) c. 27 891. Gilia californica Benth. ๑) c. 27 892. Navarretia squarrosa Hook. et. Arn. $\odot$ c. 27 893. Phlox Drummondi Hook. ๑ c. 27 894. Polemonium coeruleum L. 4 c. 27

Polygonaceae Lindl.

895. Calligonum aphyllum Gürke ћ 27

896. Emex spinosa Campd. $\odot$ c. 27 897. Fagopyrum tataricum Gärtn. $\odot$ 898. Polygonum aviculare L. $\odot$ G. 27 899. " Bistorta L. 4 c. 27 900. " $\quad$ Convolvulus $L$. $\odot$ 901. " $\quad$ dumetorum L. $\odot$ c. 24 902. " Fagopyrum L. $\odot$ c. 27 903. " " Hydropiper L. $\odot$ c. 27 904. „ lapathifolium $L$. $\odot$ c. 27

905. „ tomentosum Schrank.

906. Rheum Emodi Wall $\odot$ c. 27

907. Rheum Emodi Wall. 4 c. 27

907. ” palmatum L. 4 c. 27

908. " " Rhaponticum L. 4 c. 27

909. " undulatum L. 4 c. 27

910. Rumex acetosa L. 4 c. 27

911. , Acetosella L. 4 G. 26

912. „ aquaticus $L .4$ M.

913. " confertus Willd. 2 M. 24

914. " crispus L. 4 c. 27

915. " obtusifolius L. 4 M.

916, " $\quad$ pulcher L. 4 c. 27

917. " scutatus L. 4 c. 27

\section{Portulacaceae Lindl.}

918. Calandrina procumbens Moris. $\odot$ c. 27

919. Claytonia perfoliata Domn. $\odot$

c. 27 Portulaca grandiflora Lindl. $\odot$

921. „ oleracea L. • c. 27

922. Talinum patens Willd. 2 c. 27

\section{Primulaceae Vent.}

923. Anagallis arvensis $L$. var, coerulea Gren. et. Godr. $\odot$ c. 27

924. ," var. phoenicea Gren. et. Godr. ๑ c. 27

925.* Androsace septentrionalis $I$. $\odot$, .. c. 27

926. Asterolinon stellatum Hoffm. et. Link. • c. 27

927. Lysimachia vulgaris $L .4$ c. 27 928. Primula auricula L. 4 c. 27 929.* " cortusoides L. 4 c. 27 930. " officinalis Hill. 4 c. 27 931. Samolus Valerandi L. 4 c. 27

\section{Ranunculaceae Juss.} 932.*Aconitum ferox Wall. 4 c. 27 933. " lasiostomum Reichb. 4

934. napellus $L$ c. 27

935. Actaea spicata L. 4 c. 27

936. " " var. rubra (Bigel.) 4 c. 27

937. Adonis autumnalis L. $\odot$ c. 27

938. Anemone virginiana $L .4$ c. 27

939. Aquilegia atropurpurea W. 4

940. $\quad \quad$ formosa Fisch. 4 c. 27

941. " olympica Boiss. 4

942. ” vulgaris $L .4$ c. 27

943. Caltha palustris L. 4 G. 26

944. Clematis integrifolia $L: 4$ c. 27

945. " recta L. 4 c. 27

946. ” tangutica Korsh. 5 c. 27

947. , vitalba $L . \hbar$ C. 26

948. Delphinium Ajacis L. $\odot$ c. 27

949. „ consolida $L \odot$ c. 27

950. " " elatum L. 4 c. 27 
951.* Delphinium orientale J. Gay. $\odot$ c. 27

952.* $\quad$ rossicum Litw. c. 27 953.* Eranthis hiemalis Salsb. 4 c 27 954. Ficaria ranunculoides Roth. 4 c. 27 (fructus!)

955. Isopyrum fumarioides $L$. $\odot$ c. 27 956. Myosurus minimus $L . \odot$, $\odot$ c. 27 957. Nigella damascena $L$. $\odot$ c. 27 958. " Garidella Spenn. $\odot$ c. 27 959. „, sativa $L . \odot$ c. 27

960. Paeonia albiflora Pall. 4 c. 27 961.* " Beresowskii Komar. 4 962. Ranunculus acer L. 4 c. 27 963. " $\quad$ arvensis $L . \odot$ c. 27

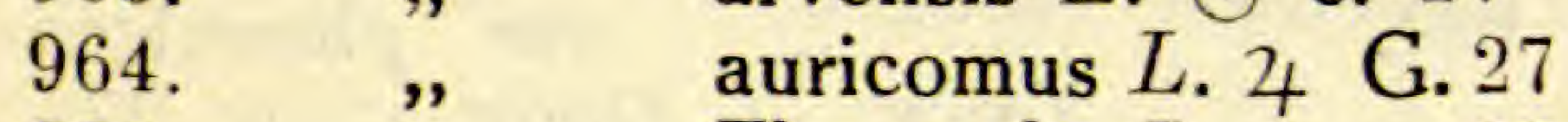
965. " $\quad$ Flammula L. 4 c. 27 966.* " $\quad$ pedatus $W . K .4$

967. $\quad$ polyanthemos $L .4$ c. 24 968. $\quad, \quad$ repens $L . ~ 4$ c. 26 969.* $\quad$ sceleratus $L$. $\odot$ c. 26 970. Thalictrum angustifolium Jacq. 4

971. claum $L$ c. 27 972. " $\quad$ minus L. 2 c. 27 973. Trollius europaeus L. 4 c. 27

Resedaceae D. C.

$974^{*}$. Reseda alba $L . \odot, 4$ c. 27 975. , crystallina Webb. et. 976. Berth. c. 27 976. " inodora $R \operatorname{chb} . \stackrel{\odot, 4}{\text { c. } 27}$ 977. " lutea $L . \odot, 4$ c. 27 978. " luteola $L . \odot$ c. 27 979. " odorata L. $\odot$ c. 27 980. " Phyteuma L. $\odot$ c. 27

Rhamnaceae S. F. Gray. 981. Rhamnus cathartica $L . \hbar$ G. 27 982. " Frangula L. $\hbar$ G. 27

\section{Rosaceae Juss.}

983. Agrimonia Eupatoria L. 4 c. 27 984. ", pilosa Led. 4 c. 27 985. Aruncus silvester Kost. 4 c. 27
986. Crataegus monogyna Jacq. $\hbar$ c. 27

987. Filipendula hexapetala Gilib 4 c. 27

988. " ulmaria Maxim. 4 c. 27

989. Fragaria indica Andr. 4 c. 27 990. " vesca $L .4$ c. 27 991. " viridis Duch. 4 c. 27 992. Geum aleppicum Jacq. 4 c. 27 993. „ molle Vis. et. Panc. 4

994. „ rivale $L .4$ c. 27 c. 27 995. ” urbanum L. 2 M. 25 996. Potentilla alpestris Hall. f. 4 c. 27

$997 . \quad \quad \quad \quad$ argentea $L .4$ c. 27
$998 . \quad \quad \quad$ bifurca L. 4 c. 27

999. " " delphinensis Gren. et. Godr. c. 27

1000. " desertorum Bge. 4 c. 27

1001. " fruticosa $L . \hbar$ c. 27

1002. ", intermedia $L .4$ c. 27

1003. " longipes Led. 4 c. 27

1004. ", palustris Scop. 4 M.

1005. ", recta L. 4 c. 27

1006. " $\quad$ rupestris L. 4 c. 27

1007. " Tormentilla Schrank. 4 c. 27

1008. , $\quad$ supina $L . \odot, \odot$ c. 27 1009. Poterium Sanguisorba L. 4 c. 27 1010. Prunus Padus L. $\hbar$ c. 27 1011. " spinosa L. $\frac{\hbar}{2}$ c. 27 1012. Rosa cinnamomea $L . \hbar$ c. 27 1013.* ” dahurica Pall. $\hbar$ W. 26 1014.* ", rugosa Thunb. 方 W. 26 1015. Rubus saxatilis L. 4 c. 27 1016. * Sanguisorba officinalis L. 4 $1017 * \quad$ W. 26 1017.* , " tenuifolia Fisch 4 W. 26

1018. Spiraea media Schm. $\hbar$ c. 27 1019. "\# salicifolia $L . \hbar$ c. 27 1020. Sorbus Aucuparia L. $\frac{1}{2}$ c. 27

$$
\text { Rubiaceae Juss. }
$$

1021. Asperula Aparine Schott. 4 1022. arvensis L. c. 27 1023. " cynanchica L. 4 c. 25 1024.* " odorata L. 4 M. 24 
1025. Asperula supina $M$. B. 4 c. 27 1026. Crucianella chlorostachys $F$. et. $M$. 1027. ", exasperata $\stackrel{\odot}{F}$. et. 27. ค c. 27

1028. Galium mollugo L. 4 c. 27 1029. " silvaticum L. 4 M. 24 1030. ” spurium L. 4 M. 24 1031. , verum L. 4 c. 25 1032.* Rubia tinctorum L. 4 c. 27 1033. Scherardia arvensis $L$. $\odot$ c. 27

\section{Rutaceae Juss.}

1034. Ruta graveolens L. 4 c. 27

\section{Saxifragaceae Dumort.}

1035.* Chrysosplenium alternifolium $L$. 4 c. 27

1036. Heuchera sanguinea Englm. 4 c. 27

1037. Parnassia palustris L. 4 c. 27

1038. Ribes alpinum L. 5 c. 27

1039. Saxifraga caespitosa L. 4 c. 27 1040 . $” \quad$ irrigua M. B. 4 c. 27 1041. Tellima grandiflora $R . B r .4$

$$
\text { c. } 27
$$

\section{Scrophulariaceae Lindl.}

1042. Alonsoa Warscewiczii Reg. •

$$
\text { c. } 27
$$

1043. Antirrhinum majus L. $\odot$ c. 27 1044. Orontium L. $\odot$ c. 27

1045. Calceolaria pinnata $L$. $\odot$ c. 27 1046. Chaenostoma antirrhinoides $L$.

$$
\text { c. } 27
$$

1047. foetidum Benth. $\odot$ c. 27

1048. Collinsia bicolor Benth. $\odot$ c. 27 1049. Cymbalaria muralis Baumg. 4 c. 27

1050. Digitalis ambigua Murr. 4 c. 27 1051. ” laevigata W. et. K. 4

1052. " Iutea L. 4 c. 27

1053. $\quad$ purpurea L. 4 c. 24

1054. Hebenstreitia dentata $L$. $\odot$ c. 27 1055. Linaria bipartita $W$. ค c. 27 1056 . canadensis Dumont. $\odot$
1057.* Linaria littoralis $W$. . c. 24

1058. " macroura M. B. 4 c. 24

1059. " tristis Mill. • c. 24

1060. " vulgaris Will. 4 c 27

1061. " " var. Biebersteinii Lindem. 2 c. 27

1062.* Lindenbergia urticifolia $\mathrm{Lehm}$. (.) c. 27

1063.* Manulea violacea LK. • c. 27

1064. Melampyrum nemorosum $L$. (.) M.

1065. Mimulus cardinalis Domgl. 4 c. 27 1066.* " cupreus Regel. var. tigrinus 4 c. 27

1067. " Langsdorffii Hort. $\odot, 4$

1068. " luteus L. ॰ c. 27

1069. ", moschathus Domgl. 4 c. 27

1070. Nemesia strumosa $\odot$ c. 27

1071. Nycterinia Capensis $\odot$ c. 27 1072. Odontites serotina Rchb. $\odot \mathrm{M}$. 1073. Pedicularis palustris $L$. ., $4 \mathrm{M}$. 1074. Scrophularia nodosa $L .2$ c. 25 1075. , umbrosa Dumort.

1076. Verbascum Blattaria L. $\stackrel{2}{ }$. c. 27 1077. ," nigrum L. .. c. 27 1078. , phlomoides L. $\odot$ c. 27

1079. , speciosum Schiad.

1080.* Veronica arvensis $L . \%$ c. 27 M. 24

1081. " Beccabunga L. 4 c. 27

1082. ", Chamaedrys L. 4

c. 27

1083. " gentianoides Vahl. 4 c. 27

1084. „, longifolia L. 4 c. 27

1085. " officinalis L. 4 c. 27

1086. - scutellata L. 4 c. 27

1087. ,,$\quad$ serpyllifolia L. 4 c. 25

1088. " $\quad$ sibirica L. 4 c. 27

1089. " spicata L. 4 M. 24

1090.,$\quad$ spuria L. 4 c. 27

1091. ," Tournefortii Gmel. $\odot$ c. 27

Solanaceae Pers.

1092. Atropa Belladonna L. 4 c. 27 1093. " caucasica Kreyer. 4 c. 27 
1094.* Atropa Paschkewiczi Kieyer. 4 c. 27

1095. Capsicum annuum L. ( ) c. 27

1096. Datura Bertolonii Parl. ( c) 27

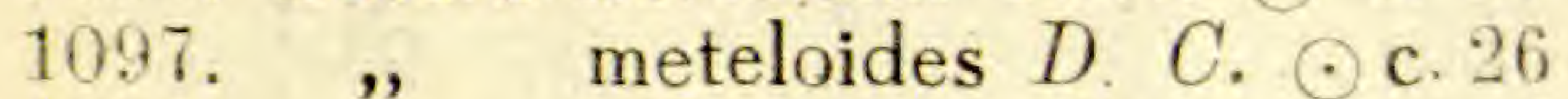
1098. ", stramonium L. ๑) c. 27 1099. ,, Wrighti Rgl. ๑ c. 27 (?) 1100. Hyoscyamus albus $L$. $\odot$ c. 27

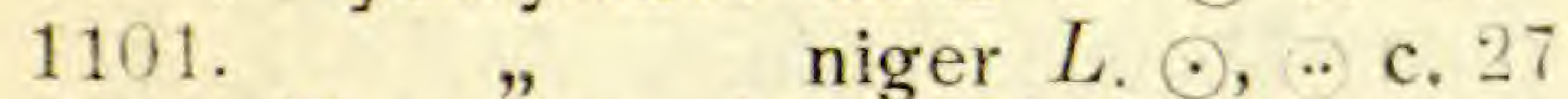
1102. Nicandra physaloides Guertn. $\odot$ c. 27

1103. Nicotiana affinis Moore. $\odot, 24$

1104. $\quad$ paniculata L. $\odot$ c. 27 1105. " rustica L. $\odot$ c. 27 1106. " Sanderiana Hort. c. 27 1107. " silvestris $L \odot$ c. 27 1108. " Tabacum L. $\odot$ c. 27 1109. Nolana prostrata L. $\odot$ c. 27 1110. Petunia nyctaginiflora Juss . c. 27

1111. Physalis Alkekengi L. 4 c. 24 1112. " angulata $L$. $\odot$ c. 27 1113. ", Francheti Masters. c. 27 1114. " pubescens L. ๑ c. 27 1115. Physochlaena orientalis G. Dom. 2 c. 27

1116. Salpiglossis variabilis Hort. • c. 27

1117. Solanum Capsicastrum Link: 市 c. 27

1118. " dulcamara L. 4 c. 27 1119. " Gilo Raddi. ค c. 27 1120. " gracile Otto. $\odot$ c. 27 1121. \# guineense Lam. () c. 27 1122. ", Hystrix R. Br. c. 27 1123. " nigrum $L$. v. villosum $L$, ( ) 27 1124. , , $\quad$ v. vulgare $L$. (.) c. 27

1125. Withania somnifera Dum. 万 c 27

Tropaeolaceae Juss.

1126. Tropaeolum majus L. $\odot$ c. 27

Umbelliferae Morison.

1127. Aegopodium alpestre Ledb. 4

1128. , Podagraria L. 4 c. 27

1129. Aethusa Cynapium L. $\odot$ c. 27
1130. Anethum graveolens L. $\odot$ c 27 1131. Angelica archangelica $L$. . c 26 1132. ", silvestris L. 4 c. 27 1133. Anthriscus silvestris Hoff. 4 c. 27 1134. Astrantia major L. 4 c. 27 1135. Bifora radians $M$. B. ๑ c. 27 1136. ,. testiculata Boiss. c. 27 1137. Bupleurum rotundifolium L. $\odot$ c. 27

1138. Carum Carvi L. .) c. 27 1139. Cenolophium Fischeri Koch. 4 c. 27

1140. Chaerophyllum aromaticum L. 7 c. 27

1141. Conium maculatum $L$. $*$ c. 26 1142. Coriandrum sativum L. $\odot$ c. 27 1143. Daucus Carota L. (spont.) $\odot$

$$
\text { c. } 27
$$

1144. Eryngium pandanifolium 4 c. 27 1145. " planum L. 4 c. 27 1146. Falcaria vulgaris Bernh. -. c. 27 1147. Heracleum barbatum Ledb. 4 c. 27

1148 , , pubescens $M . \quad$ B. . c. 27

1149. , , $\quad$ sibiricum L. .. M. 25 1150. Laserpitium pruthenicum $L$. - M. 1151. Levisticum officinale L. 4 c. 27 1152. Libanotis montana Al?. -.) c. 24 1153. , sibirica Koch. 4 c. 26 1154. Ligusticum scoticum L. 4 c. 27 1155. Myrrhis odorata Seop. 4 c. 27 1156. Pastinaca sativa L. .. c. 27 1157. " teretiuscula Boiss. .. c. 26

1158. Peucedanum cervariaefolium 1159. C.A.M. 4 c. 27 2 M. 24 1160. " palustre Monench. 4 c. 24

1161. Pimpinella Anisum L. $\odot$ c. 27 1162. " magna L. 4 M. 1163. " $\quad$ saxifraga L. 4 c. 27 1164. " " Tragium Vill. 2 c. 26 1165. Sanicula europaea L. 2+ M. 116i6. Scandix Pecten Veneris L. $\odot$ c. 27

1167. Selinum carvifolia L. \& M. 1168. Seseli Hippomarathrum L. 4 1169. " laserpitifolium Palib. c. 264 
1170. Torilis Anthriscus Gmel. $\odot$ M. 1171. Zozimia absinthifolia D. C. 4 c. $26 i$

\section{Urticaceae Endl.}

1172. ForskohIea angustifolia Retz. $\odot$ c. 26

1173. Parietaria ramiflora Moench. 4 c. 27

1174. Urtica cannabina L. 7 W. 26

1175. „, dioica L. 4 c. 27.

1176. , Dodartii L $\odot$ c. 26

1177. „, membranacea Poir. c. 27

1178. „ pilulifera L. ๑) c. 27

1179. „ urens L. $\odot$ c. 27

\section{Vacciniaceae Lindl.}

1180. Vaccinium Oxycoccus L. 4 G. 27 1181.* ", uliginosum L. 方 G. 26

\section{Valerianaceae Dumort.}

1182. Centranthus ruber D.C. 4 c. 26 1183. Fedia cornucopiae D.C. С. c. 27 1184. Valeriana palustris $K r: 4$ c. 26 1185. ", Sprygini Sm. 4 c. 27 1186. " " Wolgensis Kez. 4 c. 27

1187. Valerianella coronata D. C. . c. 27 1188. , , eriocarpa Kioli. ( c. 27
11^!). Valerianella rimosa Bastard. $\odot$ c. 27

1190. tridentata Bas. $\odot$ c. 27

\section{Verbenaceae J. St. Hil.}

1191.* Lantana flava Medic $\hbar$ c. 27 1192. Verbena arborea $H$. et. B. 5

c. 27

1193. , Aubletia Jacq. ... c. 27

1194. " hybrida Hort. $\odot$ c. 27

\section{Violaceae D. C.}

1195. Viola alpestris Wittr. 4 c. 27

1196. „, canina Rchb. 4 c. 27

1197.* ,, cornuta L. 4 c. 27

$1198 *$ * elatior Fr. 4 c. 27

1199.* ", elegantula Schoth. c. 27

1200. " epipsila Led 7 c. 27

1201. " hirta L. 4 c. 27

1202. ", mirabilis L. 4 c. 27

1203.* " Munbyana Boiss. et. Reut. var. Battandieri c. 27

1204. ', odorata L 4 c. 27

1205. „, Patrini D. C. 4 c. 27

1206. ,, tricolor Witt. $\odot, .$. c. 27

Zygophyllaceae R. Br.

1207. Peganum Harmala L. 4 c. 26

12 (18. Tribulus terrestris L. $\odot$ c. 27

1209. Zygophyllum Fabago L. 4 c. 27

Semina sporaeque plantarum nonnullarum in territor. Karadagh (Tauria orientalis) sponte crescentium, quae a prof. J. G. Wassilkov anno 1927 lecta sunt.

Семена и споры некоторых диких растений Карадагской (Восточн. Крым) флоры, собранные профессором И. Г. Васильковым летом 1927 года.
Насеньне ды зароднікі некаторых дзікіх расьлін Карғдагскае (Усході. Крым) флёры, якое сабрана прафәсарам Я. Ю. Васільковым улетку 1927 году. 
1210. Althaea ficifolia Cav.

1211. Asplenium Trichomanes $L$. 1212.*Briza spicata Sibth. et. Sm. 1213. Capparis spinosa $L$.

1214. Carpinus orientalis Mill. 1215. Cichorium Intybus $L$. 1216.* Cleome ornithopodioides $L$. 1217. Cornus mas $L$. 1218* Daucus pulcherrimus $D . C$. 1219. Echium altissimum Jacq. 1220.* Euphorbia Chamaesyce $L$.

Professor J. G. Wassilkov, Horti praefectus.
1221. Heliotropium europaeum $L$. 1222. Linaria genistaefolia Mill. 1223. Nitraria Schoberi $L$.

1224. Paeonia corallina Retz. var.

1225. Peganum Harmala $L$.

1226. Scabiosa ucrainica $L$.

1227. Scilla autumnalis $L$.

1228. Setaria verticillata $P$. $B$. 1229.*Sideritis montana $L$.

1230. Tragus racemosus Desf.

V. L. Gorsky, hortulanus.

Gorky. R P. Alborutheniae (U. S. S. R.).

Calendis Februariis MCMXXVIII. 
1

14 



\section{Jardin Botanique de I'Academie Agronomique.}

Gorky. Russie Blanche. U.S.S.R.

\section{9}

\section{ПЕРАЛПК НАСЕНЬНЯ,}

ЯКОЕ БАТАНІЧНЫ САД БЕЛАРУСКАЙ ДЗЯРЖАЎНАЙ АКАДЭМОі ここここСЕЛЬСКАЕ ГАСПАДАРКІ ПРАПАНУЕ ДА АБМЕНУここここ

\section{Delectus seminum,}

Anno MCMXXVIII (partim ac MCMXXVI et MCMXXVII) collectorum, quae Hortus Botanicus Academiae Agronomicae Rei publicae Alboruthenicae pro mutua commutatione offert.

\section{DEE ПЕРЕЧЕНЬ СЕMЯН,}

ПРЕДЛАГАЕМЫХ В ОБМЕН БОТАНИЧЕСКИМ САДОМ БЕЛОРУССКОЙ ГОСУДАРСТВЕННОЙ АКАДЕМИИ СЕЛЬСКОГО ХОЗЯЙСТВА.

ZKOI GOGUDARSTUENNOI AKADEMII SEL

[PUBLCD? ALADEM

STATE?

thoz Ta Is TUA

ASOBNYADEITAKZ ZAPISAK BELARUSW

Асобны адбітак з „Запісак Беларускай Дзяржаўнай Акадэміі С. Г.“ т. IX

Gory-Gorky. R. P. Alborutheniae (U. S. S. R.)

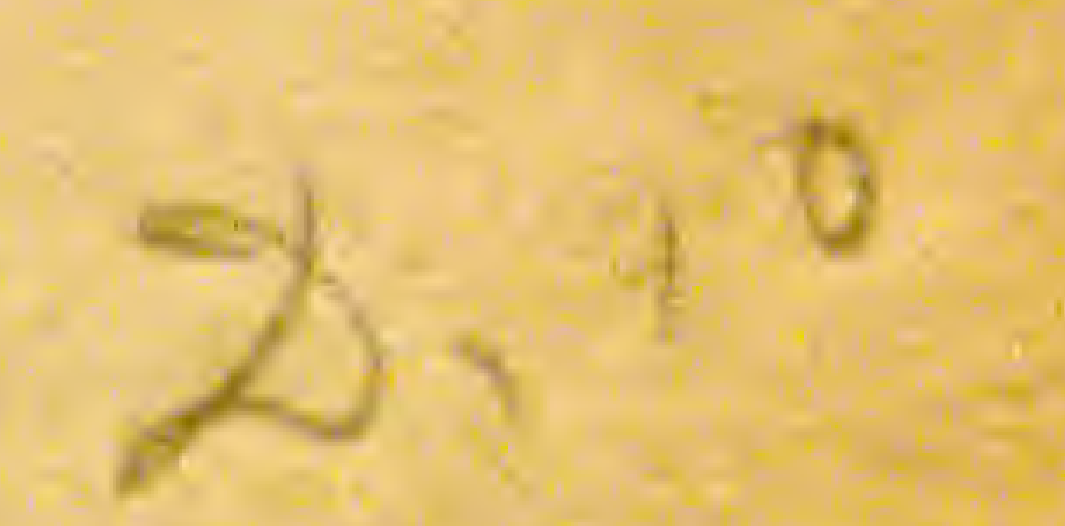

T O P K i

ДРУКАРНЯ АКАДЭМі

$\begin{array}{llll}1 & 9 & 2 & 9\end{array}$

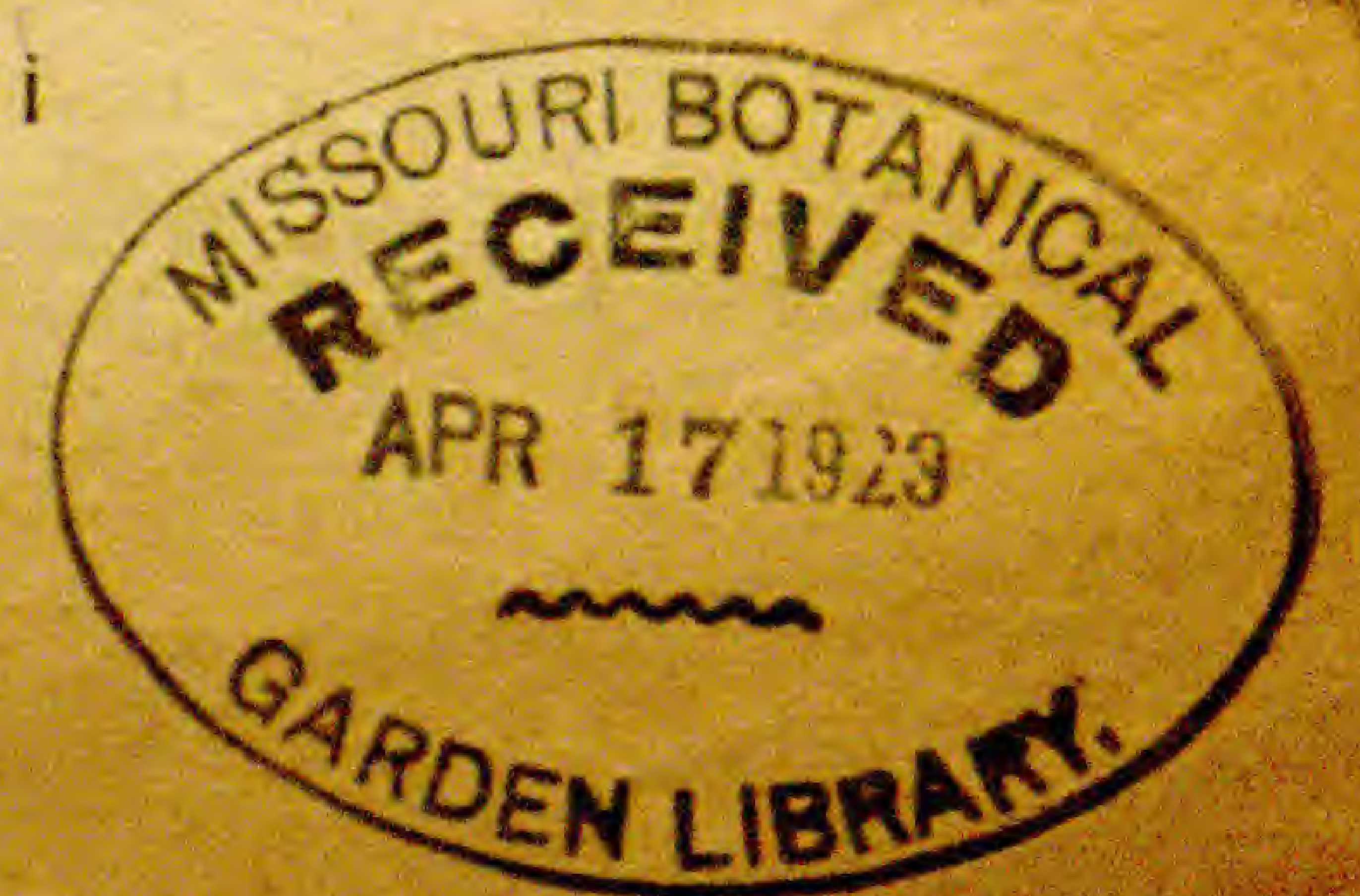





\section{Abbreviationes:}

*-paulum; K-Karadagh; W-Wladiwostok; G-Gorky; c-planta sub divo in Horto Botanico Academiae culta.

Numerus $(26,27,28)$ nomina plantarum sequens, annum, quo semina lecta sunt, significat.

\section{Скарачэньні і умоўныя знакі:}

Зоркай (*) абазначана насеньне, якое маецца ў нязначнай колькасьці. К-насеньне, якое сабрана ў Карадагу (Крым); W-навакол Ўладзівастоку; G-навакол Горак; с-з расьлін, якія разводзяџџа ў вадкрытым грунце ў Батанічным Садзе Акадэміі.

Лічбы $(26,27,28)$, якія надрукаваны пасьля назоваў расьлін, азначаюџь гәды збору насеньня.

\section{Сокращения и условные обозначения:}

Звездочкой (*) обоэначены семена, имеющиеся в малом кояичестве. K-семена собранные на Карадаге (Крым); W-в окрестностях Владивостока; $\mathrm{G}-$-в окрестностях Горок; c-c растений, культивируемых в Ботаническом Саду Академии в открытом грунту.

Џыфры $(26,27,28)$, помещенные за названиями растений, означают год сбора семян. 


\section{Pteridoph y ta.}

1. Adianthum gracillimum Moore. 4 c. 28

2. Aspidium cristatum $S w .2$ c. 28 3. " Filix-mas $S w .4$ c. 28 4. $\quad$ spinulosum $S w .4$ c. 28 5. Asplenium dimorphum Knze. 4 c. 28 6. " Filix-femina Bernh. 4

7. Trichomanes L. 4 K. 28 8. Cystopteris bulbifera Bernh. 4 c. 28
9*. Cystopteris fragilis Bernh. 4 c. 28 10. Osmunda cinnamomea $L .4$ W. 26 11. Phegopteris Dryopteris Fée. 4 c.28 12. Polypodium Phegopteris L. 4 c. 28

13. Pteris aquilina $L .4$ c. 28

14. " cretica $L .4$ c 28

15. " longifolia L. 4 c. 28

16. Scolopendrium vulgare $S m .4$ c. 28

17. Struthiopteris germanica Willd. 4 c. 28

\section{Monocotyledoneae.}

Alismataceae C. Rich. 18. Alisma Plantago L. $4 \mathrm{M}$. Araceae Juss.

19. Calla palustris $L .4$ G. 28 Commelinaceae Reich. 20. Commelina coelestis. W. $\odot$ c. 28 21. $\quad$ tuberosaL. 4 c. 28

22. Tinantia fugax Scheidw. 4 c. 28

Cyperaceae J. St. Hil. 23*. Carex acuta $L .4$ c. 28

$24^{*}$. " caespitosa $L \quad 4$ c. 28

$25^{*}$. " elongata L. 4 c 28

26*. " flava L. 4 c. 28

27. " hirta L. 4 c. 28

28. " leporina L. 4 c. 28

29. " muricata L. 4 c. 28

30. " pallescens L. 4 c. 28

31. " panicea L. 4 c. 28

$32^{*}$. ” pilosa L. 4 c. 28

$33^{*}$. " remota L. 4 c. 28

34. " rostrata Stokes. 4 c. 28

35. $\quad$ stricta Good. 4 c. 28

36. $\quad$ vesicaria $L .4$ c. 28

37. $\quad$ vulgaris $\mathrm{Fr} .4$ c. 28

38. " vulpina $L .4$ c. 28

39. Cyperus esculentus L. 4 c. 27

40. Eriophorum latifolium Hoppe. 4

G. 26

41. Scirpus silvaticus L. 4 c. 28

Graminea e Jus s.

42. Agropyrum caninum $F$. Beauv. 4 c. 28
43 Agropyrum glaucum Roem. $e^{t}$. Schult 4 c. 28 44. $\quad$ repens $P$. Beauv. 4

45. Agrostis alba $L .4$ c. 28

46. " canina L. 4 c. 28

47. " vulgaris With 4 c. 28

48. Aira capillaris Host. $\odot$ c. 28

49. " caryophyllea $L$. $\odot$ c. 28

50. Alopecurus geniculatus $L$. $\odot$ c.28

51. " pratensis L. 4 c. 28

52. $\quad$ ventricosus Pers. 4

c. 28

53. Anthoxanthum odoratum L. 4 c. 28

54. Apera spica venti $P$. Beauv. $\odot$

c. 27

55. Arrhenatherum elatius $M$. et. $\mathrm{K} 4$ c. 28

56. Asperella hystrix Humb. 4 c. 28

57. Atropis convoluta Gris. 4 c. 28

58. " distans Gris. 4 c. 28

59. Avena barbata Brot. $\odot$ c. 28

60. " pubescens L. 4 c. 27

61. " strigosa Schreb. $\odot$ c. 28

62. Beckmannia eruciformis Host. 4

c. 28

63. Brachypodium ramosum $R$. et. $S$. 4 c. 28

64. $\quad$ silvaticum $R$. et. $S$.

65. Briza maxima L. $\odot$ c. 28

66. " media $L .4$ c. 28

67. " minor L. $\odot$ c. 28

68*. " spicata Sibth. et. Sm. $\odot$ K.

$69^{*}$. " virens L. c. 28 
70. Bromus arvensis $L$. $\odot$ c. 28

71. " commutatus Schrad. $\odot$, ค.) 28

72. „ hordeaceus $L . \odot, \odot$ c.28

73. " inermis Leyss. 4 c. 28

74. ” macrostachys Desf. $\odot$

75. c. 28

„ secalinus $L . \odot, \odot$ c. 27

76. ” squarrosus $L . \odot, \odot$ c. 27

77.,$\quad$ sterilis $L . \odot, \odot$ c. 27

78. ” tectorum $L . \odot$ c. 28

79. Calamagrostis Epigeios Roth. 4 c. 28

80. Catabrosa aquatica P. B. 4 c. 27

81. Cenchrus echinatus $L$. c. 27

82. $"$ montanus Nees. c. 27

83. Chloris barbata $S w$. c. 27

84. Cinna glomerata $L$. c. 28

85. " mexicana Beauv. 4 c. 28

86. Coix lacryma Jobi $L$. $\odot$ c. 27

87. Cynosurus cristatus L. 4 c. 28 88*. " echinatus L. 4 c. 27

89. Dactylis Aschersoniana Graebn.

$\begin{array}{ll}\text { 90. } \quad ~ & 4 \text { c. } 28 \\ \text { 91. } \quad \# \quad \text { glomerata L. } 2 \text { c. } 28 \\ \text { L. fol. var. } 4\end{array}$

c. 28

92. Deschampsia caespitosa P. B. 4 c. 28

93. ” flexuosa Trin. 4 c. 27

$94^{*}$. Dinebra arabica Jacq. c. 27 95. Eleusine indica Grtn. $\odot$ c. 27 96. Elymus araliensis Rgl. 4 c. 28 97. " giganteus Vahl. 4 c. 27 98. ” sibiricus L. 4 c. 28

99. Eragrostis minor Host. $\odot$ c. 28 100. " pilosa P. B. $\odot$ c. 28 101. Eriochloa villosa $K n t h . \odot$ c. 27 102. Festuca arundinacea Sehr. 4 c. 28

103. " Beckeri Hackel. 4 c. 28 104. ” gigantea Vill. 4 c. 28

105. " heterophylla Lam. 4 c. 27

106. " ovina L. 4 c. 28

107. " pratensis Huds. 4 c. 28

108. ” rubra $L .4$ c. 28

109. " sulcata Hackel. c. 28

110. Gastridium australe $P$. B. $\odot$ c. 28

111. Glyceria spectabilis $M$. et. K. 4 c. 27

112. Hierochloë odorata Wahlb. 4
113. Holcus lanatus L. 4 c. 27

114. Hordeum jubatum L. 4 c. 28

115. \# secalinum Schreb. 4 c.28

116. Koeleria gracilis Pers. 4 c. 28

117. Lagurus ovatus $L . \odot$ c. 28

118. Lasiagrostis splendens Kunth. 4

$$
\text { c. } 28
$$

119. Leersia oryzoides $S w .4$ c. 28 120. Lolium perenne L. 4 c. 28 121. remotum Schrank. $\odot$

\section{c. 28}

122. \# temulentum $L . \odot$ c 28 123. Melica altissima $L$. 4 c. 28 124. " nutans $L .{ }^{2}$ c. 28 125. " transsilvanica Schur. 4 c. 28 $126^{*}$. Milium vernale $M . B . \odot$ c. 28 127. Miscanthus sacchariflorus Hack. 4 c. 28

128. Nardus stricta L. 4 c. 27

129. Oryzopsis holciformis Richt. 4 c 27

130. Panicum calyfornicum Benth. $\odot$ c. 27

131. ” ciliare Retz. $\odot$ c. 27

132. $” \quad$ crus galli $L . \odot$ c. 28

133. ” frumentaceum $F r$. et.

Cav. $\odot$ c. 27

134. - germanicum c. 28

135. ” miliaceum $L$. $\odot$ c. 28

136. " sanguinale L. $\odot$ c. 28

137*. Paspalum digitaria Poir. 4 c. 27

138. Phalaris arundinacea L. 4 c. 28

139. "

fol. var, v.

140* lutescens 4 c. 28

141. Phleum alpinum L. 4 c. 28

142. " Boehmeri Wib. 4 c. 28

143. ” paniculatum L. 4 c. 28

144. ” pratense $L .4$ c. 28

145. Phragmites communis Trin. 4

146. Poa alpina $L .4$ c. 28

G. 28 .

147. „ annua $L$. $\odot$ c. 28

148. " bulbosa L. 4 c. 28

149. „ caesia Sm. 4 c. 28

150. " compressa $L .4$ c. 28

151. ” glauca Vahl. 4 c. 28

152. " nemoralis L. 4 c. 28

153 ” palustris $L .4$ c. 28

154. " pratensis $L .4$ c. 28

155. " trivialis $L .4$ c. 28

156. Polypogon monspeliensis Desf.

(.) c. 28

157. Scleropoa rigida Griseb. $\odot$ c. 28 
158. Secale montanum Guss. 4 e. 28 159. Setaria verticillata $P$. B. ๑ c. 28 160. " viridis P. B. $\odot$ c. 28 161. Sieglingia decumbens Bernh. 4

162. Stipa capillata L. 4 c. 27

163. " pulcherrima C. Koch. 4

164. Tragus racemosus Desf. $\odot$ c. 28

165 Trisetum flavescens $P$. B. 4 c. 27

166. $\quad$ rigidum Trin 4 c. 28

$167^{*}$. $\quad$ sibiricum Rupr. 4 c. 27

I rid a c a e Jus s.

168. Gladiolus imbricatus L. 4 c. 28

169*. Iris corthalinia Fom. 4 c. 28

170. " Fischeriana Hort. 4 c. 28

171. " halophila Pall 4 c. 28

172. " musulmanica Fom. 4 c. 28

173. „Pseudacorus L. 4 c. 28

174. " setosa Pall. 4 c. 28

175. " sibirica L. 4 c. 28

176. " spuria L. 4 c. 28

177. Sisyrinchium anceps Cav. 4 c. 28

Jun c a c e e V e n t.

178. Juncus balticus Willd. 4 c. 28

$179^{*}$. " bufonius $L$. $\odot$ c. 28

180. " " compressus Jacq. 4 c. 28

181. " effusus $L .4$ c. 28

182. " lamprocarpus Ehrh. 4

184. " pilosa Willd 4 G. 27

Juncaginaceae Rich.

185. Scheuchzeria palustris L. 4

G. 27
$\mathrm{L}$ i 1 i a c e e $\mathrm{S}$ c o p. 186. Allium albidum Fisch. 4 c. 28 187*. " angulosum L. 4 c. 27 188. " coeruleum Pall. 4 c. 28 189. " fistulosum L. 4 c. 28 190. " " globosum Red. 4 c. 28 191. " obliquum L. 4 c 28 192. " $" \quad$ odorum L. 4 c. 28 193. ” schoenoprasum L. 2 c.28 194. ", senescens L. 4 c. 28 $195^{*}$. " ursinum $L$ क. 26 196. Ant ericum Liliago $L 4$ c. 28 197. Asparagus officinalis $L .4$ c. 28 198. Asphodelus tenuifolius Cav.. 4 c. 28 199. Bulbine annua Willd $\odot$ c 28 200*. Convallaria majalis L. 4 c. 27 $201 *$ Echeandia terniflora Ortega 4 c. 28 202\%. Gagea lutea Ker-Gawl 4 c. 28 203. Galtonia candicans Dene. 4

204. Lilium tigrinum Gaver 4 c. 28 $205 \%$. Majanthemum bifolium D. C. it $\mathrm{M}$

206. Muscari racemosum Mill. 4 c 28 207. Ornithogalum narbonense L. 4 c. 28

208. Paris quadrifolia L. $4 \mathrm{M}$.

209. Polygonatum multiflorum All. 4 c. 28 210. Puschkinia scilloides Adans. 4 c. 28

211. Scilla autumnalis L. 4 K. 27

212. " bifolia L. 4 c. 28

213. Zygadenus sibiricus A. Gray ${ }^{4}$

214. Veratrum c. 28 anum Bernh. 4 c. 28

\section{Dicotyledoneae.}

Aceracea e $\mathrm{Neck}$. 215. Acer platanoides $L$. $\hbar$ G. 28 216. " tataricum L. $\hbar_{2}$ G. 28.

A i z o a c e a e.

217. Mesembrianthemum tricolor $\odot$

218. Tetragonia expansa c. 27

. $\odot$

A m a ranth a c e e Jus s. 219. Amaranthus Blitum L. $\odot$ c. 28
220. Amaranthus caudatus $L$. $\odot$ c. 28 221 * $~^{2}$ deflexus L. 4 c. 26 222. " " retroflexus L. $\odot$ 223. " gangeticus L. $\stackrel{\odot \text { c. } 28}{28}$ Ampelidaceae H. B. K. 224. Vitis amurensis Rupr. 万 W. 26 A pocynaceae R. Br. 225. Vinca minor L. 4 c. 28 
Aristolochiaceae Blume. 226. Asarum europaeum L. 4 c. 28 Asclepiadaceae Lindl. 227. Asclepias Cornuti Decsn. 4 c.27 228. Cynanchum medium $K$. Schum. 229. Vincetoxicum ${ }^{2} \cdot{ }^{28}$ 4 c. 28

Balsaminaceae S. F. 230. Impatiens glanduligera Royle. $\odot$ $231^{*}$. \# noli tangere $L$. $\odot$ c. 28 Berberidaceae Torr. et. Gray. 232. Berberis vulgaris $L$. $\hbar$ G. 28 Betulaceae Rich.

233. Carpinus orientalis Mill. 支 K. 27 234. Corylus Avellana L. $\hbar$ G. 28 235. " Colurna L. $\hbar$ c. 28

Borragina cea e S. F. 236. Anchusa italica Retz. (.) c. 28 $237 \%$, officinalis L. 4 c. 27 238. Borrago officinalis L. $\odot$ c. 28 239. Cerinthe minor $L$. $\odot$ c. 28 240. Cynoglossum officinale L. 4 c. 27 $241^{*}$. Echium altissimum Jacq. $\odot$ c. 26 242. " vulgare $L$. $\odot$ c. 28 243. Heliotropium europaeum L. $\odot$

244. $\quad$ peruvianum L. 4 c. 27

245. Lappula Myosotis Moench. $\odot$ c. 28 246. Lindelofia spectabilis Lehm. 4 c 2 ; 247. Lithospermum officinale L. 4 248. Lycopsis arvensis L. $\odot$, $\odot$ c. 28 249. Myosotis intermedia Link. $\odot, \odot$, 250. \# silvatica Hoffm. $\odot, 2$ c. 28 251. Nonnea pulla D. C. 4 c. 27 252. versicolor $\mathrm{S} w$. c. 26 253. Omphalodes linifolia Moench. $\odot$ c. 28 $254^{*}$. Pulmonaria officinalis $L .4$ c. 28 255. Symphytum officinale L. 4 c. 28
Campanulaceae Juss. 256. Adenophora Bulleyana Diels 4 c. 28

257. Campanula abietina Gris c. 28 258. ” alliariaefolia Willd. 4 c. 28 259. \# americana $L$. $\odot$ c. 28 260. " $\quad$ carpathica Jacq. 4 c 28 261. " $\quad$ cervicaria $L . \odot, 4$ 262 c. 28 4 c. 28 263. \# erinus $L . \odot$ c. 28 264 * " glomerata L. 4 c. 28 265. " " L. v. acaulis 4 c. 28 266. " grandis Fisch.et. Mey. 4 c. 28 267. " grossekii Heuff. 4 c. 28 268. " lactiflora $M . B .4$ c.28 269. " latifolia L. 4 c. 28 270. " " Leutweinii Heldr. 4 271. longistyla Fomin 28

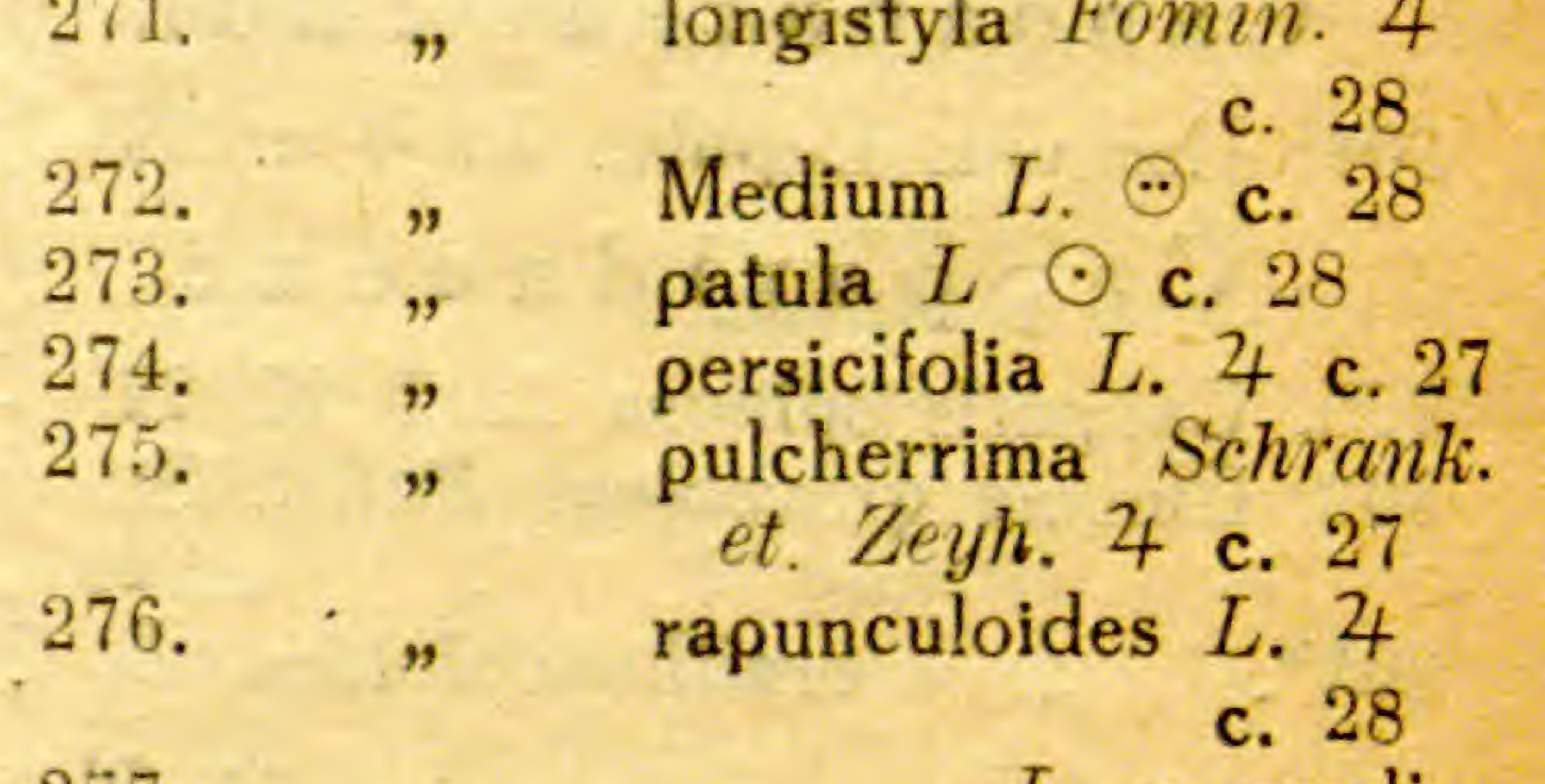

277. „ " L. v. cordifolia C. Koch. 4 c. 28 278.* " Rapunculus L. $\odot$ c.27 $279 . \quad " \quad$ Reuteriana Boiss. et. Bal. c. 28. 280. " rotundifolia L. 4 c. 28 281. " " L. v. Hostii 282. 4 c. 28 282. sarmentosa Hochst.
c. 28. 283. " Steveni M. B. 4 c. 28 284. " "Tomasiniana Koeh. 285. " $\quad$ trachelium L. 4 c. 28 286. " " Van Houttei Carr. 4 c. 28 287. Clintonia pulchella Lindl. $\odot$ c. 28 288*. Codonopsis ovata Benth. 4 c. 27 
289. Conodopsis ussuriensis Hemsl. 4 c. 28 290*. $\quad$ viridiflora Maxim. c. 28 291. Jasione montana L. $\odot$ c. 28 292. " perennis Lamarck. 4 c. 28 293*. Laurentia Michelii $A . D$. C. $\odot$ 294.* Lobelia erinus $L$. $\odot$ c. 27 295. \# inflata $L$. $\odot$ c. 28 295a. syphilitica $L .4$ c. 27 296. Phyteuma canescens W. et. Kit. 4 c. 28 297.,$\quad$ spicatum L. 4 c. 28 298. Platycodon grandiflorum $D . C$. 4 c. 28

299. Prismatocarpus strictus $D . C$.

c. 28 300. Specularia hybrida $D . C$. $\odot$ c 28 301. ” Speculum D.C. $\odot$ c.28 302. Symphyandra Hoffmannii Pant.. c. 28

303. Wahlenbergia pendula Schrad. ค) c. 28

Cannabace a e Endl. 304. Humulus Lupulus L. 4 c. 28 C a p parid a c e a e 305. Capparis spinosa $L$. $\hbar$ K. 27 306. Cleome graveolens Rafin. $\odot$ $307 * . \quad$ ornithopodioides $L . \odot$
K. 27

Caprifoliaceae Vent. 308. Adoxa Moschatellina L. 4 c. 28 309. Lonicera tatarica $L$. 万ै c. 28 310. Sambucus nigra $L . \hbar$ c. 25 311. ” racemosa $L$. मे G. 28 312. Symphoricarpus racemosa Mchx. 古 c. 28 313. Viburnum Lantana L. $\hbar$ c. 27 314. " Opulus L. 5 G. 28 Caryo $\rho$ h y lla ceae Juss. 315. Agrostemma Githago L. $\odot$ c. 28 316. Alsine pinifolia Fenzl. 4 c. 25 $317^{*}$. Arenaria graminifolia Schrad. 318 . 4 c. 28 319 " $\quad$ longifolia $M$. B. 4 c. 28 320. Cerastium arvense $L$. c. 2
321. Cerastium ca€spitosum Gilib. 4 c. 28 322. Corrigiola littoralis $L$. $\odot$ c. 28 323. Cucubalus baccifer L. 4 c. 28 324. Dianthus arenarius L. 4 c. 28 325. ” barbatus L. 4 c. 28 326. " Borbasii Vandas. 4 c. 28 327. $\quad$ campestris M.B. 4 228 c. 28 328. capitatus D. C. 4 c. 27 329. " " chinensis L. $\odot$ c. 28 330. " collinus Waldst. et. Kit. 4 c. 28 331. " deltoides L. 4 c. 28 332. " giganteus D.' Urv. 4

333. Heddewigi Hort. 28

c. 28

334. " laciniatus $\odot$ c. 28 335. " plumarius $L .4$ c. 336. " polymorphus $M$. B. 4 337. Elisanthe noctiflora $R u p r$ c, 28 338. Cypsophyla elegans $M$. B. $\odot$ 339. " muralis L. $\odot$ c. 28 $340^{*}$. Herniaria glabra L. 4 c. 26 341. Holosteum umbellatum $L$. $\odot$, $\odot$ 342. Lychnis chalcedonica L. 4 c. 28 343. $\quad$ Flos cuculi $L .4$ c. 28 344. Malachium aquaticum $R c h b .4$ 345. 345. Melandryum album Garcke. $\odot$, 346. Sagina $\odot$ c. 28 procumbens $L .4$ c. 28 347. Saponaria officinalis $L .4$ c. 26 348. Scleranthus annuus $L . \odot$ c. 28 349. Silene Armeria $L$. $\odot$ c. 28 350. " Brotherana Som. Lev. c.27 351. " commutata Guss c. 26 352. " compacta Fisch. $\odot$ c. 28 353 . " conica $L . \odot$ c. 28 354. " conoidea $L . \odot$ c. 27 355. " chlorantha Ehrh. 4 c. 26 356 . " gallica $L . \odot$ c. 28

357. " italica Pers. 4 c. 28

358. " Otites Sm. $\odot, 4$ c. 26

359. " saxatilis Sims. c. 27 $360^{*}$. " sibirica Pers. 4 c. 26 361. " tatarica Pers. 4 c. 28 362. " venosa Aschers. 4 c. 28 363. ” wolgensis Pers. 4 c. 26 
364. Spergula arvensis $L$. $\odot$ c. 28 365. Spergularia campestris Aschers. $\odot$, $\odot$ c. 28 366. Stellaria media $L$. c. 28 367. " graminea L. 4 c. 27 368. Tunica prolifera Siop. $\odot$ c. 28 369. " Saxifraga Scop. 4 c. 28 $370^{*}$. Vaccaria pyramidata Medik.๑c. 28 371. Viscaria viscosa Aschers. 4 c. 28

Celastrace a e Lind 1 . 372. Evonymus europaeus $L . \hbar$ c. 27 373. $\Rightarrow$ verrucosus Scop. 市 G. 28

Chenopodiaceae Less. 374. Atriplex hastatum $L$. $\odot$ c. 28 375. $\quad$ hortense $L . \odot$ c. 28 376. Beta patellaris Moq. $\odot$ c. 28 377. Chenopodium album $L$. $\odot$ c. 28 378. \# Botrys L. $\odot$ c. 28 379. $" \quad$ foliosum Schrad. $\odot$ : c. 28 380. \# murale L. $\odot$ c. 28 381. " polyspermum L. $\odot$ c.28 382. " vulvaria $L$. $\odot$ c. 28 383. Corispermum hyssopifolium $L$. $\odot$ c. 28 384. Kochia arenaria Roth. $\odot$ c. 27 385. " trichophylla Schmeiss. $\odot$ c. 28 386. Monolepis trifida Schrad. $\odot$ c.28 387. Spinacia oleracea $L$. $\odot$ c. 28 388. tetrandra Stev. $\odot$ c. 28 C is taceae Dun. 389. Helianthemum Chamaecistus Mill. 4 c. 28

Compositae Adans.

390. Achillea Millefolium L. 4 c. 28 390 a. $\quad$ nobilis $L .4$ c. 28 391. " Ptarmica L. 4 c. 28 392. " $\quad$ trichophylla Schrenk. $\odot$ c. 28 393. Acroclinum roseum $\odot$ c. 28 394. Ageratum mexicanum Sims. $\odot$ 395. Anthemis altissima L. $\odot$ c. 28 396. $"$ arvensis $L . \odot$ c. 28 397. Arctotis grandis Thunb. $\odot$ c. 28 $398^{*}$. Arnica montana L. 4 c. 27 399. Artemisia Absinthium $L .4$ c. 28
400. Artemisia campestris L. 4 c. 28 401. $"$ vulgaris $L .4$ c. 28 402. Aster alpinus L. 4 c. 28 403. " salicifolius Scholl. 4 c. 28 404. Baeria coronaria A: Gray. $\odot$ c. 28 405. Bellis annua L. $\odot$ c. 28 406. " perennis L. 4 c. 28 407. Bidens cernuus L. $\odot \mathrm{M}$.

408. " leucantha Willd. $\odot$ c. 28 409. " tripartitus $L . \odot$ c. 28

410. Buphtalmum speciosum Schreb. 4 c. 28

411. Calendula officinalis $L$. $\odot$ c. 28 412. Callistephus chinensis Nees. $\odot$

413*. Carduus crispus $L, 4$ c. 28 414. Carlina vulgaris $L$. *. c. 27

415. Carpesium cernuum $L .4$ c. 28 416. Centaurea axillaris Willd. 4

c. 28

417. " bella Triv. c. 28

418. " " Calcitrapa L. v. iberica Trev. $\odot$ c. 28

419. " Cyanus L. $\odot$ c. 28

420. " crocodylium $L$. $\odot$ c. $28 \rightarrow$

421.* " glastifolia L. 4 c. 28

422. " Jacea L. 4 c. 28

$423^{*}$. " Koenigii Sosn. c. 27

424. " maculosa Lam. $\odot$ c. 27

425. " reflexa Lam. 4 c. 28

426.* " Scabiosa L. 4 c. 27

427.* $" \quad$ salmantica $L . \odot$ c. 27

428. " salonitana Vis. 4 c. 28

429.* Chondrilla juncea $L .4$ c. 28

430. Chrysanthemum corymbosum $L$. 4 c. 28

431. " Leucanthemum $L$.

4 c. 28

432*. " roseum Web. et.

Mohr. 4 c. 27

433. $" \quad$ tricolor Hort. $\odot$

434. $\quad$ viscosum Desf. $\odot$ c. 28

435. Cichorium Intybus $L . ~ 4$ c. 28

436. Cirsium arvense Scop. 4 M

437. " lanceolatum Scop. $\odot$

c. 28

438. ", Maackii Max. 4 c. 28

439. " rivulare Link. 4 c. 28 .

440. Cnicus Benedictus L. $\odot$ c. 28

441. Coreopsis coronata Hoocker.c. 28 
442. Coreopsis peltata $L$. $\odot$ c. 28

443. Cosmea bipinnata Willd. $\odot$ c. 28

444. Crepis biennis $L$. $\odot$ c. 28

445. ” foetida $L$. $\odot$ c. 28

446*. ” paludosa Moench. 4 c. 27

$447^{*}$. " parviflora Desf. $\odot$ c. 27

448. " pulchra $L$. $\odot$ c. 28

449. " tectorum $L$. $\odot$ c. 28

450. Crupina vulgaris Cass. $\odot$ c. 28

451. Dahlia coccinea Cav. 4 c. 27

452. " variabilis Desf. 4 c. 27

453. Dimorphotheca aurantiaca D. C.

454 .

$\odot$ c. 28

c. 28

455. Echinops globif $\mathrm{e}$ Janka. 4 c. 28 $456^{*}$. Erigeron acer $L . \odot, 4$ c. 27 $457^{*}$.,$\quad$ alpinus $L .4$ c. 28 458. Eupatorium cannabinum L. 4 c. 28 459. Evax pygmaea Brot. $\odot$ c. 28 460. Felicia tenella Ness. $\odot$ c. 28 461. Filago spathulata Presl. $\odot$ c. 28 462.* Gaillardia picta Sweet. $\odot$ c. 27 463. Galinsoga parviflora Cav. $\odot$ c.27 464. Gnaphalium vira-vira Molina. $\odot$

465. Guizotia oleifera c. 28 466. Hedypnois cretica Willd. $\odot$ c. 28 467. Helenium Hoopesii A. Gray. 4

468. Helianthus cucumerifolius. 28 et. Gray. $\odot$ c. 28

469 . globosus $\odot$ c. 28

470. Helichrysum arenarium Moeneh. 4 c. 28

471. Hieracium Pilosella $L .4$ c. 28 472. " pratense Tausch. 4 473. - silvaticum L. 4 M. 474.*,$\quad$ umbellatum $L .4$ c. 27 475. virosum Pall. 4 c. 28 476. Hyoseris lucida $L$. $\odot$ c. 28 477. Hypochoeris radicata $L .4$ c. 28 478. Inula conyza $D . C . \odot, 4$ c. 27 479. " Helenium L. 4 c. 28 490. " magnifica Lipsky. c. 28 $481^{*}$. oculus christi $L .4$ c. 27 482*. Jurinea cyanoides Rchb. 4 c. 27 483*. Kalbfussia Salzmannii Sehulz. 4

484. Lactuca sativa $L$. $\odot$ c. 26 c. 26 485.* viminea Presl. $\odot$ c. 28
486. Lampsana communis $L . \odot$ c. 27 487. " grandiflora M. B. 4 488. Lappa amplissima Boiss. $\stackrel{\text { c. }}{-}$ c. 28 489. " tomentosa $\mathrm{Lam} . \odot$ c. 28 490. Lasiospermum pedunculare Lag. 491. c. 28 491. Layia Calliglossa Gray. $\odot$ c. 28 492. Leontodon autumnalis $L .4$ c. 28 $493^{*}$. Leontopodium alpinum Cass. 4 494.* Liatris spicata Willd 4 c. 28 495. Madia sativa $\mathrm{Mol} \odot$ c 28.25 496. Matricaria Chamomilla $L$. $\odot$ c. 28 497. $\quad \quad$ inodora $L . \odot, \odot$ c. 28 498. " $\quad$ suaveolens Buchen. $\odot$ 28

499. Onopordon Acanthium L. $\odot$ c. 28 $500 *$. Petasites officinalis Moench. 4 G. 26

501 Picris hieracioides $L . \odot, 4$ c. 28 502. Psephellus dealbatus Boiss. c. 27 503*. \# hypoleucus Boiss.c. 27 504. Pyrethrum Parthenium Sm. 4 $505 . \quad$ c. 27. 506. Rhagadiolus stellatus $D .4$ c. 2 507. Rudbeckia hirta $L$ c. 28 508. " laciniata $L .4$ c. 28 509. Scorzonera eriosperma $M . B .4$ 510. " hispanica $L .4$ c. 28 511. " laciniata $L \quad \odot, 4$ c. 28 512. Senecio erraticus Bertol. $\odot$ c. 27 513. " Jacobaea L. 4 c. 27 514. " ligularia Hook. 4 c. 27 515. " vulgaris $L . \odot . \odot$ c. 28 516. Serratula coronata $L 4$ c. 28 517. " heterophylla Desf. 4 $518^{*}$ c. 28 519. Siegesbeckia orientalis 4 c. 26 520. Silybum Marianum Gaertn. $\odot$, 52. Sonchus arvensis L. 4 c. 28 523. " asper Hill $\odot$ c. 28 523 a. $" \quad$ nleraceus $L$. $\odot$ c. 28 524. Tagetes erecta $L$. $\odot$ c. 28 525. " lucida Cav. 4 c. 28 526. " patula $L . \odot$ c. 28 527. Tanacetum vulgare $L .4$ c. 28 
$528^{*}$. Taraxacum bessarabicum Hand. Mat. 427

529. \# laevigatum D. C. 4

530. $\quad$ c. 28

c. 28

531. Tragopogon brevirostris $D . C . \odot$

532. $\quad$ coloratus C.A. $M$

533. " major Jacq. c. 28

534. " $\quad$ porrifolius L. -.) c. 27

$535 . \quad$ pratensis L. * c. 28

536. Tussilago Farfara L. 4 c. 28

537. Urospermum picroides $F$. W. Schmidt. $\odot$ c. 28

538. Ximenesia encelioides Cav. $\odot$

539. Zacintha verrucosa c. 28

c. 28

540. Zinnia elegans Jacq. $\odot$ c. 28

541. " multiflora L. $\odot$ c. 28

54\%. " tenuiflora Jacq. $\odot$ c. 28

7 Convolvulaceae Vent.

543. Convolvulus tricolor $L$. $\odot$ c. 28

544 . $”$ undulatus $C a v . \odot$ c.28

545. Ipomoea purpurea Lam. $\odot$ c. 28

Cornacea e D. C.

546. Cornus mas L. 5 K. 27

Crassulacea e D. C.

547. Crassula glomerata Berg. $\odot$ c. 28

548. Sedum acre L. 4 c. 28

549. " aizoon L. 4 c. 28

550*. " Ewersii Ledb. 4 c. 28

551. " glaucum W. et. K. 4 c. 28

552. " hybridum L. 4 c. 28

553. " kamtschaticum F. et. $M$.

554. \# pallidum M. B. $\stackrel{4}{+}$ c. 28

555. " purpureum Link. 4 c. 28

556. " spurium M. B. 4 c. 28

Cruciferae Juss.

557. Aethionema Buxbaumii D.C. $\odot$ c.28

558. Alyssum desertorum Steph. $\odot$

c. 28

559. " linifolium Steph. $\odot$ c. 28 $560^{*}$. " repens Baumg. 4 c. 27 561. Arabis albida Stev. 4 c. 28

562. \# hirsuta Scop. $\odot, 4$ c. 28
563. Arabis laxa Sibth et. Sm. c. 28 564. Barbarea stricta Andrz. *. c. 28 56.5. " vulgaris $R . \quad B r . \odot$ c. 28 $566 *$. Berteroa incana $D . C . \odot, \odot$ c. 28

567. Biscutella cicchoriifolia Loisl. $\odot$

$$
\text { c. } 28
$$

$568^{*}$. Brassica juncea Czern. $\odot$ c. 28

569. Bunias orientalis $L .4$ G. 28

570. Camelina sativa Crantz. $\odot$ c. 28

571. Capsella bursa pastoris Moench.

572. Carrichtera Vella $D$ c. 28

573. Cheiranthus nanus Merk. * c. 27

574. Chorispora tenella D. C. $\odot$ c. 28

575. Cochlearia officinalis L. 4 c. 28

576. Conringia orientalis Dum. $\odot$ c. 28

577. Crambe hispanica L. $\odot$ c. 28

578. Descurainia Sophia Webb. et.

$$
\text { Berth. } \odot \text { c. } 28
$$

579. Diplotaxis muralis $D . C$. $\odot$ c. 28

$580 *$. Draba hirta L. 4 c. 28

581\%. Erinus alpinus $L .4$ c. 28

582. Erysimum cheiranthoides $L$. $\odot$

583. \# cuspidatum D. $\stackrel{\text { C. }}{28} \odot$

$$
\text { 584. } \quad \text { pulchellum J. Gay. }
$$

$785 . \quad$ c. 28
suffruticosum c. 28

586. Eruca sativa Lam. $\odot$ c. 28

587. Erucastrum elongatum L. 2 c. 26

588. Farsetia clypeata $R . B r .4$ c. 26

$589 . \quad$ eriocarpa D. C. 4 c. 27

590. Hesperis fragrans Fisch. 4 c. 28

591. " lutea Maxim. $\odot$ c. 28

592. " matronalis L. 4 c. 28

593. Iberis pectinata Boiss. $\odot$ c. 28

594. " sempevirens L. 4 c. 27

595. \# umbeliata $L . \odot$ c. 28

596 Isatis tinctoria $L$. $\odot$ c. 28

$597 *$. Lepidium Draba L. 4 c. 28

598. " $\quad$ ruderale $L . \odot$ c. 28

599. " $"$ sativum L. $\odot$ c. 28

600. Lunaria biennis Moench. $\odot$ c. 28

$601^{*}$. $\Rightarrow \quad$ redeviva $L .4$ c. 28

602. Malcolmia africana Ait. $\odot$ c. 28

603 Matthiolla annua Sweet. $\odot$ c. 28

604. \# bicornis $\odot$ c. 28

605. Melanosinapis communis Spenn.

(.) 27

606. Myagrum perfoliatum $L$. $\odot$ c. 28

607. Nasturtium palustre D.C. 4 G. 28 
608. Neslia paniculata Desv. $\odot$ c. 28 609. Peltaria alliacea $L .4$ c. 28 610. Raphanus Raphanistrum $L$. $\odot$ c. 28

611. Rapistrum rugosum All. $\odot$ c. 27 612. Senebieria Coronopus Poir. $\odot$

613. Sinapis alba $L$. $\odot$ c. 28 c. 28

614. ” apula Tenore. $\odot$ c. 28 615. Sisymbrium Irio L. 4 c. 28 616 . $\quad$ officinale Scop. $\odot$ c. 28 617. Stenophragma Thalianum Celak. ค. 28

618. Succovia balearica Medic. $\odot$ c. 619. Thlaspi arvense $L . \odot$ G. $28^{2}$ 620. Turritis glabra $L$. $)$ c. 28

Cucurbitace a e Holl. 621. Bryonia alba L. 4 c. 28 622. Cucumis Chito Naud. $\odot$ c. 27 623 . " prophetarum L. $\odot$ c. 28 624. Cucurbita maxima Duch. $\odot$ c. 28 625., melanosperma $A$. Br. 626. " Melopepo L. $\odot$ c. 28 627. " napolitana Reg. $\odot$ c. 628. $\quad$ Рepo L. $\odot$ c. 28 629 . " verrucosa $L$. $\odot$ c. 28 630. Cyclanthera pedata Schrad. $\odot$ 631*. Ecballium elaterium Rich. $\odot$ c. 28 632. Lagenaria vulgaris Ser. $\odot$ c. 28 633. Melothria cucumerina Naud. c. $634^{*}$. \# punctata Cogn. c. 27 635. Sicyos angulata $L$. $\odot$ c. 27 D a $t$ is c a c e a 636. Datisca cannabina L. 4 c. 28 Dipsacaceae D. C. 637. Callistemma brachiatum Sibth. (.) c. 28 638*. Cephalaria media Litw. c. 27 639. $\quad$ syriaca Schrad. 4 c. 25 640. $\quad$ tatarica Schrad. 4 641. Dipsacus laciniatus $L \quad$ c. 28 642. Knautia arvensis Coult. 4 c. 28
643. Pterocephalus plumosus Coult. $\odot$ c. 26

644. Scabiosa atropurpurea $L$. $\odot$ c. 28 645. " Columbaria L. 4 c. 27 646. " micrantha Desf. $\odot$ c. 28 647. " ochroleuca L. 4 c. 27 648. " ucrainica $L . \odot$ K. 27 649. Succisa praemorsa Aschers. 4 M.

Empetraceae Lind l. 650*. Empetrum nigrum L. 万 G. 27

E ric a ce a e Lind I. 651.* Calluna vulgaris Salisb. $\hbar$ G. 28

Euphorbiaceae J. St. Hil. 652. Euphorbia Chamaesyce L. $\odot$ K 27 653. ” Esula L. 4 G. 28 $654 . \quad \Rightarrow \quad$ exigua $L$. $\odot$ c. 28 655. " " graeca Boiss. et. Sprun. $\odot$ c. 28 $656 . \quad$ helioscopia $L . \odot$ c. 28 $657^{*}$. humifusa Willd. $\odot$ 658. $\quad$ uralensis Fisch. 4 c. 28 659. Mercurialis annua $L$. $\odot$ c. 26 660. Ricinus communis $L$. $\odot$ c. 27

Gentianaceae Dumort. 661: Gentiana cruciata L. 4 c. 28 662. " Pneumonanthe L. 4 c. 28 663. Menyanthes trifoliata $L .4$ G. 26 $664^{*}$. Swertia longifolia Boiss. 4 c. 28 Geraniaceae J. St. Hil. 665. Erodium cicutarium L. Herit. $\odot$ c. 28

666. $" \quad$ gruinum Ait. $\odot$ c. 28

667. Geranium albanum M. B. 4 c. 28 668. " dissectum L. $\odot$ c. 28 669. " divaricatum Ehrh. 4 $670 . \quad$ palustre L. 4 c. 27 671. " " pratense L. 4 c. 28 672. " $"$ pusillum L. $\odot$ c. 28 $673^{*}$. " $\quad$ sanguineum L. 4 c. 28 Globulariaceae D. C. 674. Globularia trichosantha $F$. et. $M$. 4 c. 28

675. " Willkommii Nyman. 4 c.28 


\section{Guttiferae Endl.}

676. Hypericum Ascyron L. 4 c. 28 677. " montanum L. 4 c. 28 678 . " perforatum L. 4 c. 28 679. $\quad$ " quadrangulum L. 4 c. 28

Hydrophyllaceae. 680. Nemophila maculata Benth. $\odot$ c. 28 681. Phacelia bipinnatifida Mich. 4

divaricata $A$ c. 28

683. " $\quad$ tanacetifolia $\begin{aligned} & \odot \text { c. } \text { centham }^{28} \\ & \odot \text { c. } 27\end{aligned}$

Labiat a e Juss.

684. Ajuga reptans L. 4 c. 28

$685 . \quad ” \quad L$. var. albiflora Tin. 4 c. 28

686. Ballota nigra $L .4$ c. 28

687. Betonica officinalis L. 4 c. 28

688. Brunella vulgaris $L .4$ c. 28

7. 689. Dracocephalum moldavica $L$. $\odot$

690.

Ruyschiana L. 4 c. 28

691. Elscholtzia cristata Willd. $\odot$ c.28 692. Eremostachys laciniata Bge. 4 c. 28 693. Galeopsis speciosa Mill. $\odot$ c. 28 694. ” Tetrahit. L. $\odot$ c. 28 695. Glechoma hederacea L. 4 c. 28 $696^{*}$. Horminium pyrenaicum L. 4 c. 28

697. Hyssopus officinalis $L . \hbar$ c. 28 698. Lallemantia iberica Fish. et. Mey.

699. peltata F. et. M. $\stackrel{2}{28}$ c. 28

700. Lamium album L. 4 c. 28

701. ” amplexicaule $L . \odot$, $\odot$ c. 28

702*. „ Galeobdolon Crantz. 4 c. 28

703*. ” maculatum L. 4 c. 27

704. " purpureum L. $\odot, \odot$ c. 28

705. Leonurus Cardiaca L. 4 c. 28

706. " Marrubiastrum L. $\odot$ c.) 26

707. Lycopus europaeus $L .2 \perp$ c. 28 708. $\quad$ exaltatus L. 4 c. 28 $709^{*}$. Marrubium vulgare L. 4 c. 27
710. Melissa officinalis L. 4 c. 27

711. Mentha arvensis L. 4 c. 28

712*. " longifolia Huds. 4 c. 27

713. " piperita L. 4 c. 28

714. ${ }^{*}$ Monarda fistulosa $L . ~ 4$ c. 27

715. Nepeta Cataria L. 4 c. 28

.716. ” grandiflora M. B. 4 c. 28

717. ” macrantha Fisch. 4 c. 28

718. " nuda L. 4 c. 28

719*. Ocimum Basilicum L. $\odot$ c. 27

72 C. Origanum vulgare $L .4$ c. 28

721. Phlomis pungens Willd. 4 c. 27

722. " tuberosa $L .4$ c. 28

723. Plectranthus glaucocalyx Max.

724. Pycnanthemum lanceolatum c. 28 Pursh. 4 c. 28

725. Salvia Aethiopis L. 4 c. 27

726. " azurea Lam. 4 c. 28

727. " cleistogama De Bar. et.

Paul. 4 c. 28

728. " dumetorum Andrz. c. 28

729. " glutinosa L. 4 c. 28

730. " nemorosa L. 4 c. 28

731. " nilotica Murr. $\odot$ c. 28

$732 *$. " nutans L. 4 c. 27

733*. " officinalis $L$. मे c. 26

$734 *$. " scabra Thunb. 5 c. 27

735. " Sibthorpii Sm. 4 c. 28

$736^{*}$. " triangularis Thunb. ₹ c.27

737. " Verbenaca L. 4 c. 27

738. " verticillata $L .4$ c 28

739. Satureja Clinopodium Caruel. 4 c. 28

740. " " var. variega-

tum Mort. 4 c. 28

741. " hortensis $L . \odot$ c. 28

742. " serpyllifolia Briq. 5

713.

743. Scutellaria altissima $L .4$ c. 25

744. $\quad$ galericulata $L .4$ c. 28

745 . $\quad$ orientalis $L .4$ c. 28

746. Sideritis montana $L$. $\odot$ c. $28 *$

$747^{*}$. Stachys annua $L$. $\odot$ c. 28

748. - lanata Jacq. 4 c. 28

749. " palustris $L .4$ c. 28

750 . $\quad " \quad$ recta $L .4$ c. 28

751. " sideritoides $C$. Ko $\mathrm{ch}$.

- c. 28

752. \# silvatica L. 4 c. 28

753. Teucrium Chamaedrys $L .4$ c 28

754 . $"$ hyrcanicum L. 4 c. 27

755. Thymus glaber Mill. 4 c. 28 
756. Thymus serpyllum $L$. v. citriodora 4 c. 28

Le gum inos a e Jus s.

757. Amorpha fruticosa $L . \hbar$ c. 27 $758^{*}$. Astragalus Cicer L. 4 c. 28 759. $"$ glycyphyllus L. 4 $760 \%$. $\quad$ Onobrychis L. 4 c. 28 761. " sulcatus L. 4 c. 28 762. Caragana frutescens $D . C . \hbar$ c. 28

763. Colutea orientalis Mill. $\hbar$ c. 27

764 Coronilla varia $L .4$ c. 28 765. Cytisus hirsutus $L$. $\hbar$ c. 28 766. " scoparius Link. ई M. 25 767. Dolichos Lablab L. $\odot$ c. 28 768. Dorycnium herbaceum Vill. 市

769. Ervum Lens L. $\odot$ c. 28 c. 27

770. Galega orientalis Lam. 4 c. 28 771. Genista tinctoria $L . \hbar$ c. 28 772. Glycyrrhiza echinata L. 4 c. 27 $773^{*}$. Hedysarum obscurum L. 4 c. 25 774. Lathyrus Aphaca L. $\odot$ c. 27 775. " canescens Taubert. 4

776. \# Clymenum L. $\odot$ c. 28 777. „ montanus Bernh. 4

778 c. 27

779 " ochrus D. C. $\odot$ c. 28

779 . " odoratus $L$. $\odot$ с 28

780 . " palustris L. 4 c. 28

$781^{*}$. " $\quad$ pratensis L. 4 c. 28

782. $" \quad$ sativus $L . \odot$ c. 28

783. " silvestris L. 4 c. 28

$784^{*} \quad$ sphaericus Retz. $\odot$

c. 27

785. " vernus Bernh. 4 c 28

786. Lotus corniculatus L. 4 c. 28

787. "edulis $L$. $\odot$ c. 28

788. ” tetragonolobus $L$. $\odot$ c. 28

$789^{*}$. Lupinus albus $L$. $\odot$ c. 28

790. . leporinus c. 28

791. $\quad$ mutabilis $S w$. c. 28

792. " perennis L. 4 c. 28

793. " pilosus $M u r r . \odot$ c. 28

794. $\#$ sp. fl. albo c. 27

795. Medicago catalonica Schk. c. 28

796. " laciniata Mill. $\odot$ c. 28

797. " lupulina $L . \odot, 4$ c. 28

$798 *$ "

\begin{abstract}
maculata Willd. $\odot$
\end{abstract}
799*. Medicago orbicularis All. $\odot$ c. 28

800. " sativa L. 4 c. 28

801. $"$ scutellata All. $\odot$ c. 28

802. Melilotus albus Desr. $\odot$ c. 28

803. " indica All. $\odot$ c. 28

804 . " $\quad$ officinalis Desr. . c.28

805. " wolgicus Poir. $\odot$ c. 28

806. Onobrychis viciaefolia Scop. 4

c. 25

807. Ononis biflora Desf. ๑ c. 28

808. \# hircina Jacq. 4 c. 28

809. Ornithopus sativus Brot. $\odot$ c. 28

810. Pisum arvense $L$. $\odot$ c. 28

811. " elatius $M$. B. $\odot$ c. 28

812. " Jomardi Schrank. $\odot$ c. 28

813. " sativum L. $\odot$ c. 28

814. ” thebaicum $W . \odot$ c. 28

815. Robinia pseudacacia $L$. $\hbar$ c 28

816. Scorpiurus subvillosa $L$. $\odot$ c. 28

817. $" \quad$ vermiculata $L$. $\odot$ c. 28

$818^{*}$. Thermopsis caroliniana $M$. A. Curt. 4 c. 28

819. „ fabacea D.C. 4 c. 28

$820^{\circ}$. Trifolium agrarium $L$. $\odot$ c. 27

821. " angustifolium L. $\odot$ c. 28 \&

822. ", arvense L. $\odot$ c. 28

823. " " badium Schreb. $\odot$ c.

824.28

824. " $\quad$ cernuum Brot. $\odot$ c. 28

825. , fragiferum L. 4 c. 28

826. " incarnatum L. $\odot$ c. 28

827 . " $\quad$ glomeratum L. $\odot$ c. 28

828. " "upinaster L. 4 c. 28

829 . " " medium L. 4 c. 27

830. " montanum L. 4 c. 28

831. " pratense L. 4 c. 28

832. " procumbens L. $\odot$ c.28

833. " $"$ repens L. 4 c. 28

834. " " resupinatum $L \odot$, $\odot$

835 - c. 28

835. " $\quad$ spadiceum $L . \odot$ c. 28

836. " $\quad$ striatum L. $\odot$ c. 28

$837^{*}$. $"$ tomentosum L. $\odot$. c. 28

838. Trigonella coerulea Ser. $\odot$ c. 28

839. "foenum-graecum L.c $\odot$ c. 28

840. " orthoceras Kar. et. Kir. $\odot$ c. $2 \varnothing$

841. Vicia angustifolia Roth. $\odot$ c. 28

842. " atropurpurea Desf. $\odot$ c. 28

843. " cracca L. 4 c. 28

844. " Ervilia Willd. $\odot$ c. 28

845. "Faba L. $\odot$ c. 28

846. " hirsuta G. Koch. $\odot$ c. 28 
847. Vicia lutea $L$. $\odot$ c. 28

848. " Michauxii Spreng. $\odot$ c. 28

849. " monanthos Desf. $\odot$ c. 28

850. " narbonensis L. $\odot$ c. 28

851. " picta Fisch. $\odot$ c. 27

852. " sativa $L$. (sem. alb.) $\odot$ c 28

853. " sepium L. 4 c. 28

854. ” unijuga $A$. Br. 4 c. 28

$\mathrm{Li} m \mathrm{n}$ a $\mathrm{th}$ a ce a e.

855. Limnanthes alba Hartw. $\odot$ c. 28

856. " Douglasii $R . B r . \odot$

$$
\text { c. } 28
$$

Linacea e Dumort.

857. Linum Lewisii Pursh. 4 c. 27

858. " nervosum $W$ K. 4 c. 28

859 . " perenne L. 4 c. 28

860. ” usitatissimum L. $\odot$ c. 28

$\mathrm{L}$ o a $\mathrm{s}$ a c e a e.

861. Blumenbachia Hieronymi Urb. $\odot$ xa Loasa bryoniaefolia Schro 28

863. ” triphylla Juss $\odot$ c 28

864 . ” urens Juss. $\odot$ c. 28

$865 \Longrightarrow$ vulcanica Andre. $\odot$ c. 28

Lythraceae Lindl.

866. Cuphea lanceolata Ait. $\odot$ c. 28 $867^{*}$. Lythrum salicaria L. 4 c. 27 $868^{*}$. Nesea salicifolia $H . B$, et $K$. 古

$869^{*}$. Peplis Portula L. $\odot$ c. 27 c. 28

$\mathrm{M}$ a l va c e a e Jus s. $870^{*}$. Abutilon Avicennae Gaertn. $\odot$. c. 27 871. Althaea ficifolia Cav. 4 c. 28 872. " officinalis L. 4 c. 27 873. $" \quad$ rosea Cav. 4 c. 28

874. Anoda cristata Schlecht. $\odot$ c. 28

875. Hibiscus Trionum $L \odot$ c. 27

876. Lavatera thuringiaca L. 4 c. 27

877. $\quad$ trimestris $L . \odot$ c. 27

878. Malope grandiflora $\odot$ c. 28

879. Malva Alcea $L .4$ c. 27

$880 . \quad$ " crispa $L . \odot, \odot$ c. 28

$881^{*}$. " hispanica L. c. 28

882. " neglecta Wall. $\odot$ c. 28

883. " parviflora $L . \odot$ c. 28

884. " rotundifolia $L$. $\odot$ c. 28
885. Malva silvestris $L . \odot$ c. 27

886. Malvastrum peruvianum A. Gray.

$887^{*}$. Pavonia hastata $\mathrm{Cav} . \stackrel{\odot}{ }$ c. 28

Moracea e Lind 1 .

888. Morus alba $L$ 古 c. 28

Nyctagin a c a e.

889. Mirabilis Jalappa $L$. $\odot$ c. 27

890\%. \# Wrightii Dcne. $\odot$ c. 27

Oleaceae Lindl.

891. Fraxinus excelsior $L$. $\hbar$ G. 28

892. Ligustrum vulgare $L . \hbar$ c. 28

893. Syringa vulgaris $L$. मे G. 28

On a g r a ce a Lind $\mathrm{l}$.

894. Boisduvalia densiflora Wats. $\odot$

895 c. 28

895. " Douglasii Spach. $\odot$

896. Chamaenerium c. 27

896. Chamaenerium angustifolium

897. Clarkia elegans Dougb. ${ }^{2}$ G. 25
c. 28

898. Epilobium adnatum Gris. 4 c. 28

899. " hirsutum L. 4 c. 28

$900 . \quad$ " Lamyi F. Schultz 4

901 c. 28

902. " montanum L. 4 c. 27

903. " palustre L. 4 G. 27

904. Eucharidium grandiflor ${ }^{2}$ pedunculare 28

M. $\odot$ c. 28

905. Gaura parviflora Dougl. $\odot$ c. 28

906. Godetia grandiflora Lindl. $\odot$

907. c. 28 c. 28

908 c. 28

909 " biennis $L \quad$ c. 28

$910 \quad$ " fruticosa L. 4 c. 28

911. " " gigas De Vries c. 27

912 " grandiflora Ait. $\odot$ c. 27

912. " Lamarkiana L. 4 c. 28

missouriensis Sms. 4

914. c. 28

915. " muricata $L . \odot$ c. 28

916. " odorata Jacq. $\odot$ с 28

916. \# pumila L. 4 c. 28

$917^{*}$. " $\quad$ rosea Ait 4 c. 26

918. " tetraptera Cav. $\odot$ c.28

919. " $\quad$ triloba Nuttal. $\odot$ c. 28 
Oxalidaceae Lindl. 920. Oxalis corniculata $L . \odot, \odot$ c. 28 921. " stricta L. 4 c. 28

\section{Papaveraceae Jus s.}

922. Argemone mexicana L. $\odot$ c. 28 923. Chelidonium FranchetianumPrain.

924* laciniatum Mill 2

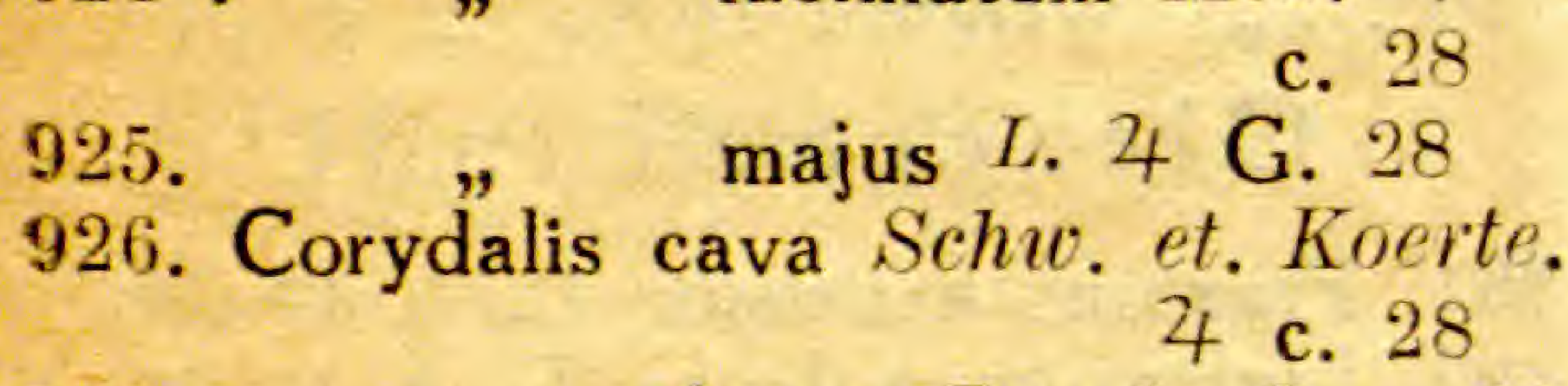

927. „ glauca Purch. $\odot$ c. 28

928. $" \quad$ ophiocarpa Hook. f. et. Thoms. 4 c. 28

929. " sempervirens Pers. $\odot$

c. 28

930. " $\quad$ vaginans Royle. c. 28

931*. " vesicaria Pers. $\odot$ c. 28

933. Eschscholtzia californica Cham.๑

c. 28

934. Fumaria officinalis $L . \odot$ G. 28

935. Glaucium flavum Crantz..$\cdot 28$

936. Hypecoum grandiflorum Benth.

937. $"$ pendulum L. $\odot$ c. 28 938. Meconopsis cambrica Vig. 4 c. 28 939. Papaver alpinum L. 4 c. 26

940. $\quad$ argemone $L$. $\odot$ c. 28

941. " atlanticum Ball. 4 c. 28

942. $"$ dubium L. $\odot$ c. 28

943. $"$ fugax Poir. $\odot$ c. 28

944. $"$ orientale L. 4 c. 28

945. " pilosum Sibth. 4 c. 28

$946 *$. " radicatum Rottb. c. 28

947. " Rhoeas L. $\odot$ c. 28

948. " somniferum L. $\odot$ c. 28

Passifloracea e.

949. Passiflora edulis Sims. $\hbar 27$

950*. \# gracilis Link. 28

951*., suberosa L. \ 27

Philade $1 \rho$ h a ce a e.

952. Philadelphus coronarius $L . \hbar$

c. 28

Ph y tol a c c a c e a e.

953. Phytolacca decandra L. 4 c. 27
Plantaginacea e Lind 1 .

954. Plantago alpina $L . ~ 4$ c. 28

955. $\quad$ altissima $L$. c. 27

956*. " $\quad$ arborescens Poir. 4

c. 28

$957 *$. $\quad$ Brownii Rafin. c. 28

958. " Candollei Rafin. c. 28

959. " Cornuti Gouan. 4 c. 28

960. " crassifolia Forsk. c. 28

961. " Cynops L. 4 c. 27

962. $\quad$ indica $L$. c. 28

963. " lanceolata L. 4 c. 28

964. ” lusitanica Willd. $\odot$ с 28

965. " major L. 4 c. 28

966. ” maritima L. 4 c. 28

967. " maxima Ait. 4 c. 28

968. " media L. 4 c. 28

969. " $\quad$ Psyllium L. $\odot$ c. 28

$970 . \quad \# \quad$ ramosa Aschers. $\odot$ c. 28

971. $\quad$ serraria L. c. 28

972. " virginica L. $\odot$ c. 28

Plumbaginaceae Lindl.

973. Plumbagella micrantha Ledeb. $2 \mathrm{R}$

974. Statice tatarica $L .4$ c. 27

$$
\text { c. } 28
$$

Polemoniaceae D. C. 975. Collomia grandiflora Dougl.

c. 28

976. " linearis Nutt. $\odot$ c. 28

977. Gilia californica Benth. $\odot$ c. 28

978. Navarretia squarrosa Hook. et. Arn. $\odot$ c. 28

979*. Phlox Drummondi Hook. $\odot$ c. 27

980. Polemonium coeruleum L. 4 c. 28

Polygonaceae Lindl. 981. Calligonum aphyllum Gürke 527 982. Emex spinosa Campd. -) c. 28 983. Fagopyrum tataricum Gärtn. $\odot$

984. Oxyria digyna Hill. 4 c. 28 985*. Polygonum alpinum $A l l .4$ c. 28 986. $\quad$ " aviculare $L$. $\odot$ G. 28 987. ", Bistnrta L. 4 c. 28 988. ", Convolvulus L. $\odot$ c. 28 989. " $\quad$ Fagopyrum $L . \odot$ 990. " Hydropiper L. $\odot$ c. 28 
991. Polygonum tomentosum Schrank.

992. Rheum Emodi Wall. 4 c. 27

993. " palmatum L. 4 c. 27

994. " Rhaponticum L. 4 c. 28

995. " undulatum L. 4 c. 28

996. Rumex acetosa L. 4 c. 28

997. \# Acetosella L. 4 G. 28

998. „ aquaticus L. 4 M.

999. ” confertus Willd. 4 M.

1000. " crispus L. 4 c. 27

1001. " obtusifolius L. 4 M.

1002. " pulcher L. 4 c. 27

1003. " scutatus L. 4 c. 28

\section{Portulacacea e Lind l.}

1004. Calandrina procumbens Moris. $\odot$ c. 28

1005. Claytonia perfoliata Donn. $\odot$

1006. Portulaca grandiflora Lindl. $\odot$ c. 28

1007. oleracea L. $\odot$ c. 27

7 1008. Taiinum patens Willd. 4 c. 27

Primulaceae Vent.

1009. Anagallis arvensis $L$. var. coerulea Gren. "et. Godr.

(.) c. 28

1010. " " var. phoenicea Gren, et. Godr. $\odot$

c. 28

1011. " grandiflora c. 28

1012. Androsace elongata L. $\odot$, $\odot$

$1013^{*}$. " septentrionalis L. $\odot$,

1014. Asterolinon stellatum Hoffm. et. Link. $\odot$ c. 28

1015. Lysimachia vulgaris L. 4 c. 28

1016. Primula auricula L. 4 c. 28

1017*. " cortusides L. 4 c. 28

$1018^{*}$. " denticulata $S w .4$ c. 28

$1019^{*}$. " elatior Hill 4 c. 28

$1020^{*}$. " $\quad$ farinosa L. 4 c. 28

1021*. ” Forbesii Franch. 4 c 28

1022*. " japonica A. Gray. 4

c. 28

1023. " officinalis Hill. 4 c. 28

1024. " Sieboldii Morr. 4 c. 28

1025*. Samolus Valerandi L. 4 c. 27
R anunculaceae Juss.

1026. Aconitum ferox Wall. 4 c. 28

1027. " lasiostomum Reichb. 4

1028. \# napellus L. 4 c. 26

1029. Actaea spicata $L$. 4 c 28

1030. " " var. rubra (Bigel.) 4 c. 28

1031. Adonis autumnalis $L$. $\odot$ c. 27

1032. Anemone multifida Poir. 4 c. 28

1033*. , nemorosa L. 4 c. 28

$1034^{*} \quad, \quad$ pratensis L. 4 c. 28

$1035^{*}$. " $\quad$ ranunculoides $L .4$

c. 28

1036. " rivularis Buch-Ham.

1037. " virginiana L. 4 c. 27

1038. Aquilegia atropurpurea W. 4

c. 28

1039. " formosa Fisch. 4 c. 28

1040. " nivea Baumg. 4 c.28

1041. " olympica Boiss 4 c. 28

1042. -" oxysepala $\mathrm{Tr}$. et. Mey.

$$
4 \text { c. } 28
$$

1043. ", vulgaris L. 4 c. 28

1044. Caltha palustris L. 4 G. 26

1045. Clematis integrifolia L. 4 c. 28

1046. , recta L. 4 c. 28

$1047^{*}$,,$\quad$ sibirica Mill. 528

1048. " " tangutica Korsh. $\hbar$ c. 28

1049. , vitalba $L . \hbar$ C. 26

1050. Delphinium Ajacis L. $\odot$ c. 28

1051. , cheilantum Fisch. 4

c. 28

1052. " $\quad$ consolida L. $\odot$ c. 27

1053. " " elatum L. 4 c. 28

$1054 *$ " $\quad$ orientale J. Gay. $\odot$

1055\% $\quad$ speciosum Bieb. 4

$1055^{*} \quad " \quad$ speciosum $\quad$ c 28

1056. Eranthis hiemalis Salsb. 4 c 28 1057. Ficaria ranunculoides Roth. 4 c. 28

1058. Hepatica triloba Gilib. 4 c. 28 1059. Isopyrum fumarioides $L$. $\odot$ c. 28 1060. Myosurus minimus L. $\odot$, $\odot$ c 28 1061. Nigella damascena $L$. $\odot$ c. 27 1062*. , Garidella Spenn. $\odot$ c. 27 1063. " sativa $L . \odot$ c. 28

1064. Paeonia albiflora Pall. 4 c. 28 1065. , corallina Retz. var. triternata Boiss. 4 K. 27 
1066. Ranunculus acer $L .4$ c. 28 1067.

1068

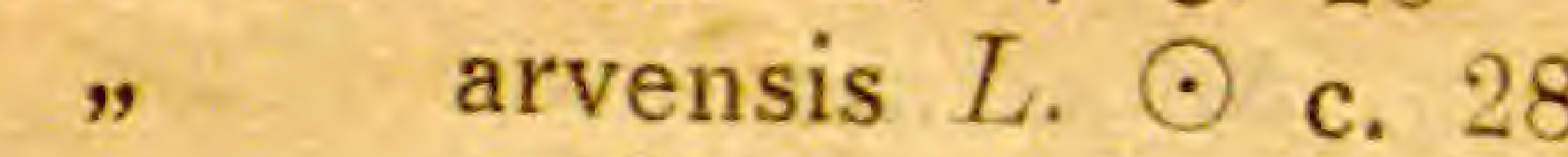
" auricomus L. $4 \underset{28}{\mathrm{G}}$

1069. " 1070*. " Cymbalaria Purch. 4 1071. 1072* " Flammula L. 4 c. 28 1073. 1073. " polyanthemos L. 4 1074. c. 24 1075. " repens L. 4 c. 28 1076 Th" sceleratus L. $\odot$ c. 28 4 c. 28
4 cacq. 1077. " flavum L. 4 c. 27 1078. " minus L. 4 c. 28 1079. Trollius europaeus L. 4 c. 28

Resedacea e D. C. $1080^{*}$. Reseda alba $L . \odot, 2$ c. 28 1081*. " complicata Brory. 4

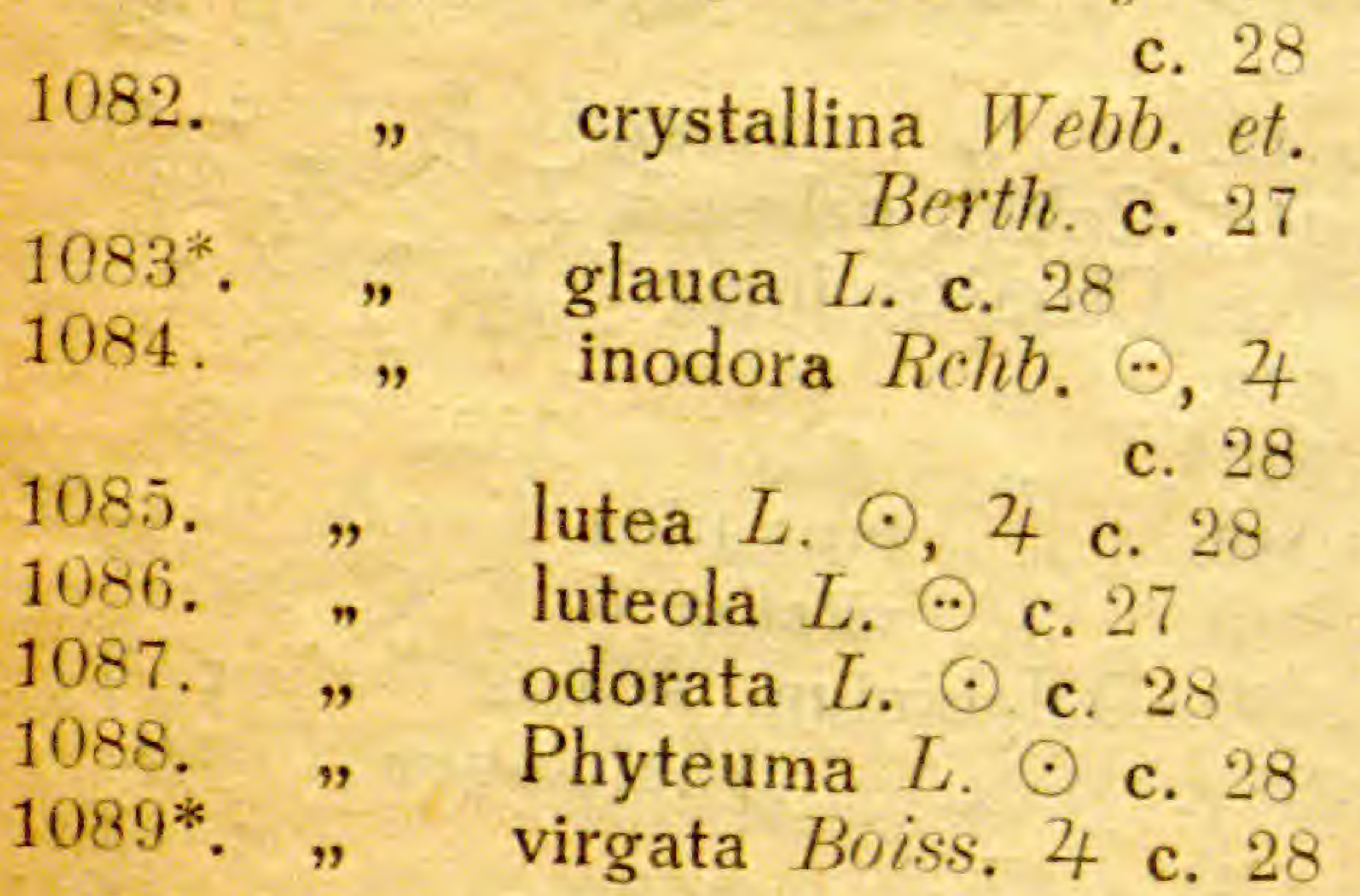

\section{Rhamnaceae S. F: Gray.} 1090. Rhamnus cathartica $L$. 5 G. 27 1091. " Frangula L. के G. 28 Ros a c a e Juss.

1092. Agrimonia Eupatoria L. 4 c. 28 1093. 1094*. Amelan pilosa Led. 4 c. 28 万ै G. 28 1095. Aruncus silvester Kost. 4 c. 28 1096*. Comarum palustre L. 4 G. 28 1097. Crataegus monogyna Jacq. मे 1098. sanguinea Pall 28 1099. Filipendula hexapetala Gilib. 4 c. 28 1100 .

, ulmaria Maxim. 4
1101. Fragaria indica $A n d r .4$ c. 28 1102. " vesca L. 4 c. 28 1103. " viridis Duch. 4 c. 28 1104. Geum aleppicum Jacq. 4 c. 28 1105. " molle Vis. et. 1 anc. 4 1106 c. 28 1107. " urbanum L. 4 M. 25 1108. Potentilla alpestris Hall. f. 4 $1109 . \quad$ c. 28 1110. " argentea L. 4 c. 28 1111 $"$ bifurea L. 4 c. 28 " delphinensis Gren. et. 1112. " desertorum Bge. 4 $1113 . \quad$ c. 28 1114 " fruticosa L. $\frac{\hbar}{2}$ c. 28 1115. fulgens Wal. 4 c. 28 1115.,$\quad$ intermedia $L .4$ c. 28 1116. " longipes Led. 4 c. 28 1117. " palustris Scop. 4 M. 1118. ” recta $L .2$ c. 28 1119. " rupestris L. 4 c. 28 1120. " Tormentilla Schrank.

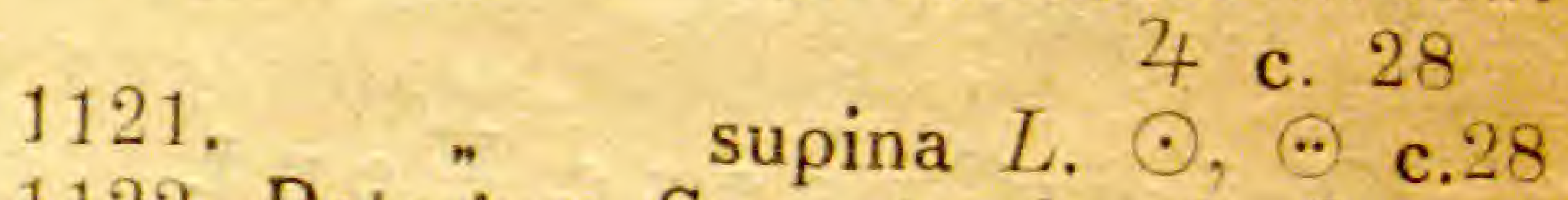
1122. Poterium Sanguisorba L. 4 c. 28 1123. Prunus Padus $L . \hbar$ c. 28 1124. $\Rightarrow \quad$ spinosa $L . \hbar$ c. 28 1125. Rosa cinnamomea $L$. $\hbar$ c. 28 1126.* " dahurica Pall. 5 W. 26 1127*. " rugosa Thunb. 只 W. 26 1128. Rubus saxatilis $L .4$ c. 28 1129 Sanguisorba officinalis $L .4$ $1130 * \quad$ c. 28 1 $"$ tenuifolia Fisch. 4 1131. Spiraea media Schm. 5 c. 28 1132. $"$ salicifolia $L .5$ c 28 1133. " sorbifolia $L$. के G. 28 1134. Sorbus Aucuparia L. $\hbar$ c. 28 1135*. " domestica Sm. है c. 28 Rubi a c e a e Jus s. 1136. Asperula Aparine Schott. 4 1137 c. 28 1138. " cynanchica $L$ L. 4 c. 28 1139. Asperula supina $M . B .4$ c. 27 1140. Crucianella chlorostachys $F$. et. 1141. M. 4 c. 28 n exasperata $F$. et. $M$. ค. c. 28 
1142. Galium mollugo L. 4 c. 28

1143. " parisiense L. $\odot$ c. 28

1144. " $\quad$ Saccharatum All ○

1145. " silvaticum L. 4 c. M. 28

1146. " spurium L. 4 M. 24

1147. " verum L. 4 c. 25

1148. Phuopsis stylosa Benth. et. Hook. 4 c. 28

1149** Rubia tinctorum L. 4 c. 27

1150. Scherardia arvensis L. $\odot$ c. 28

Rutaceae Juss.

1151. Ruta graveolens L. 4 c. 28

Saxifragaceae Dumort.

1152. Astilbe chinensis $F r$. et. Sav. 4 c. 28

1153.* Chrysosplenium alternifolium 4 c. 28

1154. Heuchera americana L. 4 c. 28 $1155 \quad$ sanguinea Englm. 4

₹ 1156. Parnassia palustris L. 4 c. 28

1157. Ribes alpinum $L . \hbar$ c. 28

1158. Saxifraga caespitosa L. 4 c. 28 1159.* " irrigua $M . B .4$ c. 27 1160. Tellima grandiflora $R . B r .4$

c. 28

Scrophulariaceae Lindl.

1161. Alonsoa Warscewiczii Reg. $\odot$

1162. Antirrhinum majus L. $\odot$ c. 28

1163. " Orontium L. $\odot$ c.

28

1164. Calceolaria pinnata L. ๑ c. 28

1165. " scabiosifolia Sims.

c. c. 28

1166. Chaenostoma antirrhinoides $L$.

1167. foetidum Benth 28

1168. Collinsia b. 28

1168. Collinsia bicolor Benth. $\odot$ c.

28

1169. Cymbalaria muralis Baumg. 4

c. 28

1170. Digitalis ambigua Murr. 4 c.

1171. " gloxinifflora c. 28

1172. " laevigata W. et. K. 4 c. 28
1173. Digitalis lutea L. 4 c. 28

1174. " media Roth. 2 c. 28

1175. " micranta Roth. 4 c. 28

1176. " nevadensis Kunze.

1177. „ purpurea L. 4 c. 28

1178. Elatinoides elatine Wettst. $\odot$

c. 28

1179. " spuria Weitst. $\odot$

1180. Hebenstreitia dentata $L$ c. $\odot$. c.

180. 28

1181* Linaria alpina Mill. 4 c. 28

$1182^{*}$. " $\quad$ aparinoides $F$. G. Dietr.

v. hybrida c. 28

1183. " bipartita W. $\odot$ c. 28

1184. " Broussonnetii Chav.

1185.0 c. 28

1185. " canadensis Dumort. $\odot$

1186. c. 28

1187 c. 28

1187. " chalepensis Mill. $\odot$

1188. " genistaefolia Mill. 28

$1189 . \quad$ c. 28

" japonica Miquel. 4
c. 28

1190. " littoralis W. $\odot$ c. 28

1191. " macroura $M . B .4$ c. 28

1192*. " marocana Hook. $\odot$ c. 28

1193. - minor Desf. $\odot$ c. 28

1194. " purpurea Mill. $\odot$ c. 28

1195. " reflexa Desf. $\odot$ c. 28

1196. " " Desf. v. aureopur-

$1197^{*}$. n reticulata Desf. c. 28

1198. " sapphirina Hoffm. et. Link. $\odot$ c. 28

1199. " spartea H. et. Lk. ๑c.28

1200. " tryphylla Mill. $\odot$ c. 28

1201. " tristis Mill. $\odot$ c. 28

1202. " vulgaris Mill. 4 c. 27

1203. " " var. Biebersteinii

Lindem. 4 c. 27

1204.* Lindenbergia urticifolia Lehm. () c. 28

1205. Manulea violacea $L k$. $\odot$ c. 28 1206.* Melampyrum nemorosum L. 4 M.

1207. Mimulus cardinalis Dougl. 4 c.27 1208*. " curpeus Regel. var, tigrinus 4 c. 28 
1209. Mimulus luteus L. $\odot$ c. 28 1210. " moschatus Dougl. 4 c. 28

1211. Nemesia strumosa $\odot$ c. 28 1212. Nycterinia Capensis $\odot$ c. 28 1213. Odontites serotina Rehb. $\odot$ M. 1214. Pedicularis palustris $L$. $\odot, 4 \mathrm{M}$. 1215. Scrophularia nodosa L. 4 c. 28 1216. " umbrosa Dumort. 4 1217. Verbascum Blattaria L. $\stackrel{\text { c. } \odot \text { c. } 28}{\text { c. } 28}$ 1218. " nigrum $L . \odot$ c. 28 1219. " phlomoides L. $\odot 27$ 1220 . ” pyramidatum $M . B$. 4 c. 28 1221. \# speciosum Schrad.27 1222*. Veronica arvensis $L . \odot, \odot$ c. 1223. " Beccabunga L. 4 1224. " Chamaedrys L. 28 c. 1225. " gentianoides Vahl. 1226*. incana L. 4 c. 28 1227. " longifolia L. 4 c. 28 $1228^{*} . \quad " \quad$ officinalis $L .4$ c. 27 1229. " scutellata L. 4 c. 28 1230. " serpyllifolia L. 4 c. 1231. " sibirica L. 4 c. 28 1232. " spicata L. 4 M. 1233. " spuria L. 4 e. 28 1234. $\quad \Longrightarrow \quad$ Tournefortii Gmel. $\odot$ c. 28

\section{Solanaceae $\rho$ ers.}

1235. Atropa Belladonna L. 4 c. 28 1236. " caucasica Kreyer. 4 c. 28

1237. Capsicum annuum $L$, $\odot$ c. 27 1238. Datura Bertolonii Parl. $\odot$ c. 28

1239. ” Leichhardtii F. Muell. $\odot$

1240. " meteloides D.C. $\odot$ c. 26

1241. " stramonium L. $\odot$ c. 28

1242. "Wrighti Rgl. ๑ c. 28

1243. Hyoscyamus albus $L$. $\odot$ c. 27

1244. $\quad$ niger $L . \odot . \odot$ c. 27

1245. Nicandra physaloides Gaertn.

c. 28

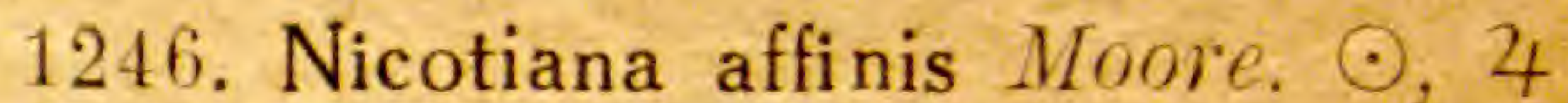
c. 28 1247. " paniculata L. $\odot$ c. 28 1248. " rustica $L . \odot$ c. 28 1249. „ Sanderiana Hort. $\odot$ 1250 c. 27 " silvestris $L . \odot$ c. 27 1251. " suaveolens Lehm. $\odot$
1252. c. 28

$"$ Tabacum L. $\odot$ c. 28 1253. Nolana prostrata $L$. $\odot$ c. 27 1254. Petunia nyctaginiflora Juss. $\odot$ 1255. Physalis Alkekengi L. 4 c. 27 1256. " angulata L. $\odot$ c. 27

1257. ” Francheti Masters. c. 28 1258. \# pubescens $L$. $\odot$ c. 28 1259. Physochlaena orientalis G. Don. 4 c. 28 1260.* Salpiglossis variabilis Hort. $\odot$ 1261. Solanum Capsicastrum Link, 28 1262 c. 28 1262. " demissum c. 28 1263. " dulcamara L. 4 c. 28 1264. " Gilo Raddi. $\odot$ c. 27 1265. " gracile Otto. $\odot$ c. 28 1266. " guineense Lam. $\odot$ c. 1267*. " Hystrix $R, B r$. c. 27 1268. " miniatus Bernh. $\odot$ c. 28 1269. " nigrum $L$. v. villosum $1270 . \quad v$ vulgare 1271. Withania somnifera c. 28 1. Withania somnifera Dun. $\hbar$ c. 27

Tropaeolaceae Juss. 1272. Tropaeolum majus $L$. $\odot$ c. 28

Umbelliferae Morison. 1273. Aegopodium alpestre Ledb. 4 1274. $\quad$ c. 28 1275. Aethusa C. 28 127. Aethusa Cynapium L. $\odot$ c. 28 1276. Anethum graveolens $L$. $\odot$ c. 28 1277. Angelica archangelica $L$. $\odot$ c. 1278.28 28 
1279. Anthriscus silvestris Hoff. 4 c. 28

1280. Astrantia major L. 4 c. 28 1281. Bifora testiculata Boiss. () c. 28 1282. Bupleurum rotundifolium $L$. $\odot$ c. 28

1283. Carum Carvi L. $\odot$ c. 28

1284. Caucalis latifolia $L$. $\odot$ c. 28

$1285^{*}$. Cenolophium Fischeri Koch. 4 c. 27

1286. Chaerophyllum aromaticum $L$. 4 c. 28 1287. " bulbosum L. $\%$ c. 28

1288. Conium maculatum L. -) c. 26

1289. Coriandrum sativum L. $\odot$ c. 28 1290. Daucus Carota L. (spont.) $\odot 27$ 1291. " grandiflorus Sc.op. $\odot$

1292. $1293^{*}$. K. 27 1294. Eryngium pandanifolium 4 c.

1295. " planum L. 4 c. 28 1296. Falcaria vulgaris Bernh. . c. 1297. Heracleum barbatum Ledb. 4

1298. c. 28

1299. pubescens M.B. $\odot$ c. 28 1300. Laserpitium pruthenicum L. .) M. 1301. Levisticum officinale L. 4 c. 28 1302. Libanotis montana All. $\odot$ c. 28 1303. " sibirica Koch. 4 c. 28 1304. Ligusticum scoticum L. 4 c. 28 1305. Myrrhis odorata Scop. 4 c. 28 1306. Pastinaca sativa $L$. - c. 28

1307. " teretiuscula Boiss. $\odot$ c. 28 1308. Petroselinum sativum Hoff. 4 c. 28 1309. Peucedanum cervariaefolium $C$. A. M. 4 c. 28

1310. " Oreoselinum Moench. $4 \mathrm{M}$.

1311. " " palustre Moench. 4 c. 24

1312.* Pimpinella Anisum L. $\odot$ c. 27 1313.* " magna L. $4 \mathrm{M}$. 1314. " $\quad$ saxifraga $L, 4$ c. 28 1315. " $\quad$ Tragium Vill. 4 c.
1316. Sanicula europaea L. 4 c. 28 1317. Scandix Pecten Veneris L. $\odot$ c. 28 1318*. Selinum carvifolia L. 4 M. 1319. Seseli Hippomarathrum L. 4 c. 28 1320. " laserpitiifolium Palib. 4 c. 26 1321. Torilis Anthriscus Gmel. $\odot$ c. 28

Urticaceae Endl.

1322. Forskohlea angustifolia Retz. $\odot$ c. 28

1323. Parietaria ramiflora Moench. 4 c. 27

1324. Urtica cannabina L. 4 c. 28 1325. " dioica L. 4 c. 28

1326. " Dodartii L. $\odot$ c. 28 1327. " membranacea Poir. $\odot$ c. 28 1328. " pilulifera $L$. $\odot$ c. 28 . 1329. " urens $L . \odot$ c. 28

Vacciniaceae Lindl. 1330. Vaccinium Oxycoccus L. 4 G. 1331* * uliginosum L. $\hbar \underset{26}{\mathrm{G}}$.

Valerianaceae Dumort. 1332. Centranthus Calcitrapa Dufr. $\odot$ c. 28 1333. $\quad$ ruber D. C. $\odot$ c. 1334. Fedia cornucopiae D. C. 4 c. 1335. Valeriana palustris $K r .4$ c. 28 1336. " Sprygini Sm. 4 c. 28 1337. " Wolgensis Kez. 4 1338. Valerianella coronata D. C. $\odot$ c. 28 1339. " eriocarpa Krok. $\odot$ c. 28 1340. \# rimosa Bastard. $\odot$ 1341. " tridentata Bas. $\odot$ c. 28 1342. " vesicaria Mnch. $\odot$ c. 28 
Verbenaceae J. St. Hil. 1343*. Lantana flava Medic. $\hbar$ c. 27 1344. Verbena arborea $H$. et. B. $\hbar$

$\begin{array}{lll}1345 . & & \text { Aubletia Jacq. } .27 \\ & \text { c. } \\ 1346^{*} . \quad \# & \text { hastata L. } 4 \text { c. } 28 \\ 1347 . & 28 \\ & & \text { hybrida Hort. } \odot \text { c. } \\ 28\end{array}$

\section{Violaceae D. C.}

1348. Viola alpestris Wittr. 4 c. 28

1349. " canina Rchib. 4 c. 28

1350. " cornuta L. 4 c. 28

1351. " elatior $\mathrm{Fr} .4$ c. 28

1352. " epipsila Led. 4 c. 28
1353. " hirta L. 4 c. 28

1354. " mirabilis L. 4 c. 28

$1355^{*}$. " Munbyana Boiss.et. Rent.

var. Battandieri c. 28

1356. " odorata L. 4 c. 28

1357. ” Patrini D. C. 4 c. 28

1358. " sagittata Ait. 4 c. 28

1359. " tricolor Wittr. $\odot, .$. c.

Zygophyllaceae R. Br.

1360. Nitraria Schoberi L. $\hbar$ K. 27

1361. Peganum Harmala L. 4 c. 26

1362. Tribulus terrestris $L \odot$ c. 27

1363. Zygophyllum Fabago L. 4 c.

Semina plantarum in provincia Austro-Ussuriense regionis naturalis Manshuriae crescentium, quae a E. J. Wassilkoff anno 1927 loco natali lecta sunt.

Насеньне, якое са6рана ўлетку 1927 г. А. Я. Васільковай у Паўднёва-Усурыйскай правінцы Манжурскае флёрыстычнае краіны.
Семена, собранные летом 1927г.ð Е. И. Васильковой в Южно-Уссурийской провинции Манжурской флористической области.
1364.* Actaea acuminata Wallich. 1365.* Agropyrum ciliare Franch. v.

1366. Allium lineare $L$.

$$
\text { pilosum Korch. }
$$

1367.,$\quad$ sacculiferum $M a x$.

1368. . sp.

1369. Ampelopsis brevipedunculata

$1370^{*}$. Anemone dichotoma $L$. Maxim.

1371. Artemisia sp.

1372. " sp.

1373. Arundinella anomala Stoud.

1374.* Astragalus uliginosus $L$.

1375. Aster fastigiatus $F$. et. $M$.

1376. " Maackii Rgl.

1377. " Scaber Thunb.

1378. Betula dahurica Pall.

1379. " japonica $H$. Wincler.

1380. " ovalifolia Rupr.

1381. Bidens Maximovicziana Oett.

1382. Cacalia aconitifolia Bunge
1383.* Cacalia hastata $L$.

1384. Calamagrostis langsdorfii Trinius

1385.* Calystegia rosea Choisy

1386. Caragana fruticosa Besser

1387* Carex limosa $L$.

1388.* " pallida C.A. Mey

1389. Cicuta virosa $L$.

1390. Cimicifuga dahurica Max.

1391. " Simplex Wormsk.

1392.* Clematis angustifolia Jacq.

1393. " fusca Turez.

1394. " manshurica Rupr.

1395. Codonopsis lanceolata B. et. $H$.

1396. " " ussuriensis Hemsley

1397. Cuscuta japonica Choisy

1398. Cynanchum amplexicaule Hem-

1399. ” atratum Bnge sley.

1400. Drosera longifolia $L$.

1401.* Elsholtzia cristata Willd. 
1402.* Epilobium palustre $L$.

1403. Erigeron canadensis $L$.

1404. Eupatorium Kirilovi Turcz.

1405.* Evonymus sp.

1406.* Galium trifidum $L$.

1407 . " verum $L$.

1408. Geranium Wlassovianum Fischer

1409. Geum aleppicum Jacq.

1410 Gypsophila muralis $L$.

1411.* Habenaria linearifolia Max.

1412. Hemerocallis flava $L$.

1413.* Hieracium umbellatum $L$.

1414. Hypericum Ascyron L.

1415.* Ipomaea sibirica $L$.

1416. Iris Kaempheri Sieb.

1417. " laevigata Fisch.

1418.* Lathyrus palustris L. v. pilosus

1419. Lilium avenaceum $L$. $L d b$.

1420. Lobelia sessilifolia Lamb.

1421.* Lonicera praeflorens Butalin.

1422.* Lychnis fulgens Fisch.

1423. Lycopus Maackianus Kom.

1424. Lysimachia dahurica $L d b$.

1425. Lythrum salicaria $L$.

1426. Melilotus suaveolens $L d b$.

1427.* Menyanthes trifoliata $L$.

1428. Mulgedium sibiricum Less.

1429.* Naumburgia thyrsiflora Duby

1430. Panicum acroanthum Stendel

1431.

crus-galli $L$.

1432. Paeonia albiflora Pall.

1433.* ” obovata Max.

1434.* Pedicularis grandiflora Fisch.

1435.* $\quad$ resupinata $L$.

1436. " Sceptrum Carolinum L.

1437.* Perilla ocymoides $L$.

1438.* Plagiospermum chinensis Oliver.

1439. Platanthera hologlottis Max.
1440. Platycodon grandiflorus A.D.C.

1441. Pleurospermum kamtschaticum

$1442 *$. $\rho_{\text {oa }}$ pratensis $L$.

Hoffm.

1443.* Pogonia ophioglossoides Ker.

1444. Polygonatum officinale All.

1445.* \# tomentosum Schrank.

1446*. Polygonum sp.

$1447 . \quad \cdots \quad$ sp.

1448* Ranunculus japonicus Thunb.

1449.* $\quad$ sceleratus $L$.

1450.* Rubia chinensis Rgl.

1451.* Rumex acetosella $L$.

1452. Sanguisorba officinalis $L$.

1453. ” tenuifolia Fich.

1454.* Scheuchzeria palustris $L$.

1455. Scutellaria angustifolia Kom.

1456.* Sedum Aizoon $L$.

$1457^{*}$. Serratula coronata $L$.

$1458 *$ Setaria viridis $P$. $B$.

1459. Smilax oldhami $\cdot$ Miq.

1460.* Sonchus arvensis $L$.

1461. Sophora flavescens Ait.

1462. Spodiopogon sibiricus Trin.

1463*. Triadenum asiaticum Max.

1464. Trifolum lupinaster $L$.

1465.* Trollius chinensis Bge.

1466. Typha latifolia $L$.

1467.* Urtica laetevirens Max.

1468. Veratrum album $L$.

1469. " Maackii $R g l$.

1470.* Veronica grandis Fich.

1471.* ” tubiflora Turcz.

1472.* Viburnum sargenti Koehne

1473.* Vicia amoena Fischer.

$1474^{*}$. " cracca $L$.

1475.* " pseudo-orobus F. et. Mey.

1476.* " unijuga $\mathrm{A} . \mathrm{Br}$.

1477.* Viola Patrini D. C.

\title{
Professor J. G. W'assillkoff, Horti praefeetus.
}

\author{
I. L. Gorsky, Hortulanus.
}

Gorky. R. P. Alborutheniae (U. S. S. R.).

Calendis Martiis anni MCMXXIX. 



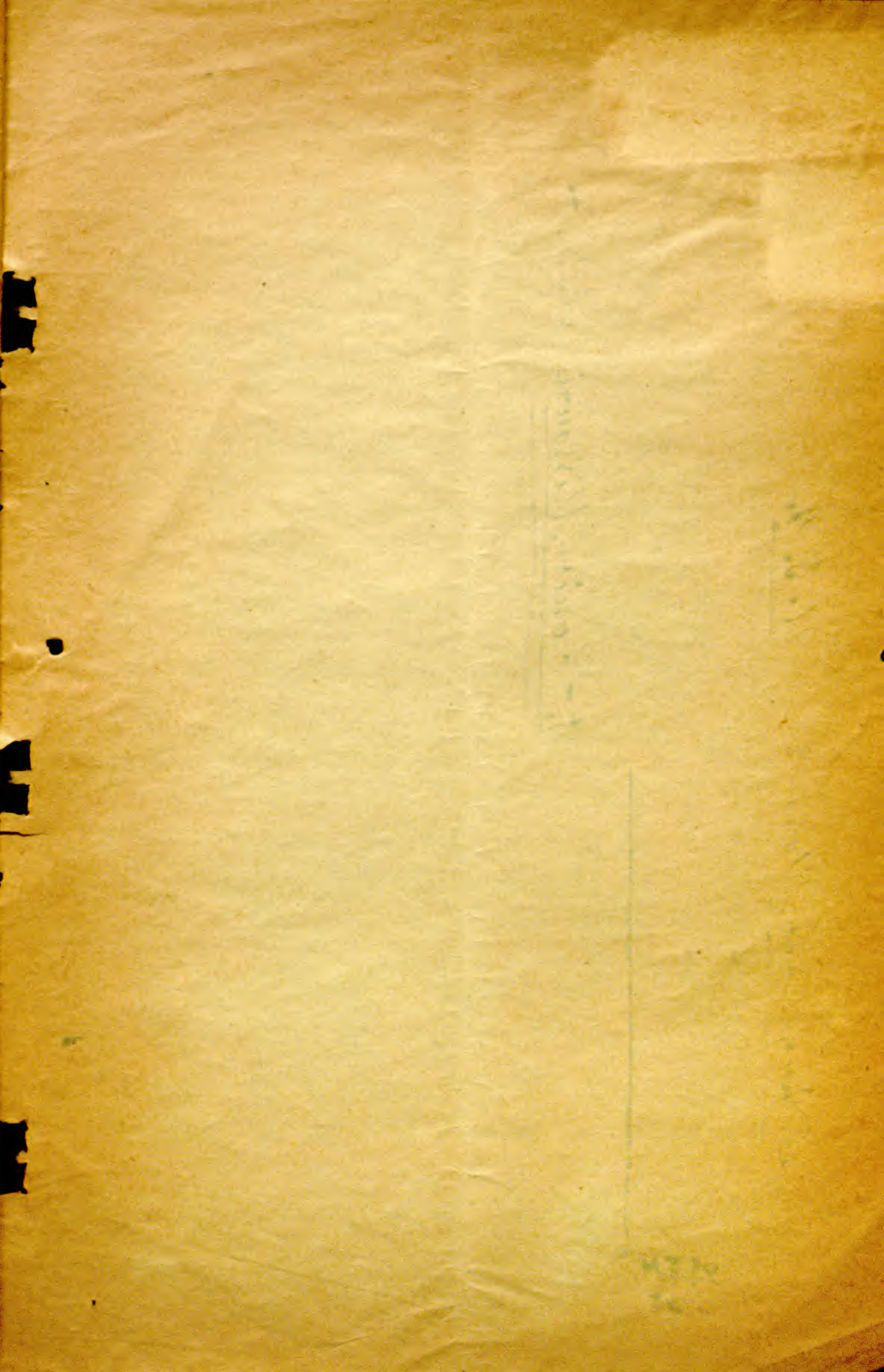




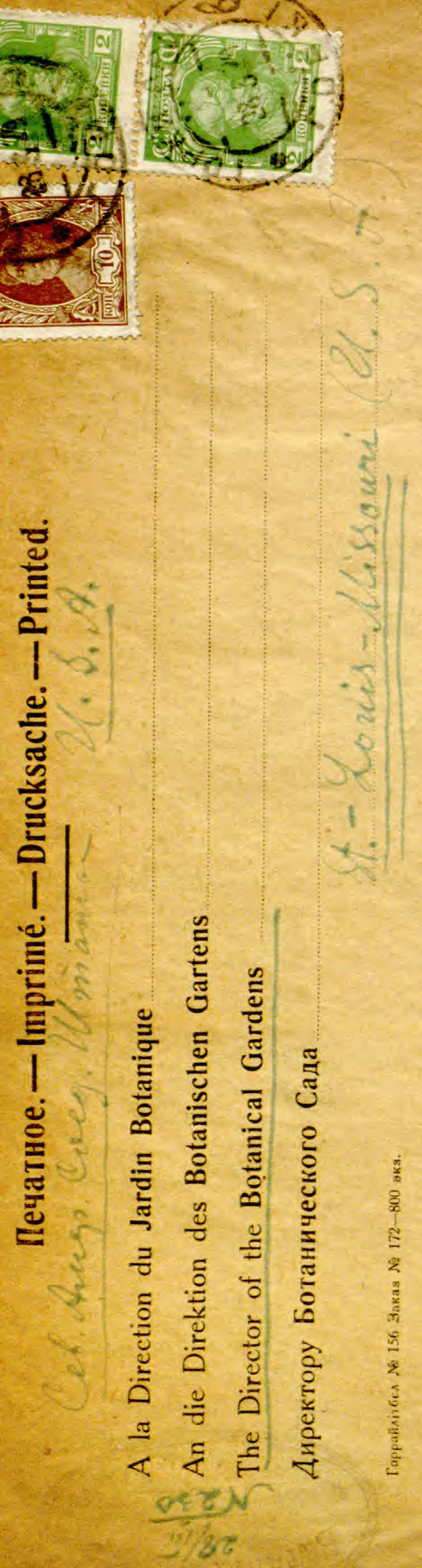


Jardin Botanique de l'Academie Agronomique Gorky. Russie Blanche. U. S, S. R.
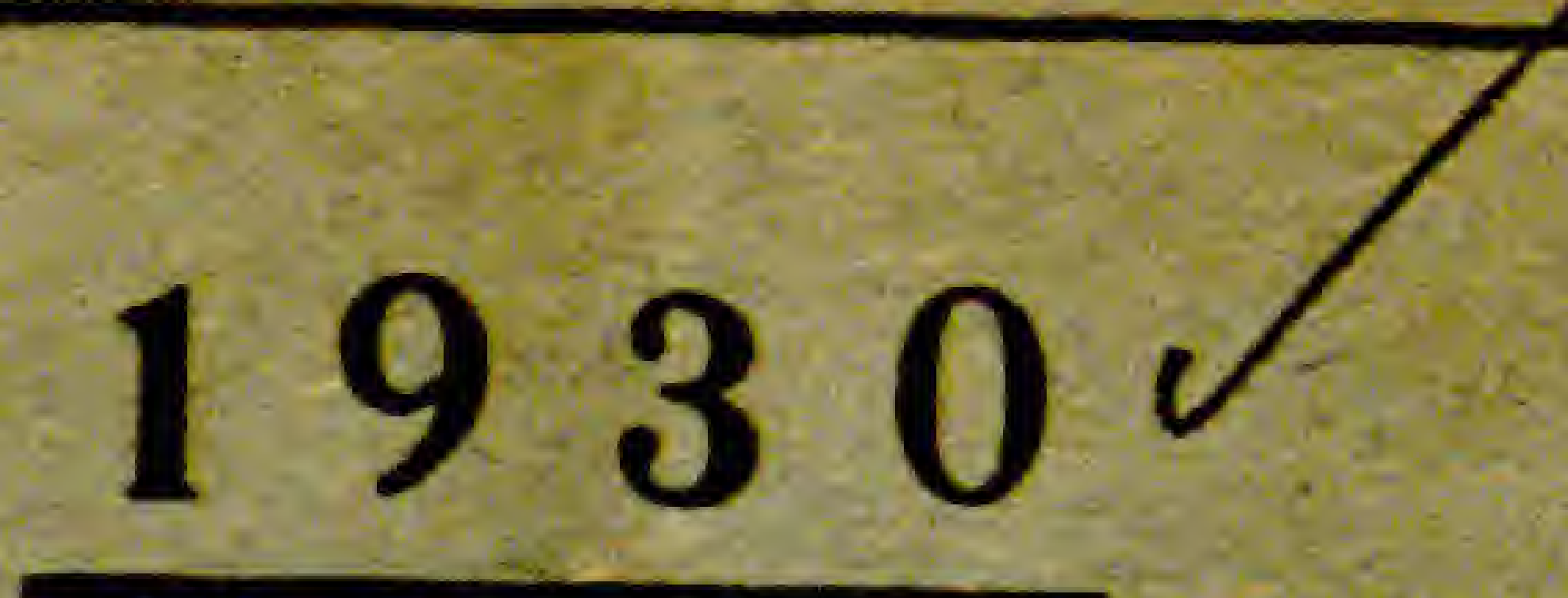

\section{ПЕРАЛІК НАСЕНЬНЯ,}

якое Батанічны Сад Беларускай Дзяржаўнай Акадэміі Сельскай Гаспадаркі прапануе да абмену

\section{DELECTUS SEMINUM,}

Anno MCMXXIX (partim ac MCMXXVI, MCMXXVII et MCMXXVIiI) collectorum, quae Hortus, Botanicus Academiae Agronomicae Rei publicae Alboruthenicae pro mutua commutatione offert

\section{ПЕРЕЧЕНЬ СЕМЯН,}

предлагаемых в обмен Ботаническим Садом Белорусской

Государственной Академии Сельского Хозяйства

Gcry Gorky. R. P. Alboruthe niae (U. S. S. R.)

Асобны адбітак 3 „3апісак Беларускай Дзяржаўнай Аvaдэніi т. XI 1930 r."

TOPKI, БCCP

ДРУКАРНЯ АКАДОМіI

1930

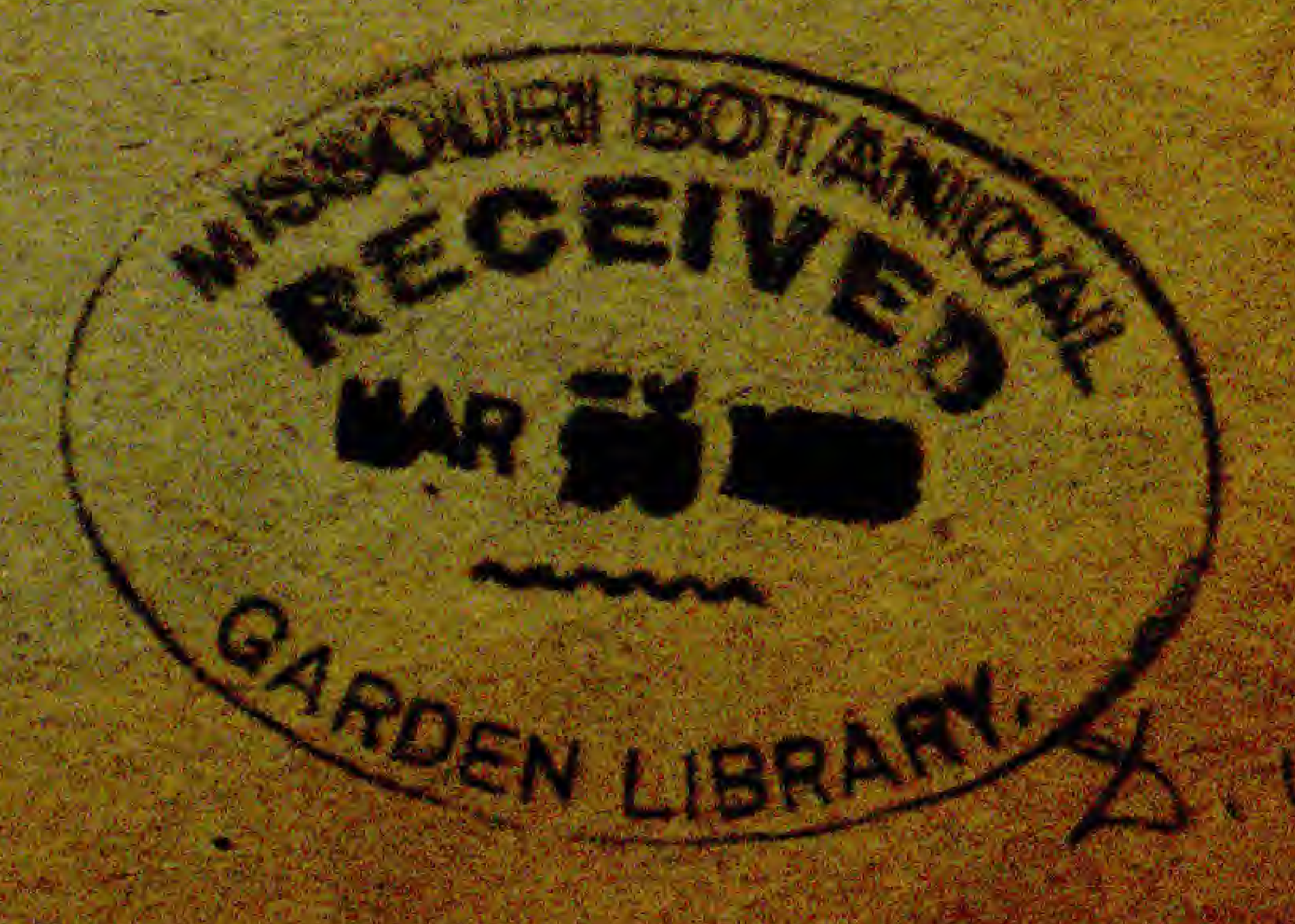



Jardin Botanique de l'Academie Agronomique

Gorky. Russie Blanche. U. S. S. R.

\section{0}

\section{ПЕРАЛІК НАСЕНЬНЯ,}

якое Батанічны Сад Беларускай Дзяржаўнай Акадэміi Сельскай Гаспадаркі прапануе да абмену

\section{DELECTUS SEMINUM,}

Anno MCMXXIX (partim ac MCMXXVI, MCMXXVII et MCMXXVIII) collectorum, quae Hortus Botanicus Academiae Agronomicae Rei publicae Alboruthenicae pro mutua commutatione offert

\section{ПЕРЕЧЕНЬ СЕМЯН,}

предлагаемых в обмен Ботаническим Садом Белорусской Государственной Академии Сельского Хозяйства

Gory-Gorky. R. P. Alborutheniae (U. S. S. R.)

ГОРКі, БСС

ДРУКАРНЯ АКАДЭМІІ

1930 



\section{A b b reviationes:}

*_paulum; K-Karadagh; W-Wladiwostok; G-Gorky; c-planta sub divo in Horto Botanico Academiae culta.

Numerus $(26,27,28)$ nomina plantarum sequens, annum, quo semina lecta sunt, significat, semina sine numerus in anno 1929 collecta sunt.

Скарачэньні і умоўныя знакі:

Зоркай (*) абазначана насеньне, якое маеџџа ў нязґачнай колькасьџі. $\mathrm{K}$-насеньне, якое сабрана ў Карадъагу (Крым); W-навакол Ўладзівастоку; G-навакол Горак; с-з расьлін, якія разводзяџџа ў вадкрытым грунце ў Батанічным Садзе Акадәміi.

Лічбы $(26,27,28)$, якія надрукаваны пасьля назоваў расьлін, азначаюџь гады збору насеньня. Насеньне без лічбаў, сабрана ў 1929 годзе.
Сокращения и условныя обозначения:

Звездочкой (*) обозначены семена, имеющиеся в малом количестве. $\mathrm{K}$ - семена, собранные на Кағадаге (Крым); W-в окрестностях Владивостока; G-в окрестностях Горок; c-c растений, культивируемых в Ботаническом Саду Академии в открытом грунту.

Џыфры $(26,27,28)$, помещенные за названиями растений, означают год сбора семян, семена без чисел собраны в 1929 году. 


\section{P t e ridophyta.}

1. Adianthum gracillimum Moore.

2*. Anogramme leptophylla $L k$. $\stackrel{\text { c. }}{\odot}$

3. Aspidium cristatum $S w .4 \stackrel{\odot}{\circ}$ c.

4. ” Filix-mas $S w .4$ c.

5. $\quad$ spinulosum $S w .4$ c.

$6 . \quad$ thelypteris $S w .4$ c.

7. Asplenium dimorphum Knze. 4 c.

8. „ Filix-femina Bernh.

9.*,$\quad$ Trichomanes L. 4

K. 27

10. Cyrtomium falcatum Prsl. 4 c. 11. Cystopteris bulbifera Bernh. 4 c. 12.* $\quad$ fragilis Bernh. 4 c.

13. Onoclea sensibilis L. 4 c.

14. Osmunda cinnamomea L. 4 W. 26

15. Phegopteris Dryopteris Fée. 4 c.

16. Polypodium Phegopteris L. 4 c.

17. Pteris aquilina $L .4$ c.

18. " cretica L. 4 c.

19. " longifolia $L .4$ c.

20. " serrulata L. 4 c.

21. " $\quad$ v. crispa 4 c.

22. " " v. cristata 4 c.

23. ” $"$ v. tenuifolia 4 c.

24. Scolopendrium vulgare Sm. 4 c.

25. Struthiopteris germanica Willd.

$4 \mathrm{c}$.

\section{Monocotyledonea e.}

Alismataceae C. Rich.

26. Alisma Plantago L. 4 M.

Araceae Juss.

27. Calla palustris $L . ~ 4$ G

Commelinaceae Reich.

28. Commelina coelestis $W$. $\odot$ c. 29.*,$\quad$ tuberosa $L .24$ r. 28 30. Tinantia fugax Scheidw. 4 c. 28

Cyperaceae J. St. Hil.

31. Carex acuta L. 4 c.

32. " caespitosa $L .4$ c.

33. " chordorrhiza Ehrh. 4 G.

34. ” diandra Schrank, 4 G.

35. " dioica $L .4$ G.

36. " elongata L. 4 c.

37. ” flava L. 4 c.

38. " hirta L. 4 c.

39. ” leporina $L .4$ c.

40. " limosa $L .4$ c.

41. ” muricata L. 4 c.

$42 *$. ” pallescens L. 4 c. 28

43. ” panicea $L, 4$. c.

44. " pilosa L. 4 c.

45.* ” remota L. 4 c. 28
46. Carex rostrata Stokes. 4 c.

47. „ stellulata Good. 4 G.

48. ” stricta Good. 4 c.

49. " vesicaria $L .4$ c.

50. ” vulgaris $\mathrm{Fr} .4 \mathrm{c}$.

51. ” vulpina $L .4$ c.

52.* Cyperus esculentus L. 4 c. 27

53. Eriophorum angustifolium Roth.

54* gracile Koch 4 G.

$55 \quad$ latifolium Hoppe 4 G.

56 * Heleocharis palustris $R$. $\mathrm{Br}$.

57. Scirpus silvaticus L. 4 c.

$$
\text { Graminea Jus s. }
$$

58. Aegilops cylindrica Host. $\odot$ c.

59. " ovata $L . \odot$ c,

60. $\Rightarrow$ triuncialis $L . \odot \mathrm{c}$.

61* Aeleuropus laevis Trin. 4 c.

62. Agropyrum caninum P. Beauv.

63. 4 c.

64. " elongatum $P . B .4$ c.

64. " glaucum $R$. et. Schult.

65. $\quad$ repens $P . B .4^{4} \mathrm{c}$

66. " tenerum Vasey. 4 c.

67. Agrostis alba L. 4 c.

68. " canina $L .4$ c.

69. ” vulgaris With. 4 c. 
70. Aira capillaris Host. $\odot$ c. 28

71. „ caryophyllea $L$. ๑ c. 28

72. Alopecurus agrestis $L$. $\odot, \odot$ c.

73.* $\quad$ fulvus $S \mathrm{~m} . \odot$ c.

74. $\quad$ geniculatus $L . \odot$ c.

75. " pratensis L. 4 c.

76. ventricosus Pers. 4 c.

77.* Andropogon Ischaemum $L$.

78. Anthoxanthum odoratum $\frac{4}{L}$ c.

4 c.

79. Apera spica venti $P . B . \odot$ c.

80. Arrhenatherum elatius $M$. et $K$.

4 c.

81. Asperella hystrix Humb. 4 c.

82. Atropis convoluta Gris. 4 c.

83. Avena barbata Brot. $\odot$ c,

84. ” chinensis Fisch. c.

85. " hirsuta Roth. $c$.

86.* " Ludoviciana Dur. c.

87. " strigosa Schreb. $\odot$ c.

88. Beckmannia eruciformis Host.

89. Boissiera bromoides Hochst.

90. Brachypodium ramosum $R$. et. $S$.

91. $\quad$ silvaticum $R$. et. $S$.

92. Briza maxima L. $\odot$ c. $28^{4+}$

93. „ media L. 4 c.

94. - minor L. $\odot$ c.

95.* „ spicata Sibth. et. Sm..

K. 27

96.* „ virens $L$. c. 28

97. Bromus arvensis $L$. $\odot$ c.

98. " commutatus Schrad. $\odot, \odot$ c.

99. $\quad$ ” hordeaceus $L . \odot . \odot$ c.

100. " inermis Leyss. 4 c.

101. " macrostachys Desf. (

102. ” maximus Desf. $\odot$ c.

103. ” secalinus $L . \odot, \odot$ c.

104. " squarrosus $L . \odot . \odot$

$105 . * \quad$ sterilis $L$. $\odot$ c. 27

106. " tectorum $L . \odot$ c.

107. ” unioloides H. B. K. ๑.

108. Calamagrostis Epigeios Roth.

109. Catabrosa aquatica P. B. ${ }_{4}^{4}$ c

c. 27

110. Cenchrus echinatus $L$. $\odot$ c.
111. Cenchrus montanus Nees.

c. 27

112. Chloris barkata $S w$, c. 27

113. Cinna glomerata $L$. c.

114. " mexicana Beauv. 4 c.

115. Coix lacryma Jobi $\bar{L}$. $\odot$ c.

116. Cynosurus cristatus L. 24 c.

117. Dactylis Aschersoniana Gaebn.

118 . 4 c.

118. " glomerata L. 4 c.

119. " " L. fol. var.

120. Deschamosia caespitosa $P^{4}$ c.

4 c.

121. Desmazeria sicula Dum. $\odot$ c.

122. Eleusine indica Grtn. (.) c. 27

123. Elymus araliensis Rgl. 4 c.

124. ” arenarius $L .4$ c.

125. " canadensis $L .4$ c.

126. $"$ excelsus Turcz. 4 c.

127. ” giganteus Vahl: 4 c.

128. " junceus Fisch. 4 c.

129. ” sibiricus L. 4 c.

130. " virginicus L. 4 c.

131. Eragrostis minor Host. $\odot$ c.

132. $"$ pilosa P. B. $\odot$ c.

133. Eriochloa villosa Knth. $\odot$ c.

134. Festuca arundinacea Schr. if c.

135. " Beckeri Hackel. 4 c.

136. ” gigantea Vill. 4 c.

137. " heterophylla Lam. 4

c. 27

138. ” ovina L. 4 c.

139. ” pratensis Huds. 4 c.

140. " rubra L. 4 c.

141. " sulcata Hackel. c.

142. Gastridium australe P. B. ๑ c.

143. Glyceria spectabilis $M$. et. K.

14 c.

144. ${ }^{*}$ Heleochloa schoenoides Itost.

$\odot$ c.

145. Hierochloë odorata Walllb. 4 c.

146.* Holcus lanatus L. 4 c. 27

147. Hordeum jubatum L. 4 c.

148. " sativum $L$. v. spontaneum $K$. Koch. $\odot$ c.

149. " secalinum Sclireb.

150. Koeleria gracilis Pers. 2f c.

151. Iagurus ovatus $L$. $\odot$ c.

152. J asiagrostis splendes hunt?.

153. I eersia oryzoides $S w,{ }^{4}$ c.

154. Lolium perenne L. 4 c. 
155. Lolium remotum Schrank. (-) c. 156. " temulentum $L$. $\odot$ c. 157. Melica altissima $L .4$ c.

158. \# nutans L. 4 c.

159. \# transsilvanica Schur.

2 c.

160. Miscanthus sacchariflorus Hack. 2 c.

161. Nardus stricta $L .4$ c.

162. Oryzopsis holciformis Richt. 4 c. 27

163. Panicum bulbosum $H$. B. K. c. 164. " calyfornicum Benth.

$165 . \quad$ ciliare Rets $\odot$ c.

$166 . \quad$ ciliare Retz. $\odot$ c.

167. " $\quad$ crus galli L. $\odot$

167. $"$ frumentaceum $\mathrm{Fr}$. et. Cav. $\odot$ c. 27

168. " germanicum c.

169. " mileaceum $L$. $\odot$ c.

170. " sanguinale L. $\odot$ c. 28

171.* Paspalum stoloniferum Desv. 4 c.

172. Phalaris arundinacea $L .4$ c.

173. " " fol. var., v. lutescens 4 c.

174. " canariensis $L$. $\odot$ c.

175. Phleum alpinum $L .4$ c.

176. " Boehmeri Wib. 4 c.

177. ” paniculatum L. 4 c. 28

178. $"$ pratense $L .4$ c.

179. Phragmites communis Trin. 4

180. Poa alpina $L .4$ c.

181. „ annua $L . \odot$ c.

182. " bulbosa L. 4 c.

183. " caesia $\mathrm{Sm} .2$ c.

184. ” compressa L. 4 c.

185. " glauca Vahl. 4 c.

186. " nemoralis $L .4$ c.

187. " palustris L. 4 c.

188. " pratensis L. 4 c.

189. . trivialis $L .4$ c.

190. Polypogon monspeliensis Desf. $\odot$

191. Scleropoa rigida Griseb. ๑ c. 28

192. Secale montanum Guss. 4 c.

193. Setaria verticillata $P . B . \odot$ c. 28

194. " viridis $P . B . \odot$ c.

195. Sieglingia decumbens Bernh. 4

196. Stipa capillata L. 4 c.

c. 28

197*. „ Graffiana Stev. 4 c.
198. Stipa pulcherrima C. Koch. 4 c. 199. Tragus racemosus Desf. $\odot$ c. 200. Trisetum flavescens $P . B .4$ c. 201. " rigidum Trin. 4 c. 202. " sibiricum Rupr. 4 c. 203. Triticum dicoccum Schrank. v. dicoccoides $\odot$ c. 205. $" \quad$ ventricosum Ces. c.

\section{I ridace a e Juss.}

206.* Gladiolus hydridus 4 c.

207. " imbricatus L. 4 c.

208. Iris corthalinia Fom. 4 c.

209. "Fischeriana Hort. 4 c.

210. " halophila Pall. 4 c.

211. " missouriensis Nutt. 4 c.

212. " musulmanica Fom. 4 c.

213. " Pseudacorus L. 4 c

214. " setosa Pall. 4 c.

215. " sibirica L. 4 c.

216. ” spuria L. 4 c.

217*. " Thunbergii E. Lundstr. 4 c. 218. Sisyrinchium anceps Cav. 4 c.

$$
\text { Juncacea e Vent. }
$$

219. Juncus atratus Krocker. 4 G. 220. " balticus Willd 4 c.

221.* " bufonius $L$. $\odot$ c.

222. " campressus Jacq. 4 c.

223. " effusus L. 4 c.

224. " lamprocarpus Ehrh. 4 c.

225. Luzula nivea D. C. 4 c.

$226^{*}$. " pilosa Willd. 4 G. 27

Juncaginaceae Rich.

227. Scheuchzeria palustris L. 4 G.

Lilla ce a e Scop.

228. Allium albidum Fisch. 4 c.

229. " angulosum L. 4 c.

230. " coeruleum Pall. 4 c.

231. " fistulosum L. 4 c.

232. ” globosum Red. 4 c.

233.* " lineare L. 4 c.

234. " obliquum L. 4 c.

235. " odorum L. 4 c.

$236 .^{*}$ " rotundum L. 4 c.

237. " schoenoprasum L. 4 c. 
238.* Allium ursinum L. 4 c.

239. Anthericum Liliago L. 4 c.

240. Asparagus officinalis L. 4 c.

241. Asphodelus tenuifolius Cav. 4 c.

242. Bulbine annua Willd. $\odot$ c. 28

243. Bulbinella latifolia Kunth. c.

$244^{*}$. Convallaria majalis L. 4 c.

245.* Echendia terniflora Ortega 4 c.

246 * Gagea lutea Ker-Gawl. 4 c. $247^{*}$. Lilium Martagon L. 4 c.

248. ” tigrinum Gawler. 4 c. 249.* Majanthemum bifolium $D$. C.

250. Muscari racemosum Mill. 4 c.

\section{D i c ot y led o n e a e}

Aceracea e $\mathrm{Neck}$.

262. Acer platanoides $L . \hbar \mathrm{G}$.

263. ” tataricum $L$. 方 G.

A izo ace a e

264. Mesembrianthemum tricolor $\odot$ c. 265. Tetragonia expansa Murr. $\odot$ c.

A maranthace ae Juss.

266. Amaranthus Blitum L. $\odot$ c.

267. " caudatus $L . \odot$ c.

268.* " deflexus L. 4 c.

269. „ gangeticus $L . \odot$ c.

$270 . \quad \cdots \quad$ retroflexus $L \odot$ c.

Apocynaceae R. Br.

271. Vinca minor $L .4$ c.

Aristolochiaceae Blume.

272. Asarum europaeum L. 4 c,

Ascle $\rho$ iadacea e Lindl.

273. Asclepias Cornuti Decsn. 4 c. 274. Cynanchum medium K. Schum.

275. $" \quad$ Vincetoxicum $R$. $\mathrm{Br}$. $4 \mathrm{c}$.
251. Ornithogalum narbonense $L$.

252. Paris quadrifolia L. $4 \mathrm{M}$

253. Polygonatum multiflorum All.

4 c.

254. Puschkinia scilloides Adans 4 c.

255. Scilla autumnalis $L .4$ K. 27

256. " bifolia $L .4$ c.

257. Smilacina stellata Desf. 4 c

258. Tulipa suaveolens Roth. 4 c.

259.* Zygadenus elegans Pursch.

260. sibiricus A. Gray. 4 c.

261. Veratrum album $L$. var. Lobelianum Bernh. 4 c.
Bals a minaceae S. F.

276. Impatiens glanduligera Royle. 277.* $\quad$ noli-tangere $L . \odot$ c.

- B erberidaceae Torr. et.

$$
\text { Gray. }
$$

278. Berberis vulgaris $L$. $\hbar \mathrm{G}$.

$$
\text { Betulaceae Rich. }
$$

279. Carpinus orientalis Mill. 5 K. 27 280. Corylus Avellana $L . \hbar$ G.

Borraginaceae S. F.

281. Anchusa italica Retz: $\odot$ c. 282. Borrago officinalis $L$. $\odot$ c. 283.* Cerinthe major $L$. $\odot$ c.

284. $\quad$ minor $L . \odot$ c.

285. Cynoglossum officinale $L \cdot 4$ c.

286. Echium vulgare $L$. $\odot$ c.

287. Heliotropium europaeum $L$. $\odot$ c. $288 . \quad$ peruvianum $L .4$ c. 289. Lappula Myosotis Moench. $\odot$

c. 28

290. Lindelofia spectabilis Lehm. 4 c. 291. Lithospermum officinale $L .4$ c 292. Lycopsis arvensis $L . \odot, \odot$ c. 293.* Mertensia echioides Benth. 4 c, 294. Myosotis intermedia Link. $\odot, \odot$. 
295. Myosotis silvatica Hoffm. $\odot, 4$ c. 296. Nonnea pulla D. C. 4 c.

297. versicolor Sw. c. 26

298. Omphalodes liniforia Moench. $\odot$

299.* Pulmonaria officinalis L. 4 c. 300. Symphytum officinale L. 4 c.

Campanulaceae Juss.

301. Adenophora Bulleyana Diels. 4 c. 302. Campanula abietina Gris. c.

303. " alliariaefolia Willd.

304. " americana $L . \stackrel{4}{ } \odot$ c.

305. " carpathica Jacq. 4

306. celtidifolia Boiss.

306. " c.

307. " cervicaria L. $\odot, 4$

308. „ Correvoniana Hort. 4 c.

309. $\quad$ erinus $L . \odot$ c.

310.* "

311.

312.

314.

315.

$316 . \quad$ "

$317 . \quad$,

318.

$319 . \quad$,

320. " longistyla Fomin. 4

321. Medium L. .) c,

322. " " michauxioides Boiss

mollis $L .2 \mathrm{c}$.

324. multiflora W. et. Kit

c.

325. " nobili-macrantha Reg

c.

326. „ patula L. (. c.

327. " " pelviformis Lam. c.

328. " persicifolia L. 4 c.

329. " pulcherrima Schrank et. Zeyh, 4 c.
330. Campanula rapunculoides L. 4

L. v, cor-

531. difolia $\overparen{C}$. Koch. 4 c.

332.* " Rapunculus L. $\odot$

c. 27

333. ” Reuteriana B.et. Bal.

334. - rotundifolia $L .4$ c.

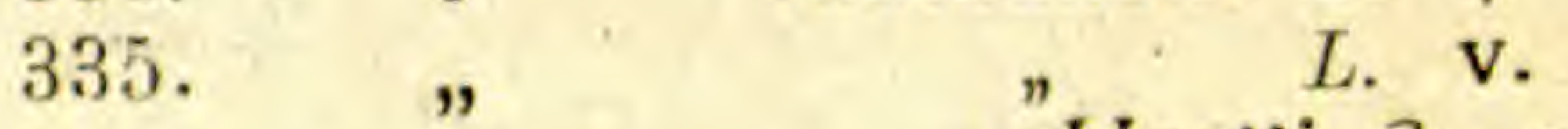

Hostii 4 c.

336. „ sarmentosa Hor.hst.

337. \# $\quad$ Scheuchzeri Lodd. 4

c.

338. ” Steveni M. B. 4 c.

339. - thyrsoides $L$. c.

340 , " Tomasiniana Koch.

c. 28

341. " trachelium L. 4 c.

342. " turbinata Schot.,

Nym. et Kotschy. 4 c.

343. \Van Houttei Carr.

344. Clintonia pulchella Lindl. $\odot$ c. 345.* Codonopsis ovata Benth. 4 c.

27

346. " ussuriensis Hemsl.

viridiflora 4 c.

* " " viridiflora Max. 4 c.

348.* Jasione montana $L$. $\odot$ c. 28

349. ” perennis Lam. 4 c.

350.* Laurentia Michelii A.D.C. $\odot$ c.

351. Lobelia erinus $L$. $\odot$ c.

352. \# inflata $L . \odot$ c.

353. * syphilica L. 4 c.

354. Phyteuma canescens W. et. Kit.

355 spicatum L. 4 c. 4 c.

356. Platycodon grandiflorum D. C.

4 c.

357. Prismatocarpus strictus D. C. c.

358. Specularia Speculum D. C. ๑ c.

359. Symphyandra Hoffmannii Pant.

360. Wahlenbergia pendula Schrad.

(.) c.

Cannabaceae Endl.

361. Humulus Lupulus L. 4 c. 
Ca $\rho \rho$ aridace a e

362. Capparis spinosa $L .5$ K. 27 363.* Cleome Candelabrum Sims. $\odot$

c.

$364 . * \quad \begin{aligned} & \text { graveolens Rafin. } \odot \text { c. } \\ & 365 *^{*} \quad \text { ornithopodioides } L . \odot \\ & 366 .\end{aligned} \quad$ K. 27
$\quad$ violacea $L . \odot$ c.

Caprifoliaceae Vent.

367. Adoxa Moschatellina L. 4 c. 368. Lonicera tatarica $L .5$ c. 369. Sambucus ebulus $L .24$ c. 370. " nigra $L .5$ c. 371. " racemosa $L . \hbar$ c. 372 Symphoricarpus racemosa Mchx. 万 c.

373. Viburnum Lantana $L . \hbar$. c. 374. $"$ Opulus $L .5$ G.

Caryophyllaceae Juss.

375. Agrostemma Githago L. $\odot$ c. 376. Alsine pinifolia Fenzl. 4 c. 377.* Arenaria graminifolia Schrad.

378. ” longifolia $M, B, 4$ c.

379. $n$ serpyllifolia $L . \odot$ c. 28

380. Cerastium arvense L. 4 c.

381. " caespitosum Gilib. 4

382. Corrigiola littoralis $L$. $\odot$ c.

383. Cucubalus baccifer $L,-4$ c.

384. Dianthus arenarius $L .4$ c.

385. " attenuatus $\mathrm{Sm} .4 \mathrm{c}$.

386. ” barbatus $L, 4$ c.

387. " Borbasii Vandas. 4 c.

388. " campestris M. B. 4 c.

389. " chinensis $L . \%$ c.

390. " collinus W. et. Kit. 4

391. deltoides $L .24$ c c

392. " giganteus D'Urv. 4 c.

393. , laciniatus : c.

394. " plumarius L. 4 c.

395. ” polymorphus M. B. 4

396. . rupicolus Biv, 4 c.

397. Elisanthe noctiflora Rupr. $\odot$ c.
398. Gypsophyla elegans M. B. $\odot$ c. 399 . muralis $L$. $\odot$ c. 28 400.* Herniaria glabra L. 4 c,

401. Holosteum umbellatum $L . \odot, \odot$ 402. Lychnis chalcedonica $L .4$ c.

403. "Flos cuculi L. 4 c. 404. " Flos-Jovis Lam. $\odot$ c, 405. " fulgens Fich. $\odot \cdot$ c.

406. Malachium aquaticum Rehb. 4 c. 407. Melandryum album Garcke. $\odot$, $\odot$

408. Sagina procumbens L. 4 c.

409. Saponaria ocymoides $L_{i} 4$ c.

410. $\quad$ officinalis $L .4$ c.

411. Scleranthus annuus $L$. $\odot$ c.

412. Silene Armeria $t . \odot$ c.

413.* " Brotherana Som. Lev.c. 27

414*. " compacta Fisch. $\odot$ c.

415. " conica $L . \odot$ c.

416.* $"$ conoidea $L . \odot$ c.

417.* " chlorantha Ehrh. 4 c. 26

418. " dichotoma Ehrh $\odot$ G.

419. " gallica L. $\odot$ c.

420.* \# italica Pers. 4 c. 28

421. " Otites Sm. .), 4 c. 26

422. " saxatilis Sims. c.

423.* " sibirica Pers. 4 c. $26=$

424. " tatarica Pers. 4 c.

425. " venosa Aschers. 4 c.

426. " wolgensis Pers. 4 c. 26

427. Spergula arvensis $L . \odot$ c.

428. Spergularia campestris Aschers.

429. Stellaria graminea $L .4$ c.

430. " media $L$. c.

431. Tunica prolifera Scop. $\odot$ c. 28

432. " Saxifraga Scop. 4 c.

433.* Vaccaria pyramidata Medik. $\odot$ c. 28

434. Viscaria viscosa Aschers. 4 c.

Celastraceae Lindl.

435. Evonymus europaeus $L .5$ c. 436. " verrucosus Scop. 5 G.

Chenopodiaceae Less.

437 Atriplex hastatum $L, \odot$ c. 438. " hortense $L . \odot$ c. 28 439. Beta patellaris Moq. $\odot$ c. 440. Chenopodium album $L . \odot$ c. 
441. Chenopodium Botrys $L . \odot$ c. 442 .

443.

444. foliosum Schrad. $\odot$ c. murale $L . \odot$ c. polyspermum $L$. $\odot$ c.

445. $\quad$ vulvaria $L . \odot$ c. 446. Corispermum hyssopifolium $L$. $\odot$ c. 447. Kochia trichophylla Schmeiss. $\odot$ c. 448. Monolepis trifida Schrad. $\odot$ c. 449. Spinacia oleracea $L . \odot$ c. 450. $\# \quad$ tetrandra Stev. $\odot$ c. Cistace a D un.

451. Helianthemum Chamaecistus 452. Mill. 4 c. guttatum Mill. (-) c.

Compositae Adans.

453. Achillea Millefolium L. 4 c. 454. " nobilis $L .24$ c. 28 455. " Pratmica L. 4 c. 456. " trichophylla Schrenk.

4:3. Acroclinum roseum $\odot \mathrm{c}$. c. 458. Ageratum mexicanum Sims. 459. Anthemis altissima $L$. $\odot$ c 460.,$\quad$ arvensis $L . \odot$ c. 461. Arctotis grandis Thunb. () c. 462. Arnica Chamissonis Less. 4 c. 463.* " montana L. 4 c.

464. Artemisia Absinthium I. 4 c. 465. - campestris L. 4 c. 466. ” vulgaris $L, 4$ c.

467. Aster alpinus L. 2 c.

468. " salicifclius Scholl. 4 c. 469. Baeria coronaria A. Gray. $\odot$ c. 470. Bellis perennis $L .4$ c.

471. Bidens leucantha Willd. $\odot$ c. 472. " tripartitus $L . \odot$ c. 473. Buphtalmum speciosum Schreb. 4 c.

474. Calendula officiralis $L . \odot$ c. 475. Callistephus chinensis Nees. $\odot$ c. 476. Carlina vulgaris $L . \odot$ c.

477. Carpesium cernuum L. 4 c. 478. Carthamus tinctorius $L . \odot$ c. 479. Centaurea axillaris Willd \& c. 480. bella Trev. c.
481. Centaurea Calcitrapa $L$. v. iberica Trev. $\odot$ c. 28

482. ” Cyanus $L . \odot$ c.

483.* " glastifolia L. 4 c.

484. " Jacea $L .2$ c.

485.* " Koenigii Sosn. c.

486. " maculosa Lam. .. c.

48\%. $*$ nigra $L$. c.

488. " reflexa Lam. 4 c. 28

489. " salonitana Vis. 4 c, 28

490,* Chondrilla juncea $L .2$ c.

491. Chrysanthemum corymbosum $L$.

$4 \mathrm{c}$

493. 4 c.

493. " tricolor Hort.

494. $\quad$ viscosum Desf.

405. Cichorium Intybus 2 c.

495. Cichorium Intybus L. 4 c.

496. Cirsium arvense Scop. $4 \mathrm{M}$.

497. ऋ hispanicum D.C. c.

498. " lanceolatum Scop. (-) c.

499. " Maackii Max. 4 c. 28

500. " rivulare Link. 4 c. 28

501. Cnicus Benedictus L. $\odot$ c.

502. Coreopsis coronata Hoock. c.

503. $"$ peltata L. $\odot$ c.

504. Cosmea bipinnata Willd. () c.

505. Cosmos diversifolius Otto. $\odot$ c.

506. Crepis biennis $L$. (;) c.

507. " foetida $L$. $\odot$ c.

508. " pulchra L. $\odot$ c.

509. " tectorum $I_{\text {. }} \odot$ c.

510. Crupina vulgaris Cass. () c.

511.* Dahlia Cactus Hort. 2 c.

512. " coccinea Cav. 2 c.

513. " variabilis Desf. 4 c.

514. Dimorphotheca aurantiaca D. C.

$515 . \quad$ pluvialis Moench. $\odot$ c.

516. Echinops globifer Janka. 4 c.

517.* Erigeron acer $L$. $\odot, 2$ c.

518* " alpinus $L .2$ c.

519. " canadensis L. $\odot, \odot$ c.

520. Eupatorium cannabinum L. 4 c.

$521 . \quad$ Kirilovi Turca. c.

522. Evax pygmaea Brot. $\odot$ c.

523. Felicia tenella Ness. $\odot$ c.

524. Filago spathulata irest. (-) c.

525.* Gaillardia picta Sweet. $\odot$ c.

526. Galinsoga parviflora Cav. $\odot$ c. 
527. Gnaphalium vira-vira Molina.

58. Guizotia ole c.

528. Guizotia oleifera D. C. ๑ c.

529. Hedypnois cretica Willd. ๑ c.

530. Helenium Hoopesii A. Gray.

531. Helianthus cucumerifolius 4 c. et. Gray. $\odot$ c.

532. $\quad$ globosus $\odot$ c.

533.* Helichrysum angustifolium D.

C. c.

534. „ arenarium Moench.

535. Hieracium Pilosella L. 4 c.

536. " pratense Tausch. 4 c.

537. " silvaticum L. 4 c.

538. " umbellatum L. 4 c.

539. virosum Pall. 4 c.

540. Hyoseris lucida $L . \odot$ c.

541. Hypochoeris radicata L. 4 c.

542. Inula conyza $D . C . \odot .4$ c.

543 * " germanica $L .4$ c.

544. "Helenium L. 4 c.

545. ” magnifica Lipsky. c.

546.* " oculus christi L. 4 c.

547. Jurinea cyanoides Rehb. 4 c.

548. Lactuca sativa $L$. $\odot$ c.

549.* „ viminea Presl. *) c. 28

550. Lampsana communis L. $\odot$ c. 27

551. " grandiflora $M$. $B$.

4 c.

552. Lappa amplissima Boiss, $\odot$ c.

553. " tomentosa Lam. $\odot$ c.

551. Lasiospermum pedunculare Lag

555. Layia Calliglossa Gray. () c.

556. Leontodon autumnalis L. 4 c.

557.* Leontopodium alpinum Cass. 4

558. Madia sativa Mol. (-) c.

c.

559. Matricaria Chamomilla L. ๑ c.

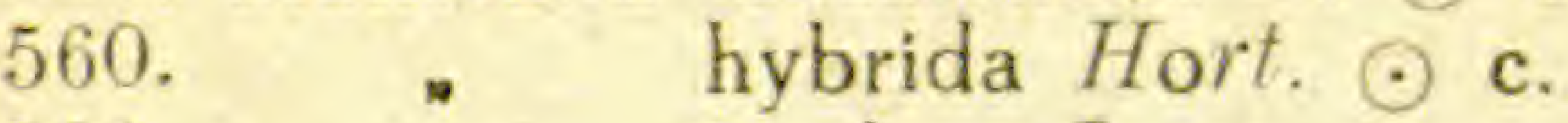

561. " inodora $L . \odot$, $\odot$ c.

562. " suaveolens Burhen.

563. Mulgedium sibiricum Less. 4 c.

564. Onopordon Acanthium L. - c c.

565. " $"$ illyricum $L$. .. c.

566.* Petasites officinalis Moench. 4

$\mathrm{G}$.

567. Picris hieracioides $L . \odot, 4$ c.

568.* Psephellus dealbatus Boiss. c. 27 569

hypoleucus Boiss. c.
570. Pyrethrum Parthenium Sm. 4

571. $\quad$ roseum $M . B .2$ c. 27

572. Rhagadiolus stellatus $D . C . \odot$ c.

573. Rudbeckia hirta $L . \odot$, $\odot$ c.

574. ” laciniata L. 4 c.

575. Scorzonera eriosperma $M$. B.

576 . $" \quad$ hispanica L. 4 c.

577. " laciniata L. $\odot, ~ 4$ c.

578. Senecio erraticus Bertol. $\odot$ c.

579. " Jacobaea L. 4 c.

580. " ligularia Hook. 4 c.

581. " vulgaris $L . \odot, \odot$ c. 28

582. Serratula coronata $L .4$ c.

$583 . \quad$ heterophylla Desf. 4 c.

584. " radiata $M . B .4$ c.

585* Siegesbeckia orientalis $L$. $\odot$ c.

586. Silybum Marianum Gaertn. $\stackrel{26}{\odot \text {. }}$

587.* Solidago virga-aurea $L . \stackrel{+}{4}$ c.

588. Sonchus arvensis L. 4 c.

$589 . \quad " \quad$ asper Hill. $\odot$ c.

590. " oleraceus $L$. $\odot$ c.

591. Tagetes erecta $L$. $\odot$ c.

592. " lucida Cav. 4 c.

593. " patula $L . \odot$ c.

594. ” signata Bartl. $\odot$ c.

595. Tanacetum vulgare $L .4$ c.

596. Taraxacum laevigatum $D$. C.

597. 4 c.

598. Tragopogon officinale Wigg. 4 c.

$599-\odot$ c.

599. " coloratus C.A. M.

600. " major Jacq. c. 28

601. $\quad$ porrifolius $L$. $\odot$ c.

$602 . \quad$ pratensis L. $\odot$ c.

603. Tussilago Farfara L. 4 c.

604. Urospermum picroides $F . W$. Schmidt. $\odot$ c.

605.* Ursinia speciosa D. C. c.

606. Ximenesia encelioides Cav. $\odot$ c.

607. Zacintha verrucosa Giertn. $\odot$

608. Zinnia elegan3 Jacq. $\odot$ c. c. 28.

609. " multiflora L. $\odot$ c.

610. " $"$ tenuiflora Jacq. $\odot$ c.

Convolvulacea e Vent.

611. Convolvulus tricolor $L$. $\odot$ c. 
612. Convolvulus undulatus $\mathrm{Cav}$. $\odot$ c. 613. Ipomoea purpurea $\mathrm{Lam}$. $\odot$ c.

\section{Cornaceae D. C.}

614. Cornus mas $L .5$ K. 27

Crassulacea e D. C.

615.* Cotyledon horizontalis Guss.

4 c.

616. Crassula glomerata Berg. $\odot$ c. 28

617. Sedum acre $L .4$ c.

618. " aizoon L. 4 c.

619. " Ewersii Ledb. 4 c.

620. " glaucum W. et. K 4 c.

621. " hybridum L. 4 c.

622. " kamtschaticum F. et. M.

4 c.

623. „ pallidum M. B. . c. c.

624. ” purpureum Link. 4 c.

625. " rupestre $L .4$ c.

626. ” spurium M. B. 4 c.

627. Sempervivum ruthenicum Koch. 2 c.

628. " tectorum L. 4 c.

Cruciferae Juss.

629. Aethionema Buxbaumii D. C.

( ) c.

630.* Alyssum desertorum Steph. $\odot$

c. 28

631.* , linifolium Steph. $\odot$ c. 28

632. Arabis albida Stev. 4 c.

633. ” hirsuta Scop. $\odot, 4$ c.

634. " laxa Sib. et. Sm. c.

635. Barbarea stricta Andrz. - c. 28

636. vulgaris $R . \mathrm{Br}$. $\odot$ c.

$637 *$ Berteroa incana $D . C . \odot, \odot$ c.

638. Brassica juncea Czern. $\odot$ c.

639. Bunias orientalis $L .2$ G.

640. Camelina sativa Crantz. $\odot$ c.

641. Capsella bursa pastoris Moench

$\odot$ c.

642. Carrichtera Velía D. C. $\odot$ c.

643. Cheiranthus nanus Merk. $\odot$ e.

644. Chorispora tenella D. C. $\odot$ c. 28

645. Cochlearia officinalis $L .4$ c.

646. Conringia orientalis Dum. $\odot$ c.

647. Crambe hispanica $L$. $\odot$ c.

648.* ” maritima L. 4 c.
649. Descurainia Sophia W. et. B.

(.) c. 28

650. Diplotaxis muralis D. C. $\odot$ c.

651.* Draba hirta L. 4 c.

652. Erinus alpinus L. 4 c.

653. Erysimum cheiranthoides $L$. $\odot$ c.

654 . $\quad$ cuspidatum D.C. $\odot$ c.

655. " pulchellum $J$. Gay.

656. suffruticosum c.

657. Eruca sativa Lam. $\odot$ c.

658. Erucastrum elongatum L. 4 c.

659. Farsetia clypeata $R$. Br. 4 c.

660. " eriocarpa $D$ C. 4 c.

661. Hesperis fragrans Fisch. 4 c.

662. " lutea Max. $\odot$ c.

663. matronalis L. 4 c.

664.* Hutchinsia alpina $R . B r .4$ c.

665 . Iberis amara $L . \odot$ c.

666 . " pectinata Boiss. $\odot$ c.

667. " sempervirens $L .2$ c. 27

668 . $~ u m b e l l a t a ~ L . \odot$ c.

669. Isatis tinctoria $L$. . c.

670.* Lepidium Draba L. 4 c. 28

$671 . \quad \% \quad$ ruderale $L$. $\odot$ c.

672. ” sativum $L$. $\odot$ c.

673. Lunaria biennis Moench. (-) c.

$674^{*}$. " redeviva $L .4$ c.

675. Malcolmia africana Ait. $\odot$ c.

676. Matthiola annua Sweet. $\odot$ c.

677 . $\#$ bicornis $\odot$ c.

6:8. Melanosinapis communis Spenn.

○. c.

679 Myagrum perfoliatum L. $\odot$ c.

680. Nasturtium palustre D.C. $\odot, 4$ G.

681. Neslia paniculata Desv. ๑ c.

682. Peltaria alliacea L. 4 c.

683. Raphanus Raphanistrum L. $\odot$ c.

684. Rapistrum rugosum All. (- c.

685. Senebieria Coronopus Poir. $\odot$ c.

686. Sinapis alba $L$. $\odot$ c.

687. $"$ apula Tenore. $\odot$ c.

688. Sisymbrium Irio L. 4 c. 28

689. - officinale Scop. () c.

690. Stenophragma Thalianum Celak.

(.) c. 28

691. Succovia balearica Medic. () c.

692. Thlaspi arvense $L . \odot$ G. 28

693. Turritis glabra $L . \odot$ c.

Cucurbitaceae Holl.

694. Bryonia alba $L .4$ c. 
695. Cucumis Chito Naud. (.) c. 27

696. „ prophetarum $L$. $\odot$ c.

697. Cucurbita maxima Duch. $\odot$ c.

698. $״$ melanosperma $\mathrm{A}$. $\mathrm{Br}$.

699. Melopepo L. $\odot$ c.

700. " napolitana Reg. $\odot$ c.

701. $~ \# \quad P e p o L . \odot$ c.

702. " verrucosa $L$. $\odot$ c.

703. Cyclanthera pedata Sinrad. $\odot$ c.

704. Ecballium elaterium Rich. ๑ c.

705. Echinocystis Wrightii Cogn. () c.

706. Lagenaria vulgaris Ser. $\odot$ c.

707. Melothria cucumerina Naud. c.

708. Sicyos angulata $L$. $\odot$ c.

D a $t$ is c a ce a e.

709. Datisca cannabina L. 4 c.

Di $\rho$ s a c a ceae D. C.

710. Callistemma brachiata Sibth.

711.* Cephalaria media Litw. c. 27

712. $\#$ syriaca Schrad. 4 c.

713. \# tatarica Schrad.

714. Dipsacus ferox Loiss. $\odot$ c.

715. „ fullonum Mill. (-) c.

716. " laciniatus L. $\odot$ c. 26

717. " plumosus Franch. :- c.

718. Knautia arvensis Coult. 4 c.

719. " atrorubens Janka 4 c.

720. Pterocephalus plumosus Coult.

721. Scabiosa atropurpurea c. 26

722. " Columbaria L. 2 c. 27

723 . " leucophylla Borb. 4 c.

724. lucida Vall. 4 c.

725. " micrantha Desf. (- c.

$726^{\circ} \quad " \quad$ ochroleuca L. 4 c. 27

727. $\quad$ ucrainica L. .. K. 27

728. Succisa praemorsa Aschers. $4 \mathrm{M}$

Empetraceae $\mathrm{Lind}$.

729. Empetrum nigrum $L . \hbar \mathrm{G}$.

Ericaceae Lind I.

730.* Arctostaphylos Uva-uris Spr. $\hbar$ 731. Calluna vulgaris Salisb. $₹$ G.
Euphorbiacea e J. St. Hil.

732. Euphorbia Balsamifera Ait. 4 c.

733. " Chamaesyce L. $\odot$

734. " Esula L. 4 G.

735. " $\quad$ exigua $L . \odot$ c.

736. ” graeca Bolss. et. Sprun. $\odot$ c.

737. $"$ helioscopia $L, \odot$ c.

738. $"$ humifusa Willd. $\odot$ c.

739. " uralensis Fisch. 4 c.

740. Mercurialís annua $L$. $\odot$ c.

741. Ricinus communis L. $\odot$ c. 27

Gentianaceae Dumort.

742. Gentiana cruciata $L .4$ c.

743. " Pneumonanthe L. 4 c.

744. Menyanthes trifoliata L. \& G.

745.* Swertia longifolia Boiss. 4 c.

Geraniaceae J St. Hil.

746. Erodium cicutarium L. Herit.

747. " gruinum Ait. $\odot$ c.

$\odot$ c.

748. Geranium albanum $M$ B. 4 c.

749. $"$ dissectum $L$. $\odot$ c.

750. " divaricatum Ehrh. 4 c.

751. ” palustre L. 4 c.

752. " pratense L. 4 c.

753. „ $\quad$ pusillum L. $\odot$ c.

$754^{*}$ " sanguineum L. 4 c.

Globulariaceáe D. C.

755. Globularia trichosantha $F$ et. $M$.

$756 \quad$ Willkommii Nyman.

$4 \mathrm{c}$.

Guttiferae Endl.

757. Hypericum Ascyron L. 4 c.

758. " montanum $L, 4$ c.

759. " $"$ perforatum L. 4 c.

760 . $\quad$ quadrangulum $L .4$ c.

Hydroph yllaceae

761. Nemophila maculata Benth. $\odot$ c. 762. Phacelia divaricata A. Gray. $\odot$ c. 
763. Phacelia tanacetifolia Bentham.(c).

Labiatae Juss.

764. Ajuga reptans L. 4 c.

765. " " L. v. albiflora

766. Ballota nigra $L .4$ c.

Tin. 4 c.

767. Betonica officinalis L. 4 c.

768. Brunella glandiflora Moench.

769. $\quad$ vulgaris $L .24$ c.

770. Dracocephalum moldavica $L$.

771.

Ruyschiana $L$.

772. Elscholtzia cristata Willd 4 c.

773. Eremostachys laciniata Bge.

774. Galeopsis speciosa Mill. $\odot$ c. 775 . $\quad$ Tetrahit $L . \odot$ c.

776. Glechoma hederacea L. 4 c.

777. Horminium pyrenaicum L. 4 c.

778. Hyssopus officinalis $L$. $\hbar$ c.

779. Lallemantia iberica $F$. et. $M$.

780.

$\odot$ c.

$$
" \quad \text { peltata } F \text {. et. M. }
$$

781. I amium album $L .4$ c.

$\odot$ c.

782. " amplexicaule $L . \odot . \odot$ c.

783.* " Galeobdolon Crantz.

784. ” maculatum L. 4 c.

785. " purpureum L. $\odot, \odot$ c.

786. Leonurus Cardiaca L. 4 c.

787. " Marrubiastrum L. $\odot$, $\odot$

788. Lycopus europaeus L. 4 c.

789. ” exaltatus $L .24$ c.

790. Marrubium praecox Janka. 4 c.

791. ” vulgare $L .2+$ c.

792. Melissa officinalis $L .2+\mathrm{c}$.

793. Mentha arvensis L. 4 c.

794.* " longifolia Huds. 4 c.

795. " Pulegium L. $\odot$ c.

796. Nepeta Cataria L. 4 c.

797. " grandiflora M. B. 4 c.

798. " macrantha Fisch. 4 c.

799. " nuda $L .4$ c.

800. Origanum vulgare $L .4$ c.

801. Phlomis pungens Willd. \& c.

802. " tuberosa $L . .4$ c.

803. Plectranthus glaucocalyx Max.
804. Pycnanthemum lanceolatum Pursh. 4 c. 28

805. Salvia Aethiopis L. 4 c. 27

806. " azurea Lam. 4 c. 28

807. " cleistogama De Bar. et.

Paul. 4 c.

808. " dumetorum Andra. c. 28

809. ” glutinosa L. 4 c.

810. " leonia Benth. 4 c.

811. " nemorosa $L .4$ c.

812. " nilotica Murr. $\odot$ c.

813.* ” officinalis $L . \hbar$ c.

814. " Regla Cav. c.

815. " Sibthorpii Sm. 4 c.

816.* " triangularis Thunb. 5 c. 27

817. " Verbenaca L. 4 c. 27

818. " verticillata $L .4$ c.

819. Satureja Clinopodium Caruel.

820.

821. tum Mort. 4 c.

822 " hortensis $L . \odot$ c.

" serpyllifolia B $* i q . \hbar$

c. 28

823. Scutellaria altissima $L .4$ c.

824. " galericulata $L .4$ c.

825.,$\quad$ orientalis $L .4$ c.

826. Sideritis montana $L$. $\odot$ c.

827. " scordioides L. 4 c.

828.* Stachys annua $L$. $\odot$ c. 28

829. " lanata Jacq. 4 c.

830. " palustris L. 4 c.

831. " recta $L .4$ c.

832. " sideritoides C. Koch. c.

833. ” $\quad$ silvatica $L .4$ c,

834. Teucrium Chamaedrys L. 4 c.

835. " hyrcanicum L. 4 c. 27

836. Thymus glaber Mill. $4 \mathrm{c}$.

837. " odoratissimus $M . B$.

838. " serpyllum L. v. citrio-

Legumin os a Jus s.

839. Amorpha fruticosa $L$. 5 c. 27

840. Astragalus Cicer L. 4 c.

841. " glycyphyllus $L . \&$ c.

842. " Onobrychis L. 4 c.

843. " sulcatus $L, 4$ c.

844. Caragana frutescens $D . C .5$ c.

845. Colutea cruenta Ait. $\hbar$ c.

846. " orientalis Mill. क c. 27 
847. Coronilla varia L. 4 c.

848. Cytisus scoparius Link. ई $\mathrm{M}$.

849. Dolichos Lablab L. $\odot$ c.

850. Dorycnium herbaceum Vill. Ђ

851. Ervum Lens L. $\odot$ c. 28 c. 27

852. Galega orientalis Lam. 4 c.

853. Genista ovata W. et. $K$. 万 c.

854. " tinctoria $L .5$ c.

855. Glycyrrhiza echinata L. 4 c.

856.* Hedysarum obscurum L. 4

c. 26

857. Lathyrus Aphaca $L \odot$ c.

858. " canescens Taubert. $4 \mathrm{c}$

859. " Clymenum $L$. $\odot$ c.

860. " montanus Bernh. 4

861. " ochrus D.C. $\odot$ c.

862. " odoratus L. $\odot$ c.

863. $"$ palustris $L .4$ c.

864. " pratensis L. 4 c.

865. " sativus L. $\odot$ c.

866. " $"$ silvestris L. 4 c.

867.* " sphaericus Retz (- c.

868. " vernus Bernh. 4 c.

869. Lotus corniculatus L. 4 c.

870. " edulis L. $\odot$ c.

871. " tetragonolobus $L . \odot$ c.

872. Lupinus mutabilis $S w$. ( c.

873. $" \quad$ perennis $L .4$ c.

874. -. pilosus Murr. () c.

875. Medicago catalonica Schk. c. 28

876. " hispida Gaertn. $\odot$ c.

877. " laciniata Mill. $\odot$ c.

878. " lupulina $L . \odot, 4$ c.

879. " maculata Willd. $\odot \mathrm{c}$,

880. " orbicularis All. $\odot$ c.

881. $\quad$ sativa L. 4 c.

882. " scutellata All. (c) c.

883. Mielilotus albus Desr: -) c.

884. $"$ indica All. $\odot$ c.

885 " officinalis Desr. . c.

886. " wolgicus Poir -. c. 28

887. Onobrychis viciaefolia Scop. 4 c. 25

888. Ononis biflora Desf. (. c. 28

889. , hircina Jacq. 4 c.

890. $"$ rotundifolia $L .5$ c.

891. Ornithopus sativus Brot. (-) c.

892. Oxitropis campestris D.C. 4 c.

893. Pisum arvense $L$. $\odot$ c.

894. " elatius $M . B . \odot$ c.

895. " Jomardi Schrank. ๑ c.

896. „ sativum L. $\odot$ c.
897. Pisum thebaicum $W$. $\odot$ c.

898. Robinia pseudacacia $L . \hbar$ c.

899. Scorpiurus subvillosa $L . \odot$ c.

900 . $" \quad$ vermiculata $L$. $\odot$ c.

901. Thermopsis caroliniana $M$. A.

902. $\quad$ furt. 4 c.
903. Tabacea D. C. 4 c.
904. Trifolium agrarium L. $\odot$ c
$905 . \quad \%$ alpestre L. 4 c.
$906 . \quad$ angustifolium L. $\odot$
c. 28

907. $\quad$ arvense L. $\odot$ c.

908.* " $"$ badium Schreb. .. c.

28

909. " cernuum Brot. $\odot$ c.

910.* $" \quad$ cherleri L. $\odot$ c.

911. " fragiferum L. 4 c.

912. " incarnatum $L$. $\odot$ c.

913.* " glomeratum L. $\odot$ c.

914. " $"$ Lupinaster L. 4 c.

915. " medium L. 4 c.

916. " montanum L. 4 c.

917. " pratense $L$ \& c.

918. " $"$ procumbens L. $\odot$

919 c. 28

920 " repens L. 4 c.

920. " resupinatum $L \odot \odot, \odot)=$

921. " $\quad$ rubens $L .4$ c.

922. ", spadiceum L. C c.

923. " $\quad$ striatum L. $\odot$ c. 28

924.* " tomentosum L. $\odot$

925.* Trigonella coerulea Ser. $\odot$ c. $2 \varepsilon$

926.,$\quad$ foenum-graecum $L$.

$927 . \quad$ orthoceras $K a r$ c.

, Kir. - c.

928. Vicia angustifolia Roth. $\odot$ c.

929. " " atropurpurea Desf. $\odot$ c.

930. " cracca L. 4 c.

931. " Ervilia Willd. ๑ c.

932. " Faba L. $\odot$ c.

933. " hirsuta G. Koch. $\odot$ c.

934. ", lutea $L$. $\odot$ c.

935. " Michauxii Spreng. $\odot$ c.

936. " monanthos Desf. $\odot$ c.

937. " narbonensis $L$. ( c.

938. " picta Fisch. $\odot$ c. 27

939. " pisiformis L. 4 c.

940. ", sativa L. (sem. alb.) $\odot$ 
941. Vicia sepium L. 4 c.

942. " unijuga $A . B r .4$ c.

Li m n a n thace e.

943. Limnanthes alba Hartw. $\odot$ c. 944. " Douglasii $R$. Br. $\odot$ c.

Linaceae Dumort.

945. Linum nervosum $W . K .4$ c. 946. " perenne $L .4$ c.

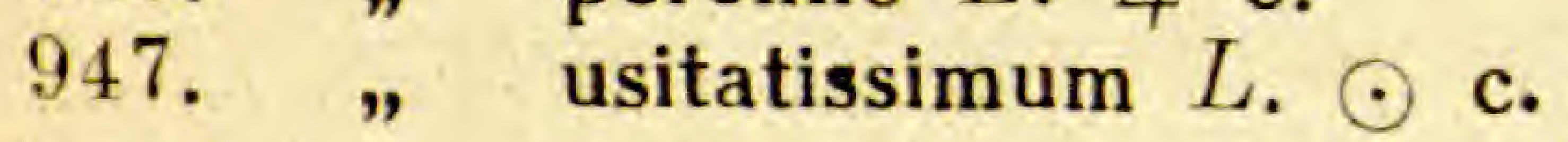

Lo a s a e a e.

948. Blumenbachia Hieronymi Urb.

949. Loasa bryoniaefolia Schrad c.

950. ” triphylla Juss $\odot$ c.

951. " vulcanica Andre. $\odot$ c.

Lythraceae Lindl.

952. Cuphea lanceolata Ait. $\odot$ c.

953. Lythrum salicaria $L .4$ c.

954. Nesea salicifolia $H$. B. et. $K$.

支.

Malvareae Juss.

955. Abutilon Avicennae Gaertn. $\odot$ c 956. „ Regneli Miq. c.

957. Althaea ficifolia Cav. 4 c.

958. " officinalis L. 4 c.

959. " rosea Cav. 4 c.

960. Anoda cristata Schlecht. $\odot$ c.

961. Hibiscus Trionum $L . \odot$ c.

962. Lavatera thuringiaca $L .4$ c.

963. ״ trimestris $L . \odot$ c.

964. Malope grandiflora $\odot$ c.

965. Malva Alcea $L .4$ c.

966.* " brasiliensis Desr. c.

967. " $\quad$ crispa $L . \odot, \odot$ c.

$968 .^{*}$ " hispanica L. c. 28

969. " neglecta Wall. $\odot$ c.

970. " parviflora L. $\odot$ c.

971. " rotundifolla $L$. $\odot$ c.

972. \# silvestris L. $\odot$ c. 27

973. Malvastrum peruvianum A. Gray.

$\odot$ c. 28
Moraceae Lind I.

974. Morus nigra $L . \hbar$ c.

Nyctagin a c e a e.

975. Mirabilis Jalappa $L$. $\odot$ c.

976. Oxybaphus glabrifolius Vahl. c.

Oleacea e Lindl.

977. Fraxinus excelsior $L . \bar{G}$ G

978. Ligustrum vulgare $L$. $\hbar$ c.

979. Syringa vularis $L$. $\hbar$ c.

On agraceae Lind I.

980. Boisduvalia densiflora Wats. ( c. 981. ” Douglasii Spach.

982. Circea alpina L. 4 c.

$\odot \mathbf{c}$.

983. Clarkia elegans Dougl. @ c.

984. Epilobium adnatum Gris. 4 c. 985.* " Dodonaei Villars. 4 c. 986. " hirsutum L. 4 c.

987. " Lamyi F. Schultz.

4 c.

988. „ montanum L. 4 c. 27

$989 . \quad$ palustre L. 4 G. 27

990. " $\quad$ pedunculare A.Cunn.

4 c.

991. Eucharidium grandiflorum $F$. et. M. $\odot$ c.

992. Gaura parviflora, Dougl $\odot$ e.

993. Godetia grandiflora Lindl. () c.

994. Jussieua peruviana $L . \odot$ c.

995. Lopezia racemosa Cav. () c.

996. Oenothera ammophila Focke.

$997 . \quad$ biennis $L$ fro

998. " " fruticosa L. 4 c.

999. " gigas De Vries. c.

1000. " glauca Michx. 4 c.

1001. " grandiflora Ait. .) c.

1002. " Lamarkiana L. 4 c.

1003.* " missouriensis Sms.

" muricata $L . \odot \mathrm{c}$.

1005. " odorata Jacq. () c.

1006. " pumila $L .4$ c.

1007. $\rightarrow$ purpurea Curt. (c) c.

1008. rosea Ait. 4 c.

1009. " tetraptera Cav. c. 
1010. Oenothera triloba Nuttal. $\odot$ c.

Oxalidaceae Lindl.

1011. Oxalis corniculata $L . \odot, \odot$ c. 1012. ” stricta L. 4 c.

Pa $\rho$ averaceae Juss.

1013. Argemone mexicana $L$. $\odot$ c. 1014. Chelidonium Franchetianum Prain. $\odot$ c. 1015. \# laciniatum Mill. 1016. " majus L. 4 c. 1017. Corydoalis cava Schw. et Koerte. 4 c.

1018. " glauca Purch. $\odot$ c. 1019. " ophiocarpa Hook, f. et. Thoms. 4 c. 1020. " sempervirens Pers. 1021. " vaginalis Royle. c. 1022. Eschscholtzia californica Cham. $\odot$ c.

1023. Fumaria officinalis $L$. $\odot$ c. 1024. Glaucium flavum Crantz .-) c. 102. Hypecoum grandiflorum Benth. 1026. pendulum $L . \odot$ c. 1027.* Meconopsis cambrica Vig. 4 c. 1028* Papaver alpinum L. 4 c. 26 1029. ” argemone $L . \odot$ c. 1030. ” atlanticum Ball. 4 c. 1031. " dubium L. $\odot$ c. 1032. " fugax Poir. $\odot$ c. 1033. " orientale L. 4 c. 1034. " pilosum Sibth. 4 c. 1035. " Rhoeas L. $\odot$ c. 1036. " somniferum L. $\odot$ c. 1037. opiiferum $\odot$ c. 1038. Raemeria violaceae Medic. $\odot$ c.

$\rho$ assifloraceae.

1039. Passiflora edulis Sims. $\hbar 27$

$\rho$ hiladel $\rho$ haceae.

1040. Philadelphus coronarius $L . \hbar$ c.
Phitolaccaceae.

1041. Phytolacca decandra L. 4 c.

Plantaginaceae Lindl.

1042. Plantago alpina L. 4 c.

1043. \# altissima $L$. c.

1014. " amplexicaulis Cav. $\odot$ c.

1045* $" \quad$ arborescens Poir. 4

1046*. " Brownii Rafin. c. 28

1047. " $\quad$ Candollei Rafin. c.

1048. " Cornuti Gouan. 4 c.

1049. " crassifolia Forsti. c. 28

1050. " Cynops L. 4 c. 27

1051. . $\quad$ indica $L$. c.

1052. " lanceolata L. 4 c.

1053. " lusitanica Willd. $\odot$ c.

1054. ” major L. 4 c.

1055. ” maritima L. 4 c.

1056. " maxima Ait. 4 c.

1057. ” media L. 4 c.

1058. " $\quad \rho_{\text {syllium } L . \odot \text { c. }}$

1059. " purpurescens Nutt.

1060. " ramosa Aschers $\odot$ c.

1061. " serpentina All. c.

1062. " $\quad$ serraria L. c. 28

1063. $\quad$ virginica $L$. $\odot$ c.

Plumbaginaceae Lindl.

1064. Armeria plantaginea Will if c. 1065. Plumbagella micrantha Ledeb.

1066. Statice tatarica L. 4 c.

Polemoniace a e D. C.

1067.* Collomia grandiflora Dougl. $\odot$ c. 2.9

1068. ” linearis Nutt. () c. 1069. Gilia californica Benth. क) c. 1070. Leptosiphon androcacers St.

(.) c.

1071. Navarretia squarrosa Hook, et. Arn. $\odot$ c.

1072. Phlox Drummondi Hook. $\odot$ c. 1073. Polemonium coeruleum L. 4 c.

Polygonacea Lindl. 1074. Calligonum ap syllum Gürke $\hbar_{2} 7$ 
1075. Emex spinosa Campd. (.) c. 1076. Fagopyrum tataricum Gärtn.

1077. Oxyria digyna Hill. 4 c.

1078. Polygonum alpinum All. 4 c.

1079. " $"$ aviculare $L . \odot$ c.

1080. " $\quad$ Bistorta L. 4 c.

1081. \# Convolvulus $L . \odot$ c.

1082. " Fagopyrum L. $\odot$ c.

1083. " Hydropiper L. $\odot$ c.

1084. " tomentosum Schrank.

$1085 *$ P. 27

1085.* Rheum Emodi Wall. 4 c. 27

1086. " palmatum L. 4 c.

1087. " Rhaponticum L. 4 c.

1088. " undulatum L. 4 c.

1089. Rumex acetosa $L .4$ c.

1090. " Acetosella L. 4 c.

1091. " aquaticus $L .4 \mathrm{M}$.

1092. \# confertus Willd. 4 M.

1093. " crispus L. 4 c.

1094. " domesticus Hartm. 4 c.

1095. " obtusifolius L. \& M.

1096. " pulcher L. 4 c.

1097. " scutatus L. \& c.

Portulacaceae Lind l.

1098. Calandrina procumbens Moris. $\odot \mathbf{c}$.

1099. Claytonia perfoliata Domn. $\odot$ c. 1100.* " sibirica $L$. c.

1101. Portulaca grandiflora Lindl. $\odot$ c. 1102. $\cdots \quad$ oleracea $L . \odot$ c.

1103. Talinum patens Willd. 4 c.

Primulaceae Vent.

1104. Anagallis arvensis $L$. var. coerulea Gren. et. Godr. $\odot$ c.

1105. Anagallis arvensis L. var. phoenicea Gren. et. Godr. $\odot$ c.

1106. Anagallis grandiflora $\odot$ c.

1107. Androsace elongata $L . \odot$, $\odot$ c.

1108. " lactiflora Pallas. $\odot$ c.

1109. " septentrionalis L. $\odot$,

1110. Asterolinon stellatum Hoffm. et. Link. $\odot$ c.

1111. Cortusa Matthioli L. 4 c.

1112.* Lubinia mauritiana Spreng. c.

1113.* Lysimachia dahurica $L d b .4$ c.

1114. " vulgaris L. 4 c.

1115.* Primula algida Adan. \& c.

1116.

$1117 *$.

1118.

1119.

1120.

1121.

1122 .

1123.

1124

1125.

1126.

1127,*

1128.* ,

1129 .

1130. auricula $L .2$ c.

Beesiana Forrest. $4 \mathrm{c}$.

columnae Tenore.

cortusoides $\stackrel{4}{L}$ c. 4 c.

denticulata Sw. 4 c.

elatior Hill. 4 c. farinosa $L .4$ c. frondosa Janka.2, c. japonica A. Gray.

$$
4 \text { c. }
$$

officinalis Hill. 4 c. paxii $4 \mathrm{c}$.

pulverulenta Duthie. 4 c.

saxatilis Kom. 4 c. Sieboldii Morr. 4 c. uralensis Fisch.

4. c.

1131, Samolus Valerandi L. 4 c. 27

Ranunculaceae Juss. ${ }^{3}$

1132. Aconitum ferox Wall, 4 c.

1133. " lasiostomum Reichb

1134. " $\quad$ napellus $L .2 \begin{aligned} & 4 \text { c. } \\ & \text { c. } 26\end{aligned}$

1135. Actaea spicata L. 4 c.

1136. " " var. rubra (Bigel.)

1137. Adonis autumnalis $L . \odot$ c.

1138. Anemone multifida Poir. 4 c.

1139.

1140.

1141.

1142 .

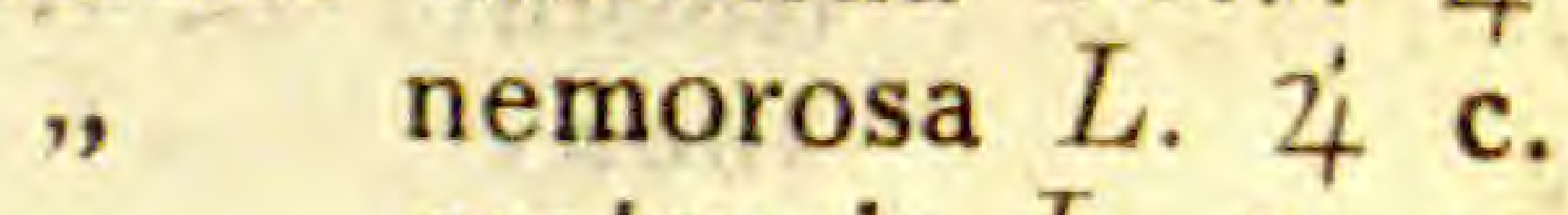

" $\quad$ nemorosa L. 4 c.

" ranunculoides $L .4$ c.

" rivularis Buch.-Ham.

4 c.

1143. " virginiana $L .4$ c.

1144. Aquilegia atropurpurea $W .4$ c.

1145. " formosa Fisch. 4 c.

1146. " nivea Baumg. 24 c.

1147. " olympica Boiss. 4 c.

1148. " oxysepala Tr.et. Mey.

1149.2 c.

1150. Calth vulgaris $L .4$ c.

1151. Clematls palustris $L .4$ c.

1151. Clematls integrifolia L. 4 c.

1152

1153

1154 recta $L .4$ c.

sibirica Mill. 528

tangutica Korsch. $\hbar \mathrm{c}$. 
1155. Clematis vitalba $L$. $\hbar$ C. 26 1156. Delphinium Ajacis L. () c. 1157.* ” Bulleyanum Forrest.

4 c. 1158. ” cashmerianum Royle. 1159. " cheilantum Fisch. 1160. consolida L. $\odot$ c. 1161. ” elatum L. 4 c. 1162.* ” orientale J. Gry. 1163. „ puniceum Pall. 4 c. 1164. ” rossicum Litw. 4 c. 1165. " speciosum Bieb. 4 c. 1166.*,$\quad$ triste Fisch. 4 c. 1167. Eranthis hiemalis Salsb. 4 c. 1168. Firaria ranunculoides $R o t h .4$ c. 1169. Hepatica triloba Gilib. 4 c. 1170. Isopyrum fumarioides $L$. $\odot$ c. 1171. Myosurus minimus $L . \odot$, $\odot$ c. 1172. Nigella damascena $L . \odot$ c. 1173. ” Garidella Spenn. () c. 1174. ” . sativa $L . \odot$ c. 1175. Paeonia albiflora Pall. 4 c. 1176.* ” corallina Retz. var. triternata Boiss. 4 K. 27

117. Ranunculus acer L. 4 c. 1178. " arvensis $L . \odot$ c. 1179. " auricomus L. 4 c. 1180. ” cassubicus L. 4 c. 1181. " Cymbalaria Purch.

1182. „ Flammula L. 4 c. 1183. " $\quad$ illyricus L. 4 c. $1184{ }^{*} \quad " \quad$ pedatus $W . K$.

1185. \# repens L. 4 c. 1186. \# $\quad$ sceleratus $L$. $\odot$ c. 28 1187. Thalictrum angustifolium Jacq. 1188. \# minus L. 4 c. 1189. Trollius europaeus L. 4 c. 1190. ” pumilus D. Don. 4 c. Resedace a e D. C.

1191. Astrocarpus sesamoides D. C. 1192. Raseda alba $L . \odot, 4$ c. 1193. " complicata Bory. 4 c. 1194. " crystallina Webb. et. 1195. " glauca L. c.
1196. . " inodora Rehb. $\odot, 4$

1197. „ lutea $L . \odot, 4$ c.

1198. " luteola $L . \odot$ c.

1199. " odorata $L$. $\odot$ c.

1200. " Phyteuma L. () c.

1201* " virgata Boiss. 4 c.

R h a m nace a e S. F. Gray.

1202. Rhamnus cathartica $L$. $\hbar$ G. 1203, ” Frangula L. ₹ G.

Ros ace a e Juss.

1204.* Acaena glabra J. Bnch. c.

1205. Agrimonia Eupatoria L. 4 c.

1206. $\quad$ pilosa Led. 4 c.

1207. Amelanchier vulgaris Mönch. 5 G.

1208. Aruncus silvester Kost. 4 c.

1209.* Comarum palustre L. 4 G.

1210. Crataegus monogyna Jacq. 4 c.

1211. " sanguinea Pall. $\hbar$ G.

1212. Filipendula hexapetala Gilib.

4 c.

1214. Fragaria indica $A n d r .4$ c.

1215. „ vesca $L .4$ c.

1216. ,. viridis Duch. 4 c.

1217. Geum aleppicum Jacq. 4 c.

1218. " molle Vis. et Pane. 4 c.

1219. " rivale L. 4 c.

1220. " urbanum L. 4 M. 25

1221. Potentilla alpestris Hall. f. 4 c.

1222. " argentea L. 4 c.

1223. " arguta Pursh. 4 c.

1224. ” bifurca $L .4$ c.

1225. ” Buccoana Clem. 2 c.

1226. " chrysantha Trev. 4 c.

1227. " delphinensis Gren. et. Godr. c.

1228. ” desertorum Bge. 4 c.

1229. " fruticosa $L$. $\hbar$ c.

1230. ” fulgens Wal. 4 c

1231. " gelida C.A.M. 4 c.

1232. ” glandulosa Lindl.

1233. " intermedia $L .4$ c.

1235. " longipes Led. 4 c.

1235. ” Meyeri Boiss. 4 c.

1236.* ” norvegica $L . \odot$ G.

1233. $\#$ nepalensis Hook. 4 c. 
1238. Potentilla palustris Scop. $4 \mathrm{M}$. 1239. " paradoxa Nutt. $\odot$ c. 1240. " pedata Willd. 4 c. 1241. " pimpinelloides L. 4 c. 1242. " procumbens Sibth.

1243. ” pulcherrima Lehm.

1244.4 c.

1245. " $\quad \begin{aligned} & \text { recta } L .4 \text { c. } \\ & \text { rupestris } L .4 \text { c. }\end{aligned}$

1246. " Tormentilla Schrank.4c.

1247. ” supina $L . \odot, \odot$ c.

1248. Poterium sanguisorba $L .4$ c. 28

1249. Prunus Padus $L . \hbar$ c.

1250. " spinosa $L . \hbar$ c.

1251. Rosa cinnamomea $L . \hbar$ c.

1252.* " dahurica Pall. $\hbar$ W. 26

1253.* " rugosa Thunb. 5 W. 26

1254. Rubus saxatilis $L .2$ c.

1255. Sanguisorba officinalis $L .4$ c.

1256. " muricata Spach.

1257. „ tenuifolia Fisch.

1258. Spiraea 4 c.

1259 Spiraea media Schm $\hbar$ c.

1260 " salicifolia $L . \hbar$ c.

1261. Sorbus Aucuparia L. $\hbar$ G.

1262. ” domestica Sm. 5 c.

Rubiacea e Juss.

1263. Asperula Aparine Schott. 4 c.

1264. " arvensis $L$. $\odot$ c.

1265. " cynanchica L. 4 c.

1266 * " odorata $L$ \&.

1267. Crucianella chlorostachys $F$. et.

1268. " exasperata $F$. et. $M$. ค. c. 28

1269. Galium mollugo L. 4 c.

1270. " parisiense L. $\odot$ c.

1271. " Saccharatum All. $\odot$ c.

1272. " uliginosum $L .4$ c.

1273. " verum $L .4$ c.

1274. Phuopsis stylosa Benth. et. Hook.

1275. Rubia 4 c.

1275. Rubia tinctorum L. 4 c.

1276. Scherardia arvensis $L$. $\odot$ c.

Rutacea e Juss.

1277. Ruta graveolens L. 4 c.
Saxifragaceae Dumort.

1278. Astilbe chinensis Fr. et. Sav. 4 c.

1279. Chrysosplenium alternifolium $L$. $4 \mathrm{c}$.

1280. Heuchera americana $L .4$ c.

1281. ” sanguinea Englm.

2 c.

1282 Parnassia palustris L. 4 c.

1283. Ribes alpinum L. $\hbar$ c.

1284. Saxifraga caespitosa L. 4 c.

1285.,$\quad$ irrigua $M . B . ~ \&$ c.

1286.* ” rotundifolia L. 4 c.

1287. " tenella Wuff. \& c.

1288. Tellima grandiflora $R$. Br. 4 c.

S c rophulariace a e Lind l.

1289. Alonsoa Warscewiczii Reg. $\odot$

c. 28

1290. Antirrhinum majus $L$. $\odot$ c.

1291. * Orontium L. ๑) c.

1292. Calceolaria pinnata $L$. $\odot$ c.

1293. " scabiosifolia Sims.

1994. Cha c.

1294. Chaenostoma antirrhinoides
c. $2 \frac{2}{8}$

1295. $"$ foetidum Benth.

1296* Chelone obliqua L. 4 c.

$\odot$ c.

1297. Collinsia bicolor Benth. $\odot$ c. 28

1298. Cymbalaria muralis Baumg. 4 c.

1299. Digitalis ambigua Murr. 4 c.

1300 .

ferruginea $L$. (.) c.

1301. " lutea L. 4 c.

1302. " media Roth. 4 c.

1303. ” micranta Roth. 4 c.

1304. " nevadensis Kanze. c.

1305. " purpurea L. 4 c.

1306. Elatinoides elatine Wettst. ()

$$
\text { c. } 28
$$

1307. " spuria Wettst.๑ c.

1308. Hebenstreitia dentata $L$. $\odot$ c.

1309. Linaria alpina Mill. 4 c.

1310 * $\quad$ " aparinoides F. G. Dietr.

v. hybrida c. 28

1311. " bipartita W. $\odot$ c.

1312. " Broussonnetii Chav. c.

1313. " canadensis Dumort. $\odot$ c.

1314. " capraria De Not. 4 c.

$1315 *^{*}$ " chalepensis Mill. $\odot$ c. 28

1316. " dalmatica Mill. $\odot$ c. 
1317. Linaria genistaefolia Mill. 4 c. 1318. ” japonica Miquel. 4 c.

1319. " littoralis $W . \odot$ c.

1320. ” macroura M. B. 4 c. 28

1321. ” marocana Hook.

1322. " minor Desf. $\odot$ c.

1323. " origanifolia D.C. 4 c.

1324. ” purpurea Mill. $)$ c.

1325. " reflexa Desf. $\odot$ c

1326. " ” Desf. var. aureopurpurea $\odot$ c. 28

1327.* " reticulata Desf. c.

1328. " sapphirina Hoffm. et. Link. $\odot$ c 28

1329. „ spartea H. et. Lk. $\odot$ c.

1330. ” tryphylla Mill. $\odot$ c.

1331. ” tristis Mill. $\odot$ c.

1332. " vulgaris Mill. 4 c.

1333. " " var. Biebersteinii Linden. 4 c. 27

1334.* Lindenbergia urticifolia Lehm.

$\odot$ c. 28

1335. Manulea violacea $L k \odot$ c 28

1336. Mimulus cordinalis Dougl. 4

c. 27

1337. " cupreus Regel. var. tig-

1338

1339. " moschatus Dougl. 4 c.

1340. Nemesia strumosa $\odot$ c.

1341. Nycterinia Capensis $\odot$ c.

1342. Odontites serotina $R c h b \odot \mathrm{M}$

1343. Pedicularis palustris $L . \odot, 4 \mathrm{M}$.

1344. Scrophularia nodosa $L \quad 4$ c.

1345 . umbrosa Dumort. $4 \mathrm{c}$.

1346. Verbascus Blattaria $L$. $\odot$ c.

1347. ” nigrum $L$. $\odot$ c.

1348. " phlomoides L. $\odot$ c.

1349. ” pyramidatum $M$. $B$. $4 \mathrm{c}$.

1350. "speciosum Schrad. $\odot$ c.27

1351. Veronica Beccabunga L. 4 c.

1352. ” Chamaedrys L. 4 c.

1353. " gentianoides Vahl. 4 c.

1354.* ” incana $L .4$ c.

1355. " longifolia $L .4$ c.

1356. " officinalis L. 4 c. 27

1357. " Ponae Gouan. 4 c.

1358. " scutellata $L .4$ c.

1359. ” serpyllifolia $L .4$ c.

1360. " sibirica L. 4 c.

1361. " spicata L. 4 M.
1362. Veronica spuria $L .4$ c.

1363. „ Tournefortii Gmel. ๑) c.

1364. Wulfenia carithiaca Jacq. 4 c.

1365. Zaluzianskya capensis Walp. c.

Solanaceae Pers.

1366. Atropa Belladonna L. 4 c.

1367. \# caucasica Kreyer. $\overrightarrow{4}$ c.

1368. Capsicum annuum $L$. $\odot$ c.

1369. Datura Bertolonii Parl. $\odot$ c.

1370. " Leichhardtii F. Muell.

1371. $\odot$ c. 28

1371. " metel $L . \odot$ c.

1372.,$\quad$ meteloides D. C. $\odot$

1373. " stramonium $L . \odot \mathbf{c}$.

1374. " Wrighti Rgl. $\odot$ c.

1375. Hyoscyamus albus $L$. $\odot$ c.

1376. " niger $L . \odot$, $\odot$ c.

1377. Nicandra physaloides Gaertn.

1378. Nicotiana affinis Moore. $\odot, 4$ c.

1379. " cerinthoides Hornem.

1380. $\quad$ " paniculata $L . \odot$ c.

1381. " $\quad$ rustica $L . \odot$ c.

1382. " silvestris $L . \odot$ c.

1383. " suaveolens Lehm.

Tabacum $L \cdot \odot$ c

1385. Nolana prostrata $L$. $\odot$ c.

1386. Petunia nyctaginiflora Juss.

1387. Physalis angulata $L . \odot$ c.

1388. " Francheti Masters. 4

1389. \# pubescens $L$. $\odot$ c. 28

1390. Physochlaena orientalis G. Don. $4 \mathrm{c}$.

1391 * Salpiglossis variabilis Hort. $\odot$ c. 28

1392. Sarachia Jaltomata Schleicht.

1393. Solanum Capsicastrum Link.

demissum c. $28^{\hbar}$ c.

1395.. " dulcamara $L .4,5$ c.

1396. " Gilo Raddi. ๑ c. 27

1397. " gracille Otto. $\odot$ c.

1398. „ guineense Lam. $\odot$ c.

1399. ” Hystrix R. Br. c. 
1400. Solanum miniatus Bernh. () c. 1401. " nigrum $L$. v. villosum L. ๑ c 28 1402. v. vulgare $L$.

1403. " sisymbriifolium Lam. $\odot$ c.

1404. Withania somnifera Dun. $\frac{1}{2}$ c.

Tropaeolaceae Juss.

1405. Tropaeolum majus $L . \odot$ c.

Umbelliferae Morison.

1406. Aegopodium alpestre $L \epsilon d b .4 \mathrm{c}$. 1407. " Podagraria L. \& c. 1408. Aethusa Cynapium L. $\odot$ c. 1409. Anethum graveolens $L$. $\odot$ c. 1410. Angelica archangelica $L . \odot$ c. 1411. " silvestris L. 4 c.

1412. Anthriscus silvestris Hoff. 4 c.

1413. Astrantia major L. 4 c.

1414. Bifora testiculata Boiss. - c.

1415. Bunium bulbocastanum L. 4 c.

1416. Bupleurum rotundifolium $L$. $\odot$

1417. Carum Carvi L. .) c.

1418.* Caucalis daucoides $L$. $\odot$ c.

1419. $\quad$ latifolia $L . \odot$ c.

1420. Cenolophium Fischeri Koch. 4

c.

1421. Chaerophyllum aromaticum $L$. 1422 bulbosum $L$. (.) c.

1423. Conium maculatum L. (-) c.

1424. Coriandrum sativum L. (.). c.

1425. Daucus Carota L. (spont) $\odot$ c.

1426. " Gingidium L. .. c.

1427. " grandiflorus Scop. () c

1428 muricatus $L . \odot$ c.

1429.* " pulcherrimus D.C. .. K 27

1430.* Eryngium pandanifolium Cham. et. Schl. 4 c. 27

1431. " planum L. 4 c.

1432. Falcaria vulgaris Bernh. $\cdots$ c.

1433. Heracleum barbatum Ledb. 4 c. 1434. $"$ pubescens M. B. (.) c.

1435. n sibiricum L. $\%$ c. 28 1436. Laserpitium pruthenicum $L$. $\odot$

1437. Levisticum officinale L. 4 c.
1438. Libanotis montana All. $\odot$ c. 1439. " sibirica Koch. 4 c. 1440. Ligusticum scoticum L. 4 c. 1441. Myrrhis odorata Scop. 4 c. 1442. Pastinaca sativa $L . \odot$ c.

1443. \# teretiuscula Boiss. :c. 28

1444. Petroselinum sativum Hoff. 4 c. 1445. Peucedanum cervariaefolium C. A. M. 4 c. 1446. $\quad " \quad$ Oreoselinum Moench. $4 \mathrm{M}$.

1447.* Pimpinella magna $L . ~ \&$ M. 1448. " saxifraga L. 4 c. 1449.* " Tragium Vill. 4 c.

1450. Sanicula europaea L. 4 c. 1451. Scandix Pecten Veneris L. $\odot$ c. 1452. Seseli Hippomarathrum L. 4 c. 1453. " laserpitifolium Palıb. 4 1454. Torilis Anthriscus Gmel. $\bigodot_{28}^{26}$ c.

Urticacea e Endl.

1455. Forskohlea angustifolia Retz. 1456. Laportea gigas Wedd. ${ }_{4} \odot \mathrm{c}$. 1457.* Parietaria officinalis $L .4$ c. 1458. ” ramiflora Moench. 4 c.

1459. Urtica cannabina 'L. 4 c.

1460. ” dioica $L .4$ c.

1461. " Dodartii L. $\odot$ c.

1462.* " laetevirens Max. 4 c.

1463. " membranaceae 1 oir.

1464. " pilulifera $L$. $\odot$ c.

1465. " urens L. $\odot$ c.

Vacciniaceae Lind I.

1466. Vaccinium Myrtillus L. $\hbar$ G. 1467. " Oxycoccus L. 5 G. 1468. $"$ uliginosum $L$. $\hbar$ G.

Valerlanaceae Dumort. 1469. Centranthus CaIcitrapa Dufr. 1470 C $\odot 28$ 1470. $\quad$ ruber $D . C . \bigodot_{26}$ c. 
1471. Fedia cornucopiae D. C. 4 c. 1472. Patrinia intermedia $R$. et. S.

$4 \mathrm{c}$.

1473. Valeriana palustris $K r .4$ c. 28 1474. " Sprygini Sm. 4 c. 1475. " Wolgensis hez. 4 c. 1476. Valerianella coronata D. C. $\odot$

1477. $\quad$ eriocarpa Krok.

1478. " rimosa Bastard.

1479. $\odot$ c.

1479. $\quad \quad \quad$ tridentata Bas.
c. 28

1480. „ vesicaria Mnch.

$\odot$ c.

Verbenacea e J. St. Hil.

1481.* Lantana flava Medic. $\hbar$ c. 27

1482. Verbena arborea $H$. et. B. 5 c.

1483. " Aubletia Jacq. (.) c.
1484.* " hastata L. 4 c.

1485. V" hybrida Hort. $\odot$ c.

1486. Viola canina Rchb. 4 c.

1487. " cornuta L. 4 c.

1488. " elatior Fr. 4 c.

1489. " epipsila Led. 4 c.

1490. " hirta L. 4 c.

1491. " mirabilis L. 4 c.

1492\%. " Munbyana B. et. $R$. var.

Battandieri c.

1493. " odorata L. 4 c.

1494. " sagittata Ait. 4 c.

1495. " tricolor Wittr. $\odot, \odot$ c.

Zy go $\rho$ hyllace a e R. B r.

1496. Nitraria Schoberi L. 5 K. 27

1497. Peganum Harmala L. 4 c.

1498. Tribulus terrestris $L$. $\odot$ c.

1499. Zygophyllum Fabago L. 4 c.

Semina plantarum in provincia Austro-Ussuriense regionis naturalis Manschuriae crescentium, quae a E. J. Wassilkoff anno 1927 loco notali lecta sunt.

Насеньне якое сабрана ўлетку 1927 г. А. Я. Васільковай у Паўднёва-Усурыйскай правінџыі Манжурскае флёрыстычнае краіны.
Семена, собранные летом 1927 г. Е. И. Васильковой в Южно-Уссурийской провинџии Манчжурской флористической области.
1500.* Actaea acuminata Wallich. 1501.* Agropyrum ciliare Franch. v.

1502. Allium lineare $L$. pilosum Korch.

1503. ” sacculiferum Max.

$1504 . \quad$ sp.

1505 * Ampelopsis brevipedunculata Max.

1506. Artemisia $s \rho$.

$1507 . \quad$ sp.

1508. Arundinella anomala Stoud.

1509.* Astragalus ulihinosus $L$.

1510. Aster fastigiatus $F$. et. $M$.

1511. „ Maackii Rgl.

1512. " scaber Thunb.

1513. Betula dahurica Pall.

1514. „ japonica $H$. Wincler.
1515. Betula ovalifolia Rupr.

1516.* Bidens Maximovicziana Oett.

1517. Cacalia aconitifolia Bunge.

1518*. ” hastata $L$.

1519. Calamagrostis langsdorfii Trinius.

1520* Calystegia rosea Choisy.

1521. Caragana fruticosa Besser.

1522.* Carex limosa $L$.

1523.* ” pallida C. A. M.

1524. Cicuta virosa $L$.

1525. Cimicifuga dahurica Max.

1526. ” Simplex Wormsk.

1527* Clematis angustifolia Jacq.

1528. " fusca Turcz.

1529. ” manschurica Rupr.

1530. Codonopsis lanceolata B. et. $H$. 
1531. Codonopsis ussuriensis Hemsley. 1532. Cuscuta japonica Choisy.

1533. Cynanchum amplexiaule Hemsley. 1534 . atratum Bnge.

1535. Drosera longifolia $L$.

1536.* Elscholtzia cristata Willd.

1537.* Epilobium palustre $L$.

1538. Erigeron canadensis $L$.

1539. Eupatorium Kirilovi Turcz.

1540.* Evonymus sp.

1541* Galium trifidum $L$.

1542. $\quad$ verum $L$.

1543. Geranium Wlassovianum Fischer.

1544. Geum aleppicum Jacq.

1545. Gypsophila muralis $L$.

1546.* Habenaria linearifolia Max.

1547. Hemerocallis flava $L$. .

1548.* Hieracium umbellatum $L$.

1549. Hypericum Ascyron $L$.

1550. Iris Kaempheri Sieb.

1551. " laevigata Fisch.

1552.* I athyrus palustris $L$. v. pilosus

1553. Lilium avenaceum $L$. $\mathrm{L} d b$.

1554. Lobelia sessilifolia Lamb.

1555** Lychnis fulgens Fisch.

1556. Lycopus Maackianus Kom.

1557. Lysimachia dahurica $L d b$.

1558. Lythrum salicaria $L$.

1559. Melilotus suaveolens $L d b$.

1560.* Menyanthes trifoliata $L$.

1561. Mulgedium sibiricum Less.

1562.* Naumburgia thyrsiflora Duby.

1563. Panicum acroanthum Steudel.

1564. crus-galli $L$.

1565. Paeonia albiflora Pall.

1566.* ” obovata Max.

1567.* Pedicularis grandiflora Fisch.

1568.* $\quad$ resupinata $L$.

1569. " Sceptrum Carolinum $L$.
1570.* Perilla ocymoides $L$.

1571.* Plagiospermum chinensis Oliver.

1572. Platanthera hologlottis Max.

1573. Platycodon grandiflorus A.D.C.

1574. Pleurospermum kamtschaticum

1575.* Poa pratensis $L$. Hoffm.

1576.* Pogonia ophioglossoides Ker.

1577. Polygonatum officinale All.

1578.* Polygonum sp.

1579. ” $\quad$ s $\rho$.

1580.* Ranunculus sceleratus $L$.

1581.* Rubia chinensis Rgl.

1582 * Rumex acetosella L.

1583. Sanguisorba officinalis $L$.

1584. " tenuifolia Fich.

1585.* Scheuchzeria palustris L.

1586. Scutellaria angustifolia Kom.

1587.* Sedum Aizoon $L$.

1588.* Serratula coronata L.

1589.* Setaria viridis $P . B$.

1590. Smilax oldhami Miq.

1591.* Sonchus arvensis L.

1592. Sophora flavescens Ait.

1593. Spodiopogon sibiricus Trin.

1594.* Triadenum asiaticum Max.

1595. Trifolium lupinaster L.

1596.* Trollius chinensis Bge.

1597. Typha latifolia L.

1598.* Urtica laetevirens Max.

1599. Veratrum album L.

1600. ” Maackii Rgl.

1601.* Veronica grandis Fischer.

1602.* " tubiflora Turcz.

1603.* Viburnum sargenti Koehne.

1604** Vicia amoena Fischer.

$1605{ }^{*} \quad$ n cracca L.

1606.* " pseudo-orobus F.et. Mey.

1607.* Viola Patrini D. C.

Professor M. N. Medzisch,

Z. N. Denissov,

Horti praefectus.

Horti inspektor

V. L. Gorsky, Assistent.

Gorky, R. P. Alborutheniae (U. S. S. R.).

Calendis Februariis anni MCMXXX.

Удоўгалоўлітбел 스 1025. Зак, № 221-1300 экя. Горкі, Арукарня Акадэміi. 

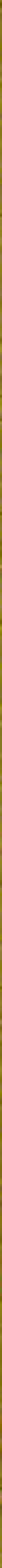

1
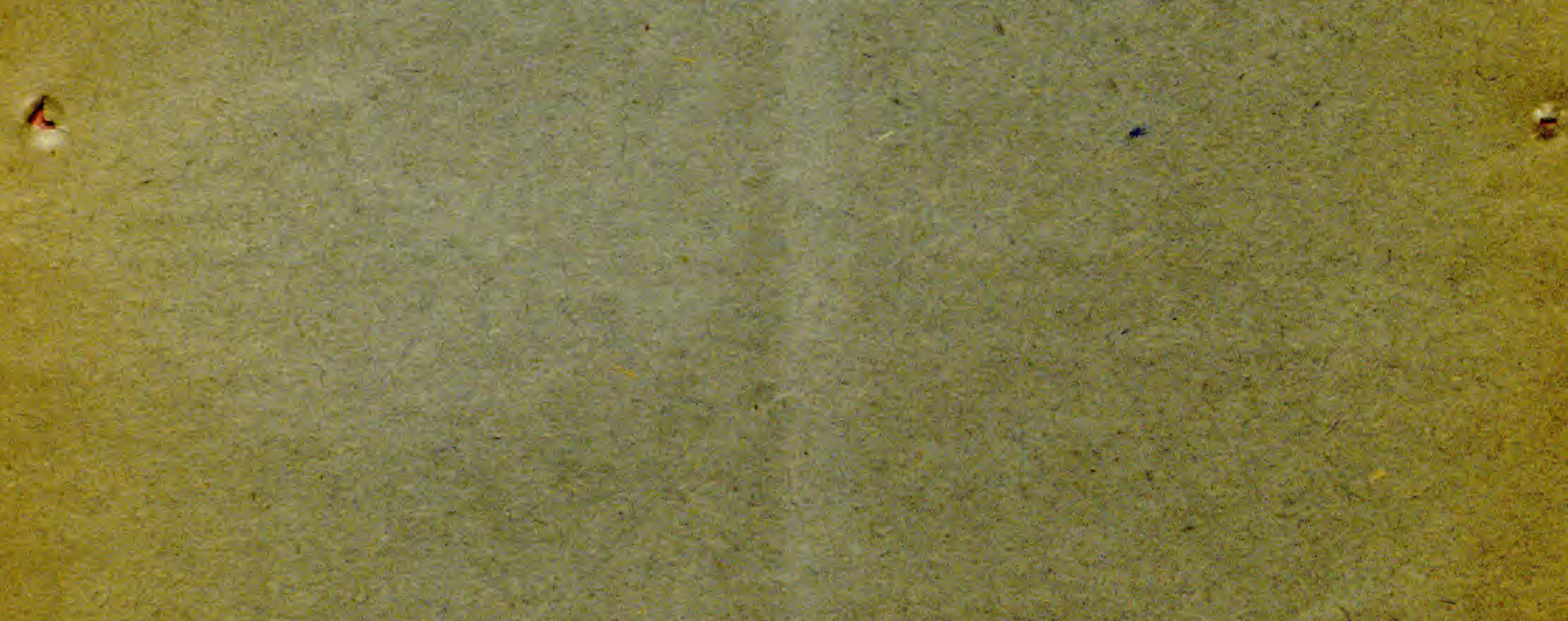

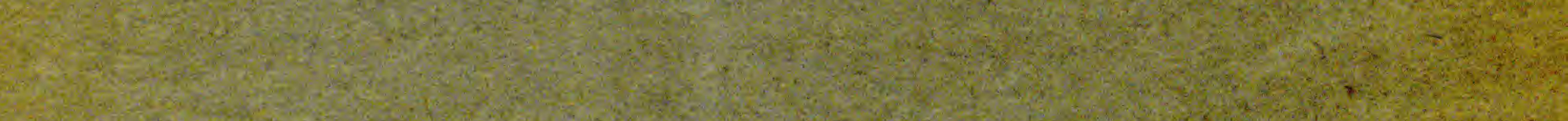

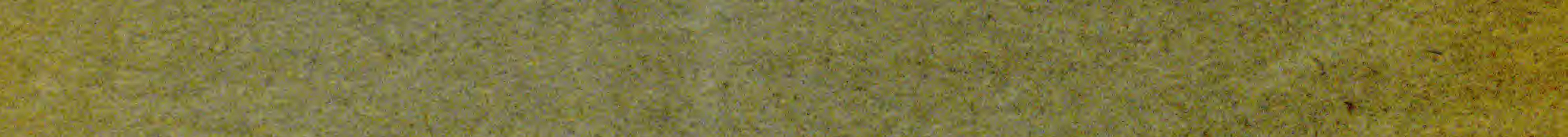

t.

-

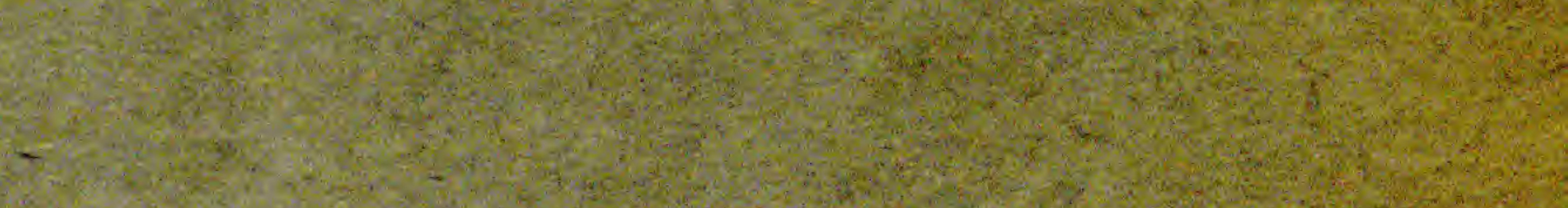

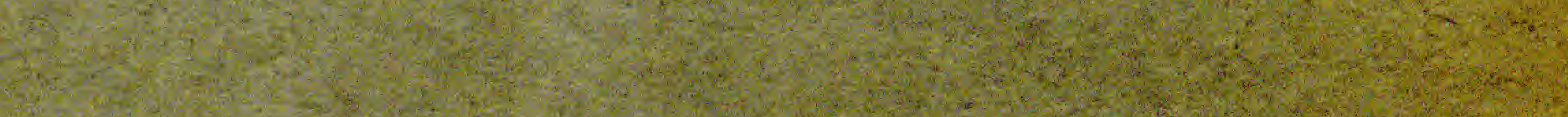

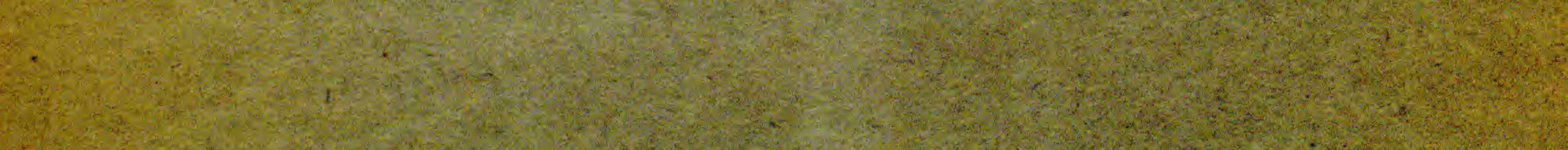

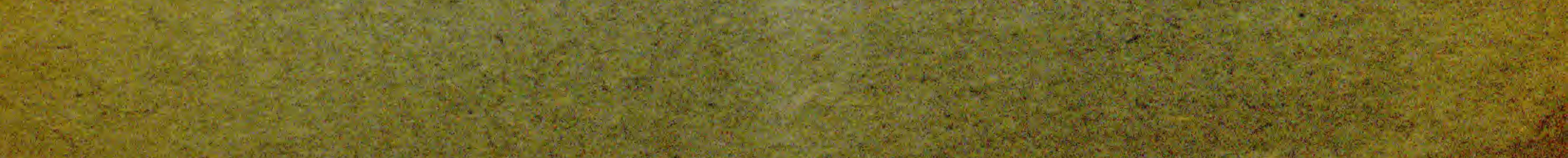

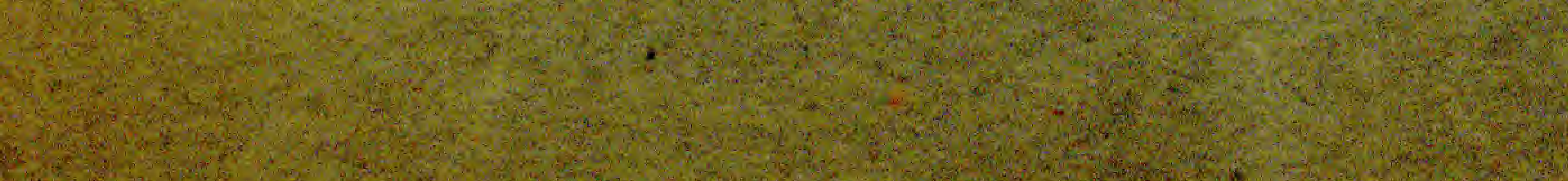



Jardin Botanique de l'Academie Agronomique Gorky. Belorussia. U. S. S. R.
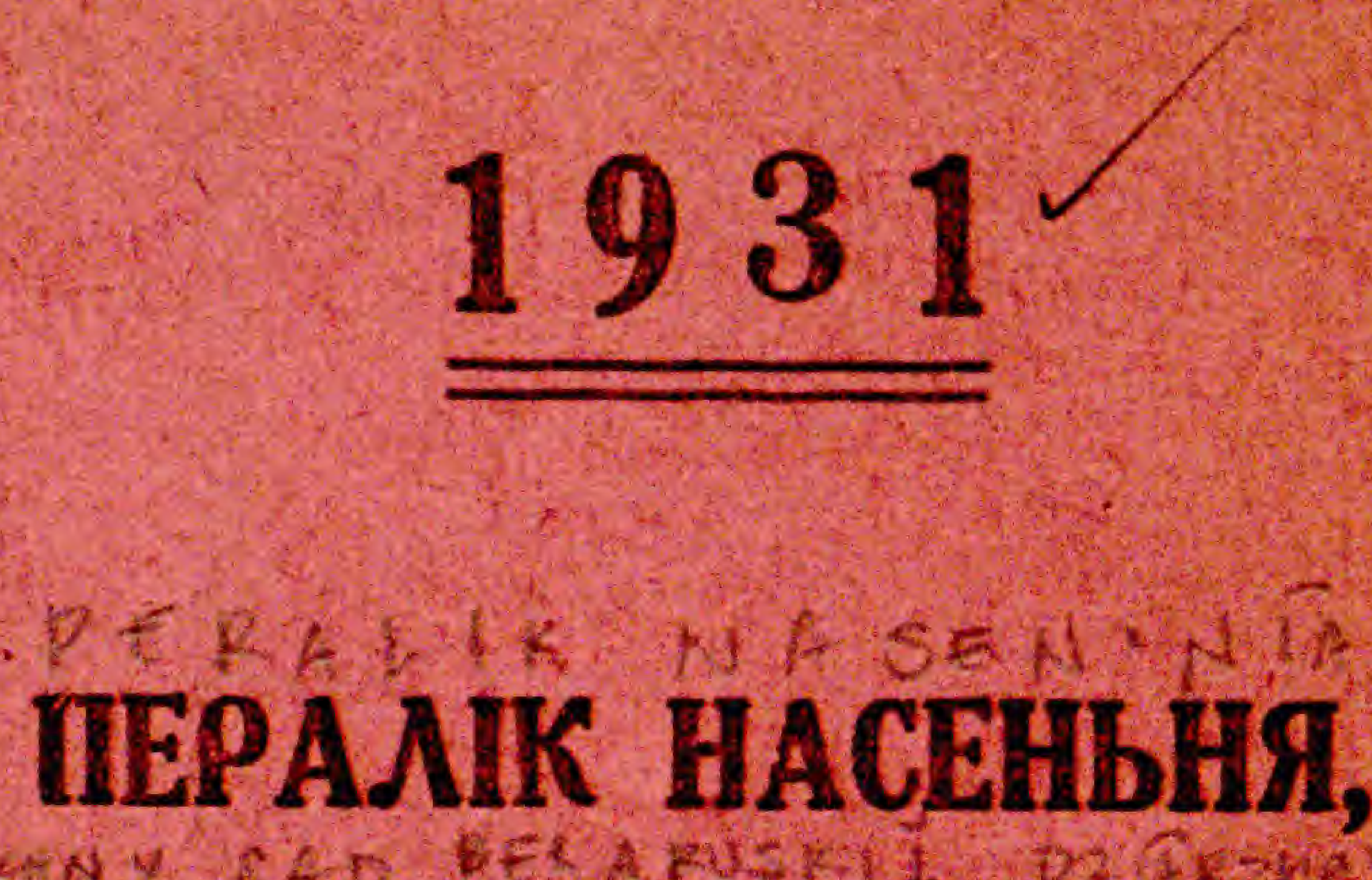

ЯКОЕ БАТАНІЧНЫ САД БЕААРУСКАЙ ДЗЯРЖАЎНАЙ АКАДӘМИ

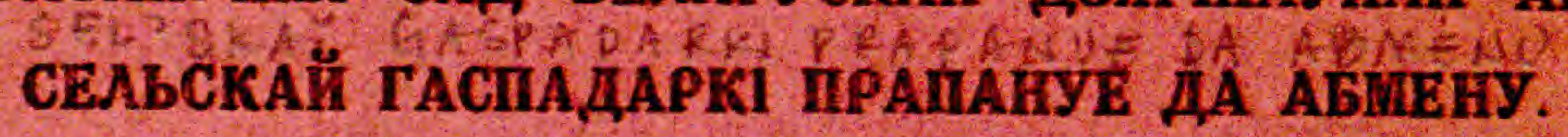

\section{DELECTUS SEMINUM,}

Anno MCMXXX ( collectorum, quae Hortus Botanicus Academiae Agronomicae Rei publicae Alboruthemicae pro mutua commutatione offert.

\section{PER}

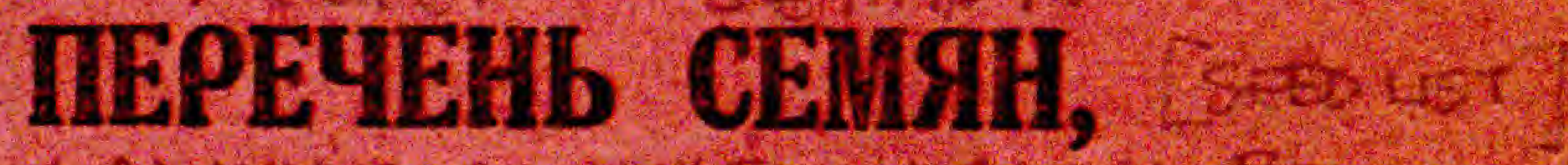

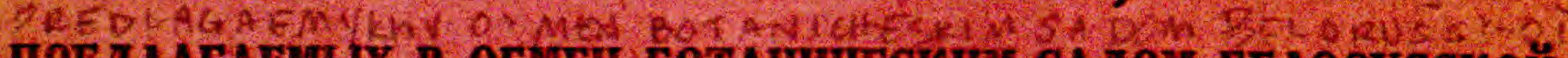
ПРЕДИАГАЕМЫХ В ОБДВН БОТАНИЧЕСКИМ САДОП БЕЛОРУССКОЙ

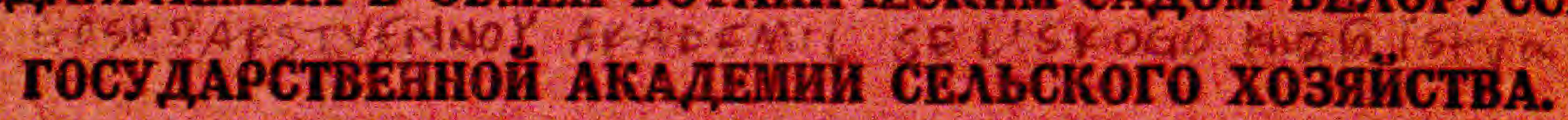

Gory-Gorky. R. P. Alhoruthenia (U. S. S. R.)

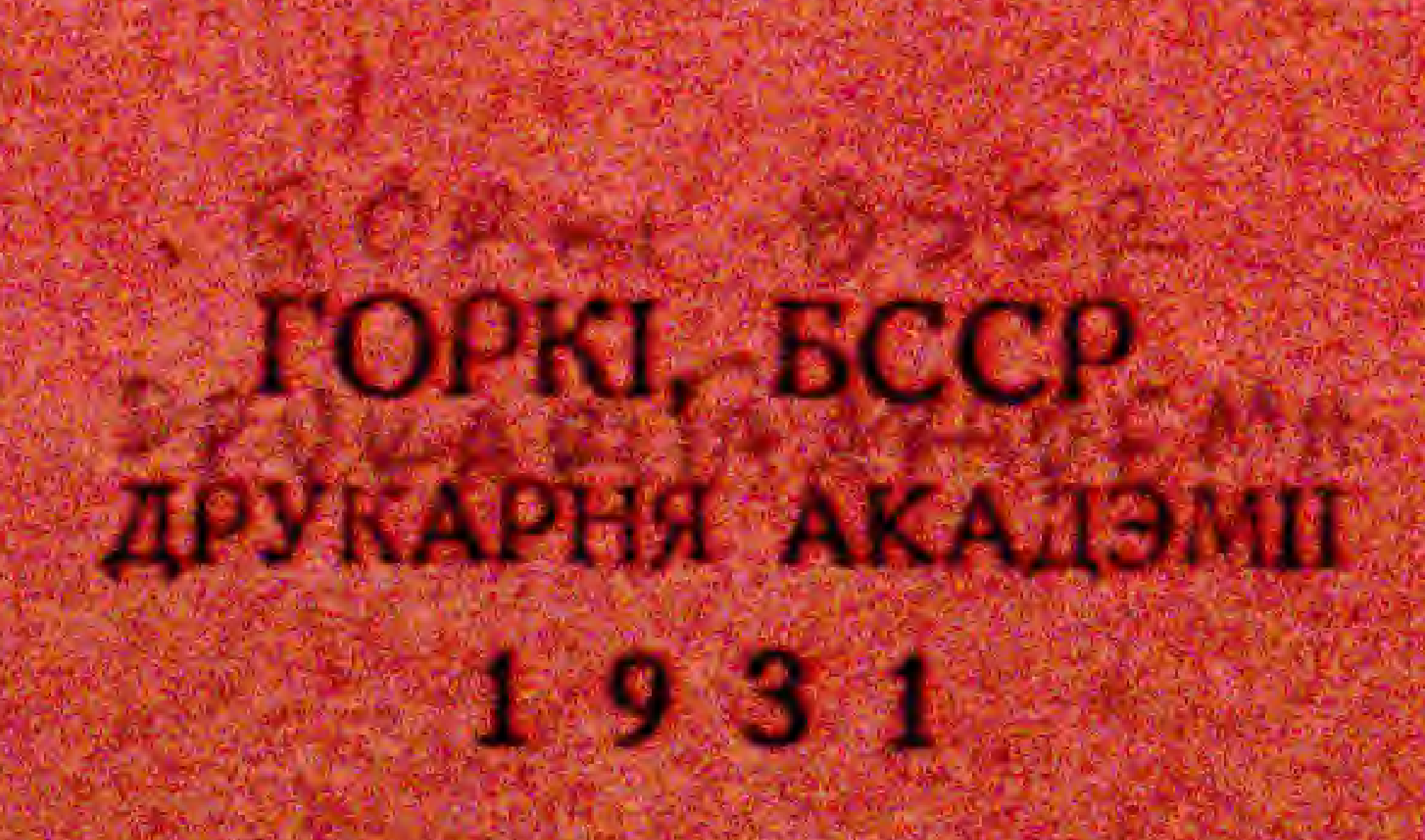

- 

Jardin Botanique de l'Academie Agronomique Gorky. Belorussia. U. S. S. R.

\section{1}

\section{IIEPANK HACEHDHЯ,}

ЯКОЕ БАТАНЧЖЫ САД, БЕААРУСКАЙ ДЗЯРЖМЎНАЙ АКАДЭМІ СЕЛЬСКАЙ ГАСПАДАРКІ ПРАПАНУЕ ДА АБМЕНУ.

\section{DELECTUS SEMINUM,}

Anno MCMXXX (partion ac MCMXXVII, MCMXXVIII et MCMXXIX) collectorum, quae Hortus Botanicus Academiae Agronomicae Rei publicae Alboruthenicae pro mutua commutatione offert.

\section{ПЕРЕЧЕНЬ СЕМЯН,}

ПРЕДМАГАЕМЫХ В ОБИЕН БОТАНИЧЕСКИМ САДОМ БЕАОРУССКОЙ ГОСУДАРСТВЕННОЙ АКАДЕМИИ СЕАЬСКОГО ХОЗЯЙСТВА.

Gory-Gorky. R. P. Alboruthenia (U. S. S. R.)

ГОРКІ, БССР

ДРУКАРНЯ АКАДЭМП

1931 
Упаўгалоўлітбел 흐 3376. Зак. № 283-500 экs.

Горкі. Арукарня Академіi. 


\section{Abbreviationes:}

*-paulum; G-Gorky; c-planta sub divo in Horto Botanico Academiae culta.

Numerus $(27,28,29)$ nomina plantarum sequens, annum, quo semina lecta sunt, significat, semina sine numerus in anno 1930 collecta sunt.

\section{Скарачәньні і умоўныя знакі:}

Зоркай (*) абазначана насеньне, якое маеџуа ў нязначнай колькасьџु, $\mathrm{G}$-насеньне, якое сабрана навакол Горак; с-з расьлін, якія разводзяцца у вадкрытым грунце ў Батанічным Садзе Акадәміi

Лічбы $(27,28,29)$, якія надрукаваны пасьля назваў расьлін, азначаюџь гады збору насеньня. Насеньне бяз лічбаў, сабрана ў 1930 годзе.
Сокрапения и условные обозначения:

Звездочкой (*) обозначены семена, имеюшиеся в махом количестве. G-семена собранные в окрестностях Горок; с-с растений, культивируемых в Ботаническом Саду Академии в открытом грунту.

Џифры $(27,28,29)$, помешенные за названиями растений, означают год сбора семян, семена без чисел собраны в 1930 году. 



\section{Pteridoph y t a.}

1. Adiantum Capillus Veneris $L$.

2. fulvum Roul 4 c.

3. " gracillimum Moore.

4. 4 c.

4. $\Rightarrow \quad$ pedatum L. 4 c.

5.* Anogramme leptophylla Lk. ๑,

6. Aspidium cristatum Sw. 4 c.

7. " Filix-mas Sw. 4 c.

8. " spinulosum Sw. 4 c.

$9 . \quad$ "thelypteris $S w .4$ c.

10. Asplenium dimorphum Knze. 4

11. " Filix-femina Bernh. ${ }^{\text {c. }}$

12* \# Trichomanes L. 4 c.

13. Cyrtomium falcatum Prsl. $4 \mathrm{c}$. 14. Cystopteris bulbifera Bernh. 4 c. 15.* " fragilis Bernk. 4 c. 16.* Doodia media $\mathrm{R} . \mathrm{Br}, 4$ c.
17.* Gymnogramme sulphurea Des. 4

18. Nephrodium molle Schott.

19. Onoclea sensibilis $L .4$ c. 29

20. Osmunda cinnamomea L. 426

21. Pellaea cordata $S m$. var. flexuosa

24.

22. Phegopteris Dryopteris Fée. 4 c.

23. Polypodium Phegopteris $L .4$ c.

24.* " Reinvwardtii Kze..4 c.

25. Pteris aquilina $L .4$ c.

26. " cretica $L .4$ c.

27. " longifolia $L .4$ c.

28. " serrulata $L .4$ c.

29. " $\quad$ v. crispa 4 c.

30. $"$ v. cristata 4 c.

31. " $" v$ v. tenuifolia 4 c.

32. " $" v$ variegata 4 c.

33. Scolopendrium vulgare $S m$. 4 c.

34. Struthiopteris germanica Willd.

\section{G y m nos per m a e.}

35. Cupressus Benthami Endl. 5 c. 36. Picea excelsa Link. 古 G.

37. Pinus silvestris $L . \hbar \mathrm{G}$.

38. " Strobus $L$. $\frac{\hbar}{2}$ c.

\section{Monocotyledone a e.}

\section{Alismataceae C. Rich.}

39. Alisma Plantago L. 4 G.

\section{Araceae Juss.}

40. Calla palustris L. 4 G.

\section{Commelinaceae Reich.}

41. Commelina coelestis W. $\odot$ c. 42.* $" \quad$ elliptica $H . B . \odot$ c.

\section{Cyperaceae J., St. Hil.}

43. Carex acuta L. 2 c.

44. " caespitosa L. 4 c.

45. " chordorrhiza Ehrh. 4 G.

46. " diandra Schrank, 4 G.
47. Carex dioica $L, 4$ G.

48. " elongata $L .4$ c.

49. " flava $L .4$ c.

50. ” hirta L. 4 c.

51. " leporina L. 4 c. 29

52. " limosa L. 4 c.

53. \# muricata $L .4$ c.

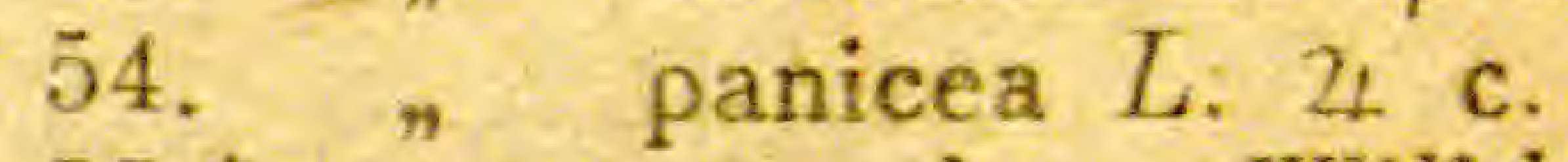

55.* " paradoxa Willd. 4 G.

56 . " pilosa L. 4 c.

57. " rostrata Stokes. 4 c.

58. " stellulata Good. 4 G.

59. " stricta Good. 4 c.

60. " vesicaria $L$. c.

61. " vulgaris Fr. 4 c.

62. " vulpina $L .4$ c.

63. Cyperus esculentus L. 4 c.

64. Eriophorum angustifolium Roth. 
65.* Eriophorum gracile Koch. 4 G. 66 . latifolium Hoppe.

67. Scirpus silvaticus $L .4$ c. 2 G.

\section{Gramineae Juss.}

68.* Aeleuropus laevis Trin. 4 c. 69. Agropyrum caninum $P . B$. if c. 70.

71.

72.

73.

74. Agrostis alba L. 4 c.

75. „ canina $L .4$ c.

76. " vulgaris With. 4 c.

77. Aira capillaris Host. $\odot$ c.

78. " caryophyllea $L$. $\odot$ c.

79. Alopecurus agrestis $L . \odot, \odot$ c.

80. $" \quad$ alpinus $S m .4$ c.

81. $"$ fulvus $\mathrm{Sm}$. $\odot$ c.

82. " geniculatus $L . \odot$ c.

83. $” \quad$ pratensis L. 4 c.

84. " ventricosus Pers. 4

85. Andropogon Ischaemum L. 4

86. Anthoxanthum odoratum L. 4

87. Apera spica ventl $P . B . \odot$ c.

88. Arrhenatherum elatius $M$. et $K$. 4 c.

89. Asperella hystrix Humb. 4 c.

90. Atropis convoluta Gris. 4 c.

91. Avena barbata Brot. $\odot$ c.

92. " hirsuta Roth. c.

93. "strigosa Schreb. $\odot$ c. 29

94. Beckmannia eruciformis Host. 4 c. 29

95. Brachypodium ramosum $R$. et $S$. 96. silvaticum $\stackrel{2}{R}$. et $S$. $4 \mathrm{c}$.

97. Briza elatior Sibth. et Sm. 4 c.

98. " maxima $L$. $\odot$ c.

99. " media L. 4 c.

100. " minor $L$. $\odot$ c.

101.* " virens $L$. c.

102. Bromus arvensis $L$. $\odot$ c. 29

103. " commutatus Schrad. $\odot$,

104. " hordeaceus $L . \odot$ c.
105. Bromus inermis Leyss. 4 c.

106. $"$ secalinus $L . \odot$, $\odot$ c.

107. " squarrosus $L . \odot$, $\odot$ c.

27

108. \# tectorum $L . \odot$ c.

109. " unioloides $H . B . K . \odot$,

110. Calamagrostis Epigeios Roth.

111. Ca 4 c.

111. Catabrosa aquatica $P . B .4$ c.

112. Cenchrus echinatus $L$. $\odot$ c. 29

113. ” montanus Nees. $\odot$ c. 27

114. Chloris barbata $S w . \odot$ c.

115. Cinna glomerata $L .4$ c.

116. " mexicana Beauv. 4 c.

117. Coix lacryma Jobi $L$. $\odot$ c. 29

118. Cynosurus cristatus L. 4 c.

119. Dactylis Aschersoniana Graebn.

4 c.

120. " glomerata L. 4 c.

121. " " L. fol. var.

122. Deschampsia caespitosa $P . \stackrel{4}{\text { C. }}$. 4 c.

123. Ehrharta erecta Lam. $\odot$ c.

124. Eleusine indica Grtn. $\odot$ c.

125. Elymus araliensis $R g l . ~ 4$ c.

126. " arenarius $L .4$ c.

127. " canadensis $L \cdot 4$ c.

128. " excelsus Turcz. 4 c.

129. " giganteus Vahl. 4 c.

130. " junceus Fisch. 4 c.

131. " sibiricus $L .4$ c.

132. " virginicus $L .4$ c.

133. Eragrostis minor Host. (i) c. 29

134. " pilosa P. B. $\odot$ c. 29

135. Festuca arundinacea $S c h r .4$ c.

136. " Beckeri Hackel. 4 c.

137. " gigantea Vill. \& c.

138. " ovina $L .4$ c.

139. " pratensis Huds. 4 c.

140. " rubra $L .4$ c.

141. " sulcata Hackel. c.

142. Gastridium australe $P . B . \odot$ c.

143. Glyceria spectabilis $M$. et $K$.

$144^{*}$. Heleochloa schoenoides Host.

145. Hierochloë odorata Wahlb. 4

146. Holcus lanatus L. 4 c.

147. Hordeum bulbosum L. 4 c.

148. " jubatum L. 4 c. 29

149. ” maritimum $L . \odot$ c. 
150. Hordeum murinum $L . \odot$ c.

151. " sativum $L$. v. spontaneum $K$. Koch. $\odot$

c. 29

152. Koeleria glauca D. C. 4 c. 153. " gracilis Pers. 4 c.

154. Lagurus ovatus $L$. $\odot$ c.

155. Lasiagrostis splendens Kunth.

156. Leersia oryzoides Sw. 4 c.

157. Lolium perenne L. 4 c.

158. " remotum Schrank. $\odot$ c.

159. " temulentum $L . \odot$ c.

160. Melica altissima L. 4 c.

161.* $" \quad$ nutans $L .4$ c.

162. „ transsilvanica Schur. 4

c.

163. Miscanthus sacchariflorus Hack. 4 c.

164. Nardus stricta $L .4$ c.

165. Oryzopsis holciformis Richt. 4 c. 27

166. Panicum bulbosun $H$. B. K. 4

167. californicum Benth.

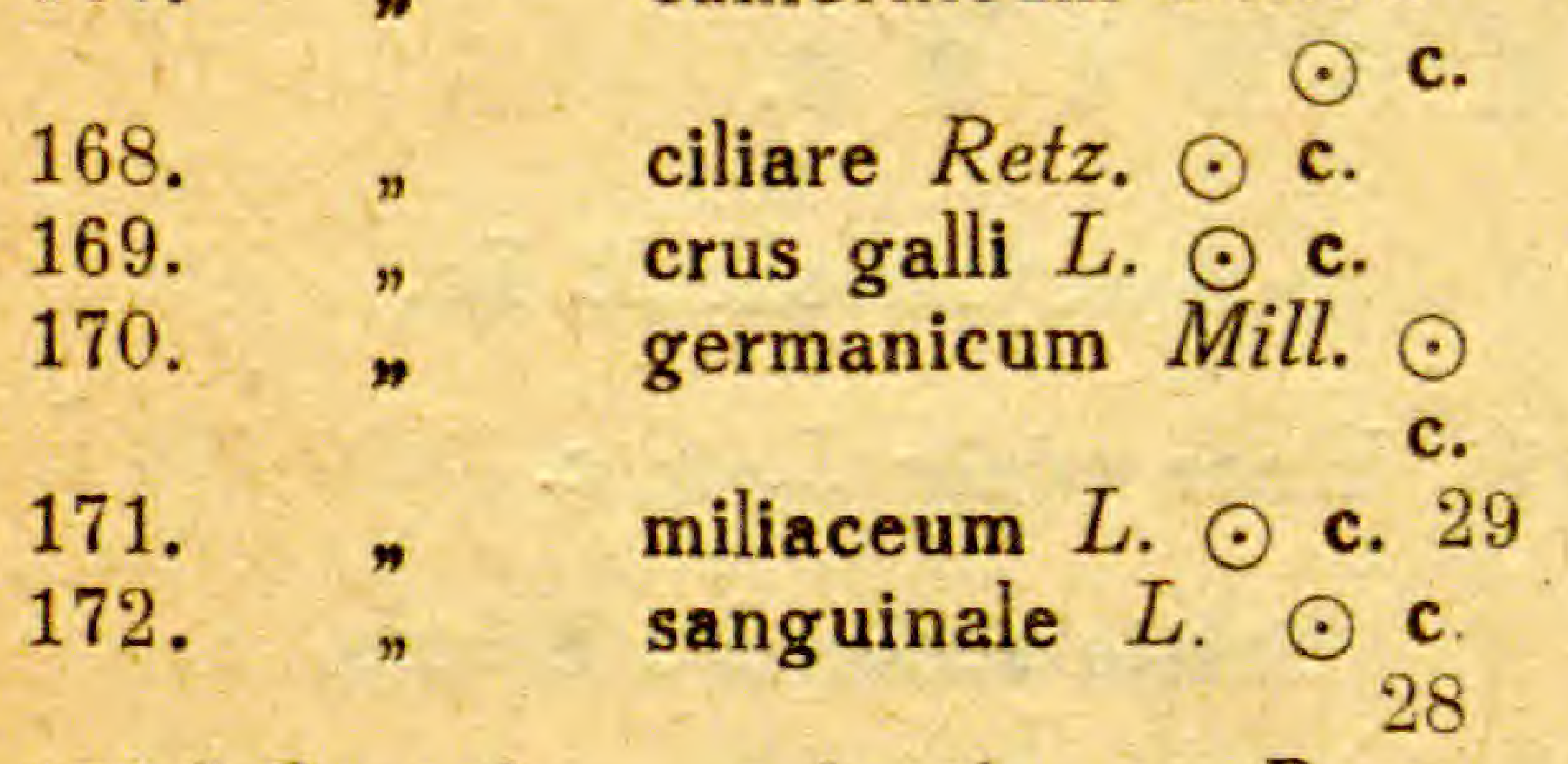

173.* Paspalum stoloniferum Desv.

174. Phalaris arundinacea $L .4^{4} \mathrm{c}$.

175. " " L. fol. var. v. lutescens 4 c.

176. " canariensis $L$. $\odot$ c.

177. " minor Retz. $\odot$ c.

178. Phleum alpinum L. 4 c.

179. " Boehmeri Wib. 4 c.

180. " paniculatum L. 4 c.

181. " pratense L. 4 c.

182. Phragmites communis Trin. 4

183. Poa alpina $L .4$ c.

184. " annua $L$. $\odot$ c. 29

185. " bulbosa L. 4 c. 29

186. " caesia Sm. 4 c.

187. " compressa L: 4 c.

188. " glauca Vahl. 4 c.

189. " nemoralis L. 4 c.
190. „ palustris L. 4 c. 29

191. " pratensis L. 4 c.

192. ” trivialis $L .4$ c.

193.* Polypogon monspeliensis Desf.

194. Scleropoa rigida Griseb. $\odot$ c.

28

195. Secale montanum Guss. 4 c.

196. Setaria verticillata $P . B . \odot$ c.

197. " viridis $P . B . \odot$ c.

198. Sorghum saccharatum Moench.

19. c.

199. Stipa capillata $L .4$ c.

200.* " Graffiana Stev. 4 c.

201. " Lessingiana Trin. et Rupr,

202. $\quad$ pulcherrima C. Koch. 4

203. Tragus racemosus Desf. $\odot_{29}$ c. 29

204. Trisetum flavescens $P$. B. 4 c.

205. $\# \quad$ rigidum Trin. 4 c

206. Triticum dicoccum Schrank. v. dicoccoides $\odot$ c.

207. " monococcum L. $\odot$ c.

208. " ventricosum Ces. c.

\section{Iridaceae Juss.}

209. Gladiolus hybridus 4 c.

210 . imbricatus L. 4 c.

211. Iris desertorum Gueld. 4 c.

212. "Fischeriana Hort. 4 c.

213. " halophila Pall. 4 c.

214. " missouriensis Nutt. 4 c.

215. " musulmanica Fom. 4 c.

216. "Pseudacorus $L .4$ c.

217. " setosa Pall. 4 c.

218. " sibirica $L .4$ c.

219. " spuria L. 4 c.

220. "Thunbergii E. Lundstr. 4

221. Sisyrinchium anceps $\mathrm{Cav} .4$ c.

\section{Juncaceae Vent.}

222. Juncus atratum Krocker. 4 G.

223. " balticus Willd. 4 c.

224 * " bufonius $L$. $\odot$ c.

225. " compressus Jacq. 4 c.

226. $"$ effusus $L .4$ c.

227. " lamprocarpus Ehrh. 4 
228. Luzula nivea D. G. 4 c.

\section{Juncaginaceae Rich.}

229. Seheuchzeria palustris $L . ~ 4 \mathrm{G}$

\section{Liliaceae Scop.}

230. Allium albidum Fisch. 4 c.

231. " angulosum $L .2 \mathrm{c}$.

232. " coeruleum Pall. 4 c.

233. " fistulosum L. 2 c.

234. " flavum L. 4 c.

235. " globosum Red. 4 c.

$236{ }^{*}$ " lineare $L .24$ c.

237. " obliquum L. 4 c.

238. " odorum L. 4 c.

239. " rotundum L. 4 c.

240. " schoenoprasum L. 4 c.

241. " sibiricum $L .2+$ c.

242. Anthericum Liliago L. 4 c. 29

243. Asparagus officinalis $L .4$ c.

244. Asphodelus tenuifolius Cav. 4
245. Bulbine annua Willd. ( c)

246. Convallaria majalis $L .4$ c.

247. Galtonia candicans Dcne. 4 c.

248. Lilium Martagon L. z c.

249. " tigrinum Gawler. 4 c.

250. Muscari botrioides Mill. 4 c.

251. " racemosum Mill. 4 c.

252. Ornithogalum narbonense $L .4$

253. " umbellatum L. 4

254,* Paris quadrifolia L. 4 M.

255. Polygonatum multiflorum All.

256. Puschkinia 4 c.

257. Scilla sibirica Andrz. 4 c.

258.* Smilacina stellata Desf. 4 c.

259. Zygadenus elegans Pursch. 4

260. $\quad$ sibiricus A. Gray. 4

261. Veratrum album $L$. var. Lobelianum Bernh. 4 c.

\section{Dicot y 1 edone a e.}

\section{Aceraceae Neck.}

262. Acer platanoides $L . \hbar$ G. 263. " tataricum $L$. $₹ \mathrm{G}$.

\section{Aizoaceae.}

264.* Mesembryanthemum tricolor Haw. $\odot$ c. 265. Tetragonia expansa Murr. $\odot$ c.

\section{Amarantaceae Juss.}

266. Amarantus albus $L$. $\odot$ c. Blitum L. $\odot$ c. 267. 268. 269.

270.

271.

272.

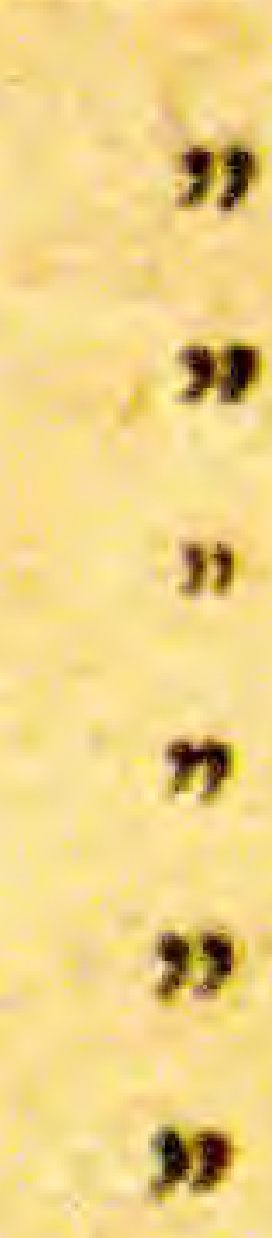

273. 274.

275 . caudatus $L . \odot$ c. deflexus L. 4 c. gangeticus $L$. $\odot$ c. lividus $L$. $\odot$ c. purpurascens Otto. $\odot$ c. retroflexus $L$. $\odot$ c. silvestris Desf. spinosus $L . \odot \mathrm{c}$.
Apocynaceae R. Br.

276. Vinca minor L. 4 c.

\section{Aristolochiaceae Blume.}

277. Asarum europaeum L. 4 c.

\section{Asclepiadaceae Lindl.}

278. Asclepias Cornuti Decsn. 4 c. 279. Cynanchum medium $K$. Schum.

280. 4 c.

" Vincetoxicum $R . B r$. 4 c.

Balsaminaceae S. F.

281. Impatiens glanduligera Royle.

$282 *$

noll-tangere $L . \odot$ c.

Basellaceae

284.* Boussingaultia basellodies H.B.K. 4 c. 
Berberidaceae Torr. et Gray.

285. Berberis vulgaris $L, \hbar$ G.

\section{Betulaceae Rich.}

286. Carpinus orientalis Mill. $\hbar$ K

287. Corylus Avellana $L . \hbar$ G.

Bignoniaceae.

288. Incarvillea Delavayi Franch. 4

Borraginaceae S. F.

289. Anchusa italica Retz. $\odot$ c. 29 290. Borrago officinalis $L$. $\odot$ c.

291. Cerinthe minor $L$. $\odot$ c.

292. Cynoglossum officinale $L .4$ c. 293. Echium italicum $L . \odot, 4$ c. 294. " plantagineum L. 4 c. 295. \# vulgare $L$. $\odot$ c.

296. Heliotropium europaeum L. $\odot$

297. peruvianum L. 4 c.

298. Lappula Myosotis Moench. $\odot$ c. 299. Lindelofia spectabilis Lehm. 4

300. Lithospermum officinale $L .4$ c. 301. Lycopsis arvensis $L . \odot, \odot$ c. 302. Myosotis alpestris Koch. $\odot$ c. 303. $~($ caespitosa Schultz. $\odot$, $\cdot c$ 304. " intermedia Link. $\odot$, $\odot$, 305, silvatica Hoffm. 306. Omphalodes linifolia Moench.

c. c. 309. Symphytum officinale L. 4 c.

Campanulaceae Juss.

310. Adenophora Bulleyana Diels. 4 311. Campanula abietina Gris. c.
312. Campanula alliariaefolia Willd.

313. 4 c.

" americana $L . \odot$ c. carpathica Jacq. 4

315 c.

315.* " vervicaria L. $\odot, 4$ c glomerata $L$. v. acaulis $4 \mathrm{c}$.

319. " gracilis Jard. 4 c. 320. \# grandis F. et M. 4

321. grossekii Heuff.

c.

322. " imeretina Rupr. 4

323. $\rightarrow \quad$ laciniata $L$. c.

324. " lactiflora M. B. 4 c.

325.,$\quad$ latifclia $L .4$ c.

$326 . \quad n \quad$ Leutweinii Heldr. 4

327. „ longistyla Fomin. 4

328. " michauxioides Boiss.

$\odot$ c. multiflora $W$. et Kit. nobili-macrantha $\stackrel{\text { Reg. }}{\text { c. }}$ c. patula $L . \odot$ c. pelviformis Lam. c. persicifolia $L .4$ c. pulcherrima Schrank. et Zeyh. 4 c. rapunculoides L. 4 336. $"$ folia C. Koch. 4 c. 337. " rotundifolia L. 4 c. 338. " $\quad$ v. Hostii 4 c.

339. " sarmatica Ker.-Gawl.

340. sarmentosa Hochst.

341. ” Scheuchzeri Lodd.

342. Steveni $M . B .4$ c.

343. " $\quad$ thyrsoldes $L$. c.

344. " $\quad$ trachelium L. 4 c.

345. " turbinata Schot., Nym. et Kotschy. 4 c. 
346. Codonopsis ussuriensis Hemsl.

347. $\quad$ viridiflora $M a x .4$ c. 348.* Jasione montana $L . \odot$ c.

349. ” perennis Lam. 4 c.

350. Lobelia erinus $L$. $\odot$ c.

351. " inflata $L$. $\odot$ c. 29

352. " syphilitica L. 4 c. 29

353. Phyteuma canescens W. et. Kit. 4 c.

354. $\quad$ spicatum L. 4 c. 29 355. Platycodon grandiflorum $D$. $C$. 356. Specularia Speculum D. C. $\odot$ c. 357. Symphyandra Hoffmannii Pant.

358. Wahlenbergia pendula Schrad. ○ c. 29

\section{Cannabaceae Endl.}

359. Cannabis sativa $L . \odot$ c.

360 . " $\quad$ ". v. indica $\odot$ 361. Hnmulus Lupulus L. 4 c.

\section{Capparidaceae.}

362. Capparis spinosa $L . \hbar$ K. 27 363. Cleome graveolens Rafin. $\odot$ c. $364^{*}$. $\quad$ violacea $L . \odot$ c. 29 Caprifoliaceae Vent.

365.* Adoxa Moschatellina L. 4 c. c. 28

366. Lonicera tatarica $L$. 5 c. 28 367. Sambucus ebulus L. 4 c. 368. \# racemosa $L .4$ c. 369. Symphoricarpus racemosus $M c h x$. 370. Viburnum Lantana $\hbar_{2}$ c. 28 371. $\quad$ Opulus $L . \hbar^{2} \mathrm{G}$

\section{Caryophyllaceae Juss.}

372. Agrostemma Githago L. $\odot$ c. 373. nicaeensis Pers. $\odot$

374. Alsine pinifolia Fenzl. 4 c. 27 375. Arenaria graminifolia Schrad. 4
376. Arenaria longifolia $M$. B. $4 \mathrm{c}$. 377.,$\quad$ serpyllifolia $L . \odot$ c. 378. Cerastium arvense $L .4$ c. 379. " caespitosum Gilib. 4 c. 29

380. \# inflatum Link. $\odot$ c. 381. Corrigiola littoralis $L$. $\odot$ c. 382. Cucuoalus baccifer $L$. 4 c. 383. Dianthus arenarius $i \quad 4 \mathrm{c}$.

384. \# attenuatus Sm. 4 c. 29 385. ” barbatus $L .4$ c.

386. " Borbasii Vandas. 4 c.

387. ” campestris $M$. B. $4^{28}$ c. 388.* $" \quad$ chinensis $L . \odot$ c. 389. " collinus W. et Kit. 4

c. 29

390.* $\quad$ deltoides $L .4$ c. 29 391. " " giganteus D'Urv. 4 c. 392. " laciniatus $\odot$ c. 29 393 . " plumarius $L .4$ c. 394 . " polymorphus $M$. B. 4 395. " rupicolus Biv. 4 c. 396. Drymaria cordata Willd. $\odot$ c. 397. Elisanthe noctiflora Rupr. $\odot$ c. 398. Gypsophyla elegans $M . B . \odot$ c. 29

399. $\quad$ muralis $L$. $\odot$ c. 28 400. Holosteum umbellatum $L . \odot, \odot$ c. 401. Lychnis chalcedonica $L .4$ c. 402. " Coeli-rosa Desr. $\odot$ c. 403. $\quad$ Flos cuculi L. 4 c. 404. Flos-Jovis Lam. $\odot$ c. 405. Malachium aquaticum Rchb. 4 c. 29 406. Melandryum album Garcke. $\odot$, 407. Minuartia caespitosa Deg. c. 408. Moehringia lateriflora Fenzl. 4.

409. Sagina procumbens $L .4$ c. 410. Saponaria officinalis $L .2$ c. 411. " $"$ L. fl. pl. 4 c. 412. Scleranthus annuus $L$. $\odot$ c. 413. Silene Armeria $L$. $\odot$ c.

414. " commutata Guss. c. 415. " compacta Fisch. $\odot$ c, 416. " conica $L$. $\odot$ c. 417. " conoidea $L$. f. cleistoga418. ma Troitzky. $\odot$ c. 419, " gallica $L . \odot$ c. " italica Pers. 4 c. 
420. Silene Otites $S m . \odot, 4$ c.

421. " saxatilis Sims. c.

422. " tatarica Pers. 4 c.

423. " vuosa Aschers. 4 c.

424. Spergularia campestris Aschers. $\odot, \odot \mathbf{c}$.

425. media Boiss. () c.

426. Stellaria graminea $L .4$ c.

427. Tunica prolifera Scop. $\odot$ c.

428. "Saxifraga Scop. 4 c.

429. Vaccaria pyramidata Medik.

430. Viscaria viscosa Aschers. 4 c.

\section{Celastraceae Lindl.}

431. Evonymus europaeus $L . \hbar$ c. 432. $\% \quad$ verrucosus Scop.

\section{Chenopodiaceae Less.}

433. Atriplexs hastatum $L$. $\odot$ c.

434. " hortense $L$. $\odot$ c. 28

435. Axyris amarantoides $L$. $\odot$ c.

436. Beta patellaris Moq. () c. 29

437. trigyna $W$. et $K i t .2$ c.

438. Chenopodium album $t$. $\odot$ c.

439. \# bonus Henricus $L$. 4 c.

440. $\quad$ Botrys $L . \odot$ c.

441. " $\quad$ ficifolium $\mathrm{Sm}$. $\odot$

442. $\quad$ foetidum Schrad.

443. „ foliosum Schrad.

444. murale $L$.

445. " " opulifolium Schrad.

$446 * \quad n \quad$ polyspermum $L$. $\odot$

447. $\quad$ vulvaria $L . \odot$ c.

448. Corispermum hyssopifolium L. $\odot$

449. Kochia trichophylla Schmeiss. \&

c.

450. Monolepsis trifida Schrad. $\odot$ c.

451. Obione sibirica Fisch. $\odot$ c.

452. Salsola Soda $L$. $\odot$ c.

453. Spinacia tetrandra Stev.

c. 29

\section{Cistaceae Dun.}

454. Helianthemum Chamaecistus Mill. 2 c.

455 . guttatum Mill. $\odot$

456. $" \quad$ ledifolium Mill.

\section{Compositae Adans.}

457. Achillea filipendulina Lam. 4 c. 458. " Millefolium $L .4$ c. 459. " Ptarmica L. 4 c. 460. Acroclinium roseum Hook. $\odot$ c. 461. Ageratum conyzoides $L$. $\odot$ c. 462. Ammobium alatum $R . B r$. $\odot$ c. 463. Anthemis altissima $L$. () c. 29 464.. , arveusis $L$. $\odot$ c. 29 465. Arctotis stoechadifolia Berg. (c)

466. Arnica Chamissonis Less. 4 c. 467. " montana $L .4$ c.

468. Artemisia Absinthium L. 4 c.

469. " annua $L$. $\odot$ c.

470. " campestris $L .4$ c.

471. " vulgaris $L .4$ c.

472. Aster alpinus L. 4 c.

473. „ salicifolius Scholl. 4 c.

474. " Tradescanti L. 4 c.

475. Baeria coronaria A. Gray. $\odot$ c.

476. Bellis perennis $L .2$ c.

477. Bidens leucantha Willd. $\odot$ c. 478. " tripartitus $L$. $\odot$ c.

479. Buphtalmum speciosum Schreb.

4 c.

480. Calendula aegyptiaca Desf. $\odot$ c.

481. $\quad \Rightarrow \quad$ arvensis $L . \odot$ c.

482. " officinalis $L . \odot$ c.

483. Callistephus chinensis Nees. $\odot$

484. Carlina vulgaris $L$. $\odot$ c. 29

485. Carpesium cernuum $L .4$ c.

486. Carthamus lanatus $L$. $\odot$ c.

487. " tinctorlus $I . \odot$ c.

488. Centaurea axillaris Willd. 4 c.

489. $"$ bella Trev. c.

490. " $\quad$ Calcitrapa $L$. v. iberica Trev. c. 28

491. " Cyanus L. $\odot$ c.

492. " " glastifolia $L .4$ c.

493. " Jacea $L .4$ c. 
494. Centaurea maculosa Lam. $\odot_{29} \mathrm{c}$.

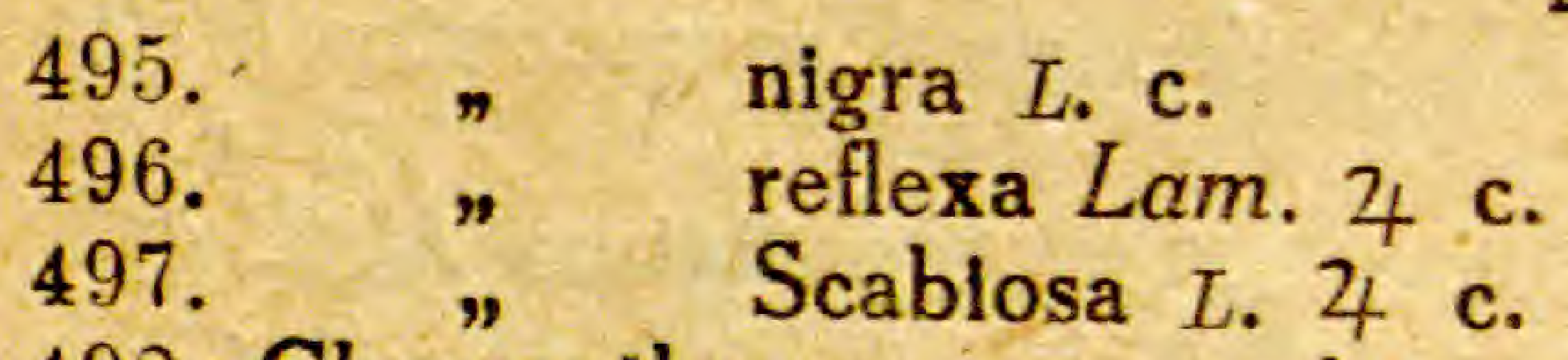
498. Chrysanthemum corymbosum $L$.

$\begin{array}{ll}499 . & \text { 4 c. } \\ 500 . & \begin{array}{l}\text { Leucanthemum } \\ \text { L. } 4 \text { c. }\end{array} \\ \text { tricolor Hort. } \odot\end{array}$

502. Cichorium Intybus L. 4 c. 503. $"$ pumilum Jacq. c. 504. Cirsium arvense Scop. 4 c. 505. " hispanicum D. C. c. 506. " lanceolatum Scop. . c. 507. rivulare Link. 4 c. 28 508. Cladanthus arabicus Cass. $\odot$ c. 509. Cnícus Benedictus $L$. $\odot$ c. 510. Coreopsis peltata $L$. $\odot$ c.

511. Cosmea bipinnata Willd. $\odot$ c. 512. Cosmos diversifolius Otto, $\odot$ c. 513. Crepis alpina $L . \odot$ c. 514. " biennis $L . \odot$ c. 515. " foetida $L$. $\odot$ c. 516. " pulchra $L$. $\odot$ c. 517. " tectorum $L$. $\odot$ c. 518. Crupina vulgaris Cass. $\odot$ c. 519. Dahlia coccinea Cav. 4 c. 29 520.* " Merckii Lehm. 4 c. 521. " scapigera Knowl. et West 522. Dimorphotheca aurantiaca ${ }^{4} D . C$. 523. " " pluvialis Moench. 524. Echinops globifer Janka $4 \mathrm{c}$. 525.* Erigeron alpinus L. $4 \mathrm{c}$. 526. canadensis $L . \odot$, $\odot$ c. 29

527. Eupatorium cannabinum $L .4$ c.

528. Evax pygmaea Brot. $\odot$ c. 29

529. Felicia tenella Ness. $\odot$ c.

530. Filago spathulata Presl. () c.

531. Galinsoga parviflora Cav. () c. 532. Gnaphalium vira-vira Molina $\odot$ c. 29

533. Guizotia oleifera $D$. C. $\odot$ c. 534. Helenium Hoopesii A. Gray. 4
535.* Helicrysum angustifolium $D . C$.

536. arenarium Moench.

537.* Hieracium alpinum L. 4 c.

538. ” Pilosella L. 4 c. 539. " pratense Tausch. 4

540.* $\quad$ tridentatum Fries.

541. 4 c.

541. $" \quad$ virosum Pall. 4 c.

542. Hyoseris lucida $L$. $\odot$ c. 29

543. Hypochoeris radicata L. 4 c. 544. Inula conyza $D . C . \Theta, 4$ c. 545. "Helenium L. 4 c. 546. " magnifica Lipsky..c. 547. " oculus christi L. 4 c.

548. Lactuca sativa $L . \odot$

549. Lampsana communis L. $\odot$ c 27 5.50. " grandiflora M. B. 4

551. Lappa amplissima Boiss (-) c. 552. \# tomentosa Lam. $\odot$ c. 29 553. Lasiospermum pedunculare Lag. ค. c. 29

554.* Layia Calliglossa Gray. $\odot$ c. 555. Leontodon autumnalis L. 4 c. 556. Leontopodium alpinum Cass. 4

557. Madia sativa Mol $\odot \mathrm{c}$.

558. Matricaria Chamomilla L. $\odot$ c. 559. " hybrida Hort. $\odot$ c. 560. " inodora $L . \odot, \odot$ c. 561. " " suaveolens Buch. $\odot$

562. Mulgedium sibiricum Less. 4 c. 563. Onopordon Acanthium L. $\odot$ c. 564 . illyricum $L$. $\odot$ c. 565. Palafoxia texacana D. C. () c. 566. Picris hieracioides $L_{0} \odot, 4$ c. 567. Psephellus hypoleucus Boiss. c. 568. Pyrethrum Parthenium Sm. 24. c.

569. $\quad$ roseum $M . B .4^{27}$ c. 570. Rhagadiolus stellatus $D$. C. $\odot$

571. Rudbeckia hirta $L$. $\odot$, $\odot$ c. 572. $"$ laciniata $L .4$ c. 573. Scorzonera eriosperma M. B. 4 574. " hispanica L. 4 c. 575. Senecio erraticus Bertol. $\odot$ c. 
576 Senecio Jacobaea L. 4 c. 27 577. \# ligularia Hook. 4 c. 578. Serratula coronata L. 4 c. 579.* Siegesbeckia orientalis $L$. $\odot$ c. 28

580. Silybum Marianum Gaertn. $\odot$, 581. Solidago virga-aurea $L . \%$ c.

582. Sonchus arvensis $L .4$ c. 29

583. $"$ asper Hill. $\odot$ c.

584. " oleraceus $L$. $\odot$ c.

585. Soilanthus oleracea $L$. $\odot$ c.

586. Tagetes erecta $L$. $\odot$ c. 29

587. " lucida Cav. 4 c. 29

588. ” patula L. $\odot$ c.

589. " signata Bartl. $\odot$ c.

590. Tanacetum vulgare $L .4$ c.

591. Taraxacum laevigatum D. C. 4

593. Tragopogon brevirostris $D . C$.

594. coloratus $C$. c.

$595 . \quad$ major $\odot$ c.

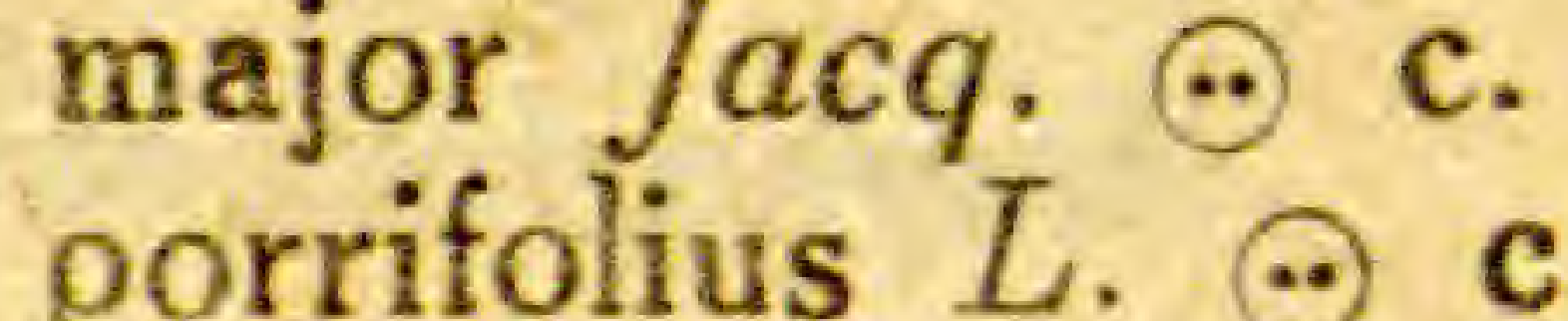

597. $\quad$ pratensis $L \odot$ c.

598. Tussilago Farfara L. 2 c.

599. Urospermum picroides $F$. W. Schmidt. $\odot$ c.

600.* Ursinia speciosa D. C. c.

601. Ximenesia encelloides Cav. $\odot$

602. Zacintha verrucosa Gaertn. $\odot$ c.

603. Zinnia elegans Jacq. $\odot$ c.

604. " Haageana Rgl. $\odot$ c.

605 . " multiflora $L$. $\odot$ c.

\section{Convolvulaceae Vent.}

606. Convolvulus tricolor $L$. $\odot$ c.

607. $\quad$ undulatus Cav. $\odot$

608. Ipomoea purpurea Lam. $\odot$ c. 609. " splendens G. Don. (c)

c.

Crassulaceae D. C.

610. Crassula glomerata Berg. $\odot$ c. 28
611. Sedum acre L. 4 c.

612. " aizoon $L$. c.

613. " " Ewersii Ledb. 4 c.

614. ” hybridum L. 4 c.

615. " pallidum $M . B . \odot$ c.

616. " purpureum Link. 4 c.

617. " rupestre $L .4$ c.

618. \# spurium $M$. B. 4 c.

619. Sempervivum patens Griseb. et

620 ruthenicum Koch

621. $\quad$ tectorum L. ${ }_{29}^{4}$ c.

\section{Cruciferae Juss.}

622. Aethionema Buxbaumii D. C.

623. Arabis albida Stev. 4 c.

624. " hirsuta Scop. $\odot, 4$ c.

625. " laxa Sib. et Sm. c.

626. " rosea D.C. c.

627. " suecica Fries (-) c.

628. Barbarea stricta Andrz. $\odot$ c.

629. vulgaris $\mathrm{R}, \mathrm{Br}$.

630. Berteroa incana D.C. $\odot, \odot$ c.

631. Brassica juncea Czern. $\odot$ c.

632. \#. nigra Koch. $\odot$ c.

633. Bunias Erucago $L$. $\odot$ c.

634. " orientalis $L .4$ c.

635. Camelina linicola Sch. et $S p$. $\odot$

636. Capsella bursa pastoris Moench.

637. Carrichera Vella D. G. $\odot$ c.

638. Cheiranthus nanus Merk. -) c.

639.* Chorispora tenella D. C. ๑ c.

28

640. Cochlearia officinalis $L .4$ c.

641. Conringia orientalis Dum. $\odot$ c.

642. Crambe hispanica $L$. $\odot$ c.

643.* " pinnatifida $R . B r .4$ c.

644. Descurainia Sophia W. et B. c.

645. Diplotaxis muralis $D . C$. $\odot$ c.

646. Draba hirta L. 24 c.

647. Erysimum cheiranthoides L. $\odot$ c.

648. " cuspidatum D.C. $\odot$

649. - pulchellum J. Gray. 4 c. 
650. Erysimum suffruticosum Spreng.

651. Eruca sativa Lam. $\odot$ c.

c.

652. Farsetia clypeata $R . B r$. 4 c.

653. " eriocarpa D.C. 4 c. 29

654. Hesperis fragrans Fisch. 4 c.

655. " lutea Max. $\odot$ c.

656. " matronalis L. 4 c.

657. Hutchinsia alpina $R . B r .4$ c.

658.* Iberis amara $L$. $\odot$ c.

659 . " umbellata $L . \odot$ c.

660. Isatis tinctoria $L . \odot$ c.

661.* Lepidium ruderale $L$. $\odot$ c.

662. $\quad$ sativum $L . \odot$ c.

663. Lunaria biennis Moench. -) c.

664. Malcolmia africana Ait. $\odot$ c.

665. Matthiola annua Sweet. $\odot$ c.

666. " bicornis D.C. $\odot$ c.

667. Myagrum perfoliatum $L . \odot$ c.

668. Neslia paniculata Desv. $\odot$ c.

669. Peltaria alliacea $L .4$ c. 29

670. Senebieria Coronopus Poir. $\odot$ c.

671. Sinapis alba $L$. $\odot$ c.

672 . " apula Tenore. $\odot$ c.

673. " chinensis $L$. c.

674. " dissecta Lag. $\odot$ c.

675. Sisymbrium Irio L. 4 c. 28 676.* $\quad \quad$ officinale Scop. $\odot$ c. 29

677. Stenophragma Thalianum Celak.

678. Thlaspi arvense $L . \odot \mathrm{G}$.

679. Turritis glabra $L$. $\odot$ c. 29

\section{Cucurbitaceae Holl.}

680. Bryonia alba $L .4$ c.

681. Cucumis prophetarum $L$. $\odot$ c.

682. Cucurbita maxima Duch. $\odot$ c.

683. $”$ melanosperma $A . B r$.

684. " Melopepo L. $\odot$ c.

" napolitana Reg. $\odot$ c.

686. " $\quad$ Pepo L. $\odot$ c.

$687 . \quad$ verrucosa $L \odot$ c.

688. Cyclanthera pedata Schrad. $\odot$

c. 29

689. Ecballium elaterium Rich. $\odot$ c.

29
691. Lagenaria vulgaris Ser. (๑) c.

692. Cicyos angulata $L$. $\odot$ c.

\section{Datiscaceae.}

693. Datisca cannabina $L .4$ c.

Dipsacaceae D. C.

694.* Callistemma brachiata Sibth. $\odot$

c.

695. Cephalaria tatarica Schrad. 4 c.

696. Dipsacus ferox Loiss. (.) c.

697. „ fullonum Mill. $\odot$ c.

698. ” laciniatus $L . \odot$ c.

699. " plumosus Franch. (.) c. 29

700. Knautia arvensis Coult. 4 c. 701.* atrorubens Janka. 4 c.

702. Scabiosa atropurpurea $L$. $\odot$ c.

703. " graminifolia $L .4$ c.

704. " leucophylla Borb. 4 c

705. " lucida Vail. 4 c.

706. $\quad$ maritima $L \odot$ c.

707. " micrantha Desf. $\odot$ c.

708. " ucrainica $L . \odot$ K. 27

Euphorbiaceae J. St. Hil.

709.* Euphorbia Balsamifera Ait. 4

710. " Esula L. 4 G.

711. " exigua $L$. $\odot$ c.

712.* $\quad$ graeca B. et $S p$.

713. helioscopia $L$ c.

714. " humifusa Willd. $\odot$

715* $\quad$ c.

716. " uralensis Fisch. 4 c.

717. Mercurialis annua $L$. $\odot$ c.

\section{Gentianaceae Dumort.}

718. Gentlana cruciata $L .4$ c.

719. " Pneumonanthe L. 4 c

720. " tibetica King 4 c.

721.* Swertia longifolia Boiss. 4 c.

Geraniaceae J. Sţ. Hil.

722. Erodium cicutarium L. Herit. $\odot$ 
723. Erodium gruinum Ait. $\odot$ c.

724. Geranium albanum $M . B .4$ c.

$725 . \quad$ " dissectum $L . \odot$ c.

726. " divaricatum Ehrh. 2t

$727 . *$

728. " pratense $L .2$ c. 29

729. $\quad$ pussillum $L$. $\odot$ c.

\section{Globulariaceae D. C.}

730. Globularia trichosantha $F$. et $M$.

Willkommii $N_{y m a}$ c. 4 c.

\section{Guttiferae Endl.}

732. Hypericum Ascyron L. 4 c. 733. " montanum L. 4 c. 734. $" \quad$ perforatum L. 4 c. 735. $\quad$ quadrangulum $L .4$ c.

\section{Hydrophyllaceae}

736. Nemophila maculata Benth. $\odot$

737. Menziesii Hook. c. Arn. $\odot$ c.

738. Phacelia congesta Hook. $\odot$ c.

739. " divaricata A. Gray. $\odot$

740. " grandiflora A. Gray. $\odot$

741. " tanacetifolia Benth. $\odot$

c.

\section{Labiatae Juss.}

742. Ajuga reptans $L .4$ c.

743. " " L. v. albiflora Tin.

744. Ballota nigra $L .4$ c.

4 c.

745. Betonica officinalis $L .2$ c.

746. Brunella grandiflora Moench. 4

747. " vulgaris L. 4 c.

c.

748. Dracocephalum moldavica $L$. $\odot$

749 .

Ruyschiana $L$.

750. Elscholtzia cristata Willd 4 c.
751. Galeopsis speciosa Mill. $\odot$ c.

752. $\quad$ Tetrahit L. $\odot$ c. 29

753. Horminum pyrenaicum L. 4 c.

754. Hyssopus officinalis $L$. 5 c.

755. Lallemantia peltata $F$. et. $M$. $\odot$

756. Lamium album L. 4 c.

757. " amplexicaule $L . \odot, \odot$

$758 . \quad$ purpureum $L$. $\odot$.

759. Lavandula multifida $L .4$ c.

760. Leonurus Cardiaca L. 4 c.

761. " Marrubiastrum $L . \odot$,

762. Lophanthus rugosus $F$. et $\oplus_{M}$. 4

763. Lycopus europaeus L. 4 c. 29

764. $\quad$ exaltatus L. 4 c.

765.* Marrubium praecox Janka 4 c.

766 . $\quad$ vulgare $L .4$ c.

767. Melissa officinalis L. 4 c.

768. Mentha arvensis $L .4$ c.

769. " longifolia Huds. 4 c.

770. Nepeta Cataria L. 4 c.

771. " grandiflora M. B. 4 c.

772. " macrantha Fisch. 4 c.

773. $\quad$ nuda $L .4$ c.

774. Origanum vulgare L. 4 c.

775. Phlomis tuberosa $L \cdot 4 \mathrm{c}$.

776. Pycnanthemum lanceolatum Pursh. 4 c. 28

777. Salvia cleistogamá De Bar. et Paul. 4 c. 29

778. \# dumetorum Andrz. c. 28

779. " glutinosa $L \cdot 4$ c.

780. " hians Royle. 4 c.

781. " hispanica $L$. $\odot . c$.

782. " leonia Benth. 4 c. 29

783. " nemorosa $L .4$ c. 29

784. " nilotica Murr. $\odot$ c.

785. ” officinalis $L \cdot \hbar$ c.

786. " Regla Cav. c.

787. " Sibthorpii Sm. 4 c.

788. " tilliifolia Vahl. $\odot$ c.

789. " verticillata $L .4$ c.

790. " virgata Ait. 4 c.

791. Satureja Clinopodium Caruel. 4 c.

792. " " var, variegatum Mort. 4 c.

793. $\quad$ hortensis $L . \odot$ c.

794. Scutellaria galericulata $L .4$ c. 795.* $\quad$ orientalis L. 4 c. 
796. Sideriits montana $L$. $\odot$ c. 29

797. scordioides L. 4 c.

798. Stachys lanata Jacq. 4 c.

799. $\quad$ recta $L .4$ c.

800. " sideritoides C. Koch. c.

801. $n$ silvatica $L .4$ c.

802. Teucrium Chamaedrys L. 4 c.

$803 . \quad$ hyrcanicum $L .2$ c. 27

804. Thymus glaber Mill. 4 c.

805. " Marschallianus Willd.

806. $\quad$ serpyllum $L$. v. citriodora $4 \mathrm{c}$.

\section{Leguminosae Juss.}

807. Amorpha fruticosa $L . \hbar$ c. 27 808. Anthyllis tetraphylla $L$. $\odot$ c. 809. Astragalus Cicer L. 4 c.

810. $\Rightarrow \quad$ glycyphyllus L. 4 c. 811. " Onobrychis L. 4 c. 812. " sulcatus L. 4 c. 29

813. Baptisia australis $\mathrm{R} . \mathrm{Br} .4 \mathrm{c}$.

814. Caragana arborescens Lam. $\hbar$

815. " frutescens D.C.

816. Colutea cruenta Ait. $\hbar$ c.

817. Coronilla varia $L .4$ c. 29

818. Dolichos Catjan $L$. $\odot$ c.

819. Dorycnium herbaceum Vill. $\hbar$

c. 27

820.* Galega orientalis Lam. 4 c.

821. Genista ovata $W$. et $K$. $\frac{\hbar}{2}$ c.

822. " sagittalis L. 4 c.

823. " tinctoria $L$. 5 c.

824. Glycyrrhiza echinata $L .4$ c. 29

825 . Hedysarum flexuosum $L$. $\odot$ c.

826. Lathyrus Aphaca L. $\odot$ c. 27

827. " canescens Taubert. 4

828. " Clymenum L. $\odot$ c.

829. - ochrus D.C. $\odot$ c.

830 . $\quad$ " odoratus $L . \odot$ c.

831. " palustris L. 4 c.

832. " " sativus $L$. $\odot$ c.

833. " silvestris L. 4 c.

834. " vernus Bernh. 4 c.

835 . Lotus ciliatus $C$. Koch. c.

836.* " coimbrensis Willd. c.

837. " corniculatus L. 4 c.

838." " edulis $L$. $\odot$ c. 29
839. Lotus Tetragonolobus $L . \odot$ c. 840. Lupinus elegans $H$. B. et $K$. $\odot$

841. " mutabilis $S w . \odot$ c. 29

842. $" \quad$ perennis L. 4 c.

843. $\quad$ pilosus Murr. $\odot$ c.

844. Medicago arabica All. $\odot$ c.

845. " denticulata Willd. $\odot$

846. " Echinus D.C. $\odot$ c.

847. " hisplda Gaertn. $\odot$ c.

848. " " laciniata Mill. $\odot$ c.

849. " lupulina $L . \odot, 4$ c.

850 .* " maculata Willd. $\odot$ c.

851. sativa $L .4$ c.

852. Melilotus albus Desr. $\odot$ c.

853. $" \quad$ indica All. $\odot$ c.

854. " officinalis Desr. $\odot$ c.

855. " sulcatus Desf. $\odot$ c.

856. " wolgicus Poir. $\odot$ c.

857. Ononis biflora Desf.

858. " hircina Jacq. 4 c. 29

859. " rotundifolia $L . \hbar$ c. 29

860. Ornithopus sativus Brot. $\odot$ c.

861. Orohus tuberosus $L .4$ c.

862.* Phaseolus caffer Haberl. $\odot$ c.

863. " helvolus $L$. $\odot$ c.

$864 .^{*} \quad " \quad$ lathyroides $L . \odot$ c.

865. " Mungo $L . \odot$ c.

866.* " Ricciardianus Ten.

867. Pisum arvense $L . \odot$ c.

868.* " elatius M. B. $\odot$ c. 29

869. " Jomardi Schrank. $\odot$ c.

870. " sativum $L . \odot$ c.

871. " thebaicum $W . \odot$ c.

872. Robinia pseudacacia $L \cdot \hbar$ c c.

873. Scorpiurus subvillosa $L$. $\odot$ c.

874. $\quad$ vermiculata $L . \odot$ c.

875. Thermopsis caroliniana $M$. $A$. Curt. 4 c.

876. " fabacea D.C. 4 c.

877. montana Nutt. 4 c.

878. Trifolium agrarium $L$. $\odot$ c. 29

879. " alpestre $L .4$ c.

$880 .^{*} \quad "$ angustifolium $L . \odot$ c.

881. " arvense $L . \odot$ c. 29

$882{ }^{*} \quad "$ dubium Freyn. $\odot$ c.

883. " fragiferum $L .4$ c. 29 
884. Trifolium incarnatum $L$. $\odot$ c.

885. " glomeratum $L . \odot$

886. " ligusticum Balb. c.

887. " Lupinaster L. 4 c.

888. " medium L. 4 c.

889. " montanum L. 4 c.

890. 29

890. " pratense $L .4$ c.

$891 .^{*} \quad " \quad$ procumbens $L . \odot$ c.

892.* " repens $L .4$ c.

893.* " $\quad$ resupinatum $L . \odot, \odot$

c. 28

894. $\quad$ rubens $L .4$ c.

895 . " striatum $L . \odot$ c. 28

896. Trigonella calliceras Fisch. $\odot$ c.

897. " foenum-graecum $L$.

898. " orthoceras Kar. et Kir. $\odot$ c. 29

899. Vicia amoena Fisch. $\odot$ c.

900. " angustifolia Roth. $\odot$ c.

901. 29

901. " atropurpurea Desf. (3) c.

" cracca L. 4 c.

903. „ Ervilia Willd. $\odot$ c.

904. " Faba L. $\odot$ c.

905. " grandiflora Scop. $\odot$ c.

906. " hirsuta G. Koch. $\odot$ c.

907. " lathyroides $L . \odot$ c.

908. " lutea $L . \odot$ c.

909. " Michauxii Spreng. $\odot$ c

910. " monanthos Desf. $\odot$ c.

911. " narbonensis $L . \odot$ c.

912. " pisiformis $L .4$ c.

913. " sativa $L$. (sem. alb.) $\odot$ c.

914.* „ „ L. v. segetalis $\odot$

915. " sepium $L .4$ c.

916. " unijuga $A . B r .4$ c.

\section{Limnanthaceae.}

917. Limnanthes alba Hartw. $\odot$ c. 29

918. $"$ Douglasii $R . B r$.

\section{Linaceae Dumort.}

919. Linum monogynum Forst. c. 920. " perenne L. 4 c.
921. Linum usitatissimum $L$. (i) c.

\section{Loasaceae.}

922. Blumenbachia Hieronymi Urb.

923.* Loasa triphylla Juss. $\odot$ c.

\section{Lythraceae LindI.}

924. Cuphea lanceolata Ait. $\odot$ c.

925. Lythrum hissopifolia $L$. $\odot$ c.

926. Nesea salicifolia H.B. et $K$. ₹ c.

\section{Malvaceae Juss.}

927. Abutilon Avicennae Gaertn. ค

928. Althaea ficifolia Cav. 2 c.

929. " officinalis $L .4$ c.

930. " rosea Gav. 4 c. 29

931. Anoda cristata Schlecht. $\odot$ c.

932. " Wrightii A. Gray. $\odot$ c.

933. Hibiscus Trionum $L$. $\odot$ c.

934. Kitaibelia vitifolia Willd. 4 c.

935. Lavatera assurgentifolia Kellog.

936. \# thuringiaca $L .2$ c. 27

937. $\quad$ trimestris $L$. $\odot$ c.

938. Malope grandiflora $\odot$ c.

939. Malva Alcea $L .4$ c.

940. " brasiliensis Desr. c.

941. " crispa $L . \odot, \odot$ c.

942. " neglocta Wall. $\odot$ c.

943. " radiata $L$. $\odot$ c.

944. " rotundifolia $L . \odot$ c.

945. Mălvastrum peruvianum $A$. Gray.

$\odot$ c.

\section{Moraceae Lindl.}

946. Morus nigra $L$. $\hbar$ c.

\section{Nyctaginaceae.}

947. Mirabilis Jalappa $L$. $\odot$ c.

948. Oxybaphus glabrifolius Vahl. c.

\section{Oleaceae Lindl.}

949. Fraxinus excelsior $L . \hbar$ G.

950. Ligustrum vulgare $L$. $\hbar$ c.

951. Syring a vulgaris $L . \hbar$ c. 


\section{Onagraceae Lindl.}

952. Boisduvalia densiflora Wats. $\odot$

953. Clarkia elegans Dougl. $\odot$ c.

954. Epilobium adnatum Gris. 4 c

955. $\leadsto$ Dodonaei Villars. 4

956. " hirsutum L. 4 c.

957. " Lamyi F. Schultz. 4

958. " montanum L. 4 c.

959. " pedunculare A. Cunn.

960. Gaura parviflora Dougl. $\odot^{4}$ c.

961. Godetia grandiflora Lindl. $\odot$ c.

962. Jussieua peruviana $L$. $\odot$ c.

963. Lopezia racemosa Cav. $\odot$ c.

964. Oenothera ammophila Focke.

$\odot$ c.

965. $\quad$ blernis $L . \odot$ c.

$966 . \quad$ - campyfolia

967. \# Drummondii Hook.

968. fruticosa $L . \odot$ c.

969. " " gigas De Vries. c.

$970 . \quad$ " grandiflora Ait. $\odot$

971. * missouriensis Sms.

972. $\quad$ muricata $L . \odot$ c.

973.* " $\quad$ odorata Jacq. $\odot$ c

974. " pumila L. 4 c.

975.,$\quad$ rosea Ait. 4 c.

976. $\quad$ tetraptera Cav. $\odot$

977. $\quad \quad$ triloba Nuttal. $\odot$

c. 29

\section{Oxalidaceae Lindl.}

978. Oxalis corniculata $L . \odot$, $\odot$ c. 979. " stricta $L .4$ c.

\section{Papaveraceae Juss.}

980. Adlumia cirrhosa Rafin. 4 c.

981. Argemone mexicana $L$. $\odot$ c.

982. Chelidonium laciniatum Mill. 4

983.

$$
\text { majus } L .4 \mathrm{c} \text {. }
$$

984. Corydalis cava Schw. et Koerte. 4 c.

985. " glauca Purch. $\odot$ c.

986. " ophiocarpa Hook. f. et Thoms. 4 c.

987. „ sempervirens Pers. $\odot$

988* $\quad$ vaginalis Royle. c.

989. Eschscholtzia californica Cham.

990. Fumaria $\odot$ c.

990. Fumaria officinalis $L$. $\odot$ c. 29

991. Glaucium flavum Crantz (-) c.

992. Hypecoum grandiflorum Benth.

-993. pendulu $\odot$ c. 29

994.* Meconopsis cambrica Vig. 4

995. Papaver argemone $L$. $\odot$ c.

996. " atlanticum Ball. 4 c.

997. " dubium $L . \odot$ c.

998. ” fugax Poir. $\odot$ c.

999. " orientale L. 24 c.

1000. " pavonium C. Meyer.

1001. " pilosum Sibth. 4 c.

1002. " Rhoeas L. $\odot$ c.

1003. " setigerum D. C. $\odot$ c.

1004. " somniferum $L . \odot$ c.

1005. " " L. v. opii-

ferum $\odot$ c. 29

1006.* " tauriculum Boiss. c.

1007. Roemeria violaceae Medic. $\odot$

c.

\section{Philadelphaceae}

1008. Philadelphus coronarius $L$. $\frac{\hbar}{2}$

c.

\section{Phytolaccaceae}

1009. Phytolacca decandra L. $4 \underset{29}{\text { c. }}$

\section{Plantaginaceae Lindl.}

1010. Plantago altissima $L$. c.

1011.* " amplexicaulis Cav. $\odot$

1012* - arborescens Poir. 29

1013. c. 28 
1014. Plantago Cornuti Gouan. 4 c. 1015. ” crassifolia Forsk. c.

1016. " indica $L$. c.

1017. " lanceolata L. 4 c.

1018. " lusitanica Willd. ๑ c.

1019. ” major L. \& c.

1020. " maritima Ait. 2 c.

1021.* " maxima Ait. 4 c.

1022. " media L. 4 c.

1023* ” montana Lam. 4 c.

1024. $\quad$ Psyllium L. $\odot$ c. 29

1025. " purpurescens Nutt.

1026. " ramosa Aschers. $\odot$ c.

1027. " serpentina All. c.

1028. ” serraria $L$. c. 28

1029. " virginica $L$. $\odot$ c.

\section{Plumbaginaceae Lindl.}

1030. Armeria plantaginea Willd. 4

1031. Plumbagella micrantha Ledeb.

1032. Statice tatarica $L .4$ c. 4 c.

\section{Polemoniaceae D. C.}

1033. Collomia grandiflora Dougl. $\odot$

c.

1034. \# linearis Nutt. $\odot$ c. 1035. Gilia androsaceae Steud. $\odot$ c. 1036. " californica Benth. $\odot$ c. 1037. " tricolor Benth. $\odot$ c.

1038. Leptosiphon androsacers St.

c.

1039. Navarretia squarrosa Hook. et Arn. $\odot \mathrm{c}$.

1040. Phlox Drummondi Hook. () c. 1041. Polemonium coeruleum L. 2

1042. " mexicanum Cerv.

1043. " pauciflorum Wats.

1044.* $" \quad$ reptans $L .{ }^{\odot}$ c. c.

Polygonaceae Lindl.

1045. Calligonum aphyllum Gürke. $\hbar$
1046. Emex spinosa Campd. $\odot$ c. 1047. Fagopyrum tataricum Gärtn. $\odot$

1048. Oxyria digyna Hill. 4 c. c. 29

1049. Polygonum alpinum All. 4 c.

1050. $\quad$ aviculare $L . \odot$ c.

1051. " Bistorta L. 4 c.

1052. " Convolvulus $L$. $\odot$

1053 Hydropiper c.

1053. c. 29

1054. " sagittatum L. $\odot$ c.

1055. " $\quad$ tomentosum Schrank.

๑) c. 27

1056. Rheum acuminatum Hook. 4

1057. " Emodi Wall. 4 c.

1058. " palmatum L. 4 c.

1059. " Rhaponticum L. 4 c.

1060. " undulatum $L .4$ c.

1061. Rumex Ácetosa L. 4 c.

1062 " Acetosella L. 4 c. 29

1063. " crispus L. 2 c.

1064. \# domesticus Hartm. 4

1065. \# pulcher L. 4 c.

1066. " scutatus L. 4 c.

\section{Portulacaceae LindI.}

1067. Calandrina procumbens Moris.

(.) c.

1068 Claytonia perfoliata Donn. $\odot$ c. 29

1069. Portulaca grandiflcra Lindl. ()

1070* oleracea $L$. c. 1071.* " $\quad$ pusilla H. B. et K. $\odot \mathbf{c}$. 1072. Talinum patens Willd. 4 c.

\section{Primulaceae Vent.}

1073. Anagallis arvensis L. var. coerulea Gren. et Godr. $\odot$ c.

1074. ” " L. var, phoenicea Gren. et Godr. $\odot$ c 29

1075. \# grandiflora Andr. $\odot$

1076. Androsace elongata $L . \odot, \odot$ c. 
1077. Androsace septentrionalis $L$. $\odot$

1079. Cortusa Matthioli $L$. 4 c.

1080. Lysimachia ciliata $L .4$ c.

1081.

Ephemerum L. 4

1082. vulgaris $L .4$ c.

1083. Primula Beesiana Forrest. 4 c.

1084. " cortusoides $L .4$ c.

1085. " denticulata Sw. 4 c.

1086. " $\quad$ elatior Hill. 4 c.

1088. " frondosa Janka. 4 c.

1089* $\quad 28$

c. 29

1090. " officinalis Hill. 4 c.

1091.* " pulverulenta Duthie 4

1092.* Samolus Valerandi L. 4 c. 29

\section{Ranunculaceae Juss.}

1093. Aconitum ferox Wail. 4 c.

1094. " lasiostomum Reichb.

1095. Actaea spicata $L .4$ c. 4 c.

1096. " " var. rubra (Bi-

1097. Adonis autumnalis gel.) $4 \mathrm{c}$.

1098. Anemone albana Stev.

1099.* $\quad$ Halleri All. 4 c.

1100. " multifida Poir. 4 c.

1101. , nemorosa L. it c.

1102. " patens L. 4 c.

1103. $\quad$ pratensis L. 4 c

1104. " rivularis Buch.-Ham.

1105.4 c.

1106. " silvestris $L .4$ c.

1107. Aq virginiana $L .4$ c.

1108. Aquilegia altaica

1109 " atrata Koch. 4 c

atropurpurea $\mathrm{W} \cdot 4$

$1110 . \quad$ c.

1111. " formosa Fisch. $4 \mathrm{c}$

1112. " nivea Baumg. 4 c.

1113. " olympica Boiss. 4 c.

" oxysepala $T r$. et Mey.

$1114.2 \mathrm{c}$
1115. Caltha palustris $L$. 4 c. 29 1116. Clematis integrifolia $L .4$ c. 1117. " recta $L .4$ c. 1118. " tangutica Korsch.

1119. Delphinium Ajacis $L$. $\odot$ c. 28 1120. " cashmerianum Roy. le. $4 \mathrm{c}$.

1121. " cheilantum Fisch.

1122.4 c.

1123.* $\quad$ elatum L. 4 c.

* c. 29

1124.* " puniceum Pall. 4

1125. " rossicum Litw.

speciosum Bieb. 4

1127. $\quad$ triste Fisch. 4 c.

1128. Eranthis hiemalis Salsb. 4 c.

1129. Ficaria ranunculoides Roth. 4

1130. Hepatica triloba Gilib. 4

1131. Isopyrum fumarioides $L . \odot$ c.

1132. Nigella damascena $L$. $\odot$ c.

1133.

1134. P" sativa $L . \odot$ c

135. Paeonia albiflora Pall. 4 c.

1135.* " tenuifolia $L .4$ c.

1136. Ranunculus acer $L .4$ c.

1137.

arvensis $L . \odot \mathbf{c}$.

1138.29

1138. " auricomus L. 4 c

1139. " cassubicus L. 4

1140. \#- Cymbalaria Purch.

1141. 4 c.

1142 " Flammula $L$ 4 c.

$1143 * \quad$ illyricus $L .4$ c.

1144.

1144. $\quad$ repens $L .4$ c. 29

1145. Thalictrum angustifolium Jacq.

1146.

1147. " minus $L .2$ c.

1148. simplex L. 4 c.

1149. Trollius europaeus $\mathrm{L} .2 \mathrm{c}$.

1149. " pumilus D. Don. 4

Resedaceae D. C.

1150. Reseda alba $L . \odot, \quad$ c c. 29 
1151. Reseda glauca $L . \odot$ c.

$\begin{array}{lll}1152 . & \text { inodora } R c h b . \odot, 4 \text { c. } \\ 1153 . & \text { lutea } L . \odot, 4 \text { c. } \\ 1154 . & \text { luteola } L . \odot \text { c. } \\ 1155 . \quad " & \text { mediterranea } L . \odot \text { c. } \\ 1156 . & \text { odorata } L . \odot \text { c. } \\ 1157 . * \quad & \text { Phyteuma } L . \odot \text { c. }\end{array}$

\section{Rhamnaceae S. F. Gray.}

1158. Rhamnus cathartica $L$. 方 G. 1159. ” Frangula $L$. $₹ \mathrm{G}$.

\section{Rosaceae Juss.}

1160*. Acaena glabra J. Buch. 4 1161. Agrimonia Eupatoria L. 4 c. 1162. " pilosa Led. 4 c. 29

1164. Amelanchier vulgaris Miönch $5 \mathrm{G}$.

1165. Aruncus silvester Kost. 4 c. 1166. Crataegus munogyna Jacq. 专 sanguinea $P$ c. G. 1168. Filipendula hexapetala Gilib. 4

1169. " ulmaria Max. 4

1170. Fragaria indica $A n d r .4$ c.

1171. " vesca $L .4$ c. 29

1172. " viridis Duch. 4 c.

1173. Geum aleppicum Jacq. 4 c.

1174. " molle Vis. et Panc. 4

1175. \# rivale $L .4$ c.

1176. Potentilla alpestris Hall. f. 4

argentea $L .4$ 1178. " $"$ arguta Pursch. 4 c. 1179. " bifurca $L .4$ c. 1180. " Buccoana Clem. 4 1181. desertorum Bgl. 4

1182. \# fruticosa $L .5$ c. 1183. " fulgens Wal. 4 c. 1184. " gelida C. A. M. 4
1185. Potentilla glandulosa Lindl. 4

1186. " intermedia $L .4$ c. 1187. " lcngipes Led. 4 c. 1188. ” Meyeri Boiss. 4 c. 1189. " nepalensis Hook. 4

1190. 1191. 29 1191. " pedata Willd. 4 c. 1192. " pimpinelloides L. 4

1193. \# procumbens Sibth. 4

1194. $\quad$ pulcherrima Lehm.

1195. 4 c.

1196. " " rupestris L. 4 c. 1197.* " Tormentilla Shrank. 1198. Poterium sanguisorba $L . \frac{1}{4}_{28}$ c.

1199. Prunus Padus $L$. $\hbar$ c.

1200. " spinosa $L$. $\frac{\hbar}{\text { c c. }}$ 1201. Rosa cinnamomea $L$. $\hbar$ c. 1202. Rubus caesius $L$. $\hbar$ c. 1203.* " leucodermis Dougl. $\hbar$

1204.* \# saxatilis L. 4 c. 1205. Sanguisorba officinalis $L$. 4 c. 1206. " muricata Spach. $\odot$ 1207. , tenuifolia Fisch. 4 c. 1208. Spiraea media $S c h m$. $\hbar$ c. 1209. " salicifolia $L$. $\frac{\hbar}{2}$ c. $1210 \quad$ sorbifolia $L$. म G.

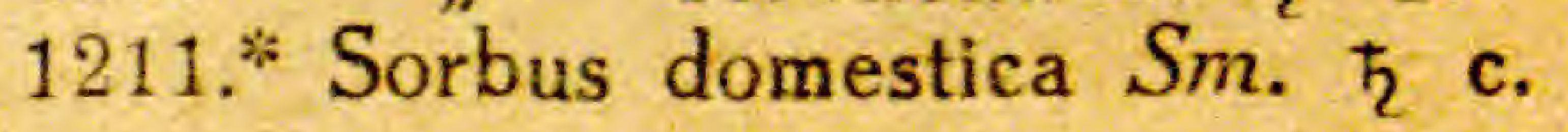

\section{Rubiaceae Juss.}

1212. Asperula Aparine Schott. 4 1213. $\quad$ arvensis L. $\odot$ c. 1214. Crucianella chlorostachys $F$. et 1215. Galium mollugo $L .4$ c.

1216. $\quad \because \quad$ parisiense $L$. $\odot$ c. 1217. " $\quad$ Saccharatum All. $\odot$ 1218." c. $1218 *^{*}$ " tricornae With. ( 1219. " uliginosum $L \cdot 4$ c. 
1220. Galium verum $L .4$ c. 1221. Phuopsis stylosa B. et Hook. 4 c. 29 1222. Rubia tinctorum L. 4 c. 29 1223. Scherardia arvensis $L$. $\odot$ c.

\section{Rutaceae Juss.}

1224.* Dictamnus Fraxinella Pers. 4 1225. Ruta graveolens $L$. \& c c.

\section{Saxifragaceae Dumort.}

1226. Astilbe chinensis Fr. et Sav. 4 c. 29 1227.* Chrysosplenium alternifolium $L$. 1228. Heuchera americana $L .24$ c. 1229. sanguinea Englm. 4

1230. Ribes alpinus $L_{0} \frac{\hbar}{2}$ c.

1231. Saxifraga caespitosa $I .4$ c. 1232.* " rotundifolia $L .4$ c. 1233. " tenella Wuff. 4 c.

1234. Tellima grandiflora $R . \quad B r .29$

\section{Scrophulariaceae Lindl.}

1235. Alonsoa Warscewiczil Reg. $\odot$ 1236. Antirrhinum majus $L$. $\odot$ c. $1237 . \quad$ Orontium L. $\odot$ c. 1238. Calceolaria pinnata $t$. $\odot$ c. 1239. „ scabiosifolia Sims. 1240. Chaenosto c. 29 1240. Chaenostoma antirrhinoides $L$. 1241. foetidum Benth.

1242. Chelone obliqua L. 4 c. 1243.* Collinsia bicolor Benth. () c. 1244.* " grandiflora Lge. $\odot$

1245. Cymbalaria muralis Baumg. 4 1246. Digitalis ambigua c. 124i Digitalis arr. 4 c " lutea L. 4 c. " media Roth. 4 c. 1249. " micranta Roth. 4 c.
1250. Digitalis nevadensis Kanze. 4 1251. $"$ purpurea L. 4 c. 1252. Elatinoides spuria Wettst. 1253. Hebenstreitia dentata $L$. $\odot$ c. 1254. Linsria bipartita $W$. $\odot$ c. 29 1255. „ Broussonnetii Chav.

1256. " canadensis Dumort. $\odot$ 1257. „ eapraria De Not. 4 1258. dalmatica Mill c. 1259. " genistaefolia Mill. 4 1260. " japonica Miquel c.

1261. " littoralis W. $\odot$ c. 1262. " marocana Hook. c. 1263.29 " minor Desf. $\odot$ c. 1264. " origanifolia D. C. 4 1265. " purpurea Mill. $\odot$ c. 1266.* " reflexa Desf. var. au120 reopurpurea $\odot$ c. 28 1267.* $~ \quad$ sapphirina Hoffm. et $1268, \quad$ Link. $\odot$ c. 28 1268. " spartea $H$. et Lk. $\odot$ $1269 . \quad$ c. 1270. " supina Desf. $\odot$ c. 1271. " tryphylla Mill. $\odot$ c. 29 1272. " $\quad$ tristis Mill. $\odot$ c. 1273. " vulgaris Mill. \& c. 1274. Mimulus alpinus Piper. 4 c. 1275. " cardinalis Dougl. 4 c. $1276 . \quad$ cupreus Regel 29 $1277 . \quad$ tigrinus 4 c. 1278. " moschatus Dougl. 4 1279 $\quad$ c. 29 1280. Nemesia strumosa Benth. $\odot$ c. 1281. Nycterinia Capens's Benth. $\odot$ 1282. Pentemon c. 1283. 
1284. Pentstemon glaber Fisch. 4 c. 1285. Scrophularia lateriflora Trautv. 4 c.

1286. " nodosa Dumort.

peregrin $4 \mathrm{c}$.

1288. „ umbrosa Dumort. $4 \mathrm{c}$.

1289. Verbascum Blattaria L. $\odot$ c. 1290. $\quad$ nigrum $L$. $\odot$ c.

1291. " $\quad$ phlomoides L. $\odot$

1292. " pyramidatum $M$. $B$ speciosum Schrad.

1294. " thyrsiflorum Hook,

1295. Veronica Beccabunga L. 4 c.

1296. " Chamaedrys L. 4 c.

1297. " gentianoides Vahl. 4

c.

1298. ” longifolia L. 4 c.

1299. " persica Poir. $\odot$ c.

1300. " Ponae Gouan. 4 c.

1301. " seutellata i. 4 c.

1302. - serpyllifolia L. 4 c.

1303. " sívirica L. 4 c.

1304 " spuria L. 4 c.

1305. " Tournefortii Gmel. $\odot$

1306. Wulfenia carithiaca Jacq. $4 \mathrm{c}$.

1307. Zaluzianskya capensis Walp. $\odot$

1308.* $\quad$ selaginoides Walp.

$\odot$ c.

\section{Solanaceae Pers.}

1309. Atropa Belladonna L. 4 c.

1310.* ” caucasica Kreyer. 4 c.

1311. Datura Bertolonii Parl. ๑ c.

1312. " Leichhardtii F. Muell.

ค c. 29

1313. " metel L. $\odot$ c. 29

1314. stramonium L. $\odot$ c.

1315. Hyoscyamus canariensis Ker.-

1316.
1317. Nicandra physeloides Gaertn.

1318. Nicotiana affinis Moore. $\odot$ c.

1319. " cerinthoides Hornen.

1320. fragrans Hort $\odot$ c.

1321. " macrophylla Lehm.

1322. paniculata $L$. c.

1323. " $\quad$ rustica $L . \odot$ c.

1324.* Tabacum L. $\odot$ c. 29

1325. Petunia hybrida Hort. $\odot$ c.

1326. " violacea $L d l$. $\odot$ c.

1327.* Physalis angulata L. $\odot$ c.

1328. " Francheti Masters. 4

1329. Physochlaena orientalis G. Don. 4 c.

1330. Salpiglossis variabilis Hort. $\odot$

1331. Sarachia Jaltomata Schleicht.

1332. Schizanthus pinnatus $R$. et $P$.

1333. Solanum demissum c.

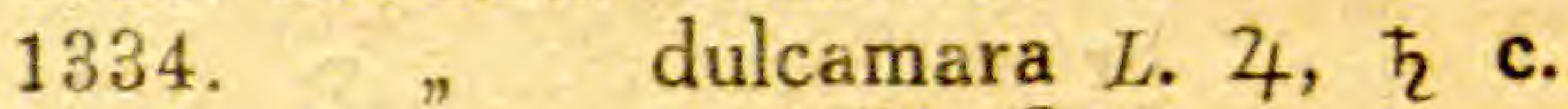

1335. „ gracille Otto $\odot$ c.

1336. " guineense Lam. $\odot$ c.

1337. " Hystrix $R . B r$. c.

1338. " miniatus Bernh. $\odot$ c.

1339. " nigrum L. v. villosum

1340. " $\quad$ v. vulgare $L$.

¿ c. 29

1341. \# Pseudocapsicum L. 万

1342. $\quad$ sisymbriifolium Lam. ๑ c. 29

\section{Tropaeolaceae Juss.}

1343. Tropaeolum Lobbianum Paxt.

1344. " majus $t$. $\odot$ c.

1345. " $\quad$ minus $L . \odot$ c.

\section{Thymeliaceae.}

1346.* Daphne mezereum $L . \hbar$ c.

\section{Umbelliferae Morison.}

1347. Aegopodium alpestre Ledb. 4 c. 
1348. Aegopodium Podagraria L. 4

1349. Aethusa Cynapium $L . \odot$ c.

1350. Ammi mayus L. $\odot$ c.

1351. Anethum graveolens $L$. $\odot$ c. 1352. Angelica archangelica $L$. $\odot$ c. 1353. Anthriscus silvestris Hoff. 4 c.

1354. Astrantia major L. 4 c.

1355. Bifora testiculata Boiss. $\odot$ c. 1356. Bupleurum rotundifolium $L$. $\odot$

1357. Carum Carvi $L . \odot$ c.

c.

1358.* Caucalis daucoides $L$. $\odot$ c. 29 1359. " latifolia $L$. $\odot$ c. 29 1360. Chaerophyllum aromaticum $L$.

$1361 . \quad 4$ c.

" bulbosum $L$.

1362. Conium maculatum $L . \odot$ c.

1363. Coriandrum melphatense Ten. et Guss. $\odot$ c.

1364. sativum $L$. $\odot$ c.

1365. Daucus Carota L. (spont.) $\odot$ c. 29

1366. " Gingidium L. $\odot$ c. 29

1367. " grandiflorus Scop. $\odot$

1368. Eryngium amethystinum L. 4

1369. campestre c.

1370. " planum L. 4 c.

1371. Heracleum barbatum Ledb. 4

1372. $\quad$ pubescens $M,{ }^{c}$.

1373. Levisticum officinale 4 c.

1374. Libanotis montana All. 4 c.

$1375 . \quad n$ sibirica Koch. 4 c.

1376. Ligusticum scoticum L. 4 c.

1377. Myrrhis odorata Scop. 4 c.

1378. Oenanthe crocata $L .4$ c.

1379.* " pimpinelloides L. 4

1380. Pastinaca sativa $L$. $\odot$ c.

1381. " teretiuscula Boiss. $\odot$

1382. Petroselinum sativum Hoff. 4

1383. Peucedanum cervariaefolium C. A. M. 4 c.

1384. Pimpinella saxifraga $L .4$ c. 1385. Sanicula europaea L. 4 c.
1386. Scandix Balansae Reut. $\odot$ c. 1387. " Pecten Veneris L. $\odot$ c. 1388. Seseli Hippomarathrum L. 4

1389. Torilis. 1389. Torilis Anthriscus Gmel. $\odot$ c. 1390. Zozimia absinthifolia D. C. 4 c.

\section{Urticaceae Endl.}

1391. Laportea gigas Wedd. 4 c. 1392.* Parietaria officinalis $L .4$ c. 1393.* $" \quad$ ramiflora Moench.

1394. Urtica cannabina $L .24$ c.

1395. " dioica $L .4$ c.

1396. " Dodartii L. $\odot$ c.

1397.* " laetevirens Max. 4 c.

1398.* " membranaceae Poir. $\odot$

1399. " pilulifera $L$. $\odot$ c.

Vacciniaceae Lindl.

1400. Vaccinium Myrtillus L. $\hbar$ G. 1401. - Oxycoccus $L . \hbar$ G. 1402. " uliginosum $L . \frac{\hbar}{2}$ G.

\section{Valerinaceae Dumort.}

1403.* Centranthus ruber D. C. 4 c. 1404. Fedia cornucopiae $D . C$. $\odot$ c. 1405. Valeriana palustris $K r .4$ c. 1406. " Sprygini Sm. 4 c. 1407. " Wolgensis Kez. 4

1408. Valerianella coronata $D . C . \odot$

1409. c.

1409. $\quad$ eriocarpa Krok. $\odot$

c. 29

$1411 . \quad$ c. 29

c.

\section{Verbenaceae J. St. Hil.}

1412. Verbena Aubletia Jacq. $\odot$ c. 1413. ${ }^{*}$ " canadensis Britt. (i) 1414.* $"$ erinoides Lam c. 1415.* " hastata L. 4 c. 1416. " hybrida Hort. $\odot$ c. 1417. " officinalis $L .4 \mathrm{c}$. 
1418.* Verbena tenera $S p r$. c.

1419. " urticifolia L. 4 c.

Violaceae D. C.

1420. Viola canina Rchb. 4 c.

1421. " cornuta L. 4 c.

1422. " elatior Fr. 4 c.

1423. " hirta $L .2$ c. 29

1424. " lutea Vill. 4 c.

1425. " mirabilis L. 4 c.

1426. " odorata L. 4 c.
1427. Viola Rothomagensis Desf. $\odot$ 1428.* " sagittata Ait. 4 c. 1429. " $"$ tricolor Wittr. $\odot, \odot$ c.

\section{Zygophyllaceae R. Br.}

1430. Peganum Harmala $L . ~ 4$ c. 29

1431. Tribulus terrestris $L$. $\odot$ c. 1432. Zygophyllum Fabago L. 4 c. c.

Professor T. N. Godnev,

Horti praefectus.

V. L. Gorsky, Assistens.

Gorky, R. P. Alborutheniae (U. S. S. R.). 


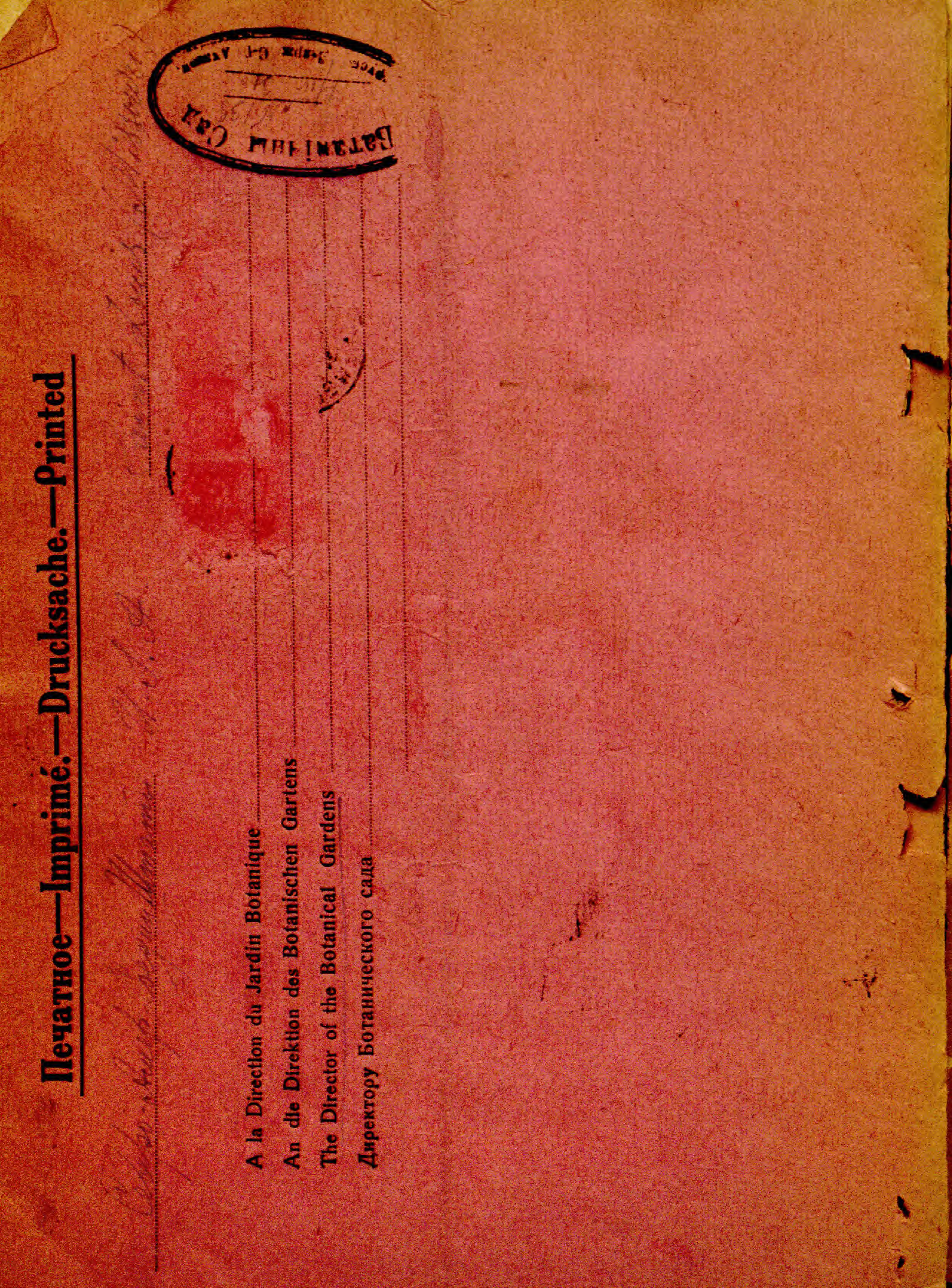
Jardin Bot nique. Gorki Belorussia U. S. S. R.

$$
1932^{\swarrow}
$$

Дадатак да пераліку насеньня 1931 году Батанічнага Саду ў Горках

Additio ad Delectum seminum anno 1931 r. Hortus Botanicus in urbe Gorky Bielorussia (U. S. S. R.).

Дополнение к перечню семян I93I года Ботанического Сада в Горках (Белоруссия) 


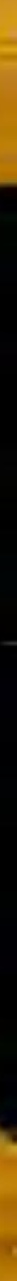

Упаўгалоўлітбел № 119. Заказ № 41-500.

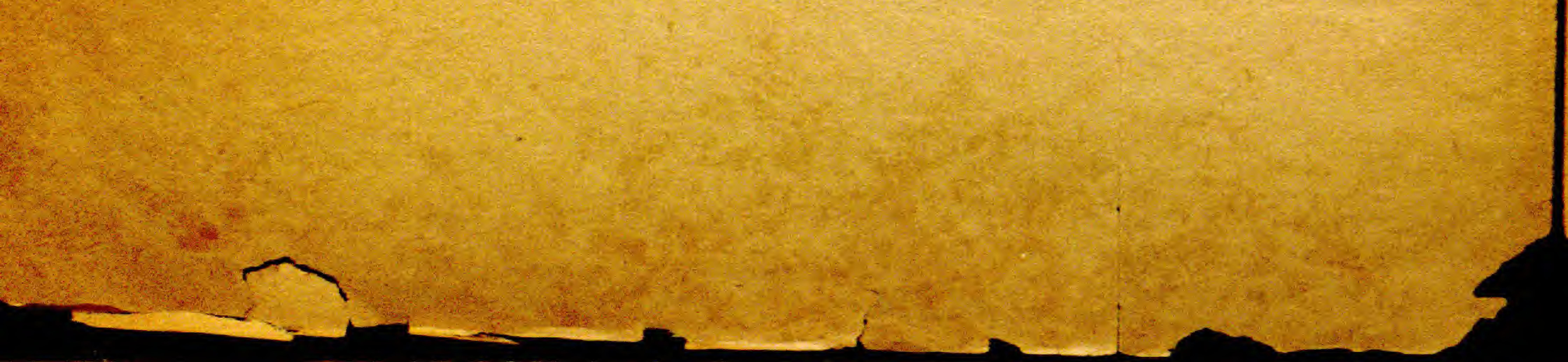


1433.* Abutilon indicum Sweet. 4

1434. Acer Ginnale Max. 5 c.

1435. " Negundo L. $\hbar$ c.

c.

1437.* Agrostis maritima Law. $\odot$ c. 1438.* , nebulosa $B$. et $R$.

1439. Alchemilla vulgaris $L, 4 \mathrm{c}$.

1440. Allium carinatum L. 4 c.

1441. "Cepa L. var. agregatum

1442. " oleraceum L. 4 c.

1443. " senescens $L .4$ c.

1444. " tríquetrum L. 4 c.

1445. " vineale $L .4$ c.

1446. Althaea armeniaca Tenore. c

447. " cannabina $L$ 4 c.

1448.* Anchusa capensis Thumb.

14.49.* Anemone alpina $L .4$ c. c.

1450. Angelica pachycarpa Lng.
1436. Aesculus Hippocastanum L. $\hbar$

1467. Campanula cerifera Hort. c. 1468. $\quad$ Pallasiana $R$. et. $S$. 1469.* 4 c. c. 1470.* Carex remota L. 4 c.

1471. Centaurea alpina $L$. c.

1472.* Cephalaria alpina R.S. 4 c. 1473. Cerinthe alpina Kit. $\odot$ c. 1474.* $\quad$ " aspera Roth. $\odot$ c. 1475. Chenopodium Quinoa Willd.

1476* Chondrilla juncea $L$. $\odot$ c. 1477. Cicuta virosa $L . ~ 4$ G.

1478. Cotoneaster lucida Schlecht. $\hbar$

1479, " multiflora Bunge.

1480. Crataegus melanocarpa $\stackrel{\hbar}{h}$. $B$.

181. Crepis

1481. Crepis aurea Cass. 4 c.

1482. " rubra $L$. $\odot$ c.

1483. Cydonia japonica Pers. 5 c.

1484. Cyperus rotundus L. c.

c.

1485. Cytisus nigricans $t$. $2 \mathrm{c}$.

1486. Dahlia variabilis Desf. 4 c.

1487. Dianthus Carthusianorum $L$.

1452. Aquilegia hispanica Borbas. 4 c.

1453. " jucunda Fisch. 4 c.

1454. Aralia calyfornica $S$. Wats, c.

1455. Arctotis calendulacea Willd.

$\odot$ c.

1456.* Armeria maritima Willd. 4

1457. Arnica foliosa Nutt. 4 c.

1458. " sachalinensis A. Gray. $4 \mathrm{c}$.

1459. Artemisia Cina Berg. c.

1460. Aubrietia deltoidea D. C. 4

1461.* Avena nuda $L$. $\odot$ c.

c.

1462. " pubescens Huds. 4

1463. Bellis mexicana A. Gray. c.

1464.* Berberis heteropoda Schrenk.

ई c.

1465. Beta maritima $L$. $\odot$ c. var. atrorubens 4

1488. " caryophyllus L. 4

1489. " grandiflorus Poir.

1490. Dioscorea caucasica Lipsky. 4

149 I. Erigeron boreale Vierh. c.

1492. Erodium moschatum $L \odot$ c.

1493, Eryngium giganteum Bieb. $\odot$

1494. " serbicum Panc. 4

1495.* Eupatorium aromaticum $L$. c. 4

1496.* Euphorbia glareosa $M . B$. c.

1497. $\quad$ procera $M . B$. c. i $^{\text {c. }}$

1498. Fagopyrum sagittatum Golib.

1466. Biscutella auriculata $L$. $\odot$ c. 
1499.* Festuca delicatula Lag. $\odot$ c. 1500. Foeniculum officinale All. 4

1501. Fragaria virginiana Duchesne. 4 c.

1502. Fraxinus americana $L$. $\hbar$ c. 1503. Geranium Endressii J. Gay. 4 1504.* , sibiricum L. 4 c. 1505. Geum japonicum Thunb, 4 c. 1506. Glycine Soja $S$. et $Z$. sem. alba

1507. " " $\quad$. et $Z$ nigra $\odot$ sem. 1508. Guizotia abyssinica Cass. $\odot$ c. 1509.* Gypsophila repens L. 4 c. $1510^{*}$. Hedisarum alpinum $L$. v. japonicum $24 \mathrm{c}$.

1511. Helenium nudiflorum Nutt. c. 1512. Helianthella quinquenervis $A$. Gray. c.

1513. Hesperis tristis $L$. $\odot$ c.

1514. Heuchera cylindrica Dougl. 4

1515. Hibiscus gossypinus Thumb. $2 \mathrm{c}$. 1516* Hieracium aurantiacum L. 2

1517. $\quad$ boreale $F_{r} .4 \mathrm{c}$. 1518.* $n$ echioides Lamnit. 1519. Hordeum hexastichum $L^{4}$. c. 1520* Humulus japonicus $S$. et. $Z$.

1521. Inula bifrons $L .2$ c. ( ) 1522.* " germanica $L .4$ c. 1523.* Iris kaempferi Siebold. 4 c. 1524. Jurinea alata Cass. $\odot$

1525. Lactuca altissima $M . B$. $\odot$ c. 1526. " sagittata $W$. et Kit.

1527. „ saligna $L . \odot$ c. - c. 1528. Lathyrus aureus Taubert. 4 1529. " pratensis $L .4$ c. 1530.* " tuberosus L. 4 c. 1531. Lavatera cretica $L$. $\odot$ c. 1532. Lonicera chrysantha Turcz. $\hbar$ 1533. $n$ xylosteum $L .5$ c. 1534.* Lupinus Cruikshankii Hook. $\odot$ c.
1535. Lupinus Douglasii Agardh. 4 c. 1536. . nanus Dougl. $\odot$ c. 1537. Lychnis coronaris Desr. $\odot$ c. 1538.* Madia capitata T. et Gray.

1539* dissitiflora Nutt $\odot$ c. 1540.* Malva cepensis $L$. $\odot$ c. 1541. " sylvestris $L .4$ c. 1542. Melissa alpina Benth. 4 c. 1543.* Mentha serrata Perard. 4

1544. $\rightarrow \quad$ suavis Guss. 4 c. 1545.* Morina longifolia Wall. 4 c. 1546. Mulgedium Plumieri $D . C .4$

1547.* Nuphar luteum Sib. et Sm. 4 1548. Papaver aurantiacum 1548. Papaver aurantiacum Loisef. 4 1549. Pennisetum villosum $R . B r$. c. 1550. Pentstemon campanulatus $W$.

1551. „ Gordonii Hook 1552. " Hailli A. Gray 15.53 9) ovatus Doug. 4

1554. Petunia axillaris Britt ๑ c. 1555. Peucedanum sativum Benth, et Hook. c.

1556. Pimpinella aromatica Bieb. 4 1557.* Platicadon Mariesii Hort. 4 1558. Polemonium gracile Willd. 4 1559** Potamogeton crispus L. 4 $1560 . \quad$ lucens $L$. G " natans $L .4$ G. 1562. Quercus pedanculata Ehrh. G.

1563. Ranunculus grandiflorus $L .4$ 1564. Rosa canina $L$. $\zeta$ c. 1565. " rugosa Thunb. 5 c. 1566. Rumex stenophyllus Ledeb. 4 c. 
1567. Salvia austriaca Jacq. 4 c.

1568. " japonica Thumb. $4 \mathrm{c}$. 1569. " lyrata $L .2$ c.

1570. Sumbucus canadensis $L . t \hbar c$. 1571. nigra $L$. $\hbar$.

1572.* Scrophularia heterophylla $W$.

c.

1573. Scutellaria macrantha Fisch. e. 1574. Sedum glaucum W. et K. 4 c. 1575.* Sempervivum annuum C. Sm. (.).

1576. Senecio Fuchsil Gmel. 4 c. 1577. Setaria italica Beaue

1578. Sidalcea candida A. Groy, 24

c.

1579. Silene japonica Rohrb. z c. 1580.* Solanum Handersoni Hort. $\frac{1}{2}$

1581. Sparganium simplex this ?

$y_{1}$

G.

Professor T. N. GODNEV,

Horti praefectus.
1582. Spiraea opulifolia $L . \hbar$ c.

1583. " ulmifolia Scop. 5 c. 1584.* Stachys alpina $L$. c.

1585.* " palustris L. 4 c.

1586.* Thalyctrum Delavayi Franch.

1587.* $\quad$ Fendleri Engelm.

1588. Trifolium hybridum $L$. v. elegans Garcke. 4

1589. Trigonella Balansae $B$. et $R$.

$\odot \mathbf{c}$.

1591. Vain coerulea Ser. $\odot$ c.

1591. Vaierianella olitoria Pallich. $\odot$

1592. Ventenata dubia c. c. 1593. Verbascum gnaphalodes $M$. $B$. 1594* Wulfenia corinthiaca Jacq. 4 c.

\section{L. GORSKY.} Assistens.

Gorky, Bielorussie, U. S. S. R. 


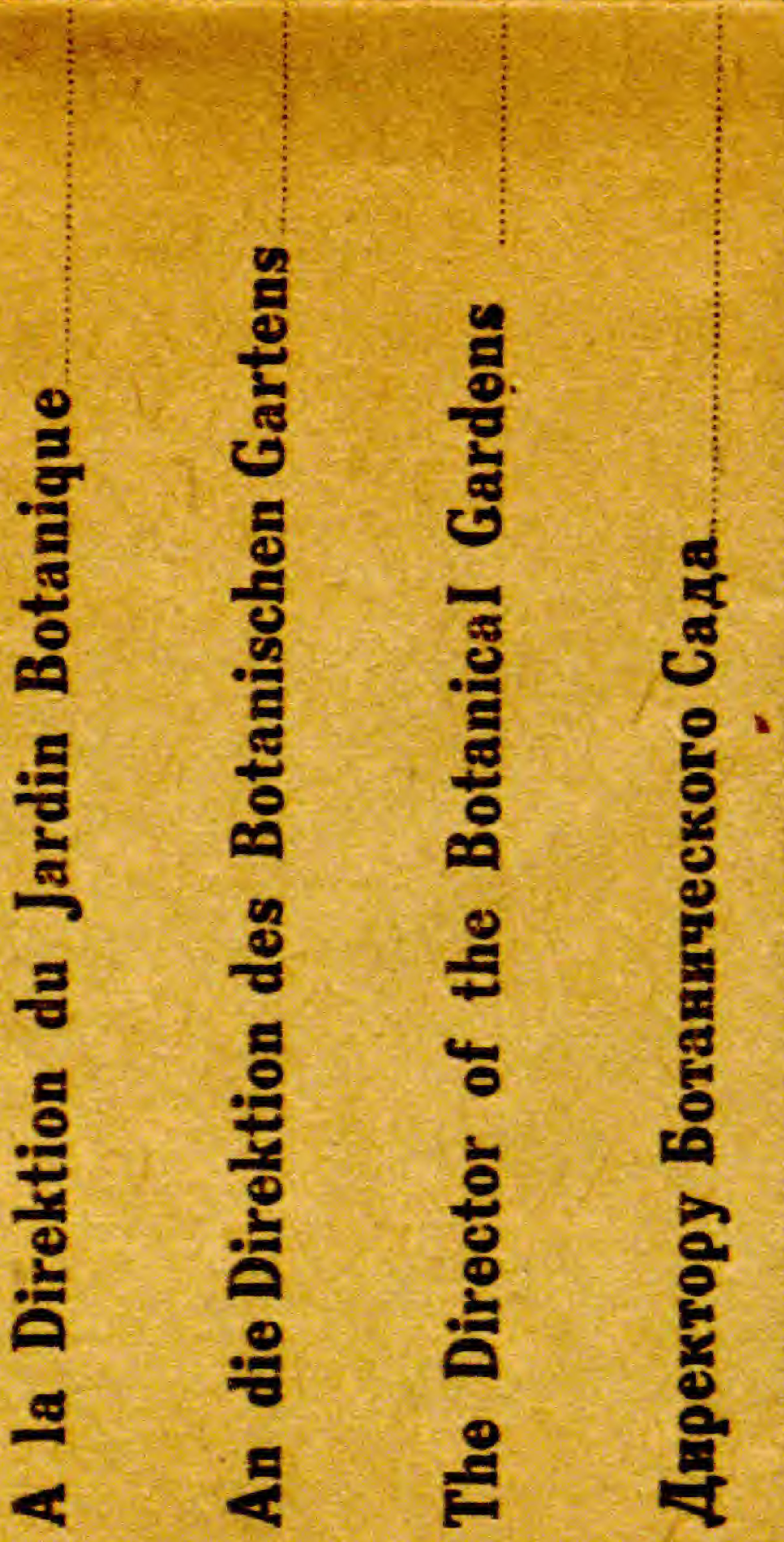


Jardin Botanique de I'lnstitut Agronomique Gorky. Belorussia USSR.

$$
1935
$$

\section{ПЕРАЛІК НАСЕННЯ,}

ЯКОЕ БОТАНІЧНЫ САД У ГОРКАХ ПРАПАНУЕ ДА АБМЕНУ.

\section{DELECTUS REMINUM,}

Anno MCMXXXIV (partim ac collectorum, quвe Hortus Bоны

\section{ПЕРЕЧЕНЬ СЕМЯН,}

\section{ПРЕДМАГАЕМЫХ В ОБШЕН БОТАНИЧЕСКИМ САДОМ В ГОРКАХ}




\section{A b breviationes:}

Numerus $(32,33)$, nomina plantarum, sequens annum, quo semina lecta sunt, significat; semina sine numerus in anno 1934 collecta sunt.

\section{Скараяяниі і ўмоўндя звакі |Сокрамения и условные обезва- \\ Лічбы $(32,33)$, якія надрукованы}

Цифры $(32,33)$, помещенные за гады збору насення; насенне без лінбаў сабрана ў 1934 годзе. сбора семян; семена без чисел собраны в 1934 году. 



\section{Pteridophyta}

1 Adiantum Capillus Veneris $L$.

2 " fulvum Raoul. 432

3 . gracillimum Moore. 432

4 Aspidium cristatum $S w .4$

5 "Filix-mas $S w .4$

6 " spinulosum $S w .4$

7 "thelypteris $\mathrm{Sw} .4$

8 Asplenium dimorphum Knze. 4

9 "Felix femina Bernh. 4

10 Cyrtomium falcatum Prsl. 432

11 Cystopteris bulbifera Bernh, 433
12 Nephrodium molle Schott. 432

13 Phegopteris Dryopteris Fèe 433

14 Polypodium Phegopteris $L .4$

15 Pteris cretica L. 432

16 " longifolia $L .432$

17 " serrulata $L .432$

18 " $\quad$ v. cristata 432

19 " $"$ v. tenuifolia 432

20 " $\quad$ v. variegata 432

21 Struthiopteris germanica Willd. 4

\section{G y mospermae}

22 Picea excelsa Link.

| 23 Pinus silvestris $L$.

Monocotyledonea e.

Araceae Juss.

24 Calla palustris I. 432

44 Alopecurus fulvus $S m$.

$45 \quad$ " geniculatus $L$. $\odot$

$47 \quad$ " pratensis L. 4

" ventricosus Pers. 4

Andronogon Ischaemum L. 2, 32

49 Anthoxanthum gracile $B r . \odot$

50 odoratum L. 4

51 Apera spica venti $P, B$. $\odot 33$

52 Aristida adscensionis $L$. $\odot 4$

53 Arrhenatherum elatius $M$. et $K$.

26 Carex hordeistichos Vill. 433

27 " ligerica Gay. 433

28 " Pseudocyperus $L .432$

29 " rostrata Stokes. 433

30 " vesicaria $L .4$

31 " vulpina $L .432$

32 Scirpus silvaticus $L .2$

Gramineae Juss.

33 Agropyrum Buonapartis Dur. et Schinz $\odot$

34

35

36

37

38

39

40

41

42

43 caninum $P . B$.
cristatum 4
Bess. 4 elongatum $P . B .4$ repens $P$. B. 4 tenerum Vasey. 4

Agrostis alba L. 4

$$
\text { canina } L .4
$$

maritima Law. $\odot$

vulgarls With. 4

Aira elegans Willd. $\odot$
54

55 Arthraxon ciliaris P. B. $\odot$

56 Asperella hystrix Humb. 4

57 Avellinia Michelii Parl. 4

58 Avena barbata Brot. $\odot$

19 " flavescens $L .4$

60 - planiculmis Schrad. 4

61 " pubescens Huds. 4

62 Beckmannia baicalensis Reg. 4

63 " eruciformis Host. 4

64 Briza media $L .4$

65 " minor $L .4$

66 Brizopyrum siculum Link. $\odot$

67 Bromus arvensis $L$. $\odot 33$

68 " ciliatus $L .4$

$69 "$ commutatus Schrad. $\odot$

70 " hordeaceus $L . \odot \odot$

71 " inermis Leyss. 4

$72 "$ secalinus $L, \odot \odot 33$ 
73 Bromus tectorum $L$. $\odot$ 74 " uniloides $H . B . K . \odot$

75 Calamagrostis Epígeios Roth. 4 76 Catapodium loliaceum Linq. $\odot$

77 Certotheca Iappacea Desv. $\odot$

78 Chaeturus faseiculatus Link. $\odot$

79 Chloris barbata $\mathrm{Sw}$. $\odot$

80 " meccana Host. $\odot$

81 Chrysurus cynosiroides Pers. $\odot$

82 Cinna glomerata L. 4

83 mexicana Beauv 4

84 Coix lacryma Jobi $L$. $\odot$

85 Ctenopsis pectinella $D$. Not. $\odot$

86 Cynosurus cristatus $L .4$

87 Dactylis glomerata L. 4

88 Deschampsia caespitosa $P$. $B$.

89 Echinaria capitata Desf. $\odot{ }^{4}$

90 Ehrharta panicea $\mathrm{Sm}$. $\odot 33$

91 Eleusine Coracana Gürtn. ()

92 " indica Grtn. $\odot$

937 Toccusa Fresen. $\odot$

94 Elvmus araliensis $\mathrm{RaJ}_{95} 2$

$96 \quad$ " $\quad$ canadensis $L .4$

97 " dahuricus Turcz.

98 " excelsus Turcz. 4

99 " junceus Fisch. 4

T09 " sibiricus $L .4$

101 " virginicus L. 4

102 Eragrostis megastachya Link $\odot$

103 " minor Host. $\odot$

104 " pilosa P. B. $\odot$

105 Festuca alpina Suter. 4

106 - arundinacea Schr. 4

107 , Beckeri Hackel. 4

108 " ciliata Pers. 4

109 " cretacea Czern. 4

110 " gigantea Vill. 4

111 . Myuros L. 4

112 " ovina $L, 4_{4}$

113 " polesica Zapal. 4

$114 "$ pratensis Huds. 4

115 " rubra $L .4$

116 " sulcata Hackel. 4

117 " varia Haenke. 4

118 Gastridium atistrale $P$. B. $\odot$

119 Gaudinia fragilis Beauv. $\odot$

120 Haynaldia villosa Schut. $\odot$

121 Hilardia rigida Vosey. $\odot$

122 Hordeum jubatum L. 4 123 m murinum Huds. $\odot$ 124 Koeleria glauca D. C. 4 125 " gracilis Pers. 4 126 Lasiagrostis splendens Kunth. 4 127 Leptochloa mucronata $K t h$. 32

128 Lolium italicum $A$. $B r .4$

129 " perenne L. 4

130 " remotum Schrank. $\odot 33$

131 " temulentum $L . \odot 33$

132 Melica altissima $L .4$

133 " transsilvanica Schur. 4

134 Miscanthus sacchariflorus Hack.

135 Nardurus Poa Boiss. $\odot 33$

136 Nardus stricta L. 4

137 Panicum bulbosum H. B. K. 4

138 " frumentaceum Roxb. $\odot$

$139 \quad " \quad$ miliaceum $L$. $\odot$

$140 \quad " \quad$ sanguinale $L$. $\odot$

141 n teneriffae $R . B r .4$

142 Pcespalum dilatatum Poir. $\odot$

143 " elegans Hort.

145

146

147

148 Phleum pratense $L .4$

149 Poa abyssinica Jacq. 4

150 ” alpina $L .4$

151 " annua $L$. $\odot$

152 " badensis Haenke. 4

153

154

155

156

157

158

159

160

161 Pollinia imberbis Nees. $\odot$

162 Polypogon maritimus Willd. $\odot$

163 " monspeliensis Desf.

164 Puccinella retroflexa (Curt).

165 Schismus - Holmb.

165 Schismus marginatus $P . B . \odot$

166 Scleropoa rigida Griseb. $\odot$

167 Sesleria argentea Savi 4

168 Setaria germanica Beauv. 
169 Setaria italica Beauv. $\odot$

170 " panicea $(L)$ Schinz et Thell. $\odot$

$171 \%$ persica P. B. ()

172 Sorghum halepense $(L)$ Pers.

173 . vulgare Pers. $\odot$

174 Sporobolus tenacissimus Beauv.

175 Tricholaene rosea Nees. $\odot$

176 Trisetum argenteum Roemt. et Schul. 4

177 Triticum dicoccum Schrant. v. dicoccoides $\odot 4$

178 " durum Desf. durospermum $\odot$

$179 \# \quad$ nordeiforme $\odot$

180 " " $~ " ~ m e l a n o p u s ~ \odot$

$181 \quad$ " spelta $L . \odot$

182 " $"$ ventricosum Ces.

183 Ventenata dubia $F$. Schulz. $\odot$

I84 Weingaertneria canescens Bernh.

\section{4}

Iridaceae Juss.
201 Juncus compressus Jacq. 4

202 Luzula nivea D. C. 4

Liliaceae Scop.

203 Allium albidum Fich. 4

$204 "$ carinatum L. 4

$205 "$ canadense $\bar{L}$. 4

$206 \%$ caeruleum Pall 433

$207 "$ fistulosum L. 4

208 " L. v. vivipa. rum 4

flavum $L .4$ globosum Red. 4 lineare $L .432$

Moly L. 4

obliquam L. 433

odorum L. 4

oleraceum L. 4 oviflorum $L .4$

Porrum L. 4

rotundum L. 432

schoenoprasum $L .4$

senescens $L .4$ vineale $L .4$

222 Anthericum Liliago L. 4

223 Asparagus officinalis L. 4

224 Funkia lancifolia Spral. 4

225 Gagea lutea K. Gavl. 432

226 Hemerocalis flava $L .4$

227 Lilium candidum L. 4

228 , Martagon L. 432

229 " tigrinum Gawler 4

230 Muscari racemosum Mill. 4

231 Ornithogalum narbonense L. 4

194 " Pseudacorus L. 4

195 " setosa Pall. 4

196 " sibirica $L, 4$

197 " spuria L. 4

198 "Thunbergii E. Lundstr. 4

199 Sisyrinchium anceps Cav. 4

Juncaceae Vent.

200 Juncus bufonius $L$. $\odot 33$

232

" $\quad$ umbellatum $L$.

233 Polygonatum multiflorum All. 4

234 Smilacina stellata Desf. 4

235 Zygadenus sibiricus A. Gray. 4 33

236 Veratrum album $L$. v. Lobelia. num Bernh. 4

\section{Dicotyledonea e}

Aceraceae Neck.

237 Acer ginnale Max.

238 239
240 Acer tataricum L.

A izoaceae

241 Tetragonia expansa Murr. $\odot$ 
A marantaceae Juss.

242 Achyranthes argentea Lam. $\odot$ 243 Amarantus albus $L . \odot 32$

244 * caudatas L. $\odot$

245 " elegantissimus Hort.

246 " 32

$248 \quad$ guineus Moq. $\odot$

n purpurascens Otto $\odot$

$249 \quad n \quad$ retroflexus $L . \odot$

$250 \quad$ - spinosus $L . \odot 32$

251 xanthostachys Willd

252 Froelichia floridana (Nutt) $\stackrel{32}{M \circ q}$.

$\odot$

A pocynaceae R. B r.

253 Vinca minor $L .4$ c.

Arali a ceae Juss.

254 Aralia californica S. Wats. $\hbar$

Aristolochiaceae Juss.

255 Asarum europaeum L. 4

L Asclepiadaceae Lindl.

256 Asclepias Cornuti Decsn. 4

$257 \quad$ phytolaccoides Pursch.

$258 \quad$ verticillata L. $4^{4}$

259 Cynanchum medium $K$. Schum.

$260, \quad$ vincetoxicum $R . B r$. 433

Ba ls a minaceae S. F.

261 Impatiens Balsamina $L$. $\odot$

262 " glanduligera Royle. $\odot$

263 " scabrida D. C. $\odot$

Bignoniaceae

264 Incarvillea variabilis Batal. hybrida 4
Borraginaceac S. F.

265 Borrago officinalis $L$. $\odot$

266 Cerinthe aspera Roth. $\odot$

267 " minor $L$. $\odot$

268 Cynoglossum officinale $L, 433$

269 Echium vulgare $L$. $\odot$

270 Heliotropium indicum $L . \odot 32$

271 Lithospermum officinale $L .4$

272 Myosotis alpestris Koch. $\odot$

273 Omphalodes linifolia Moench. $\odot$

274 Symphytum asperum Lep. 4

275

$$
n \quad \text { officinale } L .4
$$

Campanulaceae Juss.

276 Campanula abietina Gris. c. 33

277 alliariaefolia Willd.

$278 \quad$ carpathica Jack 4

$279 \quad$ erinus L. Jack. 4

280 " glomerata $\stackrel{\odot}{\text { " }}$. aca-

$281 \quad$ grandis $F$. ut 4.33 .4

283 , Medium L. $\odot$

284 " rapunculoides $L .49$

285 " $\quad$ rotundifolia $L .4$

286 " $\quad$ v. Hostii

$287 \pi$ sarmantica Ker.G $\stackrel{4}{4}$ awl.

288 sarmentosa Hochst. 33

289 Codonopsis ussuriensis Hemsl.

$290 \quad$ viridiflora Max. 4

291 Lobelia inflata $L$. $\odot$

292 " syphilitica L. 432

293 Phyteuma canescens W. et. Kit.

294 Platycodon grandiflorum $D .{ }^{\frac{\pi}{4}}$.

295 Specularia Speculum D. C. $\stackrel{4}{\circ}$

296 Symphyandra Hoffmannil Pant. $(-)$

297 Wahlenbergia lobelioides_ $L k$. $\odot$

Cannabacea_e Endl.

298 Cannabis gigantea Vass. $\odot$ $299 \quad n \quad$ sativa $L . \odot$

300 Humulus japonicus $S$, et $Z$. $\odot$

$301 \quad$ Lupulus L. 4 
C a p parid a c e a e

302 Cleome gigantea $L$. $\odot$ 303 " graveolens Rafin. $\odot$

Caprifoliaceae Vent.

304 Lonicera iberica Bieb. $\hbar 33$

$305 "$ orientalis Lam. $\frac{2}{2}$

$306 "$ tatarica $L .5$

$307 \quad$ xylosteum $L$. $\hbar$

308 Sambucus ebulus $L .4$

309 nigra $L . \hbar$

$310 \quad$ racemosa $L . \hbar$

311 Symphoricarpus racemosus $M c h x$ 312 Viburnum Lantana $L .5$

$313 \quad$ Opulus $L$. $\hbar$

Caryophyllaceaé Juss.

314 Agrostemmá Githago L. $\odot$

$315 \quad n$ nicaeensis Pers. $\odot$

316 Anychia polygonoides Rafin. $\odot$

317 Arenaria graminifolia Schrad. 2

318 serpyllifolia $L$. $\odot$

319 Cerastium Biebersteini D. C. 4

320

321 Diathus arenarius L. 4

322 " barbatus L. 4

323 " Carthusianorum $L$. v.

$324 \quad$ collinus W. et Kit. 4

325 " deltoides L. 433

326 " grandiflorus Poir. 432

327

328

329

330

331

332

333

$334 \quad$ " Steveni Fisch. 4

- plumarius L. 4

" rupicolus Biv. 4

" trifasciculatus Kit. 4

Drymaria cordata Willd. $\odot$

Gypsophila elegans Bieb. $\odot$

335. Holosteum umbellatum $L . \odot \odot$

336 Lychnis chalcedonica L. 4 33

337 " Coronaria Lmk. 433 338 Malachium aquaticum $R c h b .4$ 339 Melandrium album Garcke. 4

340 Sagina procumbens $L .4$

341 Saponaria cerastoides Fisch. $\odot$ 342 343 officinalis $L .4$,
344 Silene Armeria $L$. $\odot$

345 " gallica $L . \odot$ c.

346 ." japonica Rohrb. 4

$347 \rightarrow$ tatarica Pers. 433

348 " venosa Aschers. 433

349 Spergula maxima Weihe. $\odot$

$350 \quad$ sativa Boenn. $\odot$

351 " vulgaris Boenn. ๑ 33

352 Spergullaria media Boiss. $\odot 32$

$353 \quad n \quad$ rubra Pers. $\odot \odot 33$

354 Stellaria graminea $L .4$

355 Tunica Saxifraga Scop. 4

356 Vaccaria pyramidata Medik. $\odot$

357 Viscaria viscosa Aschers. 4

Celastraceae Lindl.

358 Evonymus europaea $L . \bar{z}$

359 " latifolia Scop. $\frac{1}{2}$

360 verrucora Scop. 5

Chenopodiaceae Less.

रिण1 Atriplex hastatumil $t$. Tि 33

$362 "$ hortense $L$. $\odot$

363 Axyris amarantoides $L$. $\odot 33$

364 Chenodopium album $L$. $\odot 32$

$365 \quad \pi \quad$ bonus Henricus

$366 \quad$ Botrys L. $\odot 32^{4}$

$367 \quad n \quad$ capitatum Aschers.

368 $\quad$ chilense Schrad.

$369 \quad \odot 32$

$370 \quad " \quad$ foetidum Schrad.

$371 \quad$ foliosum $\odot 32$

$\odot 32$

$372 \quad$ opulifolium Schrad.

373 Obione sibirica Fisch. $\odot$

374 Salsola Soda L. $\odot 32$

375 Spinacea tetrandra Stev. $\odot 33$

$$
\text { Cistaceae Dun. }
$$

376 Helianthemum Chamaecistus

Mill. 432

377

378 guttatum Mill $\odot$

ledifolium Mill.@ 
Compositae Adans

379 Achillea filipendulina Lam. 432 380 " Millefolium L. 4

381 " odorata $L: 4$

382 " Ptarmica L. 432

383 Anacyclus officinarum Hayne. $\odot$

384 Acroclinum roseum Hook. $\odot$

385 Ageratum conyzoides $L$. $\odot$

386 Ammobium alatum $R$. Br. $\odot$

387 Anthemis altissima $L$. $\odot$

388 " arvensis $L$. $\odot 33$

$389 \quad$ nobilis $L$. $\odot 32$

390 Antennaria tomentosa Hort. 4

391 Arctotis calendulaceae Willd. $\odot$ 392 , stoechadifolia Berg. $\odot$ 393 Arnica Chamissonis Less. 4 394 Artemisia Absinthium L. 4 $395 \quad n \quad$ vulgaris $L .4$ 396 Aster alpinus L. 4

397 " salicifolius Scholl. 4 398 " Tradescanti L. 4

399 Baeria coronaria A. Gray. (๑) 33 400 Bellis mexicana A. Gray. 401 Bidens leucantha Willd. () c.

402 Buphtalmum speciosum Schreb.

404 Calendula officinalis $L$. $\odot$

405 Calimeris incisa D. C. 33

406 Calliopsis bicolor Bosse. $\odot$

407 Callistephus chinensis Nees. $\odot$

408 Carlina vulgaris $L$. $\odot$

409 Carpesium cernuum L. 4

410 Carthamus lanatus $L$. $\odot$

411 Centaurea alpina $L .4$

$\begin{array}{lll}412 & n & \text { axillaris Willd. } 4 \\ 413 & n & \text { Cyanus L. } \odot 33 \\ 414 & " & \text { glastifolia } L .4 \\ 415 & \text { Jacea } L .433 \\ 416 & \text { nigra L. } 432 \\ 417 & \text { reflexa Lam. } 433\end{array}$

418 Chardinia heterophylla Cass. () 419 n xeranthemoides Desf.

420 Chrysanthemum coccineum Willd

421

422

4

423
424 Cichorium Inrybus $L .4$ 425 " pumilum Jacq. 33

426 Cladanthus arabicus Cass. $\odot$

427 Cnicus Benedictus $L$. $\odot 33$

428 Cosmea bipinnata Willd. $\odot$

429 Cosmos diversifolius Otto. $\odot$

430 Crepis alpina L. $\odot 32$

431 " aurea Cass. 432

432 " biennis $L . \odot$

433 " foetida $L$. $\odot$

434 " pulchra L. $\odot 32$

435 " rubra $L . \odot$

436 Crupina vulgaris Cass. $\odot$

437 Dahlia variabilis Desf. 4

438 " "Rosenelfe

439 " " Mirjam

440

441

442

443

444

445

446

447

448

\section{9}

450

451

$"$

Black Knigt

" Erlkönig

" Krymchilde

Krymchilde

Red Cross.

Weltfrieden

Hindenburg

"Zitionenvogel

Ir. Fried Fal-

4 อั 2

kenhagen

King Horold

Schneeberg

Marie Haupt-

Faschingsfee

man

453 Dimorphotheca pluvialis Moench.

454 Echinops globifer Janka. 4

$455 \quad$ Szowitzii Fisch. et Mey.๑)

456 Emilia sonchifolia $D . C$.

457 Erigeron coulteri Port. et Coult.

458 Euaptorium aromaticum L. ${ }_{4}^{4}$

459 " cannabinum L. 4

460 Felicia tenella Ness. $\odot$

461 Filago spathulata Presl. $\odot$

462 Gaillardia aristata Pursch. 4

463 Galinsoga parviflora Cav. ๑ 32

464 Gnaphalium uliginosum $L$. () $\mathbf{3 3}$

465 vira-vira Molina $\odot$

466 Grindelia squarrosa Dun. 433

467 Guizotia abyssinica Cass. ๑. c. 33

468 Helenium Hoopesii $A$. Gray. 4 c.

469 " nudiflorum Nutt. c.

470 Heliantella quinquenervis $A$.

Gray. 33 
471 Helichrysum arenarium Moench.

472 Hieracium alpinum $L .4$

$473 \quad \rightarrow \quad$ boreale $\mathrm{Fr} .4$

$474 \quad " \quad$ Pilosella $L .4$

$475 \quad$ " pratense Tausch. 4

$476 \quad$ " tridentatum Fries. 4

$477 \quad$ virosum Pall. 4

478 Hyoseris lucida $L$. $\odot$

479 Inula bifrons $L .432$

480 " germanica $L .433$

481 " Helenium $L .4$

482 " magnifica Lipsky.

483 Lactuca altissima $M$. $B$. $\odot$

$484 "$ perennis $L .433$

485 " sagittata W. et Kit.

486 " saligna $L$. $\odot$

$487 \quad$ " sativa $L . \odot$

488 Lampsana grandiflora M. B. 4

489 Lappa amplissima Bois. $\odot 33$

490 tomentosa Lam. $\odot$

491 Lostenia glabrata $L d b \odot$

492 Layia eleganns Torr. et Gray.
519 Sol dago altissima $L .2$ 520 macrophylla Pursh. 4 521 Sonchcus arvensis $L .4$ 522 Spilanthes oleracea $L$. $\odot$ 523 Stevia ovata $\operatorname{Lag} .432$ 524 " purpurea Pers. 4 525 Tagetes lucida Cav. 433

526 : patula $L . \odot 33$ 527 " signata Bartl. $\odot$ 528 Tanacetum vulgare $L .4$ 529 Taraxacum officinale Wigg. 4 530 Tragopogon brevirostris D. C. $\odot$ 531 Uropappus clevelandii Greene.

532 Urospermum picroldes $F . \stackrel{\odot}{W}$. Schmidt. $\odot$

533 Xanthium canadense Mill $\odot 33$

534 Ximenesia encelioides Cav. $\odot$

535 Zacintha verrucosa Gaertn. $\odot$

536 Zinia elegans Jacq. $\odot$

537 "Haageana Rgl. $\odot$

538 " multiflora L. $\odot 33$

$539 "$ verticillata Andrz. $\odot$

Convolvulaceae Vent.
494 Leontopodium alpinum Cass. 4

495 Madia capitata $T$. et Gray. $\odot$

496 - dissiflora Nutt. $\odot$

497 " sativa Mol. $\odot$

498 Matricaria chamomilla $L . \odot$

$499 \quad$ $\quad$ inodora $L . \odot \odot 33$

$500 \quad$ suaveolens Buch.

501 Mulgedium Plumieri D. C. ${ }_{4}^{33} 32$ 502 sibiricum Less. 4

503 Onopordon Acanthinum $L$. $\odot$

504 Palafoxia texacana $D$. C. $\odot 32$

505 Picris hieracioides $L$. $\odot-432$

506 Prenanthes purpnrea $L .4$

507 Pyrethrum Parthenium Sm. 4

508 roseum M. B. 433

509 Rhagadiolus edulis Gaertn. ()

510 Rudbeckia hirta $L$. $\odot \odot$

511 n laciniata L. 4

512 . $\quad$ purpurea L. 432

513 Scorzonera hispanica L. 4

514 Senecio Fuchsii Gmel. 4

515 - ligularia Hook. 4

516 Serratula coronata $L .433$

517 Silphyum integrifolium Mich. 4 518 perfoliatum L. 4
540 Convolvulus tricolor L. $\odot 32$

$541 \quad$ undulatus Cav. $\odot 33$

542 Evolvulus latifolius Ker. Gavol.

433

Cornaceae D. C.

543 Cornus sanguinea $L .5$

Crassulaceae D. C.

544 Sedum acre $L .433$

$545 \Rightarrow$ aizoon $L .4$

$546 \quad n \quad$ boloniense Loisl. 4

547 "Ewersii Ledb. 4

548 " hybridum $L .4$

$549 ; \quad$ pallidum $M . \stackrel{B}{B} . \odot$

$550 \quad$ n purpureum Link. 4

$551 \quad n \quad$ rupestre $L .4$

552 " spurium $M . B .4$

553 Sempervivum patens Griseb. et

554 Schenk. 4

555 ruthenicum Koch.

- soboliferum $\stackrel{4}{4}$ Sims.

4 
Cruciferae Juss.

556 Arabis albida Stev. 4

557 . hitsuta Scop. $\odot 4$

558 " rosea D.C. 33

559 " suecica Fries. $\odot$

560 Barbarea stricta Andrz..-

561 " vulgaris $R . B r$. $\odot$

562 Berteroa incana $D . C . \odot \odot 33$

563 Biscutella auriculata $L$. $\odot$

564 " raphanifolia Poir. $\odot$

565 Brassica juncea Czern. $\odot$

$566 \rightarrow$ napus $L$. v. oleifera Moench. $\odot$

567 „ nigra Koch. $\odot$

$568 "$ rapa $L$. V. oleifera Mo-

569 Bunias Erucago L. $\odot 32$

$570 \quad$ orientalis $L .4$

571 Calepina Corvini (All) Desv. $\odot$

572 Camelina Alyssum (Mill) Thell.

$573 \quad$ linicola Sch. et $S p$. 574 macrocarpa Vierzs. $\odot$
599 Sesquerella gracilis $S$. Wats.

600 Sinapis alba $L$. $\odot$

601 " apula Tenore. $\odot$ c.

602 " arvensis $L . \odot$

$603 \quad$ " dissecta Lag. $\odot$

604 Vogelia paniculata Hornem. $\odot$

Cucurbitaceae Holl.

605 Bryonia alba $L .4$

606 Cyclanthera explodens Naud. ()

607 pedata Schrad. $\odot$

608 Ecballium elaterium Rich. $\odot$

Dilleniaceae Sal.

609 Actinidia arguta Miq.

D i psac ace a D. C.

610 Callistemma brachiata Sibth. $\odot$

611 Cephalaria alpina $R$. S. 4

612 " tatarica Schrad. 4

613 Dipracus fullonum Mill.
576 Capsella bursa pastoris Moench.

577 Carrichera Vella $D$. C. $\odot$

578 Cheiranthus Allioni Hort. $\odot 32$

579 Draba scandinavica Liudblon. 4

580 Erysimum cheiranthoides $L$. $\odot$

581 cuspidatum D. C.

582 Eruca sativa Lam. $\odot$

583 Hesperis dinarica G. Beck. 4

$584 \quad$ fragrans Fisch. 4

585 " matronalis $L ., 4$

586 tristis $L .4$

587 Hutchinsia alpina $\mathrm{R} . \mathrm{Br} .4$

588 Iberis amara $L$. $\odot$

589 " pectinata Boiss. $\odot$

590 Isatis tinctoria $L$. (.) 33

591 Lepidium latifolium $L .4$

592 . ruderale $L . \odot$

593 " sativum $L$. $\odot$

594 Myagrum perfoliatum $L$. $\odot 32$

595 Notoceras canariensis $R$. Br.

596 Raphanus sativus $L$.

597

v. oleiferus

Mentzger. $\odot$

598 Senebiera Coronopus Poir. $\odot$
615 Knautia arversis Coult. 433

616 atrorubens Janka. 4

617 Morina longifolia Wall. $\overline{4}_{4}$

618 Scabiosa atropurpurea $L$. $\odot$

619 " graminifolia L. 4

$620 "$ lucida Vall. 4

Europhorbiaceae J. St. Hil.

621 Euphorbia Esula L. 4

622 , procera $M . B .4$

623 Ricinus communis $L$. v. viridis

624 Securinega ramiflora Moench. $\odot$

Gentianaceae Dumort.

625 Gentiana cruciáta $L .4$

626 - $\quad$ frigida Haenke. 4

627 , macrophylla Pall. 4

628 " Pneumonanthe L. 4

$629 \quad " \quad$ tibetica King. 4

$630 \quad n \quad$ Walujewii Rgl. et

$$
\text { Schmalh. } 4
$$

Geraniaceae J. St. Hil.

631 Erodium cicutarium L. Herit. $\odot$ 
632 Erodium gruinum Ait. ()

633 moschatum $L$

634 Geranium dissectum $L$.

635 n divaricatum Ehrh, 4

$636 \quad$ " palustre L. 4

637 " pratense L. 4

638 " pusillum L. $\odot$

639 " sanguineum L. 433

$640 \quad n \quad$ sibiricum L. 433
641 Globularia Willkommii Nyman. 433

Guttiferea Endl.

fit2 Hypericum Ascyron L. 4

fi43 " hirsutum L. 4

$644 \quad " \quad$ perforatum L. 4

$645 \quad$ " quadrangulum L. 433

Hydrophyllaceae
646 Nemophila maculata Benth. 9 647 Phacelia tanacetifolia Benth. $\odot$

Hippocastanaceae D. C. 648 Aesculus Hippocastanum L. $\hbar$

Labiatae Juss.

649 Agastache nepetoides (L.) O.K. 650 Ajuga reptans $L$. \& C.

i51 $n \quad$ L. V albiflora Tin. 2. 33

652 Ballota nigra L. 433

653 Betonica grandiflora Stev. 432

$654 \quad$ officinalis $L .4$

655 Dracocephalum austriacum L. 4

656 moldavica $L$. $\odot$

$657 \quad$ Ruyschiana L. 4

658 Elscholtzia cristata Willd. $\odot$

659 Hyssopus officinalis $L$. $\hbar$

660 Lamium maculatum $L .4$

661 Leonurus Cardiaca L. 4

662 Lophanthus rugosus $F$. et M. 4 663

scrophulariaefolius

Benth. 4

605 Lycopus exaltus L. 433

666 Marubium praecox Janka. 432

667 Melissa officinalis $L .7$

668 Mentha crispa $L .4$

$669 \quad \cdots \quad$ lo nzifolia Huds. 4

$670 \quad, \quad$ rotundifolia $L .4$

671 n viridis $L .24$

672 Monarda Bradburiana Beck. 4

673 Nepeta Cataria L. 4

674 . grandiflora M. B. 4

675, macrantha Fisch. 4

676 , nuda $L .4$

677 Origanum vulgare $L .4$

678 Plectranthus indicus Spring.

679 Phlomis tuberosa $L, 4$

680 Salvia austriaca Jacq. 4

681 "glutinosa $L .4$.

682 " hispanica $L_{=} \odot$

683 " japonica Thunb. 4

684 " pratensis $L .4$

$68.5 *$ Regla Cav. 433

$686 \sim$ tilifolia Vahl. ()

686 , verticillata $L .433$

-688 Satureia Clinopodium Caruel. 4

\section{(i)}

690

691

692

693

694

695

696
697
698

698

699 Teucrium chamaedrys L. 4

700 Thymus glaber Mill. 4

Leguminos a e Jutss.

701 Adesmia muricata D. C. 4

702 Anthyllis tetraphylla $L$. $\odot$

703 Astragalus alpinus L. 4

$704 \quad n \quad$ Cicer $L \cdot 4$

705

706

707

708

709

710

711

712
" galegiformis 2. 4

n glycyphyllus $L$.

n Onobrychis $L .4$

Atylosia barbata Baker. ()

Baptisia australis $R . B r .4$

Biserulla Pelecinus $L$. $\odot$

Caragana arborescens Lam. $\hbar$ frutescens $D . C . \frac{5}{2}$ 
713 Cicer pinnatifidum Jaub. of

714 Colutea cruenta Ait. 5

$\$ 15$ Coronilla varia L. 433

716 Cytisus ratisbonensis Schaeff. 5

717 Desmodium canadense $D$. C. T

718 Dolichos umbellatus Thunb.

619 Galega officinalis L. 4

720 Genista ovata $W$. ot $K$. 万

721 " sagittalis $L, 4$

$722 "$ tinctoria $L$. $\frac{\hbar}{2}$

723 Glycyrrhiza echinata $L .4$

724 " lepidota Pursch. 4

725 Glycine Soja S. et Z. .

726 Hippocrepis multisiliquosa $E$. C 33

727 Hymenocarpus circinnatus Saw.

728 - nummularius

729 Lathyrus annuus $L$. $\odot$

$730 \quad$ " articulatus $L$..

731 " canescens Taubert

$733 \quad$ "Clymentm L. $\odot$

734 " latifolius L. 4

$735 \quad$ Nissolia $L . \odot$

$736 \quad, \quad$ Ochrus $D . C . \odot$

$737 \quad n \quad$ sativus L. $\odot$

$738 \quad, \quad$ silvestris $L .4$

739 " tingitanus L. $\odot$

$740 \quad$ tuberosus $L .4$

741 " vernus Bernh. 4

742 Lens nigricans Godr. $\odot$

743 Lespedeza bicolor Turez. 5

744 Lotus conjugatus $L$. $\odot$

745, corniculatus $L .4$

746

747

748 Lupinus angustifolius $L$. $\odot$

749 "elegans $H$. B. et $K$. $\odot$

750 " hybridus Hort. $\odot$

751

752

753

754

755

756

757 Medicago arabica All.

758

759
" luteus $L$. $\odot$

" perennis L. 4

" polyphyllus $\mathrm{Ldl}, 4$

" pubescens Benth. $\odot$

" succulentus Dougl. $\odot$

" varius $L_{\text {. }} \odot$

" denticulata Willd.

" Eehinus D. C. ๑ 32
Mectícago globulosa Desv. $\odot$

grimmi 4

hispidit Gaertn (2)

Iitpulina $L . \odot 4$

Pironae Vis. $\because$

praecox D. C. $\odot$

sativa $L .4$

tribuloides Desr. $\odot$

Melilotus albus Desr

769 " indicus All.

770 officinalis Desr. .

771 Onobrychus petraea Desv. 4

$772^{\circ} \quad$ " vaginalis C.A.Mey. 4

773 " viciaefolia Ssop. 4 c,

$774 \quad " \quad$ v. gracilis

775 Ononis biflora Desf. $\odot$ (Bess.) 4 c.

776 " hircina Jacq. 4

777 Ornithopus sativus Brot. $\odot 32$

778 Phaseolus tuberosus $L$. $\odot$

$779 \quad$ " vulgaris $L . \odot$

780 nightianus $R$. Grah $\odot$

781 Pisum abysinicum $R, \mathrm{Br}$. .
784 " Jomardi Schrank. $\odot$

785 " Sativum L.

786 " thebaicum $W . \odot$

787 Pocockia cretica $D C$

788 Robinia Pseudacacia $L$. 万

789 Scorpiurus sulcatus L. $\odot$

790 Securinega Coronilla D.C. $\odot$

791 Tetragonolobus purpurea Mo792 Thermopsis caroliniana $M$. $A$.

793 Curt. 2

794 Trifoliun

795 Tholium arvense $L$, \&

796 " fragiferum L. 4

$797 \quad$ " Lupinaster $L 4$

" maritimum Huds

798 " montanum L. 4

$799 \quad " \quad$ pratense L. 4

$800 \quad n$ repens $L, 4$

$801 * \quad$ rubens $t .4$

$802 \quad$ " striatum $L . \odot$

803 Trigonella Balansae $B$. et $R$. $\odot$

$804 \quad " \quad$ calliceras Fisch. $\odot$

$805 "$ coerulea Ser.

806 - foenum graecum L. $\odot$

$807 \quad " \quad$ ovalis Boiss. $\odot$

808 Vicia atropurpurea Desf. $\odot$. 
809 Vicia cordata Wulf. ๑

$810 》 \quad$ cornigera Comb. $\odot$

811 " Ervilia Willd. (-)

813 " monanthos Desf.

814 " Nissoliana Lo $\odot$

$816 \Rightarrow$ pisiformis $L .4$

$818 "$ " (sem alb.) $\odot$

819 " sepium L. 4

820 " unijuga $\mathrm{A}, \mathrm{Br}, \mathrm{Z}_{4}$.

821 Vigna capensis Walp. $\odot$

822 " catjang Walf. $\odot$

Linaceae Dumort.

823 Linum perenne $L .4$

824 "usitatissimum $i$. $\odot$

Loas aceac.

825 Blumenbachia Hieronymi Urb. $\odot$
$812 "$ hirsuta G. Koch.

$815 "$ ochroleuca

817 "sativa $L$.

848 Modiola Napaea dioica Cav. 4

849 Sida anarthra $I$. Km. 432

850 " spinosa $L .4$

Martyni aceae

851 Martynia lutea (Lindl) Stapf. ()

Moraceae Lindl.

852 Morus alba $L \cdot \hbar$

Nyctaginaceae

853 Mirabilis jalapa L. $\odot$

854 Oxybaphus glabrifolius Vahl. 4

Oleaceae Lindl.

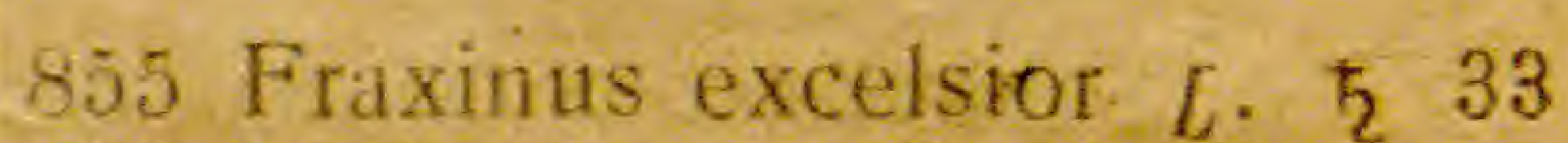

856 Ligustrum vulgare $L$. $\hbar$

857 Syringa persica $L$. $\hbar$

$858 \rightarrow$ vulgaris $L \cdot \frac{\hbar}{2}$

\section{Onagraceae Lindl.}

826 Cuphea procumbens Cav.

827 Lythrum hyssopifolia $L$. $\odot$

$$
\text { Malvaceae Juss. }
$$

828 Abutilon Avicennae Gaertn. $\odot$ 829 Althaea armeniaca Terone.

830 " cannabina $L .4$

831 - ficifolia Cav 4

$832 \pi$ officinalis L. 4.32

833 " pulchella Birnh. ()

834 " rosea Cav. 4

$835 \quad " \quad$ v. nigra 4

836 Anoda ciistata Sehlecht. ()

- 837 Hibiscus trionum L. ()

838 Kitaibelia vitifolia Willd. 4

839 Lavatera cretica $L$. ()

$840 \quad$ "trimestris L. $\odot$

841 Malope trifida Cav. $\odot$

842 Malva brasiliensis Desi.

$.8+3$ " crispa L. $\odot$.

844 " neglecta Wall. $\odot 32$

845 " silvestris L. 4

st6 Malvastrum peruvianum A. Gray.

(.)

859 Boisduvalia densiflora Wats. $\odot$

860 Clarkia elegans Dougl. (-)

861. Epilobium adnatum Gris. 433

862 " Dodanaei Villars. 4

$863 \quad n \quad$ hirsutum L. 4

864 Lamyi $E$ Schultz. 4

865 Eucharidium grandiflortum Fisch.

8006 et Mey. (2)

866 Godetia grandiflora Lindl. ()

867 Lopezia racemosa Cav. (s)

868 Oenothera ammophila Focke. $\odot$

C. 33

$869 \quad \quad \quad$ biennis $L . \odot$

870 . $n$ fruticosa L. 4

$871 \quad " \quad$ Gigas De Vires.

$872 \quad " \quad$ Lamarckiana Ser. $\odot$

$873 \quad$ " muricata $L . \odot$

874 " $\quad$ pumila L. 4

Oxalidaceae Lindl.

875 Oxalis corniculata $L$. Q $\odot$

876 " stricta $L .4$

Papaveraceac Juss.

847 Modiola caroliniana G. Don. ๑) 877 Adlumia cirrhosa Rafin. 433 
878 Chelidonium laciniatum Mill. 4 879 majus L. 4 880 Corydalis cheilantifolia Hemst. 881 " glauca Purch. $\odot$ 882 " vaginalis Royle.

883 Eschscholtzia californica Cham.

884 Fumaria capreolata $L$. $\odot$

$885 \quad$ officinalis $L$. . $\odot$

886 Glaucium flavum Crantz. .. 32

887 " phoeniceum Crantz. ()

888 Papaver argemone $L$. $\odot$

889 " aurantiacum Loisef. 4

32

890 " dubium L. $\odot$

891 . fugax Poir. ()

$892 \quad$ orientale $L .4$

893 " pavonium $C$. Meyer.

$894 \quad " \quad$ Rhoeas L.. c.

895 " Schinzianum Ferlde 4

33

896 " setigerum D. C. $\odot 33$

897 n somniferum I. V. opij

iferum $\odot$

898 n v. nigrum

899 " tauriculum Boiss. 32

Philadelphaceae.

900 Philadelphus coronarius $L . \quad \hbar_{2} 32$

Plantaginaceae Lindl.

901 Plantago Candollei Rafin. 4

$902 \quad "$ lanceolata L. 4

903 " Psyllium L. $\odot 33$

$904 n$ purpurescens Nutt.

905 ramosa Aschers. ${ }^{32}$

Plumbaginaceae Lindl.

906 Armeria alliacea Roem. et

arctica Wchult 4

908 " vulgaris Willd. 27

909 Plumbagella micrantha Ledeb. 4

Polemoniaceae D. C.

910 Collomia linearis Nutt. $\odot$
911 Gilia californica Benth. $\odot$

912 " tricolor Benth. ()

913 Leptosiphon androsaceus St.

914 Polemonium coeruleum L. 4

915 " gracile Wille. 4

$916 \quad n \quad$ reptans L. 4

Polygonaceae Lindl.

917 Emex spinosa Campd. .

918 Fagopyrum sagittatum Golib. $\odot$

919 " tataricum Gïrtn. (

920 Oxyria digyna Hill. 4

921 Polygonum alpinum All. 4

$922 \quad n \quad$ Bistorta L. 4

$923 \quad$ Convolvulus $L . \odot$

y24 " $"$ Hydropiper L.

925 " $"$ tomentosum Schrank

926 Rheum compactum L. 432

927 n macropterum Mart. 4

928 " palmatum L. 433

929 " Rhaponticum L. 4 und 11 atum

$932 \rightarrow$ Acétosella L. 432

933

" Patientia L. 4

$934 "$ stenophyllus Ledeb. 4

Portulacaceae Lindl.

335 Calandrina procumbens Moris $\odot$

936 Claytonia perfoliata Donn. $\odot$

937 Portulaca grandiflora Lindl. ()

938 n oleracea $L$. ( 32

- Primulaceae Vent.

939 Anagallis arvensis $L$. v. coerulea Gren et Godr. $\odot 32$

$940 \quad, \quad$ grandiflora Andr. (?)

941 Androsacae elongata $L$. $\odot \odot 33$

942 Cortusa Matthioli L. 4

943 Lysimachia ciliata $L .4$

$944 \quad n \quad$ dahurica $L d b .432$

945 " vulgaris L. 4

946 Primula algida Adam. 4

$947 \rightarrow \quad$ elatior Hill. 433

948

949

(1)

japonica A. Gray. 432

" offic nalis Hill. 4 
Ranunculaceae Juss.

950 Aconitum ferox Wall. 4

$951 \quad$ Napellus $L .4$

952 Acthaea spicata $L .4$

953 Anemone albana Stev. 4

954 " coronaria L. 4

$955 \quad \because \quad$ patens $L .4$

956 " $\quad$ pratensis L. 4

957 \# rivularis Buch.-Ham.

$958 \quad$ silvestris L. $2^{4}$

960 Aquilegia altaica 33

961 " atrata Koch. 433

962 " $"$ formosa Fisch. 433

963 " $\quad$ hispanica Barbas. 4 33

964 , jucunda Fisch. 433

965 "vulgaris L. 433

966 Clematis integrifolia $L .7$

967 r recta $L .4$

968 " $"$ tangutica Korsch. 33
$959 \quad$ \# virginiana $L .433$

992 Thalictrum aquilegifolium L. 4

993 " Delavayi Franch.

$994 \quad x \quad$ FendleriEngelm. 433

995 * minus L. 4

996 . $\quad$ pubescens

$997 \quad$ " simplex L. 4

998 Trollius chinensis Bge. 4

999 " $\quad$ europaeus L. 4

$1000 \quad " \quad$ pumilus D. Don 4

Resedaceae D. C.

1001 Reseda alba $L$. $\odot$

1002. " glauca L. (.) 33

$1003 \quad "$ lutea L. $\odot 2$

1004 " odorata L. $\odot$

Rhamnaceae S. F. Gray

1005 Rhamnus cathartica $L$. 5

1006 , Frangula $L .5$

Rosaceae Juss.

ம07 Agrimonia Eupatoria L. 4 \begin{tabular}{l|lll} 
Royle. 4 & $1008 \quad$ odorata Mill. 4
\end{tabular}

970 . $\quad$ cheilantum Fisch.

971 elatum L. 24

$972 \quad n \quad$ puniceum Pall. 4

973 " rossicum Litw. 4

$974 \quad$ " speciosum Bieb. 4

975 " $\quad$ triste Fisch

976 Myosurus minimus L. $\odot . \odot 33$

977 Nigella damascena $L$. $\odot$

$978 \quad "$ sativa $L . \odot$

979 Paeonia albiflora Pall. 433

980 Ranunculus acer $L .4$

$981 \quad \because$ asvensis L. ¿ 33

982 " auricomus L. 433

$983 \quad n \quad$ cassubicus $L .2$

$984 \quad n \quad$ Cymbalaria Purch.

$985 \quad$ Flammula $2.4 \frac{33}{4}$

986 " grandiflorus L. 4

987

illyrisus $L .4$

988 " pedatus W.K. 4

$989 \quad \% \quad$ repens $L .4$

$990 \quad-\quad$ sceleratus $L$. $\odot$

991 Thalictrum angustifolium Jacg.
1009 , " pilosa Led. 4

$1010 \quad n$ repens $[$. 4

1011 Amelanchier vulgaris Mönch. 5

1012 Crataegus monogyna Jack. †

$1013 \quad " \quad$ Oxyacantha Pers. 5

$1014 " \quad$ Sanguinea Pall. $\hbar$

1015 Duchesnea indica Focke. 4

1016 Filipendula hexapetala Gilib. 4

1017 UImaria Max. 4

1018 Fragaria vesca $L .4$

1019 \# viridis Dueh. 4

1020 Geum aleppicum Jacq. 433

$1021=$ japonicum Thunb. 4 .

$1022 "$ molle Vis. et Panc. 433

1023 " rivale $L .4$

1024 Physocarpus amurensis Max, $\frac{\hbar}{2}$

1025 Potentilla alpestris Hall. f. 4

1026 \# argentea L. 4

1027 \# Anserina L. 4

$1028 \quad " \quad$ biturca L. 433

$1029, \quad$ Bucroana Clem. 4

1030

1031

1032

1033

1034

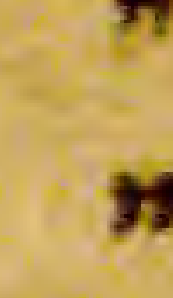

"

n

$n$ fruticosa $J$. $\hbar$ fulgens Wal. 432 glandulosa Lindl. 4 intermedia $L .4$ longipes Led. 4 
1035 Potentilla Meyeri Boiss. 4

$1036 \quad " \quad$ nepalensis Hook. 4

-1037 " pedata Willd. 4

1038 " pimpinelloides L. 24

1039 " pulcherrima Eehm. 4

1040 \# $\quad$ recta $L .2$

1041 . $\quad$ rupestris L. 4

1012 Prunus Cerasus $L, \hbar$

$1043 \#$ Padus $L . \hbar 33$

1044 spinosa $L . \hbar_{2}$

1045 Rubus caesus $L .5$

$1046^{\circ}$ " idaeus $L$. $\hbar$

1017 " leucodermis Dougl $5 \mathrm{c}$.

$1048 \%$ odoratus $L . \quad$

1049 " xanthocarpus Bureau et Franch. 5

1050 Sanguisorba oficinalis $L .24$

105

1052

1053 Sorbu" Aucuparia $L$. muricata spach.

1054 Spiraea media Schm. 5

1055 " opulifolia $L . \hbar$

1056 " salicifolia L. क

Ra biacea e Juss.

1058 Asperula Aparine Schott. 4

$1059 \quad-\quad$ arvensis L. $\odot 33$

1060 Cephalanthus occidentalis $L_{.}$.

1061 Crucianella chlorostachys $F$.

1042 Galium mollugo $L .4$

1063 " saccharatum All. (

$1064 "$ tricorne With. $\odot$

1065 " verum L. 4

16.6 Rubia tinctorum $L_{4} 4$

1067 Scherild a arvensis $L . \odot$

1068 Vaillantia fispida $L$. () Rntaceae Juss.

1069 Ptelea trifoliata $L$.

1070 Ruta graveolens L. 4

Saxifragaceae D umert.

1071 Astilbe chinensis Fr. et Sav. z 1072 n grandis Stapf. 4

1073 " rubra Hook fil.

1074 Heuchera americana L. 2

1075 Heuchera cylindrica Dougl. 4 $1076 \quad n \quad$ sanguinea Englm. 4 1077 Ribes alpinum L. 5

1078 Saxifraga caespitosa $L .4$ 1079 Tellima grandiflora $R, B r, 4$

Scrophulariaceae Lindl.

1080 Antirrhinum majus L. $\odot$

1081 Calceolaria pinnata $L$. $\odot$

1082 Cymbalaria muralis Baumg. 4

1083 Digitalis ambigua Murr. 433

1084 " media Roth. 24

1085 " nevadensis Kanze, 4

1086 " purpurea L. 24

1087 Hebonstreitia dentata $L$. $\odot$

1088 Linatia magoccana Hook. $\odot$

1089 " vulgaris Mill. 4

1090 Mimulus Tilingii $R g l$. $\odot$

1091 Pentstemon barbatus Roth. 4

1092 . diffusus Dougl. 4

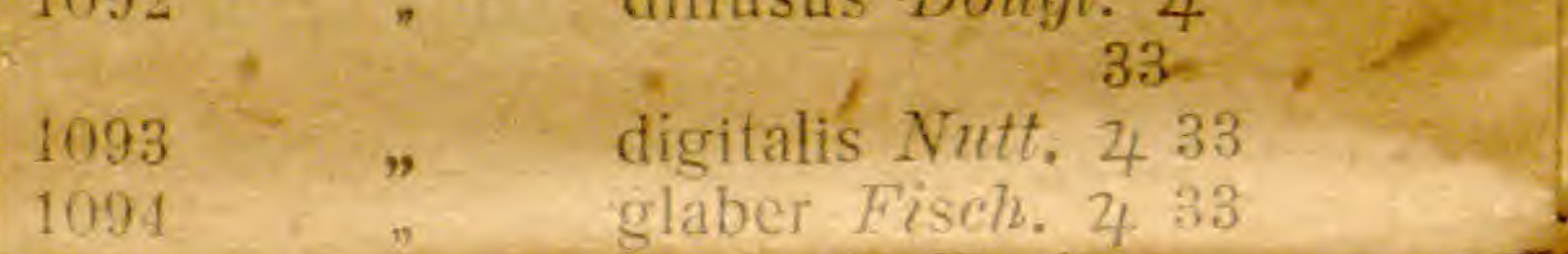

1096

1097

1098 Scrophularia lateriflora Trautv. Menziesii Hook. 4 ovatus Doug. 4

iogeg $>2$

$1099 \quad$ nodosa Dumort. If $_{4}$

1100 Verbascum guaphalodes $M . \vec{B}$.

1101 \# nigrum L. .32

1102 " $"$ phlomoides L. . 32

$11033^{*}$ " pyramidatum M. B.

1104 Veronica Beccabunga L. ${ }_{4}$

1105 "Chamaedrys $L \quad$ \&

$1106 \quad " *$ genqanoides 5 ahl. 4

$1507 n$ longifolia L. 4

1108 ". Ponae Gollan. 4

1109

1110

1111

n. $\quad$ sibirica $L .4$

\# spuria $L .4$

" Tournefortii Gmel. $\odot$

Solanaceae Pers.

1112 Datura aegyptiaca 'Fesl. ()

1113 " stramonium L. $\odot$

1114 Hyoscyamus niger $L . \odot \odot$

1115 Nicandra physaloides Gaertn. $\odot$

11 i6 Nicotiana affinis Moore. $\odot{ }_{4}$ 
1117 Nicotiana fragrans Hort. (\%) 32 1118 -

1119

1120

1121

1122 Physalis

1123

1124

1125

1126 Physochlaena orientalis G. Don. 4

1127 Sarachia Jaltomata Sehleicht . $\odot^{33}$

1128 Schizanthus pinnatus R. et P. $\odot$

1129 Solanum Dulcamara L. 4 . 万h

$1130 \quad n \quad$ gracile Otto. $\odot$

1131 " guinense Lam. ()

1132 " heterodoxum Dun ๑33

1133 " Lycopesicum $\vee$. grassularium $\odot$

1134 , nigrum L. $\odot$

$1135 "$ persicum W.

1136 " sisymbrifolium Lam. (.)

$1137 \quad$ Sodomeum L. (.)

$1138 \quad, \quad$ Tomatillo Phil. $\odot$
1156 Cryptotaenia canadensis D.C. 432 e

1157 Coriandrum satixum $L$. $\odot$

1158 Eryngium campestre L. 4

1159 " giganteum Biel. ..

1160 " planum L. 4

1161 " serbicum Panc. 4

1162. Falearia vulgaris Bern $l_{l}$. ..

1163 Heracleum barbatum Ledl. 4

1164 " pubescens $M$. B. .

116.5 " sibiricum L. 4

1166 Levisticum officmale $L, 4$

1167 Libanotis montana All. .)

1168 . sibirica Koch. 432

1169 Ligusticum scoticum $I_{\text {. }} 4$

1170 Myrrhis odorata Scop. 4

1171 Pastinaca satira $L$. (*)

1172 - teretiuscula Boiss. (32

1 T73 Pimpinella aromatica Bieb. 433

1174 Scandix Pecten veneris $L$ ○

1175 Seseli Hippomarathrum L. 4

Urticaceae Lind!

1176 Urtica cannabina $L .4$ cubensis Klotzsch. $\odot$

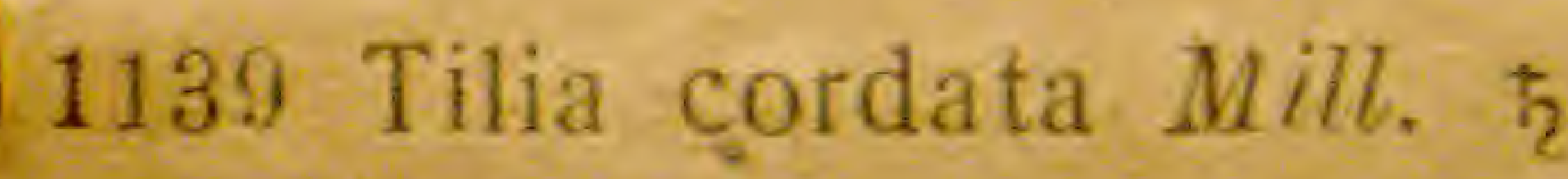

Tropaeolaceae Iuss.

1140 Tropaeolum Lobbianum Paxt. $\odot$

1141 i, majus $L . \odot$

$1142 \quad n$ minus $L$. $\odot$

Umbelliferae Morison.

1143 Aegopodium alpestre Ledb. 432
1144
Podagraria $L .4$

1145 Aenthusa Cynapium L. $\odot$

1146 Ammi majus $L$. $\odot$

1147 Anenthum graveolens $L$. $\odot$

1148 Angelica Archangelica L. $\odot$,

1149 Anthriscus silvestris Hoff. 4

1150 Astrantia major L. 4

1151 Bifora testiculata Boiss. $\odot$

1152 Bupleurum rotundifolium $L$. $\odot$

1153 Carum Carvi $L$. $\odot$

1154 Chaerophyllum aromaticum L. 4

1155 Cicuta virosa $L .4$ diocia $L \cdot 4$

\section{1}

1179 " gracilis Ait. 4

1180 " urens $L . \odot$

Valerianáceae Dumort.

1181 Valeriana palustris $\mathrm{Kr} .4$

1182 . Wolgensis Kez. 4

1183 Valerianella coronata $D . C$. $\odot$

1184 on eriocarpa Krok. (

$1185 \quad n \quad$ olitoria Pallich. ()

Verbenaceae J. St. Hil.

1186 Verbcnal Aubletia Jacq.

1187 \ \# hastata L. 4

$1188 \quad$ officinalis $L .432$

$1189 \quad " \quad$ urticifolia $L \cdot 432$

Violaceac D. C.

1190 Viola cornuta L. 4.

1191 , elatior Fr. 4

1192 " hirta $L \cdot 4$

1193 " mirabilis $L \cdot 433$

$1194 \%$ odorata L. 4

1195 ", tricolor Wittr. $\odot \odot$

\section{Professor N. F. NIKOLAEFF}

Horti praefectus. Assistens N. O. ZETTERMAN 


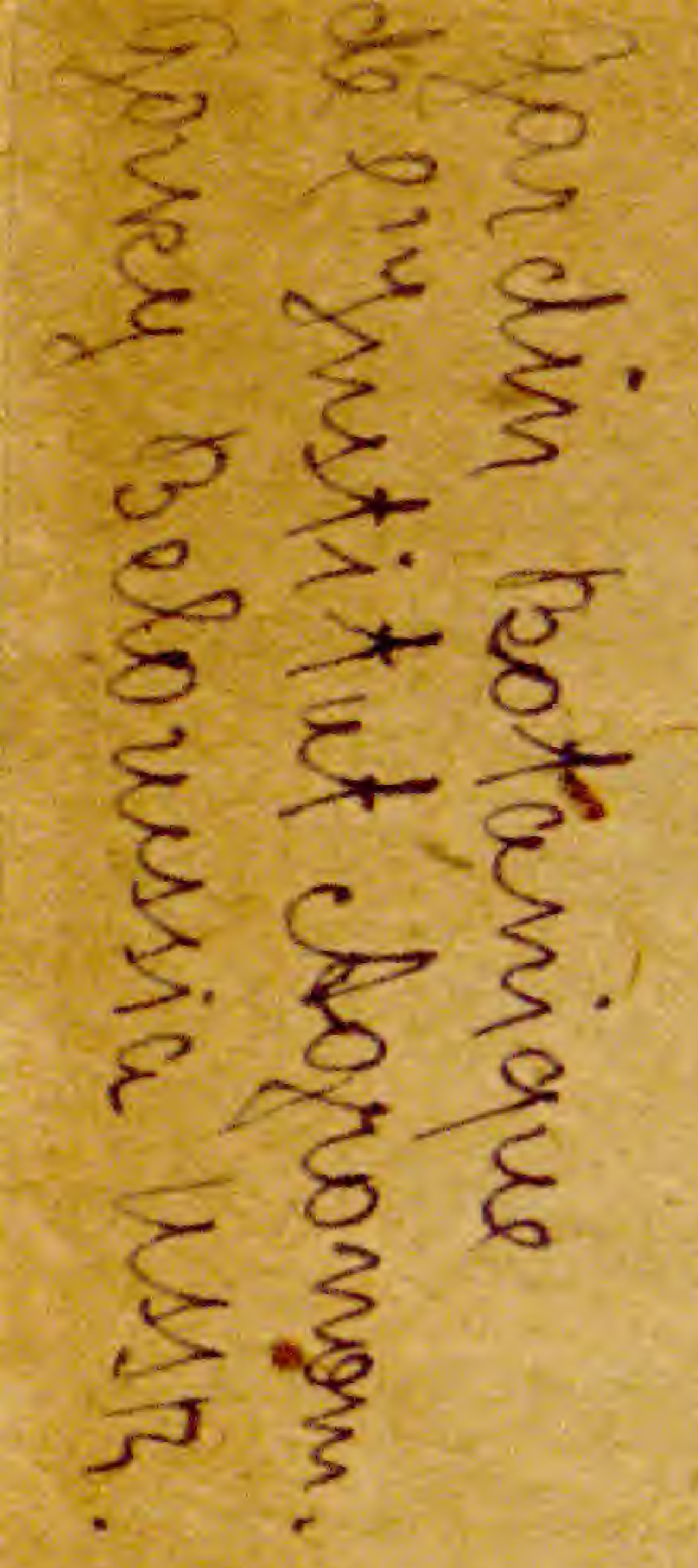

:

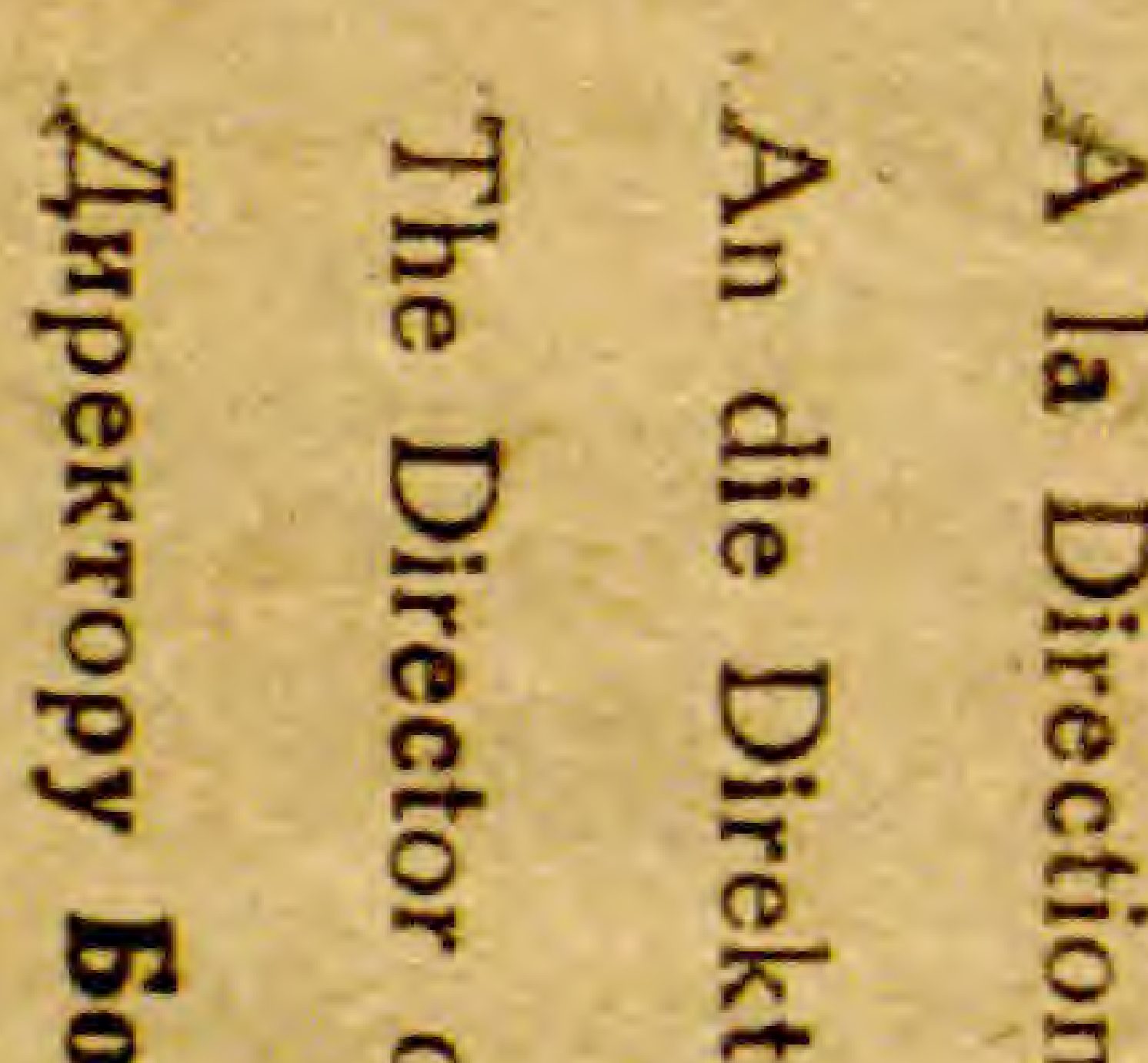

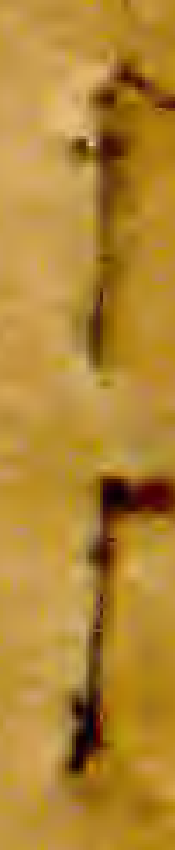

유 ढ

F

$\begin{array}{lll}0 & 8 \\ 0 & 0\end{array}$

훙 을

:

눌

| 10

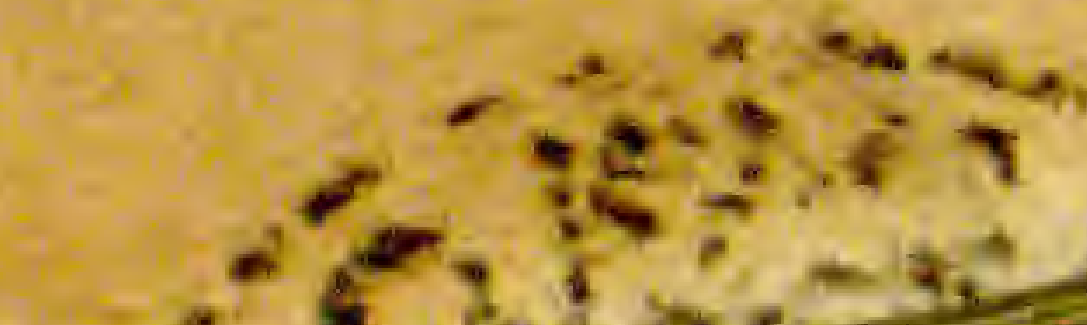
$(x)+(x)$ is 0 retit
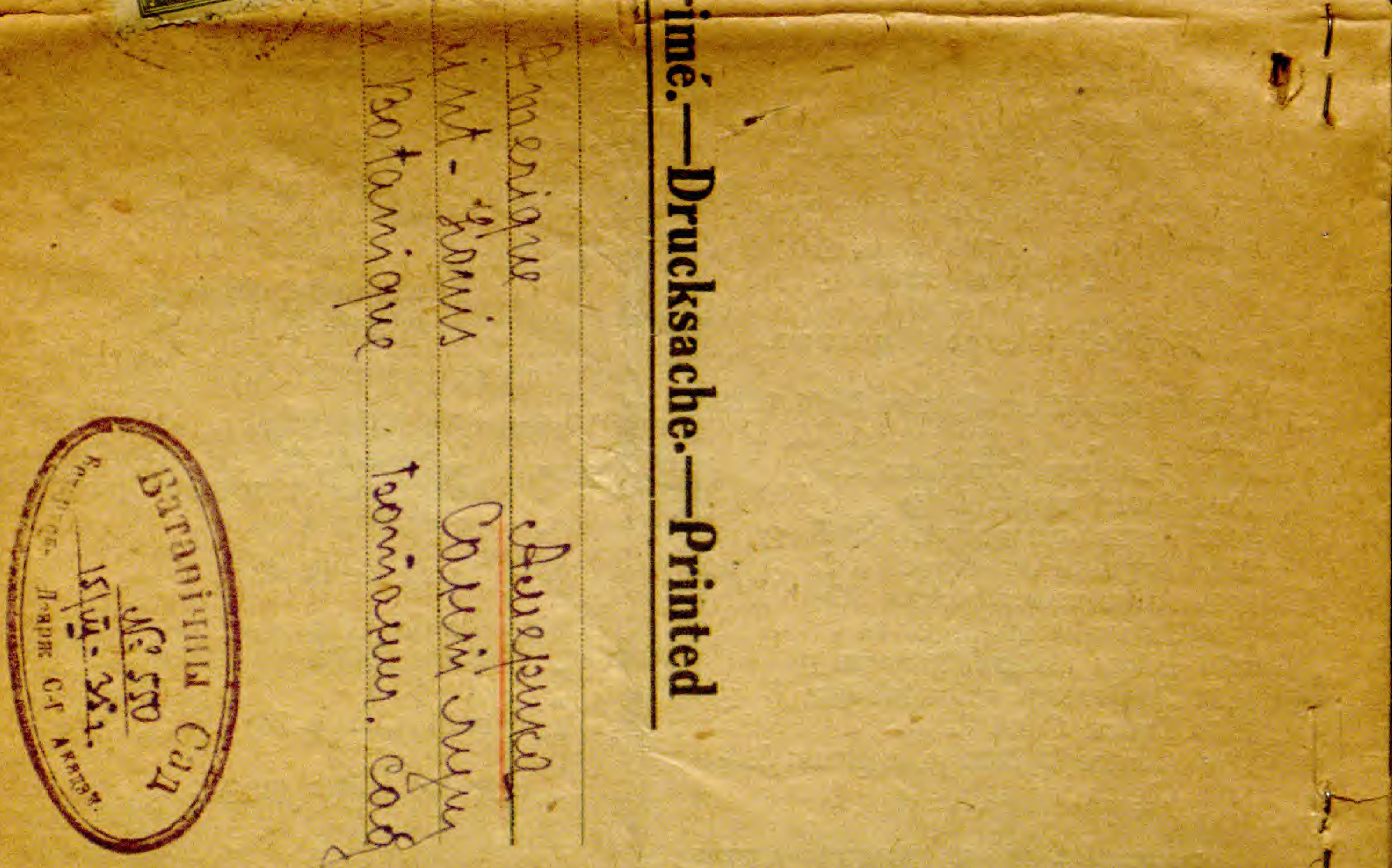

19 
JARDIN BOTANIQUE DE L'INSTITUT AGRONOMIQUE GORKY, BELORUSSIA, USSR

\section{6}

\section{DELECTUS SEMINUM}

Anno MCMXXXV (partim ac MCMXXXIV) collectorum quae Hortus Botanicus in Gorky pro mutua commutatione offert

\section{ПЕРАЛІК НАСЕННЯ,}

якое ботанічны сад у Горках прапануе да абмену

ГОРКИ БССР

ТИПОГРАФИЯ С.-Х. ИНСТИТУТА

\section{ПЕРЕЧЕНЬ СЕМЯН,}

предлагаемых в обмен ботаническим садом в Горках

$\begin{array}{llll}1 & 9 & 3 & 6\end{array}$ 


\section{P T E R I D O P H Y T A}

1 Aspidium cristatum $S w, 4$

2 Filix-mas $S w .4$

3 spinulosum $S w$. v. genuinum Milde 4

4 Asplenium dimorphum Knze. 4
Felix-femina Bernh. 4

6 Cystopteris bulbifera Bernh. 4

7 Onoclea sensibilis $L .4$

8 Polypodium Phegopteris L. 4

9 Struthiopteris germanica Willd. 4

\section{MONOCOT Y LEDONEAE}

\section{Commelinaceae Reich.}

10 Commelina coelestis $W$. $\odot$

\section{Cyperaceae J. St. Hil.}

11 Carex vesicaria $L .4$

12 Scirpus silvaticus $L .4$

\section{Gramineae Juss.}

13 Agropyrum Buonapartis Dur, et. Schinz. $\odot$

11 caninum $P . B .4$

16 elongatum P. B. 4

17 glaucum $R$. et Schult. 4

18 repens $P . B .4$

19 tenerum Vasey. 4

20 Agrostis alba $L .4$

21 maritima Law. $\odot$

22 vulgaris With 4

23 Aira elegans Willd. $\odot$

24 Alopecurus arundinaceus Poir. 4

25 fulvus $\mathrm{Sm}$. $\odot$

26 lasiostachys Link. 4

27 pratensis $L .4$

28 ventricosus Pers. 4

29 Anthoxanthum gracile $\mathrm{Br}$. $\odot$

30 Arrhenatherum erianthum Boiss. et Reut. 4

31 Arthraxon ciliaris $P$. B. $\odot$

32 Beckmania eruciformis Host, 4

33 Briza maxima $L .4$

34 media $L .4$

35 minor L. 4

36 Bromus ciliatus $L .4$

37 commutatus Schrad. () $\odot$

38 giganteus $L . \quad 4$

39 hordęaceus $L$. $\odot \odot$
40 Bromus japonicus Thunb. $\odot$

41 unioloides $H$. B et $K$. $\odot \odot$

42 Ceratochloa pendula Thell.

43 Dactylis glomerata $L .4$

44 hispanica Roth. 4

45 Deschampsia caespitosa $P$. B. 4

46 Diarrhena mandshuíica

47 Echinochloa Crus galli $P$. B. $\odot$

48 Eleusine indica Grtn. $\odot$

49 Toccusa Fresen. $\odot$

50 Elymus angustus 4

51 araliensis $R g l .4$

52 arenarius $L .4$

53 canadensis $T$

o4 220 Platycodon grandiflorum D.C. 4

55 exelsus Turcz. 4

56 junceus Fisch. 4

57 sibiricus $L .4$

58 virginicus $L .4$

59 Eragrostis megastachya Link. $\odot$

60 minor Host. $\odot$

61 pilosa $P . B$. $\odot$

62 Festuca alpina Suter. 4

63 arundinacea Schreb. 4

64 capillata Lam. 4

65 ciliata Pers. 4

66 fuegiana $\mathrm{Hook}$. 4

67 ovina $L .4$

68 polesica Zapal. 4

69 pratensis Huds. 4

70 psammophila Fritsch.

71 rubra $L .4$

72 supina Schur. 4

73 varia Haenke. 4

74 Haynaldia villosa Schur.

75 Heleochloa schoenoides Host. $\odot$

76 Hilardia rigida Vosey. $\odot$

77 Hordeum jubatum L. 4

78 murinum $L$. $\odot$

79 Koeleria glauca D. C. 4 
80 Koeleria gracilis Pers. 4

81 Lamarckia aurea Moench. ()

82 Leptochloa polistachya $K h$.

83 Lolium italicum $A$. Br. 4

84 linicola Sond. $\odot$

85 perenne $L .4$

86 Melica altissima $L .4$

87 transsilvanica Schur. 4

88 Nardus stricta $L .4$

89 Oplismenus frumentocius $\mathrm{Fr}$.

90 Panicum bulbosum $H$. B. K, 4

91 californicum Benth. $\odot$

92 colonum L. $\odot$

93 frumentaceum Roxb. $\odot$

94 filiforme Gcke. $\odot$

95 miliaceum $L$. $\odot$

96 Paspalum elegans Hort. $\odot$

97 Phalaris canariensis $L$. $\odot$

98 minor Retz. $\odot$

99 Phleum nodosum L. 4

100 pratense $L \quad 4$

101 Poa abyssinica Jacq. 4

102 alpina $L .4$

103 badensis Haente 4

104 caesia $\mathrm{Sm} .4$

105 compressa L. 4

\section{Iridaceae Juss.}

124 Iris aphylla $L .4$

125 aurea Lindl. 4

126 Clarkei Bak. 4

127 Delavayi Micheli 24

128 desertorum Gldst. 4

129 Fischeriana Hort. 4

130 Pseudacorus L. 4

131 setosa Pall. 4

132 sibirica $L .4$

133 Sisyrinchium anceps Cav. 4

134 angustifolium Mill. 4

\section{Juncaceae Vent.}

135 Juncus alpinus Vill. 4

136 balticus Willd. 4

137 compressus Jacq. 4

138 Luzula nivea $D$. C. 4

\section{Liliaceae Scop.}

139 Allium albidum Fisch. 4

140 canadense $L .4$

141 carinatum $L, 4$

142 coeruleum Pall, 4

143 fistulosum L. 4

145 globosum Red. 4

146 jesdianum $L \quad 4$

147 Moly $L .4$

148 nigrum L. 4

149 odorum L. 4

150 oleraceum $L .4$

151 Porrum L. 4

152 Schoenoprasum $L \quad{ }_{4}$

153 senescens $L .4$

154 trigustrum $L .4$

155 Anthericum Liliago L. 4

156 Asparagus officinalis $L .4$

157. Funcia lancifolia $S p r .4$

158 Hemerocallis flava $L .2$

159 Lilium tigrjnum Gawler. 4

160 Muscari racemosum Mill. 4

161 Smilacina stellata Desf, 4

162 Veratrum album L. 4

163 Zygadenus sibiricus A. Gray. 4

\section{DIC OT Y L E D ONEAE}

\section{Aceraceae Neck.}

164 Acer tataricum L. $\hbar$
Aizosceae

165 Tetragonia expansa Murr. $\odot$ 


\section{Amarantaceae Juss.}

166 Achyranthes argentea Lam. $\odot$

167 Amarantus albus $L$. $\odot$

168 aureus Dietr. $\odot$

169 caudatus $L$. $\odot$

170 chrostachys Willd. $\odot$

171 elegantissimus Hort. (

172 flavus $L$. $\odot$

173 gangeticus $L . \odot$

174 hypochondriacus $L$. $\odot$

175

176

177

178

179 paniculatus $L$. $\odot$ purpurascens Otto $\odot$ retroflexus $L$. $\odot$ speciosus Simp. $\odot$ xantostachys Willd. $\odot$

\section{Apocynaceae R. Br.}

180 Amsonia latifolia M. E. Jones 4 181 Tabernaemontana Walt. 4 182 Vinca minor $L .4$

\section{Araliaceae Juss.}

183 Aralia californica $S$. Wats. 184 mandshurica Rupr. ${ }_{2}$

\section{Aristolochiaceae Juss.}

186 Asarum europaeum L. 4

\section{Asclepiadaceae Lindl.}

187 Asclepias Cornuti Deesn. 4 188 - phytolaccoides Pursch. 4 189 rerticillata $L .4$

190 Periploca graeca L. 4

\section{Balsaminaceae S. F.}

191 Impatiens Balsamina $L$. () 192 glanduligera Roule. $\odot$

193 scabrida D. C. $\odot$

\section{Bignoniaceae}

194 Incarvillea variabilis Batal. hybrida 4

Borraginaceae S. F.

195 Anchusa capensis Thunb. $\odot$
196 Borrago officinalis $L$. ()

197 Cerinthe minor $L$. $\odot$

198 Cynoglossum officinale $L .4$

199 Echium vulgare $L$. $\odot$

200 Omphalodes linifolia Moench. $\odot$

201 Simphytum asperum Lep. 4

202 offtcinale $L .4$

\section{C mpaunlaceae Juss.}

203 Adenophora palustris

204 Campanula alliariaefolia Willds 4 205 alpina Jaeq. 4

206 Erinus L. $\odot$

207 grandis $F$. et $M .4$

208 Grossekii Heuff. 4

209 latifolia L. 4

210 Morettiana Rehb. 4

211 Pollasiana $R$. et $S .4$

212 pulcherrima Schr. et Zeyl 4

213 Raineri Perp. 4

214 rotundifolia L. v. Hostii 4

215 Codonopsis pilosula Nannf.

216 ussuriensis Hemsl. 4

217 viridiflora Max. 4

218 Lobelia inflata $L$. $\odot$

219 Phyteuma canesens $W$. et Kit 2

220 Platycodon grandiflorum D.C. 4

221 Mariesii Hort. 4

222 Specularia Speculum A. D. C. $\odot$

223 Symphyandra Hoffmannii Pant $\odot$

224 Wahlenbergia lobelioides $L k$. $\odot$

\section{Capparidaceae}

225 Cleome candelabrum Sims. (226 gigantea $L$. $\odot$

\section{Carpifoliaceae Vent.}

227 Lonicera Carfifolium $L .5$

228 tatarica $L .5$

229 Sambucus Ebulus L. 4

230 Viburnum Opulus $L .5$

\section{Caryophyllaceae Juss.}

231 Agrostemma Githago L. $\odot$

232 nicaeensis Pers. .

233 Arenaria graminifolia Sehrad. 4

234 long!folia $M$. B. 4

235 Cerastium Biebersteinii D. C. 4 $236 \quad$ Inflatum Link. $\odot$ 
237 Dianthus arenarius $L .4$

238 barbatus I. 4

239 bicolor Hort. 4

240 deltoides $L .24$

241 giganteus D'urv. 4

242 trifasciculatus Kit. 4

243 Gypsophila repens $L$. $\odot$

244 Steveni Fisch. 4

245 Lychnis Flos Jovis Desr. -

246 grandiflora Jacq. 4

247 Malachium aquaticum Rehb. 4

248 Melandrium album Garcke 4

249 Saponaria cerastioides Fisch $\odot$

250 officinalis $L .4$

251 Scleranthus annuus $L$. $\odot$

252 Silene Armeria $L$. $\odot$

253 gallica $L$. $\odot$

254 japonica Rohib. 4

255 Spergula maxima Weihe $\odot$

256 sativa Boenn. $\odot$

257 Viscaria viscosa Aschers. 4

\section{Chenopodiace Less.}

258 Acroglochin chenopodiores

Schrad. 4

259 Atriples hortense $L$. $\odot$

260 Chenopodium Eonus-fienricus

$$
\text { L. } 4
$$

261 Obione sibirica Fizcll. $\odot$

\section{Cistaceae Dun}

262 Helianthemum guttatum Mill. $\odot$ 263 ledifolium Mill. $\odot$

\section{Compositae Adans.}

264 Achillea Millefolium L. 4

265 odorata $L .4$

266 Ageratum conyzoides $L$. $\odot$

267 Ammobium alatum $R$. Br.

268 Anacyclus officinarum Hayne

269 Anaphalis yeddoensis Maxim.

270 Anthemis altissima $L . \odot$

271 Aplopappus Parryi A. Gray. 4

272 Arctotis calendulaceae Willd. $\odot$

273 stoechadifolia Berg. ()

274 Arnica Chamissonis Less. 4

275 foliosa Nutt. 4

276 sachalinensis Gray. 4

277 Artem ssia Absinthium L. 4

278 sacrorum Ledb. 4

279 Artemissia vulgaris L. 4
280 Aster altaicus Willd. 4

281 salicifolius Scholl. 4

282 Tradescanti $L .4$

283 Baeria coronaria A. Gray. $\odot$

284 Bellis mexicana $A$. Gray. 4

285 Bidens leucantha Willd. $\odot$

286 Buphtalmum speciosum Schreb.4

287 Calendula officinalis $\Sigma$. $\odot$

288 Calimeris incisa D. C.

289 Calliopsis bicolor Bosse. $\odot$

290 Callistephus chinensis Ness. $\odot$

291 Carpesium cernuum L. 4

292 Carthamus lanatus $L$. $\odot$

293 Centaurea alpina $L .4$

294 - Cyanus L. $\odot$

295 glastifolia $L .4$

296 Chondrilla panciflora 4

297 Chrysanthemum caucasicum Pers. 4

298 coccineum Willd. 4

299 corymbosum $L .4$

$300 \quad$ Leucenthemum L. 4

301 viscosum Desf. $\odot$

302 Cichorium Intibus L. 4

303 Cirsium arvense Scop. 4

304 Cladanthus arabicus Cass. $\odot$

305 Coreopsis peltata $L$. $\odot$

307 Cosmos diversifolius Otto $\odot$

308 Crepis biennis $L$. $\odot$

309 rubra $L$. $\odot$

310 tectorum $L . \odot \odot$

311 Dahlia variabilis Desf. 4

312 Dimorphotheca pluvialfs Moench $\odot$

313 Echinops globifer Janka 4

314 Szowitzii Fisch. et Mey.

315 Emiila sonchifolia D. C. $\odot$

316 Erigeron coulteri Port, etCoult. 4

317 Eupatorium aromaticum L. 4

318 odoratum $L \cdot 4$

319 Gaillardia aristata Purch. 4

320 lanceolata Michx. 4

321 Gazania longiscapa $\odot 4$

322 Gnaphalium vira-vira Molina $\odot$

323 Guizotia abyssinica Cass $\odot$

324 Helenium Hoopesii A. Gray. 4

325 Heliantella quinquenervis

\section{A. Gray. 4}

327 boreale $\mathrm{Fr} .4$

328 Pilosella $L .4$

329 pratense Tausch. 4

330 virosum Poll. 4 
331 Hipochoeris radicata $L .4$

332 Inula bifrons $L .4$

333 Heleniuu L. 4

334 magnifica Lipsky. 2

335 Lactuca altissima $M$. B. $\odot$

336 saligna $L \cdot \odot$

337 sativa $L$. v. oleifera $\odot$

338 Lappa tomentosa Lam. $\odot$

339 Lasthenia glabrata $L d b$. $\odot$

340 Layia elegans Torr. et Gray. $\odot$

341 Leontodon autumnalis $L .4$

342 Leontopodium alpinum Cass. 4

343 Liatris spicata Willd. 4

344 Madia capitata T, et Gray. $\odot$

345 dissiflora Nutt. $\odot$

346 sativa $\mathrm{Mol}$. $\odot$

347 Matricaria Chamomilla $L$. $\odot$

348

349 Myriactis nepalensis Less. 4

350 Onopordon Acanthium $L$. $\odot$

351 Prenanthes purpurea $L$. 4

352 Psephellus dealbatus Boiss. 4

353 Pyrethrum Parthenium Sm. 4

354 Rudbeckia laciniata $L \cdot 4$

355 maxima Nutt. 4

356 Scorzonera hispanica $L .4$

357 Jaquiniana Boiss. 4

359 Senecio Fuchsii Gmel. 24

360 Ligularia Hook. 4

361 Serratula coronata $L \cdot 4$

362 radiata Bieb. 4

363 Silphyum fntegrifolium Michx. 4

364 perfoliantum $L \cdot 2 t$

365 Solidago altissima $L \cdot 4$

366 Sonchus asper Will. $\odot$

367 Tagetes lucida Cav. 4

368 signata Bartl. $\odot$

369 Tanacetum pseudachillea $C$.

370 Winkl. 4

371 Taraxacum officinale Wigg. 4

372 Tragopogon floceosum 4

373 tommasinii 4

374 Uropappus clevelandii Greene. $\odot$

375 Urospermum picroides $F$. $W$. Schmidt. $\odot$

376 Ximenesia encelioides Cav. $\odot$ 377 Zacintha verrucosa Gaertn. $\odot$

378 Zinnia elegans Jacq. $\odot$

379 Haageana Regel. $\odot$

380 multiflora $L \cdot \odot$

381 verticillata Andrz. $\odot$

\section{Convolvulaceae Vent.}

382 Convolvulus elongatus Willd.

383

384

385

386 Ipomea coccineá $L$. $\odot$

387 Lederagalia $L$. $\odot$

388 - purpurea Roth. $\odot$

389 rubricoerulea Hook. $\odot$

390 sibirica Pers. $\odot$

391 splendens Sims.

392 Pharbitis hispida Choizi.

Cornaceae D. C.

393 Cornus alba $L$.

Crassulaceae D. C.

394 Sedum aizoon $L .4$

395 boloniense Loisl. 4

396 carpaticum 4

397 hybridum $L .4$

398 purpureum Link. 4

399 rupestre L. 4

400 spurium $M . B .4$

4णी Sempervivum annum C.Sm. $\odot$ 402 patens Griseb et Schenk. 4

403 ruthenicum Koch. 4

\section{Cruciferae Juss.}

404 Arabis albida Stev. 4

405 rosea $D . C .4$

406 suecica Fries. (?)

407 Barbarea stricta Andrz.

408 vulgaris $R$. Br..

409 Biscutella auriculata $L$. $\odot$

410 raphanifolia Poir. $\odot$

411 Brassica Napus L. v. oleifera Moench. $\odot$

412 rapa $L$. v. oleifera $D . C$. $\odot$

413 Bunias orientalis L. 4

414 Calepina Corvini (All) Desv. $\odot$

415 Camelina Alyssum (Mill) Thell. $\odot$

416 linicola Sch. $\epsilon S$ Sp. $\odot$

417 microcarpa Andrz. $\odot$

418 Capsella Bursa pastoris Moench.๑

419 Carrichtera Vellac D. C. $\odot$

420 Cheiranthus Allioni Hort. $\odot$

421 Cochlearia officinalis $L$.

422 Crambe abyssinica Horh. 
423 Crambe hispanica $t$.

424 Diplotaxis muralis $D$. $C$.

425 Draba scandinavica Liudblon. 4

426 Eruca sativa $D$. C. $\odot$

427 Erysimum sufruticosum Spreng.

428 Hesperis alpina Schur.

429 dinarica $G$. Beck. 4

430 fragrans Fisch. 4

431 matronalis $L .4$

432 tristis $L .4$

433 violacea Boiss 4

434 Iberis amara $L$. $\odot$

435 Isatis tiuctoria $L$. $\odot$

436 Lepidium latifolium L. 4

437 sativum $L$. $\odot$

438 Notoceras canariense $R$. Br. $\odot$

439 Raphanistrum silvestre Aschers. $\odot$

440 Raphanus sativus L. v. oleiferus Mentzger $\odot$

441 Senebiera Coronopus Poir. $\odot$

442 Sesquerella gracilis $S$. Wats.

443 Sinapis alba $L$. $\odot$

444 apula Tenore. $\odot$

445 arvensis $L$. $\odot$

446 chinensis $L . \odot$

447 dissecta Lag. ()

448

44 y v. cispliolia

450 " $\quad$ v. japonica

451 , v. mongolica

$452 \quad$ v. sareptana

453 " v. subintegrifolia

$454 \quad$ " v. subsareptana

455 nigra $\odot$

456 Thlaspi arvense $L$. $\odot$

457 Vogelia paniculata Hornem. $\odot$

\section{Cucurbitaceae Holl.}

458 Bryonia alba L. 4

459 Cyclanthera explodens Naud. $\odot$

\section{Dipsacaceae D. C.}

460 Callistemma brachiatum Sibth. () 461 Cephalaria alpina $R$. S. 4

462 corniculata Roem, et Schult. 4

463 tatarica Schrad 4

464 Dupsacus fullonum Mill. (-)

465 Iaciniatus $L$. $\odot$

466 Knautia atropurpurea 4

467 Scabiosa australis Wulf.
Euphorbia ceae J. St. Hil.

468 Euphorbia Esula L. 4

469 procera $M . B .4$

\section{Gentianaceae Dumort.}

470 Erythraea Centaurium Pers.

471 Gentiana alba Miihlbg. 4

472 Cruciata L. 4

473 Fetisowii Regel et Wahl.

474 frigida Haenke. 2

475 Kesselringii Regel 4

476 macrophylla Pall. 4

477 Pneumonanthe L. 4

478

479

480

481

straminea Maxim. 4 thianschanica Rupr. 4 tibetica King. 4

Walujewii Rgl. et Schmalh.

Geraniaceae J. St. Hil.

482 Erodium cicutarium L'Herit $\odot$

483 gruinum Ait. $\odot$

484 moschatum $L$. $\odot$

485 Geranium dissectum $L$. $\odot$

486 palustre $L .4$

487 pratense $L, 2$

488 pussillum $L$. $($ )

489. sibiricum L. 4

\section{Guttiferae Engl.}

490 Hypericum Ascyron L. 4

491 calmianum $\overline{5}$

492 hirsutum L. 4

493 perforatum L. 4

\section{Hydrophyllaceae}

494 Ellisia Nyctelaca $L$. $\odot$

495 Nemophila maculata Benth. $\odot$

496 Pnacelia tanacetifolia Benth. ()

\section{Labiatae Juss.}

497 Ajuga reptans $L .4$

498 Anthyllis Polyphylla

499 Betonica officinalis $L .2$

500 Dracocephalum moldavica $L$. $\odot$

501 Ruyschiana $L .4$

502 Hormium pyrenaieum $L, 4$

503 Hyssopus otfficinalis $L$. $\hbar$ 
504 Laminnm maculatum L. 4

505 Lophantus rugosus $F$. et $M$.

506 scrophulariaefolius Bent. 4

507 urticifolia

508 Melissa officinalis $L .4$

509 Mentha candicus 4

510 crispa L. 4

511 piperita L. 4

512 viridis $L .4$

513 Monarda fistulosa L. 4

514 Nepeta Cataria L 4

515 grandiflora $M, B, 4$

516. macrantha Fisch. 4

517 nuda $L .4$

518 Origanum vulgare $L .4$

519 Phlomis tuberosa L. 4

520 Salvia austriaca Jacq. 4

521 coccinea $L$.

522 glutinosa $L .4$

523 hians Royle. 4

524 japonica Thunb. 4

525 pratensis $t .4$

526 Satureja Clinopodium Caruel. 4

527

528 Scutellaria alpina $L, 4$

$j 29$ dependene saxim 4

si scordioide 2. 2

532 Stachys alpina $T \cdot 4$

533 silvatica $L .4$

534 Teucrium Chamderys L. 4

535 Thymus glaber Mill. 4

\section{Leguminosae Juss.}

536 Anthyllis tetraphylla $L$. $\odot$

537 Astragalus Cicer $L \cdot 4$

538 glycyphyllus $L \cdot \hbar$

539 gummifer Labill.

540 hamosus $L \cdot \odot$

541 Baptisia australis $R . B r .4$

542 Biserrula Pelecinus- $L$. $\odot$

543 Caragana arborescens Lam. h

544 Cicer pinnatifidum Jaub. et Spach. $\odot$

545 Colutea cruenta Ait. 5

546 Desmodium canadense $D . C$. 5

547 Ervum abyssinicum Hort.

548 Galega officinalis $L .4$

549 Genista ovata $W$. et $\pi$, 5

550 tinctoria $L . \hbar_{2}$

c51 Glycyrrhiza lepidota Purseh. 4
552 Hymenocarpus nummularius

Willd $\odot$

553 Lathyrus aphaca $L$. $\odot$

554 articulatus $L$. $\odot$

555 cicera $L . \odot$

556 Clymenum $L$. $\odot$

557 latifolius $L .4$

558 Nissolia $L_{\text {. }} \odot$

559 sativus $L$. $\odot$

560 silvestris L. 4

561 tingitanus $L$. $\odot$

562 vernus Bermh. 4

563 Lotus corriculatus L. 4

564 ornithopodioides $L$. ()

565 Lupinus angustifolius $I$. .

566 luteus $L_{\text {. }} \odot$

567 perennis $L .4$

568 polyphylus LAl. 4

569 varius T. .

570 Maackia amuriensis

571 Medicago denticulata Willd $\odot$

572 globulosa Desv. •

573 grimmi 4

574 hispida Gaertn. $(8$

575 lupulina $t . \odot 4$

576 Pironae Vis. $\odot$.

577 praecox D.C.C

578 sativa L. 4

579 Melilotus albus Desr. (-)

580 indicus All. $\odot$

581 Onobrychis altissima

582 petraea Desv. 4

583 Sativa Lam.

584 vaginalis C. A. Mey. 4

585 Ononis biflora Desf. $\odot$

586 hircina Jacq. 4

587 Orobus vernus L. 4

588 Fhaseolus acutifolius A. Gray.

589 capensis Thunb. $\odot$

590 Pisum abyssinicum $R . B r . \odot$

591 arvense $L$. $\odot$

592 sativum $L$. $\odot$

thebaicum H.

594 Pocockia cretica $D . C$. $)$

595 Robinia Pseudacacia L. 5

596 Scorpiurus subvillosus $L$. (-)

597 Securinega Coronilla $D$. C. C

598 Tetragonolobus purpureus

Moench.

599 Thermopsis caroliniana $\mathbf{M}$. $A$. Curt. 4

600 fabacea $D . C$.

f01 montana Nutt. 
602 Trifolium arvense $\Gamma$. .

603 fradiferum L. 4

604 Lipinaster $L .24$

605 maritimum Huds. .

606 medium L. 2

607 montanum $\mathrm{I} \cdot \mathrm{Z}$

608 rubens L. 4

609 Trigonella Balansae $B$. t.t. $K$. $\odot$

610 caeliceras Fisch. $\odot$

611 coerulea Ser. $\odot$

612 cretica $\odot$

613 foemum graecum $\boldsymbol{L}$. $\odot$

614 ovalis Boiss. $\odot$

615 Vicia amphiocarpa $D$. ()

616 atropurpurea Desf. $\odot$

617 bengnalensis $L$. $\odot$

618 calcarota Desf. $\odot$

619 Cracca L. 4

620 disperma D. C. $\odot$

621 elegantissima $\odot$

622 ervilia (L) Willd. (

623 ferruginea $\odot$

624 fulgens Battand. ()

625 globosa Rets. $\odot$

626 hybrida $L$. $\odot$

627 michauxii Spr. ()

628 narbonensis $L$. $\odot$

629 peregrina $L . \odot$

630 picta Fisch, et Mey. $\odot$

631 Sativa L. $\odot$

632 sepitum $L .4$

633 striata $M . \vec{B}$.

684 tricolor Sebast.

635 unijuga $A . B r$.

636 vestita Boiss.

637 Vigna capensis Walp. $\odot$

\section{Linaceae Dumort.}

638 Linum africanum

639 altaicum Fisch, 4

940 arboreum L. 4

641 crepitans Dum.

642 gallicum L. ๑

643 grandiflorum Desf. 0

644 Muelleri Moris. $z_{4}$

645 narbonense $L .4$

646 nervosum W. et Kit. 4

647 perenne $L .4$

648 usitatissimum $t$. $\odot$

\section{Loosaceae}

69. Blumenbachia Hieronimi Urb

\section{Lythraceae Lindl.}

650 Cuphea procumbens Cav. $\odot$

651 Lithrum hyssopifolia $L$. $\odot$

\section{Malvaceae Juss.}

652. Althaea cannabina $I . \cdot 4$

653 ficifolia Cav, 4

654 officinalis $L .2$

655 narbonensis Pourr. 4

656

657

pulchella Birnh. $\odot$

rosea Cav. 4

$658 \quad$ v. nigra 4

659 Anoda cristata Schlecht.

660 Hibiscus gossypinus Thunb.

661 trionum $L$. $\odot$

662 Lavatera cretica $L$. $\odot$

663 trimestris $\boldsymbol{L}$. $\odot$

664 Malope trifida Cav.

665 Malva brasiliensis Desr.

666 crispa $L$. $\odot \odot$.

667 silvestris L. 4

668 Malvastrum peruvianum

A. Gray. ()

669 Modiola caroliniana $G$. Don.

670 Napaea dioict-C 67 Palava malvaemolia cot.

672 Sida spinosa $L, 4$

\section{Martyniaceae}

673 Martynia lutea (Lindi) Stapf.

\section{Moraceae Lindl.}

674 Humulus japonicus $S$. et Z C 675 Lupulus $L$. $\odot$

\section{Nictaginaceae}

676 Oxybaphus glabrifolius Vahl. 4

\section{Oleaceae Lindl.}

677 Siringa persica L. 5

678 vulgaris $L$. $\hbar$

\section{Onagraceae Lindl.}

679 Boisduvalia densiflora Wats, 680 Epilobium Dodanaei Villars. 4 681 hirsutum L. 7 
682 Epilobium Lamyi E. Schultz. 24 683 Eucharidium grandiflorum Fisch.

684 Oenothera biennis $L$ et Mey. (2)

685 fruticosa L. 2

686 muriticata $L$. (.)

\section{Oxalidaceae Lindl.}

687 Oxalis corniculata $L . \odot \odot$ 688 stricta L. 4

\section{Papaveraceae Juss.}

689 Chelidonium laciniatum Mill. 4 690 majus L. ${ }_{4}$

691 Corydalis cheilantifolia Hemsl. 4 692 glauca Purch. $\odot$

693 ophiocarpa Hook, et Thoms. 4 694 vaginalis Royle

695 Eschscholtzia californtca Cham $\odot$

696 Fumaria officinalis $L$. $\odot$

697 Glaucium phoenicem Crantz. (:) 698 Papaver argemone $L$. $\odot$

699 fugax Poir. .

$700 \quad$ Rhoeas

I. L. v. upiferum 6

\section{Plantaginaceae Lindl.}

703 Plantago lanceolata $L .24$

704 ramosa Aschers. $\odot$

\section{Plumbaginaceae Lindl.}

705 Armeria alliacea Roem. et

* Schult. 4

706 aretica Wall. 4

707 longistilla 4

708 maritima Willd, 4

709 vulgaris Willd. 4

710 Plumbagella micrantha Ledeb. 4

\section{Polemoniaceae D. C.}

711 Collomia linearis Nutt. (c)

712 Gilia californica Benth. $\odot$

713 tricolor Benth.

714 Leptosiphon androsaceus St. (.)

7 I5 Polemonium coeruleum L. 4

716 gracile Willd. 4

717 Richardsonii v. humil. Gray.

\section{Polygouaceae Lindl.}

718 Emex polygonum

719 spinosa Campd. ..

720 Fagopyrum sagittatum Golib. ()

721 tataricum Gürtn. (

722 Oxyria digyna Hill. 4

723 Polygonum alpinum All. 4

724 Bistorta $L, 2$

725 tomentosum Schrank. 4

726 Rheum palmatum L. 4

727 Rhaponticum L. 4

728 undulatum $L, 4$

729 Rumex Acetosa $L .2$

730 stenophyllus Ledeb. 4

\section{Portulacaceae LindI.}

731 Portulaca grandiflora Lindl. ()

732 oleracea $L$. $\odot$

\section{Primul teae Lindl.}

733 Lysimachia ciliata L. 4

734 vulgaris L. 2

735 Primula algida Adam. 4

736 cortusoides $L .4$

737 elatior Hilt. 4

738 officinalis Hill. 4

739 Veitchil Duthie 4

\section{Ranunculaceae Juss.}

740 Aconitum barbatum 4

741 ferox Wall. 4

742 Acthaea spicata L. 4

743 Anemone albana Stev. 4

744 coronaria L. 4

745 cylindrica $A$ Gray. 4

746 mexicana H. B. et Kit. 4

747 silvestris L. 2

748 Aquilegia altaica 4

749 chrysantha A. Gray. 4

750 formosa Fisch. 4

751 hispanica Barbas 4

752 jucunda Fisch, 4

753 vulgaris 4.4

754 Clematis integrifolia L. 4

755 recta $L \quad 4$

756 serratifolia Rohb. 2

757 tangutica Korsch, 5

758 Delphinium barbatum 4

759 caschmerianum Royle. 4 
760 Delphinium cheilantum Fiseh, 4

761 chinense 4

762 elatum L. 4

763 rossicum Litw. +

764 sinensis 4

765 triste Fisch. 4

766 Nigella damascena $L, \odot$

767 sativa $t . \odot$

768 Paconia albiflora Pall. 4

769 Ranunculus acer L. 4

770 cassubicus $L \cdot 4$

771 grandiflorus $\boldsymbol{L}$. 4

772 Thalictrum angustifolium Jacq. 4

773

774

775

776

777

778 Trollius chinensis Bge. 4

779 europaeus $L .4$

780 pumilus $D$. Don. 4

Resedaceae D. C.

781 Reseda alba $L$. $\odot$

782 lutea $L$. $\odot$

\section{Rosacoae Juss.}

783 Agrimonia Eupatoria L. 4

784

785

786

787 Duchesnea indica Focke. 4

788 Filipendula hexapetala Gilib. 4

789 Ulmaria (L) Max. 4

790 Fragaria vesca $L .4$

791 virginiana Duchesn 4

792 Geum japonicum Thunb. 4

793 rivale $L .4$

794 Physocarpus opulifolius Max.

795 Potentilla alpestris Hall. 4

796 anserina $L .4$

797 argentea $L .4$

798 Buccoana Clem. 4

799 fruticosa $L .4$

800 glandulosa Lindl. 4

801 intermedia $L .4$

802 longipes Led. 4

803 pedata Willd. 4

804 pimpinelloides $L .4$

805 pulcherrima Lehm. 4

806 recta $L .4$
807 Potentilla supina $L .24$

808 Prunus spinosa $L$. 5

809 Rubus caesus $L$. $\hbar$

810 leucodermis Dougl. 5

811 odoratus $L$ h

812 Sanguisorba muricata Spach.

813 officinalis L. 4

814 tenuifolia Fisch.

815 Spiraea media Sehm. 5

\section{Rubiaceae Juss.}

816 Asperula Aparine Schott. 4

817 asvensis $L$. $\odot$

818 orientalis Boiss. et Hohen.

819 Crueianella chlorostachys

820 Galium mollugo L. 4 F. et. M. 4

821 parisiense $L$. $\odot$

822 saccharatum Alt. $\Theta$

823 tricorne With. $\odot$

824 verum L. 4

825 Rubia tinetorum $L .4$

826 Sherardia arvensis $L$. $\odot$

827 Vaillantia hispida $L$. $\odot$

\section{Rutacege Juse}

828 Ruta graveolens L. 4

\section{Saxifragaceae Dumort.}

829 Astilbe ehinensis Fr. et Sav.

830 grandis Stapf. $4^{\circ}$

831 rubra Hook. fil.

832 Heuchera Drummondi Hort. 4

833 hispida Purseh. 4

834 micrantha Dougl.

835 sanguinea Engelm. 2 f

836 Ribes alpinus $L$. $\hbar$

837 Saxifraga caespitosa $L$. 5

838 conifera Coss. et Dur. 4

839 decipiens Ehrh. 4

\section{Scrophulariaceae Lindl.}

840 Antirrhinum craniifolium

841 Digitalis lutea $L, 4$

842 media Roth. 4

843 nevadensis Kanze. 4

844

845 Hebenstreitia dentata $L$. $\odot$

846 Linaria vulgaris Mill. 4

847 Mimulus Tilingii Rgl. $\odot$ 
848 Pentstemon barbatus Roth. 4

849 glaber Fisch. 4

850 Hallii A. Gray. 4

851 Menziesii Hook. 4

852 orizonicus 4

853 ovatus Doug. 4

854 Lcrophularia lacteriflora Trautv. 4

855 nodosa $L .4$

856 Verbascum grandiflorum Gueld.:

857 hexiroseum

858 Veronica Beccabunga L. 4

859 gentianoides Vahl. 4

860 longifolia $L .4$

861 sibirica $L .4$

862 spuria $L .4$

\section{Solonaceae Pers.}

863 Atropa Belladonna L. 4

864 Browallia grandiflora

865 Datura aegyptiaca Desl. $\odot$

866 Betrolonii Parl. $\odot$

867 stramonium L. $\odot$

868 Hyoscyanum niger $L . \odot \odot$

869 Nicandra physaloides Gaertn.

870 Nicotiana affinis Aloore. $\odot$

\section{3}

874

875 Physalis edulis Sims. $\odot$

876

877

878

879 Physochlaena orientalis G.Don.4

880 Schizantus pinnatus $R$. et $P$. $\odot$

881 Solanum citrullifoliцm $A$. $B r$.

882 Dulcamara $L .4$

883 gracile Otto. $\odot$

884 guinense Lam. $\odot$

885 heterodoxum Dun. -

886

887

888

889

890

891

892

Lycopersicum v. grassularium $\odot$ mincatum

nigrum $L . \odot$

persicum $W$. h

sisymbrifolium Lam. $\odot$

Sodomeum $L$. $\odot$

Tomatillo Phil. $\odot$

\section{Tropaeolaceae Juss.}

893 Tropaeolum Lobbianum Paxt. 894 majus $L_{0} \odot$
805 Tropacolum minus L. (?)

\section{Umbelliferae Morison.}

896 Aegopodium podagria $L .4$

897 Ammi majus L. $\odot$

898 Anenthum giaveolens L. $($.

899 Angelica Archangelica $L$. $\odot$

900 purpurea -

901 Anthriscus silvestris Hoff. 4

902 Astrantia major L. 4

903 Bifora testiculata Boiss. $\odot$

904 Bupleum rotundifolium $L$. $\odot$

905 Carum Carvi $L$. .

906 Chaerophyllum aromaticum L. 4

907 bulbosum

908 Cicuta virosa $L .4$

909 Coriandrum melphitense

910 sativum $L$. $\odot$

911 Eryngium campestre L. 4

912 giganteum Bieb. -

913 planum $L .4$

914 Heracleum barbatum Ledl. 4

915 pubesens $M$. B. $\odot$

916 Levisticum officinale $L .4$

917 Libanotis montana All. $\odot$

918 sibirica Koch. 4

919 Ligusticum scoticum $L .4$

920 Myrrhis odorata Scop. 4

921 Pastinaca sativa $L$. $)$

922 Scandix Pecten veneris $L$. $\odot$

923 Seseli Hippomarathrum L. 4

\section{Urticaceae Lindl.}

924 Urtica cannabina L. 24

925 dioica $L .4$

926 gracilis Ait. 4

927 urens $L$. $\odot$

\section{Valerianaceae Dumort.}

928 Valeriana officinalis $L .4$

929 Wolgensis Kez. 4

930 Valerianella coronata $D . C$. $\odot$

931 olitoria !Pallich. $\odot$

Verbenaceae J. St. HiI.

932 Verbena hastata L, 4 
Violaceae D. C.

933 Viola bosniaca 4

934 cornuta $L .4$

935 elatior Fries. 4
936 hirta L. 4

937 odorata L. 4

938 palmata $L . Z_{4}$

939 palustris L. 4

940 tricolor Wittr. $\odot \odot$

Assistens N. M. IWANO

Professor N. F. NIKOLAEV

Horti praefectus

Index seminum desideratorum ante calendas Aprilias rogamus. 


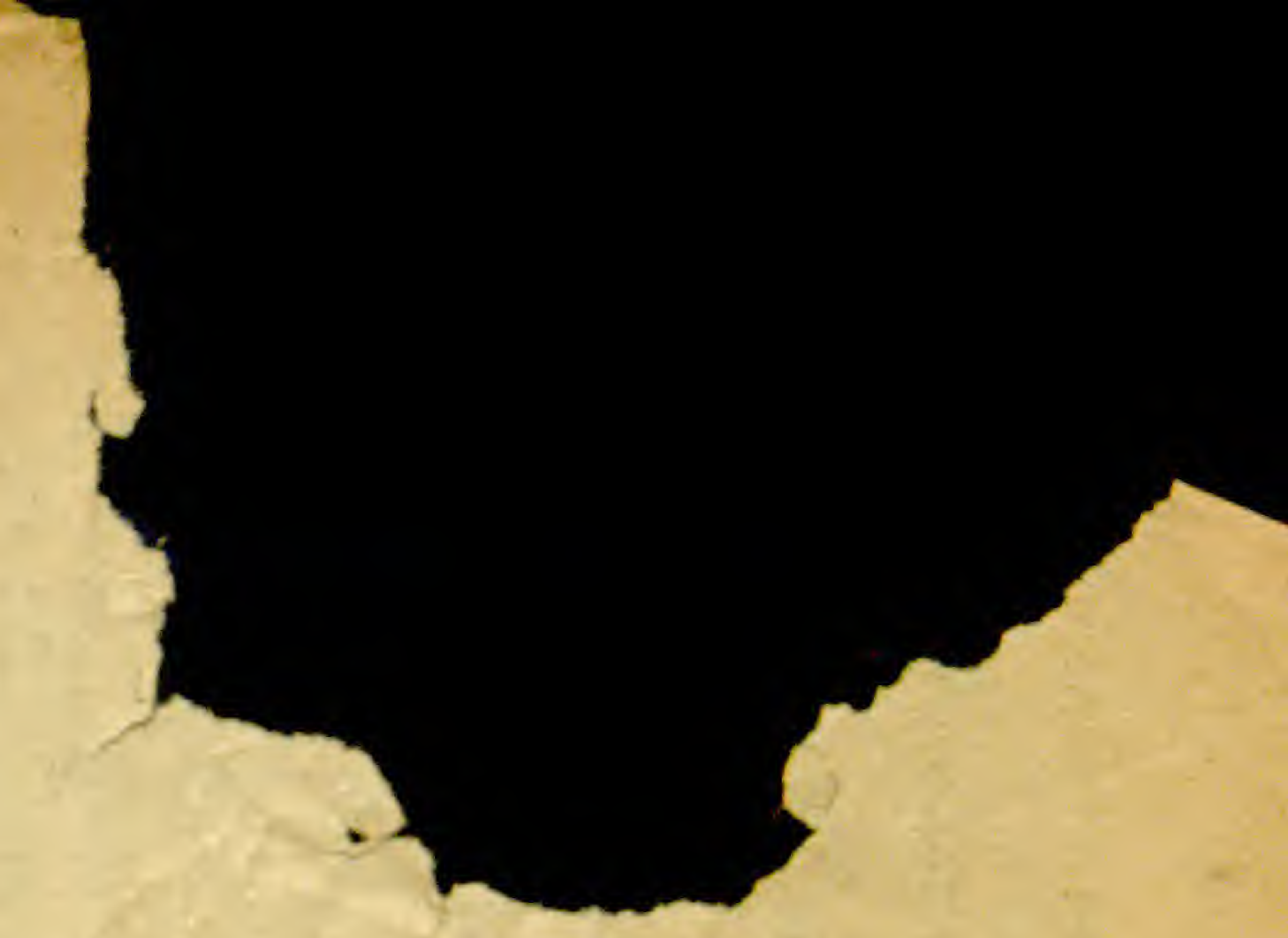


Violaceae D, C.

- Viola bosniaca 4

o cornuta L. 4

ogtior Fries. 4

造 $\frac{2}{2} 5$

G $\cong$ त्र. F.NIBn

0
0
0
0
0
0
0
0
0
0
0

ㅇ. $\overrightarrow{0}$.

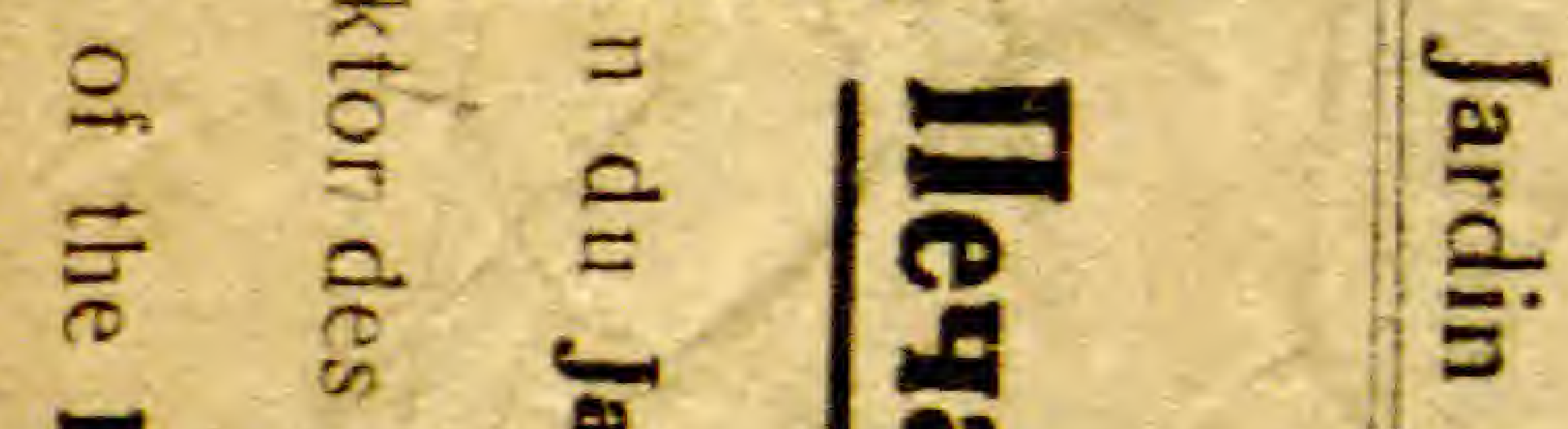

ष

르 है

ह.

๑ ह है

ล

ส

$\curvearrowright$

is

is

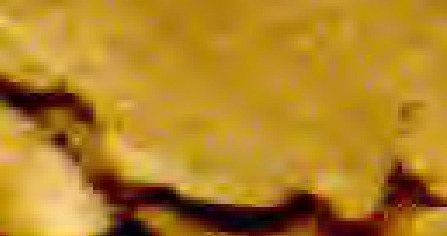

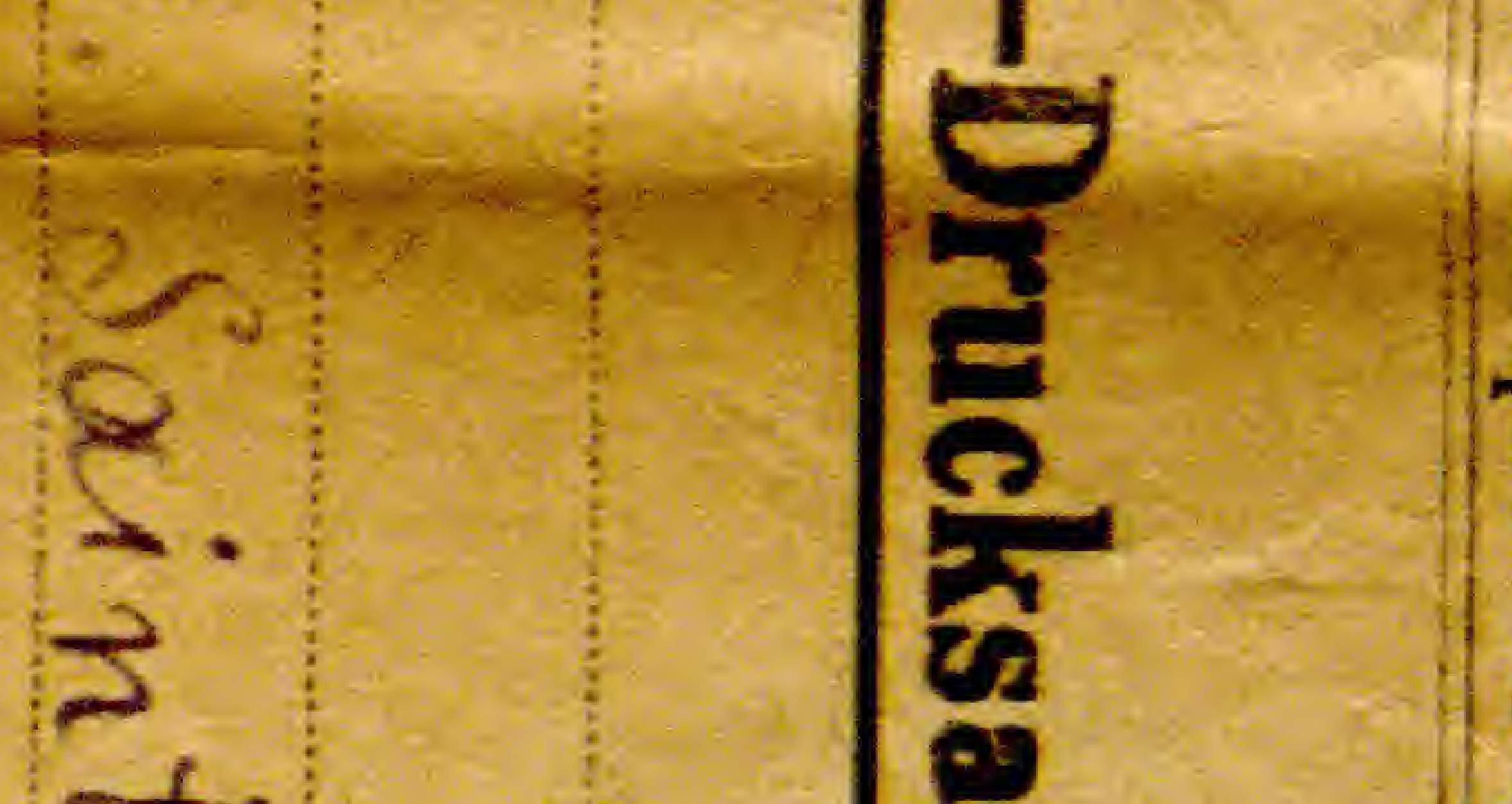

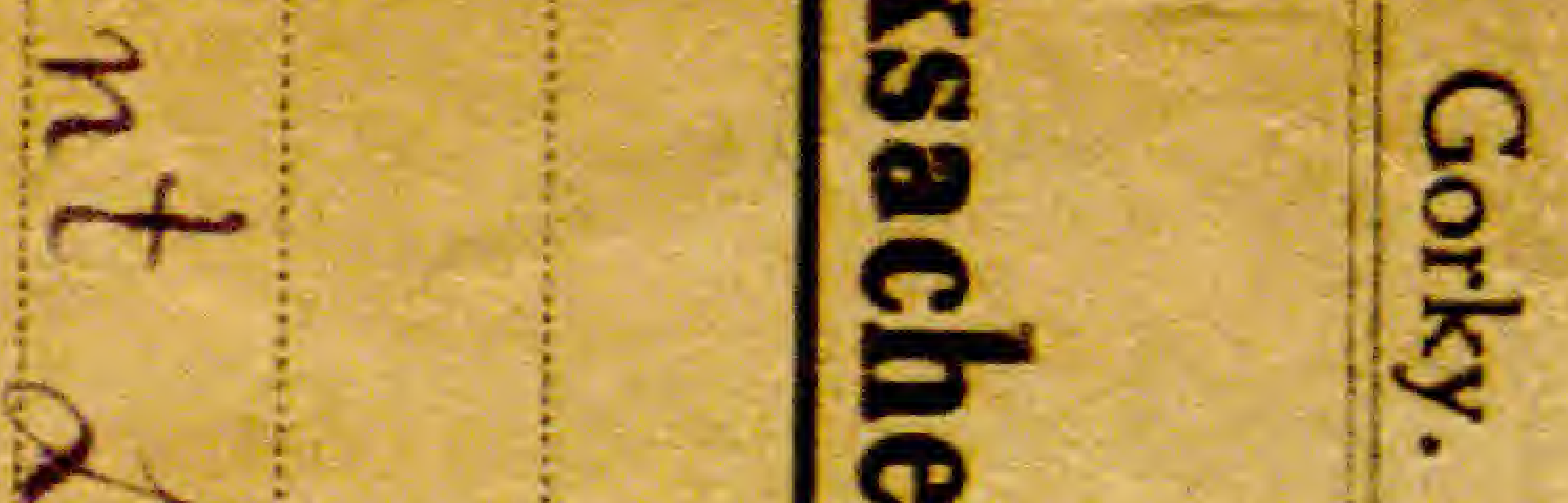

o 1

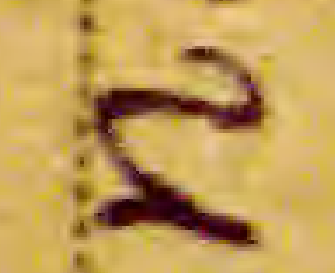

$\sqrt{n}$.

$\sum_{\infty} \quad \frac{\text { a. }}{8}$

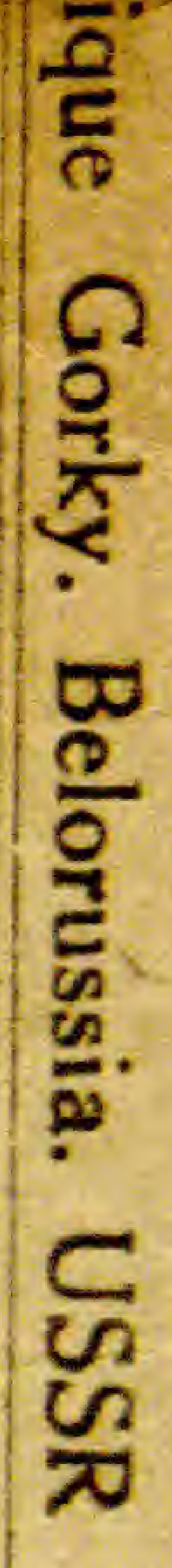

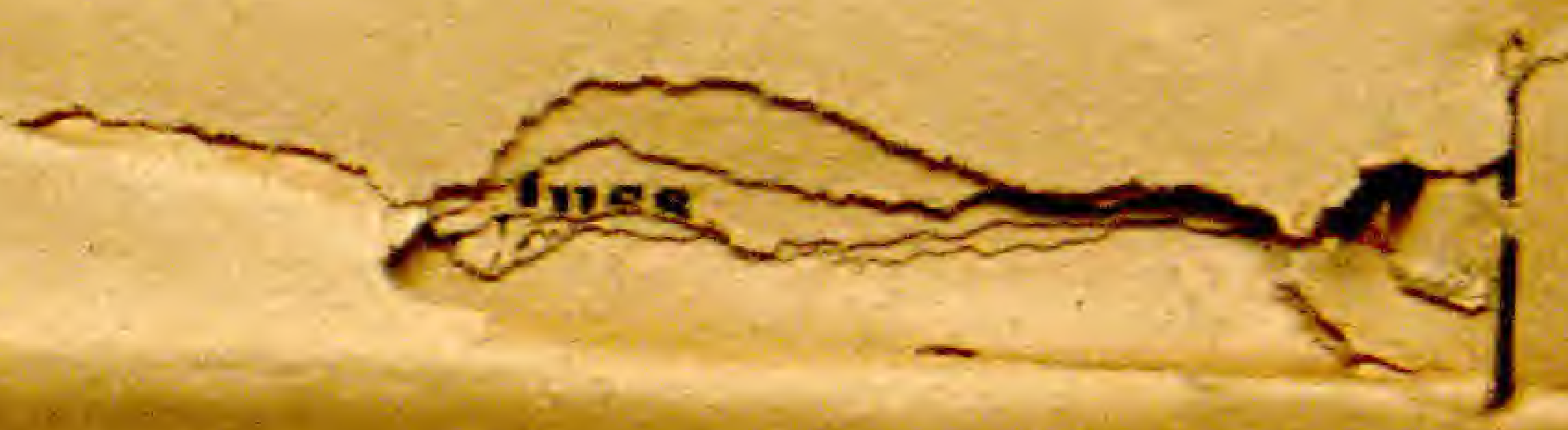

ई. 


\title{
DELECTUS SEMINUM
} ANNO MCMXXXVI COLLECTORUM QUAE

HORTUS BOTANICUS

UNIVERSITATIS GORKIJENSIS

MUTUA COMMUTATIONE

OFFERT

\author{
PRO
}





\section{SECTIO GENERALIS.}

I. Polypodiaceae.

1.* Athyrium crenatum Rupr. ${ }^{1}$

2.* A. filix femina Roth.

3.* Cystopteris fragilis Bernh.

4.* Dryopteris Linneana Christ.

5.*D. Phegopteris Christ.

6.* D. spinulosa O. Ktze.

7.* D. Thelypteris A. Gray.

8.* Polysticum Braunii Fée.

II. Pinaceae.

9.* Picea excelsa Link.

10. Pinus Strobus L.

III. Cupressaceae.

11.* Juniperus communis L.

12. Thuja occidentalis L.

IV. Gramineae.

13.* Briza media L.

1 Genera et species in ordine alphabetico disposite sunt.

* Plantae spontaneas. 
14. ${ }^{*}$ Brachypodium silvaticum P. B.

15. Bromus carinatus Hook. et Arw.

16. * B. inermis Leyss.

17. B. mollis L.

18.* Dactylis glomerata L.

19.* Deschampsia caespitosa P. B.

20. Elymus hirsutiglumis Sorib.

21. E. sibiricus L.

22. Festuca alpina Sut.

23. F. bromoides hort.

$24{ }^{*} \mathrm{~F}$. rubra L.

$25{ }^{*}$ F. sulcata L.

26. Hordeum jubatum L.

27. Lolium perenne L.

28. Melica altissima L.

29."M. nutans L.

30.* Milium effusum L.

31. Phalaris paradoxa L.

32. Phleum Michaeli All.

33.* Ph. pratense L.

34. Poa alpina L.

35. Sesleria coerulea Arduin.

36." Setaria viridis Beauv.

37. Stipa capillata L.

38.* S. Joannis Cel.

39.* Triticum repens $\mathrm{L}$.

40. Zea mays L.

V. Alismaceae.

41.* Alisma Plantago L 
VI. Araceae.

42." Calla palustris L.

VII. Liliaceae.

43. Allium albidum Fisch.

44. A. fistulosum L.

45.* A. Schoenoprasum L.

46. Bulbine annua Willd.

47.* Convallaria majalis $\mathrm{L}$.

VIII. Betulaceae.

48. Alnus incana Moench. f. acuminata Reg.

49. Betula lutea Michx.

50. B. verrucosa Ehrh.

IX. Fagaceae.

51.* Quercus pedunculata Ehrh.

$X$. Ulmaceae.

52.* Ulmus pedunculata Foug.

53.* U. scabra Mill.

XI. Urticaceae.

54. Cannabis sativa $L$.

55. Humulus Lupulus L.

XII. Polygonaceae.

56. Polygonum lapathifolium L.

57. P. molle D. Don.

58. Rheum officinalis Baill.

59. Rh. palmata L. v. tanguticum Maxim. 
60.* Rumex acetosa L.

61. R. acetosella L.

62. R. confertus Willd.

63.* R. crispus L.

64. R. hydrolapathum Huds.

65. R. hymenosepalus Torr.

66. R. ucrainicus Fisch.

XIII. Chenopodiaceae.

67.* Chenopodium album L.

68. Ch. Botrys L.

69. Ch. capitatum Aschers.

70. Ch. Quinqua Willd.

71. Corispermum hyssopifolium L.

XIV. Amarantaceae.

72. Amarantus Blitum L.

73. A. gangeticus hort.

74. A. lividus L.

75. Celosia cristata L. v. sanguinea hort.

$X V$. Nyctaginaceae.

76. Mirabilis Jalapa L.

77. Oxybaphus nyctagineus Sweet.

XVI. Portulacaceae.

78. Portulaca grandiflora Hook.

XVII. Phytolaccaceae.

79. Phytolacca decandra L. 
XVIII. Caryophyllaceae.

80.* Agrostemma Githago L.

81. Cucubalus baccifer L.

82. Dianthus barbatus L.

83. D. caryophyllus L.

84. D. chinensis L.

85.* D. deltoides $\mathrm{L}$.

86. Gypsophila acutifolia Fisch.

87. G. paniculata L.

88. Lychnis diurna Sibth.

89. L. coronaria Desr.

90. L. chalcedonica L.

91. L. Flos-cuculi L.

92. L. viscaria L.

93.* Melandrium album Garcke.

94. Saponaria cerastioides Fisch.

95. Silene Armeria L.

96. S. Bergiana Lindm.

97. S. conica L.

98. S. cucubalus Wieb.

99. S. Hormemannii Steud.

100. S. gallica L.

101. S. lacta A. Br.

102. S. mellifera Boiss. et Reut.

103. S. muscipula L.

104. S. venosa Aschers.

105.* Stellaria nemorum L.

XIX. Ranunculaceae.

106.* Aconitum excelsum Rchb.

107. A. Lycoctonum L. 
108. Aquilegia canadensis L.

109. A. vulgaris L.

110. Delphinium Cheilanthum Fisch.

111* D. consolida L.

112. D. elatum L.

113. D. e. v. alpinum Hegi.

114. D. e. v. tirolense Hegi.

115. D. grandiflorum L. fl. alba hort.

116. Isopyrum fumarioides L.

117. Myosurus minimus L.

118. Nigella arvensis L.

119. N. damascena L.

120. N. sativa L.

121. Ranunculus abortivus $\mathrm{L}$.

122.* R. auricomus L.

123.* R. cassubicus L.

124. R. dissectus M. B.

125. R. muricatus L.

126.* Thalictrum minus L.

$X X$. Papaveraceae.

127. Adlumia cirrhosa Raf.

128.* Chelidonium majus L.

129. Ch. laciniatum Mill.

130. Escholtzia californica Cham.

131. Glaucium flavum Crantz.

132. G. squamigerum Kar. et Kir.

133. Papaver alpinum L.

134. P. altaicum Ball.

135. P. dubium L.

136. P. nudicaule L. 
137. Papaver orientale L.

138. P. rhoeas L.

139. P. somniferum L.

140. P. somniferum (ex Tatrespublica).

141. P. somniferum (ex Kasachstan).

142. P. somniferum (ex Extremo Oriente).

143. P. somniferum (ex Tjan-Schjan).

144. P. somniferum (ex Sajsan).

XXI. Fumariaceae.

145. Corydalis semperflorens Pers.

XXII. Cruciferae.

146. Aethionema Buxbaumii D. C.

147. Ae. pulchellum Boiss.

148. Barbarea orthoceras hort.

149. Biscutella auriculata L.

150** Berteroa incana D, C.

151. Brassica juncea Czern.

152. B. nigra Roch.

153. Camelina dentata Wallr.

154. ${ }^{*}$ C. linicola N. Zing.

155. C. microcarpa Andrz.

156. ${ }^{*}$ C. sativa Crantz.

157. Capsella Bursa pastoris Moench.

158. Carrichtera vella D. C.

159. Cheiranthus Allioni hort.

160. Cochlearia officinalis L.

161. Crambe abyssinica Rochst.

162. C. hyspanica L.

163. Erysimum canescens Roth. 
219. Lathyrus odoratus L.

220.* L. pratensis L.

221.* L. silvestris L.

222. Lupinus elegans H. B. et K.

223. L. ornatus Dougl.

224. L. polyphyllus Lindl.

225. L. p. Lindl. f. coerulea hort.

226. L. p. Lindl. f. rosea hort.

227.* Lotus corniculatus L.

228. Medicago hispida Gaertn.

229.* M. falcata L.

230. M. orbicularis All.

231. M. sativa L.

232. M. sativa $\mathrm{L}$.

233.* Melilotus albus Desr.

234. M. indica All.

235.* M. officinalis Desr.

236. Ornithopus sativus Brot.

237.* Orobus vernus Bernh.

238.* Oxytropis pilosa D. C.

239. Phaseolus Mungo L.

240. Tetragonolobus purpureus Moench.

241.* Trifolium agrarium $\mathrm{L}$.

$242{ }^{*} \mathrm{~T}$. arvense $\mathrm{L}$.

243. T. maritimum Huds.

244.* T. montanum L.

245.* T. pratense L.

$246{ }^{*} \mathrm{~T}$. repens $\mathrm{L}$.

247. T. spadiceum L.

248. Trigonella coerulea Ser.

249. Securigera coronilla D. C. 
250. Sophora alopecuroides L.

251. Vicia americana Muhl.

252* V. cracca L.

$253 *^{*}$ V. silvatica $\mathrm{L}$.

254. V. macrocarpa Bert.

XXVII. Geraniacene.

255. Erodium cicutarium L'Herit.

256. E. Hoefftianum C. A. M.

XXVIII. Tropaeolaceae.

257. Tropaeolum majus $\mathrm{L}$.

$X X I X$. Linaceae.

258. Linum austriacum $\mathrm{L}$.

259. L. campanulatum L.

260. L. extraxillare Kit.

261. L. grandiflorum Desf.

262. L. perenne L.

263. L. perenne L. v. sibiricum D. C.

264. L. usitatissimum L.

$X X X$. Euphorbiaceae.

265. Ricinus communis $\mathrm{L}$.

XXXI. Empetraceae.

266. Empetrum nigrum L.

XXXII. Celastraceae.

267. Evonymus europaea L.

268.* E. verrucosa Scop. 
XXXIII. Aceraceae.

269. Acer ginnala Maxim.

270. A. Negundo L.

271.* A. platanoides L.

272. A. tataricum L.

XXXIV. Balsaminaceae.

273. Impatiens balsamina L. fl. plena alba hort.

274. I. b. f1. plena rosea hort.

275. I. b. fl. plena rubra hort.

XXXV. Rhamnaceae.

276.* Rhamnus Frangula L.

277. Rh. cathartica L.

XXXVI. Tiliaceae.

278.* Tilia cordata Mill.

279. T. plathyphyllus Scop.

XXXVII. Malvaceae.

280. Abutilon Avicennae Gaertn.

281. Althea officinalis L.

282. A. rhytidocarpa Trautv.

283. A. rosea Cav.

284. A. r. fl. alba hort.

285. A. r. fl. lutea hort.

286. A. r. fl. nigra hort.

287. A. r. fl. nitiduscula-rosea hort.

288. A. r. fl. rosea hort.

289. A. r. fl. rubra hort.

290. Anoda lavatheroides Med. 
291. Kitaibelia vitifolium Willd.

292. Lavathera thuringiaca L.

293. Malachra radiata $\mathrm{L}$.

294. Malva alcea L.

295. M. brasiliensis Desr.

296. M. crispa L.

297. M. flexuosa hort.

298. M. mauritiana L.

299. M. meluca hort.

300. M. moschata L.

301. M. pusilla Withering.

302. M. verticillata L.

303. Palana flexuosa Mast.

XXXVIII. Hypericaceae.

304. * Hypericum perforatum L.

XXXIX. Violaceae.

305. Viola arborescens L.

306. V. Bosniaca hort.

307. V. delphinifolia Nutt.

308* V. tricolor. L. v. arvensis Koch.

309. V. t. v. hybrida hort.

$X L$. Begoniaceae.

310. Begonia semperflorens Link. et Otto.

XLI. Lythraceae.

311. Cuphea viscosissima Jacq.

XLII. Onagraceae.

312.* Epilobium angustifolium L. 
313. Epilobium collinum Gmel.

314. * E. hirsutum L.

315. E. Lamyi Schultz.

316. Euch ridium grandiflorum Fisch. et Mey.

317. Oenothera biennis L.

XLIII. Umbelliferae.

318. Aegopodium Podagraria L.

319.* Angelica silvestris L.

320. Anethum graveolens Benth.

321* Anthriscus silvestris Hoffm.

322. Bupleurum rotundifolium $\mathrm{L}$.

323. Callipeltis cuccullaria hort.

324. Carum Carvi L.

325. Cicuta virosa L.

326.* Conium maculatum L.

327. Coriandrum sativum L.

328. Duucus Carota L

329.* Eryngium planum L.

330.* Libanotis montana All.

331. Pimpinella Anisum L.

332. Trinia Henningii Hoffm.

333. Verbesina enceloides Benth. et Hook.

XLIV. Vacciniaceae.

334. Vaccinium Vitis idaea L.

XLV. Primulaceae.

335. "Lysimachia vulgaris L.

336.* Primula officinalis $L$. 
XLVI. Oleaceae.

337. Fraxinus pensylvanica March.

338. Syringa vulgaris L.

XLVII. Convolvulaceae.

339. Convolvulus rhynchosepmus Hochst.

XLVIII. Polemoniaceae.

340. Phlox Drummondii Hook.

341. Gilia aggregata Spreng.

XLIX. Hydrophyllaceae.

342. Phacelia congesta hort.

343. Ph. loasaefolia Torr.

344. Ph, viscida Torr.

L. Borraginaceae.

345. Borrago officinalis L.

346. Cynoglossum officinale L.

LI. Verbenaceae.

347. Verbena hybrida hort.

LII. Labiatae.

348. Brunella pienina Ullepitsch.

349.* Brunella vulgaris L.

350. Dracocephalum Moldavica L.

351. Hyssopus officinalis L.

352. H. o. fl. coerulea hort.

353. Lallemantia ibericum Fisch. et Mey.

354. L. peltata Fisch. et Mey.

355. Leonurus glaucescens Bye. 
356. Leonurus marrubiastrum L.

357. Lophanthus anisatus Benth.

358. Melissa offìcinalis L.

359. Monarda fistulosa Poir.

360. Salvia Aethiopis L.

361. S. grandiflora Benth.

362. S. Horminum L.

363. S. officinalis L.

364. S. Przewalskii Max.

365. S. Sclarea L.

366. S. verticillata $\mathrm{L}$.

367. Stachys annua $\mathrm{L}$.

368. S. lanata Jacq.

369. S. nivea Benth.

370. S. palustris L.

371. S. plumosa Griseb.

LIII. Solanaceae.

372. Atropa Belladonna L.

373. Datura aegyptiaca Vesl.

374. D. Stramonium L.

375. Hyoscyamus agrestis Kit.

376.* H. niger $L$.

377. Nicandra physaloides Gaertn.

378. Nicotiana affinis hort.

379. N. rustica L.

380. N. Sanderae hort.

381. Petunia hybrida hort.

382. Physalis peruviana $\mathrm{L}$.

383. Salpiglossis variabilis hort, 384. Solanum nigrum $L$, 


\section{Scrophulariaceae.}

385.* Alectrolophus major Rchb.

386. Antirrhinum Coulterianum Benth.

387. A. majus L. fl. rubra hort.

388. A. m. fl. alba hort.

389. Celsia cretica L.

390. Digitalis lanata Ehrh.

391. D. lutea L.

392. D. purpurea L.

393. Linaria alpina Mill.

394. L. bipartita Willd.

395. L. chalepensis Mill.

396.* L. vulgaris Mill.

397. Nemesia foetans Vent.

398. Scrophularia aquatica L.

399.* S. nodosa $\mathrm{L}$.

400. Verbascum phlomoides L.

401. V. tapsiphorme Schrad.

402. Veronica spicata L.

$L V$. Orobanchaceae.

403. Orobanche Libanotidis Rupr.

LVI. Plantaginaceae.

404. Plantago arenaria W. et $\mathrm{K}$.

405.* P. lanceolata L.

406. P. major L.

407. P. Psyllium L.

408. P. tibetica Hook. et Thoms. 
LVII. Rubiaceae.

409. Asperula arvensis L.

410.* Galium boreale L.

411.* G. palustre L

412. G. purpureum L.

413. G. tricorne Stok.

LVIII. Caprifoliaceae.

414. Lonicera tatarica L.

415.* Viburnum Opulus L.

416. V. Lantana L.

LIX. Valerianaceae.

417. Valeriana nitida $\mathrm{Pr}$.

418. V. officinalis L.

419. V. rossica Sm.

LX. Dipsacaceae.

420. Cephalaria alpina Schrad.

421. C. tatarica Schrad.

422. Dipsacus fullonum Mill.

423. D. silvestris Huds.

424. D. strigosus Willd.

425. Scabiosa Olivieri hort.

LXI. Cucurbitaceae.

426. Cucumis Melo L.

427. C. sativus L.

428. Cucurbita Pepo L. 
LXII. Campanulaceae.

429. Adenophora denticulata Fisch.

430. Campanula carpatica Jacq.

431.* C. latifolia L.

432. C. Leutweinii Heldr.

433. C medium L.

434. C. ranunculoides $\mathrm{L}$.

435.* C. Trachelium L.

LXIII. Lobeliaceae.

436. Lobelia Erinus L.

437. L. E. v. compacta hort.

438. L. hybrida hort.

439. L. inflata L.

LXIV. Compositae.

440.* Achillea Millefolium L.

441. A. nobilis L.

442. Ageratum conyzoides L. v. Mexicanum.

443. Anacyclus officinarum Hayne.

444. Arctium Lappa L.

445. Arnica montana L.

446. Anthemis candidissima Willd.

447. A. cotula L.

448. A. nobilis L.

449.* Artemisia Absinthium L.

450 . $^{*}$ A. vulgaris L.

451. Bidens dahlioides Wats.

452. B. pilosa L.

453.* B. tripartitus $L$.

454. Calendula algeriensis Boiss, et Reut. 
455. Calendula officinalis L.

456. C. maroccana Ball.

457. C. stellata Cav.

458. Calliopis bicolor Rchb.

459. Carduus Marianum L.

460. Centaurea cyanus L.

461. C. stenolepis Kern.

462. C. sulphurea Willd.

463. Chrysanthemum corymbosum L.

464. Ch. Parthenium Bernh.

465. Cichorium Inthybus L.

466.* Cirsium arvense Scop.

467. Cnicus benedictus L.

468. Coreopis grandiflora Nutt.

469. Cosmos bipinnatus Cav.

470. Dimorphotheca pluvialis Moench.

471. Echinops Ritro L.

472. E. spherocephalus L.

473.* Erigeron canadensis L.

474. Eupatorium cannabina L.

475. Helianthus annus L.

476. H. bicolor hort.

477. Inula Helenium L.

478. Lactuca altissima M. B.

479. L. sativa L.

480. L scariola L.

481. L. virosa L.

482. Laya platyglossa A. Gray.

483. Leontodon asperrimus Boiss.

$484{ }^{*} \mathrm{~L}$. autumnalis L.

485. L. crispus Vill. 
486. Leontodon pyrenaicus Gouan.

487. L. saxatilis Lam.

488. Leucanthemum vulgare Lam.

489. Madia sativa Don.

490. Matricaria Chamomilla L.

491** M. discoidea D. C.

492. Onopordon acanthium L.

493. Picridium tingitanum Desf. et Hook.

494.* Picris hieracioides L.

495. Psephellus carthalinicus.

496. Senecio sonchifolius Moench.

497. Serratula coronata L.

II. SEMINA PLANTARUM SPONTANEARUM collectore a $P$. N. Smirnov in Kologriv regione (Gorkij prov.), anno 1936 lecta.

I. Polypódiaceae.

498. Athyrium filix femina Roth.

499. Dryopteris spinulosa O. Ktze.

500. Pteridium aquilinum Kuhn.

501. Struthiopteris germanica Kuhn.

II. Lycopodiaceae.

502. Lycopodium clavatum L.

503. L. complanatum L.

III. Cupressaceae.

504. Juniperus communis L. 


\section{Alismaceae.}

505. Alisma plantago L.

506. Saggittaria sagittifolia L.

V. Butomaceae.

507. Butomus umbellatus L.

VI. Gramineae.

508. Setaria viridis P. B.

VII. Cyperaceae.

509. Carex elongata $\mathrm{L}$.

510. C. Goodenowii Gay.

511. C. vesicaria L.

512. C. vulpina $\mathrm{L}$.

513. Eriophorum polystachyum L.

VIII. Orchidaceae.

514. Orchis maculata L.

IX. Polygonaceae.

515. Polygonum Convolvulus L.

516. Rumex confertus Willd.

$X$. Caryophyllaceae.

517. Dianthus superbus L.

518. Lychnis flos cuculi L.

519. Melandrium album Garcke.

520. Silene nutans L.

521. Stellaria Holostea L.

522. Viscaria viscosa $A_{\text {schers. }}$ 
XI. Ranunculaceae.

523. Aconitum excelsum Rchb.

524. Anemone nemorosa L.

525. A. ranunculoides $\mathrm{L}$.

526. Atragene sibirica Reg. et Til.

527. Caltha palustris L.

528. Ranunculus acer L.

529. R. repens L.

530. Thalictrum minus L.

XII. Papaveraceae.

531. Chelidonium majus $\mathrm{L}$.

XIII. Fumariaceae.

532. Corydalis solida Sm.

XIV. Crassulaceae.

533. Sedum maximum Sut.

$X V$. Saxifragaceae.

534. Chrysosplenium alternifolium L.

535. Ribes rubrum L.

XVI. Rosaceae.

536. Comarum palustre L.

537. Geum rivale L.

538. G. urbanum L.

539. Filipendula Ulmaria Maxim.

540. Fragaria vesca L. 
541. Prunus Padus L.

542. Rubus arcticus L.

543. R. Chamaemorus L.

544. R. idaeus L.

545. R. saxatilis L.

XVII. Leguminosae.

546. Lathyrus silvestris L.

547. Orobus vernus $L$.

548. Trifolium agrarium L.

549. T. spadiceum L.

550. Vicia cracca L.

XVIII. Geraniaceae.

551. Geranium pratense L.

XIX. Euphorbiaceae.

552. Euphorbia Esula L.

$X X$. Celastraceae.

533. Evonymus verrucosa Scop.

XXI. Balsaminaceae.

554. Impatiens noli tangere $\mathrm{L}$.

XXII. Rhamnaceae.

555. Rhamnus Frangula L.

XXIII. Guttiferae.

556. Hypericum parforatum L. 
XXIV. Violaceae.

557. Viola canina L.

558. V. tricolor L.

$X X V$. Thymelaeaceae.

559. Daphne Mezereum L.

$X X V I$. Onagraceae.

560. Chamenerium angustifolium Scop.

XXVII. Umbelliferae.

561. Angelica silvestris L.

562. Anthriscus silvestris Hoffm.

563. Heracleum sibiricum L.

564. Libanotis sibirica C. A. M.

565. Pimpinella Saxifraga L.

566. Carum Carvi L.

XXVIII. Pirolaceae.

567. Chimaphila umbellata Nutt.

568. Pirola rotundifolia $\mathrm{L}$.

569. Ramischia secunda Garcke.

XXIX. Ericaceae.

570. Andromeda polifolia L.

571. Arctostaphylos uva ursi Spr.

572. Ledum palustre L.

573. Vaccinium Myrtillus L.

574. V. uliginosum L. 
XXX. Polemoniaceae.

575. Polemonium coeruleum $\mathrm{L}$.

XXXI. Borraginaceae.

576. Myosotis caespitosa Schult.

XXXII. Labiatae.

577. Ajuga reptans L.

578. Origanum vulgare $\mathrm{L}$.

579. Stachys palustris L.

$X X X I I I$. Solanaceae.

580. Solanum Dulcamara L.

XXXIV. Scrophulariaceae.

581. Alectrolophus major Rchb.

582. Linaria vulgaris Mill.

583. Pedicularis palustris L.

584. Scrophularia nodosa L.

585. Verbascum Thapsus L.

XXXV. Caprifoliaceae.

586. Lonicera coerulea L.

587. L. Xylosteum L.

XXXVI. Valerianaceae.

588. Valeriana officinalis $\mathrm{L}$.

XXXVII. Dipsacaceae.

589. Knautia arvensis Coult. 
XXXVIII. Campanulaceae.

590. Campanula patula $\mathrm{L}$.

591. C. rotundifolia L.

$X X X I X$. Compositae.

592. Achillea Millefolium L.

593. Antennaria dioica Gaertn.

594. Artemisia vulgaris L.

595. Bidens tripartita L.

596. Centaurea scabiosa L.

597. Solidago virga aurea L.

598. Tanacetum vulgare $\mathrm{L}$.

599. Taraxacum officinale L.

III. SEMINA PLANTARUM GRANEARUM ET FABACEARUM IN HORTO BOTANICO CULTA.

1. Gramineae.

600. Avena sativa L. v. aristata Kr. „Zemchujina”. 601. A. s. a. „Ligovo”.

602. A. s. a. "Miraz".

603. A. s. a. „Moskovskoy A 315”.

604. A. s. a. $\mathrm{n}^{\circ} 06522$.

605. A. s. a. $\mathrm{n}^{\circ} 08115$.

606. A. s. a. $\mathrm{n}^{\circ} 08121$.

607. A. s. v. aurea Körn. „Zolotoy Dojd".

608. A. s. a. "Leitevizkiy".

609, A. s. "Lochovskiy”. 
610. Avena sativa "Stepniak 648". 611. A. s. v. mutica Al. „Pobeda”. 612. A. s. m. "Shatilovskiy". 613. A. s. v. obtusata Al. "Magistral". 614. Hordeum distichum L. v. abissinicum. 615. H. d. v. leocritum. 616. H. d. v. medicum Körn. $\mathrm{n}^{\circ} 26$. 617. H. d. m. Körn. n? 46. 618. H. d. v. nigricans. 619. H. d. v. nudum L. „Byloe”. 620. H. d. n. $n^{\circ} 105$. 621. H. d. n. $n^{\circ} 110$. 622. H. d. n. $\mathrm{n}^{\circ} 155$. 623. H. d. v. nutans Schübl. „Europaeum 313/133”. 624. H. d. n. „Viner”. 625. H. d. n. "Zolotoy Dojd". 626. H. d. n. colchicum. 627. H. d. n. $\mathrm{n}^{\circ} 17$. 628. H. d. v. pallidum Sir. 629. H. d. p. "Viatsky 1009". 630. H. d. p. "Chersonetz". 631. H. d. p. n 032. 632. H. d. p. $\mathrm{n}^{\circ} 045$. 633. H. d. v. parallelum. 634. H. d. v. persicum $\mathrm{n}^{\circ} 062$. 635. Panicum miliaceum contractum Al.v. aureum $n^{\circ} 01$. 636. P. m. c. v. dacicum Körn. $n^{\circ} 03$. 637. P. m. c. v. sanguineum $\mathrm{n}^{\circ} 0853$. 638. P. m. effusum Al. v. subcoccineum $\mathrm{n}^{\circ} 742$. 639. P. m. e. v. subflavum $\mathrm{n}^{\circ} 204$. 640. Triticum durum Desf. „Kubanka”. 
641. Triticum durum ,Tunika 22".

642. L. d. v. affine Körn. ${ }^{\circ} 309$.

643. T. d. a. $\mathrm{n}^{\circ} 769$.

644. T. d. v. cerulescens Bayle.

645. T. d. v. leucurum Al. B. G. 26.

646. T. d. 1. B. G. 33.

647. T. d. v. Libycum Körn.

648. T. d. v. hordeiforme Host. $\mathrm{n}^{\circ} 04$.

649. T. d. hordeiforme $\mathrm{n}^{\circ} 010$.

650. T. d. h. $\mathrm{n}^{\circ} 189$.

651. T. d. h. $\mathrm{n}^{\circ} 432$.

652. T. d. h. $\mathrm{n}^{\circ} 496$.

653. T. d. h. $\mathrm{n}^{\circ} 675$.

654. T. d. h. Tr. f. lutescens sorrubra.

655. T. d. v. melanopus Al. „Palestrina”.

656. T. d. m. $\mathrm{n}^{\circ} 069$.

657. T. d. m. „Blizkaja” et v. falcatum „Kipr 17853”.

658. T. d. m. $\mathrm{n}^{\circ} 886$.

659. T. d. v. murciense Körn.

660. T. d. v. Reichenbachii Körn.

661. T. dicoccum Schübl. v. rufum Schübl.

662. T. monococcum L.

663. T. vulgare Vill. v. albidum Al, $\mathrm{n}^{\circ} 0676$.

664. T. v. v. alborubrum Körn.

665. T. v. v. caesium Al. $\mathrm{n}^{\circ} 011$.

666. T. v. v. dicoccum.

667. T. v. v. erythrospermum.

668. T. v. v. e. Körn.

669. T. v. e. B. $n^{\circ} 047$.

670. T. v. e. „Zemna”.

671. T. v. e. „Kooperatorka”. 
672. Triticum vulgare e. "Novinka”.

673. T. v. e. „Ukrainka”.

674. T. v. e. $\mathrm{n}^{\circ} 82 / 02$.

675. T. v. e. $\mathrm{n}^{\circ} 341$.

676. T. v. e. $\mathrm{n}^{\circ} 841$.

677. T. v. e. $n^{\circ} 17854$ Pappar Payvntör (Kipr.).

678. T. v. v. ferrugineum Al. ,Moskovskaia 2411".

679. T. v. f. $\mathrm{n}^{\circ} 146$.

680. T. v. ferrugineum $n^{\circ} 150$.

681. T. v. v. graecum. Körn. P 80-5.

682. T. v. v. Hostianum Clem. $\mathrm{n}^{\circ} 237$.

683. T. v. v. lutescens Al. "Garnet”.

684. T. v. 1. „Kitchener”.

685. T. v. 1. "Leda”.

686. T. v. 1. "Marquis".

687. T. v. 1. $\mathrm{n}^{\circ} 062$.

688. T. v. 1. $\mathrm{n}^{\circ} 329$.

689. T. v. 1. $\mathrm{n}^{\circ} 479$.

690. T. v. 1. n $1060 / 10$.

691. T. v. $1, \mathrm{n}^{\circ} 1484$.

692. T. v. v. milturum Al.

693. T. v. m. A-40.

694. T. v. m. B. 80.

695. T. v. m. $\mathrm{n}^{\circ} 321$.

696. T. spelta L.

II. Leguminosae.

697. Cicer arietinum L.

698. Ervum lens L. v. dupnyensis Bar. Dw.

699. E. 1. v. macrosperma f. nummularia Al. „Dymchataia". 
700. Ervum lens macrosperma n. "Obiknovennaia”. 701. E. 1. m. n. „Saratovskaia n $120 ”$.

702. E. 1. m. n. "Saratovskaia n 140 ".

703. E. 1. m. n.' ",Tarelochnaia n 39 ".

704. E. 1. v. persica Bar. „Persidskaia”.

705. E. 1. v. pseudomarmorata Bar. „Rosovaia $44 / 3$ ".

706. E. 1. v. Pulmani Bar. „Dymchataia”.

707. E. 1. v. vulgaris Al. „Mironovskaia”.

708. Lathyrus sativus L. $\mathrm{n}^{\circ} 823$.

709. Lupinus albus L. $\mathrm{n}^{\circ} \mathrm{y}-26$.

710. L. angustifolius L. $\mathrm{n}^{\circ} 374$.

711. L. luteus L. $\mathrm{n}^{\circ} 530$.

712. L. mutabilis $\mathrm{n}^{\circ} 97$.

713. Phaseolus vulgaris L. v. ellipticus „Stennaia”.

714. $\mathrm{Ph}$. v. e. $\mathrm{XPh}$. sphaericus v. unicolus f, atropurpureus "Zolotaja Gora".

715. Pisum sativum L. v. coronatum Al. ,Schtampovij”.

716. P. s. v. glaucospermum "Victoria Zelenaja".

717. P. s. g. "Folger-Gayne”.

718. P. s. v. melillucum A1. „Bismark”.

719. P. s. v. vitellinum Al. „Voskovoj”.

720. P. s. v. vulgatum Körn. „Victoria Mondorf”.

721. P. s. v. "Victoria rozovaja".

722. P. s. v. "Kapital”.

723. Vicia Faba L. v. minor L. „Bournet”.

724. V, sativa L. var. typica Reg.

725. V. s. t. „Kolibri Schvedskaja”.

\section{Addenda.}

726. Fragaria vesca L.

727. Heracleum villosum Fisch. (ex Tauria). 
Е ПЛАтно

\section{ПЕРЕЧЕНЬ СЕМЯН .} СОБРАННЫХ В 1936 ГОДУ

И ПРЕДЛАГАЕМЫХ В ОБМЕН БОТАНИЧЕСКИМ САДОМ ГОРЬКОВСКОГО ГОСУДАРСТВЕННОГО УНИВЕРСИТЕТА

\section{g(b)}

ГОРЬКОВСКОЕ ИЗДАТЕЛЬССТВО 1937 


\section{DELECTUS SEMINUM}

ANNO MCMXXXVII COLLECTORUM

QUAE

\section{HORTUS BOTANICUS}

UNIVERSITATIS GORKIJENSIS

PRO

MUTUA COMMUTATIONE

OFFERT

GORKIJ MCMXXXVAR I TOng 



\section{SEMINA PLANTARUM HERBACEUM.}

I. Polypodiaceae.

1.* Athyrium crenatum Rupr. ${ }^{1}$

2. A. filix femina Roth.

3. Polystichum Braunii Fée.

4. Cystopteris fragilis Bernh.

5.* Dryopteris filix mas (L.) Schott.

6.* Dryopteris Linneana Christ.

7.*D. phegopteris Christ.

8* D. spinulosa O. Ktze.

9.*D. thelypteris A. Gray.

10.* Pteridium aquilinum Kuhn.

II. Gramineae.

11. Agropyrum desertorum Fisch.

12. Alopecurus lasiostachys Link.

$13 .{ }^{*} \mathrm{~A}$. ventricosus Pers.

14. Andropogon ischaemum L.

15. A. i. ssp. Wilcommi hort.

16. Aristida adscensionis L.

17. A. plumosa L.

18. Avena elatior L.

19. Boutelona racemosa Lag.

1 Genera et species in ordine alphabetico disposite sunt.

* Plantae spontaneae. 
20.* Brachypodium silvaticum P. B.

21.* Bromus inermis Leyss.

22. B mollis L.

23. B. unioloides Humb.

24. Chaeturus fasciculatus Link.

25. Dactylis Aschersoniana Graebn.

26. D. glomerata L.

27. D. hispanica Koch.

28.* Deschampsia caespitosa P. B.

29. D. flexuosa Trin.

30. Elymus akmolensis Drob.

31. E. dahuricus Turcz.

32. E. excelsus Turcz.

33. E. sibiricus L.

34. Hordeum bulbosum L.

35. H. jubatum L.

36. Lolium canadense Micht.

37. Melica altissima L.

38. M. transsilvanica Schur.

39.* Milium effusum L.

40. Phalaris paradoxa L.

41. Phleum alpinum $\mathrm{L}$.

42. P. ambigum Griesb.

43. P. Michelii All.

44.* $\mathrm{P}$. nodosum $\mathrm{L}$.

45.* P. pratense L.

46. Poa alpina L.

47. P. bulbosa $L$.

48.* Setaria viridis P. B.

49. Trisetum distichophyllum P. B.

50.* Triticum repens $\mathrm{L}$. 
III. Alismaceae.

51. Alisma plantago L.

IV. Liliaceae.

52. Allium albidum Fisch.

53.* A. angulosum $\mathrm{L}$.

54. A. fistulosum L.

55. A. montanum F. W. Schm.

56. A. odoratum L.

57. A. paradoxum G. Don.

58. Bulbine annua Willd.

59.* Convallaria majalis L.

60. Smilacina stellata Frist.

V. Urticaceae.

61. Urtica Dodarti L.

62. Parietaria officinalis $L$.

VI. Polygonaceae.

63.* Polygonum Hydropiper L.

64. Rheum Emodi Wall.

65. R. Rhaponticum L

66.* Rumex acetosa L

67. R. acetosella L.

68. R. callosus Reidringer.

69.* R. confertus Willd.

70. R. hydrolapathum Huds.

71. R. hymenosepalus Torr.

72. R. mexicanus Meiss.

73. R. microcarpus Brychn. 
74. Rumex nepalensis Spreng.

75. R. pallidus Bigch.

76. R. stenophyllus Ledeb.

77. R. thyrsiflorus Fingerh.

\section{Chenopodiaceae.}

78.* Chenopodium album L.

79. C. aristatum L.

80. C. Bonus-Henricus L.

81. C. botrys L.

82. C. capitatum Aschers.

83. C. quinoa Willd. (1936).

84.* Corispermum hyssopifolium L.

VIII. Amaranthaceae.

85. Amaranthus aurea T. A. Desf.

86. A. blitum L.

87. A. caudatus $L$.

88. A. lividus $\mathrm{L}$.

89. Celosia cristata L. v. sanguinea hort.

90. Gomphrena Haageana Klotszch.

IX. Nyctaginaceae.

91. Mirabilis jalapa $\mathrm{L}$.

92. Oxybaphus nyctagineus Sweet.

X. Portulacaceae.

93. Calandrinia elegans Schrd.

94. C. grandiflora Lindl.

95. Talinum pratens Willd. 
XI. Phytolaccaceae.

96. Phytolacca decandra L.

XII. Caryophyllaceae.

97. Cerastium tomentosum L.

98. Dianthus barbatus L.

99. D. callizornus Schott. et Kotschy.

100. D. capitatus D. G.

101. D. caryophyllus L,

102. D. cruentus Griseb.

103. D. cyri F. et Mey.

104. D. gallicus Pers.

105. D. giganteus Urv.

106. D. hungaricus Pers.

107. D. tatrae Borb.

108. Gypsophila acutifolia Fisch.

109. G. elegans M. B.

110. G. e. v. grandiflorum hort.

111. G. perfoliata $\mathrm{L}$.

112. G. repens $\mathrm{L}$.

113. G. Stevenii Fisch.

114. Lychnis chalcedonica L.

115. L. coeli-rosea Desf.

116. L. haageana Lemaire.

117. L. flos-cuculi L.

118.* Melandrium album Garcke.

119. M. noctifolium Fr.

120. M. dioceum Sch. et Thell.

121. Saponaria calabrica Guss.

122. S. cerastiodies Fisch. 
123. Saponaria officinalis L.

124. Silene armeria L.

125. S. Bergiana Lindm.

126. S. conica L.

127. S. cucubalus Wiebl.

128. S. gallica L.

129. S. grandiflora Franch.

130. S. Hornemmannii Steud.

131. S. lacta A. Br.

132. S. maritima With.

133. S. mellifera Boiss. et Reut.

134. S. muscipula L.

135.* S. nutans L.

136 S. pseudo-otites Bess.

137. S. venosa Aschers.

138. Tunica Saxifraga Scop.

XIII. Ranunculaceae.

139.* Aconitum excelsum Rchb.

140. A. Lycoctonum L.

141. Anemone decapetala L.

142. A. reflexa Steph.

143. A. tetrasepala Royle.

144. Aquilegia canadensis L.

145. A. flabellata Sieb. et Zucc.

146. A. olympica Boiss.

147. A. Skinneri Hook.

148. A. vulgaris L.

149. A. v. v. nivea.

150. Delphinium cheilanthum Fisch.

151.* D. consolida L. 
152. Delphinium elatum L.

153. D. e. v. alpinum Hegi.

154. D. e. v, tirolense Hegi.

155. D. hybridum Steph.

156. D. h. „Prinz Gustav” hort.

157. D. Maakkianum hort.

158. D. trolleifolium A. Gray.

159. Isopyrum fumarioides $\mathrm{L}$.

160. Nigella arvensis L.

161. N. damascena $L$.

162. N. sativa L.

163. Ranunculus abortivus $\mathrm{L}$.

164. R. bruticus L.

165.* R. cassubicus L.

166. R. dissectus B. M. v, grandiflora C. A. M.

167. R. muricatus L.

168. R. trilobus Desf.

169. R. Villarsii DC.

170. Thalictrum elatum Jacq.

171. T. glaucum Desf.

172. ${ }^{*}$ T. minus L.

XIV. Papaveraceae.

173. Adlumia cirrhosa Raf.

174. Bocconia cordata Willd.

175. B. japonica Andre.

176. B. microcarpa Maxim.

177. Chelidonium laciniatum Mill,

178 * C. majus L.

179. Escholtzia californica Cham.

180. Glaucium corniculatum Curt. 
181. Glaucium flavum Crantz.

182. Papaver alpinum $L$.

183. P. bracteatum Lindl.

184. P. dubium L.

185. P. nudicaule L.

186. P. orientale $\mathrm{L}$.

187. P. Rhoeas L.

188. P. somniflorum L.

$X V$. Fumariaceae.

189. Corydalis nobilis Pers.

190. C. semerflorens Pers.

XVI. Cruciferae.

191. Aethionema Buxbaumii D. C.

192. A. pulchellum Boiss.

193. Alyssum alpinum Scop.

194. A. campestre L.

195. A. desertorum Stapf.

196. A. montanum L.

197. A. lenense D. C.

198. A. Szoavitsianum F. et $M$.

199. Barbarea orthoceras Ledeb.

200. Biscutella auriculata L.

201. B. laevigata L.

202. Brassica juncea Czern.

203. B. nigra Koch.

204. Camelina dentata Wallr.

205. Capsella Bursa pastoris L.

206. Carrichtera vella D. C.

207. Cheiranthus Allionii Hort. 
208. Cochlearia officinalis $\mathrm{L}$ :

209. Crambe abissinica Kochst.

210. C. hispanica L.

211. C. Koctebelica Bush.

212. C. maritima L.

213. Erucastrum arabicuin Fisch. et Mey.

214. Erysimum canenscens Roth.

215. E. cuspidatum D. C.

216. E. ochroleucum D. C.

217. E. perovskianum Fisch. et Mey.

218. E. pulchellum J. Gay.

219. Hesperis alpina Schur.

220. H. dinarica G. Beck.

221. H. fragrans Fisch.

222. H. matronalis L.

223. H. violacea Boiss.

224. Huntschinsia petraea Ait.

225. Iberis amara $\mathrm{L}$.

226. I. coronaria D. Don.

227. I. intermedia Guers.

2228. I. odorata L.

229. I. pectinata Boiss.

230. Isatis alpina All.

231. I. glauca Boiss.

232. I. tinctoria $\mathrm{L}$.

233. I. t. v. campestre Koch.

234. Lepidium cordatum Willd.

235. Malcomia maritima $\mathrm{R}$. Br.

236. Peltaria turcmena Lipsky.

237. Rapistrum rugosum All.

238. Sinapis alba L. 
239. Sinapis arvense L.

240. Sisymbrium officinale Scop.

241 * S. Thalianum J. Gay.

$242{ }^{*}$ Thlaspi arvense L.

243. T. densiflorum Boiss. et Ky.

244. Turritis glabra L.

XVII. Resedaceae.

245. Reseda alba $L$.

246. R. lutea L.

XVIII. Crassulaceae.

247. Sedum anapetalum D. C

248. S. hybridum L.

249. S spurium M. B.

250. S. s. v. splendens hort.

251. Sempervirum ruthenicum Koch (plant. juv.).

252* S. soboliferum Sims (plant. juv.).

$X I X$. Saxifragaceae.

253. Heuchera cylindrica Dougl.

254. H. glabra Willd.

255. H. hispida Pursh.

256. H. pubescens Pursh.

257. Mitlella pentandra Hook.

258. Tiarella cordifolia L.

XX. Rosaceae.

259. Agrimonia pilosa Led.

260. A. Eupatoria L.

261. Filipendula hexapetala Gilib. 
262. Filipendula Ulmaria Maxim.

263. Fragaria indica Andr.

264. G. Eupatorium L. sp. odorata hort.

265. G. japonicum Thunb.

266. G. macrophyllum Willd.

267. G. tyrolense Kern.

268. G. urbanum L.

269. G. virginianum L.

$270{ }^{*}$ Potentilla aurea $\mathrm{L}$.

271. P. argentea L.

272. P. chrisantha Trev.

273. P. gracilis Dougl.

274. P. rupestris $\mathrm{L}$.

275. P. splendens Ram.

276. Poterium canadense A. Gray.

277. P. Sanguisorba L.

278. P. sitchense S. Wats.

279. Sanguisorba minor Scop.

280. S. officinalis L.

281. S. tenuifolia Fisch.

$X X I$. Leguminosae.

282. Anthyllis tetraphylla L.

283. Astragalus canadensis L.

284. A. Cicer L.

285. A. galegiformis $\mathrm{L}$.

286. A. lacticus $\mathrm{L}$.

287. Baptisia australis R. Br.

288. Cicer arietinum L.

289. Desmodium canadense D. C.

290. Galega officinalis L. 
291. Galega orientalis Lam.

292. Glycine max. Merr.

293.* Lathyrus latifolius L.

294. L. montanus Bernh.

295. L. odoratus L.

296. Lotus ornithopodioides L.

297. Lupinus elegans $H$. B. et $K$.

298. Medicago coerulea Less.

299.* M. falcata L.

300. M. hispida Gaertn.

301. M. radiata $L$.

302. M. scutellaria All.

303. M. indica All.

304. Melilotus coeruleus Desr.

305.* M. officinalis Desr.

306. Onobrychis altissima L.

307. O. arenaria D. C.

308. O. montana D. C.

309. Ononis arvensis $\mathrm{L}$.

310 * O. hircina Jacq.

311. O. spinosa $L$.

312. Ornithopus sativus Brit.

313. O. perpusillus L.

314. Securigera coronilla D. C.

315. Tetragonolobus purpureus Moench.

316.* Trifolium agrarium L.

317. T. alpestre L.

318.* T. arvense $\mathrm{L}$.

319. T. Lupinaster L.

320. T. maritimum Huds.

321.* T. montanum L. 
322. Trifolium pratense L.

323. T. rubens $\mathrm{L}$.

324. Trigonella coerulea Ser.

325. Vicia americana Muhl.

326. V. macrocarpa Bert.

327* V. pisiformis L.

XXII. Geraniaceae.

328. Geranium albanum Bieb.

329. G. albiflorum Hook.

330. G. dissectum L.

331. $\mathrm{G}$. pratense $\mathrm{L}$.

332. G. tuberosum L.

333. Erodium cicutarium L.'Herit.

334. E. Hoefftianum C. A. M.

XXIII. Linaceae.

335. Linum altaicum Fisch.

336. L. campanulatum L.

337. L. grandiflorum Desf.

338. L. perenne L. v. sibiricum D. C.

XXIV. Malvaceae.

339. Abutilon Avicennae Gartn.

340. Althaea cannabina L.

341. A. kurdica Schl.

342. A. rhytidocarpa Trautv.

343. A. rosea Cav.

344. A. r. fl. albo hort.

345. A. r. fl. luteo hort.

346. A. r. fl. nigra hort. 
347. Althaea rosea fl. rosea Hort. 348. A, r. fl. rubra hort.

349. A. sulphurea Boiss. et Hohen.

350. Anoda lavatheroides Med.

351. A. hastata Cav.

352. Hibiscus Trionum L.

353. Kitaibelia vitifolium Willd.

354. Lavatera assurgentiflora Kellog.

355 . L. thuringiaca L.

356. L. trimestris L.

357. Malachra radiata $\mathrm{L}$,

358. Malva alcea L.

359. M. brasilensis Desr.

360. M. crispa L.

361. M. moschata L.

362. M. pusilla Withering.

363. $M$. verticillata $L$.

364. Polana flexuosa Mast.

365. Sidalcea oregona A. Gr.

$X X V$. Cistaceae.

366. Helianthemum polyfolium D. C.

367. H. pulverulentum D. C.

368. H. roseum Lam. et D. C.

369. H. variabile Spach.

XXVI. Hypericaceae.

370. Hypericum Ascyron L.

371. H. erectum Thunb.

372. H. hirsutum L.

373.* H. perforatum L. 
374. Hypericum tetrapterum Fries.

375. $\mathrm{H}$. tomentosum $\mathrm{L}$.

\section{Violaceae.}

376. Viola arborescens L.

377. V. Bosniaca hort.

378. V. tricolor L.

379.* V. arvensis Koch.

XXVIII. Lythraceae.

380. Cuphea viscosissima Jaq.

381. Lythrum alatum Pursh.

$X X I X$. Onagraceae.

382. Clarkia elegans Dougl.

383. C. pulchella Pursh.

384. Epilobium angustifolium L.

385. E. Lamyi Schultz.

386. Oenothera fruticosa L.

387. O. missouriensis Semp.

388. O. speciosa Nutt.

$X X X$. Umbelliferae.

389.* Aegopodium podagraria L.

390.* Angelica silvestris L.

391. Ammi majus L.

392. Bupleurum filicaule Brot.

393. B. petraeum L.

394. Coriandrum sativum L.

395. Didiscus pilosus Benth.

396.* Eryngium planum L.

397. E. oliverianum Delar. 
398. Ferula sulcata Desf.

399. Libanotis montana All.

400. Trinia vulgaris D. C.

\section{Primulaceae.}

401. Lysimachia vulgaris $L$.

402. Primula lejcophylla Pax.

403. P. officinalis L.

404. P. o. fl. luteo hort.

405. P. o. fl, rubro hort.

XXXII. Asclepiadaceae.

406. Asclepias phytoloccoides Pursh.

407. A. syriaca L.

XXXIII. Polemoniaceae.

408. Gilia aggregata Spreng.

409. G. capitata Dougl.

410. Polemonium boreale Adams.

411. P. campanulatum Fr.

412. P. gracile Willd.

413. P. grandiflorum Benth.

414. P. himalayanum Baker.

415. P. mexicanum Cerv.

416. P. occidentale Greene.

417. P. pulchellum Bungl.

418. P. Richardsonii R. Grah. v. humilis Gray.

419. P. sibiricum D. Don.

420. P. Var-Bruntiae Britt.

$X X X I V$. Hydrophyllaceae.

421. Nemophila insigne Bent. 
422. Phacelia campanularia A. Gray.

423. P. congesta Hook.

424. P. loasaefolia Torr.

425. P. Whitlavia A. Gray.

$X X X V$. Borraginaceae.

426. Anchusa officinalis L.

427. Borrago laxiflora Willd.

428. Cerinthe alpina Rit.

429. Cynoglossum officinale $\mathrm{L}$.

430. Echium creticum L.

431. E. rubrum Jaques.

432. Lithospermum officinale L.

433. Myosotis alpestris F. W. Schmidt.

434. Symphytum asperum Lepech.

XXXVI. Verbenaceae.

435. Verbena urticifolia L.

XXXVII. Labiatae.

436. Betonica grandiflora St.

437. Ballota nigra L.

438. Brunella grandiflora Moench.

439. B. pienina Ullepitsch.

440 ** B. vulgaris $\mathrm{L}$.

441. Dracocephalum Moldavica L.

442. Hyssopus officinalis L.

443. Lallemantia canescens Fisch. et Mey.

444. L. peltata Fisch. et Mey.

445.* Lamium maculatum L.

446. Leonurus glaucescens Bge. 
447. Leonurus marrubisatrum L.

448. Lophanthus anisatus Benth.

449. Lycopus europaeus L.

450. Marrubium vulgare $L$.

451. Monarda fistulosa L.

452. M. violacea Desf.

453. Nepeta azurea R. Br.

454. N. cataria L.

455. N. grandiflora MB.

456. N. macrantha Fisch.

457. N. nuda L

458. N. n ssp. pannonica Gams.

459.* Origanum vulgare L.

460. Phlomis chrysophylla Boiss.

461. Ph. tuberosa L.

462. Ph. viscosa Poir.

463. Salvia pratensis L.

464. S. Przewalskii Max.

465. S. trians Royle.

466. S. verticillata L.

467. S. hortensis L.

468. Scutellaria altissima L.

469. Sideritis hyssopifolia L.

470. S. scordioides L.

471. Stachys lanata Jacq.

472. S. nivea Labill.

473. S. grandiflora Benth.

474. S. officinalis Trety.

475. S. recta L.

476. Teucrium scorodonia L.

477. Ziziphora capitata L. 


\section{Solanaceae.}

478. Atropa Belladonna L.

479. Datura aegiptiaca Vesl.

480. D. Stramonium L.

481. Hyoscyamus agrestis Rit.

482. H. aurens $\mathrm{L}$.

483. $\mathrm{H}$. niger $\mathrm{L}$.

484. Nicandra physaloides Gaertn.

485. Nicotiana rustica L.

486. Physalis peruviana $\mathrm{L}$.

487. P. Franchetti Mast.

488. Salpiglossis variabilis hort.

489. Scopolia lurida Dun.

490.* Solanum dulcamara L.

491* S. nigrum L.

XXXIX. Scrophulariaceae.

492.* Alectrolophus major Kehb.

493. Antirrhinum Coulterianum Benth.

494. A. majus L.

495. A. orontium L.

496. Celsia cretica L.

497. Digitalis ambigua Murr.

498. D. lutea L.

499. D. media Roth.

500. D. nevadensis Kunze.

501. Linaria alpina Mill.

502. L. bipartita Willd.

503. Linaria chalepensis Mill.

504. L. genistefolia Mill.

505. L. purpurea Mill. 
506.* Linaria vulgaris Mill.

507. Mimulus luteus L.

508. Pentestemon alpinus Torr.

509. P. cobaea Nutt.

510. P. digitalis Nutt.

511. P. hirsutus Willd.

512. P. laevidatus Soland.

513. P. ovatus Dougl.

514. P. Watsonii A. Gray.

515.* Scrophularia alata Gilb.

516. S. chrysantha Jaub. et Spach.

517. Verbascum lychnitis L.

518. V. 1. "Miss Willmott" hort.

519. V. phlomoides L.

520. V. nigrum L.

521. V. phoeniceum L.

522. V. Wilsonii hort.

523. Veronica candida Lood.

524. V. orchidea Crantz.

525.* V. spicata L.

526. V. spuria L.

527. Wulfenia carinthiaca Jacq.

$X L$. Orobanchaceae.

528. Orobanche libanotidis Rupr.

XLI. Plantaginaceae.

529. Plantago arenaria W. et $\mathrm{K}$.

530. P, asiatica L.

531. P. kamtschatica Hook.

532. P. major L. 
533. Plantago Rugelii Dcne.

534. P. purpurascens Willk.

535. P. Schwarzenbergiana Schur.

536. P. serpentina Link.

537. P. tibetica Hook et Shows.

XLII. Rubiaceae.

538. Asperula arvensis L.

539. A. ciliata Rochel.

540. Crucianella angustifolia L.

541." Galium boreale L.

542. G. palustre L. (1936).

543. G. purpureum L.

544. G. tricorne Stok (1936).

545. Ophiorrhiza Mungos. L.

546. Rubia tinctorum L.

XLIII. Valerianaceae.

547. Patrinia intermedia Room. et Schult.

548. Valerianella coronata D. C.

XLIV. Dipsacaceae.

549. Cephalaria alpina Schrad.

550. C. ambrosioides R. et Sch.

551. Dipsacus fullonum Mill.

552. D. silvestris Huds.

553. D. strigosus Willd.

554. Knautia orientalis L.

555. Scabiosa atropurpurea $L$.

556. S. fumarioides Vis. et Ponc.

557. S. maritima L. 
558. Scabiosa micrantha Desf.

559. S. olivieri Coult.

560. S. pyrenaica All.

561. S. stellata L.

$X L V$. Campanulaceae.

562. Adenophora denticulata Fisch.

563. Campanula alliariaefolia Willd.

564. C. amabilis Leichtl.

565. C. americana L.

566. C. barbata L.

567. C. caespitosa Scop.

568. C. carpathica Jacq. fl. albo hort.

569. C. c. fl. cooruleo hort.

570. C. collina Bieb.

571. C. lactiflora Bieb.

$572{ }^{*}$ C. latifolía L.

573. C. Lentwennii Heldr.

574. C. medium L.

575. C. Morettiana Reichb.

576. C. rapunculoides L.

577. C. sarmatica Ker.-Gawl.

578. C. Scheuchzeri Vill.

579. Platicodon grandiflorum A. D. C.

580. IVahlenbergia lobelioides Link.

XLVI. Compositae,

581. Achillea distans $W$. et $K$.

582. A. nobilis L.

583. Anaphalis margaritacea Benth. et Hook. 584. Anthemis altissima L. 
585. Anthemis cotula L.

586. A. macedonica Boiss.

587. A. parnassia Nym.

588. A. ruthenica Bieb.

589. Arctium lappa L.

590. Arnica montana L.

591. Artemisia lanata Willd.

592. Aster altaicus Willd.

593. A. amethystinus Nutt.

594. A. incisus Fisch.

595. A. Maackii Regel.

596. A. Novae-Belgii L.

597. A. peregrinus Pursh.

598. Astericus maritimus Less.

599. Bidens dahlioides S. Wal.

600.* B. tripartitus L.

601. Calendula aegyptiaca L.

602. C. algeriensis Boiss. et Bent.

603. C. arvensis L.

604. C. maroccana Bull.

605. C. stellata Cav.

606. Calliopsis bicolor Rchb.

607. Carduus Marianum L.

608. Centaurea alpina L.

609. C. cyanus L.

610* C. jacea L.

611. C. montana L.

612. C. nigra L.

613.* C. ruthenica Lam.

614. C. scabiosa L.

615. C. sulphurea Willd. 
616. Chrysanthemum alpinum L.

617. C. arcticum L.

618. C. caucasicum Pers.

619. C. coronarium L.

620.* C. leucanthemum L.

621. C. maximum Ramond.

622.* Cichorium Inthybus L.

623.* Cirsium arvense Scop.

624. Cnicus benedictus L.

625. Coreopsis bicolor Reichb.

626. C. grandiflora Wutl.

627. C. pubescens Ell.

628. C. tinctoria Nutt.

629. Crepis rubra L.

630. Dimorphotheca annua Less.

631. D. aurantiaca D. C.

632. D. pluvialis Moench.

633. Doronicum pardolianches L.

634. Dracopis amplexicaulis Cass.

635.* Erigeron acer L.

636.* E. canadensis L.

637. E. coulteri Porter.

638. Eupatorium cannabina L.

639. Gailardia aristata Pursh.

640. G. lanceolata Michs.

641. Grindelia robusta Nutt.

642. Helianthus annuus $L$.

643. H. bicolor hort.

644. Heliopsis laevis Pers.

645. Helipterum roseum Benth.

646. $\mathrm{H}$. foliosum Waldst. et Kit. 
647. Helipterum pulmonarioides Will.

648. H. rigidum Hartm.

649. H. robustum Fr.

650. Hypochaeris radicata $L$.

651. Inula macrocephala Boiss. et Kotschy.

652. I. magnifica Lipsky.

653. Jurinea alata Cass.

654. Lactuca altissima H. B.

655. L. scariola L.

656. L. sativa $\mathrm{L}$.

657. L. virosa L.

658. Laya platiglossa A. Gray.

659.* Leontodon autumnalis $\mathrm{L}$.

660 . L. crispus Will.

661. L. pyrenaicus Gown.

662. L. saxatilis Lam.

663. Matricaria chamomilla L.

664.* M. discoidea D. C.

665. M. eximia hort

666. Onopordon acanthium $L$.

667. Picridium tingitanum Desf. et Kit.

668. Pulicaria dysenterica Gaerth.

669. Pyrethrum carneum Bieb.

670. P. niveum Zag.

671. P. corymbosum Willd.

672. $\mathrm{P}$. roseum $M$. B.

673. P. parteniium Sm.

674. Rudbeckia laciniata L.

675. Sanvitalia procumbens Lam.

676. Senecio nemorensis L.

677. S. sonchifolius Moench. 
678.* Serratula coronata L.

679. Silphium perfoliatum L.

680. Solidago canadensis L.

681. S. nemoralis A. H.

682.* S. virga aurea L.

683. Sonchus arvensis $L$.

684. Statice Gmelini Willd. Dlumbaginacea

685. Tagetes erecta $\mathrm{L}$.

686. T. lucida Cav.

687.* Tanacetum vulgare L.

688. Taraxacum officinale Weber.

689. Tolpis barbata Gaertn.

690. Tragopogon brevirostis D. C.

691. T. major Jacq.

692. T. orientalis L.

693.* T. pratensis L.

694. Tridax trilobata Hemsl.

695. Troximon gracilis Gray.

696. Xeranthemum annum $\mathrm{L}$.

697. Zinnia uniflora L.

II. SEMINA PLANTARUM SPONTANEARUM A VARIIS LOCALITATIS IN DIVERSIS REGIONIBUS COLLECTA.

1. J. I. Cos -in Naltschic regione CabardinoBalcariae Reipublicae.

I. Polypodiaceae.

698. Scolopendrium vulgare Sm. 
II. Ranunculaceae.

699. Thalictrum minus $\mathrm{L}$.

III. Leguminosae.

700. Ononis hircina Jacq.

IV. Malvaceae.

701. Althea ficifolia Cav.

V. Convolvulaceae.

702. Convolvulus silvaticus Waldst. et Kit.

VI. Gentianaceae.

703. Erythrea centaurium Pers.

VII. Labiatae.

704. Betonica officinalis L.

705. Salvia verticillata $\mathrm{L}$.

706. Teucrium Chamaedrys L.

707. T. Polium L.

VIII. Compositae.

708. Centaurea ovina Pall.

709. Inula cordata Boiss.

710. I. Helenium L.

2. A. D. Smirnova-in Pjanae fluvii regione, Gorkij prov.

1. Araceae.

711. Calla palustris L. 
II. Liliaceae.

712. Paris quadrifolia L.

III. Caryophyllaceae.

713. Melandrium album Garcke.

IV. Ranunculaceae.

714. Aconitum excelsum Rchb.

715. Actaea spicata L.

$V$. Leguminosae.

716. Vicia angustifolia Roth.

717. V. sepium L.

718. V. silvatica L.

VI. Thymelaeaceae.

719. Daphne Mezereum L.

VII. Umbelliferae.

720. Heracleum sibiricum L.

VIII. Primulaceae.

721. Primula officinalis $\mathrm{L}$.

IX. Borraginaceae.

722. Cynoglossum officinale L.

$X$. Labiatae.

723. Clinopodium vulgare $\mathrm{L}$.

724. Dracocephalum Ruyschiana L.

28 
XI. Solanaceae.

725. Solanum Dulcamara L.

XII. Compositae.

726. Picris hieracioides $\mathrm{L}$.

727. Sonchus arvensis L.

3. E.J. Tscherbakova-per clivum septentrionalem montis Alatau-

Kirgisiensis, duo vel tria milia in altum super planitiem maris (prope urb. Frunze).

I. Gramineae.

728. Alopecurus platensis L.

729. Dactylis glomerata L.

730. Koeleria gracilis Pers.

731. Poa pratensis L.

732. Festuca Ganeschini Drob.

733. Phleum phleoides Simk.

II. Liliaceae.

734. Tulipa Sinaidae Vved.

735. T. dasystemon Rgl.

III. Polygonaceae.

736. Polygonum polymorphum Lab.

737. Rheum Wittrockii Zundster. 
IV. Caryophyllaceae.

738. Silene tenuis Willd.

739. S. venosa Aschers.

$V$. Ranunculaceae.

740. Trollius alataicus C. A. M.

741. Hegemone lilacina Bge.

742. Aconitum songoricum Stapf. f. nudum.

743. A. songoricum Stapf. f. pubescens.

744. A. rotundifolium Kar. et Kir.

745. A. nemorum M. Pop.

746. Anemone narcissiflora L.

747. A. albana Stev.

748. Ranunculus Alberti Rgl.

749. R. Severzovii Rgl.

750. Aquilegia Karelini O. et B. Fedtsch.

VI. Papaveraceae.

751. Papaver nudicaule L.

VII. Leguminosae.

752. Trifolium platense L.

753. Onobrychis arenaria D. C.

VIII. Umbelliferae.

754. Angelica brevicaulis B. Fedtsch.

IX. Primulaceae.

755. Primula algida Adam.

756. P. Kaufmanniana Rgl. 
$X$. Gentianaceae.

757. Gentiana aurea L.

$X I$. Labiatae.

758. Nepeta nuda L.

759. Phlomis oreophila Kar. et Kir.

760. Origanum vulgare L.

XII. Scrophulariaceae.

761. Verbascum songoricum Schrink.

762. Veronica spicata L. .

763. V. spuria L.

XIII. Campanulaceae.

764. Campanula glomerata L.

$X I V$. Compositae.

765. Ligularia macrophylla D. C.

766. Achillea filipendulina Lam.

\section{SEMINA PLANTARUM LIGNOSARUM ET FRUTICUM.}

I. Cupressaceae.

767. Thuja occidentalis $\mathrm{L}$.

II. Betulaceae.

768.* Alnus glutinosa Gaertn.

769. A. incana Moench. f. acuminata Reg. 
770. Betula lutea Michx.

771.* B. verrucosa Ehrh.

772.* Corylus Avellana L.

III. Fagaceae.

773.* Quercus pedunculata Ehrh.

IV. Ulmaceae.

774. Ulmus campestris L (ex Tauria - leg. N. M. Tschernova).

775.* Ulmus pedunculata Foug.

776.* U. scabra Mill.

$V$. Ranunculaceae.

777.* Atragene sibirica Reg. et Til.

VI. Saxifragaceae.

778.* Ribes nigrum L.

779. R. rubrum L.

VII. Rosaceae.

780. Amelanchier vulgaris $\mathrm{L}$.

781. Crataegus monogyna Jacq.

782. Physocarpus opulifolius Ledeb.

783. Prunus divaricata Ledeb.

784.* P. Padus L.

785.* Rubus arcticus L.

786.* R. idaeus L.

787.* R. saxatilis $\mathrm{L}$.

788.* Sorbus Aucuparia L.

789. Spiraea japonica L. 
790. Spiraca j. v. pumila.

791. S. salicifolia L.

VIII. Leguminosae.

792. Caragana arborescens Lam.

793. Colutea arborescens L.

794. Cytisus supinus L.

795.* Genista germanica L.

796. G. ovata Waldst. et Kit.

797. G tinctoria L.

IX. Celastraceae.

798. Evonimus europea L.

799.* E. verrucosa Scop.

800. E. latifolia Scop.

$X$. Aceraceae.

801. Acer ginnala Maxim.

802. A. negundo $L$.

803. A. tataricum L.

XI. Rhamnaceae.

804.* Rhamnus Frangula L.

805.* R. cathartica L.

XII. Tiliaceae.

806.* Tilia cordata Mill.

807. T. plathyphyllus Scop.

XIII. Thymelaeceae.

808.* Daphne Mezereum L. 
XIV. Vacciniaceae.

809.* Ledum palustre L.

$810 *$ Vaccinium vitis idaea $\mathrm{L}$.

811* V. myrtillus L.

$X V$. Oleaceae.

812. Fraximis pensylvanica March.

813.* F. excelsior L.

814. Syringa vulgaris L.

815. S. Josikaea Jacq.

XVI. Caprifoliaceae.

816. Lonicera coerulea L.

817. L. tatarica L.

818. Sambucus racemosa L.

819. Symphoricarpus racemosus Michx.

820. Viburnum Opulus L.

821. V. Lantana L.

IV. SEMINA PLANTARUM DECORANTIUM.

I. Polipodiaceae.

822. Adiantum Capillus Veneris L.

823. Asplenium bulbiferum Forst.

824. Nephrolepis tuberosa Presl.

825. Pteris cretica L.

826. P. C. v. albo-lineata.

II. Cyperaceae.

827. Cyperus natalensis Hochet.

828. C. papyrus L. 
III. Liliaceae.

829. Lilium tigrinum Gawl (bulbi).

IV. Iridaceae.

830. Gladiolus hybridus hort. (bulbi).

831. G. primulinus Bauer (bulbi).

V. Aizoaceae.

832. Mesembrianthemum cordifolium L.

VI. Portulacaceae.

833. Portulaca grandiflora Hook.

VII. Caryophyllaceae.

834. Dianthus barbatus L.

835. D. sinensis L.

VIII. Cruciferae.

836. Alyssum Benthamii Lam. v. compactum hort. 837. Matthiola bicornis hort.

IX. Crassulaceae.

838. Sedum hispanicum L.

839. Bryophyllum Laygremontianum (viv.). 840. B. tubiflorum Aaw. (viv.).

$X$. Leguminosae.

841. Lupinus polyphyllus Lindl.

842. L. p. f. alba hort.

843. L. p. f. coerulea hort.

844. L. p. f. rosea hort. 


\section{Oxalidaceae.}

845. Oxalis corniculata L.

XII. Tropaeolaceae.

846. Tropaeolum majus L. v. nanum hort.

XIII. Balsaminaceae.

847. Impatiens balsamina L. fl. pleno hort.

$X I V$. Violaceae.

848. Viola tricolor L. v. hybrida hort.

XV. Begoniaceae.

849. Begonia semperflorens Linkc. et Otto v. Albert Martin.

850. B. s. v. Marienburg hort.

851. B. s. v. Stringans hort.

852. B. s. v. vernon compacta hort.

853. B. s. v. Vulcan hort.

854. B. s. v. Zauberin hort.

855. B. s. v. Domtaube hort.

XVI. Cactaceae.

856. Cereus peruvianus Mill. v. monstrosus D. C. (viv.)

857. Phyllocactus phyllantoides Link.' (viv.)

$X$ VII. Onagraceae.

858. Oenothera missuriensis Sims.

XVIII. Polanoniaceae.

859. Phlox Droomondii Hook. 
XIX. Asclepiadaceae.

860. Asclepias incarnata L.

$X X$. Borraginaceae.

861. Anchusa italica Betz.

862. Heliotropium corymbosum R. et P.

XXI. Verbenaceae.

863. Verbena hybrida hort. fl. coerulea hort. 864. V. h. „Defiance” hort.

XXII. Solanaceae.

865. Nicotiana affinis hort.

866. N. colossca Andre.

867. N. Sanderae hort. v. atrosanguinea hort.

868. N. tabacum L. ,Havanna" hort.

869. N. t. v. Wigandioides hort.

870. Petunia hybrida hort. v. grandiflora hort.

871. P. h. "Aurora" hort.

872. P. h. "Alba” hort.

873. P. h. "Brilliant Rose" hort.

874. P. h. "Coerulea" hort.

875. P. h. "Krimhilde" hort.

876. P. h. „Stalblau” hort.

877. Salpiglossis variabilis hort.

XXIII. Scrophulariaceae.

878. Alousoa Warscewiczii Rgl. var. compactus hort. 879. Antirrhinum majus L. fl. albo hort. 880. A. m. "Apricose" hort. 
881. Antirrhinum majus v. nanum grandiflorum „Karminkönigin” hort.

882. A. m. ,,Purpurkönig" hort.

883. A. m. fl. rosea hort.

884. A. m. ,,Tschernyi platsch" hort.

885. Calceolaria pinnata $R$. et $P$.

886. Schizanthus hybridus hort. v, grandiflora hort.

XXIV. Gesneriaceae.

887. Gloxinia hybrida hort.

888. Atreptocarpus polyanfha L.

$X X V$. Sesameae.

889. Martynia proboscidea Glok.

XXVI. Cucurbitaceae.

890. Echinocystis lobata $\mathrm{Tr}$. et Gr.

XXVII. Campanulaceae.

891. Lobelia Erinus L.

892. L. E. „Crystal Palace” hort.

XXVIII. Compositae.

893. Ageratum conyzoides L. v. mexicanum hort.

894. Brachycome iberidifolia But.

895. Calendula officinalis L fl. pl. „Favorit Bucartes” hort.

896. C. o. v. grandiflora "Orange Königen" hort. 897. C. o. „Zolotij Schar" hort.

898. Callistephus chinensis Nees et Esb. „Krassavitza Rynka" scharlachovaja hort. 
899. Callistephus chinensis "Strausovoje pero" karminno-rosovoje hort.

900. C. ch. "Strausovoje pero" goluboje hort.

901. Gailardia hybrida hort.

902. Dahlia Mekri Lehm.

903. Pyrethrum parthenifolium L. v. aureum hort.

904. Solidago canadensis L.

905. Tagetes crecta L.

906. T. patula L. „Liliput” hort.

907. Zinnia elegans Jacq.

V. SEMINA PLANTARUM OFFICINALIUM.

I. Liliaceae.

908. Allium Schoenoprasum L.

II. Iridaceae.

909. Iris sibirica L.

III. Urticaceae.

910. Cannabis sativa L.

IV. Polygonaceae.

911. Rheum officinalis Baill.

912. Rh. palmata L. v. tanguticum Maxim.

913. Rumex acetosa L.

V. Chenopodiaceae.

914. Chenopodium ambrosioides L. 
VI. Caryophyllaceae.

915. Agrostemma Githago L.

916. Saponaria officinalis L.

VII. Ranunculaceae.

917. Nigella arvensis L.

918. N. damascena L.

919. N. sativa L.

VIII. Papaveraceae.

920. Papaver rhoeas L.

921. P. somniferum L. (ex Extrema Oriente).

922. P. s. (ex Marriinsk).

923. P. s. (ex Tatarstan).

924. P. s. (ex Tjan-Tschjan).

925. P. s. (ex Sajsan).

IX. Cruciferae.

926. Brassica juncea Czern.

927. Brassica nigra Roch.

928. Sinapis alba L.

929. Thlaspi arvense L.

$X$. Capparidaceae.

930. Cleome gigantea L.

XI. Crassulaceae.

931. Sedum acre L.

XII. Rosaceae.

932. Geum urbanum L.

933. Sanguisorba officinalis L. 
XIII. Leguminosae.

934. Melilotus officinalis Desr.

XIV. Rutaceae.

935. Ruta graveolens L.

$X V$. Euphorbiaceae.

936. Ricinus communis L.

XVI. Malvaceae.

937. Abutilon Avicennae Gaertn.

938. Althea officinalis $\mathrm{L}$.

939. A. rosea Cav. fl. nigra hort.

940. Malva alcea L.

941. M. crispa L.

942. M. mauritiana L.

943. M. moschata L.

944. M. verticillata $L$.

XVII. Violaceae.

945. Viola tricolor L. v. arvensis Koch.

XVIII. Umbelliferae.

946. Carum Carvi L.

947. Conium maculatum $\mathrm{L}$.

948. Pimpinella Anisum L.

XIX. Primulaceae.

949. Primula officinalis $\mathrm{L}$. 
$X X$. Borraginaceae.

950. Cynoglossum officinale $L$.

XXI. Labiatae.

951. Dracephalum Moldavica L. fl. alba.

952. Hyssopus officinalis $L$.

953. Lallemantia ibericum Fisch. et Mey.

954. Lophanthus anisatus Benth.

955. Melissa officinalis L.

956. Mentha piperita L.

957. Nepeta Cataria L.

958. Ocimum Basilicum L.

959. Salvia officinalis L.

960. Thymus vulgaris $\mathrm{L}$.

XXII. Solanaceae.

961. Atropa Belladonna L.

962. Datura aegiptiaca Vesl.

963. D. inermis Jacq.

964. D. Stramonium L.

965. Hyoscyamus agrostis Kit.

966. H. niger L.

967. Nicotiana rustica L.

968. Solanum nigrum L.

XXIII. Scrophulariaceae.

969. Digitalis ambigua Murr.

970. D. lanata Ehrh.

971. D. ferruginea L.

972. D. lutea L.

973. D. purpurea L. 
974. Verbascum phlomoides L.

975. V. tapsiphorme Schrad.

XXIV. Plantaginaceae.

976. Plantago Cynops L.

$X X V$. Valerianaceae.

977. Valeriana nitida $\mathrm{Pr}$.

978. V. officinalis L.

979. V. rossica Sm.

XXVI. Lobeliaceae.

980. Lobelia syphilitica L.

XXVII. Compositae.

981. Achillea Millefolium L.

982. A. nobilis L.

983. Anacyclus officinarum Hayne.

984. Arnica montana L.

985. Anthemis nobilis L.

986. A. tinctoria L.

987. Artemisia Absinthium L.

988. A. vulgaris $\mathrm{L}$.

989. Bellis perennis L.

990. Calendula officinalis L.

991. Carduus Marianum L.

992. Cephalophore aromatica Schrad.

993. Centaurea cyanus L.

994. C. c. L. fl. rosea.

995. Gnaphalium uliginosum L.

996. Grindelia robusta Nutt. 
997. Helianthus annuus L.

998. Inula Helenium L.

999. Lactuca sativa $\mathrm{L}$.

1000. L. virosa L.

1001. Madia sativa Don.

1002. Matricaria Chamomilla L.

1003. M. discoidea D. C.

1004. M. suaveolens Buchen.

1005. Tanacetum boreale Fisch.

1006. T. vulgare $\mathrm{L}$.

\section{SEMINA PLANTARUM OLEARUM ET TECHNICARUM.}

I. Chenopodiaceae.

1007. Beta vulgaris L.

II. Cruciferae.

1008. Brassica juncea Czern.

1009. B. nigra Roch.

1010. Camelina dentata Wallr.

1011. C. sativa Crantz.

III. Euphorbiaceae.

1012. Euphorbia Lathyrus L.

1013. Ricinus communis L.

1014. R. c. "Kavkasskaja ulutschennaja”.

1015. R. c. „R. P.”.

1016. R. c. $n^{\circ} 5$. 
IV. Umbelliferae.

1017. Carum Carvi L.

1018. Coriandrum sativum L.

1019. C. s. v. aureum

1020. C. s. v. giganteum

1021. C. s. v. microcarpum

1022. C. s. v. nanum

$V$. Labiatae.

1023. Lallemantia ibericum Fisch. et Mey.

VI. Compositae.

1024. Carthamuus lanatus L. $\mathrm{n}^{\circ} 5686$.

1025. Helianthus annuus L.

1026. H. a. "Gigant”

1027. H. a. "Fuzinca"

1028. H. a. (ex Missuori).

1029. H. a. $\mathrm{n}^{\circ} \mathrm{A}-41$.

1030. H. a. $n^{\circ} 169$.

1031. H. a. $\mathrm{n}^{\circ} 1846$.

1032. H. a. $\mathrm{n}^{\circ} 1975$.

1033. Madia sativa Don.

VII. Urticaceae.

1034. Cannabis sativa E. „N. Severskaja”

1035. C. s. "Russko-Brodskaja"

1036. C. s. "Trubtschevskaja"

1037. C. s. "Zolotonoschskaja"

VIII. Linaceae.

1038. Linum perenne $\mathrm{L}$. 
1039. Linum usitatissimum L.

1040. L. u. "Baryschovsky"

1041. L. u. "D-33"

1042. L. u. "N-79"

1043. L. u. "D-83"

1044. L. u. „N-107.

1045. L. u. "N-109"

1046. L. u. "N 113”,

1047. L. u. "N 120"

1048. L. u. "N 262"

1049. L. u. "N 266"

1050. L. u. "N $806 / 3$ "

1051. L. u. "N 1288/12”

IX. Malvaceae.

1052. Abutilon Avicennae Gaertn.

1053. A. A. $\mathrm{n}^{\circ} 479$.

1054. Malva crispa L.

1055. M. c. $\mathrm{n}^{\circ} 4762$.

1056. M. c. n ${ }^{\circ} 132129$.

1057. M. c. $\mathrm{n}^{\circ} 133543$.

1058. M. c. $\mathrm{n}^{\circ} 142263$.

1059. M. c. $\mathrm{n}^{\circ} 148401$.

1060. M. mauritania L.

1061. M. m. n 476 .

1062. M. m. n 138762 .

1063. M. meluca L.

1064. M. m. (ex Tschecho-Slovakej).

1065. M. m. n 136630 .

1066. M. m. n 139239.

1067. M. m. $\mathrm{n}^{\circ} 142522$. 
1068. Malva verticillata $\mathrm{L}$.

1069. M. v. $\mathrm{n}^{\circ} 4760$.

1070. M. v. $\mathrm{n}^{\circ} 119264$.

1071. M. v. $\mathrm{n}^{\circ} 128580$.

1072. M. v. $\mathrm{n}^{\circ} 133551$.

1073. M. v. $n^{\circ} 139930$.

\section{SEMINA PLANTARUM GRANEARUM ET LEGUMINOSARUM.}

I. Gramineae.

1074. Avena nuda L.

1075. A. sativa "Lochovski"“

1076. A. s. "Stepniak 648"

1077. A. s. v. aristata Kr. „Zemchujina”

1078. A. s. a. „Ligovo"

1079. A. s. a. ",Miraj"

1080. A. s. a. Moskovskoj A-315.

1081. A. s. a. $\mathrm{n}^{\circ} 06522$.

1082. A. s. a. $\mathrm{n}^{\circ} 08115$.

1083. A. s. a. "Leitevizki”

1084. A. s. a. "Zolotoj Dojd"

1085. A. s. a. K-5057.

1086. A. s. v. mutica "Pobeda"

1087. A. s. m. „Severianin"

1088. A. s. m. "Schatilovski"

1089. A. s. v. obtusata „Magistral".

1090. Hordeum distichum L. v. abissinicum

1091. H. d. v. leocritum.

1092. H. d. v. medicum Körn. $\mathrm{n}^{\circ} 26$. 
1093. Hordeum distihum m. Körn. $\mathrm{n}^{\circ} 46$.

1094. H. d. v. nigrum.

1095. H. d. v. nudum L. „Byloje”.

1096. H. d. n. $n^{\circ} 105$.

1097. H. d. n. $\mathrm{n}^{\circ} 110$.

1098. H. d. v. nutans Schübel, ,Europaeum 313/133”.

1099. H. d. n. „Viner”.

1100. H. d. n. v. colchicum.

1101. H. d. n. $\mathrm{n}^{\circ} 17$.

1102. H. d. v. pallidum Sir.

1 103. H. d. p. ,,Viatsky 1009".

1104. H. d. p. "Chervonetz".

1105. H. d. p. $n^{\circ} 032$.

1106. H. d. p. $\mathrm{n}^{\circ} 045$.

1107. H. d. v. parallelum.

1108. H. d. v. persicum $\mathrm{n}^{\circ} 062$.

1109. Panicum miliaceum contractum Al. v. aureum $n^{\circ} 01$.

1110. P. m. c. v. dacicum Körn. nº 03.

1111. P. m. effusum Al. v. subcoccineum $\mathrm{n}^{\circ} 742$.

1112. P. m. e. v. subflavum $n^{\circ} 204$.

1113. Triticum durum v. affine Körn $\mathrm{n}^{\circ} 309$.

1114. T. d. a. $n^{\circ} 7 \curvearrowleft 9$.

1115. T. d. v. africanum.

1116. T. d. v. cerulescens Bayle.

1117. T. d. v. leucurum Al. Br. 26.

1118. T. d. 1. Br. 33 .

1119. T. d. v. Libycum Körn.

1120. T. d. v. hordeiforme Host. $\mathrm{n}^{\circ} 04$.

1121. T. d. h. $\mathrm{n}^{\circ} 010$.

1122. T. d. h. n 189. 
1123. Triticum durum h. $\mathrm{n}^{\circ} 432$.

1124. T. d. h. n 496.

1125. T. d. h. $\mathrm{n}^{\circ} 675$.

1126. T. d. h. $\mathrm{n}^{\circ} 694$.

1127. T. d. h. $n^{\circ} 695$.

1128. T. d. lutescens $\mathrm{n}^{\circ} 0$.

1129. T. d. $1 . \mathrm{n}^{\circ} 323$.

1130. T. d. $1 . \mathrm{n}^{\circ} 786$.

1131. T. d. $1 . \mathrm{n}^{\circ} 1484$.

1132. T. d. $1 . \mathrm{n}^{\circ} 2351$.

1133. T. d. 1. $n^{\circ} 3221$.

1134. T. d. $1 . \mathrm{n}^{\circ} 9113$.

1135. T. d. melanopus $\mathrm{n}^{\circ} 069$.

1136. T. d. m. n 886 .

1137. T. d. v. murciense Körn.

1138. T. dicoccum Schubl. v. rufum Schübl.

1139. T. monococcum L.

1140. T. vulgare Will. v. albidum Al. $\mathrm{n}^{\circ} 0676$.

1141. T. v. v. alborubrum Körn.

1142. T. v. v. caesium Al. $\mathrm{n}^{\circ} 0111$.

1143. T. v. erythrospermum "Lenina”.

1144. T. v. e. "Kooperatorka”.

1145. T. v. e. "Novinka”.

1146. T. v. e. „Ukrainka”.

1147. T. v. e. $\mathrm{n}^{\circ} 841$.

1148. T. v. v. ferrugineum Al. "Moskovskaja 2411".

1149. T. v. f. $\mathrm{n}^{\circ} 146$.

1150. T. v. f. $\mathrm{n}^{\circ} 150$.

1151. T. v. v. lutescens Al. „Garnet”.

1152. T. v. 1. „Kitchener”.

1153. T. v. 1. „Leda”. 
1154. Triticum v. 1. "Marquis".

1155. T. v. $1 . \mathrm{n}^{\circ} 062$.

1156. T. v. $1 . n^{\circ} 328$.

1157. T. v. $1 . \mathrm{n}^{\circ} 479$.

1158. T. v. $1 . \mathrm{n}^{\circ} 1060 / 10$.

1159. T. v. $1 . \mathrm{n}^{\circ} 1484$.

1160. T. v. 1. milturum Al. B. 80 .

1161. T. v. m. $\mathrm{n}^{\circ} 321$.

1162. T. v. m. n 321 .

1163. T. spelta L.

II. Leguminosae.

1164. Ervum lens L.

1165. E. 1. (ex Arabia).

1166. E. 1 (ex Kabardinia).

1167. E. 1. (ex Marocco).

1168. E 1. (ex Penza).

1169. E. 1. "Saratovskaja n ${ }^{\circ} 013$ ".

1170. E. 1. "Saratovskaja n ${ }^{\circ} 120^{\prime \prime}$.

1171. E. 1. "Saratovskaja n ${ }^{\circ} 140$ ".

1172. E. 1. "Seraja” n $32 / 5$.

1173. E. 1. (ex Syria).

1174. L. 1. v. macrosperma f. nummularia A. „Dymchataja”.

1175. E 1. v. pseudomarmorata Bar. „Rosovaja 44/3”.

1176. Lupinus albus L. $\mathrm{n}^{\circ} \mathrm{y}-26$.

1177. L. angustifolius „Maloalkaloidnij”.

1178. L. a $\mathrm{n}^{\circ} 173$.

1179. L. a. $\mathrm{n}^{\circ} 374$.

1180. L. a. $n^{\circ} 418$.

1181. L. a. $\mathrm{n}^{\circ} 484$. 
1182. Lupinus albus $n^{\circ} 531$.

1183. L. a. $\mathrm{n}^{\circ} 586$.

1184. L. a. $\pi^{\circ} 983$.

1185. L. luteus $\mathrm{n}^{\circ} 345$.

1186. L. 1. $\mathrm{n}^{\circ} 374$.

1187. L. 1. $\mathrm{n}^{\circ} 4645$.

1188. L. 1. $\mathrm{n}^{\circ} 4665$.

1189. L. 1. n 4666.

1190. L. mutabilis $\mathrm{n}^{\circ} 97$.

1191. L. pilosus L.

1192. Phaseolus vulgaris $L$.

1193. Ph. v. „Arabka”.

1194. Ph. v. "Bomba belaja n 033 ”.

1195. Ph. v. "Gorohovidnaja".

1196. Ph. v. „Izumrud”.

1197. Ph. v. "Risovaja K-30”.

1198. Ph. v. "Sam-sto”.

1199. Ph. v. "Triumf".

1200. $\mathrm{Ph}$ v. $\mathrm{n}^{\circ} 063$.

1201. Ph. v. ,Tschedrij”.

1202. Ph. v. ellipticus $\mathrm{Ph}$. sphaericus v. unicolus f. atropurpureus ,Zolotaja Gora”.

1203. Pisum arvense $\mathrm{L}$.

1204. P. a. $\mathrm{n}^{\circ} 08$.

1205. P. a. $\mathrm{n}^{\circ} 33$.

1206. P. a. $\mathrm{n}^{\circ} 039$.

1207. P. a. $\mathrm{n}^{\circ} 042$.

1208. P. a. $\mathrm{n}^{\circ} 043$.

1209. P. a. $n^{\circ} 128$.

1210. P. a. $n^{\circ} 9 / 376$.

1211. P. a. $\mathrm{n}^{\circ} 5 / 518$. 
1212. Pisum arvense $n^{\circ} 2 / 546$.

1213. P. sativum L.

1214. P. s. „Ambrozia”.

1215. P. s. $\mathrm{n}^{\circ} 06$.

1216. P. s. $\mathrm{n}^{\circ} 087$.

VIII. SEMINA PLANTARUM PABULATORIUM

I. Gramineae.

1217. Agropyrum cristatum Roem. et Schult.

1218. A. desertorum Schult.

1219. A. d. $\mathrm{n}^{\circ} 01$

1220. A. d. $n^{\circ} 62$.

1221. Agrostis alba L.

1222. Alopecurus pratensis L.

1223. Bromus inermis Leyss.

1224. B. mollis L.

1225. Dactylis glomerata L.

1226. D. g. $\mathrm{n}^{\circ} 200$.

1227. D. g. $\mathrm{n}^{\circ} 202$.

1228. D. g. $\mathrm{n}^{\circ} 204$.

1229. Elymus gigantens Vahl.

1230. E. sibiricus L.

1231. Festuca ovina L. $\mathrm{n}^{\circ} 58349$.

1232. F. pratensis Huds.

1233. F. rubra L.

1234. Lolium italicum R. Br.

1235. L. perenne L

1236. Phleum pratense $\mathrm{L}$.

1237. Ph. p. $\mathrm{n}^{\circ} 1117$. 
1238. Phleum pratense $\mathrm{n}^{\circ} 1234$.

1239. Ph. p. $\mathrm{n}^{\circ} 3281$.

1240. Poa pratense L.

1241. Zea mays L.

II. Leguminosae.

1242. Cicer arietinum L.

1243. C. a. „Korolevski”.

1244. C. a. (ex Media Asiae).

1245. C. a. ,Tschorni" $n^{\circ} 418$.

1246. C. a. (ex Ucraina).

1247. C. a. $\mathrm{n}^{\circ} 77$.

1248. C. a. $\mathrm{n}^{\circ} 157 / 2$.

1249. Lathyrus sativus $\mathrm{L}$.

1250. L. s. (ex Abissinia).

1251. L. s. (ex Africa):

1252. L. s. (ex Africa).

1253. L. s. (ex Baschkiria).

1254. L. s. (ex Buchara).

1255. L. s. (ex California).

1256. L. s. (ex France) K-1112.

1257. L. s. (ex Georgia) K-965.

1258. L. s. (ex Germania) K-240.

1259. L. s. (ex Italia) K-708.

1260. L. s. (ex Kiev) K-1153.

1261. L. s. (ex Kuban) K-931.

1262. L. s. (ex Kuban) K-954.

1263. L. s. (ex Odessa) K-287.

1264. L. s. (ex Odessa) K-398.

1265. L s. (ex Rostov-Nachitschevanj).

1266. L. s. (ex Sardinia). 
1267. Lathyrus sativus (ex Sardinia).

1268. L s. (ex Sardinia).

1269. L s. (ex Sibiria).

1270. L. s (ex Ukraina).

1271. L. s. (ex Tschernigov).

1272. L. s. (ex Turkestan).

1273. Medicago denticulata Willd. $\mathrm{n}^{\circ} 130$.

1274. M. d. $\mathrm{n}^{\circ} 183$.

1275. M. d. $n^{\circ} 1123$.

1276. M. falcata L. n 11714.

1277. M. media v. hybridum hort.

1278. M. sativa (ex Fergana) K-8/2.

1279. M. s. (Peruvianskaja) K-230.

1280. Melitotus alba L. (ex America).

1281. M. a. $\mathrm{n}^{\circ} 1143$.

1282. Onobrychis sativa Lam," n $19 / 2$.

1283. O. s. $\mathrm{n}^{\circ} 70$.

1284. O. s. $\mathrm{n}^{\circ} 74$.

1285. Trifolium hybridum $\mathrm{L}$,

1286. T. pratense L. ,Jaroslawski”.

1287. T. p. „Krasnoufimski”.

1288. T. p. "Mariusinski”.

1289. T. p. "Novozybkovski".

1290. T. p. "Raszvetajevski".

1291. T. p. $\mathrm{n}^{\circ} 150$.

1292. T. p. n 1103 .

1293. T. p. n $^{\circ} 1435$.

1294. T. p. n 15691 .

1295. Vicia Faba L.

1296. V. sativa L. (ex Archangelsk).

1297. V. s. "Obyknovennaja". 
1298. Vicia sativa (ex Sverige).

1299. V. s. (ex Turkei).

1300. V. s. "Ulianovskaja".

1301. V. s. "Ulianovskaja" n 184.

1302. V. s. $\mathrm{n}^{\circ} 163$.

\section{Prof. Dr. S. S. Stankov.}

\section{Horti Director.}

Docent D. S. Awerkiev

Docent $L$. W. Tichov

Ass. Ph. A. Alexandrov Ass. L. M. Smirnova Ass. E. I. Tscherbakowa Ass. A. P. Lomakovia

Ass. M. A. Gaidukova
J. S. Ktemansky

S. A. Suvorov

N. N. Klemanskaja

Horti Hortulani 
Перечень семян, собранных в 1937 г. и предлагаемых в обмен Ботаническим сад д горь. ковского Государственного Уни в е р ситета. ОГИЗ. Горьковское Издательство 1938. Редактор проф. C. С. Стакков. Техн. редактор Л. Н. Немченко. Корректор Р. А. Долганова. Сдано в набор 15/1-38 г. Подписано к печати 17/II-38 г. Формат $60 \times 94 / 32$. Тираж 650 экз. Изд. л. $1^{3 / 4}$. Бум. л. ๆ/8. Уч.-авт. л. 1,70 Инд. СХ-3-в № 1079. Знаков в бум. л. 89000 . Уполномоченный Обллита 스 A 12 , Горьковский Полиграф, гор. Горький, ул. Фигнер, 32. Заказ 스 6622. 

БЕ СПЛ А Т H O

ПЕРЕЧЕНЬ СЕМЯН,

СОБРАННЫХ В 1937 ГОДУ

И ПРЕДЛАГАЕМЫХ В ОБМЕН

БОТАНИЧЕСКИМ САДОМ

ГОРЬКОВСКОГО ГОСУДАРСТВЕННОГО

УНИВЕРСИТЕТА

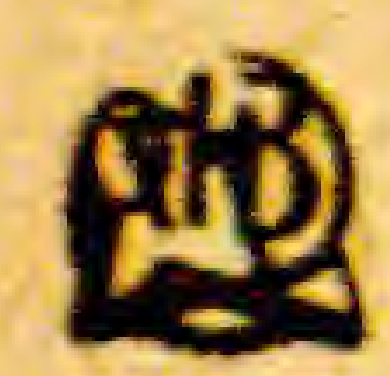

ГОРЬКОВСКОЕ ИЗДАТЕЛЬСТВО 1938 


\section{$a_{e} \beta 47$}

\section{DELECTUS SEMINUM}

ANNO MCMXXXVIII COLLECTORUM

QUAE

HORTUS BOTANICUS

UNIVERSITATIS GORKIJENSIS

PRO

MUTUA COMMUTATIONE

OFFERT

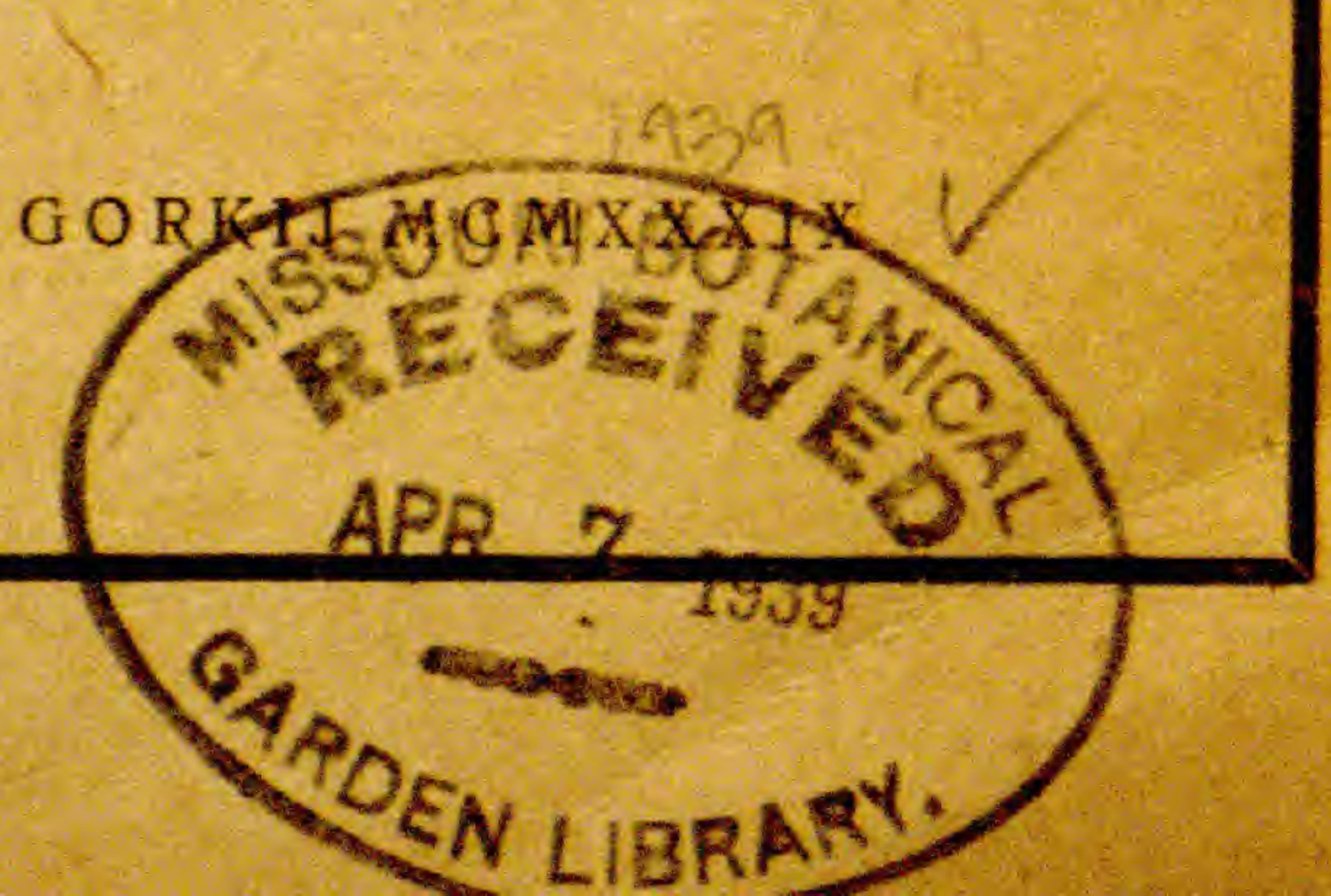





\section{DELECTUS SEMINUM}

ANNO MCMXXXVIII COLLECTORUM

QUAE

HORTUS BOTANICUS

UNIVERSITATIS GORKIJENSIS

PRO

MUTUA COMMUTATIONE

OFFERT

GORKIJ MCMXXXIX 



\section{SEMINA PLANTARUM LIGNOSARUM ET FRUTICUM.}

I. Cupressaceae.

1. Juniperus communis $\mathrm{L}$.

2. Thuja occidentalis L.

II. Betulaceae.

3. Betula verrucosa Ehrh.

4. Corylus Avellana L.

III. Fagaceae.

5. Quercus pedunculata Ehrh.

IV. Berberidaceae.

6. Berberis heteropoda Schrenk. v. oblonga Rgl.

V. Saxifragaceae.

7. Hydrangea Bretschneideri Dipp.

8. Ribes nigrum $L$.

VI. Rosaceae.

9. Amelanchier vulgaris Moench. 
11. Crataegus monogyna Jacq.

11. Physocarpus opulifolius Maxim.

12. Prunus Padus L.

13. Prunus pumila $L$.

14. Sorbaria assurgens Vilm, et Boiss.

15. Sorbus aucuparia L.

16. Spiraea callosa Froebeli hort.

17. S. Douglasii Hook v. rusea hort.

18. S. japonica L.

19. S. j. v. pumila.

20. S. salicifolia L.

21. S. syringiflora Lemoine.

22. S. Wilsonii Duthie.

VII. Leguminosae.

23. Amorpha fruticosa L.

24. Caragana arborescens Lam.

25. Colutea arborescens L.

26. C. media Willd.

27. Cytisus supinus $L$.

28. Genista germanica L.

29. G. ovata Waldst. et Kit.

30. G. tinctoria L.

VIII. Rutaceae.

31. Ptelea trifoliata L.

IX. Euphorbiaceae.

32. Securinega ramiflora Muell. 
$X$. Celastraceae.

33. Evonymus europaea L.

34. E. verrucosa Scop.

XI. Aceraceae.

35. Acer Negundo L.

36. A. platanoides $L$.

37. A. tataricum L.

XII. Rhamnaceae.

38. Rhamnus cathartica L.

39. R. Frangula L.

XIII. Vitaceae.

40. Ampelopsis quinquefolia Michx.

$X I V$. Tiliaceae.

41. Tilia cordata Mill.

42. T. plathyphyllos Scop.

$X V$. Guttiferae.

43. Hypericum patulum Thunb.

XVI. Oleaceae.

44. Fraxinus excelsior $L$.

45. F. pennsylvanica Marsh.

46. Ligustrum vulgare L. v. pyramidale Späth.

XVII. Caprifoliaceae.

47. Diervilla rivularis Gatt. 
48. Lonicera amoena Zbl.

49. L. coerulea L.

50. L. microphylla Willd.

51. L. nervosa Maxim.

52. L. tatarica L.

53. L. Xylosteum L.

54. Sambucus racemosa $L$.

55. Symphoricarpus occidentalis Hook.

56. Viburnum Lantana L.

57. V. Opulus L.

II. SEMINA PLANTARUM HERBACEUM.

I. Polypodiaceae.

58.* Athyrium crenatum Rupr. (1937)

59.* A. filix femina Roth.

60* Cystopteris fragilis Bernh.

61.* Dryopteris filix mas Schott.

62.* D. Linnaeana Christ.

63.* D. phegopteris Christ.

64.* D. spinulosa O. Ktze.

65.* D. thelipteris A. Gray.

66.* Polystichum Braunii Fee.

67.* Pteridium aquilinum Kuhn.

68." Struthiopteris germanica Willd. (1937)

* Plantae spontaneae. 
II. Lycopodiaceae.

69. Lycopodium clavatum L.

III. Alismaceae.

70.* Alisma plantago-aquatica L.

IV. Gramineae.

71. Agropyrum cristatum Gaertn.

72. A. desertorum Schult.

73. Agrostis borealis Hartm.

74. Alopecurus lasiostachys Link.

75. A. ventricosus Pers.

76. Andropogon ischaemum L.

77. A. i. ssp. Wilcommii.

78.* Anthoxanthum odoratum L.

79. Aristida adscensionis L.

80. A. plumosa L.

81. Asperella Hystrix Humb.

82. Avena elatior L.

83. A. planiculmis Turcz.

84. A. pubescens Huds.

85. A. Schelliana Hack.

86. Beckmannia baicalensis Hulten.

87.* Brachypodium silvaticum P. B.

88. Bromus carinatus Hook et Arn.

89.* B. inermis Leyss.

90. B. mollis L.

91. B. ramosus Huds. 
92. B. unioloides Humb.

93. Catabrosa humilis Trin.

94. Chaeturus fasciculatus Link.

95. Corynephorus canescens P. B.

96. Dactylis Aschersoniana Graebn.

97.* D. glomerata L.

98. D. hispanica Roth.

99.* Deschampsia caespitosa P. B.

100. D. flexuosa Trin.

101. Deyeuxia lapponica Kunth.

102. Diarrhena americana Beauv.

103. Elymus akmolensis Drob.

104. E. dahuricus Turcz.

105. E. excelsus Turcz.

106. E. hirsutiglumis Scribn.

107. Elymus sibiricus L.

108. Festuca alpina Suter.

109. F. pratensis Huds.

110. F. scoparia Kerner et Hackel.

111. F. varia Haenke

112. Holcus lanatus L.

113. Hordeum bulbosum L.

114. H. jubatum L.

115. Lolium canadense Michx.

116. L. perenne $\mathrm{L}$.

117. Melica altissima $L$.

118. M. transsilvanica Schur.

119. Milium effusum $L$.

120. Muehlenbergia mexicana Trin. . 
121. Phalaris paradoxa L.

122. Phleum alpinum $\mathrm{L}$.

123. Ph. Michelii All.

124. $\mathrm{Ph}$. paniculatum Huds.

125.* $\mathrm{Ph}$. pratense $\mathrm{L}$.

126. Poa alpina L.

127. P. annua L.

128. P. bulbosa L.

129.* P. nemoralis L.

130. R. versicolor Bess.

131. Sesleria robusta Schott.

132. Setaria viridis P. B.

133. Stipa capillata L.

134. Triodia decumbens Pall.

135.* Triticum repens $\mathrm{L}$.

V. Cyperaceae.

136. Carex glauca Scop.

VI. Liliaceae.

137. Allium angulosum $\mathrm{L}$.

138. A. caesium Schrenk.

139. A. fistulosum L.

140. A. giganteum Reg.

141. A. glaciale Vved.

142. A. hymenorrhizum Ledeb.

143. A. moly L.

144. A. montanum F. W. Schmidt.

145. A. narcissiflorum Vill. 
146. A. nutans $\mathrm{L}$.

147. A. odorum L.

148. A. paradoxum G. Dont:

149.* A. schoenoprasum $L$.

150. Anthericum ramosum $\mathrm{L}$.

151. Asparagus officinalis $L$.

152. Lilium candidum $\mathrm{L}$.

153. L. regale Wils.

154. L. tigrinum $\mathrm{Ker}-\mathrm{Gaw}$ (bulbi).

155. Muscari racemosum Mill.

156. Ornithogalum umbellatum $\mathrm{L}$.

157.* Paris quadrifolia $\mathrm{L}$.

158.* Polygonatum multiflorum Alt.

159. Scilla cernua Red.

160. Smilacina stellata Desf.

VII. Iridaceae.

161. Iris aphylla $\mathrm{L}$.

162. I. chamaeiris Bertol.

163. I. falcifolia Bunge

164. I. gueldenstaedtiana Lepech.

165. I. kaempferi Sieb.

166. I. lutescens Lam.

167. I. missouriensis Nutt.

168. I. prismatica Pursh.

169. I. ruthenica Ker-Gawl.

170. I. setosa Pall.

171.* I. sibirica L.

172. I. sogdiana Bunge. 
173. I. Thunbergii E. Lundstr.

VIII. Moraceae.

174. * Humulus Lupulus L.

IX. Urticaceae.

175. Urtica Dodartii L.

$X$. Polygonaceae.

176. Oxyria digyna Hill.

177.* Polygonum convolvulus L.

178.* P. hydropiper L.

179. Rheum Emodi Wall.

180. R. officinale Baill.

181. R. palmatum L. v. tanguticum Maxim.

182. R. Rhaponticum L.

183. R. undulatum L.

184.* Rumex acetosa L.

185.* R. acetosella L.

186. R. alpinus L.

187. R. callosus Reidringer.

188. R. confertus Willd.

189. R. hydrolapathum Huds.

190. R. hymenosepalus Torr.

191. R. mexicanus Meiss.

192. R. microcarpus Bryhn.

193. R. nepalensis Spreng.

194. R. pallidus Bigel.

195. R. stenophyllus Ledeb. 
196. R. thyrsiflorus Fingerh.

197. R. ucrainicus Fisch.

XI. Chenopodiaceae.

198. Chenopodium album L.

199. Ch. aristatum L.

200. Ch. Bonus-Henricus L.

201. Ch. botrys L.

202. Ch. capitatum Aschers.

203. Ch. quinoa Willd.

204. Corispermum hyssopifolium L.

XII. Amaranthaceae.

205. Amaranthus aurea T. A. Desf.

206.* A. blitum L.

207. A. gangeticus L.

208. Celosia cristata L. v. sanguinea Hort.

209. Gomphrena Haageana Klotszch.

XIII. Nyctaginaceae.

210. Oxybaphus nyctagineus Sweet.

XIV. Portulacaceae.

211. Calandrinia grandiflora Lindl.

$X V$. Phytolaccaceae.

212. Phytolacca decandra L.

$X V I$. Caryophyllaceae.

213. Cerastium alpinum $\mathrm{L}$. 
214. C. a. v. lanatum.

215. C. Biebersteinii D. C.

216. C. grandiflorum Waldst. et Kit.

217. C. Lerschenfeldianum Schur.

218. Cucubalus baccifer L. v. japonicum Miq.

219. Dianthus alpinus L.

220. D. barbatus L.

221. D. b. fl. nigro.

222. D. bebius Vis.

223. D. bicolor hort.

224. D. Boissieri Willk.

225. D. caesius Sm.

226. D. c. v. grandiflorum.

227. D. callizonus Schott. et Kotschy.

228. D. capitatus D. C.

229. D. Carthusianorum $\mathrm{L}$.

230. D. cinnabarinus Sprun.

231. D. cruentus Griseb.

232.* D. deltoides L.

233. D. gallicus Pers.

234. D. hungaricus Pers.

235. D. Knappii Aschers.

236. D. tatrae Borb.

237. D. tegestinus Kern.

238. D. terier Balb.

239. Gypsophila acutifolia Fisch.

240. G. elegans M. B.

241. G. e. v. grandiflorum hort.

242. G. fastigiata L. 
243. G. Stevenii Fisch.

244. G. tianschanica M. Pop. et Schischk.

245. Heliosperma quadrifidum Rchb.

246. Lychnis alpina L.

247. L. chalcedonica L.

248. L. coeli-rosa Desr.

249. L. Coronaria Desr.

250. L. flos-jovis Desr.

251. L. Haageana Regel.

252. L. mongolica Maxim.

253. L. viscaria $L$.

254.* Melandrium album Garcke.

255. M. rubrum Garcke.

256. M. Zawadskii A.B.

257. Saponaria cerastioides Fisch.

258. S. ocymoides L.

259. S. officinalis L.

260. S. o. fl. pleno.

261. Silene alpina L.

262. S. alpestris Jacq.

263. S. armeria L.

264. S. Bergiana Lindm.

265. S. ciliata Pourr.

266. S. conica L.

267. S. cucubalus Wiebb.

268. S. flavescens W. K.

269. S. gallica L.

270. S. grandiflora Franch.

271. S. Hornemannii Steud. 
272. S. italica Pers.

273. S. maritima With.

274. S. meliifera Boiss. et Reut.

275. S. muscipula L.

276. S. pseudo-otites Bess.

277. Tunica Saxifraga Scop.

278. Vaccaria pyramidata Med.

XVII. Ranunculaceae.

279.* Aconitum excelsum Rchb.

280. A. lycoctonum L.

281. A. orientale Mill.

282. Anemone baldensis L.

283. A. coronaria L.

284. A. decapetala Arduini.

285. A. fulgens J. Gay.

286. A. Leveillei Ulbr.

287. A. multifida Poir.

288. A. pedata Rafin.

289. A. tetrasepala Royle.

290. Aquilegia akitensis Huth.

291. A. alpina L.

292. A. canadensis L.

293. A. chrysantha A. Gray.

294. A. Einseleana Fr. Schultz.

295. A. flabellata Sieb. et Zucc.

296. A. formosa Fisch.

297. A. fragrans Benth.

298. A. glauca Lindl, 
299. A, grata Moly.

300. A. hispanica Borbas,

301. A. hybrida hort. fl. pleno.

302. A. jucunda Fisch. et Mey.

303. A. Kitaibelii Schott.

304. A. lactiflora Kar. et Kir.

305. A. moorcroftiana Wall.

306. A. nevadensis Boiss. et Reut.

307. A. olympica Boiss.

308. A. Ottonis Orph.

309. A. oxicephala Trautv. et Mey.

310. A. oxysepala Trautv. et Mey.

311. A. pubiflora Wall.

312. A. pyrenaica D. C.

313. A. sibirica Lam,

314. A. skinneri Hook.

315. A. stellata hort. .

316. A. vulgaris $L$.

317. Clematis tangutica Andre.

318. Delphinum bicolor Nutt.

319. D. Brunnonianum Royle.

320. D. cheilanthum Fisch.

321. D. chinense Fisch.

322 * D. consolida L.

323. D. elatum L. v. alpinum Hegi.

324. D. e. v. tyrolense Hegi.

325. D. formosum Boiss. et Huet.

326. D. hybridum Willd.

327. D. h. ,Prinz Gustav" hort.

16 
328. D. Maakianum Regel.

329. D. Przewalskii Huth.

330. D. Pylzowi Maxìm.

331. D. sulphureum Boiss.

332. D. Thunbergii Huth.

333. D. triste Fisch.

334. Isopyrum fumarioides $\mathrm{L}$.

335. Nigella arvensis L.

336. N. damascena L.

337. N. sativa L.

338. Ranunculus abortivus $\mathrm{L}$.

339. R. acer L. ssp. Steveni Hegi.

340. R. brutius Tenore

341 * $^{*}$ R. cassubicus $\mathrm{L}$.

342. R. caucasicus Bieb.

343. R. dissectus M. B. v. grandiflorus C. A. M.

344. R. muricatus L.

345. R. serbicus Vis.

346. R. trilobus Desr.

347. R. Villarsii D. C.

348. Thalictum adiantifolium Besser.

3.49. Th. elatum Jacq.

350. Th. foetidum L.

351. Th. glaucum Desf.

352. * Th. minus L.

XVIII. Papaveraceae.

353. Adlumia cirrhosa DC.

354. Bocconia cordata Willd. 
355. B. japonica Andre.

356. B. microcarpa Maxim.

357.* Chelidonium majus L.

358. Ch. m. v. laciniatum Mill.

359. Escholtzia californica Cham.

360. Glaucium corniculatum Curt.

361. G. squamigerum Kar. et Kit.

362. Meconopsis Wallichii Hook.

363. Papaver alpinum L.

364. P. bracteatum Lindl.

365. P. dubium $L$.

366. P. lateritium C. Koch.

367. P. nudicaule L.

368. P. orientale L.

369. P. Rhoeas L.

370. P. somniferum L.

XIX. Fumariaceae.

371. Corydalis sempervirens Pers.

$X X$. Cruciferae.

372. Aethionema Buxbaumii D. C.

373. A. grandiflorum Boiss. et Hohen.

374. A. pulchellum Boiss, et Huet.

375. Asyssum argenteum All.

376. A. campestre L.

377. A. desertorum Stapf.

378. A. montanum L.

379. A. saxatile L. 
380. Arabis albida Stev.

381. A. sicula Huet.

382. Aubrietia erubescens Griseb.

383. A. Iibanotica Boiss, et Hohen.

384. Barbarea orthoceras Ledeb.

385. Biscutella laevigata L.

386. Brassica nigra Koch.

387. Camelina dentata Wallr.

388. C. sativa Crantz.

389.* Capsella bursa pastoris Medic.

390. Carrichtera vella D. C.

391. Cheiranthus alpinus L.

392. Chorispora tenella D. C.

393. Cochlearia officinalis $L$.

394. Crambe abyssinica Kochst.

395. C. hispanica L.

396. C. Koctebelica Bush.

397. C. maritima L.

398. Erucastrum arabicum Fisch. et Mey.

399. Erysimum canescens Roth.

400. E. ochroleucum DC.

401. E. perofskianum Fisch. et Mey.

402. E. pulchellum Boiss.

403. Hesperis alpina Schur.

404. H. dinarica G. Beck.

405. H. fragrans Fisch.

406. H. glutinosa Vis.

407. H. matronalis L.

408. H. violacea Boiss. 
409. Hierschfeldia nasturtiifolia Fritsch.

410. Iberis intermedia Guers.

411. I. odorata L.

412. Isatis cordifolia Sweet.

413. I. glauca Boiss.

414. I. tinctoria L.

415. I. t. v. campestre Koch.

416. Lepidium cordatum Willd.

417. Mathiola bicornis hort.

418. Peltaria turcmena Lipsky.

419. Schiwereckia podolica Andr. et Boiss.

420. Sinapis alba L.

421. Sisymbrium Thalianum Gay.

422.* S. Sophia L.

423. Thlaspi arvense L.

424. T. densiflorum Boiss. et Ky.

425.* Turritis glabra L.

$X X I$. Capparidaceae.

426. Cleome speciosissima Deppl.

XXII. Resedaceae.

427. Reseda alba L.

428. R. lutea L.

XXIII. Crassulaceae.

4.9. Sedum aizoon $L$.

430. S. anopetalum D. C.

431. S. crassipes Wall. 
432. S. hybridum L.

433. S. Kamtschaticum Fisch. et Mey.

434. S. Kirilowii Rgl.

435. S. Maximowiczii Rg1.

436. S. spurium M. B.

437. S. s. v. splendens hort.

438. S. Stephanii Cham.

XXIV. Saxifragaceae.

439. Heuchera cylindrica Dougl.

440. H. sanguinaea Engelm.

441. H. s. ,Titanic" hort.

442. Mitella caulescens Nutt.

443. M. pentandra Hook.

444. Saxifraga crassifolia L.

445. S. geranioides L.

446. Tellima grandiflora $\mathrm{R}$. Br.

447. Tiarella cordifolia L.

XXV. Rosaceae.

448.* Agrimonia Eupatoria L.

449. A. E. ssp. odorata Hook.

450. A. pilosa Led.

451. Coluria potentilloides $\mathrm{R}$. $\mathrm{Br}$.

452. Eupatorium album L.

453. Filipendula camtschatica Maxim.

454. ${ }^{*} \mathrm{~F}$. hexapetala Gilib.

455. F. Ulmaria Maxim.

456. Fragaria indica Andr. 
457.*F. vesca $L$.

458. F. v. var. americana.

459. F. virginiana Duschesne.

460. Geum album Gmel.

461. G. canadense Kossy.

462. G. chiloense Balb.

463. G. macrophyllum Willd.

464. G. tyrolense Kern.

465.* G. urbanum L.

466. G. virginianum L.

467. Potentilla argentea L.

468. P. aurea L.

469. P. calabra Ten.

470. P. chrysantha Trev.

471. P. Degeni Siegfr.

472. P. Dombeyi Nestl. .

473. P. gracilis Dougl.

474. P. hybrida hort.

475. P. inclinata Vill.

476. P. pennsylvanica L.

477. P. pyrenaica Ram,

478. $\mathrm{P}$. recta $\mathrm{L}$.

479. P. rupestris $L$.

480. P. splendens Ram.

481. P. tanacetifolia Willd.

482. P. Thurberi A. Gray

483. P. transcaspica Th. Wolf.

484. Poterium sanguisorba $\mathrm{L}$.

485. P. sitchense S. Wats. 
486. Sangusorba minor Scop.

487. S. officinalis $L$.

488. S. tenuifolia Fisch.

489. Sibbaldia procumbens L.

XXVI. Leguminosae.

490. Anthyllis tetraphylla L.

491. Astragalus baeticus L.

492. A. canadensis L.

493. A. danicus Retz.

494. A. galegiformis L.

495. A. sulcatus Link.

496. Baptisia australis R. Br.

497. Cicer arietinum $L$.

498. Desmodium canadense DC.

499. Galega officinalis L.

500. Glycine max Merr.

501. Lathyrus latifolius $\mathrm{L}$.

502.* L. silvestris L.

503. Lupinus elegans $H . B$. et $K$.

504. L. polyphyllus Lindl.

505. Medicago coerulea Less.

506. M. hispida Gaertn.

507. M. radiata $L$.

508. M. scutellaria Mill.

509. Melilotus coereleus Desr.

510. M. officinalis Desr.

511. Onobrychis altissima L.

512. O. arenaria D. C. 
513. O. montana D. C.

514. Ornithopus sativus Brot.

515.* Orobus vernus Bernh.

516. Thermopsis caroliniana Curt.

517. T. fabacea D. C.

518. T. montana Nutt.

519. Tetragonolobus siliquosus Scop.

$520{ }^{*}$ Trifolium agrarium $\mathrm{L}$.

521. T. alpestre $\mathrm{L}$.

522. T. canescens Willd.

523. T. Lupinaster L.

524. ${ }^{*} \mathrm{~T}$. montanum $\mathrm{L}$.

525. T. pannonicum L.

$526 * \mathrm{~T}$. pratense L.

527. T. rubens $\mathrm{L}$.

528. T. spadiceum $L$.

529. Trigonella coerulea Ser.

530. Vicia americana Muhl.

$531 . * \mathrm{~V}$. pisiformis L.

$532{ }^{*} \mathrm{~V}$. sepium L.

XXVII. Geraniaceae.

533.* Erodium cicutarium L'Herit.

534. E. Hoefftianum C. A. M.

535. Geranium albanum Bieb.

536. G. albiflorum Hook.

537. G. dissectum L.

538.* G. sylvaticum L.

539. G. tuberos Lum. 
XXVIII. Linaceae.

540. Linum altaicum Fisch.

541. L. campanulatum $\mathrm{L}$.

542. L. extraxillare Kit.

543. L. flavum L.

544. L. gallicum L.

545. L. grandiflorum Desf.

546. L. perenne $\mathrm{L}$.

547. L. p. var. sibiricum D. C.

\section{Rutaceae.}

548. Ruta graveolens $\mathrm{L}$.

XXX. Malvaceae.

549. Althea nudiflora Lindl.

550. A. pallida W. et $\mathrm{K}$.

551. A. rosea Cav.

552. A. rosea fl. pleno

553. Kitaibelia vitifolium Willd.

554. Lavatera thuringiaca L.

555. L. assurgentiflora Kellog.

556. Malachra radiata L.

557. Malva alcea L.

558. M. moschata L.

559. M. pusilla With.

560. M. verticillata $L$.

561. Napaea dioica L.

562. Palava flexuosa Mast.

563. Sidalcea oregona A. Gr. 
XXXI. Cistaceae.

564. Helianthemum canadense Micht.

565. H. ovatum Dun.

566. H. polyfolium D. C.

567. H. pulverulentum D. C.

568. H. roseum Lam. et D. C.

569. H. variabile Spach.

XXXII. Hypericaceae.

570. Hypericum calicynum L.

571 . H. quadrangulum $\mathrm{L}$.

XXXIII. Violaceae.

572. Viola arborescens L.

573. V. tricolor L.

$574 . *$ V. arvensis Koch.

XXXIV. Datiscaceae.

575. Datisca cannabina $L$.

XXXV. Lythraceae.

576. Lythrum alatum Pursh.

XXXVI. Oenotheraceae.

577. Clarkia elegans Dougl.

578. C. pulchella Pursh.

579.* Epilobium angustifolium L.

580. E. Lamyi Schultz

581. Oenothera fruticosa L. 
582. O. missuriensis Sims.

583. O. speciosa Nutt.

XXXVII. Umbelliferae.

584, Aegopodium podagraria L.

$585^{*}$ Angelica silvestris L.

586. Anethum graveolens L.

587. Carum alpinum Spreng.

588. Cryptotaemia canadensis DC.

589. Eryngium Bourgati Gouan.

590. E. giganteum Bieb.

591. E. Oliverianum Delar.

592.* E. planum L.

593. Heracleum barbatum Ledeb.

594. H. flavescens Boiss.

595. H. mantegazzianum Lev. et Somm.

596. H. Olgae Rgl. et Schmalh.

597. H. villosum Fisch.

598. Levisticum officinale Koch.

599. Libanotis montana All.

600. Peucedanum alsaticum L.

601.* Pimpinella saxifraga $L$.

XXXVIII. Pirolaceae.

602.* Pirola secunda L.

XXXIX. Primulaceae.

603.* Lysimachia vulgaris $L$.

604. Primula officinalis Jacq. 
605. P. o. fl. rubro

$X L$. Plumbaginaceae.

606. Acantholimon glumaceum Boiss.

XLI. Asclepiadaceae.

607. Asclepias syriaca L.

XLII. Polemoniaceae.

608. Gilia aggregata Spreng.

609. G. capitata Dougl.

610. Polemonium boreale Adams.

611. P. campanulatum Fr.

612. P. coeruleum L.

613. P. filicinum Greene.

614. P. flavum Greene

615. P. gracile Willd.

616. P. grandiflorum Benth.

617. P. himalayanum Baker.

618. P. mexicanum Cerv.

619. P. occidentale Greene.

620. P. pulchellum Bunge.

621. P. reptans L.

622. P. Richardsonii R. Grah. v. humilis Gray.

623. P. sibiricum D. Don.

624. P. Van-Bruntiae Britt.

XLIII. Hydrophyllaceae.

625. Nemophila insignis Dougl.

28 
626. Phacelia campanularia A. Gray.

627. Ph. fimbriata Michx.

628. $\mathrm{Ph}$. loasaefolia Torr.

629. Ph. whitlavia A. Gray.

$X L I V$. Borraginaceae.

630. Anchusa officinalis $L$.

631. Borrago laxiflora Willd.

632. Cerinthe alpina Kit.

633. C. major L.

634. Cynoglossum cheirifolium L.

635. C. officinale $\mathrm{L}$.

636. Echium creticum L.

637. E. rubrum Jacq.

638. Lindelofia spectabilis Lehm.

639. Lithospermum officinale $\mathrm{L}$.

640. Mertensia dahurica Don.

641. Myosotis alpestris F. W. Schmidt.

642. M. a. var. "Victoria".

643.* Pulmonaria officinalis $\mathrm{L}$.

$X L V$. Verbenaceae.

644. Verbena urticifolia L.

XLVI. Labiatae.

645. Ballota nigra $L$.

646. Betonica grandiflora Steph.

647. Brunella grandiflora Jacq.

648. B. pienina Ullepitsch. 
649. B. pyrenaica Philip.

650. Dracocephalum Moldavica L.

651. D. nutans L. v. alpinum

652. Hyssopus officinalis L.

653. Lallemantia canescens Fisch. et Mey.

654. L. ibericum Fisch. et Mey.

655. L. peltata Fisch. et Mey.

656. Leonurus glaucescens Bge.

657. L. marrubiastrum L.

658. Lophanthus anisatus Benth.

659. Monarda fistulosa L.

660. M. violacea Desf.

661. Nepeta azurea R. Br.

662. N. cataria L.

663. N. cocainiaca Regl.

664. N. grandiflora M. B.

665. Nepeta macrantha Fisch.

666. N. nuda L.

667. N. n. ssp. pannonica Gams.

668. Origanum heracleifolium L.

669.* O. vulgare $L$.

670. Phlomis alpina Pall.

671. Ph. chrysophylla Boiss.

672. $\mathrm{Ph}$. tuberosa L.

673. Ph, viscosa Poir.

674. Salvia argentea L.

675. S. dumetorum Andrz.

676. S. hians Royle.

677. S. nutans $\mathrm{L}$. 
678. S. pratensis L.

679. S. Przewalskii Max.

680. Scutellaria alpina L.

681. S. altissima L.

682. Sideritis hyssopifolia L.

683. Stachys lanata Jacq.

684. S. nivea Labill.

685. S. officinalis Trev.

686. S. recta L.

687. Teucrium scorodonia L.

688. Thymus glaber Mill.

689. Ziziphora capitata L.

XLVII. Solanaceae.

690. Atropa Belladonna L.

691. Datura aegyptica Vesl.

692. D. stramonium L.

693. Nicandra physaloides Gaertn.

694. Nicotiana rustica L.

695. Physalis peruviana $L$.

696. Ph. Franchetti Mast.

697. Physochlaina orientalis G. Don.

698.* Solanum Dulcamara L.

\section{Scrophulariaceae.}

699. Antirrhinum Coulterianum Benth.

700. Digitalis ambigua Murr.

701. D. lanata Ehrh.

702. D. Iutea L. 
703. D. media Roth.

704. D. nevadensis Kunze.

705. D. purpurea L.

706. Erinus alpinus L.

707. Linaria alpina Mill.

708. L. bipartita Willd.

709. L. chalepensis Mill.

710. L. genistefolia Mill.

711. L. purpurea Mill.

712. L. vulgaris Mill.

713. Mimulus luteus $\mathrm{L}$.

714. Pentastemon alpinus Torr.

715. P. cobaea Nutt.

716. P. deustus Dougl.

717. P. digitalis Nutt.

718. P. glaber Pursh.

719. P. glaucus Grah.

720. P. hirsutus Willd.

721. P. ovatus Dougl.

722. P. procerus Dougl.

723. P. tubiflorum Nutt.

724. P. Watsonii A. Gray.

725. Rhinanthus crista galli L.

726. Scrophularia alata Gil.

727. S. chrysantha Jaub. et Spach.

728. Verbascum lychnitis L.

729. V. 1. ,Miss Willmott" hort.

730. V. nigrum L.

731. V. phlomoides L. 
732. V. phoeniceum L.

733. V. Wilsonii hort.

734. Veronica gentianoides Wahl.

735. $V$. latifolia $L$.

736. V. spicata L.

737. V spuria L.

XLIX. Plantaginaceae.

738. Plantago kamtschatica Hook.

739.* P. major L.

740.* P. media L.

741. P. purpurascens Willk.

742. P. Rugelii Decne.

743. P. Schwarzenbergiana Schur.

744. P. tibetica Hook, et Thoms.

L. Rubiaceae.

745. Asperula ciliata Rochel.

746. Galium saccharatum All.

LI. Dipsacaceae.

747. Cephalaria alpina Schrad.

748. C. ambrosioides R. et Sch.

749. C. gigantea Barb.

750. C. procera Fisch.

751. C. syriaca Schrad.

752. C. tatarica Schrad.

753. Dipsacus fullonum $\mathrm{L}$.

754. D. Silvestris Huds. 
755. D. strigosus Willd.

756.* Knautia arvensis Coult.

757. Scabiosa atropurpurea L.

758. S. caucasica Bieb.

759. S. graminifolia L.

760. S. micrantha Desf.

761. S. Olivieri Coult.

LII. Cucurbitaceae.

762. Bryonia alba L.

LIII. Campanulaceae.

763. Adenophora denticulata Fisch.

764. Campanula alliariaefolia Willd.

765. C. americana $\mathrm{L}$.

766. C. barbata L.

767. C. caespitosa Vill.

768. C. carpatica Jacq.

769. C. collina M. B.

770. Campanula grandis Fisch. et Mey.

771. C. Grossekii Heuff.

772. C. lactiflora $M$. Bieb.

773. C. latifolia L.

774. C. Leutweinnii Heldr.

775. C. medium L.

776. C. Morettiana Reichb.

777. C. nobilis Lindl.

778. C. patula L.

779. C. Portenschlagiana Roem. at Schult. 
780. C. pulcherrima Schrank.

781. C. punctata Lam.

782. C. Raineri Perpenti.

783. C. ranunculoides L.

784. C. sarmatica Ker-Gawl.

785. C. turbinata Schott.

786. C. waldsteiniana $\mathrm{R}$. et S.

787. Codonopsis clematidea C. B. Clarke.

788. C. ovata Benth.

789. C. ussuriensis Hemsl.

790. Jasione perennis Lam.

791. Phyteuma Halleri All.

792. P. orbiculare L.

793. P. Scheuchzeri All.

794. Platycodon grandiflorum A. DC.

795. Symphiandra armena A. DC.

796. S. Hofmannii Pant.

797. S. pendula A. DC.

798. S. Wanneri Heuff.

LIV. Compositae.

799. Achillea cartilaginea Led.

800. A. coarctata Poir.

801. A. distans W. et $\mathrm{K}$.

802. A. filipendulina Lam.

803. A. macrophylla L.

804. A. nobilis L.

805. A. ochroleuca Ehrh.

806. A. odorata L. 
807. A. pseudo-pectinata Janka.

808. A. sibirica Ledeb.

809. Anaphalis margaritacea Benth. er. Hook.

810. Anthemis altissima L.

811. A. candidissima Willd.

812. A. macedonica Boiss.

813. A. ruthenica Bieb.

814. Arnica Chamissonis Less.

815. A. foliosa Nutt.

816. A. longifolia Eaton.

817. A. montana L.

818. A. sachalinensis A. Gray.

819. Aster altaicus Willd.

820. A. foliaceus Lindl.

821. A. himalaicus C. B. Clarke.

822. A. paniculatus Lam.

823. A. peregrinus Pursh.

824. A. salignus Willd.

825. A. tibeticus Hook.

826. A. junnanensis Franch.

827. Biedens dahlioides Wats.

828. Calendula aegyptica Desf.

829. C. algeriensis Boiss, et Bent.

830. C. arvensis $L$.

831. C. officinalis L. "Meisterstück".

832. C. stellata Cav.

833. Calimeris incisa D. C.

834. Centaurea atropurpurea W. et K.

835. C. macrocephala Puschk.

36 
836. C. montana L

837. C. nigra L.

838. C. ruthenica Lam.

839. C. scabiosa L.

840. Chrysanthemum alpinum L.

841. Ch. coccineum Willd.

842.* Cirsium arvense Scop.

843. Cnicus benedictus L.

844. Coreopsis auriculata Schkuhr.

845. C. grandiflora Nutt.

846. Cosmos bipinnatus Cav.

847. Dimorphotheca aurantiaca D. C.

848. D. pluvialis Moench.

849. Echinops albicaulis Bgl.

850. E. Gmelini Turcz.

851. E. persicus Stev.

852. Erigeron alpinus L. ssp. glabratus Brig.

853. E. Coulteri Porter et Coult.

854. E. glabellus Nutt.

855 E. macranthus Nutt v. superbus hort.

856. Eriophyllum caespitosum Dougl.

857. Gaillardia aristata Pursh.

858. G. grandiflora hort.

859. Helenium Hoopesii A. Gray.

860. Helianthella quinquenervis A. Gray.

861. Helichrysum lanatum D. C.

862. Heliopsis laevis Pers.

863. Hieracium boreale Fr.

864. H. canadense Michx. 
865. H. pictum Scheicher.

866. H. robustum Fr.

867. Inula macrocephala Boiss et Kotschy.

868. I, magnifica Lipsky.

869. I. Royleana D. C.

870. Jurinea arachnoidea Bge.

871. Lactuca scariola L.

872. L. virosa L.

873.* Leontodon autumnalis L.

874. L crispus Vill.

875. L. pyrenaicus Gouan.

876. L. saxatilis Lam.

877. Leontopodium alpinum Cass.

878. Madia sativa Mol.

879.* Matricaria discoidea D. C.

880. Palafoxia texana D. C.

881. Picris hieracioides L.

882. Pyrethrum corymbosum Willd.

883. P. parthenium Sm.

884. Rudbeckia flava Greene.

885. R. hirta L.

886. Saussurea alpina D. C.

887. Scorzonera hispanica L.

888. S. purpurea L.

889. Senecio adoniifolius Lois.

890. S. alpinus Scop.

891. S. ambraceus Turcz.

892. S. suaveolens E11.

893. S. vulgaris L.

38 
894. Serratula coronata $\hat{L}$.

895. S. Gmelini Ledeb.

896. Silphium perfoliatum L.

897. Solidago gigantea Ait.

898. S. hybrida hort. "Goldstrahl".

899. S. multiradiata Ait. v. arctica.

900. S. squarrosa Muhl.

901.* Sonchus arvensis $\mathrm{L}$.

902. Tanacetum huronense Nutt.

903.* T. vulgare $\mathrm{L}$.

904.* Taraxacum officinale Weber.

905. Troximon gracilens Gray.

906. Verbesina enceloides Benth. et Hook.

907. Zinnia uniflora L.

\section{SEMINA PLANTARUM DECO- RANTIUM.}

1. Polypodiaceae.

908. Adiantum capillus Veneris L.

909. Asplenium bulbiferum Forst.

910. Nephrolepis tuberosa Hook.

911. Pteris cretica L.

912. P. C. v. albo-lineata,

II. Cyperaceae.

913. Cyperus natalensis Hochst. 
914. C. papyrus L.

III. Iridaceae.

915. Gladiolus hybridus hort (bulbi).

916. G. primulinus Bak (bulbi).

IV. Amaranthaceae.

917. Celosia cristata L.

V. Portulacaceae.

918. Portulaca grandiflora Hook.

VI. Caryophyllaceae.

919. Dianthus barbatus L.

920. D. chinensis L.

VII. Cruciferae.

921. Alyssum Benthamil Lam. v. compactum hort,

VIII. Oxalidaceae.

922. Oxalis corniculata $\mathrm{L}$.

IX. Tropaeolaceae.

923. Tropaeolum majus $L$, v. nanum hort.

$X$. Balsaminaceae.

924. Impatiens balsamina L. fl. pleno hort.

XI. Violaceae.

925. Viola tricolor L. v. hybrida hort. 
XII. Begoniaciae.

926. Begonia: semperflorens Link. et Otto v. ${ }_{n} \mathrm{Al}$ bert Martin".

927. B. s. v. "Marienburg" hort.

928. B. s. v. "Strigans" hort.

929. B. s. v. "Vernon compacta" hort.

930. B. s. v. "Vulcan" hort.

931. B. s. v. "Lauberin" hort.

932. B. s. v. "Domtaube" hort.

XIII. Cactacéae.

933. Cereus peruvianus Mill.

v. monstrosus D. C. (viv.)

934. Phyllocactus phyllanthoides Link. (viv.).

XIV. Onagraceae.

935. Godetia amoena Lilja hybrida ,Sybil Sherwood".

936. Oenothera missuriensis Sims.

$X V$. Asclepiadaceae.

937. Asclepias incarnata $L$.

XVI. Polemoniaceae.

938. Phlox Drummondii Hook.

XVII. Borraginaceae,

939. Anchusa italica Retz.

940. Heliotropium corymbosum Ruiz, et Pav. 
X VIII. Verbenaceae.

941. Verbena hybrida hort. fl. coerulea hort.

942. V. h. "Defiance" hort.

943. V. h. mamontovaja, „Lucifera", Scharlachovaja.

$X I X$. Solanaceae.

944. Nicotiana affinis T. Moore.

945. N. colossea André.

946. N. Sanderae hort. v. atrosanguinea hort.

947. N. tabacum L. „Havanna" hort.

948. N. t. v. Wigandioides hort.

949. Petunia hybrida hort. v. grandiflora hort.

950. P. h. "Aurora" hort.

951. P. h. "Alba“ hort.

952. P. h. "Brilliant Rose" hort.

953. Petunia hybrida "Coerulea" hort.

954. P. h. "Stalblau" hort.

955. Salpiglossis variabilis hort.

956. S. v. ",Velvet red".

$X X$. Scrophulariaceae.

957. Alonsoa Warscewiczii Rgl. var. compactus hort.

958. Antirrhinum majus L. fl. albo hort.

959. A. m. v. "Weisses Wunder".

960. A. m. v. nanum grandiflorum „Karminkönigin" hort. 
961. A. m. „Purpurkönig“ hort.

962. A. m. fl. rosea hort.

963. A. m. "Tschernyi Platsch" hort.

964. Calceolaria pinnata $\mathrm{L}$.

965. Schizanthus hybridus hort v. grandiflora hort.

XXI. Martyniaceae.

966. Martynia proboscidea Glox.

XXII. Gesneriaceae.

967. Gloxinia hybrida hort.

968. Streptocarpus polyanthus Hook.

XXIII. Cucurbitaceae.

969. Echinocystis lobata Torr. et Gr. (1937).

XXIV. Campanulaceae.

970. Lobelia erinus L.

971. L. e. "Crystal Palace" hort.

$X X V$. Compositae.

972. Ageratum conyzoides L. v. mexicanum hort. 973. Brachycome iberidifolia Benth.

974. Calendula officinalis L. fl. pl. „Favorit Bucartes".

975. C. O. "Zolotij Shar", hort.

976. Callistephus chinensis Nees „Krassavitza Rynka" scharlachovaja hort. 
977. C. c. ,Straussovoje pero6 karminno-rosovoje hort.

978. C. c. "Straussovoje pero" goluboje hort. 979. Dahlia Merkii Lehm.

980. Gaillardia hybrida hort.

981. Pyrethrum parthenifolium Willd. v. aureum hort.

982. Solidago canadensis $\mathrm{L}$.

983. Tagetes erectus L.

984. T. patulus L. "Lilliput" hort.

985. Zinnia elegans Jacq.

IV. SEMINA PLANTARUM OFFICINALIUM.

Liliaceae.

986. Asparagus officinalis $\mathrm{L}$.

Iridaceae.

987. Iris sibirica L.

Polygonaceae.

988. Rheum palmatum L. v. tanguticum Maxim.

989. R. rhaponticum L.

990. R. undulatum L.

Chenopodiaceae.

991. Chenopodium ambrosioides $\mathrm{L}$. 
992. Ch. Quinoa Willd.

Caryophyllaceae.

993. Agrostemma Githago L.

994. Lychnis chalcedonica L.

Ranunculaceae.

995. Adonis autumnalis L.

996. Nigella damascena L.

997. N. sativa L.

998. Ranunculus acer $\mathrm{L}$.

Papaveraceae.

999. Chelidonium majus L.

1000. Papaver somniferum L. „Cardinal“.

1001. P. s. fl. monstrosum.

1002. P. s. fl. pleno.

1003. P. s. v. polycephalum.

1004. P. s. v. setigerum.

1005. P. s. (ex Barnaul).

1006. P. s. (ex Extrema Oriente).

1007. P. s. (ex Germany).

1008. P. s. (ex Mariinsk).

1009. P. s. (ex Tatarstan).

1010. P. s. (ex Tatrespublik).

1011. P. s. (ex Tjan-Tschjan).

1012. P. s. (ex Sajsan).

Cruciferae.

1013. Capsella Bursa pastoris Medic. 
1014. Cochlearia officinalis $\mathrm{L}$.

1015. Hesperis matronalis $L$.

1016. Iberis amara L.

1017. Isatis tinctoria L.

1018. Thlaspi arvense $\mathrm{L}$.

Crassulaceae.

1019. Sedum acre L.

Rosaceae.

1020. Geum urbanum L.

1021. Poterium sanguisorba L.

1022. Sanguisorba officinalis $\mathrm{L}$.

Geraniaceae.

1023. Geranium silvaticum L.

Leguminosae.

1024. Melilotus officinalis Desr.

Rutaceae.

1025. Ruta graveolens L.

Malvaceae.

1026. Althea armeniaca Tenore.

1027. A. officinalis $\mathrm{L}$.

1028. A. rosea Cav. v. nigra hort.

1029. A. r. v. n. fl. pleno.

1030. Malva alcea L. 
1031. M. moschata L.

Umbelliferae.

1032. Anethum graveolens L.

1033. Carum Carvi L.

1034. Conium maculatum L.

1035. Coriandrum sativum L. v. aureum.

1036. C. s. v. melfitense.

1037. C. s. v. microcarpum.

1038. C. s. v. nanum.

1039. Daucus carota L.

1040. Eryngium planum L.

1041. Foeniculum officinale All. f. italiana.

1042. Foeniculum vulgare Mill.

1043. Levisticum officinale Koch.

1044. Pastinaca sativa L.

1045. Pimpinella Anisum L.

Primulaceae.

1046. Primula officinalis L.

Polemoniaceae.

1047. Polemonium coeruleum L.

Borraginaceae.

1048. Cynoglossum officinalis L.

1049. Lithospermum officinale $\mathrm{L}$.

Labiatae.

1050. Dracocephalum Moldavica L. 
1051. D. M. fl. albo.

1052. Hyssopus officinalis $\mathrm{L}$.

1053. Lophanthus anisatus Benth.

1054. Melissa officinalis L.

1055. Monarda didyma L.

1056. M. fistulosa L.

1057. Nepeta bucharica Lipsky.

1058. N. cataria L.

1059. N. macrantha Fisch.

1060. Ocimum basilicum L.

1061. O. canum Sims.

1062. O. minimum L.

1063. Salvia officinalis $\mathrm{L}$.

1064. S. Sclarea L.

1065. S. s. fl. albo.

1066. Saturea hortensis L.

1067. Thymus vulgaris L.

Solanaceae.

1068. Atropa Belladonna L.

1069. Capsicum annum $L$.

1070. Datura metel L.

1071. D. quercifolia H. B. K.

1072. D. stramonium L.

1073. D. tatula Mnch.

1074. D. t. M. v. gigantea hort.

1075. Hyoscyamus agrestis Kit.

1076. H. pallidus $W$. et $K$.

1077. Nicandra physaloides Gaertn. 
1078. Physalis pubescens 56250 .

1079. P. p. 133708.

1080. Solanum nigrum L.

Scrophulaceae.

1081. Digitalis ambigua Murr.

1082. D. ferruginea L.

1083. D. hybrida Katsch.

1084. D. lanata Ehrh.

1085. D. purpurea L.

1086. Verbascum phlomoides L.

1087. V. tapsiphorme Schrad.

Plantaginaceae.

1088. Plantago lanceolata L.

Valerianaceae.

1089. Patrinia intermedia Roem. et Sch.

1090. Valeriana nitida Pr.

1091. V. officinalis L.

1092. V. rossica Sm.

Campanulaceae.

1093. Campanula latifolia L.

1094. C. Trachelium L.

1095. Lobelia inflata $\mathrm{L}$.

1096. L. syphilitica L. 
Compositae.

1097. Achillea Millefolium L.

1098. A. nobilis L.

1099. Anthemis nobilis L.

1100. Arctium majus Bernh.

1101. A. minus Bernh.

1102. A. tomentosum Schrank.

1103. Arnica montana L.

1104. Artemisia absinthium L.

1105. A. maritima L. v. astrachanica Kasak.

1106. A. vulgarts $\mathrm{L}$.

1107. Bellis perenneis L.

1108. Calendula officinalis $\mathrm{L}$.

1109. Centaurea cyanus L.

1110. C. c. f1. rosea.

1111. Cephalophora aromatica Schrad.

1112. Chordrilla juncea $\mathrm{L}$.

1113. Chrysanthemum carneum Steud.

1114. Ch. cinerariaefolium Vis.

1115. Ch. corymbosum L.

1116. Ch. macrophyllum W. et $\mathrm{K}$.

1117. Ch. partenifolium B. Fedtsch.

1118. Ch. roseum Web. et Mohr.

1119. Cnicus benedictus Gaertn.

1120. Grindelia robusta Nutt.

1121. Inula britanica L.

1122. I. Helenium L,

1123. Lactuca virosa L. 
1124. Matricaria chamomilla L.

1125. M. discoidea D. C.

\section{SEMINA PLANTARUM OLEARUM ET TECHNICARUM.}

Moraceae.

1126. Cannabis sativa L.,,Kamenetz-Podolskaja".

1127. C. s. "Kawkazsko-Luninskaja“.

1128. C. s. "Italiansko-Luninskaja“.

1129. C. s. "Novgorod-Severskaja".

1130. C. s. "Stavropolsakja“.

Polygonaceae.

1131. Rumex acetosa L.

1132. R. confertus Willd.

Cruciferae.

1133. Brassica juncea Czern.

1134. B. nigra Koch.

1135. Sinapis alba L.

Resedaceae.

1136. Reseda luleola L.

Saxifragaceae.

1137. Bergenia crassifolia L. 
Linaceae.

1138. Linum perenne L.

1139. L. usitatissimum L. „D-83“.

1140. L. u. "Omega“.

1141. L. u. "Pobeditel "

1142. L. u. "Priadiltschik"

1143. L. u. "Reproduktia II"

1144. L. u. "Stachanovetz".

Euphorbiaceae.

1145. Euphorbia Lathyrus L.

1146. Ricinus communis L.

1147. R. c. "Mandshuria“

1148. R. c. "R. P."

1149. R. c. "Sang 142 ".

1150. R. c. "Sch. D".

Malvaceae.

1151. Abutiolon Avicennae Gaerth. (ex Gallia)

1152. A. A. (ex Mandshuria).

1153. A. A. (ex Zürich).

1154. A. A. $n^{\circ} 8094$.

1155. Malva brasiliensis Desr.

1156. M. crispa $n^{\circ} 4762$ (ex Belgique).

1157. M. mauritania L.

1158. M. meluca $n^{\circ} 136630$.

1159. M. m. n 139239.

1160. M. m. n 140522.

1161. M. pusilla With. 
1162. M. verticillata L. $\mathrm{n}^{\circ} 4760$.

1163. M. v. $n^{\circ} 112260$.

1164. M. v. $11^{\circ} 128580$.

1165. M. v. n 133551.

1166. M. v. n 13990 .

Asclepiadaceae.

1167. Asclepias Cornuti Decne.

1168. Phacelia kipinnatifida Michx.

Labiatae.

1169. Lallemantia iberica Fisch. et Mey.

Cucurbitaceae.

1170. Cucurbita Pepo L.

1171. C. P. v. annulata.

1172. C. P. v. caerifero.

1173. C. P. v. aurautiaca.

1174. C. P. v. maxima Duchesne.

1175. C. P. v. oviformis alba.

1176. C. P. v. verrucosa.

Compositae.

1177. Anthemis tinctoria L.

1178. Carthamnus tinctorius $L$.

1179. Lactuca sativa L.

1180. Helianthus agrophyllus Torr.

1181. H. annuus L.

1182. H. a, v. californicus. 
1183. H. a. v. "Fuxinca".

1184. H. a. v. purpurea.

1185. H. a. "Gryzovoi".

1186. H. a. "A-41".

1187. H. a. $\mathrm{n}^{\circ} 762$.

1188. H. a. $\mathrm{n}^{\circ} 1846$.

1189. H. a. $\mathrm{n}^{\circ} 1975$.

1190. H. macrophyllus Willd. v. giganteus.

1191. Madia sativa Don.

\section{SEMINA PLANTARUM GRANE- ARUM ET LEGUMINOSARUM.}

\section{Gramineae.}

1192. Agropyrum cristatum Bess.

1193. A. desertorum Schult.

1194. Agrostis alba L.

1195. Alopecurus pratensis L.

1196. Andropogon Sorghum Brot. "Belyi Kefir“.

1197. A. s. "Gaolian“

1198. Avena Ludoviciana Dur.

1199. A. nuda L.

1200. A. sativa L. v. aristata Kr. "Zhemtschuschina".

1201. A. S, a. "Ligovo $\mathrm{II}^{*}$.

1202 A. s. a. "Mirash".

1203. A. s. a. "Moskovskii A - 315 ".

54 
1204. A. s. a. aurea Körn "Leitevizki".

1205. A. s. a. "Lochovski".

1206. A. s, d. "Stepniak n ${ }^{\circ} 648^{\prime \prime}$.

1207. A. s. a. "Zolotoj Dozhd".

1208. A. s. a $\mathrm{n}^{\circ} 5056$.

1209. A. s. v. brunnea Körn.

1210. A. s. v. mutica Al. "Dippe“.

1211. A. s. m. "Pobeda“.

1212. A. s. m. .Schatilovski“.

1213. A. s. m. Severianin ".

1214. A. s. v. obtusata Al. "Magistral".

1215. A. strigosa Schreb.

1216. Bromus inermis Leyss.

1217. B. erectus Huds.

1218. Dactylis glomerata L. $n^{\circ} 204$.

1219. Elymus sibiricus L.

1220. Festuca ovina L.

1221. F. pratensis Huds.

1222. F. rubra L.

1223. Hordeum distichum L. v. abyssinicum Ser.

1223'. H. d. v. erectum Schübl. "Prima“.

1224. H. d. v. Leocrinum.

1225. H. d. v. medicum Körn n 26.

1226. H. d. v. $\mathrm{m} \mathrm{n}^{\circ} 46$.

1227. H. d. v. nudum L. $n^{\circ} 110$.

1228. H. d. v. $n^{\circ} 105$.

1229. H. d. v. nutans Schübl. " Colchicum $10 / 30^{\circ}$ 1230. H. d. v. "Europaeum $353 / 133^{\text {". }}$

1231. H. d. v. n. "Viner". 
1232. H. d. n. $\mathrm{n}^{\circ} 17$.

1233. H. d. n. n 7054 .

1234. H. d. v. persicum Körn $n^{\circ} 064$.

1235. Hordeum vulgare $L$. v. coeleste $L$. $n^{\circ} 46$.

1236. H. v. v. pallidum Ser. "Pioner".

1237. H. v. p. "Tschervonetz".

1238. H. v. p. "Tulunski“.

1239. H. v. p. "Viatsky $\mathrm{n}^{\circ} 1009^{\prime}$.

1240. H. v. p. $\mathrm{n}^{\circ} 915$.

1241. H. v. v. perallelum Körn.

1242. H. v. v. trifurcatum.

1243. Lolium perenne $\mathrm{L}$.

1244. L. italicum A. Br.

1245. Phleum pratense L.

1246. Secale cereale L.

1247. S. c. v. vulgare "Avangard".

1248. S. c. v, "Besentschukskaja".

1249. S. c. v. "Eliseevskaja".

1250. S. c. v. "Lissizina".

1251. S. c. v. "Suprunenko".

1252. S. c. v. "Taraschanskaja".

1253. Triticum compactum Host.

1254. T. dicoccum Schübl. v. rufum.

1255. T. durum Desf. v. affine Korn $n^{\circ} 309$.

1256. T. d. v. $\mathrm{n}^{\circ} 796$.

1257. T. d. v. Hordeiforme Host $\mathrm{n}^{\circ} 04$.

1258. T. d. v. H. $\mathrm{n}^{\circ} 010$.

1259. T. d. H. $n^{\circ} 189$. 
1260. T. d. H. $n^{\circ} 432$.

1261. T. d. H. $n^{\circ} 496$.

1262. T. d. H. n० 675 .

1263. T. d. H. n 694 .

1264. T. d. v. leucurum $\mathrm{Al}, \mathrm{Bg}-26^{4}$.

1265. T. d. 1. "Bg $-33^{\prime}$.

1266. T. d. v. libycum Körn.

1267. T. d. v. melanopus Al. $\mathrm{n}^{\circ} 069$.

1268. T. d. m. $\mathrm{n}^{\circ} 826$.

1269. T. d. m. n 886 .

1270. T. monococcoum L. (ex America).

1271. T. spelta $L$.

1272. T. vulgare Host. v. albidum Al. n 676 .

1273. T. v. v. alborubrum Korn "Sarrubra ".

1274. Triticum vulgare v. caesium Al. $\mathrm{n}^{\circ}$ III.

1275. T. v. v. erythrospermum Körn „,Durabl“".

1276. T. v. e. "Cooperatorka".

1277. T. v. e. "Novinka“.

1278. T. v. e. "Ucrainka".

1279. T. v. e. "Zarja'.

1280. T. v. e. "Zemka".

1281. T. v. e. $n^{\circ} 46 / 131$.

1282. T. v. e. $n^{\circ} 1114$.

1283. T. v. V. ferrugineum Al. "Moskovskaja“ $n^{\circ} 2411$.

1284. T. v. f. "Moskovskaja $n^{\circ} 2453^{\prime \prime}$.

1285. T. v. f. "Simbileevskaja $146^{\circ "}$.

1286. T. v. f. $\mathrm{n}^{\circ} 150$.

1287. T. v. v. hostianum Clem. $\mathrm{n}^{\circ} 237$. 
1288. T. v. v. lutescus Al. $\mathrm{n}^{\circ} 053$.

1289. T. v. $1 . \mathrm{n}^{\circ} 062$.

1290. T. v. $1 . \mathrm{n}^{\circ} 323$.

1291. T. v. $1 . \mathrm{n}^{\circ} 329$.

1292. T. v. $1 . \mathrm{n}^{\circ} 479$.

1293. T. v. $1 . \mathrm{n}^{\circ} 786$.

1294. T. v. $1 . \mathrm{n}^{\circ} 1060 / 10$.

1295. T. v. 1. "Beta-Stojkaia“.

1296. T. v. 1. "Charkovskaja $n^{\circ} 436$ ".

1297. T. v. 1. "Engelgardt".

1298. T. v. 1. "Garnet“".

1299. T. v. 1. „Kasanskaja $76^{\text {" }}$.

1300. T. v. 1. "Saratovskaja“.

1301. T. v. 1. "Sarmatka“.

1302. T. v. 1. "Teiskaja“.

1303. T. v. 1. "Pyreini-Pschenitschnii Gybrid“.

1304. T. v. 1. "Viatskaja $\mathrm{n}^{\circ} 05^{\prime \prime}$.

1305. T. v. 1. "Viatskaja $\mathrm{n}^{\circ} 120^{\prime \prime}$.

1306. T. v. 1. ",Viatskaja n ${ }^{\circ} 529$ “.

1307. T. v. $1 . \mathrm{n}^{\circ} 27 / 36$.

1308. T. v. $1 . \mathrm{n}^{\circ} 46 / 137$.

1309. T. v. $1 . \mathrm{n}^{\circ} 118$.

1310. T. v. $1 . \mathrm{n}^{\circ} 328$.

1311. T. v. $1 . \mathrm{n}^{\circ} 434$.

1312. T. v. $1 . \mathrm{n}^{\circ} 434 / 154$.

1313. T. v. v. milturum Al.

1314. T. v. m. $n^{\circ} 321$.

1315. Zea mays L, indurata v, alba Al. „Korol Philipp“. 
1316. Z. m. i. v. ,Severodacotskaja“"

1317. Z. m. 1. v. flavorubra Körn "Brownconti“",

1318. Z. m. i. f. "Minnesotta $n^{\circ} 13^{4}$.

1319. Z. m. i. v. multicolor Al. "Burlei Caunti“"

1320. Z. m. i. v. saccharata Körn subdulcis Kuleschov et Koshuchov.

Leguminosae.

1321. Cicer arietinum L. v. albus "Belyi“.

1322. C. a. a. "Belyi n ${ }^{\circ} 157 / 2^{\prime \prime}$.

1323. C. a. a. "Krasnyi“".

1324. C. a. a. "Tschornyi".

1325. C. a. a. $\mathrm{n}^{\circ} 77$.

1326. C. a. v. rotundatum.

1327. Ervum lens L. v. macrosperma Bor. f. nummularia L. "Obyknovennaja".

$1327^{\prime}$. E. 1. m. n. "Saratovskaja n ${ }^{\circ} 13^{\prime \prime}$.

1328. E. 1. m. n. "Saratovskaja $n^{\circ} 23^{\prime \prime}$.

1329. E. 1. m. n. "Saratovskaja n ${ }^{\circ} 140^{\prime \prime}$,

1330. E. 1. m. f. glaucosperma Tschern. „Zelonaja Srednaja".

1331. E. 1. m. g. "Seraja $\mathrm{n}^{\circ} 32 / 5^{\prime \prime}$.

1332. E. 1. v. microsperma Bar. "Coritschnevaja Melkosemiannaja".

1333. Glycine hispida Maxim. „Belozerkovskaja $n^{\circ} 26^{\prime \prime}$.

1334. G. h. "Belozerkovskaja $n^{\circ} 55^{\prime \prime}$.

1335. G. h. "Belozerkovskaja $\mathrm{n}^{\circ} 58^{\prime \prime}$.

1336. G. h. ,Chabarovskaja $118^{\prime \prime}$. 
1337. G. h. $n^{\circ} 07$.

1338. Lathyrus sativus L. (ex Gornaja Buchara).

1339. L. s. (ex. Srednaja Volga).

1340. L. s. $\mathrm{n}^{\circ} 152$.

1341. L. S. $\mathrm{n}^{\circ} 285$.

1342. L. s. $\mathrm{u}^{\circ} 539$.

1343. L. S. $\mathrm{n}^{\circ} 792$.

1344. L. s. $n^{\circ} 924$.

1345. L. s. n 990.

1346. L. s. $\mathrm{n}^{\circ} 1156$.

1347. Lupinus albus L. "Belyi".

1348. L. angustifolius L. $\mathrm{n}^{\circ} 143$.

1349. L. a. $\mathrm{n}^{\circ} 374$.

1350. L. a. $n^{\circ} 418$.

1351. L. a. $\mathrm{n}^{\circ} 983$.

1352. Lupinus luteus L. $\mathrm{n}^{\circ} 345$.

1353. L. $1 . \mathrm{n}^{\circ} 530$.

1354. L. mutabilis L.

1355. L. pilosus $\mathrm{L}$.

1356. L. polyphyllus Lindl.

1357. Phaseolus vulgaris L. "Belaja".

1358. Ph. vulgaris ,Isumrudnaja“.

1359. Ph. v. "Rissovaja".

1360. Ph. v. "Stepnaja Zasuchoustoichivaja“.

1361. Ph. v. "Schedraja Amerikanskaja“.

1362. Ph. v. "Zolotaja Gora".

1363. Pisum arvense $L$. „Peluschka $n^{\circ} 32^{\prime}$.

1364. P. a. „Peluschka $\mathrm{n}^{\circ} 33^{\prime \prime}$.

1365. P. a. "Peluschka $n^{\circ} 40 "$. 
1366. P. a. "Peluschka $n^{\circ} 43^{\prime \prime}$

1367. P. sativum L. v. coronatum Al. „Schtambovyi".

1368. P. s. v. glaucospermum Al, ,Folger Heine". 1369. P. s. g. ,Moskovskii B - 559".

1370. P. s. g. "Ranniy Zelonij".

1371. P. s. g. "Victoria Zelonaja".

1372. P. s. v. saccharatum "Rannij Bismark". 1373. P. s. v. vulgatum Al. „Ambrosia“.

1374. P. s. v. "Beliy Marafet".

1375. P. s. "Irlandetz".

1376. P. s. v. „Kapital“.

1377. P. s. v. "Victoria Mandorfskaja“.

1378. P. s. v. "Victoria Rosovaja".

1379. Vicia sativa L. ,Belaja $n^{\circ} 163^{\prime \prime}$.

1380. V. s. ,Ploskosemiannaja".

Addenda .

1381. Aegilops cylindrica Host.

1382. Althaea sulphurea Boiss.

1383. Aquilegia californica A. Gray.

1384. Corydalis nobilis Pers.

1385. Delphinium grandiflorum L.

1386. Dianthus plumarius L.

1387. Festuca bromoides L.

1388. Gypsophila paniculata L.

1389. G. perfoliata L.

1390. Lychnis flos cuculi $L$.

1391. Lycopus europaeus L. 
1392. Poterium canadense A. Gray.

1393. Ranunculus bulbosus $\mathrm{L}$.

1394.* Silene venosa Aschers.

1395. Thalictrum rugosum Ait.

1396. Trinia vulgaris D. C.

\section{SEMINA PLANTARUM SPONTANE- ARUM A VARIIS LOCALITATIS IN DIVERSIS REGIONIBUS COLLECTA.}

J. I. Cos Respubl. Cabardino-Balcaria.

1397. Acer. Trautvetteri Medw.

1398. Aconitum orientale Mill.

1399. Allium ursinum L.

1400. Althaea ficifolia Cav.

1401. Anthemis rigescens Willd.

1402. Aruncus silvester Kostel.

1403. Asparagus verticillatus L.

1404. Asperula odorata L.

1405. Azalea pontica L.

1406. Betonica grandiflora Willd.

1407. Convolvulus silvaticus $\mathrm{W}$. et $\mathrm{K}$.

1408. Cynodon Dactylon Pers.

1409. Daphne Mezereum L.

1410. Eupatorium cannabinum L.

1411. Gentiana asclepiadea L.

1412. Heracleum pubescens M. B.

1413. Myricaria germanica Desv. 
1414. Nicandra physaloides Gertn.

1415. Paeonia tenuifolia L.

1416. Rhamnus Pallasii Fisch. et Mey.

1417. Rosa pimpinellifolia L.

1418. Salvia glutinosa L.

1419. S. verticillata L.

1420. Sedum oppositifolium Sims.

1421. Stachys germanica $\Gamma$.

1422. Swertia iberica F. et M. var. albida C. A. M.

1423. V. peduncularis MB. var. glabra Kusnez.

1424. Tamus communis L.

1425. Telekia speciosa Baumg.

1426. Teucrium polium L.

Prof. Dr. S. S. Stankov.

Horti Director

Ass. B. S. Rzhanov

Vice-Director

Docent cand.

D. S. Averkiev

S. S. Klemanskij

N. I. Nikolaev

Docent cand. L. V. Tichov Ass. cand.

$F$. A. Alexandrov Horti $\mathrm{Hortulani}$

Ass. L. M. Smirnova

Ass. E. I. Stsherbakova

Ass. M. A. Gajdukova

Horti Botanici 
Перечен с смян, собранных в 1938 г.и предлагаемых в обмен Ботаническим садом Горьковского Государственного Университета * ОГНЗ. Горьковское Издательство. 1939. * Редактор проф. С. С. Станков. Техн. редактор Л. Н. Немченко. Корректор Р. А. Долган ова. * Сдано в набор 11/XII 1938 r., подписано к печати 8/II 1939r. Формат $60 \times 92 / 32$. Тираж 600 экзем. Изд. л. 2, бум. л. 1, уч.-авт. л. 1,75 , знаков в б.л. 76.000. Инд. СХ-3-(3). А У полном. Обллита 스 Б-19 Ж ПГлиграф, т. Горький, ул. Фигнер, 32. Заказ 스 9116. 


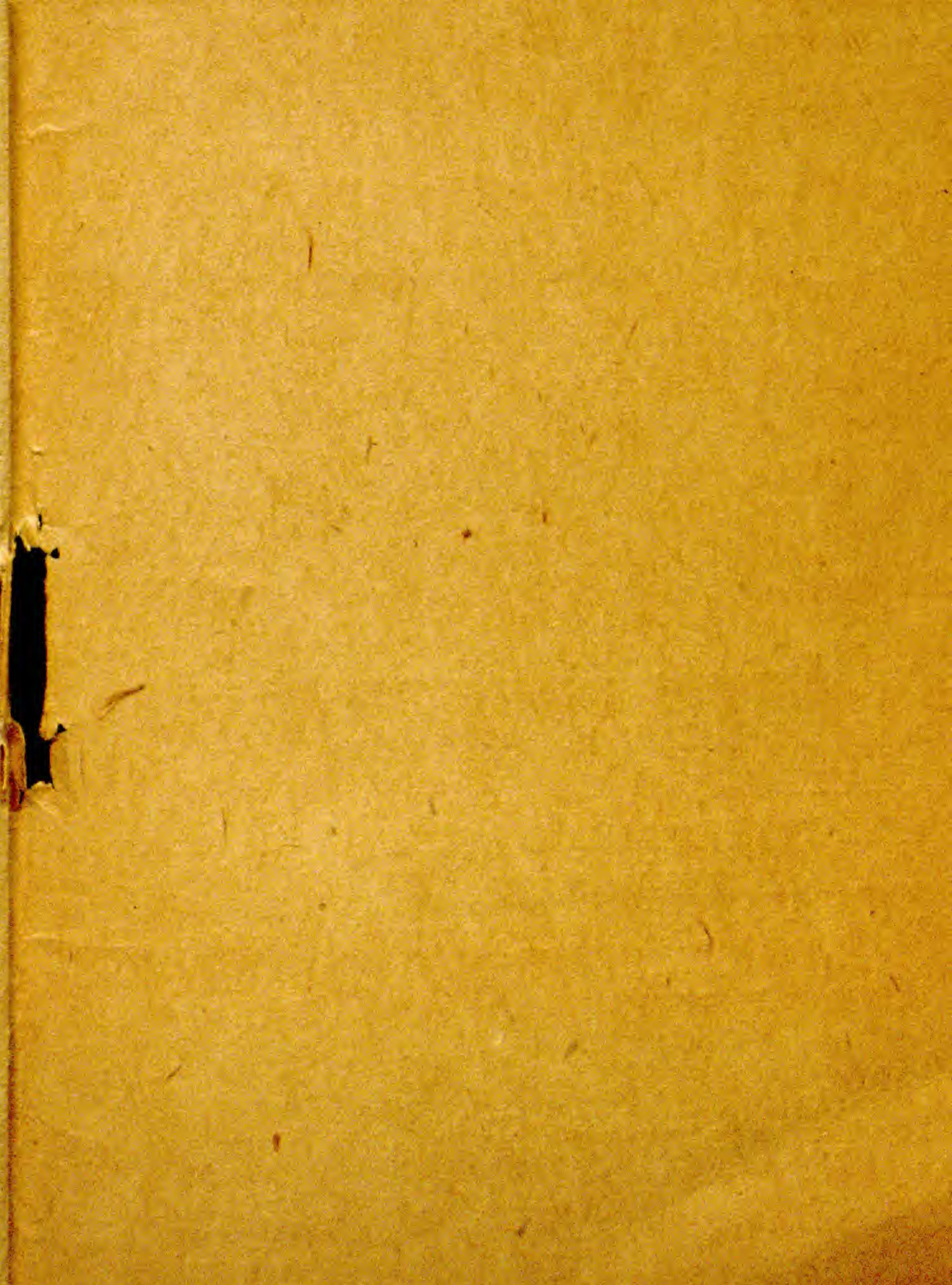


БЕСПЛАТНО

ПЕРЕЧЕНЬ СЕМЯН, СОБРАННЫХ В 1938 ГОДУ

И ПРЕДЛАГАЕМЫХ В ОБМЕН БОТАНИЧЕСКИМ САДОМ ГОРЬКОВСКОГО ГОСУДАРСТВЕННОГО УНИВЕРСИТЕТА

ГОРЬКОВСКОЕ ИЗДАТЕЛЬСТВО 1939 


\section{ar 3}

\section{DELECTUS SEMINUM}

ANNO MCMXL COLLECTORUM

\section{QUAE}

\section{HORTUS BOTANICUS} .

UNIVERSITATIS GORKIJENSIS

$$
\text { PRO }
$$

MUTUA COMMUTATIONE

OFFERT

GORK MCMAR 211941 



\section{DELECTUS SEMINUM}

ANNO MCMXL COLLECTORUM

QUAE

HORTUS BOTANICUS

UNIVERSITATIS GORKIJENSIS

PRO

MUTUA COMMUTATIONE

OFFERT

GORKIJ MCMXLI 



\section{İ. SEMINA PLANTARUM LIGNOSARUM ET FRUTICUM.}

Betulaceae.

Betula verrucosa Ehrh.

Alnus sitchensis Sarg.

Fagaceae.

Quercus pedunculata Ehrh.

Berberidaceae.

Berberis amurensis Rupr.

B. Fischeri hort.

B. heteropoda Schrenk.

B. h. v. oblonga Reg.

B. ilicifolia Forst.

B. sibirica Pall.

B. vulgaris $\mathrm{L}$.

B. v. f. atropurpurea.

B. Thunbergii DC.

Mahonia aquifolium Nutt.

Saxifragaceae.

Philadelphus pubescens Lois.

$\mathrm{Ph}$. coronarius L.

Ribes alpinum $\mathrm{L}$.

R. Warscewiczii Jancz. 
Rosaceae.

Fallugia paradoxa Endl.

Physocarpus opulifolius Maxim.

Spiraea bumalda Burvenich. v. "Antony Waterer".

S. japonica L. f.

S. japonica L. f. pumila.

S. syringaeflora Lemoine.

S. Wilsonii Duthie.

Crataegus Maximowiczii Schneid.

Sorbaria assurgens Rehd.

Sorbus aucuparia $L$.

S. heterophylla Dur.

Amelanchier vulgaris L.

Rosa amblyotis C. A. Mey.

R. centifolia L.

R. gutensteinensis Jacq.

R. kamtchatica Reg.

R. moschata Herrm.

R. micrantha Sm.

R. pomifera Herrm.

R. pimpinellifolia L.

S. rubrifolia Vill.

R. rugosa Thunb.

Prunus pumila L.

P. pennsilvanica $L$.

P. virginiana $L$.

P. Maackii Rupr.

$P$. avium $L$. 
Leguminosae.

Genista alba Lam.

G. germanica L.

G. ovata Waldst. et Kit.

G. sibirica L.

G. radiata Scop.

Amorpha canescens Nutt.

A. califorrica Nutt.

A. fruticosa L.

Caragana arborescens L.

C. frutex DC.

Colutea orientalis Mill.

C. media Willd.

Rutaceae.

Ptelea trifoliata L.

Euphorbiaceae.

Securinega flueggeoides Muell.

S. ramiflora Muell.

Anacardiaceae.

Cotinus coggygria Scop.

Rhus toxicodendron $\mathrm{L}$.

Celastraceae.

Evonymus verrucosa Scop.

Celastrus orbiculatus Thunb. 
Aceraceae.

Acer platanoides L.

A. tataricum L.

A. Negundo L.

Vitaceae.

Ampelopsis quinquefolia Planch.

Rhamnaceae.

Rhamnus cathartica L.

$\mathrm{Rh}$. imeretina Booth.

$\mathrm{Rh}$. tinctoria Waldst. et Kit.

$\mathrm{Rh}$. rupestris Scop.

Tiliaceae.

Tilia cordata Mill.

T. platyphyllos Scop.

Cornaceae.

Cornus stolonifera Michx.

Cornus alba L.

Oleaceae.

Syringa Josikaea Jacq.

S. villosa Vahl.

Caprifoliaceae.

Viburnum opulus L.

Symphoricarpus racemosus Michx.

6 
Lonicera tatarica L.

L. xylosteum L.

L. caprifolium L.

II. SEMINA PLANTARUM HERBACEUM. COLLECTA 1940.

Gramineae.

Agropyrum desortorum (Fisch.) Schult. A. sibiricum Beauw.

A. tenerum Vasey.

Andropogon ischaemum L.

Bromus canadensis hort.

B. erectus Huds.

B. uniloides Humb.

Cynosurus echinatus $\mathrm{L}$.

Elymus mollis Trin.

E. sibiricus L.

Erianthus Ravennae Schreb.

Hordeum secalinum Schreb.

Koeleria crassipes Lange.

K. gracilis Pers.

Lolium canadense Michx.

Phleum paniculatum Huds.

Roegneria tianschanica (Drob.) Nevski.

Setaria viridis P. B.

Stipa capillata L. 
Stipa sibirica Lam.

S. spartea Trin.

Triodia decumbens Pall.

\section{Liliaceae.}

Allium albidum Künth.

A. altaicum Pall.

A. angulosum $\mathrm{L}$.

A. caesium Schrenk.

A. canadense L.

A. fistulosum $\mathrm{L}$.

A. galanthum Kar. et Kir.

A. giganteum Reg.

A. glaciale Vved.

A. globosum MB.

A. hymenorrhizum Ledeb.

A. lineare L.

A. Moly L.

A. narcissiflorum Vill.

A. odorum $\mathrm{L}$.

A. paradoxum G. Don.

A. pulchellum G. Don.

A. tataricum L.

Anthericum liliago L.

A. ramosum $\mathrm{L}$.

Asparagus officinalis L.

Eremurus Olgae Rgl.

Galtonia candicans Decne.

Hemerocallis flava $L$. 
Litium concolor Salisb.

L. martagon $\mathrm{L}$.

L. tenuifolium Fisch.

L. Wilmottiae Wils.

Ornithogallum umbellatum $\mathrm{L}$.

Iridaceae.

Iris chamaeiris Bertol.

I. cordata Willd.

1. Gueldenstaedtiana Lepech.

I. lutescens Lam.

I. mellita Janka.

I. pumila L.

I. setosa Pall.

I. sibirica L.

I. soongorica Schrenk.

I. xiphioides Ehrh.

Sisyrinchium anceps Cav.

S. angustifolium Mill.

S. montanum hort.

Urticaceae.

Urtica cannabina L.

U. Dodarti L.

Polygonaceae.

Oxyria digyna (L.) Hill,

Rumex acetosa $\mathrm{L}$.

R. hydrolapathum Huds. 
Rumex hymenosepalus Torr.

$\mathrm{R}$. mexicanus Meiss.

R. pallidus Bigel.

R. patientia L.

R. stenophyllus Ledb.

$R$. thyrsiflorus Fingerh.

Chenopodiaceae.

Chenopodium aristatum $\mathrm{L}$.

Ch. botrys L.

Amaranthaceae.

Amaranthus aurea T. A. Desf.

A. blitum L.

A. caudatus L. f. atropurpureus.

A. gangeticus L.

A. purpureus hort.

Nyctaginaceae.

Oxybaphus nyctagineus Sweet.

Phytolaccaceae.

Phytolacca americana L.

Portulacaceae.

Calandrinia grandiflora Lindl.

Caryophyllaceae.

Agrostemma Githago L.

10 
Arenaria capillaris Poir.

A. graminifolia Schrad.

A. sajanensis Willd.

A. saxatilis L.

Cerastium alpinum $\mathrm{L}$.

C. Biebersteinii DC.

C. grandiflorum Waldst. et Kit.

C. Lerchenfeldianum Schur.

C. tomentosum $\mathrm{L}$.

Cucubalus baccifer L. v. japonicus.

Dianthus arenarius L.

D. banaticus Heuff.

D. barbatus L.

D. bicolor hort.

D. Boissieri Willk.

D. caesius $\mathrm{Sm}$.

D. carthusianorum L.

D. glacialis Haenke.

D. Hoeltzeri Winkl.

D. praecox Willd.

D. superbus L. v. grandiflorus.

D. tegestinus Kern.

Gypsophila acutifolia Fisch.

G. altissima L.

G. elegans Bieb.

G. elegans Bieb, v. grandiflorum hort.

G. fastigiata $L$.

G. pacifica Kom.

G. perfoliata $M$. B. 
G. Steveni Fisch.

Heliosperma quadridentatum (Murray) Schinz. et Thellung.

Lychnis alpina L.

L. chalcedonica L.

L. coronaria Desr.

L. mongolica Maxim.

Melandrium album (Mill.) Garcke.

Saponaria ocymoides L.

S. officinalis L.

Silene alpina L.

S. alpestris Jacq.

S. armeria L.

S. Bergiana Lindm.

S. conica L.

S. cucubalus Wieb.

S. gallica L.

S. grandiflora Franch.

S. japonica Rohrb.

S. laeta A. Br.

S. mellifera Boiss.

S. muscipula L.

S. tenuis Willd.

S. venosa (Gilib.) Aschers.

Tunica saxifraga (L.) Scop.

Vaccaria pyramidata Med.

Ranunculaceae.

Aconitum lycoctonum L.

12 
Anemone decapetala L.

A. fulgens J. Gray.

A. multifida Poir.

A. riparia Fernold.

Aquilegia canadensis L.

A. flabellata Sieb. et Zucc.

A. glandulosa Fisch.

Clematis Bergeronii hort.

C. tangutica Andr.

Delphinium bicolor Nutt.

D. carolinianum Walt.

D. elatum L. v. alpinum Hegi.

D. hybridum Willd.

D. intermedium DC.

Nigella arvensis $\mathrm{L}$.

N. damascena L.

N. sativa L.

Ranunculus acer L. ssp. Steveni Hegi.

$R$. anemonifolius DC.

R. serbicus Vis.

R. Villarsii D. S.

Thalictrum adiantifolium Besser.

Th. elatum Jacq.

Th. Fendleri Engelm.

Th. flexuosum Bernh.

Th. foetidum $\mathrm{L}$.

Th. minus $\mathrm{L}$.

Th. nigricans Jacq.

Th. rugosum Ait. 
Papaveraceae.

Chelidonium Franchettianum Prain.

Ch. majus L. v. laciniatum Mill.

Eschscholtzia californica Cham.

Papaver californicum A. Gray.

P. lateritium C. Koch.

P. nudicaule L.

P. pyrenaicum A. Kern. ssp. raeticum Fedde.

P. somniferum $\mathrm{L}$.

Crucifereae.

Aethionema Buxbaumii D. C.

Alyssum argenteum All.

A. desertorum Stapf.

A. sinuatum L.

Arabis auricula Lam.

Biscutella laevigata L.

Camelina dentata Wallr.

C. Sativa Crantz.

Carrichtera vella D. C.

Crambe hispanica $\mathrm{L}$.

Hesperis dinarica G. Beck.

H. matronalis $\mathrm{L}$.

H. violacea Boiss.

Iberis intermedia Guers.

I. odorata L.

I. pectinata Boiss.

Radicula silvestris Druce.

Thlaspi densiflorum Boiss. et Ky. 
Resedaceae.

Reseda alba L.

Crassulaceae.

Sedum aizoon $\mathrm{L}$.

S. album L.

S. anopetalum DÇ.

S. carpaticum Reuss.

S. crassipes Wall.

S. fabaria Koch.

S. hispanicum L.

$\mathrm{S}$. hybridum $\mathrm{L}$.

S. kamtschaticum Fisch. et Mey.

S. Kirillowii Rgl.

S. Maximowiczii Rgl.

S. spurium Bieb.

S. Stephani Cham.

Sempervivum tectorum L.

S. soboliferum L.

Saxifragaceae.

Bergenia cordifolia (Haw.) A. Br.

B. crassifolia L.

B. pacifica Kom.

Heuchera alba Zabel.

A. americana L.

H. americana v. gracilior Rgl.

H. americana v. hybrida hort.

H. cylindrica Dougl. 
Heuchera glabra Willd.

H. hispida Pursch

H. pilosissima Fisch. et Mey

H. pubescens Purch.

$H$. sanguinea Engelm.

H. villosa Mchx.

Rosaceae.

Agrimonia Eupatoria L. ssp. dahurica O. Kunthe.

A. Eupatoria L. ssp. odorata Hook.

Filipendula hexapetala Gilib.

Fragaria indica Andr.

F. moschata Duchsne.

F. orientalis Los.

F. vesca L. v. americana.

F. virginiana Duchsne.

Geum album Gmel.

G. canadense Kossy.

G. chiloense Balb.

G. coccineum Sibth. et Sm.

G. hispidum Fries.

G. japonicum Thunb.

G. Jankae G. Beck.

G. tyrolense Kern.

G. urbanum L.

G. virginianum $\mathrm{L}$.

G. Willdenowii Fisch.

Potentilla abchasica Fomin.

P. adscharica Somm. 
Potentilla alchimilloides Lop.

P. alpestre Hall.

$P$. argentea $L$.

P. argyrophylla Wall.

P. aurea L.

P. canadensis L.

P. Dombeyi Nestl.

P. gelida C. A. Mey.

P. geoides Bieb.

$\mathrm{P}$. intermedia L.

P. montenegrina Pant.

P. nevadensis Boiss.

P. nivea $L$.

P. pennsylvanica $L$.

P. pyrenaica Ram.

$P$. rupestris $\mathrm{L}$.

Poterium canadense A. Gray.

Sanguisorba carnea Fisch.

S. officinalis L.

S. sitchensis C. A. M.

S. tenuifolia Fisch.

Leguminosae.

Anthyllis variegata hort.

Astragalus asper Jacq.

A. baeticus L.

A. cicer L.

A. glycyphyllos L.

A. onobrychis $\mathrm{L}$. 
Astragalus sulcatus $\mathrm{L}$.

A. Trautvetteri Bge.

A. uliginosus $\mathrm{L}$.

Baptisia australis R. Br.

Cicer arietinum $\mathrm{L}$.

Lathyrus odoratus L.

Lotus ornithopodioides $\mathrm{L}$.

Lupinus elegans $H$. B. et $K$.

L. perennis L.

L. polyphyllus Lindl.

Medicago coerulea Less.

M. falcata L.

M. hispida Gauertn.

M. platycarpos Trautw.

$M$. radiata $L$.

M. scutellaria Mill.

Onobrychis altissima L.

O. arenaria D. C.

O. viciaefolia Scop.

Ononis hircina Jacq.

Ornithopus sativus Brot.

Oxytropis villosa D. C.

Phaseolus multiflorus L.

$\mathrm{Ph}$. multiflorus L. v. bicolor hort.

Scorpiurus vermiculata $L$.

Securigera coronilla D. C.

Tetragonolobus purpureus Moench.

Trifolium alpestre $\mathrm{L}$.

$T$. arvense $L$. 
Trifolium incarnatum L.

T. lupinaster $\mathrm{L}$.

T. medium L.

Trigonella coerulea Ser.

Vicia americana Muhl.

V. ervilia (L.) Willd.

Geraniaceae.

Erodium cicutarium L'Herit.

E. Stephanianum Willd.

Geranium albanum Bieb.

G. tuberosum L. v. macrostylum.

Linaceae.

Linum altaicum MB.

L. austriacum L.

L. flavum $\mathrm{L}$.

L. grandiflorum Desf.

L. perenne L.

L. perenne L. V. sibiricum DC.

Malvaceae.

Abutilon Avicennae Presl.

Althaea sulphurea Boiss.

Anoda hastata Lav.

Lavatera thuringiaca $L$.

L. trimestris L.

Malachra radiata $L$.

Malva crispa L. 
Malva pusilla With.

$M$. verticillata $L$.

Palana flexuosa Mast.

Sidalcea malviflora A. Gray.

S. oregona A. Gray.

S. neo-mexicana A. Gray.

S. spicata Greene.

\section{Cistaceae.}

Helianthemum chamaecistus Mill.

H. hirtum Mill.

H. lavandulaefolium Mill.

H. ovatum Dun.

H. rosmarinifolium Purch.

H. vineale Pers.

Hypericaceae.

Hypericum ascyron $L$.

H. calycinum L.

H. grandiflorum L.

Violaceae.

Viola arborescens L.

V. bosniaca Form.

Lythraceae.

Cuphea viscosissima Jacq.

Lythrum salicaria L. 


\section{Oenotheraceae.}

Clarkia elegans Dougl.

Epilobium hirsutum L.

Oenothera mollissima L.

O. tetraptera Cav.

Umbelliferae.

Anethum graveolens L.

Coriandrum sativum $\mathrm{L}$.

Peucedanum alsaticum L.

Pimpinella majus Huds.

Seseli hyppomarathrum $\mathrm{L}$.

S. Pallasii Bess.

Primulaceae.

Lysimachia acroadenia Max.

L. clethroides Duby.

L. lobeloides Wall.

L. verticillata Pall.

Primula Clusiana Tausch.

P. denticulata Sm.

Plumbaginaceae.

Armeria alpina Willd. v. ovata.

A. arctica Wall.

A. labradorica hort.

A. maritima Willd.

A. plantaginea Willd.

A. setacea Del. 
Armeria sibirica Turcz.

A. vulgaris Willd.

Statice Gmelini Willd.

Gentianaceae.

Gentiana crinita Froel.

G. macrophylla Pall.

G. tianschanica Rupr.

Apocynaceae.

Amsonia Tabernaemontana Walt.

Asclepiadaceae.

Vincetoxicum fuscatum Reichb.

V. medium Decsn.

V. purpurascens Morr. et Decne.

V. Rehmanni Boiss.

Polemoniaceae.

Gilia capitata Dougl.

Polemonium coeruleum $\mathrm{L}$.

P. filicinum Greene.

P. himalayanum Baker.

P. reptans $L$.

Hydrophyllaceae.

Nemophila insignis Dougl.

Phacelia campanularia A. Gray.

$\mathrm{Ph}$. congesta Hook. 
Phacelia fimbriata Michx.

$\mathrm{Ph}$. loasaefolia Torr.

$\mathrm{Ph}$. Whitlavia A. Gray.

Boraginaceae.

Anchusa officinalis L.

Eritrichum strictum Hort.

Labiatae.

Brunella grandiflora Jacq.

B. pyrenaica Philip.

Dracocephalum moldavica $\mathrm{L}$.

D. nutans L. V. alpinum.

D. Ruyschiana L.

Hyssopus officinalis $L$.

Lallemantia peltata Fisch. et Mey.

Leonurus cardiaca L.

Lophanthus anisatus Benth.

Monarda fistulosa $\mathrm{L}$.

Nepeta cataria L. v. citriodora.

N. grandiflora $M B$.

N. kokanica Regl.

N. macrantha Fisch.

N. nuda $L$.

N. nuda L. v. pannonica Gams.

N. spicata Benth.

Phlomis alpina Pall.

$\mathrm{Ph}$. cashemiriana Royle.

$\mathrm{Ph}$. chrysophylla Boiss. 
Phlomis tuberosa L.

$\mathrm{Ph}$. viscosa Poir.

Salvia atropotana Bge.

S. aethiopis L.

S. nemorosa L.

S. nutans L.

S. pratensis L.

S. Przewalski Maxim.

$\mathrm{S}$. utilis A. Br.

Scutellaria alpina L.

S. altissima L.

Sideritis hyssopifolia L.

S. scordioides L.

Stachys germanica L.

S. lanata Jacq.

S. officinalis Trev. .

S. nivea Labill.

Ziziphora capitata L.

Solanaceae.

Datura aegyptiaca Vesl.

D. stramonium L.

Nicandra physaloides Gaertn.

Nicotiana rustica L.

Physalis Franchetti Mast.

$\mathrm{Ph}$. pubescens L.

Solanum Dulcamara L.

S. nigrum L. 
Scrophulariacèae.

Antirrhinum Coulterianum Benth.

Digitalis ambigua Murr.

D. media Roth.

D. nevadensis Kunze.

D. purpurea L.

D. viridiflora hort.

Linaria bipartita Willd.

L. purpurea Mill.

L. triphylla Mill.

L. vulgaris Mill.

Pentstemon albidus Nutt.

P. confertus Dougl.

P. deustus Dougl.

P. diffusus Dougl.

P. glaucus Grach.

P. hirsutus (L.) Willd.

P. procerus Dougl.

Verbascum soongoricum Schrenk.

V. Wilsonii hort.

Veronica candida Lodd.

V. fruticans Jacq.

V. incana $L$.

V. spuria L.

Globulariaceae.

Globularia cordifolia L.

G. vulgaris L.

G. Willkommii Nym. 
Plantaginaceae.

Plantago alpina L.

P. Psyllium L.

P. Schwarzenbergiana Schur.

Rubiaceae.

Asperula ciliata Rochel.

A. tinctoria L.

Galium saccharatum All.

Ophiorrhiza Mungos L.

Valerianaceae.

Vaierianella coronata D. C.

Dipsacaceae.

Dipsacus ferox Loisel.

Scabiosa graminifolia L.

S. micrantha Desf.

S. Olivieri Coult.

S. pyrenaica All.

Succisa inflexa Jund.

Campanulaceae.

Adenophora communis Fisch.

Campanula amabilis Rupr.

C. barbata L.

C. carpatica Jacq.

C. edulis hort.

C. elegans Roem. et Schult.

26 
Campanula eriocalix Adam.

C. gracilis Jard.

C. imeretina Rupr.

C. Kolenatiana C. A. M.

C. lactiflora Bieb.

C. latifolia D. C.

C. medium L.

C. Pallasiana R. et S.

C. Portenschlagiana Roem. et Schult.

C. primulaefolia Brot.

C. persicifolia L.

C. pulcherrima Lam.

C. Raddeana Rupr.

C. Raïneri Perpent.

C. rapunculoides $L$.

C. Steveni Bieb.

Jasione humilis Lois.

Phyteuma Halleri All.

$\mathrm{Ph}$. orbiculare $\mathrm{L}$.

Ph. Scheuchzeri All.

Platycodon grandiflorum D. C.

Compositae.

Achillea cartilaginea Led.

A. pseudopectinata Janka.

Anthemis altissima L.

A. candidissima Willd.

A. cotula L.

A. macedonica Boiss. 
Anthemis ruthenica Bieb.

Aster himalaicus C. B. Clarke.

A. tibeticus Hook.

Bidens dahliodes Wats.

Calendula aegyptica Desf.

C. algeriensis Boiss. et Bent.

C. arvensis $\mathrm{L}$.

C. officinalis L. "Radio" hort.

C. o. "Opunti King" hort.

C. stellata Cav.

Carduus hamulosus Ehrh.

Centaurea atropurpurea W. et K.

C. banatica Kork.

C. macrocephala Puschk.

C. nigra L.

C. ossica C. Koch.

C. scabiosa L.

C. uniflora L.

Chrysanthemum maximum Ramond.

Cnicus benedictus L.

Cosmos pipinnatus Cav.

Dimorphotheca aurantiaca D. C.

D. pluvialis Moench.

Erigeron Coulteri Porter. et Coult.

E. glabellus Nutt.

E. grandiflorus Hook.

Hieracium boreale Fries.

H. robustum $\mathrm{Fr}$.

Hypochoeris radiata $\mathrm{L}$. 
Inula Helenium L.

Jurinea arachnoidea Bge.

Lactuca scariola L.

Layia platyglossa A. Gray.

Ptarmica impatiens D. C.

Serratula coronata L.

S. Gmelini Ledeb.

S. radiata $M$ Bieb.

Silybum Marianum Gaertn.

Tanacetum huronense Nutt.

Troximon gracilens Gray.

COLLECTA 1939.

Gramineae.

Agropyrum acutum R. et S.

A. junceum Beauv.

A. rigidum $P$. B.

A. trichophorum (Link.) Richt.

A. ugamicum Drob.

Alopecurus lasiostachys Link.

A. pratensis $L$.

A. ventricosus Pers.

Arrhenatherum bulbosum Presl.

Asperella hystrix Humb.

Arundinaria anomala Steud.

Avena elatior $\mathrm{L}$.

A. Schelliana Hack.

Calamagrostis argentea D. C.

Catabrosa humilis Trin. 
Dactylis glomerata L.

D. hispanica Roth.

Diarrhena americana Beauv.

Elymus akmolensis Drob.

E. canadensis $\mathrm{L}$.

E. dahuricus Turcz.

E. excelsus Turcz.

Festuca alpina Suter.

F. appenina De Not.

F. arundinacea Schreb.

F. bromoides hort.

F. capillata Lam.

F. frigida Hack.

F. Ganeschini Drob.

$\mathrm{F}$. glauca Bess.

F. polesica Zapal.

F. pungens Kit.

F. sulcata Hack.

F. vallesiaca Gaudin.

Melica altissima $\mathrm{L}$.

M. transsilvanica Schur.

Orysopsis holciformis (M. B.) Richter.

Phleum nodosum L.

Poa bulbosa L.

P. caesia Sm.

P. versicolor Bess.

Secale montanum Gun.

Stipa tenacissima L.

Trisetum distichophyllum $\mathrm{Pd}$. 
Liliaceae.

Allium montanum F. W. Schmidt.

A. nutans $\mathrm{L}$.

A. tricoccum Ait.

Iridiaceae.

Iris aphylla L.

I. columnaris hort.

I. missouriensis Nutt.

I. ruthenica Ker-Gawl.

I. sordida Willd.

Polygonaceae.

Polygonum hydropiper L.

P. polymorphum Ldb.

Rheum acuminatum Hook.

Rh. Emodi Wall.

$\mathrm{Rh}$. officinale Baill.

Rh. palmatum L. v. tanguticum Maxim.

Rh. rhaponticum L.

Rumex callosus Reidringer.

Chenopodiaceae.

Chenopodium Bonus Henricus L.

Caryophyllaceae.

Arenaria cephalotes MB.

A. Koriniana Fisch. 
Arenaria longifolia $M$. B.

Dianthus bebius Vis.

D. callizoides Schott. et Kotschy.

D. calocephalus Boiss.

D. capitatus DC.

D. cinnabarinus Sprun.

D. cruentus Griseb.

D. fragrans M. Bieb.

D. gallicus Pers.

D. giganteus Urv.

D. gloriosus hort.

D. hungaricus Pers.

D. Knappii Aschers.

D. Pancici Velen.

D. pungens $\mathrm{L}$.

D. serotinus Waldst

D. strictus Sibth. et Sm.

D. tatrae Borb.

D. tener Balb.

Gypsophyla Gmelini Bunge.

Heliosperma alpestre Rchb.

Lychnis coeli-rosa Desr.

Melandrium dioicum Coss. et Germ.

Silene supina MB.

Ranunculaceae.

Aconitum orientale Mill.

Anemone alpina L.

A. baldensis $\mathrm{L}$. 
Anemone coronaria L.

A. cylindrica Gray.

A. magellanica Warley.

A. pedata Rafin.

Aquilegia Skinneri Hook

Delphinium Brunnonianum Royle.

D. caschemirianum Royle.

D. cheilanthum Fisch.

D. elatum L.

D. grandiflorum L.

D. Maakianum Regel.

D. Przewalskii Huth.

D. Pylzowi Maxim.

D. triste Fisch.

Ranunculus bulbosus L.

R. trilobus Desr.

Papaveraceae.

Chelidonium majus L.

Papaver bracteatum Lindl.

P. orientale L.

Cruciferae.

Alyssum lenense DC.

Aubrietia erubescens Griseb.

A. libanotica Boiss. et Hohen.

Barbarea officinalis $\mathrm{R}$. Br.

Crambe koktebelica Busch.

C. maritima L. 
Erysimum cuspidatum DC.

Hesperis runcinata Waldst.

Isatis alpina All.

I. glauca Boiss.

I. tinctoria $\mathrm{L}$.

I. tinctoria v. campestre Koch.

Resedaceae.

Reseda lutea L.

Rosaceae.

Potentilla Degeni Siegfr.

P. gracilis Dougl.

P. hybrida hort.

P. nepalensis Hook.

P. pulcherrima Lehm.

P. splendens Ram.

P. Tabernaemontani Aschers.

P. transcaspica Th. Wolf.

P. virosa Donn.

Leguminosae.

Astragalus canadensis L.

A. danicus Retz.

A. falcatus Lam.

Desmodium canadense DC.

D. marilandicum Boott.

Lathyrus silvester L.

L 
Onobrychis carpatica DC.

$O$. oxytropoides Bunge.

Oxytropis campestris DC.

O. nigrescens Fisch.

Thermopsis caroliniana Curt.

Th. fabacea DC.

Vicia pisiformis L.

Linaceae.

Linum extraxillare Kit.

L. gallicum L.

Malvaceae.

Lavatera assurgentiflora Kellog.

Cistaceae.

Helianthemum canadense Micht.

Hypericaceae.

Hypericum attenuatum Choisy.

H. debile Salisb.

H. tetrapterum Fries.

$H$. virginicum $L$.

Oenotheraceae.

Oenothera rubrinervis D.-Vries.

O. speciosa Nutt. 
Umbelliferue.

Eryngium alpinum L.

E. planum $\mathrm{L}$.

Heracleum Mantegazzianum Lever. et Som.

H. Orsinii Ten.

Libanotis montana All.

Peucedanum elegans Kom.

P. oreoselinum Moench.

Primulaceae.

Primula macrocalyx Bunge.

P. officinalis Jacq.

Plumbaginaceae.

Armeria caespitosa Boiss. "

A. elongata Hoffm.

Asclepiadaceae.

Vincetoxicum luteum Hoffm.

Polemoniaceae.

Polemonium Richardsonii R. Grach. v. humilis

Gray.

P. Van-Bruntiae Britt.

Boraginaceae.

Echium rubrum Jacq.

36 
Labiatae.

Ballota nigra L.

Nepeta mandshuriensis S. Moore.

Salvia silvestris $\mathrm{L}$.

Thymus glaber Mill.

Solanaceae.

Physochlaina orientalis G. Don.

Scrophulariaceae.

Digitalis lanata Ehrh.

Pentstemon Bradburyi Pursh.

P. cobaea Nutt.

P. digitalis Nutt.

P. gracilis Nutt.

P. ovatus Dougl.

Verbascum nigrum L.

$\mathrm{V}$. phoeniceum L.

Veronica orchidea Crantz.

Valerianaceae.

Valeriana hispida Turcz.

V. rossica P. Smirn.

\section{Dipsacaceae.}

Cephalaria alpina Schrad.

C. ambrosioides R. et Sch.

C. corniculata R. et S. 
Cephalaria gigantea (Led.) Barb.

C. procera Fisch.

Cucurbitaceae.

Bryonia alba L.

Campanulaceae.

Companula abietina Griseb.

C. betonicaefolia Siebt. et Sm.

C. caespitosa Vill.

C. collina MB.

C. grandis Fisch. et Mey.

C. Grossekii Heuff.

C. intermedia Rupr.

C. Leutweinnii Heldr.

C. Morettiana Reichb.

C. saxatilis L.

C. sibirica L.

C. turbinata Schott.

C. Waldsteiniana R. et S.

Codonopsis ussuriensis Hemsl. Symphiandra pendula A. D. C.

Compositae.

Achillea asplenifolia Vent.

A. distans W. et $K$.

A. filipendulina Lam.

A. grandiflora Trev.

A. macrophylla L. 
Achillea nobilis L.

A. odorata L.

Alfredia stenolepis Kar. et Kir.

Anaphalis margaritacea Benth. et Hook.

Arnica Chamissonis Less.

A. foliosa Nutt.

A. longifolia Eaton.

A. montana L.

A. sachalinensis A. Gray.

Aster altaicus Willd.

A. incisus Fisch.

A. paniculatus Lam.

A. pyrenaicus D. C.

Centaurea leucophylla MB.

C. ruthenica Lam.

C. spinulosa Kork.

C. stenolepis Kerner.

Chrysanthemum caucasicum Pers.

Ch. corymbosum L.

Coreopsis pubescens Ell.

Dimorphotheca annua Less.

Echinops exaltatus Schrad.

E. Gmelini Turcz.

Erigeron macranthus Nutt. v. superbus hort.

Filago germanica L.

Helenium Hoopesii A. Gray.

H. mexicanum H. B. et Nov.

Helianthella quinquenervis A. Gray.

H. uniflora Torr. et Gray. 
Heliopsis helianthoides Sweet.

H. laevis Pers.

Hieracium alpinum L.

H. canadense Michx.

H. Heldreichii Boiss.

H. pictum Schleicher.

H. rigidum Hartm.

H. virosum Pall.

Inula magnifica Lipsky.

I. Royleana D. C.

Lactuca perennis L.

L. virosa $\mathrm{L}$

Leontodon crispus Vill.

L. hastilis $\mathrm{L}$.

L. pyrenaicus Gouan.

Madia sativa Don.

Matricaria discoidea DC.

Palafoxia texana DC.

Pyrethrum roseum Lindl.

$\mathrm{P}$. niveum $\mathrm{Zaq}$.

Rudbeckia hirta L.

R. fulgida Ait.

R. laciniata L.

R. maxima Nutt.

R. purpurea L.

Scorzonera albicaulis Bge.

S. purpurea L.

Senecio adoniifolius Lois.

Solidago canadensis $L$. 
S. hybrida hort. "Goldstrahl".

S. rigida $\mathrm{L}$.

S. Shortii T. et G.

Tanacetum vulgare $L$.

Zinnia uniflora L.

\section{SEMINA PLANTARUM DECO- RANTIUM.}

Polypodiaceae (sporii).

Adianthum capillus Veneris L.

Asplenium bulbiferum Forst.

Nephrolepis tuberosa Hook.

Pteris cretica L. v. albo-lineata hort.

P. cretica L.

Cyperaceae.

Cyperus natalensis Hochst.

C. papyrus L.

Iridaceae.

Gladiolus hybridus hort. (bulbi.).

Portulacaceae.

Portulaca grandiflora Hook.

Caryophylaceae.

Dianthus chinensis L. 
Begoniaceae.

Begonia semperflorens Link. et Otto. B. semperflorens "Albert Martin" hort.

B. semperflorens "Vulcan" hort.

B. semperflorens "Lauberin" hort.

Polemoniaceae.

Phlox Drummondii Hook.

Solanaceae.

Nicotiana affinis T. Moore.

N. colossea André.

N. tabacum L. "Havanna" hort.

N. tabacnm "Wigandioides" hort.

Petunia hybrida hort. v, grandiflora hort.

P. hybrida "Alba" hort.

P. hybrida "Brilliant Rose" hort.

P. hybrida "Krymchylda" hort.

Scrophulariaceae.

Antirrhinum majus L. fl. alba hort.

A. m. "Weisses Wunder' hort.

A. m. 'Purpurkönig" hort.

A. m. "Tschernyi Platsch" hort. .

A. m. fl. rosea hort.

Calceolaria pinnata L.

Gesneriaceae.

Streptocarpus polyanthus Hook. 
Campanulaceae.

Lobelia erinus L.

Compositae.

Ageratum conyzoides L. v. mexicanum hort. Brachycome iberidifolia Beath.

Gaillardia hybrida hort.

Pyrethrum parthenifolium Willd. v, aureum hort. Solidago canadensis $\mathrm{L}$.

\section{SEMINA PLANTARUM OFFICI- NALIUM.}

Liliaceae.

Asparagus officinalis $L$. Allium schoenoprasum L.

Moraceae.

Humulus lupulus L.

Cannabis sativa L. v. indica.

Polygonaceae.

Rheum palmatum L. v. tanguticum Max. Rh. rhaponticum L. $\mathrm{Rh}$. undulatum $\mathrm{L}$. 
Caryophyllaceae.

Agrostemma Githago L.

Lychnis chalcedonica L.

Saponaria officinalis $\mathrm{L}$.

Ranunculaceae.

Nigella arvensis L.

$\mathrm{N}$. damascena $\mathrm{L}$.

N. sativa $L$.

Ranunculus acer L.

Papaveraceae.

Chelidonium majus $\mathrm{L}$.

Eschscholtzia californica Cham.

Papaver somniferum L.

P. dubium L.

P. rhoeas L.

Cruciferae.

Capsella bursa pastoris Medic.

Capparidaceae.

Cleome gigantea L.

Rosaceae.

Geum urbanum L.

Poterium sanguisorba $L$. Sanguisorba officinalis L. 
Leguminosae.

Coronilla varia $\mathrm{L}$.

Glycyrrhiza echinata L. Melilotus coeruleus Desr.

M. officinalis Desr.

Malvaceae.

Althaea armenica Tenore.

A. officinalis $\mathrm{L}$.

A. rosea Cav. v. nigra.

Malva alcea $\mathrm{L}$.

M. moschata L.

Umbelliferae.

Anethum graveolens L.

Carum carvi L.

Conium maculatum L.

Coriandrum sativum $\mathrm{L}$.

Eryngium planum L.

Levisticum officinale Koch

Pastinaca sativa $L$.

Primulaceae.

Primula officinalis $\mathrm{L}$.

Boraginaceae.

Anchusa italica Retz.

Borago officinalis $L$. 
Lithospermum officinale $L$.

Alkanna tinctoria Tausch.

Solanaceae.

Atropa Belladonna L.

Datura ceratocaula Jacq.

D. inermis Jacq.

D. stramonium $\mathrm{L}$.

Nicotiana rustica $L$.

Scrophulariaceae.

Digitalis ambigua Murr.

D. ferruginea $L$.

D. purpurea L.

Linaria vulgaris $\mathrm{L}$.

Verbascum tapsiphorme Schrad.

Veronica orientalis Mill.

Plantaginaceae.

Plantago lanceolata L.

P. major L.

P. psyllium L.

Valerianaceae.

Patrinia intermedia Roem. et Sch.

Valeriana nitida Pr.

$\mathrm{V}$. officinalis L.

V. rossica $\mathrm{Sm}$. 
Campanulaceae.

Lobelia inflata $\mathrm{L}$.

Compositae.

Achillea millefolium L.

A. nobilis L.

Anthemis nobilis L.

A. tinctoria L.

Arctium minus Bernh.

A. tomentosum Schrank.

Arnica montana L.

Artemisia absinthium L.

A. vulgaris L.

Bellis perennis $L$.

Calendula officinalis $\mathrm{L}$.

Cephalophora aromatica Schrad.

Centaurea cyanus L.

Chrysanthemum carneum Steud.

Ch. cinerariaefolium Vis.

Ch. corymbosum L.

Ch. macrophyllum $W$, et $K$.

Ch. partenifolium B. Fedtsch.

Chondrilla juncea L.

Cnicus benedictus Gaerth.

Grindelia robusta Nutt.

G. squarrossa Dunal.

Inula britanica L.

I. Helenium L.

Matricaria inodora L. 
M. chamomilla L.

Tanacetum boreale Fisch.

T. vulgare $\mathrm{L}$.

Taraxacum parnassicum Dahelst.

T. stenoschistum Dahelst.

\section{SEMINA PLANTARUM TECHNI- CARUM ET OLEARUM.}

Moraceae.

Cannabis sativa $\mathrm{L}$.

Polygonaceae.

Rumex acetosa $\mathrm{L}$.

R. confertus L.

Portulacaceae.

Portulaca oleracea $\mathrm{L}$.

Cruciferae.

Brassica juncea Czern.

B. nigra Koch.

Camelina sativa Crantz.

Crambe abyssinica Kochst.

C. hispanica L.

Sinapis alba $L$.

Resedaceae.

Reseda luteola L. 
Saxifragaceae.

Bergenia crassifolia L.

Leguminosae.

Genista tinctoria L.

Euphorbiaceae.

Euphorbia Lathyris L.

Ricinus communis L. (Manczschuria).

R. C. L. Kruglik No. 5.

R. c. L. No. 142 .

\section{Malvaceae.}

Abutilon avicennae Gaertn. No. 45372.

A. a. (ex Manczschuria)

A. a. (ex Lenkhoran).

A. a. No. 8094 .

A. a. No. $10204 / a$.

Anoda cristata Schl.

A. hastata Cav.

Malva brasiliensis Desr.

$M$. crispa L.

M. mauritania L.

M. meluca L.

Malva pusilla With.

$M$, verticillata $L$.

Sida napaea Cav. 
Cucurbitaceae.

Cucurbita maxima Duschesne.

C. pepo L.

C. p. v. moschata.

C. p. Kabatzki.

Cucumis sativus L.

Labiatae.

Lallemantia iberica Fisch. et Mey.

Compositae.

Carthamus tinctorius $\mathrm{L}$.

Cichorium intybus $\mathrm{L}$.

Lactuca sativa L.

Helianthus annuus L: No. 1846.

H. a. No. 8281 .

H. a. No. 1975.

H. a. No. 4036.

H. a. No. 3519.

Madia sativa Molin.

\section{Umbelliferae.}

Coriandrum sativum L. v. nanum.

C. s. v. aureum.

C. s- v. giganteum.

C. s. v. melphytense.

C. s. v. microcarpum.

50 


\section{SEMINA PI.ANTARUM GRAMINEA- RUM ET LEGUMINOSARUM.}

Gramineae.

Agropyrum cristatum Bess.

A. desertorum Schult.

A. intermedium R. Beauv.

A. glaucum Roem. et Schult.

Agrostis alba L.

Alopecurus pratensis L.

Avena byzantina Koch.

A. brevis R. Roth.

A. Ludoviciana DuF.

A. nuda L.

A. nuda L. v. inermis Kr. Liberly.

A. sativa L. v. aristata Kr. Jemtschujina.

A. s. L. v. a. Ligovo II.

A. s. L. v. a. Mirage.

A. s. L. v. a. Moskowskij A-315.

A. s. L. v. a. Zvezda.

A. s. v. a. Leitevitski.

A. s. v. a. Zolotoj Dozd.

A. s. v. a. Lochovskij.

A. s. v. a. Pflüg.

A. s. v. a. Amerikanskij.

A. s. L. v. a. No. 8154 .

A. s. v. a. No. 9757.

A. s. v, a. Povoljie 2131. 
Avena sativa L. v. a. Zolotistij.

A. s. L. v. a. Grenadier.

A. s. L. v. a. Echo.

A. s. L. v. a. Cherson.

A. s. L. v. a. Charkovski 0596.

A. s. L. v. a. Marcton.

A. s. L. v. a. Riegland.

A. S. L. v. a. No. 5056 .

A. s. L. v. a. No. 5057.

A. s. L. v. a. Stepniak 648

A. s. L. v. a. No. 6522 .

A. s. L. brunnea Körn.

A. S. L. br. Korn "Brie".

A. S. L. Kasanensis Vav. Polbianka.

A. s. L. Krausei "Kuto".

A. S. L. montana Al.

A. s. L. m. v. "Barenhafer".

A. s. L. m. utica v. "Albion".

A. s. L. m. v. "Aliaska".

A. s. L. m. v. "Dunne"

A. S. L. m. v. Gretchen.

A. S. L. v. mutica Al "Orel".

A. S. L. v. m. "Pobeda".

A. s. L. v. m. 'Romania".

A. s. L. V. m. Shatilovskij.

A. s. L. v. orientalis Mordv. v. pugnax A. Blaek Tart.

A. S. L. v. O. v. eligulata Vav.

A. s. L. v. u. obtusata "Gentleman". 
Avena s. L. v. o. tristis Al.

A. S. 1.. v. Adelaar.

A. S. L. v. Beedenhafer.

A. s. L. V. Golden Regen.

A. S. L. V. Grisen Kr.

A. s. L. v. Zagehafer

A. S. L. v. Orion.

A. s. L. v. nigre $Z$ warte President

A. s. L. v. volgensis Vav.

A. strigosa Schreb.

Bromus arvensis $\mathrm{L}$.

B. inermis Leyss.

B. erectus Huds.

Beckmania cruciformis Host.

Dactylis glomerata L.

Elymus sibiricus L.

Festuca ovina L.

F. rubra L.

Hordeum distichum L. v. abyssinicum Sch.

H. d. L. v. erectum Schubl. Prima.

H. d. L. v. medicum Körn No. 26.

H. d. L. v. m. No. 46 .

H. d. L. v. nigricans Ser.

H. đ. L. v. nudum Körn. No. 110.

H. d. L. v. n. No. 105.

H. d. L. v. nutans Schubl. "Colchicum" $10 / 30$.

H. d. L. v. n. Europeum $353 / 133$.

H. d. L. v. n. Viner.

H. d. L. v. ก. No. 17. 
Hordeum d. L. v. n. No. 7054.

H. d. L. v. n. Hanna Lossdorffskaia.

$H$. vulgare $L$. v. astrachanense.

H. v. L. v. caeleste No. 46.

H. v. L. v. nigrum Willd.

H. y. L. v. pallidum Ser. "Pioner".

H. v. L. v. Tschervonetz.

H. v. L. v. Toulounskij.

H. v. L. v. Viatskij 1009.

H. v. L. v. Trebe.

H. v. L. v. parallelum Körn.

H. v. L. v. Horsfordianum.

H. v. L. v. Stendelii Kr.

H. v. L. v. Trifurcatum Schlecht.

Lolium perenne $\mathrm{L}$.

L. italicum R. Br.

Panicum mileaceum contractum Al. v. Aureum No. 01.

P. m. c. Al. dacicum Korn No. 03.

P. m. c. Al. sanguineum Al No. 0853.

P. m. c. Al. Kazanskaja 506.

P. m. c. Al. Voroneshskoje 0198.

P. m. c. Al. Orapajevskoje 367.

P. m. c. Al. Kazanskoje 01 .

P. m. compactum Al. 19/27.

P. m. v. effusum Al. Toidenskoje 215.

P. m. v. eff. subcoccineum Saratovskoje 146.

P. m. v. eff. Al. Voroneshskoje 215.

P. italicum L. 
Poa pratensis L.

Secale cereale $\mathrm{L}$.

Triticum compactum Host. v. araratica.

T. c. Host. v. echinopoides.

T. c. Host. v. erynaceum Kr.

T. c. Host. v. Flaksbergerii Tum.

T. c. Host. v. hrasdanicum Tum.

T. c. Host. v. Humboldti Körn.

T. c. Host. v. pseudo-rubra.

T. dicoccum Schubl.

T. d. v. Schubl. noricum inflatum.

T. d. Schubl. v. rufum.

$\mathrm{T}$. dicoccoides $\mathrm{Kr}$.

T. durum Desf. v. obscurum.

T. d. Desf, v. affine Körn No. 309.

T. d. Desf. v. coeruleum.

T. d. Desf. v. hordeiforme No. 04.

T. d. Desf. v. h. No. 10.

T. d. Desf. v. h. No. 189.

T. d. Desf. v. h. No. 432.

T. d. Desf. v. h. No. 496.

T. d. Desf. v. h. No. 0675.

T. d. Desf. v. h. No. 695.

T. d. Desf. v. libicum Körn.

T. d. Desf. v. leucurum БГ-26.

T. d. Desf. v. 1. БГ-33.

T. d. Desf. v. leocomelan Al.

T. d. Desf. v. melanopus Al. No. 069.

T. d. Desf. v. m. Al. No. 826 . 
Triticum d. Desf. v. m. Al. No. 886 ,

T. d. Desf. v. murciense.

T. d. Desf. v. Reichenbachii $\mathrm{Kr}$.

T. monococcum L. (ex America).

T. polonicum L.

T. p. L. v. villosum.

T. persicum Vav. v. fulginosum Zhuk.

T. p. Vav. v. stramineum Zhuk.

T. pseudo-hostianum.

T. spelta $\mathrm{L}$

T. S. L. V. coeruleum.

T. turgidum $\mathrm{Kr}$.

T. vulgare Host. albidum Al. No. 676 .

T. v. Host. v. alborubrum Kr. Cappubra.

T. v. Host. v. a. Kr. Capposa.

T. v. Host. v. caesium Al No. 111.

T. v. Host. v. Delfii Kr.

T. v. Host. v. erythrospermum Kr. Durable.

T. v. Host. v. e. Kooperatorka.

T. v. Host. v. e. Novinka.

T. v. Host. v. e. Zarja.

T. v. Host. v. e. Zemka.

T. v. Host. v. e. Ukrainka.

T. v. Host. v. e. Moskovskaja.

T. v. Host. v. e. No. 1114.

T. v. Host. v. e. Charkovskaja 496.

T. v. Host. v. e. Kazanksaja $0 / 6$.

T. v: Host. v. e. No. 118.

T. v. Host. v. e. Viatskaja 529. 
Triticum v. Host. v. ferrugineum Al. Moskovskaja 2411.

T. v. Host. v. f. Al. Moskovskaja 2453.

T. v. Host. v. f. Al. Simbileewskaja 146.

T. v. Host. v. f. Al. No. 150.

T. v. Host. v. f. Al. "Engelgardt".

T. v. Host. v. f. Al. "Sarmatka".

T. v. Host. v. hostianum No. 237.

T. v. Host. v. lutescens No. 053.

T. v. Host. v. 1. Al. 479.

T. v. Host. v. 1. Al. 040.

T. v. Host. v. 1. Al. 062.

T. v. Host. v. 1. A1. 3221.

T. v. Host. v. 1. Al. 323.

T. v. Host. v. 1. Al. 786.

T. v. Host. v. 1. Al. 9113.

T. v. Host. v. 1. Al. Garnet.

T. v. Host. v. I. Al. Leda.

T. v. Host. v. 1. Al. Marki 3.

T. v. Host. v. 1. Al. Kitchener.

T. v. Host. v. 1. Beta stojkaja.

T. v. Host. v. 1. Viatskaja 05 .

T. v. Host. v. 1. Viatskaja 120.

T. v. Host. v. 1. No. 329.

T. v. Host. v. 1. Zolotaja.

T. v. Host. v. 1. 1060/10.

T. v. Host, v. 1. Teiskaja.

T. v. Host. v. 1. $27 / 36$.

T. v. Host. v. I. 434/154. 
Triticum v. Host. v. milturum Al.

T. v. Host. v. m. Saratovskaja 129.

T. v. Host. v. m, Novinka.

T. v. Host. v. m. 841 .

T. v. Host. v. m. 10,78 .

T. v. Host. v. m. 321 .

Zea mays $\mathrm{L}$.

Leguminosae.

Cicer arientinum L, v. Album hort.

C. a. "Tschernij".

C. a. No. 277 .

C. a. 16 .

C. a. No, 185 .

C. d, No. 199 .

Ervum lens L. v. macrosperma Bor, v. nummularia "Obyknovennaja".

E. 1. v, m. Saratovskaja 13.

E. I. v, m. Saratovskaja 23.

E. 1. v. m. Saratovskaja 140.

E. 1. v. m. "Ddymtschataja".

E. 1. v. microsperma Bar.

E. 1. v. m. glaucosperma. Seraja $32 / 5$.

E. 1. (ex Italia).

E. 1. (ex Marocco).

E. 1. (ex Sicilia).

E. 1. (ex Syriá).

E. 1. (ex India).

E. 1. (ex Francia).

58 
Ervum 1. (ex Afghanistan).

E. I. (ex Cabarda).

E. 1. "Nariadnaia"

Glicine hispida Maxim.

Lathyrus sativus L. No. 19/42.

L. s. No. 398.

L. s. Gornaja Buchara.

L. s. Srednaja Volga.

L. S. No. 729 .

L. s. Nu. 732 .

L. s. No. $16 / 28$.

L. S. No, 829 "Sibirj".

L. s. Ukraina.

L. S, Azerbaidjan.

L. S. No. 702 .

L. S. Kubanj.

L. s. No. 539.

L. s. No, 423 .

L. S, No. 22.

L. S. No. 1136.

L. s. No. 287.

L. S. No. 12.

I. s. No. 277 .

L. s. No. 129.

Lupinus albus $\mathrm{L}$.

L. a. $y-26$.

L. angustifolius L. G. D. C.

L. a. 645 .

L. a, 174 . 
Lupinus a. 418.

L. a. maloalkaloidnij 01.

L. luteus L. 4666.

L. 1. "Pioner'.

L. 1. No. 345.

L. 1. No. 530 .

L. mutabilis $\mathrm{L}$.

L. pilosus L.

L. polyphyllus L.

Phaseolus multiflorus Willd "Lopata".

Ph. vulgaris L. No. 294.

Ph. v. No. 663. L.

Ph. v. No. 1374.

$\mathrm{Ph}$. v. amerikanskaja.

$\mathrm{Ph}$. v. Zolotaja Gora.

$\mathrm{Ph}$. v. Bolgaria.

Ph. v. No. 287.

Ph. v, No. 1362.

Ph. v. No. 1009.

$\mathrm{Ph}$. v. Bomba bielaja.

Ph. v. Poltavskaja.

Ph. v. Bielaja Kanadskaja.

$\mathrm{Ph}$. v. No. 663.

Ph. v. No. 12863.

Ph. v. Rosovaja 39.

Ph. v. Rosovo-pestraja.

Ph. v. Krasnodarskaja.

$\mathrm{Ph}$. v. Izumrudnaja.

$\mathrm{Ph}, \mathrm{v}$. Jeltaja zasuchoustoichivaja. 
Phaseolus v. olivkovaja.

Ph. v. No, 1198.

Pisum arvense L. "Peliushka" 32.

P. a. "Peliushka" 40.

P. a. 'Peliushka" 42.

P. a. No. 9/376.

P. sativum L. V. coronatum Schtambovij.

P. s. v. glaucospermum Al. Folger-Heine.

P. s. v. g. Moskovskij Al 559.

P. s. v. g. Victoria zelenaja.

P. s. V. saccharatum "Rannij Bismark".

P. s. v. s. 'Konjek Gorbunok".

P. s. v. s. "Sacharnij zelenij".

P. s. v. vulgatum "Ambrosia".

P. s. v. v. "Victoria rosovaja".

P. s. v. v. Victoria Mandorffskaja.

P. s. v. v. "Capital".

P. s. No. 06.

P. s. T $25 \times 103$.

P. s. Irlandetz.

P. s. G-25.

P. s. 047 .

P. s. 087.

P. s. Tom Thumb.

P. s. Mushung.

P. s. Rychlik.

P. s. Atar.

P. s. olivkovij gibrid.

P. s. Tschudo Kelvidonia. 
Pisum sativum Albanskij 06.

P. s. neistoschimij 0195.

P. s. Thomas Lakston.

P. s. The Wonder of Amerika.

P. s. Bismark 372 .

P. s. Urojainij.

P. s. Zolotoj No. 2.

Vicia Faba L.

V. Faba L. v. minor Bul.

V. narbonensis L.

V. sativa L. Belaja 163.

V. s. v. durra.

V. s. Archangelskaja.

V. s. ploskosemiannaja.

V. s. Saratovskaja.

Vigna capensis Walp.

V. glabra Savv.

Dolichos bicontortus Dur. 
Prof. Dr. S. S. Stankov.

Horti Director

Ass, A, S. Zhuravleva.

$$
\text { Vice-Director }
$$

Docent. cand.

D. S. Averkiev (Morphol, et Systemat.).

Docent. cand.

M. L. Stelmachovicz (Dendrolog. et Plantae decorant.).

Docent. cand.

L. V. Tichov (Plantae officinal. et technic.).

Ass. M. V. Alalykina (Pomologia)

Horti Botanici et Hortulani 
П еречень семян, собранных в 1940 г. и предлагаемых в обмен Ботаническим садом Горьковского Государственного Уннверситета \# ОГИЗ. Горьковское Издательство. 1941 г. № 1332.

Редактор проф. С. С. Спанков. Техн. редактор Л. Н. Немченко. Корректор В. М. Плотникова.

Сдано в набор 29/XI-40 г,, подписано к печати 6/I-4I г. Формат $60 \times 92 / 32$. Тираж 400 экзем. Печ. л. 2, бум. л. 1, уч.-изд. л. 1,85, знаков в п. л. 38000 . Инд. СХ-3 * МЦ 1. Полиграф, город Горький, улища Фигнер, 32.

Зак. 소 10339. 

БЕСПЛАТ НО

ПЕРЕЧЕНЬ СЕМЯН,

СОБРАННЫХ В 1940 ГОДУ

И ПРЕДЛАГАЕМЫХ В ОБМЕН

БОТАНИЧЕСКИМ САДОМ

ГОРЬКОВСКОГО ГОСУДАРСТВЕННОГО

УНИВЕРСИТЕТА

ГОРЬКОВСКОЕ ИЗДАТЕЛЬСТВО 1941 


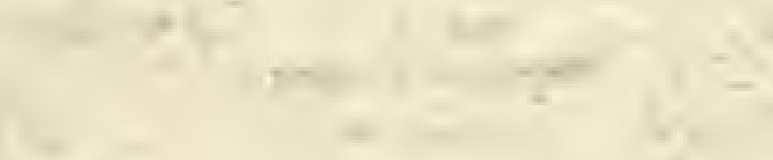

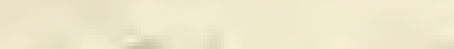

$$
\text { - }
$$

-

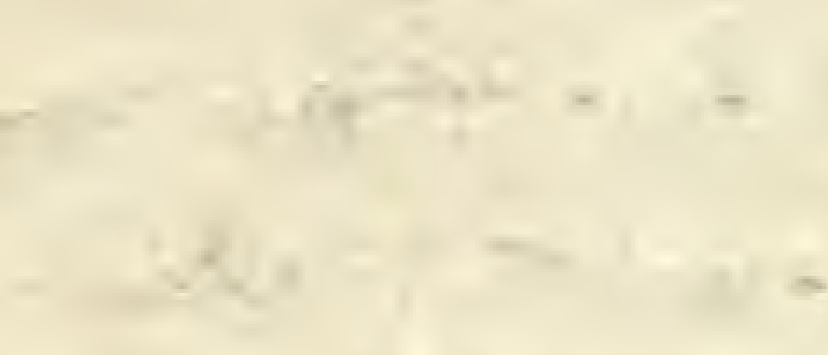

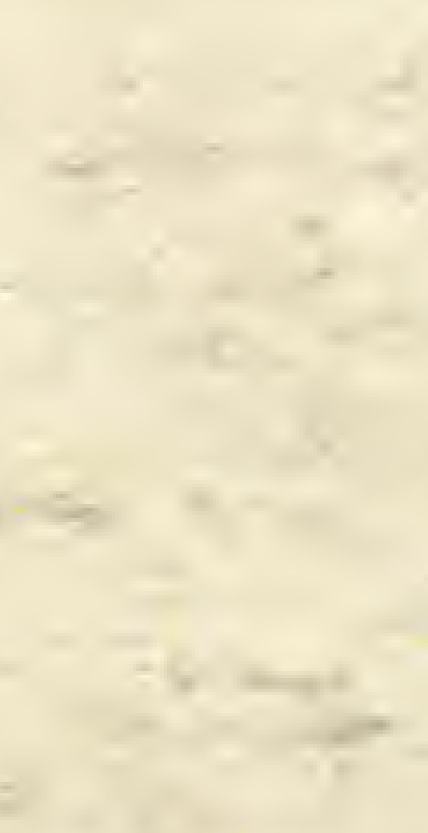

\section{$x^{2}$}
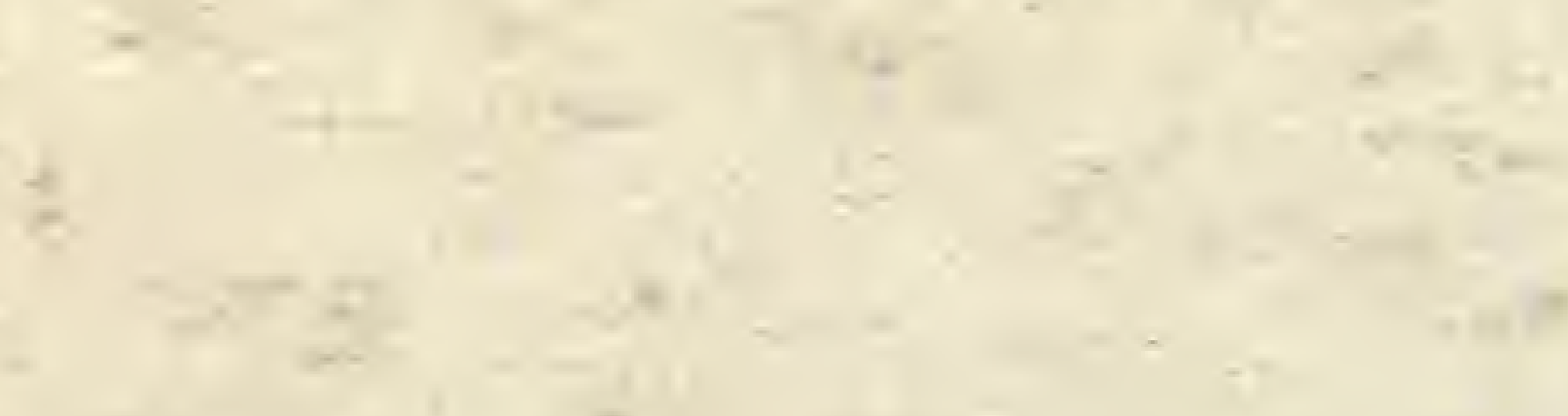

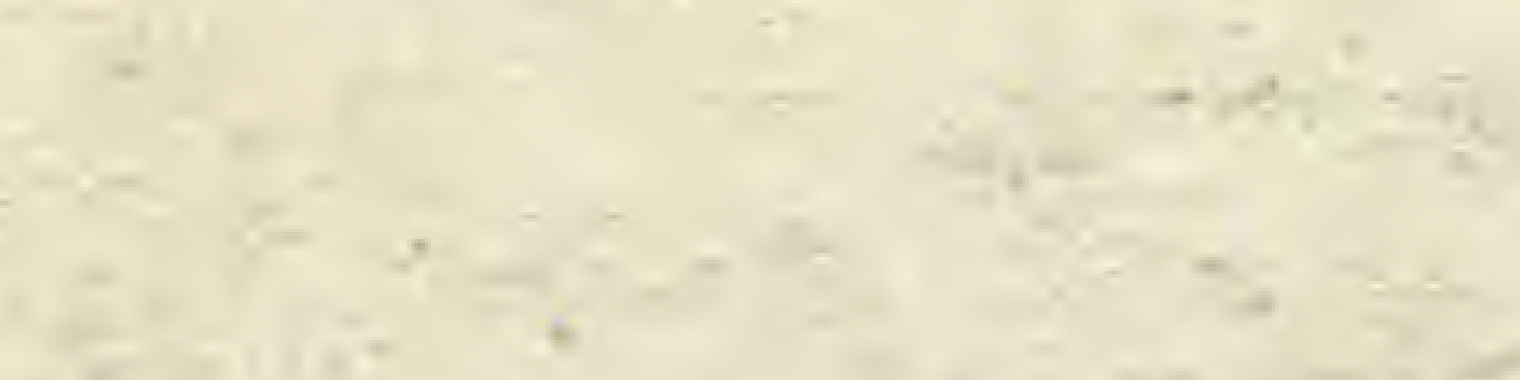

$i^{3}$

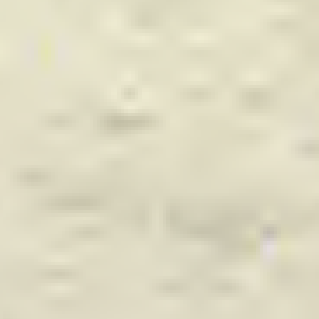
$\rightarrow$

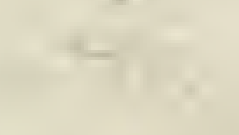

$r^{4}+2-7=$ 


$$
\begin{aligned}
& \text { VII } \\
& \text { DELECTUS SEMINUM } \\
& \text { ANNO MCMXLI COLLECTORUM } \\
& \text { QUAE } \\
& \text { HORTUS BOTANICUS } \\
& \text { UNIVERSITATIS GORKIJENSIS } \\
& \text { PRO } \\
& \text { MUTUA COMMUTATIONE } \\
& \text { OFFERT }
\end{aligned}
$$$$
\text { GORKIJ MCMXLII }
$$ 


\section{2}




\section{SEMINA PLANTARUM LIGNOSARUM ET FRUTICUM. \\ Betulaceae.}

1. Alnus sitchensis Sarg.

Berberidaceae.

2. Berberis amurensis Rupr.

3. B. heteropoda Schrenk.

4. B. h. v. oblonga Reg.

5. B. ilicifolia Forst.

6. B. Thunbergii D. C.

7. Mahonia aquifolium Nutt.

Saxifragaceae.

8. Philadelphus coronarius $L$.

9. $\mathrm{Ph}$. pubescens Lois.

10. Ph. p. f. grandiflura hort.

11. Ribes alpinum $\mathrm{L}$.

12. R. aureum Pursch.

13. R. worscewiczii Jancz.

Rosaceae.

14. Amelanchier vulgaris $L$.

15. Aronis melanocarpa E11. 
16. Cotoneaster acuminata Lindl.

17. C. humifusa Duthie.

18. C. integerrima Med.

19. Physocarpus opulifolius Maxim.

20. Prunus pumila L.

21. Rosa altaica Willd.

22. R. amblyotis C. A. Mey.

23. R. centifolia L.

24. R. moschata Herm.

25. R. pimpinellifolia L.

26. R. rubrifolia Vill.

27. R. rugosa Thunb.

28. R. r. v. alba Rehd.

29. Rubus odoratus L.

Leguminosae.

30. Amorpha californica Nutt.

31. A. fruticosa L.

32. Caragana arborescens L.

33. C. frutex D. C.

34. Colutea arborescens Linn.

35. C. media Willd.

36. Cytisus albus Hacq.

37. C. supinus Linn.

38. Genista tinctoria L.

Rutaceae.

39. Ptelea trifoliata L. 


\section{Euphorbiaceae.}

40. Securinega fluggeoides Muell.

41. S. ramiflora Muell.

Anacardiaceae.

42. Cotinus coggygria Scop.

43. Rhus toxicodendron L.

Celastraceae.

44. Evonymus verrucosa Scop.

45. Celastrus orbiculatus Thunb.

Aceraceae.

46. Acer Negundo L.

47. A. tataricum L.

Rhamnaceae.

48. Ceanothus americanus Linn.

49. Rhamnus cathartica L.

50. R. imeretina Booth.

Tiliaceae.

51. Tilia cordata Mill.

52. T. platyphyllus Scop.

Cornaceae.

53. Cornus alba L.

54. C. Baileyi Coult et Evans. 


\section{Oleaceae.}

55. Liustrum vulgare $\mathrm{L}$.

\section{Caprifoliaceae.}

56. Lonicera caprifolium L.

57. L. dioica Linn.

58. L. micrantha Regel.

59. L. muendeniensis Rehd.

60. L. tatarica L.

61. L. t. nana Alphand fl. alba

62. L. xylosteum L.

63. Symphoricarpus racemosus Michx.

64. Viburnum opulus $L$.

II. SEMINA PLANTARUM HERBACEUM, collecta 1941.

Gramineae.

65. Agropyrum tenerum Vasey.

66. A. ugamicum Drob.

67. Agrostis borealis Hartm.

68. Alopecurus ventricosus Pers.

69. Avena planiculmis Turcz.

70. A. pubescens Huds.

71. Arrhenantherum elatius $M$. et $K$.

72. Asperella hystrix Humb.

73. Bromus erectus Huds. 
74. Bromus inermis Leyss.

75. Corynephorus canescens Beauw.

76. Dactylis Aschersoniana Graebn.

77. D. glomerata L.

78. Elymus akmolensis Drob.

79. E. canadensis L.

80. E. dahuricus Turcz.

81. E. excelsus Turcz.

82. E. sabulosus M. B.

83. E. sibiricus L.

84. Fectuca glauca Bess.

85. F. sulcata Hack.

86. Hordeum bulbosum L.

87. Koeleria gracilis Pers.

88. Lolium perenne L.

89. Melica transsilvanica Schuhr.

90. Phleum pratense L.

91. Poa alpina $L$.

92. Roegneria tianschanica (Drob.) Nevski

93. Sesleria Heufleriana Sch.

94. Stipa capillata L.

95. S. calamagrostis Wahlenb.

96. S. Lessingiana Trin.

97. S. stenophylla Czern.

98. S. viridula Trin.

99. Triodia decumbens Pall.

100. Trisetum sibiricum Rupr. 


\section{Cyperaceae.}

101. Carex cuprina Nendtv.

102. C. leporina L.

Liliaceae.

103. Allium angulosum $\mathrm{L}$.

104. A. caesium Schrenk.

105. A. fistulosum $\mathrm{L}$.

106. A. hymenorrhizum Ledeb.

107. Allium schoenoprasum L.

108. A. sibiricum L.

109. Anthericum liliago $\mathrm{L}$.

110. Asparagus officinalis L.

111. Eremurus altaicus Stev.

112. E. Olgae Regel.

113. Galtonia candicans Decne.

114. Hemerocallis flava $L$.

115. Lilium concolor Salisb.

116. L. martagon L.

117. L. tenuifolium Fisch.

118. Lilium tigrinum Ker.-Gawl. (bulb.)

119. Muscari botryoides Mill.

120. Ornithogallum umbellatum L.

121. Scilla sibirica Andr.

Iridaceae.

122. Iris aphylla L.

123. I. halophila Pall. 
124. Iris Hookeri Penny.

125. I. illirica Somm.

126. I. pumila L.

127. I. setosa Pall.

128. I. sibirica L.

129. I. sogdiana Bunge.

130. I. sulphurea Koch.

131. I. variegata $\mathrm{L}$.

132. 1. virsicolor L.

133. Sisyrinchium anceps Cav.

Polygonaceae.

134. Polygonum polymorphum Ldb.

135. Rheum crassinervium Fisch.

136. R. Emodi Wall.

137. R. officinale Baill.

138. R. undulatum L.

139. Rumex acetosa L.

140. R. callosus Reidr.

141. R. hydrolapathum Huds.

142. R. hymenosepalus Torr.

143. R. Patientia L.

Chenopodiaceae.

144. Chenopodium Bonus-Henricus L.

Phytolaccaceae.

145. Rivina humilis L. 
Caryophyllaceae.

146. Arenaria graminifolia Schrad.

147. Cerastium alpinum L.

148. C. Biebersteinii D. C.

149. C. Lerchenfeldianum Schur.

150. Gypsophila elegans Bieb.

151. G. elegans Bieb. v. grandiflorum.

152. Lychnis chalcedonica L.

153. L. coronaria Desr.

154. Saponaria caespitosa D. C.

155. Silene armeria L.

156. S. tenuis Willd.

157. Viscaria alpina (L.) Don.

158. V. vulgaris Roeh1.

\section{Ranunculaceae.}

159. Aconitum lasiostomum Rchb.

160. A. vulparia Rchb.

161. Anemone hudsoniana Richards.

162. Aquilegia canadensis L.

163. A. flabellata Sieb. et Zucc.

164. A. olympica Boiss.

165. Clematis tangutica Korsch.

166. Delphinium elatum L.

167. D. grandiflorum L.

168. D. hybridum Willd.

169. Nigella arvensis L. 
170. Nigella damascena $L$.

171. N. sativa L.

172. Pulsatilla rubra Delarb.

173. Ranunculus acer L. ssp. Steveni Hegi.

174. R. bulbosus L.

175. R. polyanthemus L.

176. R. propinquus C. A. Mey.

177. Thalictrum minus $\mathrm{L}$.

178. Trollius altaicus C. A. Mey.

179. T. pumilus Don.

\section{Papaveraceae.}

180. Chelidonium laciniatum Mill.

181. Eschscholzia californica Cham.

182. Papaver lateritium C. Koch.

183. P. orientale L.

184. P. rhoeas L.

\section{Cruciferae.}

185. Aethionema Buxbaumii D. C.

186. Alyssum argenteum All.

187. A. desertorum Stapf.

188. Arabis albida Stev.

189. A. alpina L.

190. A. brevifolia D. C.

191. A. sicula Huet.

192. Aubrietia erubescens Griseb. 
193. A. libanotica Borss. et Hohen.

194. Biscutella laevigata L.

195. Carrichtera vella D. C.

196. Draba repens M. B.

197. Hesperis matronalis L.

198. H. tristis L.

199. Iberis intermedia Guers.

200. l. odorata L.

201. Isatis alpina All.

202. I. tinctoria L.

203. Malcolmia maritima R. Br.

204. Roripa silvestris (L.) Bess.

Crassulaceae.

205. Sedum album L.

206. S. Ewersii Ledeb.

207. S. Maximowiczii Regel.

208. S. rupestre L.

209. S. spurium Bieb.

210. Sempervivum tectorum L.

Saxifragaceae.

211. Bergenia crassifolia L.

212. Heuchera americana L.

213. H. cilindrica Dougl.

214. Mitella caulescens Nutt.

215. Saxifraga altissima Kerner. 


\section{Rosaceae.}

216. Filipendula hexapetala Gilib.

217. Fragaria indica Andr.

218. F. moschata Duchesne.

219. F. vesca $L$.

220. Geum coccineum Sibth. et Sm.

221. Potentilla crantzii Beck.

222. P. geoides Bieb.

223. P. hybrida hort.

224. P. Meyeri Boiss.

225. P. rupestris L.

226. P. sitchense S. Wats.

227. Sanguisorba officinalis L.

\section{Leguminosae.}

228. Anthyllis Boissieri Sag.

229. A. alopecurus Pall.

230. A. cicer L.

231. A. danicus Retz.

232. A. falcatus Lam.

233. A. galegiformis L.

234. A. glycyphyllos L.

235. A. onobrychis $L$.

236. A. sulcatus L.

237. A. uliginosus L.

238. Galega orientalis Lam.

239. Glycyrrhiza echinata L. 
240. Hedysarum sibiricum Poir.

241. Lathyrus latifolius L.

242. L. niger $\mathrm{L}$.

243. L. odoratus L.

244. Lupinus elegans H. B. et K.

245. L. polyphyllus Lindl.

246. Medicago falcata L.

247. M. hispida Gaertn.

248. M. radiata $L$.

249. M. sativa L.

250. M. scutellaria Mill.

251. Onobrychis arenaria D. C.

252. Oxytropis campestris D. C.

253. Scorpiurus vermiculata $L$.

254. Securigera coronilla D. C.

255. Tetragonolobus purpureus Moench.

256. Thermopsis fabacea D. C.

257. Th. fabacea D. C.

258. Trifolium alpestre $L$.

259. T. incarnatum L.

260. T. lupinaster L.

261. T. medium L.

262. Trigonella coerulea Ser.

263. Vicia pisiformis L.

Linaceae.

264. Linum altaicum M. B. 
265. Linum grandiflorum Desf.

266. L. perenne L.

Malvaceae.

267. Abutilon indicum (L.) G. Don.

268. Althaea ficifolia Cav.

269. A. rosea Cav.

270. A. sulphurea Boiss.

271. Hibiscus abelmoschus L.

272. Kitaibelia vitifolia Willd.

273. Lavatera thuringiaca L.

274. Malachra radiata $\mathrm{L}$.

275. Malva alcea L.

276. M. moschata L.

277. Palaua flexuosa Mast.

278. Sidalcea candida A. Gr.

279. S. malvaeflora A. Gr.

Cistaceae.

280. Cistus symphytifolius Lam.

Hypericaceae.

281. Hypericum ascyron L.

282. H. perforatum L.

Lythraceae.

283. Cuphea viscosissima Jacq.

284. Lythrum salicaria L. 


\section{Oenotheraceae.}

285. Clarkia elegans Dougl.

286. Epilobium hirsutum L.

Umbelliferae.

287. Heracleum dissectum Ledeb.

288. Libanotis montana All.

289. Seseli Osseum Crantz.

Primulaceae.

290. Androsace lactea L.

291. Lysimachia punctata L.

292. Primula algida Adams.

Plumbaginaceae.

293. Armeria sibirica Turcz.

294. Statice Gmelini Willd.

Polemoniaceae.

295. Collomia coccinea Lehm.

296. Gilia capitata Dougl.

297. Polemonium coeruleum L.

298. P. Van-Bruntiae Britt.

Hydrophyllaceae.

299. Nemophila insignis Dougl.

300. Phacelia campanularia A Gray.

301. Ph. congesta Hook. 
302. Ph. losaefolia Torr.

303. Romanzoffia sitchensis Bong.

\section{Boraginaceae.}

304. Cerinthe minor L.

305. Myosotis alpestris F. W. Schmidt.

306. Symphytum asperum Lepech.

\section{Labiatae.}

307. Brunella pyrenaica Philip.

308. Dracocephalum altaiense Laxm.

309. D. nutans L.

310. D. Ruyschiana L.

311. Hyssopus officinalis L.

312. Lallemantia peltata Fisch. et Mey.

313. Leonurus cardiaca L.

314. Lophanthus anisatus Benth.

315. Monarda fistulosa L.

316. Nepeta cataria L. v. citriodora.

317. N. kokanica Regl.

318. N. macrantha Fisch.

319. N. nuda $L$.

320. Origanum vulgare L.

321. Phlomis tuberosa $\mathrm{L}$.

32). Salvia nemorosa L.

323. S. silvestris L.

324. S. stepposa Des.-Chost. 
325. Salvia verticillata L.

326. Scutellaria altissima L.

327. Sideritis hyssopifolia L.

328. S. scordioides L.

329. Stachys germanica L.

330. S. officinalis Trev.

331. S. setifera C. A. Mey

332. Thymus Marschallianus Willd.

333. Ziziphora capitata L.

Solanaceae.

334. Atropa Belladonna L.

335. Physalis Franchettii Mast.

336. Physochlaena orientalis G. Don.

337. Solanum Dulcamara L.

Scrophulariaceae.

338. Antirrhinum Coulterianum Benth.

339. Digitalis ambigua Murr.

340. D. purpurea L.

341. Linaria bipartita Willd.

342. L. vulgaris Mill.

343. Nemesia strumosa Benth.

344. Pentstemon hirsutus Willd.

345. P. diffusus Dougl.

346. P. procerus Dougl.

347. Schizanthus pinnatus Ruiz. et Pav. 
348. Verbascum orientale M. B.

349. Veronica candida Lodd.

350. V. gentianoides Vahl.

351. V. incana $L$.

352. V. longifolia L.

Bignoniaceae.

353. Incarvillea grandiflora Bur. et Franch. 354. I. Olgae Regel.

\section{Globulariaceae.}

355. Globularia Willkommii Nym.

Plantaginaceae.

356. Plantago lanceolata L.

357. P. purpurascens Willk.

Rubiaceae.

358. Asperula tinctoria L.

Valerianaceae.

359. Patrinia rupestris Juss.

360. Valeriana hispida Turcz.

361. V. rossica P. Sm.

362. Valerianella cor nata D. C.

Dipsacaceae.

363. Scabiosa micrantha Desf. 


\section{Campanulaceae.}

364. Campanula alliariaefolia Willd.

365. C. carpathica Jacq.

366. C. persicifolia L.

367. C. sarmatica Ker.-Gawl.

368. Codonopsis clematidea Clarke.

369. Jasione humilis Lois.

370. Phyteuma Scheuchzeri All.

371. Platycodon grandiflorum D. C.

Compositae.

372. Achillea asplenifolia Vent.

373. A. cartilaginea Led.

374. A. filipendulina Lam.

375. A. millefolium L.

376. A. sibirica Ledeb.

377. A. tomentosa L.

378. Anaphalis margaritacea Benth. et Hook. 379. Antennaria nevadensis Greene.

380. Anthemis cotula L.

381. Arnica montana L.

382. Aster longifolius Lam.

383. Aster umbellatus Mill.

384. Buphtalmum salicifolium L.

385. B. speciosum Schreb.

386. Cacalia hastata L.

387. Calendula aegyptica Desf. 
388. Calendula algeriensis Boiss, et Bent. 389. C. stellata Cav.

390. Carthanus tinctorius $L$.

391. Centaurea dealbata Willd.

392. C. macrocephala Puschk.

393. C. scabiosa L.

394. Chrysanthemum corymbosum L.

395. Cosmos bipinnatus Cav.

396. Dimorphotheca annua Less.

397. D. aurantiaca D. C.

398. D. pluvialis Moench.

399. Doronicum orientale Hoffm.

400. Erigeron asper Nutt.

401. E. speciosus D. C.

402. Gaillardia aristata Pursh.

403. Helichrysum bracteatum Willd.

404. Heliopsis helianthoides Sweet.

405. H. Scabra Dun.

406. Hieracium pilosella L.

407. Inula Helenium L.

408. Layia platyglossa A. Gray.

409. Leontopodium alpinum Cass.

410. Madia sativa Don.

411. Ptarmica impatiens D. C.

412. Pyrethrum cinerarifolium Trev.

413. P. roseum Lindl.

414. Rudbeckia laciniata L. 
415. Scorzonera purpurea L.

416. Silybum Marianum Gaertn.

417. Solidago canadensis L.

418. S. multiradiata Ait.

419. Tanacetum buronense Nutt.

420. T. vulgare $\mathrm{L}$.

421. Troximon gracilens Gray.

\section{OLLECTA 1940}

422. Agropyrum desertorum (Fisch.) Schult.

423. Andropogon ischaemum L.

424. Bromus unioloides Humb.

425. Cynosurus echinatus L.

426. Elymus mollis Trin.

427. Hordeum secalinum Schreb.

428. Koeleria crassipes Lange.

429. Lolium canadense Michx.

430. Setaria viridis P. B.

Liliaceae.

431. Allium albidum Künth.

432. A. altaicum Pall.

433. A. galanthum Kar. et Kir.

434. A. giganteum Reg.

435. A. globosum M. B.

436. A. lineare L. 
437. Allium narcissiflorum Vill.

438. A. odorum L.

439. A. paradoxum G. Don.

440. A. pulchellum G. Don.

441. A. tataricum L.

442. Anthericum ramosum L.

Iridaceae.

443. Iris cordata Willd.

444. I. Songorica Schrenk.

445. I. xiphioides Ehrh.

446. Sisyrinchium montanum hort.

Urticaceae.

447. Urtica cannabina L.

Polygonaceae.

448. Oxyria digyna (L.) Hill.

449. Rumex mexicanus Meiss.

450. R. pallidus Bigel.

451. R. stenophyllus Ledb.

Chenopodiaceae.

452. Chenopodium aristatum L.

453. Ch. botrys L.

Amaranthaceae

454. Amaranthus aurea T. A. Desf. 455. A. blitum L. 
456. Amaranthus caudatus L. f. atropurpureus. 457. A. gangeticus L.

458. A. purpureus hort.

\section{Nyctaginaceae.}

459. Oxybaphus nyctagineus Sweet.

Caryophyllaceae.

460. Agrostemma Githago L.

461. Arenaria longifolia M. B.

462. A. sajanensis Willd.

463. Cucubalus baccifer L. v. japonicus.

464. Dianthus arenarius $\mathrm{L}$.

465. D. barbatus L.

466. D. Boissieri Willk.

467. D. carthusianorum L.

468. D. Hoeltzeri Winkl.

469. D. praecox Willd.

470. Gypsophila acutifolia Fisch.

471. G. altissima L.

472. G. fastigiata $\mathrm{L}$.

473. G. pacifica Kom.

474. G. perfoliata M. B.

475. G. Steveni Fisch.

476. Heliosperma alpestre (Jacq.) Rchb.

477. Melandrium album (Mill.) Garcke 478. Saponaria ocymoides L. 
479. Saponaria officinalis L.

480. Silene alpina L.

481. S. Bergiana Lindm.

482. S. conica $L$.

483. S. gallica L.

484. S. grandiflora Franch.

485. S. laeta $\mathrm{A}$. Br.

486. S. mellifera Boiss.

487. S. muscipula L.

488. S. venosa Aschers.

489. Vaccaria pyramidata Med.

Ranunculaceae.

490. Aconitum lycoctonum L.

491. Anemone decapetala L.

492. A. hudsoniana Richards.

493. A. multifida Poir.

494. A. riparia Fernold.

495. Clematis Bergeronii hort.

496. Ranunculus propinquus C. A. M.

497. Thalictrum minus L.

498. Th. Fendleri Engelm.

499. Th. flexuosum Bernh.

500. Th. nigricans Jacq.

501. Th. rugosum Ait.

Papaveraceae.

502. Chelidonium Franchettianum Prain. 
503. Papaver californicum A. Gray. 504. P. nudicaule L.

505. P. pyrenaicum A. Kern. ssp. raeticum Fedde

506. P. somniferum L.

Cruciferae.

507. Alyssum sinuatum L.

508. Camelina dentata Wallr.

509. Crambe hispanica L.

510. Hesperis dinarica G. Beck.

511. Iberis pectinata Boiss.

512. Thlaspi densiflorum Boiss, et Ky.

\section{Crassulaceae.}

513. Sedum aizoon L.

514. S. anopetalum D. C.

515. S. carpaticum Reuss.

516. S. crassipes Wall.

517. S. fabaria Koch.

518. S. Kamtschaticum Fisch. et Mey

519. S. Kirillowii Rgl.

520. S. Stephani Cham.

Saxifragaceae.

521. Heuchera glabra Willd.

522. H. hispida Pursch.

523. H. pilosissima Fisch. et Mey 


\section{Rosaceae.}

524. Agrimonia odorata Mill.

525. Fragaria virginiana Duchsne.

526. Geum aleppicum Jacq.

527. G. canadense Kossy.

528. G. chiloense Balb.

529. G. hispidum Fries.

530. G. Jankae G. Beck.

531. G. tyrolense Kern.

532. G. urbanum L.

533. G. virginianum L.

534. Potentilla abchasica Fomin.

535. P. adscharica Somm.

536. P. alchimilloides Lop.

537. P. argentea L.

538. P. Dombeyi Nestl.

539. P. gelida C. A. Mey.

540. P. intermedia L.

541. P. nevadensis Boiss.

542. P. nivea L.

543. P. pyrenaica Ram.

544. Poterium sanguisorba L.

545. S. sitchensis C. A. M.

Leguminosae.

546. Anthyllis variegata hort.

547. Astragalus baeticus L. 
548. Baptisia australis R. Br.

549. Cicer arietinum L.

550. Lotus ornithopodioides L.

551. Onobrychis altissima $\mathrm{L}$.

552. Ornithopus sativus Brot.

553. Trifolium arvense $\mathrm{L}$.

554. Vicia americana Muhl.

555. V. ervilia (L.) Willd.

Geraniaceae.

556. Erodium cicutarium L'Herit.

Linaceae.

557. Linum perenne L. v. sibiricum D. C. Malvaceae.

558. Anoda hastata Lav.

559. Lavatera trimestris L.

560. Malva crispa L.

561. M. pusilla With.

562. $M$. verticillata $L$.

563. Sidalcea neo-mexicana A. Gray

564. S. spicata Greene.

\section{Cistaceae.}

565. Helianthemum ovatum Dun. 566. H. rosmarinifolium Purch. 567. $\mathrm{H}$. vineale Pers. 


\section{Oenotheraceae.}

568. Oenothera fruticosa L.

\section{Umbelliferae.}

569. Coriandrum sativum L.

570. Peucedanum alsaticum L.

571. Pimpinella majus Huds.

\section{Primulaceae.}

572. Lysimachia acroadenia Max.

573. L. lobeloides Wall.

574. L, verticillata Pall.

Plumbaginaceae.

575. Armeria arctica Wall.

576. A. maritima Willd.

577. A. plantaginea Willd.

Gentianaceae.

578. Gentiana cruciata L.

Apocynaceae.

579. Amsonia Tabernaemontana Walt. Asclepiadaceae.

580. Vincetoxicum fuscatum Reichb.

581. V. medium Decsn.

582. V. officinale Mnch. 


\section{Hydrophyllaceae.}

583. Phacelia fimbriata Michx. 584. $\mathrm{Ph}$. Whitlavia A. Gray.

\section{Borraginaceae.}

585. Eritrichum strictum Hort.

\section{Labiatae.}

586. Nepeta grandiflora $M$. B.

587. Phlomis cashemiriana Royle.

588. Ph. chrysophylla Boiss.

589. Ph. viscosa Poir.

590. Stachys nivea Labill.

\section{Solanaceae.}

591. Datura aegyptiaca Vesl.

592. D. stramonium L.

593. Nicandra physaloides Gaertn.

Scrophulariaceae.

594. Digitalis nevadensis Kunze.

595. D. viridiflora hort.

596. Linaria purpurea Mill.

597. L. triphylla Mill.

598. Verbascum Wilsonii hort. 
599. Veronica fruticans Jacq. 600. V. spuria L.

\section{Globulariaceae.}

601. Globularia vulgaris L.

Plantaginaceae.

602. Plantago alpina L.

603. P. Psyllium L.

Rubiaceae.

604. Asperula ciliata Rochel. 605. Galium saccharatum All.

606. Ophiorrhiza Mungos L.

Dipsacaceae.

607. Dipsacus ferox Loisel. 608. Scabiosa Olivieri Coult. 609. S. pyrenaica All.

Campanulaceae.

610. Campanula amabilis Rupr.

611. C. elegans Roem. et Schult.

612. C. eriocalix Adam.

613. C. gracilis Jard.

614. C. imeretina Rupr.

615. C. pulcherrima Lam. 
616. Campanula ranunculoides $L$.

617. C. Steveni Bieb.

618. Phyteuma Halleri All.

\section{Compositae.}

619. Achillea pseudopectinata Janka.

620. Anthemis candidissima Willd.

621. A. tinctoria L.

622. A. ruthenica Bieb.

623. Aster tibeticus Hook.

624. Bidens dahlioides Wats.

625. Calendula arvensis L.

626. C. officinalis L. "Radio" hort.

627. C. officinalis L. "Opunti King" hort.

628. Carduus hamulosus Ehrh.

629. Centaurea nigra L.

630. C. ossica C. Koch.

631. C. uniflora L.

632. Chrysanthemum maximum Ramond.

633. Cnicus benedictus L.

634. Hieracium boreale Fries.

635. H. robustum Fr.

636. Hypocheoris radiata L.

637. Jurinea arachnoidea Bge.

638. Lactuca scariola L.

639. Serratula coronata L.

640. S. Gmelini Ledeb. 
III. SEMINA PLANTARUM DECORANTIUM. Iridaceae.

641. Gladiolus hybridus hort. (bulbi) 642. G. primulinus Bok. (bulbi).

\section{Caryophyllaceae.}

643. Gypsophila paniculata Linn.

Papaveraceae.

644. Papaver somniferum L. f. paeoniflorum hort.

Portulacaceae.

645. Portulaca grandiflora Hook.

Begoniaceae.

646. Begonia semperflorens Link. et Otto Primulaceae.

647. Primula obconica Hauce.

Polemoniaceae.

648. Phlox Drummondii Hook.

Solanaceae.

649. Nicotiana affinis T. Moore 650. Petunia hybrida Hort. 


\section{Scrophulariaceae.}

651. Antirrhinum majus L. fl. rosea hort.

652. A. m. "Tschernyi Platsch“.

653. Nemesia strumosa Benth. v. grandiflora hort.

Campanulaceae.

654. Lobelia erinus L.

Compositae.

655. Callistephus chinensis Nees "Triumph", "Krasa SSSR":

656. С. ch. N. „Triumph“, лиловые.

657. C. ch. N. nana, кармазинные.

658. C. ch. N. "Straussovoje pero", albi.

659. C. ch. N. "Straussovoje pero", sangin.rubri.

660. C. ch. N. „Straussovoje pero", argent.rosei.

661. C. ch. N. "Straussovoje pero", caerulei. 662. C. ch. N. "American Beauty", pallidecaerulei.

663. C. ch. N. albi, paeoniformi albi. 664. C. ch. fusco-rubri.

665. C. ch. violacei.

666. Helichrysum bracteatum Andr, 667. Solidago canadensis L, 
668. Tagetes erectus L.

669. T. signatus Bartl.

670. Zinnia elegans L.

IV. SEMINA PLANTARUM OFFICINALIUM.

Liliaceae.

671. Asparagus officinalis $L$.

672. Allium schoenoprasum L.

Iridaceae.

673. Iris sibirica L.

Moraceae.

674. Cannabis sativa L.

Polygonaceae.

675. Rheum officinalis Bail.

676. R. undulatum L.

677. R. Rhaponticum L.

Chenopodiaceae.

678. Chenopodium ambrosioides L.

679. Atriplex hortensis L. v. rubra

Caryophyllaceae.

680. Lychnis chalcedonica $L$.

681. Saponaria officinalis $L$. 


\section{Ranunculaceae.}

682. Nigella arvensis L. 683. N. damascena L.

684. N. sativa L.

685. Ranunculus acer $\mathrm{L}$.

Papaveraceae.

686. Papaver dubium L.

687. P. rhoeas L.

688. P. somniferum L.

689. Chelidonium majus L.

Capparidaceae.

690. Cleome gigantea L.

Crassulaceae.

691. Sedum acre L.

Rosaceae.

692. Geum urbanum L.

693. Sanguisorba officinalis L.

Leguminosae.

694. Glycyrrhiza echinata L.

695. Melilotus officinalis $L$.

Malvaceae.

696. Althaea officinalis L. 
697. Althaea rosea Cav. v. nigra. 698. Malva alcea L.

Umbelliferae.

699. Anethum graveolens L.

700. Carum carvi L.

701. Conium maculatum L.

702. Coriandrum sativum $L$.

703. Eryngium planum L.

704. Levisticum officinale Koch.

705. Pastinaca sativa L.

Boraginaceae.

706. Alkanna tinctoria Tausch.

707. Anchusa itlica Retz.

708. Cynoglossum officinale L.

Labiatae.

709. Hyssopus officinalis L.

710. Lallemantia ihericum Fisch.

711. Lophantus anisatus Benth.

Solanaceae.

712. Atropa Belladonna L.

713. Datura inermis Jacq.

714. D. stromonium L. 
Scrophulariaceae.

715. Digitalis ambigua Murr.

716. D. ferruginea L.

717. D. lutea L.

718. D. purpurea L.

719. D. purpurea L. v. alba

720. D. sibirica L.

Plantaginaceae.

721. Plantago lanceolata L.

Valerianaceae.

722. Patrinia intermedia Roem. et Sch.

723. Valeriana nitida $\mathrm{Pr}$.

724. V. officinalis $\mathrm{L}$.

725. V. rossica Sm.

Compositae.

726. Achillea Millefolium L.

727. A. nobilis L.

728. Anacyclus officinarum Hayne

729. Artemisia absinthium L.

730. A. vulgaris L.

731. Bellis perennis L.

732. Calendula officinalis $L$.

733. Carduus marianus L. 
734. Chondrilla juncea L.

735. Cephalophora aromatica Schrad.

736. Grindelia robusta Nutt.

737. Helianthus annuus L.

738. Inula Helenium L.

739. Pyrethrum cinerariaefolium Trev.

740. P. roseum Lindl.

741. P. carneum Bieb.

742. Matricaria chamomilla L.

743. Tanacetum boreale L.

744. T. vulgare L.

745. Taraxacum parnassicum Dahelst.

746. T. stenoschistum Dahelst.

V. SEMINA PLANTARUM GRAMINEARUM ET LEGUMINOSARUM.

Gramineae.

747. Avena nuda L.

748. A. sativa v. aurea "Lochovski ".

749. A. s. v. a. "Stepniak".

750. A. s. v. a. "Zolotoj Dojd“.

751. A. s. v. a. "Amerikanskij".

752. A. s. v. a. "Kherson".

753. A. s. v. a. "Zwezda".

754. A. s. v. a. "Pflug". 
755. Avena s. v. a. „Povoljie 2131“. 756. A. s. v. a. "Zolotistij “.

757. A. s. v. a. ${ }_{n}$ Echo ${ }^{4}$.

758. A. s. patula brunnea Korn.

759. A. s. p. b. Brie.

760. A. s. v. aristata "Lygovo II".

761. A. s. v. a. "Miraj".

762. A. s. v. a. "Moskovskij A-315“.

763. A. s. v. a. "Jemtschujina“.

764. A. s. v. a. "Severianin".

765. A. s. v. Kasanensis "Polianka“.

766. A. s. v. k. "Marcton“.

767. A. s. v. mutica "Dippe".

768. A. s. v. m. "Albion".

769. A. s. v. m. "Aliaska ${ }^{\circ}$.

770. A. s. v. m. "Gretchen".

771. A. s. v. m. "Orel".

772. A. s. v. m. "Romania".

773. A. s. v. m. "Shatilovsky".

774. A. s. v. orientalis Mordv. v. pugnax

A. Black Tart.

775. A. s. v. o tristis Al.

776. A. s. "Adelaar".

777. A. s. "Bidenhafer".

778. A. s. "Golden Regen“.

779. A. s. "Orion".

780. A. s. v. nigra "Zwarte President". 
781. Avena s, v. volgensis.

782. A. strigosa Schreb.

783. Bromus arvensis L.

784. B. inermis Leyss.

785. Dactylis glomerata L.

786. Elymus sibiricus L.

787. Festuca ovina L.

788. F. rubra L.

789. Hordeum distichum L. v. abyssinicum Sch. 790. H. d. v. erectum Schubl.

791. H. d. v. medicum "Prima " Korn No. 26.

792. H. d. v. m. "Prima" No. 46.

793. H. d. v. nigrum

794. H. d. v. nudum „Byloje“.

795. H. d. v. n. No. 105.

796. H. d. v. n. No. 110.

797. H. d. v. nutans Schubel. Europeum

$$
313 / 133 \text {. }
$$

798. H. d. v. n. No. 17.

799. H. d. v. n. „Viner“.

800. H. d, v. n. Colchicum 10/78.

801. H. d. v. n. Hanna Loosdorffskaja.

802. H. vulgare L. v. astrachanense.

803 . H. v. v. caeleste No. 46.

804. H. v. v. nigrum Willd.

805. H. v. v. pallidum Ser. "Pioneer ${ }^{4}$. 806. H. v. v. p. "Tschervonez". 
807. Hordeum v. v. p. "Toulounskif". 808. H. v. v. p. Trebi.

809. H. v. v. p. "Viatsky 1009 “.

810. H. v. No. $1398 / 5$.

811. H. v. No. $13198 / 5$.

812. H. v. No $905 / 4$.

813. H. v. v. trifurcatum Schlecht.

814. H. v. Hasfordianum.

815. Lolium italicum R. Br.

816. L. perenne L.

817. Panicum miliaceum contractum Al. v.

aureum No. 01.

818. P. m. c. v. dacicum Korn No. 03.

819. P. m. c. sanguineum Al. No. 0853 .

820. P. m. c. Kazanskoje 506.

821. P. m. c. Voroneshskoje 0198.

822. P. m. c. Oropajevskoje 367.

823. P. m. c. Kazanskoje 01.

824. P. m. compactum Al. No. 19/27.

825. P. m. c. "Krasnokutskoje“.

826. P. m. c. No. 0775.

827. P. m. c. No. 0144 .

828. P. m. v. effusum Al. Toidenskoje 215. 829. P. m. v. e. subcoccineum Saratovskoje 146.

830. P. m. v. e. Voroneshskoje 215. 831. P. m. v. e. No. 0585. 
832. Panicum m. v. e. No. 2889. 833. Poa pratensis $L$.

834. Secale cereale L.

835. Triticum dicoccum Schubl.

836. Tr. d. v. noricum inflatum.

837. Tr. d. v. rufum.

838. $\mathrm{Tr}$. dicoccoides $\mathrm{Kr}$.

839. Tr. durum Desf. v. obscurum.

840. Tr. d. v. affine Körn No. 309.

841. Tr. d. v. coeruleum.

842. Tr. d. v. hordeiforme No. 04 .

843. Tr. d. v. h. No. 10.

844. Tr. d. v. h. No. 189.

845. Tr. d. v. h. No. 432.

846. Tr. d. v. h. No. 496.

847. Tr. d. v. h. No. 0675.

848. Tr. d. v. h. No. 694.

849. Tr. d. v.

850. Tr. d. v. libicum Körn.

851. Tr. d. v. leucuum $\mathrm{B} \Gamma-26$.

852. Tr. d. v. 1. $\mathrm{B} \Gamma-33$.

853. Tr. d. v. leocomelan Al.

854. Tr. d. v. melanopus Al. No. 069.

855. Tr. d. v. m. Al. No. 826.

856. Tr. d. v. m. Al. No. 886 . 857. Tr. d. v. Reichenbachii Kr. 858. Tr. monococcum L. (ex America) 
859. Triticum persicum Vav. v. fulginosum Zhuk.

860. Tr. p. v. stramineum Zhuk.

861. Tr. spelta L.

862. Tr. s. v. coeruleum.

863. Tr. vulgare Host. albidum Al. No. 0676. 864. Tr. v. alborubrum $\mathrm{Kr}$ Sarrubra.

865. Tr. v. a. Kr. „Sarrosa ${ }^{4}$.

866. Tr. v. caesina Al. No. 111.

867. Tr. v. v. Delfii Kr.

868. Tr. v. erythrospermum Kr. Durable.

869. Tr. v. v. e. "Kooperatorka“.

870. Tr. v. v. e. "Zarja“.

871. Tr. v. v. e. "Zemka“.

872. Tr. v. v. e. "Ukrainka".

873. Tr. v. v. e. "Moskovskaja".

874 . Tr. v. v. e. No. 1114.

875. Tr. v. v. e. "Charkovskaja " 496.

876. Tr. v. v. e. "Kazanskaja 016 ".

877. Tr. v. v. e. No. 118.

878. Tr. v. v. e. "Viatskaja 529 “.

879. Tr. v. v. ferrugineum Al. „Moskovskaja

$2411^{\prime \prime}$.

880. Tr. v. v. f. "Moskovskaja $2453^{4}$. 881. Tr. v. v. f. "Simbileewskaja 146 ". 882. Tr. v. v. f. No. 150. 883. Tr. v. v. f. "Engelgardt". 
884. Triticum v. v. f. "Sarmatka". 885. Tr. v. v. hostianum No. 237. 886. Tr, v. v. lutescens Al. No. 053. 887. Tr. v. v. 1. No. 479. 888. Tr. v. v. 1. No. 040. 889. Tr. v. v. 1. No. 062. 890. Tr. v. v. 1. No. 3221. 891 . Tr. v. v. 1. No. 323. 892. Tr. v. v. 1. No. 786. 893. Tr. v. v. 1. No. 9113. 894. Tr. v. v. 1. "Garnet". 895. Tr. v. v. I. "Leda". 896. Tr. v. v. 1. "Markiz". 897. Tr. v. v. 1. "Kitchener". 898. Tr. v. v. 1. "Beta stojkaja". 899. Tr. v. v. 1. "Viatskaja $05^{\circ}$. 900. Tr. v. v. 1. "Viatskaja 126 . 901. Tr. v. v. 1. No. 329. 902. Tr. v. v. 1. "Zolotaja 903. Tr. v. v. 1. No. 1060/10. 904. Tr. v. v. 1. "Teiskaja". 905. Tr. v. v. 1. $27 / 36$, 906. Tr. v. v. 1. $434 / 154$. 907. Tr. v. v. milturum Al. 908. Tr. v. v. m. Saratovskaja 129. 909. Tr. v. v. m. "Novinka". 910. Tr. v. v. m. No. 841 . 
911. Triticum v. v. m. No. $10 / 78$. 912. Tr. v. v. m. No. 321 .

\section{Leguminosae.}

913. Cicer arientinum L. v. Album hort. 914. Cicer ar. "Tschernij".

915. C. ar. v. rotundatum.

916. C. ar. No. 277.

917. C. ar. No. 77.

918. C. ar. No. 199.

919. Ervum lens L. v. macrosperma Bor. v. nummularia "Obyknovennaja“.

920. E. 1. v. m. "Saratovskaja $13^{*}$. 921. E. 1. v. m. "Saratovskaja $23^{4}$. 922. E. 1. v. m. "Saratovskaja 140 ". 923. E. 1. v. m. "Narjadnaja $03^{4}$. 924. E. 1. v. microsperma Bar. 925. E. 1. v. glaucosperma "Seraja $32 / 5^{4}$. 926. E. 1. v. macrosperma Du-pui. 927. E. culinaris Luna 09. 928. E. 1. (ex Italia). 929. E. 1. (ex Sicilia). 930. E. 1. (ex Syria). 931. E. 1. (ex India). 932. E. 1. (ex Francia). 933. E. 1. (ex Cabarda). 934. E. 1. (ex Aphghanistan). 
935. Lathyrus sativus L. No. 19/42. 936. L. s. No. 398.

937. L. s. "Gornaja Buchara".

938. L. s. No. 729.

939. L. s. No. 732.

940. Lathyrus s. No. $16 / 28$.

941. L. s. No. $829{ }_{\text {"Sibirj }}{ }^{a}$.

942. L. s. "Ukraina“.

943. L. S. "Azerbaidjan".

944. Lupinus albus $\mathrm{L}$.

945. L. a. $\mathrm{y}-26$.

946. L. angustifolius L. G. D. C.

947. L. a. No. 645.

948. L. a. No. 174.

949. L. a. No. 418.

950. L. luteus No. 4666.

951. L. 1. No. 345.

952. L. 1. No. 530.

953. L. polyphyllus L.

954. Phaseolus vulgaris L. No. 294.

955. P. v. No. 663.

956. P. v. No. 1198.

957. Pisum arvense L. "Peliushka $32^{*}$.

958. P. a. "Peliushka $40^{4}$.

959. P. a. "Peliushka $42 "$.

960. P. a. $9 / 376$.

961. P. sativum v. coronatum „Stambovij“. 
962. Pisum s. v. glaucospermum Al. Folger Heine.

963. P. s. v. g. "Moskovskij A-559".

964. P. s. V. g. "Victoria zelenaja".

965. P. s. v. saccharatum "Rannij Bismark". 966. P. s. v. s. "Konjek Gorbunok".

967. P. s. v. vulgatum "Ambrosia“.

968. P. s. v. v. "Victoria rosovaja“.

969. P. s. v. v. "Victoria Mandorffskaja".

970. P. S. v. V. "Capital".

971. P. s. No. 06.

972. P. s. No. T $25 \times 103$.

973. P. s. T-25.

974. P. s. "Irlandetz".

975. P. s. 047.

976. P. s. 087.

977. P. S. "Tom Thumb".

978. P. s. "Olivkovij Gibrid".

979. P. s. "Tschudo Kelvidonia".

980. P. S, "Albansky $06^{\star}$.

981. P. S. "Thomas Lakston".

982. P. s. "The Wonder of America ".

983. P. s. "Bismark No. $372^{\text {" }}$

984. P. s. "Urojainij".

985. Vicia Faba L.

986. V. F. v. minor.

987. $\mathrm{V}$. harbonensis L. 
988. Vicia sativa L. "Belaja 163". 989. V. s. v. durro. 990. V. s. "Archangelskaja".

991. V. s. v. leucosperma.

992. V. s. v. platysperma.

993. V. s. v. typica.

994. V. s. v. tricolor.

Prof. Dr. S. S. Stankov

Horti Director.

Ass, A. S.

Zhuravleva

Vice-Director.
Cand. M. L.

Stelmachovicz

Hortulanus doctus.

Docent. Cand. D. S. Averkiev (System.).

Cand. M. L. Stelmachovicz

(Dendrolog. et Decoran.).

Prof. Dr. L. V. Tichov

(Medicin, et Technik. Plantae). Ass. M. V. Alalykina (Pomolog.). 
Перечень семян, собранны х в 1941 г. и п редл агае мых в об мен Ботаническим садом Горьков ского Госуд а рственного Ун ив е рс ите та. ОГй, Горьковское Издательство, 1942 г. Изд. № 1495. Индекс $\mathrm{CX}-3$.

เู้

Редактор проф. С. С. Станков, техн. редактор Л. И. Немченко, корректор в. М. Плотникова. Сдано в набор 31/XII-41 г. Піолиисано к печ. 26/ I-42 г.

- Формат 82X108 е4. Тираж 400 экз. Объем: 0,375 бум. л., $1^{1} / 2$ печ. л., 1,25 уч.-изд. л. Зн. в печ. л. 31616.

5

МЦ 208.

की

Полиграф, г. Горький, ул. Фигнер, 32. Заказ 스 10057. 

Б E C П Л А T H O

ПЕРЕЧЕНЬ СЕМЯН,

СОБРАННЫХ В 1941 ГОДУ

И ПРЕДЛАГАЕМЫХ В ОБМЕН БОТАНИЧЕСКИМ САДОМ

ГОРЬКОВСКОГО ГОСУДАРСТВЕННОГО УНИВЕРСИТЕТА

\section{ОГи}

ГОРЬКОВСКОЕ ИЗДАТЕЛЬСТВО 1942 
Shmileit ths nead Ams MICMXL11

VIII Check with
DELECTUS SEMINUM
ANNO MCMXLII COLLECTORUM
QUAE
HORTUS BOTANICUS

waviener

UNIVERSITATIS GORKIJENSIS

PRO

MUTUA COMMUTATIONE

OFFERT

1943

GORKIJ M EMTIII

SUNAI BOTANIC 



$$
\text { - VIII }
$$

\section{DELECTUS SEMINUM}

ANNO MCAXLII COLLECTORUM

\section{QUAE}

\section{HORTUS BOTANICUS}

UNIVERSITATIS GORKIJENSIS PRO

MUTUA COMMUTATIONE

OFFERT 
Перечен семян, co6pa He

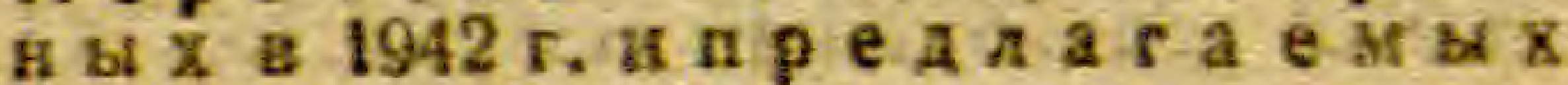

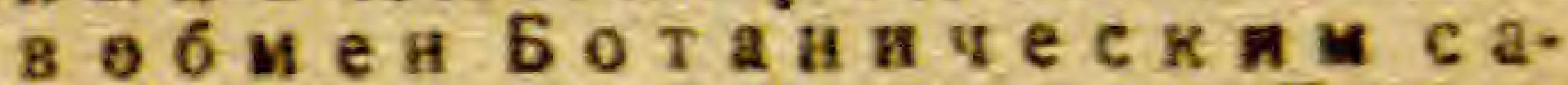

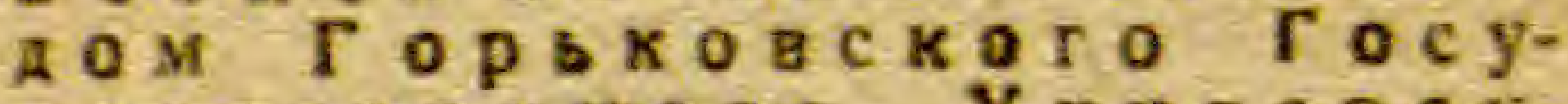

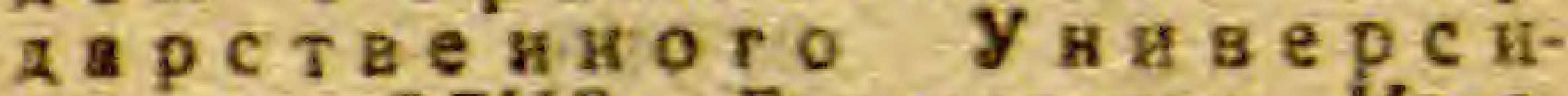
тета. огиз. Горьковское Издательство. 1943 r. Изд. Ne 1697. Нндекс $\mathrm{CX}-3$.

Peдaxtop npoф. C. C. Cmarkos.

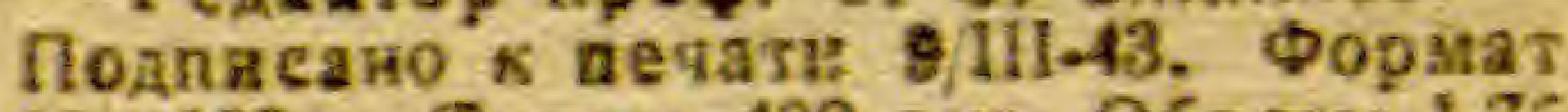
$82 \times 108$ e*. Tираж 400 экз. Объем: 1,75

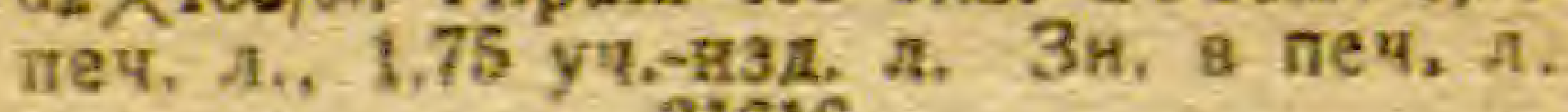
31616.

\author{
* \\ M4 09355. \\ क
}

Полиграф, г. Горькнй, ул. Фигнер, 32. 3axas aㅡ i. 
1. SEMINA PLANTARUM LIGNOSARUM ET FRUTICUM.

\section{Cen. Betulacede.}

1. Alnus sitchensis Sarg.

2. Betula Gmelini Bge.

3. B. verrucosa Ehrh.

Cен. Fagaceae.

4. Quercus pedunculata Ehrh.

Сем. Berberidaceae.

5. Berberis vulgaris $L$.

6. B. heteropoda Schrenk.

7. B. Thunbergil DC.

Ces. Saxifragaceae.

8. Deutzia parviflora Bge.

9. Hydrangea Bretschneideri Dipp.

10. Philadelphus coronarius $L$.

11. $\mathrm{Ph}$. pubescens Lois.

12. $\mathrm{Ph}$. satsumanus $\mathrm{Miq}$.

13. Ph. tenuifolius Rupr.

14. Ribes alpinum $L$. 
15. R. aureum Pursh.

16. R. Warscewiczii Jancz.

\section{Cen, Rosaceae.}

17. Amelanchier spicata K. Koch.

18. Amygdalus nana L.

19. Aronia melanocarpa Elliot,

20. Chaenomeles maulei Schneid. $v$, alpina Maxim.

21. Cotoneaster acutifolia Turcz.

22. Crataegus Maximowiczi Schneid,

23. Phisocarpus opulifolius Maxim.

24. Prunts pennsylvanica $L$.

25. P. pumila L.

26. P. virginiana $L$.

27. P. Bessey Bailey.

28. P. fruticosa Pail.

29. Pyrus baccata $E$.

30. Rosa cinnamonea $L$.

31. R. spinosissima $L$.

32. R. rubrifolia Vill.

33. R. rugosa typica Reg.

34. R. multiflora Thunb.

35. Rubus odoratus L.

36. Sibiraea laevigata Maxim.

37. Sorbus aucuparia L.

38. S. heterophylla Dur. 
39. Sorbaria assurgens Rehd.

40. Spiraea media Schmidt.

41. S. Douglasii Hook.

42. S. Menziesii Dougl.

43. S. chamaedryfolia L.

44. S. latifolia Borkh.

45. S. densiflora Nutt.

46. S , betulifolia Pall.

47. S. bumalda Burvenich V., "Antony

Waterer ${ }^{a}$.

48. S. japonica L. $\mathrm{f}$.

49. S. crenifolia C. A. Mey.

50. Spiraea salicifolia L.

51. S. alba Dur.

Cen. Leguminosae.

52. Amorpha californica Nutt.

53. A. fruticosa L.

54. Caragana arborescens L.

55. C. frutex DC.

56. Colutea arborescens L.

57. C. media Willd.

58. Cytisus albus Haeq.

59. Genista tinctoria L.

Cen. Rutaceae.

60. Ptelea trifoliata L. 


\section{Cem. Euphorbiaceae.}

61. Securinega ramillora Muell.

Сем. Anacardiaceae.

62. Cotinus coggigria Scop.

63. Rhus toxicodendron $\mathrm{L}$.

64. $\mathrm{Rh}$. typhina $\mathrm{L}$.

Cen. Celastraceae.

65. Evonymus verrucosa Scop.

Сем. Aceraceae.

66. Acer ginnala Maxim.

67. A. Negundo L.

68. A. platanoides L.

69. A. tataricum L.

Сем. Vitaceae.

70. Ampelopsis quinquefolia Planclr. Cen. Rhamnaceae.

71. Rhamnus cathartica L.

72. Ceanothus americanus $L$.

Сем. Tiliaceae.

73. Tilia cordata Mill.

Сен. Cornaceae.

74. Cornus alba L.

75. C. tatarica Mill. 


\section{Cen. Oleaceae.}

76. Syringa Josikaea Jacq.

77. S. villosa Vahl.

78. S. vulgaris L.

Ces. Caprifoliaceae.

79. Lonicera caprifolium L.

80. L. chrisantha Turcz.

81. L. dioeca L.

82. L. orientalis Lain.

83. L. Ruprechtiana Reg.

84. L. tatarica L.

85. L. xylosteum L.

86. Symphoricarpus occindentalis Hook. 87. S. racemosus Michx.

88. Viburmum opulus $\mathrm{L}$.

11. SEMINA PLANTARUM HERBACEUM COLLECTA 1942.

Gramineae.

89. Agropyrum acutum R. et $\$$, 90. A. desertorum Schult.

91. A. fragile Nevski.

92. A. junceum Beauw.

93. A pectiniforme Roem. et Schult. 
94. Agropyrum tenerum Vasey.

95. A, Turczaninowii Drob.

96. A. ugamicum Drob.

97. Agrostis borealis Hartm.

98. Alopecurus ventricosus Pers.

99. Arrhenatherum elatius $M$. et $K$.

100. Asperella hystrix Humb.

101. Avena alpina Suter.

102. A. planiculmis "Turcz.

103. A. pubescens Huds.

104. A. Schelliana Hack.

105. Bromus erectus Huds.

106. B. inermis Leyss.

107. Dactylis Aschersoniana Graebn.

108. D. glomerata L.

109. Deschampsia flexuosa Trin.

110. Elymus canadensis L.

111. E. dahuricus Turcz.

112. E. excelsus Turcz.

113. E, mollis Trin.

114. E. sabulosus $M$. B.

115. E. sibiricus L.

116. Festuca glauca Bess.

117. F. picta Kit.

118. F. polesica Zapal.

119. F, sulcata Hack.

120. F, vallesiaca Schl. 
121. Hordeum bulbosum $L$.

122. H, secalinum Schreb.

123. Koeleria crassipes Lange.

124. K. glauca D. C.

125. K. gracilis Pers.

126. Lolium perenne $\mathrm{L}$.

127. Melica ciliata L.

128. M. transsilvanica Schuhr.

129. Nardus stricta L.

130. Oryzopis holciformis (M. B.) Richt?

131. Phleum pratense L.

132. Phalaris arundinacea $\mathrm{L}$.

133. Poa alpina $L$.

134. P. compressa $L$.

135. P. nemoralis $\mathrm{L}$.

136. Roegneria canina (L.) Nevski.

137. R. tianschanica (Drob.) Nevski.

138. Sesleria Heufleriana Sch.

139. Stipa calamagrostis Wahlenb.

140. S. capillata L.

141. S. Joannis Celak.

142. S. Lessingiana Trin.

143. S. pulcherrima C. Koch.

144. S. ncrainica P. Smirn.

145. Triodia decumbens Pall.

146. Trisetum pratense Pers.

147. T. sibiricum Rupr. 


\section{Cyperaceae.}

148. Carex Amelli Christ.

149. C. cuprina Nendtv.

150. C. leporina L.

Liliaceae.

151. Allium angulosum $\mathrm{L}$.

152. A, fistulosum L.

153. A. giganteum Regel.

154. A. glaciale Vved.

155. A. globosum M. B.

I56. A. hymenorrhizum Ledeb.

157. A. lineare $\mathrm{L}$.

158. A. odorum $\mathrm{L}$.

159. A. schoenoprasum $L$.

160. A. sibiricum L.

161. Antheticum liliago L.

162. A, ramosum $L$.

163. Asparagus officinalis $L$.

164. A. tenuffolius Lam.

165. Eremurus altaicus Stev.

166. E. Olgae Regel.

167. Hemerocallis flava $L$.

168. Lilium concolor Salisb.

169. L. martagon L.

170. L. tenuifolium Fisch.

771. Muscarf bortyoides Mill. 
172. Muscari racemosutn (L.) Mill.

173. Paradisia liliastrum Bertol.

174. Scilla sibirica Andr.

Amaryllidaceáe.

175. Ixiolirion tataricum Pall.

Iridaceae.

176. Itis aphylla $\mathrm{L}$.

177. 1. halophilla Pall.

178. 1. Hookeri Penny.

179. 1. illirica Somm.

180. I. pumila L.

181. I, setosa Pall.

182. 1. sibirica $\mathbf{L}$.

183. I. sogdiana Bunge-

184. t. sulphurea Koch.

185. I. variegata $L$.

186. 1. versicolor $\mathrm{L}$.

187. Sisyrinchium anceps Cav.

Polygonaceae.

188. Polygonum polymorphum Ldb.

189. Rheum acuminatum Hook.

190. Rh, crassinervium Fisch.

191. Rh. Emodi Wall.

192. Ph, officinale Bail. 
193. Rheum palmatum L.

194. Rh. undulatum $\mathrm{L}$.

195. Rumex acetosa $\mathrm{L}$.

196. R. hydrolaphatum Huds.

197. R. hymenosepalus Torr.

198. R. Patientia L.

Chenopodiaceae.

199. Chenopodium Bonus-Henricus L.

Nyctaginaceae.

200. Oxybaphus nyctagineus Sweet-

Phytolaccaceae.

201. Phytolacca americana $\mathrm{L}_{\text {r }}$

Caryophyllaceae.

202. Arenaria longifolia $M, B$.

203. Cerastium alpinum $L$.

204. C. Biebersteinii D. C.

205. C. Lerschenfeldianum Scluhr.

206. Dianthus barbatus $\mathbf{L}$.

207. D. deitoides L.

208. Gypsophila paniculata L.

209. G. elegans M. B.

210. G. elegans M. B. v. grandiflorum.

211. Lychnis chalcedonica $\mathrm{L}$. 
212. Saponaria officinalis $L$.

213. Silene armeria L.

214. Viscaria alpina (L.) Don.

215, V. vulgaris Roehl.

\section{Ranunculaceae.}

216. Aconitum lasiostomum Rchb.

217. A. vulparia Rchb.

218. Anemone silvestris L.

219. A. virginiana $L$.

220. Aquilegia canadensis L.

221. A. flabellata Sieb. et Zucec.

222. Clematis integrifolia $L$.

223. C. manshurica Rupr.

224. C. recta L.

225. C. tangutica Korsh.

226. Delphinium elatum L.

227. D. grandiflorum L.

228. D. hybridum Willd.

229. Nigella sativa $L$.

230. Paeonia anomala L.

231. P. decora Anders.

232. Pulsatilla rubra Delarb.

233. Ranunculus acer L, ssp. Steveni Hegi.

234. R. bulbosus L.

235. R. polyanthemus L.

236. R. propinquus C. A. Mey. 
237. Thalictrum adianthifolium Bess.

238. Th. minus $\mathrm{L}$.

239. Trollius altaicus C. A. Mey.

240. T. laxus Salisb.

Papaveraceae.

241. Chelidonium laciniatum Mill.

242. Papaver lateritium C. Koch.

243. Papaver nudicaule L.

244. P. oreophyllum Rupr.

Cruciferae.

245. Alyssum argenteum All.

246. Aubrietia erubescens Griseb.

247. Bunias orientalis $L$.

248. Iberis gibraltarica L.

249. I. odorata L.

250. Isatis alpina All.

251. Sisymbrium strictissimum L.

252. Thlaspi densiflorum Boiss. et $K y$. Resedaceae.

253. Reseda lutea L.

Crassulaceae.

254. Sedum aizoon L.

255. S. Ewersii Ledeb.

256. S. fabaria Koch. 
257. Seđum kamtschaticum Fisch, et Mey. 258. S. Maximowiczii Regel.

259. S. spurium Bieb.

260. Sempervivum tectorum $\mathrm{L}$.

Saxifragaceae.

261. Bergenia crassifolia L.

262. B. pacifica Kom.

263. Heuchera americana L.

264. H. cylindrica Dougl.

265. Heuchera hispida Pursh.

266. Mitella caulescens Nutt.

267. Saxifraga altissima Kerner.

Rosaceae.

268. Agrimonia odorata Mill.

269. Aruncus silvester Kostel.

270. Filipendula hexapetala Gilib.

271. F. Ulmaria (L.) Maxim.

272. Potentilla argentea $L$.

273. P. atrosanguinea Lodd.

274. P. gelida C. A. Mey.

275. P. geoides Bieb.

276. P, hybrida hort.

277. P. nivea L. v. elongata.

278. P. rupestris L.

279. Sangisorba officinalis L. 


\section{Leguminosae.}

280. Anthyllis variegata hort.

281. Astragalus alopecurus Pall.

282. A, cicer L.

283. A, falcatus Lam.

284. A. galegiformis L.

285. A. glycyphyllos L.

286. A. uliginosus $\mathrm{L}$.

287. Desmodium canadense D. C.

288. Galega orientalis Lam.

289. Glycyrrhiza echinata L.

290. Hedysarum sibiricum Poir.

291. Lathyrus latifolius $\mathrm{L}$.

292. L. niger L.

293. L. odoratus L.

294. L. silvestris L.

295. Lupinus elegans $H$. B. et $K$.

296. L. polyphyllus Lindl.

297. Lotus corniculatus L.

298. Medicago faicata $L$.

299. M. sativa L.

300. Melilotus albus Desr.

301. Onobrychis arenaria D. C.

302. O. montana Lam, et D. C.

303. O. viciaefolia Scop.

304. Ononis spinosa L.

305. Scorpiurus vermiculata L.

16. 
306. Thermopsis caroliniana M. A. Curt. 307. Th. fabacea D. C.

308. Trifolium alpestre $L$.

309. T. incarnatum L.

310. T. lupinaster $L$.

311. T. medium L.

312. Vicia multicaulis Led.

313. V. pisiformis L.

\section{Geraniaceae.}

314. Geranium albiforum Hook.

315. G. pratense L.

Linaceae.

316. Linum flavum $L$.

317. L, grandiflorum Desf.

318. $\mathrm{L}$. perenne $\mathrm{L}$.

Rutaceae.

319. Dictamnus fraxinella Pers, 320. Ruta graveolens L:

\section{Malvaceaè.}

321. Altliae a ficifolia Cav.

322. A, nudiflora Lindl.

323. A. officinalis $\mathrm{L}$.

324. A, rosea Cav. 
325. Althaea sulphurea Boiss.

326. Kitaibelia vitifolia Willd.

327. Lavatera thuringiaca $L$.

328. Malva alcea $L$.

329. M. moschata L.

330. Sidalcea candida A. Gr.

331. S. malvaeflora A. Gir.

332. S. neo-mexicana A. Gr.

Hypericaceae.

333. Hypericum ascyron L.

334. $\mathrm{H}$. perforatum $\mathrm{L}$.

335. H. quadrangulum L.

Lythraceae.

336, Lythrum salicaria L.

Oenotheraceae.

337. Clarkia elegans Dougl.

Umbelliferae.

338. Angelica brevicaulis B. Fedtsch.

339. A, Kamtschatica Rupr.

340. Archangelica officinalis Hoffm.

341. Astrantia carniolica Wulf.

342. Buplearum aureum Fisch.

343. Eryngium alpinum $L$. 
344. Eryngium planum L.

345. Heracleum dissectum Ledeb.

346. H. sibiricum $L$.

347. H. villosum Fisch,

348. Laserpitium latifolium L.

349. Libanotis montana All.

350. Pleurospermum Golaka Rchb.

351. Seseli osseum Crantz.

352. Siler aquilegifolium Gaertn.

\section{Primulaceae.}

353. Lysimachia punctata L.

354. L. verticillata $M$. B.

355. Primula algida Adams.

\section{Plumbaginaceae.}

356. Armeria caespitosa Boiss.

357. A. leucocephala Salzm.

358. A. plantaginea Willd.

359. A. sibirica Turez.

360. Statice Gmelini Willd.

Gentianaceae.

361. Gentiana cruciata L.

362. G. Kirilowii Turcz.

363. G. phlogifolia Schot. et Kotsehy.

364. G. tianschanica Rupr. 


\section{Asclepiadaceae.}

365. Vincetoxicum officinale Moench.

Convolvulaceae.

366. Calystegia sepium (L.) R. Br.

Polemoniaceae.

367. Gilia capitata Dougl,

Hydrophyllaceae.

368. Nemophila insignis Dougl.

369. Phacelia congesta Hook.

370. Ph. loasaefolia Torr.

Boraginaceae.

371. Anchusa officinalis L.

372. Echium rubrum Jacq.

373. Mertensia sibirica G. Don.

Labiatae.

374. Ballota nigra L.

375. Brunella grandiflora Jacq.

376. Dracocephalum altaiense Laxm.

377. D, nutans L.

378. D. Ruyschiana L.

379. Horminum pyrenaicum L.

380. Hyssopus officinalis $\mathrm{L}$.

381. Lallemantia peltata Fisch. et Mey.

382. Leonurus cardiaca $\mathrm{L}$. 
383. Lophanthus anisatus Benth. 384. Nepeta cataria L. v. citriodora.

385. N. grandiflora M. B.

386. N. Kokanica Rgl.

387. N. macrantha Fisch.

388. N. nuda $L$.

389. Origanum vulgare $L$.

390. Phlomis tuberosa L.

391. Salvia aethiopis L.

392. S. limbata C. A. M.

393. S. nemorosa L.

394. S. stepposa Des-Chost.

395. S. verticillata $L$.

396. Scutellaria albida L.

397. S. altissima L.

398. Sideritis hyssopifolia L.

399. S, scordioides L.

400. Stachys germanica L.

401. S. grandiflora Benth.

402. S. lanata Jacq.

403. S. officinalis (L.) Trev.

404. S. setifera C. A. Mey.

405. Thymus glaber Mill.

406. Th. Marschallianus Willd.

- Solanaceae.

407. Atropa Belladonna L. 
408. Physalis Franchettii Mast. 409. Physochlaena orientalis G. Dots. 410. Solanum Dulcamara L.

\section{Scrophulariaceae.}

411. Antirrhinum Coulterianum Benth.

412. Digitalis ambigua Murr.

413. D. lutea L.

414. D. media Roth.

415. D. sibirica $L$.

416. Linaria triphylla Mill.

417. Pentstemon glaber Pursh.

418. P. hirsutus Willd.

419. P. diffusus Dougl.

420. P. procerus Dougl.

421. Verbascum orientale $M$. B.

422. Veronica fruticans Jacq.

423. V. gentianoides Vahl.

424. V. incana $L$.

425. V. longifolia L.

426. V. orchidea Crantz.

427. V. spuria L.

428. Wulfenia carinthiaca Jacq.

Bignoniaceae.

429. Incarvillea compacta Max. 
Globulariaceae.

430. Globularia Willkommi Nym. Plantaginaceae.

431. Plantago alpina $L$.

432. P. lanceolata L.

433. P. purpurascens Willk.

Rubiaceae.

434. Asperula tinctoria L.

435. Galium verum $\mathrm{L}$.

Valerianaceae.

436. Valeriana hispida Turcz.

437. V. rossica P. Sm.

Dipsacaceae.

438. Cephalaria gigantea E. Bobr. 439. C. procera Fisch. 440. Scabiosa australis Wulf.

441. S. graminifolia L.

442. Succisa praemorsa Aschers.

Cucurbitaceae.

443. Bryonia alba $L$.

Campanulaceae.

444. Adenophora communis Fisch. 445. A. verticillata Fisch. 
446. Campanula alliaridefolia Willd.

447. C. carpathica Jacq.

448. C. persicifolia-L.

449. C. sarmatica Ker.-Gawl.

450. C. Steveni Bieb.

451. Codonopsis clematidea Clatke.

452. C. ovata Benth.

453. Jasione perennis L.

454. Phyteuma Scheuchzeri All.

455. Platycodon grandiflorum D. C.

Compositae.

456. Achillea asplenifolia Vent.

457. A. cartilaginea Led.

458. A. filipendulina Lam.

459. A. millefolium L.

460, A. nobilis L.

461. A. sibirica Ledeb.

462. Alfredia cernua Cass.

463. Anaphalis margaritacea Benth.et Hook.

464. Antennaria nevadensis Greene.

465. Anthemis cotula L.

466. A. tinctoria L.

467. Arnica chamissonis Less.

468. A. foliosa Nutt.

469. A. montana L.

470. A. sachalinensis A. Gray. 
471. Artemisia dracunculus $L$.

472. Aster incisus Fisch.

473. A, longifolius Lam.

474. A. paniculatus Lam.

475. A. salignus Willd.

476. A. umbellatus Mill.

477. Bellis mexicana A. Gray.

478. Buphtalmum salicifolium $L$.

479. B. speciosum Schreb.

480. Cacalia aconitifolia Bge.

481. C. hastata L.

482. Carduus hamulosus Ehrh.

483. Centaurea dealbata Willd.

484. C. leucophylla M. B.

485. C. macrocephala Puschk.

486. C. nigra L.

487. C. scabiosa L.

488. Chrysanthemum corymbosum L.

489. Ch. maximum Ramond.

490. Coreopsis lanceolata L.

491. Cosmos bipinnatus Cav.

492. Dimorphotheca annua Less.

493. Doronicum macrophyllum Bernh.

494. D. orientale Hoffm.

495. Exigeron asper Nutt.

496. E. speciosus D. C.

497. Eupatorium purpureum L. 
498. Gaillardia aristata Pursh.

499. Helianthella quinquenervis A. Gr.

500. Helichrysum arenarium (L.) Moench.

501. H. lanatum D. C.

502. Heliopsis helianthoides Sweet.

503. H. scabra Dun.

504. Inula helenium L.

505. Layia platyglossa A. Gray.

506. Leontodon hispidus, $L$.

507. Leontopodium alpinum Cass.

508. Liatris spicata Willd.

509. Madia sativa Don.

510. Ptarmica impatiens D. C.

511. Pyrethrum carneum Bieb.

512. P. cinerarifolium Trev.

513. P. parthenifolium Willd.

514. P. roseum Lindl.

515. Rudbeckia laciniata L.

516. R. occidentalis Nutt.

517. Senecio altaicus $M$. B.

518. S. Doria L.

519. Silphium perfoliatum L.

520. Silybum Marianum Gaertn.

521. Solidago canadensis $L$.

522. S. multiradiata Ait.

523. S. rigida L.

524. S. Shortil Torr. 
525. Tanacetum vuigare $L$.

526. Troximon gracilens Gray.

$$
\text { COLLECTA } 1941
$$

Gramineae.

527. Corynephorus canescens Beatw. 528. Elymus akmolensis Drob. 529. Stipa stenophylla Czern. 530. Stipa viridula Trin.

\section{Liliaceae.}

531. Allium caesium Schrenk. 532. Galtonia candicans Decne. 533. Ornithogalum umbellatum $\mathrm{L}$. Poligonacede.

534. Rumex callosus Reidr. Caryophyllaceae.

535. Arenaria graminifolia Schrad. 536. Lychnis coronaria Desr. 537. Saponaria caespitosa D. C. 538. Silene tenuis Willd.

\section{Ranunculaceae.}

539. Anemone hudsoniana Richards. 
540. Aquilegfa oly mpica Boiss.

541. Nigella arvensis $\mathcal{L}$.

542. N. damascena L.

\section{Papaveraceae.}

543. Papaver orientale L.

Cruciferae.

544. Aethionema Buxbaumii D. C.

545. Alyssum desertorum Stapt.

546. Arabis albida Stev.

547. A. alpina L.

548. A, brevifolia D. C.

549. A. sicula Huet.

550. Aubrietia libanotica Boiss, et Hohen.

551. Biscutella laevigata $L$.

552. Carrichtera vella D. C.

553. Draba repens $M$. B.

554. Hesperis matronalis $L$.

555. Iberis intermedia Guers.

556 . Isatis tinctoria L.

557. Malcolmia matitima R. Br.

558. Roripa silvestris (L.) Bess.

Crassulaceae.

559. Sedum album $L$.

560 . S. rupestre L. 
Rosaceae.

561. Fragaria indica Andr.

562. F. vesca $L$.

563. Geum coccineum Sibth. et Snts

564. Potentilla Meyeri Boiss.

565. Poterium sitchense $\mathrm{S}$. Wats.

\section{Leguminosae.}

566. Anthyllis Boissieri Sag.

567. Astragalus danicus Retz.

568. A. onobrychis $L$.

569. A. sulcatus L.

570. Medicago hispida Gartn.

571. M. radiata L.

572. M. scutellaria Mill.

573. Oxytropis campestris D. C.

574. Securigera coronilia $D$. C.

575. Tetragonolobus purpureus Moench.

576. Trigonellá coerulea Ser.

\section{Malvaceae.}

577. Malachra radiata $\mathrm{L}$.

578. Palana flexuosa Mast.

Cistaceae.

579. Cistus symphitifolius Lam. 


\section{Oenotheraceae.}

580. Epilobium hirsutum L.

Primulaceae.

581. Androsace filiformis Retz.

Polemoniaceae.

582. Collomia coccinea Lehm.

583. Polemonium coeruleum L.

584. P. Van. Bruntiae Britt.

\section{Hydrophyllaceae.}

585. Romanzoffia sitchensis Bong.

\section{Boraginaceae.}

586. Cerinthe minor L.

587. Symphytum asperum Lepech.

Labiatae.

588. Monarua fistulosa L.

589. Salvia silvestris L.

590. Ziziphora capitata L.

Serophulariaceae.

591. Digitalis purpurea L.

592. Linaria bipartita Willd.

593. L. Vulgaris Mili.

594. Schizanthus pinnatus Ruiz. et Pay. 595. Veronica candida Lodd. 
Bignoniaceae.

596. Incarvillea grandiffora Bur. et Franch. 597. I. Oigae Regel.

Valerianaceae.

598. Patrinia rupestris Juss.

599. Valerianella coronata D. C.

Dipsacaceae.

600. Scabiosa micrantha Desf.

Campanutacene.

601. Jasione humilis Lois.

Compositae.

602. Achillea tomentosa L.

603. Calendula aegyptica Desf.

604. C. algeriensis Boiss. et Bent.

605. C. stellata Cav. .

606. Carthamus tinctorius $L$.

607. Dimorphotheea aurantiaca D. C.

608. D. pluvialis Moench.

609. Helichrysum bracteatum Willd.

610. Hieracium pilosella L.

611. Scorzonera purpurea $L$.

612. Tanacetum huronense Nutt. 


\section{COLLE T A 1940}

\section{Gramineae.}

613. Andropogon ischaemum L.

614. Bromus unioloides Humb.

615. Cynosurus echinatus L.

616. Lolium canadense Michx.

617. Setaria viridis P. B.

Liliaceae.

618. Allium albidum Kunth.

619. A. altaicum Pall.

620. A. galanthum Kar, et Kir.

621. A. paradoxum G. Don.

622. A. pulchellum G. Gon.

623. A. tataricum L.

\section{Urticaceae.}

624. Urtica cannabina L.

\section{Polygonaceae.}

625. Oxyria digyna (L.) Hill.

626. Rumex mexicanus Meiss.

627. R. pallidus Bigel.

Chenopodiacede.

628. Chenopodium aristatum L. 629. Ch. botrys $\mathrm{L}$. 
Amaranthaceae.

630. Amaranthus aurea T. A. Desf. 631. A, caudatus L. f. atropurpureus.

632. A. gangeticus $L$.

Caryophyllaceae.

633. Agrostemma Githago L.

634. Arenaria sajanensis Willd.

635. Cucubalus baccifer L. v. japonicus.

636. Dianthus carthusianosum L.

637. D. Hoeitzeri Winkl.

638. D. praecox Willd.

639. Gypsophila altissima $L$.

640. G. fastigiata L.

641. Heliosperma alpestre (Jacd.) Rchb.

642. Melandrium album (Mill.) Garcke.

643. Silene alpina $L$.

644. S. Bergiana Lindm.

645. S. gallica L.

646. S. muscipula L.

647. S. venosa Aschers.

648. Vaccaria pyramidata Med.

Ranunculaceae.

649. Anemone decapetala L.

650. A. multifida Poir.

651. A. riparia Fernold. 
652. Thalictrum flexuosum Bernh. 653. Th. rugosum Ait.
Papaveraceae.

654. Chelidonium Franchettianum Prain. 655. Papaver pyrenaicum A. Kern. ssp. raeticum Fedde.

656. P. somniferum $L$.

\section{Cruciferae.}

657. Alyssum sinuatum L. 658. Camelina dentata Wallr.

659. Crambe hispanica $L$. 660. Iberis pectinata Boiss.

Crassulaceae.

661. Sedum anopetalum D. C.

662. S, carpaticum Reuss.

663. S. crassipes Wall.

664. S. Kirillowii Rgl.

Saxifragaceae.

665 . Heuchera glabra Willd. 666. H. pilosissima Fisch. et Mey,

Rosaceae.

667. Geum aleppicum Jacq. 
668. Geum hispidum Fries.

669. G. virginianum $\mathrm{L}$.

670. Potentilla intermedia $\mathrm{L}$.

Leguminosae.

671. Astragalus baeticus L.

672. Baptisia australis R. Br.

673. Cicer arietinum L.

674. Lotus ornithopodioides L.

675. Ornithopus sativus Brot.

676. Trifolium arvense $L$.

677. Vicia americana Muhl.

678. V. ervilia (L.) Willd.

Geraniaceae.

679. Erodium cicutarium l'Herit.

Linacede.

680. Linum perenne L. v. sibiricum D. C. Malvaceae.

681. Anoda hastata Lav.

682. Malva crispa $L$.

683. M. pusilla With.

684. $M$. verticillata $L$.

685. Sidalcea spicata Greene. 


\section{Cistaceae.}

686. Helianthemum ovatum Dun. 687. H. vineale Pers.

Denotheraceae.

688. Oenothera fruticosa L.

Umbelliferae.

689. Coriandrum sativum $\mathrm{L}$.

690. Peucedanum alsaticum $\mathrm{L}$.

691. Pimpinella majus Huds.

Plumbaginaceae.

692. Armeria aretica Wall.

Apocynaceae.

693. Amsonia Tabernaemontana Walt.

Boraginaceae.

694. Eritrichum strictum-iort.

Labiatae.

695. Stachys nivea Labill.

\section{Solanaceae.}

696. Datura aegyptiaca Vesl. 697. Nicandra physaloides Gaertn. 


\section{Scrophulariaceae.}

698. Digitalis nevadensis Kunze.

699. Linaria purpurea Mill.

700. Verbascum Wilsonii hort.

Globulariaceae.

701. Globularia vulgaris L.

Rubiaceae.

702. Asperula ciliata Rochel.

703. Galium saccharatum All.

704. Ophiorrhiza Mungos L.

Dipsacaceae.

705. Dipsacus ferox Loisel.

706. Scabiosa Olivieri Coult.

Campanulaceae.

707. Campanula imeretina Rupr.

Compositae.

708. Achillea pseudopectinata Janka.

709. Anthemis candidissima Willd.

710. A. ruthenica Bieb.

711. Bidens dahlioides Wats.

712. Calendula arvensis $L$.

713. C. officinalis L. "Radio" hort.

714. Centaurea ossica C. Koch.

715. Cnicus benedictus L. 
716. Hieracium robustum Fr.

717. Jurinea arachnoidea Bge.

718. Lactuca scariola L.

719. Serratula coronata $L$.

720. S. Gmelini Ledeb.

IiI. SEMINA PLANTARUM DECORANTIUM.

Iridaceae.

721. Gladiolus hibridus hort. (bulbi).

722. G. primulinus Bok. (bulbi).

Portulacaceae.

723. Portulaca grandiflora Hook. Papaveraceae.

724. Eschscholtzia californica Cham.

725. Papaver somniferum L. f. paeoniflorum hort.

Caryophyllaceae.

726. Dianthus barbatus L.

727. Gypsophila paniculata Linn.

Resedaceae.

728. Reseda alba L. 
Leguminosae.

729. Lathyrus odoratus L.

730. Phaseolus multiflorus $L$.

Tropaeolociae.

731. Tropaeolum majus $\mathrm{L}$.

Violaceae.

732. Viola tricolor maxima hort.

Begoniaceae.

733. Begonia semperflorens Link. et Otto. Primulaceae.

734. Primula obconica Hauce.

Convoloulaceae.

735. Ipomea purpurea Roth.

Polemoniaceae.

736. Phlox Drummondi Hook,

Solanaceae.

737. Nicotiana affinis T. Moore.

738. N. rustica L.

739. N. wigandioides hort.

740. Petunia hybrida hort.

Scrophulariaceae.

741. Antirrhinum majus L. fi, alba hort. 
742. Antirrhinum majus "Primadonna "hort. 743. A, m. "Tschernyi Platsch" hort. 744. Nemesia strumosa Benth. grandiflora hort.

\section{Campanulaceae.}

745. Lobelia erinus L.

Compositae.

746. Ageratum conyzoides L. v. mexicanum hort.

747. Callistephus chinensis Ness. „Triumph" "Krassa SSSR".

748. C. ch. N. "Triumph" lylovyie.

749. C. ch. N. nana karmazynnyie.

750. C. ch. N. "Straussovoje Pero ${ }^{4}$ sanguin. rubri.

751. C. ch. N. "Straussovoje Pero argent. roseo.

752. C. ch. N. "Straussovoje Pero" coerulei. 753. C. ch. N, "Straussovoje Pero" fioletovije 754. C. ch. N. "American Beauty" pallidicoerulei.

755. C. ch. N. paeoniformis albi.

756. C. ch. N. paeoniformis vishnevije. 757. Pyrethrum parthenifolium Willd. $y$. aureum hort. 
758. Solidago canadensis L.

759. Tagetes erectus $L$.

760. T. signatus Bartl. pumila hort.

761. T. patula, nana hort.

IV. SEMINA PLANTARUM OFFICINALIUM.

Liliaceae.

762. Asparagus officinalis L.

763. Allium schoenoprasum L.

Iridaceae.

764. Iris sibirica L.

Moraceae.

765. Cannabis sativa $L$.

Polygonaceae.

766. Rheum officinalis Bail.

767. R. undulatum L.

768. R. rhaponticum L.

Caryophillaceae.

769. Saponaria officinalis $\mathrm{L}$. 


\section{Ranunculaceae.}

770. Nigella arvensis L.

771 . N. damascena L.

772. N. sativa L.

773. Ranunculus acer L.

\section{Papaveraceae.}

774. Papaver dubium L.

775 , P. rhoeas L.

776. P. somniferum L.

777. Chelidonium majus L.

Crassulaceae.

778. Sedum acre L.

Rosaceae.

779. Geum urbanum L.

Malvaceae.

780. Althaea officinalis $L$.

781. A, rosea Cav. v. nigra,

782. Malva alcea L.

Umbelliferae.

783. Anethum graveolens L.

784. Conium maculatum L.

785. Coriandrum sativum $L$.

786. Erynginum planum L. 
787. Levisticum officinale Koch. 788. Pastinaca sativa L.

\section{Boraginaceae.}

789. Alkanna tinctoria Tausch.

790. Anchusa italica Ritz.

791. Cynoglossum officinale L.

Labiatae.

792. Hyssopus officinalis $L$.

793. Lallemantia ibericum Fisch.

Solanaceae.

794. Datura inermis Jacq.

795. D. stramonium L.

Scrophulariaceae.

796. Digitalis sibirica L.

Valerianaceae.

797. Valeriana officinalis $L$.

Compositae.

798. Chondrilla juncea L.

799. Helianthus annuus L.

800. Pyrethrum cinerariaefolium Trev.

801. P. roseum Lindl. 
802. Pyrethrum carnetum Bieb.

803. Matricaria chamomilla L.

804. Tanacetum boreale L.

805. T, vulgare $\mathrm{L}$.

V. SEMINA PLANTARUM OLEARUM ET TECHNICARUM.

\section{Moraceae.}

806. Cannabis sativa $L$.

Polygonaceae.

807. Rumex acetosa L.

808. R. confertus E.

Cruciferae.

809. Brassica juncea Czern.

810. B. nigra Koch.

811. Crambe hispanica L.

812. Sinapis alba $L$.

Resedaceae.

813. Reseda luteola L.

\section{Malvaceae.}

814. Abutilon avicennae Gaertn. No. 45372 . 815. A. a, No. 8094 . 
816. Malva crispa $\mathrm{L}$.

817. $M$. verticilata $L$.

818. Sida napaea Cav.

Labiatae.

819. Lallemantia iberica Fisch. et Mey-

\section{Compositae.}

820. Cichorium intybus $\mathrm{L}$.

821. Helianthus annutus L. No. 1975.

822. H. a. No. 4036.

823. Madia sativa Molin.

Umbelliferae.

824. Coriandrum sat, v. giganteum.

VI. SEMINA PLANTARUM GRAMINEARUM ET LEGUMINOSARUM.

\section{Gramineae.}

825. Avena nuda $\mathrm{L}$.

826. A. sativa L. v. aurea , Lochoyski'. 827. A. s. v. a. ,Stepniak".

828. A. s. v. a. Zolotoj Dojd . 829. A. s. v. a. "Amerikanskij". 
830. Avena s. v. a. "Kherson". 831. A. s. v. a. "Pflug". 832. A. s. v. a. "Povoljie 2131". 833 . A. s. v. a. "Echo". 834. A. s. patula brunnea Korn. 835. A. s. p. b. Brie. 836. A. s. v. aristata "Lygovo 11 ". 837. A. s. v. a. "Miraj ". 838. A. s. v. a. "Moskovskij A-315". 839. A. s. v. a. "Jemtschujina". 840. A. s. v. a. ${ }_{n}$ Severianin ${ }^{*}$. 841. A. s. v. mutica "Dippe". 842. A. S. v. m. "Romania". 843. A. s. v. m. "Shatilovsky ". 844. A. strigosa Schreb. 845. Bromus arvensis $L$. 846. B. inermis Leyss. 847. Dactylis glomerata L. 818. Elynits sibiricus L. 849. Festuca avina L. 850. F. rubra L.

851. Hordeum distichum L. จ. abyssintcutn Sch.

852. H. d. v. erectum Schub1. 853. H. d. v. medicum "Prima* Korn No. 26.

854. H. d. v. m. "Prima ${ }^{4}$ No. 46. 
855. Hordeum dstichum v, nigrum. 856. H. d. V. nudum ,Byloje". 857. H. d. v. n. No. 105. 858. H. d. v. n. No. 110.

859. H. d. v. nutans Schubl. Europeum $313 / 133$.

860. H. d. v. n. No. 17. 861. H. d. V. n. "Viner". 862. H. d. v. n. Colchicum $10 / 78$. 863. H. d. v. nutans Hanna Loosdorfskaja864. H. vulgare L. v. astrachanense. 865. H. v. V. caeleste No. 46. 866 . H. v. v. nigrtum Willd. 867. H. v. v. pallidum Ser. "Pioner". 868. H. v. v. p. "Tschervonez". 869. H. v. v. p. "Toulounsky". 870. H. v. v. p. Trebi.

871. H. v. v. p. "Viatsky $1009 \%$.

872. H. v. No. $13198 / 5$.

873. H. v. No. $905 / 4$.

874 . H. v. v. trifurcatum Schlecht.

875 . H. v. Hasfordianunt.

876. Lotium italicum R. Br.

877. L. perenne L.

878. Panicum miliaceum $L$, contraclam

Kazanskoje 506.

879. P. m. c. Voroneshskoje 0198. 
880. Panicum in. c. Oropajevskoje 367. 881. P. m, compactum Al. No. $19 / 27$. 882. P. m. c. No. 0144 .

883. P. m. v. effusum Ai. Tordenskoje $-215$.

884. P. m. v. e. subcoccineum Saratovskoje 146 .

885. P. m. v. e, Voroneshskoje 215. 886. P. m. v. e. No. 0585 .

887. Poa pratensis $L$.

888. Secale cereale L.

889. Triticum dicoccum Schubł.

890. T. d. v. noricum inflatum.

891. T. d. v.rufum.

892. T. dicoccoides $\mathrm{Kr}$.

893. T. durum Desf. v. obscurum.

894. T. d. v. affine Korn, No. 309.

895. T. d. v. coeruleum.

896. T. d. v. hordiitorme No. 70.

897. T. d. v. h. No. 189.

898. T. d. v. h. No. 496 ,

899. T. d. v. No. 694.

900 . T. d. v. libicum Korn.

901. T. d, v, leucurum BГ-26.

902. T. d. v. 1. BГ- -33 .

903, T. d. v, leucomelan Al.

904. T. d. v. melanopus A1. No. 069. 
905. Triticum d. v, m. No, 826 . 906. T. d. v. m. No. 886. 907. T. d. v. Reichenbachii Kr. 908. T. monococcum L. (ex Amerika). 999. T., persicum Vav. v. fulginosum Zhuk. 910. T. spelta L.

911. T. s. v. coeruleum.

912. T. vulgare Host. albidum Al. No. 0676 913. T. v. alborubrum $\mathrm{Kr}$. Sarrubra.

914. T. v. a. Kr. "Sarrosa“.

915. T. v. caesina Al. No. 111.

916. T. v. v. Delfii Kr.

917. T. v. erythrospermum Kr. Durable.

918. T. v. v. e. "Kooperatorka".

919. T. v. v. e. "Zarja".

920. T. v. v. e. "Zemka".

921. T. v. v. e. "Ukrainka“.

922. T. v. v. e. "Moskovskaja".

923. T. v, v. e. No. 1114.

924. T. v. v. e. "Viatskaja 529 ".

925. T. v. v. ferrugineum A1. „Moskovskaja $2411^{4}$.

926. T. v. v. f. "Moskovskaja $2453^{\text {". }}$ 927. T. v. v. f. Simbileewskaja $146^{\circ}$.

928. T. v. v. f. No. 150. 929. T. v. v. f. "Engelgardt". 930. T. v. v. f. "Sarmatka". 
931. Triticum v. v. hostiantm No. 237 . 932. T. v. v. lutescens Al. No, 053. 933. T. v. v. 1. No. 479. 934. T. v. v. I. No, 040 . 935. T. v. v. I. No. 062. 936. T. v. v. 1. No. 3221. 937. T. v. v. 1. No. 323 . 938. T. v. v. 1. No. 786 . 939. T. v. v. I. No. 9113. 940. T. v. V. 1. "Garnet". 941. T. v. v. I. "Leda ${ }^{\text {. }}$ 942. T. v. v. 1. "Markiz". 943. T. v. v. 1. "Kitchener". 944. T. v. v. 1. "Beta stojkaja". 945. T. v. v. I. "Viatskaja $05^{4}$. 946. T. v. v. I. "Viatskaja $126^{\circ}$. 947. T. v. v. 1. No. 329. 948. T. v. v. 1. "Zolotaja". 949. T. v. v. I. No. $1060 / 10$. 950. T, v. v. L. "Teiscaja". 951. T. v. v. 1. $27 / 36$. 952 , T. v. V. $1.434 / 154$. 953. T. v. v. milturum Al, 954. T. y. v. in. Saratovskaja 129. 955. T. v. v. m. "Novinka ${ }^{4}$. 956. T. v. v. m, No. 841 . 957. T. v. v. m. No. $10,78$.

50. 
958. Triticum v. v. m. No. 321. Leguminosae.

959. Cicer arietinum L. v. Album hor. 960. C. a. v, rotundatum. 961. C. a. No. 277. 962. C. a. No. 199. 963. Ervum Lens L. v. macrosperma Bor. vnummularia, "Obyknovennaja" 964. E. L. V. m. "Saratovskaja $23^{*}$. 965. E. L. v. m. "Saratovskaja 140". 966. E. L. v. glaucosperma "Seraja $32 / 5 "$. 967. E. L. v. macrosperma Du-pui. 968. E. L. (ex Italia). 969. E. L. (ex Sicilia). 970. E. L. (ex Syria). 771. E. L. (ex India). 972. E. L. (ex Francia). 973. E. L. (ex Cabarda). 974. E. L. (ex Aphghanistan). 975. Lathyrus sativus L. No. 19/42. 976. L. s. No. 398. 977. E. s. "Gornaja Buchara ${ }^{4}$

978. L. S. No. 729 .

979. L. s. No. 732 .

980. L. \&. No. $16 / 28$.

981. L. \&. No. 829 "Sibiry“. 
482. Lathyrus s. "Ukraina".

983. L. S. "Azerbaidjan".

984. Lupinus albus $L$.

985. L. a. $y-26$.

986. L. a. angustifolius L. G. D. C.

987. L. a. No. 645.

988. L. a. No. 174.

989. L. a. No. 418.

390. L. luteus No. 4666.

991. L. 1. No, 345.

992. L. 1. No. 530.

993. L. polyphyllus L.

994. Phaseolus vulgaris L. No. 294.

995. P. v. No. 663.

996. Pisum arvense L. "Peliuschka $32 \%$.

997. P. a. "Peliuschka 40 ".

998. P. a. "Peliuschka $42 "$.

999. P. sativum L. V. coronatum „Stambovij".

1000. P. S. v. glaucospermum Al. Folger Heine.

1001. P. s. v. g. ${ }_{n}$ Moskovskij A $\cdot 559^{\circ}$. 1002. P. s, v. g. "Victoria zelenaja". 1003. P. s. V. saccharatum "Rannij

Bismark".

1004. P. s. v. S. "Konjek Grorbunok". 1005. P. S. V. vulgatum "Ambrosia*. 
1006. Pisum s. v. v. "Capital". 1007. P. s. No. 06 .

1008. P. S. T-25.

1009. P. s. "Irlandetz",

1010. P. s. 047.

1011. P. s, 087.

1012. P. s. "Tom Thumb".

1013. P. s, "Olivkovij Gibrid".

1014. P. S. "Tschudo Kelvidonia .

1015. P. S. "The Wonder of America".

1016. P. s. "Urojainij".

1017. Vicia Faba L.

1018. V. F. v. minor.

1019. V. harbonensis $L$.

1020. V. sativa L. "Belaja $163^{\circ}$.

1021. V. S, V. durro.

1022. V. s. "Archangelskaja".

1023. V. s. v. leucosperma.

1024. V. s, v. platysperma.

1025. V. s. v, tyrica.

1026. V. s, v, tricolor. 


\section{Prof. Dr. S. S. Stankov \\ Horti Director.}

Cand. M. L. Stelmachovicz Hortulanus doctus.

Docent Cand. D. S. Averkiev (System.). Cand. M. L. Stelmachovicz (Dendrolog. et Decoran.).

Ass. A. V. Knjaserskaja (Medicin. et Technik. Plantae) Ass. M. V. Alalykina (Pomolog.). 


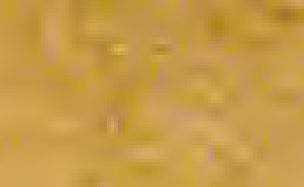

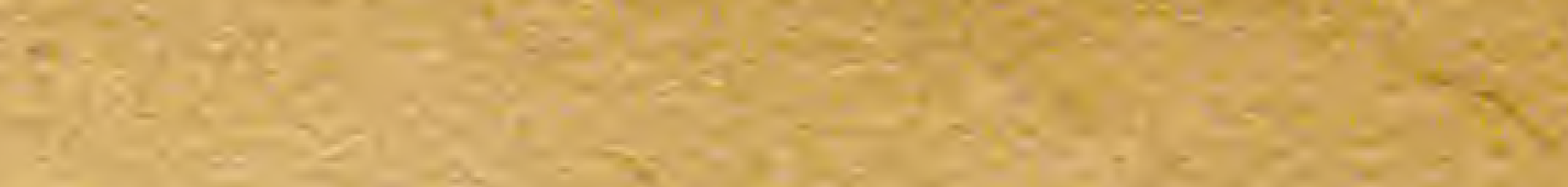
20

(3)

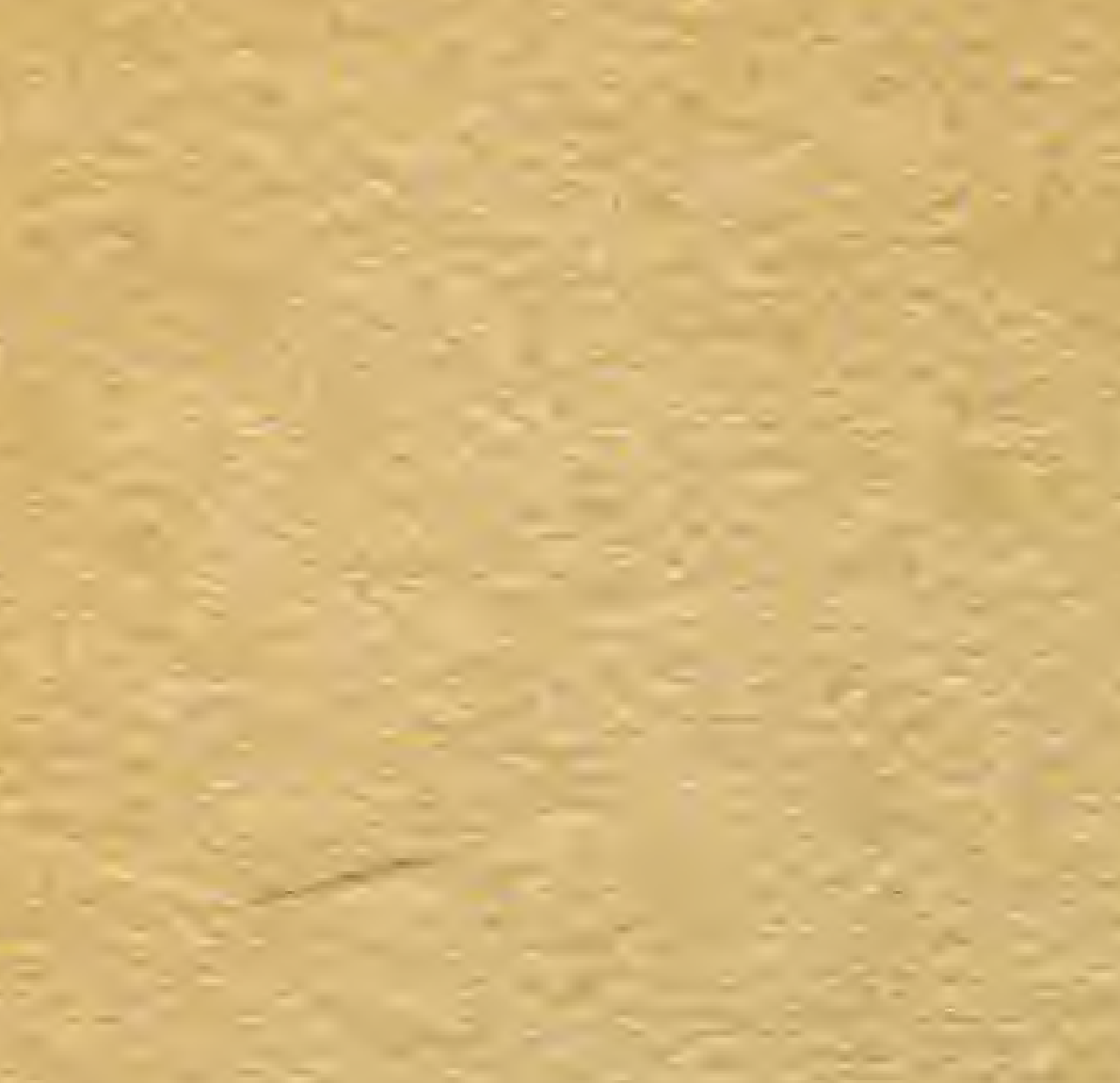

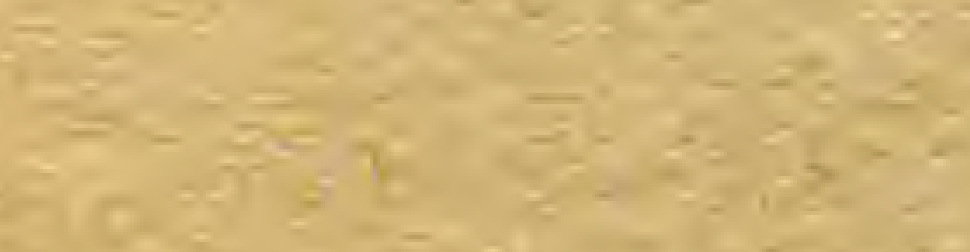

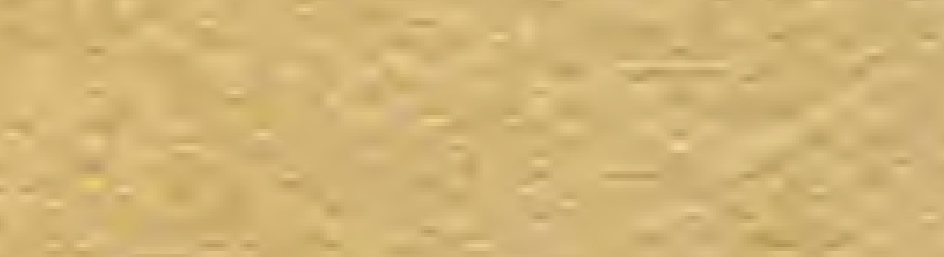

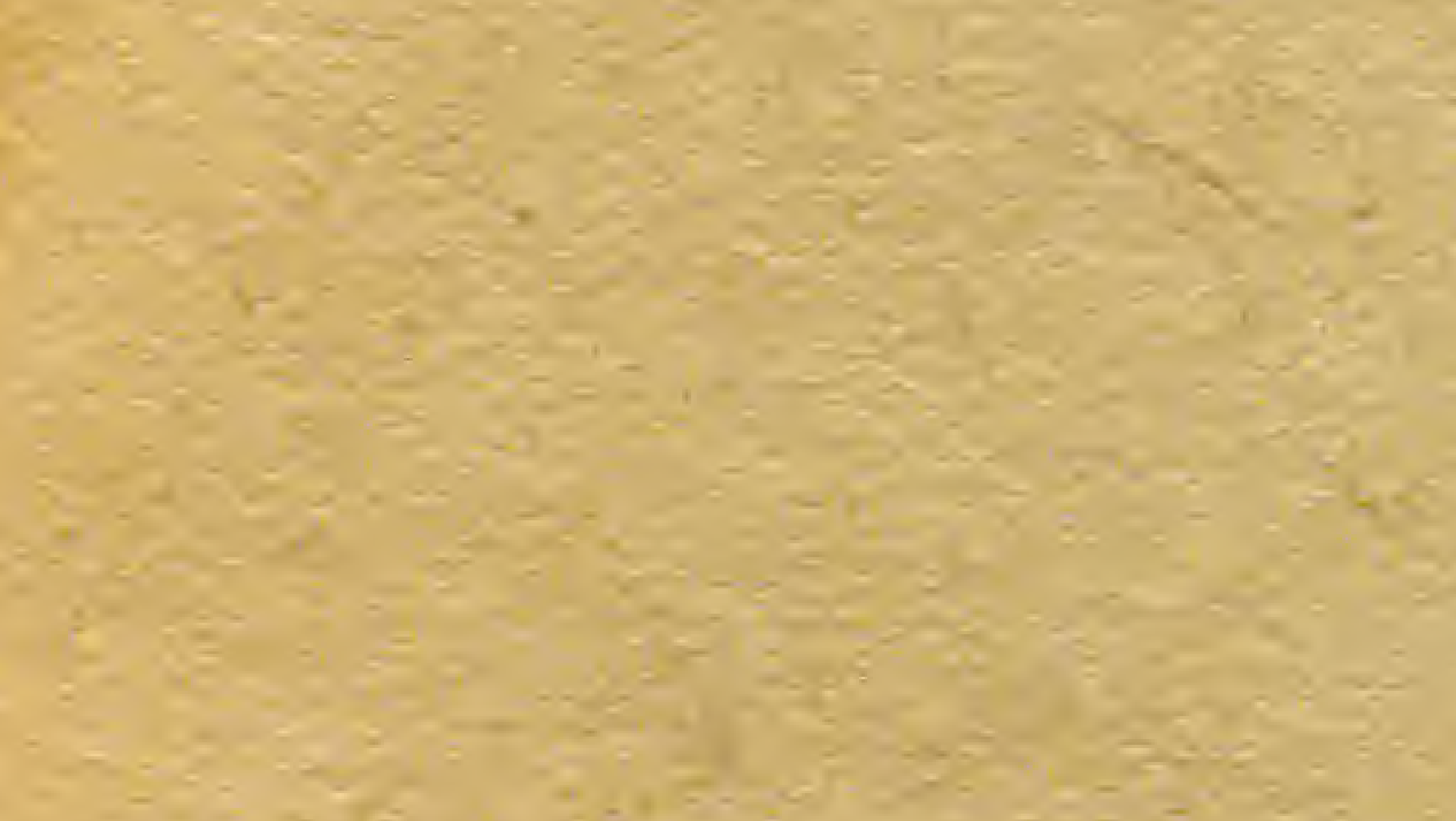




BECMЛATHO

ПЕРЕЧЕНЬ СЕМЯН,

СОБРАННЫХ В 1942 ГОДУ

И ПРЕДЛАГАЕМЫХ В ()БМЕН

БОТАНИЧЕСКИМ САДОМ

ГОРЬКОВСКОГО ГОСУДАРСТВЕННОГО

УНИВЕРСИТЕТА

\section{Огиз}

ГОРЬКОВСКОЕ ИЗДАТЕЛЬСТВО 1943 


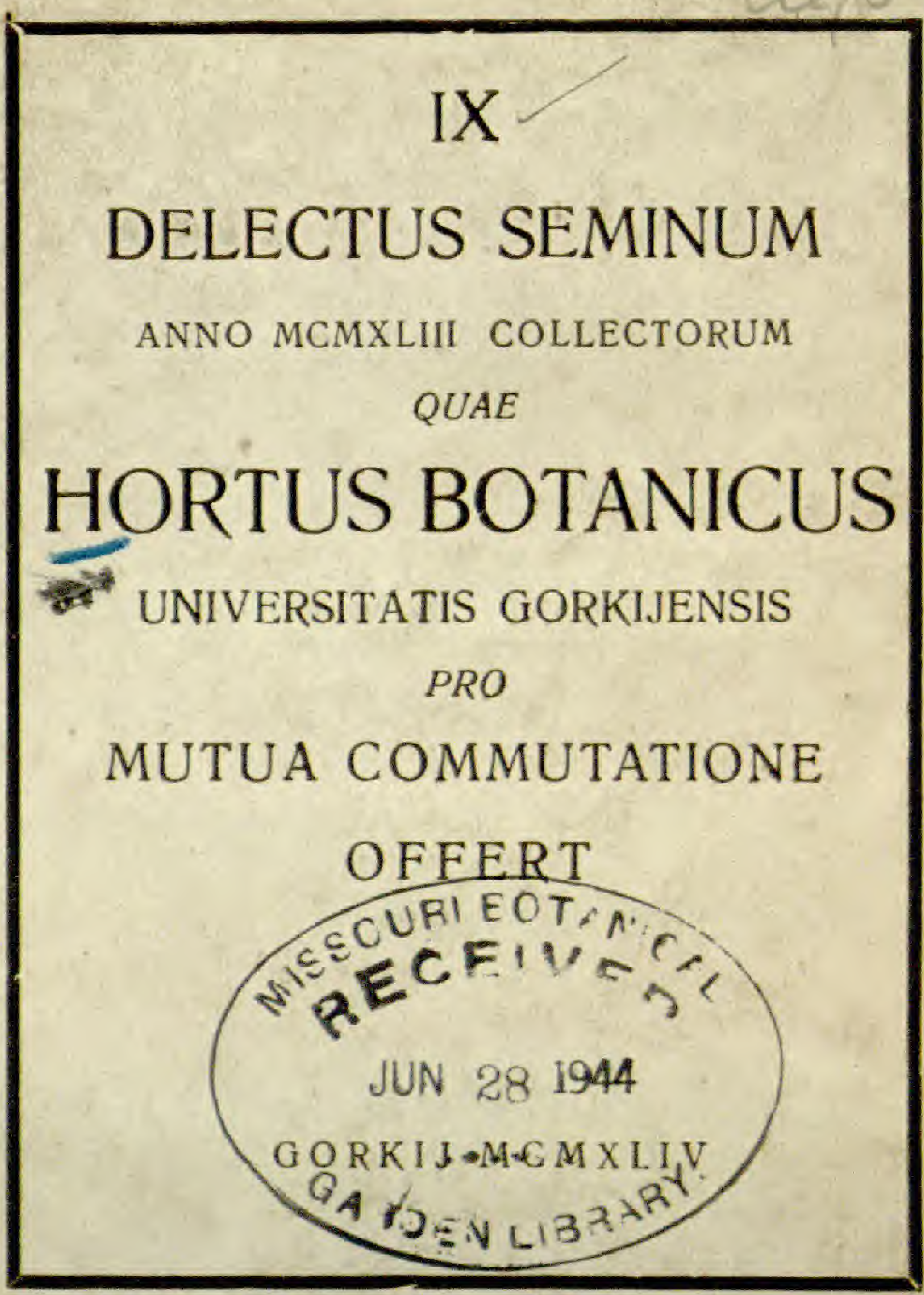





\title{
IX
}

\section{DELECTUS SEMINUM}

ANNO MCMXLIII COLLECTORUM

\author{
QUAE \\ HORTUS BOTANICUS \\ UNIVERSITATIS GORKIJENSIS \\ PRO \\ MUTUA COMMUTATIONE \\ OFFERT
}

GORKIJ MCMXLIV 
Перечень семян, собран-

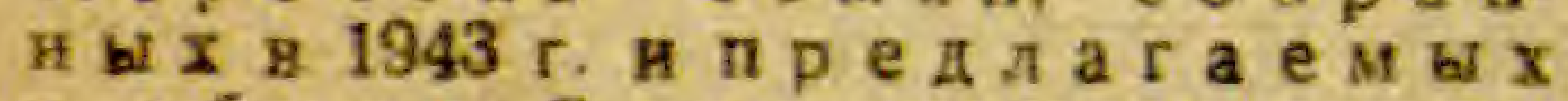
в обмен Ботаническим садом Горьковското Гос дарственното университета. ОГИз. Горьковское Издательство 1944 г. Изд. 스 1645. Нндекс $\mathrm{CX}-3$.

Peдактор проф. C. C. Cmankos. Подписано к пөчати 15/11. Формат 82 $108 /$ s. Тираж 400 экз. Объем: 1,75

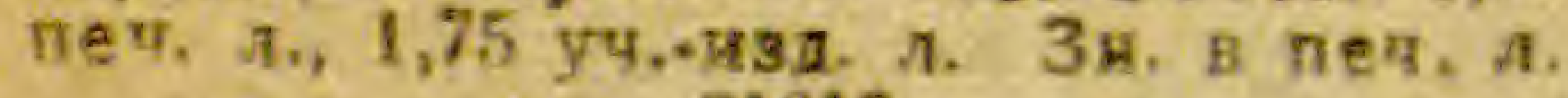
31616.

\section{МЦ 08280.}

Пөлиграф, г, Горький, ул. фитнер, 32. Заказ Ме 6978 


\section{SEMINA PLANTARUM LIGNOSARUM ET FRUTICUM.}

*)-Collecta 1942.

Betulaceae.

1. Alnus sitchensis Sarg. *)

2. Betula Gmelini Bge.

3. B. verrucosa Ehrh.

Fagaceae.

4. Quercus pedunculata Ehrh.

Berberidaceae.

5. Berberis vulgaris L.

6. B. heteropoda Schrenk.

7. B. Thunbergii DC.

Saxifragaceae.

8. Deutzia parviflora Bge.

9. Hydrangea Bretschneideri Dipp.*)

10. Philadelphus coronarius L. *)

11. Ph. pubescens Lois.")

12. $\mathrm{Ph}$. satsumanus Miq. $\left.{ }^{*}\right)$

13. Ph. tenuifolius Rupr.*)

14. Ribes alpinum L. 
15. Ribes aureum Pursh.

16. R. Warscewiczii Jancz.

Rosaceae.

17. Amelanchier spicata K. Koch.

18. Amygdalus nana L.

19. Aronia melanocarpa Elliot.

20. Chaenomeles maulei Schneid. v. alpina Maxim.

21. Cotoneaster acutifolia Turcz.*)

22. Crataegus Maximowiczi Schneid.*)

23. Phisocarpus opulifolius Maxim.

24. Prunus pennsylvanica L.*)

25. P. pumila L.*)

26. P. virginiana $L . *$ )

27. P. Bessey Bailey.*)

28. P. fruticosa Pall.")

29. Pyrus baccata L.*)

30. Rosa cinnamomea L.

31. R. spinosissima L.

32. R. rubrifolia Vill.

33 . R. rugosa typica Reg.

34. R. multiflora Thunb.*)

35. Rubus odoratus $\mathrm{L}$.

36. Sibiraea laevigata Maxim.

37. Sorbus aucuparia L.

38. S. heterophylla Dur.*)

39. Sorbaria assurgens Rehd.

40. Spiraea media Schmidt.*) 
41. Spiraea Douglasii Hook.*)

42. S. Menziesii Doug1.*)

43. S. chamaedryfolia L.*)

44. S. latifolia Borkh.*)

45. S. densiflora Nutt.*)

46. S. betulifolia Pall.*)

47. S. bumalda Burvenich v. "Antony

Waterer ${ }^{*} *$ )

48. S. japonica L. f.*)

49. S. crenifolia C. A. Mey.*)

50. S. salicifolia L.")

51. S. alba Dur.*)

Leguminosae.

52. Amorpha californica Nutt.

53. A. fruticosa L.

54. Caragana arborescens L.

55. C. frutex DC.

56. Colutea arborescens L.*)

57. C. media Willd.*)

58. Cytisus albus Haeq.*)

59. Genista tinctoria L.*)

Rutaceae.

60. Ptelea trifoliata L.

Euphorbiaceae.

61. Securinega ramiflora Muell.*) 


\section{Anacardiaceae.}

62. Cotinus coggigria Scop.

63. Rhus toxicodendron $\mathrm{L}$.

64. Rh. typhina $\mathrm{L}$.

Celastraceae.

65. Evonymus verrucosa Scop.

Aceraceae.

66. Acer ginnala Maxim.

67. A. Negundo L.

68. A. platanoides L.

69. A. tataricum L.

Rhannaceae.

70. Rhamnus cathartica L.

71. Ceanothus americanus L.

Tiliaceae.

72. Tilia cordata Mill.

Cornacede.

73. Cornus alba L.

74. C. tatarica Mill.

Oleaceae.

75. Syringa Josikaea Jacq.

76. S. villosa Vahl. 
77. Syringa vulgaris L.

\section{Caprifoliaceae.}

78. Lonicera caprifolium L.

79. L. chrisantha Turcz.

80. L. dioeca $L$.

81. L. orientalis Lam.

82. L. Ruprechtiana Reg.

83. L. tatarica L.

84. L. xylosteum L.

85. Symphoricarpus occidentalis Hook.

86. S. racemosus Michx.

87. Viburnum opulus L.

II. SEMINA PLANTARUM HERBACEUM COLLECTA 1943.

Gramineae.

88. Agropyrum acutum R. et $S$.

89. A, desertorum Schult.

90. A. fragile Nevski.

91. A. intermedium (Host.) P. B.

92. A. pectiniforme Roem. et Schult.

93. A. sibiricum (Willd.) P. B.

94. A. tenerum Vasey.

95. A. trichophorum Richt.

96. A. Turczaninowii Drob.

97. A. ugamicum Drob. 
98. Agrostis alba L. v. gigantea

99. A. borealis Hartm.

100. Alopecurus lasiostachys Link.

101. A pratensis L.

102. A, ventricosus Pers.

103. Arrhenatherum elatius $M$. et $K$.

104. Asperella hystrix Humb.

105. Avena planiculmis Turcz.

106. A. pubescens Huds.

107. A. Schelliana Hack.

108. Bromus erectus Huds.

109. B. inermis Leyss.

110. Dactylis Aschersoniana Graebn.

111. D. glomerata L.

112. Deschampsia flexuosa Trin.

113. Elymus arenarius L.

114. E. canadensis L.

115. E. dahuricus Turcz.

116. E. hirsutiglumis Scribn.

117. E. sabulosus M. B.

118. E. sibiricus L.

119. Festuca capillata Lam.

120. F. frigida Hack.

121. F. glauca Bess.

122. F. picta Ait.

123. F. pungens Kit.

124. F. sulcata Hack.

125. F. vallesiaca Schl.

126. Hordeum bulbosum L.

8 
127. Koeleria crassipes Lange.

128. K. glauca D. C.

129. K. gracilis Pers.

130. Lolium perenne L.

131. Melica ciliata L.

132. M. transsilvanica Schuhl.

133. Nardus stricta L.

134. Phalaris arundinacea $\mathrm{L}$.

135. Phleum pratense L.

136. Poa alpina L.

137. P. nemoralis $L$.

138. Roegneria canina (L.) Nevski.

139. R. tianschanica (Drob.) Nevski

140. Sesleria Heufleriana Sch.

141. Spondiopogon sibiricus Trin.

142. Stipa calamagrostis Wahlenb.

143. S. capillata L.

144. S. effusa Nakai.

145. S. Joannis Celak.

146. S. Lessingiana Trin.

147. S. pulcherrima C. Koch.

148. S. stenophylla Czern.

149. S. ucrainica P. Smirn.

150. S. viridula Trin.

151. Triodia decumbens Pall.

152. Trisetum pratense Pers.

153. T. sibiricum Rupr. 


\section{Cyperaceae.}

154. Carex Arnelli Christ.

155. C. cuprina Nendtv.

156. C. leporina L.

\section{Liliaceae.}

157. Allium altaicum Pall.

158. A, angulosum $\mathrm{L}$.

159. A. fistulosum L.

160. A. galanthum Kar. et K.

161. A. giganteum Regel.

162. A. glaciale Vved.

163. A. globosum $M$. B.

164. A. hymenorrhizum Ledeb.

165. A. Jineare L.

166. A. odorum L.

167. A. schoenoprasum L.

168. A, senescens L.

169. A. sibiricum L.

170. A. tataricum L.

171. A. tulipaefolium Ledeb.

172. Anthericum liliago L.

173. A. ramosum L.

174. Asparagus officinalis $L$.

175. A. tenuifolius Lam.

176. Eremurus altaicus Stev.

177. E. Olgae Regel.

178. Galtonia candicans Decne.

179. Hemerocallis flava $L$. 
180. Hosta coerulea Tratt.

181. Lilium martagon $\mathrm{L}$.

182. Ornithogalum pyrenaicum L.

183. Paradisia liliastrum Bertol.

184. Polygonatum multiflorum (L.) All. Amaryllidaceae.

185. Ixiolirion tataricum Pall.

\section{Iridaceae.}

186. Iris aphylla L.

187. I. ensata Thunb.

188. I. halophilla Pall.

189. I. Hookeri Penny.

190. I. illirica Somm.

191. I. Kaempferi Sieb.

192. I. orientalis Thnb.

193. I. setosa Pall.

194. I. sibirica L.

195. I. sogdiana Bunge.

196. l. sulphurea Koch.

197. I. versicolor $\mathrm{L}$.

198. Sisyrinchium anceps Cav.

\section{Urticaceae.}

199. Urtica cannabina $L$.

\section{Polygonaceae.}

200. Polygonum polymorphum Ldb. 201. Rheum acuminatum Hook. 
202. Rheum crassinervium Fisch.

203. Rh. undulatum L.

204. Rumex acetosa L.

205. R. hydrolapathum Huds.

206. R. hymenosepalus Torr.

207. R. pallidus Bigel.

208. R. Patientia L.

Chenopodiaceae.

209. Chenopodium Bonus-Henricus L.

Amaranthaceae.

210. Amaranthus caudatus L.

Caryophyllaceae.

211. Arenaria graminifolia Schrad.

212. A. grandiflora L.

213. A. longifolia M. B.

214. A. sajanensis Willd.

215. A. Syreistschikowii P. Smirn.

216. Cerastium alpinum L.

217. C. Biebersteinii D. C.

218. C. Lerschenfeldianum Schuhr.

219. Dianthus carthusianorum L.

220. Gypsophila acutifolia Fisch.

221. G. elegans $M$. B.

222. G. paniculata $L$.

223. Lychnis chalcedonica L.

12 
224. Melandrium album Garcke.

225. Saponaria officinalis $\mathrm{L}$.

226. Silene armeria L.

227. Scleranthus perennis L.

228. Viscaria vulgaris Roeh1.

\section{Ranunculacede.}

229. Aconitum barbatum Pers.

230. A. lasiostomum Rchb.

231. A. uncinatum $\mathrm{L}$.

232. A. vulparia Rchb.

233. Anemone cylindrica A. Gray.

234. A. decapetala Ard.

235. A. virginiana $L$.

236. Aquilegia canadensis $L$.

237. A. flabellata Sieb, et Zucc.

238. A. olympica Boiss.

239. Clematis Bergeronii Lav.

240. C. integrifolia L.

241. C. manschurica Rupr.

242. C. recta L.

243. Delphinium cheilanthum Fisch.

244. D. elatum L.

245. D. hybridum Willd.

246. Nigella arvensis L.

247. Pulsatilla rubra Delarb.

248. Ranunculus polyanthemus L.

249. R. propinquus C. A. Mey. 
250. Thalictrum aquilegifolium $\mathrm{L}$. 251. Th. glaucum Desf.

252. Th. minus $\mathrm{L}$.

253. Th. rugosum Ait.

\section{Papaveraceae.}

254. Papaver lateritium C. Koch.

255. P. nudicaule $\mathrm{L}$.

256. P. orientale L.

257. P. oreophyllum Rupr.

258. P. rhoeas L.

Cruciferae.

259. Alyssum alpestre L.

260. A. argenteum All.

261. Arabis albida Stev.

262. Biscutella laevigata L.

263. Carrichtera vella D. C.

264. Iberis intermedia Guers.

265. I. odorata L.

266. Sisymbrium strictissimum L.

\section{Crassulaceae.}

267. Sedum aizoon L.

268. S. fabaria Koch.

269. S. kamtschaticum Fisch. et Mey.

270. S. Maximowiczii Regel.

271. S. spurium M. B.

272. Sempervivum valesiacum Lag. 


\section{Saxifragaceae.}

273. Heuchera americana L.

274. H. cylindrica Dougl.

275. H. hispida Purch.

276. Saxifraga altissima Kerner.

\section{Rosaceae.}

277. Agrimonia odorata Mill.

278. Filipendula hexapetala Gilib.

279. F. Ulmaria (L.) Maxim.

280. Geum aleppicum Jacq.

281. G. urbanum L.

282. Potentilla atrosanguinea Lodd.

283. P. hybrida Hort.

284. P. inclinata Vill.

285. P. rupestris $L$.

286. Poterium sanguisorba L.

287. Sanguisorba officinalis L.

Leguminosae.

288. Astragalus alopecurus Pall.

289. A. cicer L.

290. A. falcatus Lam.

291. A. galegiformis $L$.

292. A. glycyphyllos L.

293. A. uliginosus $L$.

294. Baptisia australis R. Br.

295. Desmodium canadense D. C. 
296. Galega orientalis Lam.

297. Hedysarum sibiricum Poir.

298. Lathyrus latifolius L.

299. L. niger $L$.

300. Lupinus elegans H.B. et $K$.

301. L. polyphyllus Lindl.

302. Medicago falcata L.

303. B. hispida Gaertn.

304. $M$. radiata $L$.

305. M. sativa L.

306. M. scutellaria Mill.

307. Onobrychis altissima.

308. O. arenaria D. C.

309. O. oxytropoides Bunge.

310. O. transcaucasica Gr.

311. O. viciaefolia Scop.

312. Ononis spinosa L.

313. Oxytropis campestris D. C.

314. Scorpiurus vermiculata $L$.

315. Tetragonolobus purpureus Moench. 316. Thermopsis caroliniana M. A. Curt.

317. Th. fabacea D. C.

318. Trifolium arvense $\mathrm{L}$.

319. T. alpestre L.

320. $\mathrm{T}$, incarnatum $\mathrm{L}$.

321. T. lupinaster L.

322. T. medium L.

323. T. rubens L.

324. Trigonella coerulea Ser.

16 
325. Vicia ervilia Willd.

326. V. pisiformis L.

Geraniaceae.

327. Geranium platypetalum Fisch. et Mey. 328. G. pratense L.

Linaceae.

329. Linum altaicum M. B.

330. L. austriacum L.

331. L. flavum L.

332. L. perenne L.

Malvaceae.

333. Althaea ficifolia Cav.

334. A. officinalis $L$.

335. A. rosea Cav.

336. A. sulphurea Boiss.

337. Kitaibelia vitifolia Willd.

338. Lavatera thuringiaca $L$.

339. Malachra radiata $L$.

340. Malva alcea L.

341. M. crispa L.

342. Palaua flexuosa Mast.

343. Sidalcea malvifolia Fisch, et Mey.

344. S. spicata Greene.

Hypericaceae.

345. Hypericum ascyron $L$. 
346. Hypericum perforatum L. 347. $\mathrm{H}$. quadrangulum $\mathrm{L}$.

\section{Cistaceae.}

348. Helianthemum chamaecistus Mill. 349. H. hirtum Mill. 350. H. ovatum Dun. 351. H. nummularium Mill. 352. H. vineale Pers.

Violaceae.

353. Viola arborescens L.

Lythraceae.

354. Lythrum salicaria L.

Oenotheraceae.

355. Clarkia elegans Dougl.

356. Oenothera fruticosa L.

Umbelliferae.

357. Angelica brevicaulis B. Fedtsch. 358. Archangelica officinalis Hoffm. 359. Astrantia carniolica Wulf. 360. Bupleurum aureum Fisch. 361. Chaerophyllum hirsutum L. 362. Eryngium alpinum L.

363. E. planum L, 364. Heracleum dissectum Ledeb. 18 
365. Heracleum orsinii Ten.

366. H. sibiricum L.

367. H. villosum Fisch.

368. Laserpitium latifolium L.

369. Pleurospermum Golaka Rchb.

370. Seseli osseum Crantz.

371. Siler montanum Crantz.

Primulaceae.

372. Lysimachia punctata L.

373. L. verticillata $M$. B.

Plumbaginaceae.

374. Armeria caespitosa Boiss.

375. A. sibirica Turcz.

376. Statice Gmelini Willd.

377. S. latifolia Sm.

Gentianaceae.

378. Gentiana cruciata L.

Apocynaceae.

379. Amsonia Tabernaemontana Walt.

Asclepiadaceae.

380. Asclepias Cornuti Decne.

381. Vincetoxicum fuscatum Rchb.

382 . V. officinale Moench, 


\section{Polemoniaceae.}

383. Collomia coccinea Lehm. 384. Gilia capitata Dougl.

\section{Hydrophyllaceae.}

385. Nemophila insignis Dougl.

386. Phacelia congesta Hook. 387. Ph. loasaefolia Torr.

Boraginaceae.

388. Anchusa officinalis L.

389. Cerinthe minor L.

390. Cynoglossum cheirifolium L.

391. C. furcatum Wall.

392. Echium rubrum Jacq.

393. Mertensia sibirica G. Don.

394. Symphytum asperum Lepech.

\section{Labiatae.}

395. Ballota nigra L.

396. Brunella grandiflora Jacq.

397. Dracocephalum altaiense Laxm.

398. D. grandiflorum L.

399. D. Ruyschiana L.

400. Hyssopus officinalis $L$.

401. Lallemantia peltata Fisch, et Mey.

402. Leonurus cardiaca $L$.

403. Lophanthus anisatus Benth. 
- 404. Marrubium peregrinum L. 405. Monarda fistulosa $\mathrm{L}$. 406. Nepeta cataria L. V. citriodora 407. N. grandiflora M. B. 408. N. kokanica Regel. 409. N. marcantha Fisch. 410. N. nuda L. 411. Origanum vulgare L. 412. Phlomis tuberosa L. 413. Salvia aethiopis $L$. 414. S. limbata C. A. M. 415. S. nemorosa L. 416. S. utilis A, Br. 417. S. verticillata $\mathrm{L}$. 418. Scutellaria alpina $L$. 419. S. altissima L. 420. Sideritis hyssopifolia L. 421. S. scordioides $\mathrm{L}$. 422. Stachys germanica L. 423. S. grandiflora Benth. 424. S. lanata Jacq. 425. S. officinalis (L.) Trev. 426. S. setifera C. A. Mey. 427. Thymus glaber Mill. 428. Th. Marschallianus Willd.

\section{Solanaceae.}

429. Physalis Franchettii Mast. 
430. Physochlaena orientalis G. Don. 431. Solanum Dulcamara L.

\section{Scrophulariaceae.}

432. Digitalis ambigua Murr.

433. D. lutea L.

434. D. media Roth.

435. D. nevadensis Kunze.

436. D. sibirica L.

437. D. viridiflora

438. Linaria triphylla Mill.

439. L. vulgaris Mill.

440. Pentstemon confertus Dougl.

441. P. diffusus Dougl.

442. P. digitalis Nutt.

443. P. glaber Pursh.

444. P. hirsutus Villd.

445. P. ovatus Dougl.

446. P. procerus Dougl.

447. Schizanthus pinnatus Ruiz. et Pav.

448. Verbascum orientale M. B.

449. Veronica incana L.

450. V. longifolia L.

451. V. teucrium L.

\section{Bignoniaceae.}

452. Incarvillea compacta Max. 453. I. Olgae Regel. 


\section{Plantaginaceae.}

454. Plantago alpina L.

455. P. lanceolata $\mathrm{L}$.

456. P. purpurascens Willk.

Rubiaceae.

457. Asperula tinctoria $L$.

458. Galium verum L.

Caprifoliaceae.

459. Sambucus Ebulus L.

Valerianaceae.

460. Valeriana bispida Turcz.

461. V. nitida $\mathrm{Kr}$.

462. V. rossica P. Sm.

Dipsacaceae.

463. Cephalaria corniculata R. et S.

464. C. gigantea E. Bobr.

465. C. procera Fisch.

466. Scabiosa caucasica Bieb.

467. S. inflexa Beck.

468. S. praemorsa Achers.

\section{Campanulaceae.}

469. Adenophora communis Fisch. 470. A. verticillata Fisch. 
471. Campanula alliariaefolia Willd.

472. C. carpathica Jacq.

473. C. persicifolia L.

474. C. sarmatica Ker-Gavl.

475. Codonopsis clematidea Clarke.

476. Phyteuma canescens W. et K.

477. $\mathrm{Ph}$. Scheuchzeri All.

478. Platycodon grandiflorum D. C.

479. Symphyandra Hoffmannii Pant.

Compositae.

480. Achillea asplenifolia Vent.

481. A, filipendulina Lam.

482. A. millefolium $\mathrm{L}$.

483. A. nobilis $\mathrm{L}$.

484. A. tanacetifolia A11.

485. Alfredia cernua Cass.

486. A. stenolepis Benth.

487. Anaphalis margaritacea Benth. et Hook. 488. Anthemis cotula L.

489. A. tinctoria L.

490. Arnica chamissonis Less.

491. A. foliosa Nutt.

492. A. montana L.

493. Artemisia dracunculus L.

494. A. rupestris L.

495. Aster incisus Fisch.

496. A. longifolius Lam.

497. A. praecox. 
498. Aster salignus Willd,

499. A. trinervis Desf.

500. A umbellatus Mill.

501. Atractylodes ovata D. C.

502. Boltonia asteroides L'Herit.

503. Bupthalmum speciosum Schreb.

504. Cacalia aconitifolia Bge.

505. C. hastata L.

506. Calendula aegyptiaca Desf.

507. C. algeriensis Boiss. et Bent.

508. Centaurea banatica.

509. C. dealbata Willd.

510. C. leurcophylla M. B.

511. C. macrocephala Puschk.

512. C. nigra L.

513. C. Ossica Boiss.

514. C. scabiosa L.

515. Chrysanthemum corymbosum $\mathrm{L}$.

516. Ch. maximum Ramond.

517. Coreopsis lanceolata L.

518. Cosmos bipinnatus Cav.

519. Dimorphotheca annua Less.

520. D. aurantiaca D. C.

521. Echinops sphaerocephalus L.

522. Erigeron asper Nutt.

523. E. speciosus D. C.

524. Helianthella quinquenervis A. Gr.

525. Helichrysum lanatum D. C. 526. Heliopsis helianthoides Sveet. 
527. Heliopsis scabra Dun. 528. Inula glandulosa Willd.

529. I. helenium L.

530. Layia platyglossa A. Gray.

531. Leontodon hispidus L.

532. Leontopodium alpinum Cass.

533. Madia sativa Mol.

534. Pyrethrum roseum Lindl.

535. Rudbeckia laciniata L.

536. Senecio adonidifolius Lois.

537. S. altaicus $M$. B.

538. S. clivorum Max.

539. S. Doria L.

540. S. suaveolens Ell.

541. S. Wilsonianus Hemsl.

542. Serratula coronata L.

543. S. Gmelini Led.

544. S. radiata Bieb.

545. Silphium perfoliatum L.

546. Silybum Marianum Gaertn.

547. Solidago canadensis L.

548. S. sparsiflora Gray.

549. Tanacetum vulgare $L$.

$$
\text { C OLLE C T A } 1942 .
$$

Gramineae.

550. Avena alpina Suter.

551. Hordeum secalinum Schreb.

26 
552. Oryzopsis holciformis (M. B.) Richt. 553. Poa compressa L.

\section{Liliaceae.}

554. Lilium concolor Salisb.

555. L. tenuifolium Fisch.

556. Muscari botryoides Mill.

557. M. racemosum (L.) Mill.

558. Scilla sibirica Andr.

Iridaceae.

559. Iris variegata $L$.

Poligonaceae.

560. Rheum Emodi Wall.

561. Rh. officinale Bail.

562. Rh. palmatum L.

Nyctaginaceae.

563. Oxybaphus nyctagineus Sweet.

Phytolaccaceae.

564. Phytolacca americana L.

Caryophyllaceae.

565. Dianthus barbatus L.

566. D. deltoides L.

567. Viscaria alpina (L.) Don.

Ranunculaceae.

568. Anemone silvestris L. 
569. Clematis tangutica Korsh.

570. Delphinium grandiflorum L.

571. Nigella sativa $L$.

572. Paeonia anomala L.

573. P. decora Anders.

574. Ranunculus acer L. ssp. Steveni Hegi.

575. R. bulbosus L.

576. Thalictrum adianthifolium Bess.

577. Trollius altaicus C. A. Mey.

578. T. laxus Salisb.

\section{Papaveraceae.}

579. Chelidonium laciniatum Mill.

\section{Cruciferae.}

580. Aubrietia erubescens Griseb.

581. Bunias orientalis L.

582. Iberis gibraltarica L.

583. Isatis alpina All.

584. Thlaspi densiflorum Boiss. et Ky.

Resedaceae.

585. Reseda lutea L.

Crassulaceae.

586. Sedum Ewersii Ledeb.

587. Sempervivum tectorum -L.

Saxifragaceae.

588. Bergenia crassifolia (L) Fritsch.

28 
589. Bergenia pacifica Kom. 590. Mitella caulescens Nutt.

Rosaceae.

591. Aruncus silvester Kostel.

592. Potentilla argentea L.

593. P. gelida C. A. Mey.

594. P. geoides Bieb.

595. P. nivea L. v. elongata.

Leguminosae.

596. Anthyllis variegata hort.

597. Lathyrus odoratus L.

598. L. silvestris L.

599. Lotus corniculatus L.

600. Melilotus albus Desr.

601. Onobrychis montana Lam. et D. C.

602. Vicia multicaulis Led.

Geraniaceae.

603. Geranium albiflorum Hook.

Rutaceae.

604. Dictamnus fraxinella Pers.

605. Ruta graveolens L.

Malvaceae.

606. Althaea nudiflora Lindl.

607. Malva moschata L. 
608. Sidalcea candida A. Gr. 609. S. neo-mexicana A. Gr.

Umbelliferae.

610. Angelica kamtschatica Rupr.

611. Libanotis montana All.

612. Siler aquilegifolium Gaertn,

Primulacéae.

613. Primula algida Adams.

Plumbaginaceae.

614. Armeria leucocephala Salzm.

615. A. plantaginea Willd.

Gentinaceae.

616. Gentiana Kirilovii Turcz.

617. G. phlogipholia Schot. et Kotschy.

618. G. tianschanica Rupr.

Labiatae.

619. Dracocephalum nutans L.

620. Horminum pyrenaicum L.

621. Salvia stepposa Des.-Chost.

Solanaceae.

622. Atropa Belladonna L. 


\section{Scrophulariaceae.}

623. Antirrhinum Coulterianum Benth.

624. Veronica fruticans Jacq.

625. V. gentianoides Vahl.

626. V. orchidea Crantz.

627. Wulfenia carinthiaca Jacq.

Globulariaceae.

628. Globularia Willkommii Nym.

Dipsacaceae.

629. Scabiosa graminifolia L.

Cucurbitaceae.

630. Bryonia alba L.

Campanulaceae.

631. Campanula Steveni Bieb.

632. Codonopsis ovata Benth.

633. Jasione perennis L.

Compositae.

634. Achillea cartilaginea Led. 635. A. sibirica Ledeb.

636. Antennaria nevadensis Greene. 637. Arnica sachalinensis A. Gray. 638. Aster paniculatus Lam. 639. Bellis mexicana A. Gray. 640. Buphtalmum salicifolium L. 
641. Cardutus hamulosus Ehrh. 642. Doronicum orientale Hoffm.

643. Eupatorium purpureum L.

644. Gaillardia aristata Pursh.

645. Helichrysum arenarium (L.) Moench.

646. Liatris spicata Willd.

647. Ptarmica impatiens D. C.

648. Pyrethrum carneum Bieb.

649. P. cinerarifolium Trev.

650. P. parthenifolium Willd.

651. Rudbeckia occidentalis Nutt.

652. Solidago multiradiata Ait.

653. S. shortii Torr.

654. Troximon gracilens Gray.

\section{COLLECTA 1941}

Gramineae.

655. Corynephorus canescens Beauw.

Liliaceae.

656. Allium caesium Schrenk.

Polygonaceae.

657. Rumex callosus Reidr.

Caryophyllaceae.

658. Lychnis coronaria Desr. 659. Saponaria caespitosa D. C.

32 
660. Silene tenuis Willd.

\section{Ranunculaceae.}

661. Anemone hudsoniana Richards. 662. Nigella damascena $L$.

\section{Cruciferae.}

663. Aethionema Buxbaumii D. C.

664. Alyssum desertorum Stapf. 665. Arabis alpina L.

666. Aubrietia libanotica Boiss. et Hohen. 667. Draba repens M. B.

668. Isatis tinctoria $\mathrm{L}$.

669. Malcolmia maritima R. Br.

670. Roripa silvestris (L.) Bess.

Crassulaceae.

671. Sedum album L.

672. S. rupestre L.

Rosaceae.

673. Fragaria indica Andr.

674. F. vesca L.

675. Geum coccineum Sibth. et Sm.

676. Potentilla Meyeri Boiss.

\section{Leguminosae.}

677. Anthyllis Boissieri Sag. 678. Astragalus danicus Retz. 
679 Astragalus onobrychis L.

680. A. sulcatus L.

681. Securigera coronilla D. C.

Malvaceae.

682. Malachra radiata $\mathrm{L}$.

Cistaceae.

683. Cistus symphitifolius Lam.

Oenotheraceae.

684. Epilobium hirsutum L.

Primulaceae.

685. Androsace filiformis Retz.

Polemoniaceae.

686. Polemonium coeruleum L.

687. P. Van-Bruntiae Britt.

Hydrophyllaceae.

688. Romanzoffia sitchensis Bong.

Labiatae.

689. Salvia silvestris L.

690. Ziziphora capitata L.

Scrophulariaceae.

691. Digitalis purpurea L.

34 
692. Linaria bipartita Willd. 693. Veronica candida Lodd.

\section{Bignoniaceae.}

694. Incarvillea grandiflora Bur. et Franch.

\section{Valerianaceae.}

695. Patrinia rupestris Juss.

696. Valerianella coronata D. C.

\section{Dipsacaceae.}

697. Scabiosa micrantha Desf.

\section{Campanulaceae.}

698. Jasione humilis Lois.

\section{Compositae.}

699. Achillea tomentosa L. 700. Calendula stellata Cav. 701. Carthamus tinctorius L.

702. Dimorphotheca pluvialis Moench. 703. Helichrysum bracteatum Willd. 704. Hieracium pilosella $\mathrm{L}$. 705. Scorzonera purpurea L. 706. Tanacetum huronense Nutt. 
III. SEMINA PLANTARUM DECORANTIUM.

\section{Iridaceae.}

707. Gladiolus hibridus hort. (bulbi).

708. G. primulinus Bok. (bulbi).

Portulacaceae.

709. Portulaca grandiflora Hook.

Papaveraceae.

710. Papaver somniferum L. f. paeoniflorum hort.

Caryophyllaceae.

711. Dianthus barbatus L.

712. Gypsophila paniculata L.

Leguminosae.

713. Phaseolus multiflorus $L$.

Tropaeolaceae.

714. Tropaeolum majus $\mathrm{L}$.

Violaceae.

715. Viola tricolor maxima hort.

Begoniaceae.

716. Begonia semperflorens Link. et Otto. 36 
Primulaceae.

717. Primula obconica Hauce.

Convolvulaceae.

718. Ipomea purpurea Roth.

Polemoniaceae.

719. Phlox Drummondi Hook.

Solanaceae.

720. Nicotiana affinis T. Moore.

721. N. rustica $L$.

722. N. wigandioides hort.

723. Petunia hybrida hort.

Scrophulariaceae.

724. Antirrhinum majus L. fl. alba hort. 725. A. m. L. f1. "Primadonna" hort.

726. A. m. L. fl. "Tschernyi Platsch" hort.

Campanulaceae.

727. Lobelia erinus L.

Compositae.

728. Ageratum conyzoides L. v, mexicanum hort.

729. Callistephus chinensis Nees. "Triumph", "Krassa USSR". 
730. Callistephus ch, N. nana (lylovyie.). 731. C. ch. N. nana (Karmazynnyie). 732. C. ch. N. "Straussovoje Pero" (sanguin. rubri).

733. C. ch. N. "Straussovoje Pero" (argent. roseo).

734. C. ch. N. "Straussovoje Pero" (coerulei). 735. C. ch. N. "Straussovoje Pero " (fioletovoje). 736. C. ch. N. "American Beauty" (pallidicoerulei).

737. C. ch. N. paeoniformis albi.

738. C. ch. N. paeoniformis vishnevije.

739. Pyrethrum parthenifolium Willd.v. aureum hort.

740. Solidago canadensis $L$.

741. Tagetes erectus L.

742. T. signatus Bartl. pumila hort.

743. T. patula nana hort.

IV. SEMINA PLANTARUM OFFICINALIUM. Liliaceae.

744. Asparagus officinalis L. 745. Allium schoenoprasum L.

Iridaceae.

746. Iris sibirica L. 38 
Moraceae.

747. Cannabis sativa L.

Polygonaceae.

748. Rheum officinalis Bail.

749. $\mathrm{Rh}$, undulatum $\mathrm{L}$.

750. Rh. rhaponticum L.

751. Rh. tanguticum L.

Caryophyllaceae.

752. Saponaria officinalis $L$.

Ranunculaceae.

753. Nigella arvensis L.

754. N. sativa L.

755. Ranunculus acer L.

Papaveraceae.

756. Papaver dubium L.

757. P. somniferum L.

758. Chelidonium majus L.

Crassulaceae.

759. Sedum acre L.

Rosaceae.

760. Geum urbanum L. 


\section{Malvaceae.}

761. Althaea officinalis $\mathrm{L}$.

762. Malva alcea L.

763. Althaea rosea Cav. v. nigra.

Umbelliferae.

764. Anethum graveolens L.

765. Conium maculatum L.

766. Coriandrum sativum L.

767. Levisticum officinale Koch.

768. Pastinaca sativa L.

Boraginaceae.

769. Anchusa italica Ritz.

770. Cynoglossum officinale $\mathrm{L}$.

Labiatae.

771. Hyssopus officinalis L,

772. Lallemantia ibericum Fisch.

Scrophulariaceae.

773. Digitalis sibirica L.

774. D. purpurea L.

Valerianaceae.

775. Valeriana officinalis $L$.

Compositae.

776. Chondrilla juncea L.

40 
777. Helianthus annuus L.

778. Pyrethrum cinerariaefolium Trev.

779. P. roseum Lindl.

780. P. carneum Bieb.

781. Matricaria chamomilla L.

782. Tanacetum boreale L.

783. T. vulgare $\mathrm{L}$.

\section{SEMINA PLANTARUM OLEARUM ET TECHNICARUM.}

Moraceae.

784. Cannabis sativa $L$.

Polygonaceae.

785. Rumex acetosa $\mathrm{L}$.

786. R. confertus L.

- Cruciferae.

787. Brassica juncea Czern.

788. B. nigra Koch.

789. Crambe hispanica L.

790. Sinapis alba L.

Resedaceae.

791. Reseda luteola L.

Malvaceae.

792. Abutilon avicennae Gaertn. No. 45372. 
793. Abutilon a. No. 8094.

794. Malva crispa L.

795. M. verticillata L.

796. Sida napaea Cav.

Labiatae.

797. Lallemantia iberica Fisch. et Mey.

Compositae.

798. Cichorium inthybus L.

799. Helianthus annuus L. Saratovsky

No. $\mathrm{P}-27$.

800. H. a. L. Barnaulsky No. 1501.

801 . H. a. L. Saratovsky No. 19.

802 . H. a. L. Saratovsky No. 808.

803. H. a. L. Saratovsky No. 169.

804. Madia sativa Molin.

Umbelliferae.

805. Coriandrum sativum L. giganteum.

VI. SEMINA PLANTARUM GRAMINEARUM ET LEGUMINOSARUM.

Gramineae.

806. Avena nuda L.

807. A. sativa L. v. aurea "Lochovski ${ }^{a}$.

808. A. s. v. a. "Stepniak“.

809. A. s. v. a. "Zolotoj Dozd“. 
810. Avena s. v. a. "Amerikanskij". 811. A. S. v. a. "Kherson". 812. A. s. v. a. "Pflug". 813. A. s. v. a. "Povoljie 2131 ". 814. A. s. v. a. "Echo" 815. A. s. patula brunnea Körn. 816. A. s. v. Brie. 817. A. S. v. aristata "Lygovo $11^{\text {". }}$ 818. A. s. v. a. "Miraj". 819. A. s. v. a. "Moskovskij A-315". 820. A. s. V. a. "Jemtschujina". 821. A. s. v. a. "Severianin". 822. A. s. v. mutica "Dippe". 823. A. s. v m. "Romania". 824. A. s. V. m. "Schatilovsky". 825. A. strigosa Schreb. 826. Hordeum distichum L. v. abyssinicum Sch. 827. H. d. v. medicum ${ }_{n}$ Prima" Körn No. 26. 828. H. d. v. m. "Prima" No. 46. 829. H. d. v. nigrum. 830. H. d. v. nudum "Byloje“. 831. H. d. v. nudum No. 105. 832. H. d. v. nudum No. 110. 833. H. d. v. nutans Schubl. Europaeum $313 / 133$.

834. H. d. v. nutans No. 17. 835. H. d. v. n. "Viner". 836. H. d. v. n. Colchicum $10 / 78$. 837. H. d. v. ח. "Hanna Loosdorfskaja ${ }^{\mu}$. 
838. Hordeum vulgare L. v. astrachanense. 839. H. v. v. caeleste No. 46.

840. H. v. v. nigrum Willd.

841. H. v. v. pallidum Ser. "Pioner".

842. H: v. v. p. "Tschervonetz".

843. H. v. v. p. "Toulonsky“.

844. H. v. v. p. Trebi.

845. H. v. v. p. "Viatsky 1009 “.

846. H. v. No. $13198 / 5$.

847. H. v. No. $905 / 4$.

848. H. v. v. trifurcatum Schlecht.

849. H. v. Hasfordianum.

850. Lolium italicum R. Br.

851. Panicum miliaceum L. contractum Kazanskoje 506.

852. P. m. contractum Voroneshskoje 0198.

853. P. m. c. Voropajevskoje 367.

854. P. m. compactum Al. No. 19/27.

855. P. m. c. No. 0144.

856. P. m. v. effusum Al. Tordenskoje 215.

857. P. m. v. e. Voroneshskoje 215.

858. P. m. v. e. No. 0585.

859. Secale cereale L.

860. Triticum dicoccum Schübl.

861. T. d. v. rufum.

862. T. dicoccoides $\mathrm{Kr}$.

863. T. durum Desf. v. obscurum.

864. T. d. v. affine Körn No. 309.

865. T. d. v. coeruleum. 
866. Triticum d. v. hordiiforme No. 10. 867. T. d. v. h. No. 189.

868. T. d. v. h. No. 496.

869. T. d. v. h. No. 694.

870. T. d. v. libicum Körn.

871. T. d. v. leucurum $\mathrm{Br}-26$.

872. T. d. v. $1 . \mathrm{Br}-33$.

873. T. d. v. leucomelan Al.

874. T. d. v. melanopus A1. No. 069.

875. T. d. v. m. No. 826 .

876. T. d. v. m. No. 886.

877. T. d. v. Reichenbachii Kr.

878. T. monococcum L. (ex Amerika).

879. T. persicum Vav. v. fulginosum Zhuk.

880. T. spelta L.

881. T. s. v. coeruleum.

882. T. vulgare Host. albidum Al. No. 0676.

883. T. v. alborubrum Kr. "Sarrubra“.

884. T. v. a. Kr. "Sarrosa“.

885. T. v. caesina A1. No. 111 .

886. T. v. v. Delfii $\mathrm{Kr}$.

887. T. v. erythrospermum Kr. Durable.

888. T. v. v. e. "Kooperatorka".

889. T. v. v. e. "Zaria".

890. T. v. v. e. "Zemka".

891. T. v. v. e. "Ukrainka“.

892. T. v. v. e. "Moskovskaja".

893. T. v. v. e. No. 1114.

894. T. v. v. e. "Viatskaja $529^{\circ}$. 
895. Triticum v. v. ferrugineum Al. „Moskovskaja $2411^{\prime \prime}$.

896. T. v. v. f. "Moskovskaja $2453^{\circ}$. 897. T. v. v. f. "Simbileevskaja $146^{\circ}$. 898. T. v. v. f. No. 150. 899. T. v. v. f. "Engelgard". 900. T. v. v. f. "Sarmatka". 901. T. v. v. hostianum No. 237. 902. T. v. v. lutescens Al. No. 053. 903. T. v. V. 1. No. 479. 904. T. v. v. 1. No. 040. 905. T. v. v. 1. No. 062. 906. T. v. v. 1. No. 3221. 907. T. v. v. 1. No. 323. 908. T. v. V. 1. No. 786. 909. T. v. v. 1. No. 9113. 910. T. v. v. 1. "Garnet" 911. T. v. v. 1. "Leda". 912. T. v. v. 1. "Markiz". 913. T. v. v. 1. "Kitchener". 914. T. v. v. 1. "Beta stojkaja". 915. T. v. v. 1. "Viatskaja $05 \%$. 916. T. v. v. 1. "Viatskaja 126 ". 917. T. v. v. 1. No. 329. 918. T. v. V. I. "Zolotaja ${ }^{\circ}$. 919. T. v. v. 1. No. 106010. 920. T. v. v. I. "Teiskaja“. 921. T. v. v. 1. 2736. 922. T. V. V. 1. $434 / 154$. 
923. Triticum v. v. milturum Al.

924. T. v. v. m. Saratovskaja 129.

925. T. v. v. m. "Novinka“.

926. T. v. v. m. No. 841.

927. T. v. v. m. No. 1078.

928. T. v. v. m. No. 321.

Leguminosae.

929. Cicer arietinum L. v. Albium hort.

930. C. a. v. rotundatum.

931. C. a. No. 277.

932. C. а. No. 199.

933. Ervum Lens L. v. macrosperma Bor. v. nummularia "Obyknovennaja".

934. E. L. v. m. "Saratovskaja $23^{a}$. 935. E. L. v. m. "Saratovskaja 140 “. 936. E. L. v. glaucosperma "Seraja $32 / 5$ ". 937. E. L. v. macrosperma Du-pui. 938. E. L. (ex Italia).

939. E. L. (ex Sicilia).

940. E. L. (ex Syria).

941. E. L. (ex India).

942. E. L. (ex Francia).

943. E. L. (ex Cabarda).

944. E. L. (ex Aphghanistan).

945. Lathyrus sativus L. No. 19/42.

946. L. s. No. 398.

947. L. s. "Gornaja Buchara". 
948. Lathyrus s. No. 729.

949. L. s. No. 732 .

950. L. s. No. $16 / 28$.

951. L. s. No. 829 "Sibirj".

952. L. s. "Ukraina".

953. L. s. "Azerbaidjan".

954. Lupinus albus L.

955. L. a. $\mathrm{y}-26$.

956. L. a. angustifolius L. G. D. C.

957. L. a. No. 645.

958. L. a. No. 174.

959. L. a. No. 418.

960. L. lutens No. 4666.

961. L. 1. No. 345.

962. L. 1. No. 530.

963. L. polyphyllus L.

964. Phaseolus vulgaris L. No. 294.

965. Ph. v. No. 663.

966. Pisum arvense L. „Peliuschka 32 ".

967. P. a. "Peliuschka 40 “.

968. P. a. "Peliuschka $42^{\star}$.

969. Pisum sativum L. v. coronatum "Stambovij".

970. P. s. v. glaucospermum Al. Folger Heine.

971. P. s. v. g. "Moskovskij A-559".

972. P. s. v. g. "Victoria zelenaja".

973. P. s. v. saccharatum "Rannij Bismark ".

974. P. s. v. s. "Konjek Gorbunok".

975. P. s. v. vulgatum "Ambrosia".

976. P. s. v. v. "Kapital“.

48 
977. P. s. No. 06.

978. P. s. $\mathrm{T}-25$.

979. P. s. "Irlandetz".

980. P. s. 047.

981. P. s. 087.

982. P. s. "Tom Thumb".

983. P. s. "Olivkowij Gibrid.

984. P. s. "Tschudo Kelvidonia“.

985. P. S. "The Wonder of America"

986. P. s. "Urojainij“.

987. Vicia Faba L.

988. V. F. v. minor.

989. V. narbonensis L.

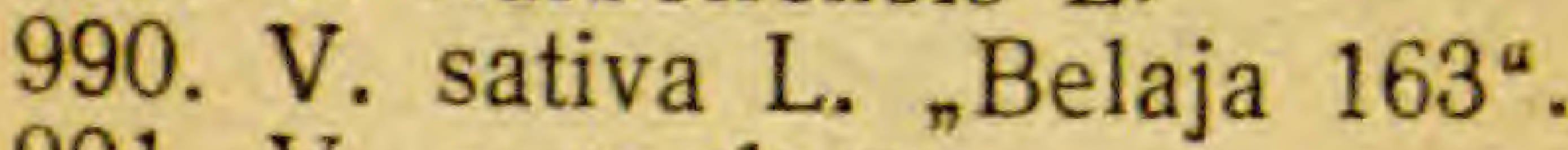

991. V. s. v. durro.

992. V. s. v. leucosperma.

993. V. s. v. platysperma.

994. V. s. v. typica.

995. V. s. v. tricolor.

\section{SEMINA PLANTARUM OLERACEUM.}

Solanaceae.

996. Physalis Alkekengi L. "Grabovski No. 1

997. $\mathrm{Ph}$.

998. Ph.

999. Ph.

1000. Ph.

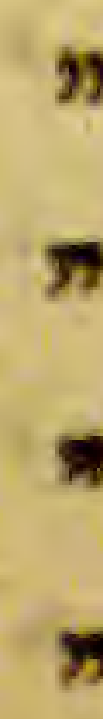

No. 2

No. 4

No. 5 
1001. Lycopersicum esculentum Mill "Daveland Grand $^{*}$

1002. L. esculentum Mill "Derbentskij“

1003. L. esculentum Mill "Glob".

1004. L. esculentum Mill

1005. L. esculentum Mill

1006. L. esculentum Mill

1007. L. esculentum Mill

1008. L. esculentum Mill

1009. L. esculentum Mill

1010. L. esculentum Mill

1011. L. esculentum Mill

1012. L. esculentum Mili

1013. L. esculentum Mill Lotharingii".

1014. L. esculentum Mill "Duga".

1015. L. esculentum Mill

1016. L. esculentum Mill

1017. L. esculentum Mill

1018. L. esculentum Mill

1019. L. esculentum Mill

1020. L. esculentum Mill

1021. L. esculentum Mill

1022. L. esculentum Mill

1023. L. esculentum Mill

1024. L. esculentum Mill

1025. L. esculentum Mill

1026. L. esculentum Mill

1027. L. esculentum Mill

"Radio".

"Oktiabrjenok".

"Alakrit".

"Persikovij".

"Marzak".

"Budennovka".

"Sokrais".

"Santa Rosa“.

"Columbia".

"König Humbert".

"Geterozigotnij".

"Bonny Best".

"Boldouin ${ }^{\alpha}$. 
1028. Lycopersicum esculentum Mill "Bison". 1029. L. esculentum Mill "Brek-o-deij“. 1030. L. esculentum Mill "John Bear". 1031. L. esculentum Mill "Sparks Erliana" 1032. L. esculentum Mill „Vishnevovidnij“. krasnij “.

1033. L. esculentum Mill "Petscherskij". 1034. L. esculentum Mill "Marglob". 1035. L. esculentum Mill "Northon" 1036. L. esculentum Mill "Izobilije“. 1037. L. esculentum Mill "Datskij Export". 1038. L. esculentum Mill "Pioner" 1039. L. escuelntum Mill "Akme“. 1040. L. esculentum Mill "Abydice". 1041. L. esculentuu Mill "Karlikovij ston" 1042. L. esculentum Mill "Castellato-Prima". 1043. L. esculentum Mill "Ponderosa". 1044. L. esculentum Mill "Italjanskij “. 1045. L. esculentum Mill "Pobeditel Severa“. 1046. L. esculentum Mill "Gudson-Willy". 


\section{Prof. Dr. S. S. Stankov \\ Horti Director.}

Cand. M. L. Stelmachovicz

Hortulanus doctus.

Docent Cand. D. S. Averkiev (System.).

Cand. M. L. Stelmachovicz

(Dendrolog. et Decoran.).

Ass. A. V. Knjaseroskaja

(Medicin. et Technik. Plantae)

Ass. M. V. Alalykina (Pomolog.). 

БЕ С П Л А Т H O

\section{ПЕРЕЧЕНЬ СЕМЯН,}

\section{СОБРАННЫХ В 1943 ГОДУ}

И ПРЕДЛАГАЕМЫХ В ОБМЕН

БОТАНИЧЕСКИМ САДОМ

ГОРЬКОВСКОГО ГОСУДАРСТВЕННОГО УНИВЕРСИТЕТА

ОГИз

ГОРЬКОВСКОЕ ИЗДАТЕЛЬСТВО 1944 